\title{
Visible-Light-Induced Dearomatization of Indoles/Pyrroles with Vinylcyclopropanes: Expedient Synthesis of Structur- ally Diverse Polycyclic Indolines/Pyrrolines
}

\author{
Min Zhu, ${ }^{\dagger, \dagger}$ Xu-Lun Huang, ${ }^{\dagger, t}$ Shuo Sun, ${ }^{\dagger}$ Chao Zheng, ${ }^{*, \dagger}$ and Shu-Li You, ${ }^{* \dagger, \dagger}$ \\ $\dagger$ State Key Laboratory of Organometallic Chemistry, Center for Excellence in Molec- \\ ular Synthesis, Shanghai Institute of Organic Chemistry, University of Chinese Acad- \\ emy of Sciences, Chinese Academy of Sciences, 345 Lingling Lu, Shanghai 200032, \\ China \\ ${ }^{\ddagger}$ School of Physical Science and Technology, ShanghaiTech University, 100 Haike \\ Road, Shanghai 201210, China \\ Email: zhengchao@sioc.ac.cn and slyou@sioc.ac.cn
}

\section{Table of Contents}

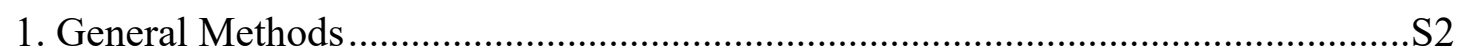

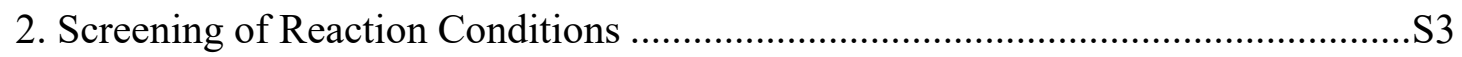

3. General Procedures and Characterization Data ...................................................S4

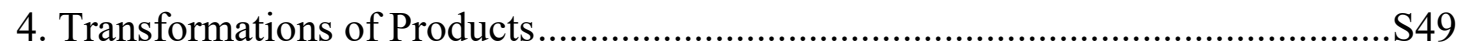

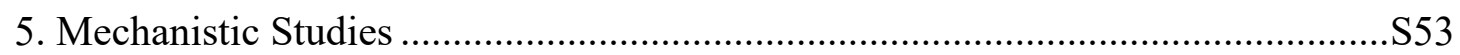

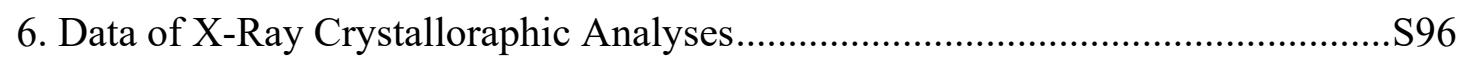

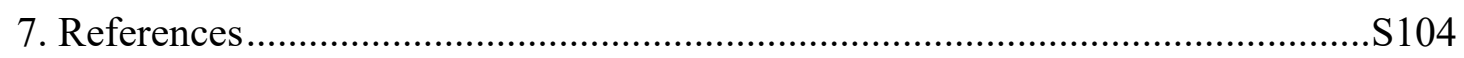

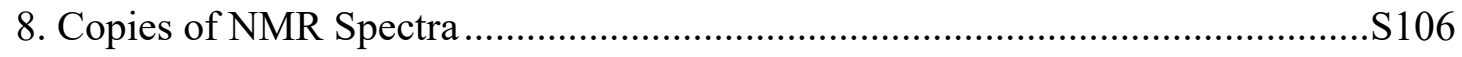




\section{General Methods}

Unless stated otherwise, all reactions were carried out in flame-dried glassware under a dry argon atmosphere. All solvents were purified and dried according to standard methods prior to use.

${ }^{1} \mathrm{H}$ and ${ }^{13} \mathrm{C}$ NMR spectra were recorded on an Agilent instrument $(400 \mathrm{MHz}$ and 100 $\mathrm{MHz}$, respectively) or an Agilent instrument (600 MHz and $150 \mathrm{MHz}$, respectively) and internally referenced to tetramethylsilane signal or residual protio solvent signals. ${ }^{19} \mathrm{~F}$ NMR spectra were recorded on an Agilent instrument $(376 \mathrm{MHz})$ and referenced relative to $\mathrm{CFCl}_{3}$. Data for ${ }^{1} \mathrm{H}$ NMR are recorded as follows: chemical shift $(\delta, \mathrm{ppm})$, multiplicity $(\mathrm{s}=$ singlet, $\mathrm{d}=$ doublet, $\mathrm{t}=$ triplet, $\mathrm{m}=$ multiplet or unresolved, $\mathrm{br}=$ broad singlet, coupling constant(s) in $\mathrm{Hz}$, integration). Data for ${ }^{13} \mathrm{C} N M R$ are reported in terms of chemical shift $(\delta, \mathrm{ppm})$.

Substrates 1, 3, 5 and 7 were synthesized according to the literature procedures. ${ }^{1}$ Reagents were purchased from Alfa Aesar, and used without further purification.

Blue LEDs $\left(24 \mathrm{~W}, \lambda_{\max }=455 \mathrm{~nm}\right)$ were used for light irradiation.

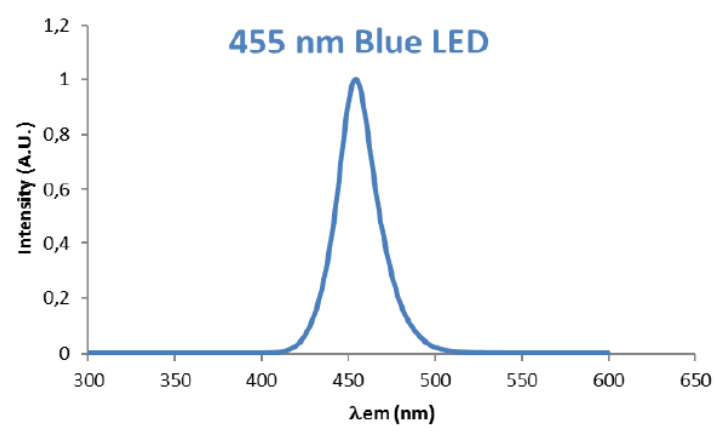




\section{Screening of Reaction Conditions}

Table S1. The evaluation of reaction conditions for the dearomatized [5+2] cycloadditions. ${ }^{a}$<smiles>CCOC(=O)c1cc2ccccc2n1C(=O)CC/C=C/[C@@H]1CC1(COC)C(C)=O</smiles>

1a $(E / Z=5.5 / 1)$

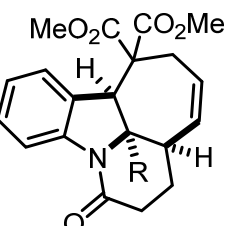

$2 \mathrm{a}\left(\mathrm{R}=\mathrm{CO}_{2} \mathrm{Et}\right)$

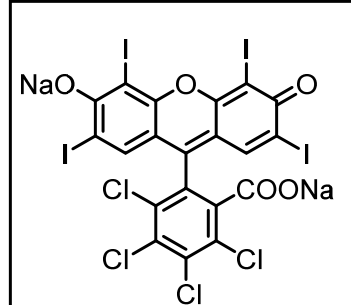

Rose Bengal (I)

$\mathrm{E}_{1 / 2}\left(\mathrm{RB}^{*} / \mathrm{RB}^{-}\right)=+0.99 \mathrm{~V}$ $\mathrm{E}_{1 / 2}\left(\mathrm{RB}^{+} / \mathrm{RB}^{*}\right)=-0.68 \mathrm{~V}$ $\Delta G\left(\mathrm{~T}_{1}-\mathrm{S}_{0}\right)=40.9 \mathrm{kcal} / \mathrm{mol}$

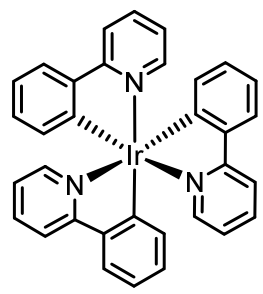

$$
\operatorname{Ir}(\text { ppy })_{3}(\mathbf{I V})
$$

$\mathrm{E}_{1 / 2}\left(\mathrm{Ir}^{\mathrm{III}} / \mathrm{Ir}^{\mathrm{II}}\right)=+0.31 \mathrm{~V}$

$\mathrm{E}_{1 / 2}\left(\mathrm{Ir}^{\mathrm{IV}} / \mathrm{Ir}^{\mathrm{III}}\right)=-1.73 \mathrm{~V}$ $\Delta G\left(\mathrm{~T}_{1}-\mathrm{S}_{0}\right)=57.8 \mathrm{kcal} / \mathrm{mol}$

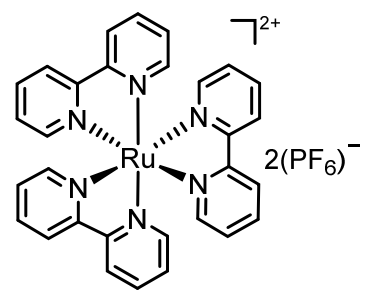

$\mathrm{Ru}(\mathrm{bpy})_{3}\left(\mathrm{PF}_{6}\right)_{2}$ (II) $\mathrm{E}_{1 / 2}\left(\mathrm{Ru}^{\mathrm{II}} / \mathrm{Ru} \mathrm{u}^{\mathrm{I}}\right)=+0.77 \mathrm{~V}$ $\mathrm{E}_{1 / 2}\left(\mathrm{Ru}^{\mathrm{III}} / \mathrm{Ru}^{\mathrm{II}}\right)=-0.81 \mathrm{~V}$ $\Delta G\left(\mathrm{~T}_{1}-\mathrm{S}_{0}\right)=46.5 \mathrm{kcal} / \mathrm{mol}$

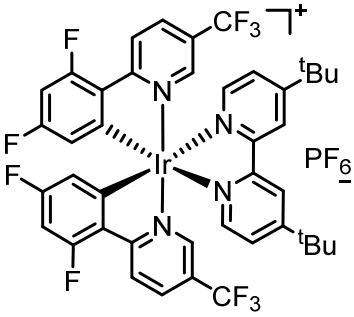

$\operatorname{Ir}\left(\mathrm{dFCF}_{3} \text { ppy }\right)_{2}(\mathrm{dtbbpy}) \mathrm{PF}_{6}(\mathbf{V})$ $\mathrm{E}_{1 / 2}\left(\mathrm{Ir}^{\mathrm{III}} / / \mathrm{Ir}^{\mathrm{II}}\right)=+1.21 \mathrm{~V}$ $\mathrm{E}_{1 / 2}\left(\mathrm{Ir}^{\mathrm{IV}} / \mathrm{Ir}^{\mathrm{III*}}\right)=-0.89 \mathrm{~V}$ $\Delta G\left(\mathrm{~T}_{1}-\mathrm{S}_{0}\right)=60.8 \mathrm{kcal} / \mathrm{mol}$

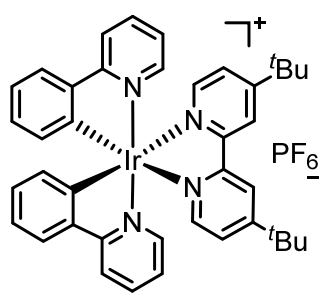

$\operatorname{Ir}(\text { ppy })_{2}($ dtbbpy $) \mathrm{PF}_{6}($ (III) $\mathrm{E}_{1 / 2}\left(\mathrm{Ir}^{\mathrm{III}} / \mathrm{Ir}^{\mathrm{II}}\right)=+0.66 \mathrm{~V}$ $\mathrm{E}_{1 / 2}\left(\mathrm{Ir}^{\mathrm{IV}} / \mathrm{Ir}^{\mathrm{III}}\right)=-0.96 \mathrm{~V}$ $\Delta G\left(\mathrm{~T}_{1}-\mathrm{S}_{0}\right)=49.2 \mathrm{kcal} / \mathrm{mol}$<smiles></smiles>

$\operatorname{Ir}(\mathrm{dFppy})_{3}(\mathbf{V I})$

$\mathrm{E}_{1 / 2}\left(\mathrm{II}^{\mathrm{III}} / \mathrm{II}^{\mathrm{II}}\right)=+0.43 \mathrm{~V}$ $\mathrm{E}_{1 / 2}\left(\mathrm{Ir}^{\mathrm{IV}} / \mathrm{Ir}^{\mathrm{III*}}\right)=-1.39 \mathrm{~V}$ $\Delta G\left(\mathrm{~T}_{1}-\mathrm{S}_{0}\right)=63.5 \mathrm{kcal} / \mathrm{mol}$

\begin{tabular}{ccccc}
\hline entry & PS & solvent & time $(\mathrm{h})$ & yield $(\%)^{b}$ \\
\hline 1 & I & DCM & 3 & 0 \\
2 & II & DCM & 3 & 0 \\
3 & III & DCM & 3 & 15 \\
4 & IV & DCM & 3 & 21 \\
5 & $\mathbf{V}$ & DCM & 3 & 75 \\
6 & $\mathbf{V}$ & DCM & 3 & 68 \\
7 & $\mathbf{V}$ & MeOH & 3 & 70 \\
8 & $\mathbf{V}$ & acetone & 3 & 71 \\
9 & $\mathbf{V}$ & $\mathrm{CH} C \mathrm{CN}$ & 3 & 84 \\
10 & $\mathbf{V}$ & DMF & 3 & 83 \\
11 & $\mathbf{V}$ & EtOAc & 3 & 67 \\
12 & $\mathbf{V}$ & DMSO & 3 & 63 \\
$\mathbf{1 3}^{c}$ & $\mathbf{V}$ & $\mathbf{C H}_{3} \mathbf{C N}$ & $\mathbf{2}$ & $\mathbf{9 1 ( 8 3 ^ { d } )}$ \\
$14^{e}$ & - & $\mathrm{CH}_{3} \mathrm{CN}$ & 24 & 0 \\
$15^{f}$ & $\mathbf{V}$ & $\mathrm{CH}_{3} \mathrm{CN}$ & 24 & 0 \\
\hline
\end{tabular}

${ }^{a}$ Reaction conditions: a solution of $1 \mathbf{a}(0.1 \mathrm{mmol})$ and PS $(1 \mathrm{~mol} \%)$ in solvent was irradiated by blue LEDs ( $24 \mathrm{~W}, \lambda_{\max }=455 \mathrm{~nm}$ ) at room temperature under argon. PS $=$ photosensitizer. ${ }^{b} \mathrm{NMR}$ 
yield using $\mathrm{C}_{2} \mathrm{H}_{2} \mathrm{Cl}_{4}$ as the internal standard. ${ }^{c} c=0.01 \mathrm{M}$. ${ }^{d}$ Isolated yield. ${ }^{e}$ Absence of a photosensitizer. ${ }^{f}$ In dark.

\section{General Procedures and Characterization Data}

\subsection{General procedure for the synthesis of the substrates}

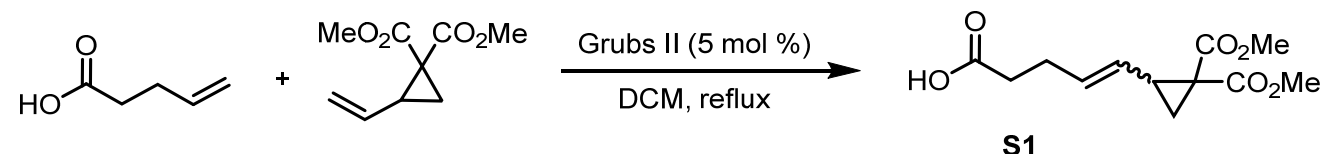

The corresponding carboxylic acids were prepared according to the previous report. ${ }^{1}$ To a solution of allylacetic acid $(1.0 \mathrm{~mL}, 10 \mathrm{mmol})$ in anhydrous DCM $(30 \mathrm{~mL})$ were added substituted vinylcyclopropanes $(1.9 \mathrm{~mL}, 12 \mathrm{mmol})$ and Grubbs II catalyst (300 $\mathrm{mg}, 0.5 \mathrm{mmol}$ ) under argon. Then, the mixture was refluxed for $6 \mathrm{~h}$ at $60{ }^{\circ} \mathrm{C}$. After the reaction was complete (monitored by TLC), it was quenched with water. The aqueous layer was extracted with EtOAc $(30 \mathrm{~mL} \times 3)$. The combined organic layers were washed with brine, dried over $\mathrm{Na}_{2} \mathrm{SO}_{4}$, filtered and concentrated by rotary evaporation. Then the residue was purified by silica gel column chromatography $(\mathrm{PE} / \mathrm{EA}=1 / 1)$ to afford the desired product $\mathbf{S 1}(1.6 \mathrm{~g}, 62 \%$ yield).

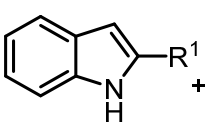

A

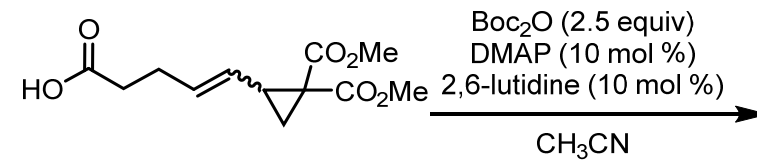

S1

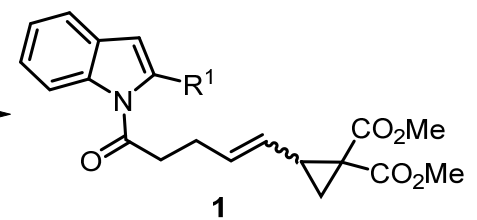

In a round bottom flask, indole derivative $\mathbf{A}(11 \mathrm{mmol})$ was dissolved in reagent grade $\mathrm{CH}_{3} \mathrm{CN}(30.0 \mathrm{~mL})$. After the addition of substituted carboxylic acid $\mathbf{S 1}$ (1.4 g, 5.5 mmol), DMAP (60 mg, $0.5 \mathrm{mmol}), 2,6$-lutidine (0.06 mL, $0.5 \mathrm{mmol})$ and $\mathrm{Boc}_{2} \mathrm{O}(3.2$ $\mathrm{mL}, 13.8 \mathrm{mmol}$ ) were added in sequence. After the reaction was complete (monitored by TLC), it was quenched with water. The aqueous layer was extracted with EtOAc (30 $\mathrm{mL} \times 3$ ). The combined organic layers were washed with brine, dried over $\mathrm{Na}_{2} \mathrm{SO}_{4}$, filtered and concentrated by rotary evaporation. Then the residue was purified by silica gel column chromatography $(\mathrm{PE} / \mathrm{EA}=20 / 1)$ to afford the desired substrates $\mathbf{1}, \mathbf{3}, \mathbf{5}$ or 7 as mixtures of $E / Z$ isomers. ${ }^{2}$ The analytical data of the products are summarized below, and the peaks of minor isomers are indicated by an asterisk $(*)$. 


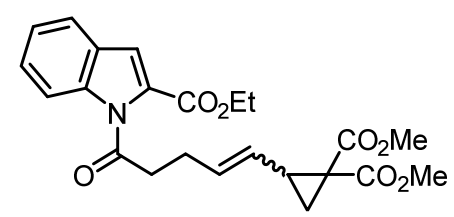

1a, yellow oil, $2.0 \mathrm{~g}, 86 \%$ yield (5.5 mmol scale), $E / Z=5.5 / 1 .{ }^{1} \mathrm{H}$ NMR (400 MHz, $\left.\mathrm{CDCl}_{3}\right) \delta 8.04-8.00(\mathrm{~m}, 1 \mathrm{H}), 7.60(\mathrm{~d}, J=7.6 \mathrm{~Hz}, 1 \mathrm{H}), 7.41(\mathrm{t}, J=7.6 \mathrm{~Hz}, 1 \mathrm{H}), 7.31(\mathrm{~s}$, $1 \mathrm{H}), 7.25(\mathrm{t}, J=7.6 \mathrm{~Hz}, 1 \mathrm{H}), 5.74-5.66(\mathrm{~m}, 1 \mathrm{H}), 5.58-5.52\left(\mathrm{~m}, 1 \mathrm{H}^{*}\right), 5.10-5.04(\mathrm{~m}, 1 \mathrm{H})$, 4.94-4.89 (m, 1H*), 4.39 (q, $J=7.2 \mathrm{~Hz}, 2 \mathrm{H}), 3.72\left(\mathrm{~s}, 6 \mathrm{H}^{*}\right), 3.70(\mathrm{~s}, 6 \mathrm{H}), 3.00-2.89$ (m, 2H), 2.78-2.42 (m, 3H), 1.63-1.55 (m, 1H), 1.50-1.46 (m, 1H), $1.40(\mathrm{t}, J=7.2 \mathrm{~Hz}, 3 \mathrm{H})$. ${ }^{13} \mathrm{C}$ NMR $\left(100 \mathrm{MHz}, \mathrm{CDCl}_{3}\right) \delta 173.4,169.7,167.6,167.5,161.4,138.0,132.4,132.1$, $129.2,129.1,127.5,126.8,125.9,123.3,122.2,117.6,114.5,61.4,52.42,52.35,52.2$, $38.9,35.3,35.2,30.5,28.1,26.2,23.6,21.5,20.4,14.0$. IR (thin film): $v_{\max }\left(\mathrm{cm}^{-1}\right)=$ 2983, 2953, 1713, 1607, 1533, 1474, 1437, 1395, 1375, 1276, 1196, 1146, 1124, 1035, 1014, 965, 898, 863, 746, 704, 437. HRMS (ESI) calcd for $\mathrm{C}_{23} \mathrm{H}_{25} \mathrm{NNaO}_{7}[\mathrm{M}+\mathrm{Na}]^{+}$: 450.1523. Found: 450.1531 .

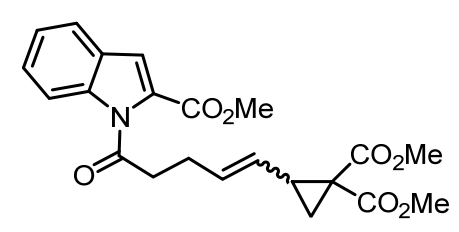

1b, yellow oil, $0.85 \mathrm{~g}, 85 \%$ yield (2.5 mmol scale), $E / Z=5.3 / 1 .{ }^{1} \mathrm{H}$ NMR (400 MHz, $\left.\mathrm{CDCl}_{3}\right) \delta 8.01-7.97(\mathrm{~m}, 1 \mathrm{H}), 7.58(\mathrm{~d}, J=7.6 \mathrm{~Hz}, 1 \mathrm{H}), 7.41-7.37(\mathrm{~m}, 1 \mathrm{H}), 7.28(\mathrm{~s}, 1 \mathrm{H})$, $7.23(\mathrm{t}, J=7.6 \mathrm{~Hz}, 1 \mathrm{H}), 5.71-5.64(\mathrm{~m}, 1 \mathrm{H}), 5.57-5.49\left(\mathrm{~m}, 1 \mathrm{H}^{*}\right), 5.08-5.02(\mathrm{~m}, 1 \mathrm{H})$, 4.94-4.86 (m, 1H*), $3.89(\mathrm{~s}, 3 \mathrm{H}), 3.86\left(\mathrm{~s}, 3 \mathrm{H}^{*}\right), 3.70\left(\mathrm{~s}, 6 \mathrm{H}^{*}\right), 3.67(\mathrm{~s}, 6 \mathrm{H}), 2.96-2.85$ (m, 2H), 2.72-2.39 (m, 3H), 1.59-1.44 (m, 2H). ${ }^{13} \mathrm{C}$ NMR (100 MHz, $\left.\mathrm{CDCl}_{3}\right) \delta 173.4$, 169.7, 167.5, 161.9, 138.0, 132.4, 132.1, 128.7, 127.6, 126.9, 126.0, 123.4, 122.3, 117.9, 114.6, 52.4, 52.32, 52.26, 38.9, 35.4, 35.3, 30.5, 28.1, 27.3, 27.2, 26.3, 23.6, 21.5, 20.4. IR (thin film): $v_{\max }\left(\mathrm{cm}^{-1}\right)=3001,2952,1715,1607,1531,1475,1434,1373,1313$, 1275, 1203, 1146, 1124, 1035, 1009, 964, 900, 867, 839, 746, 704. HRMS (ESI) calcd for $\mathrm{C}_{22} \mathrm{H}_{23} \mathrm{NNaO}_{7}[\mathrm{M}+\mathrm{Na}]^{+}: 436.1367$. Found: 436.1373 . 


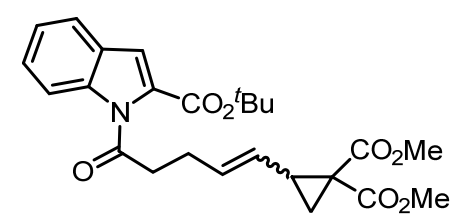

1c, yellow oil, $0.98 \mathrm{~g}, 75 \%$ yield (2.7 mmol scale), $E / Z=5.9 / 1 .{ }^{1} \mathrm{H}$ NMR (400 MHz, $\left.\mathrm{CDCl}_{3}\right) \delta 8.00-7.95(\mathrm{~m}, 1 \mathrm{H}), 7.62-7.50(\mathrm{~m}, 1 \mathrm{H}), 7.39-7.33(\mathrm{~m}, 1 \mathrm{H}), 7.21-7.12(\mathrm{~m}, 2 \mathrm{H})$,

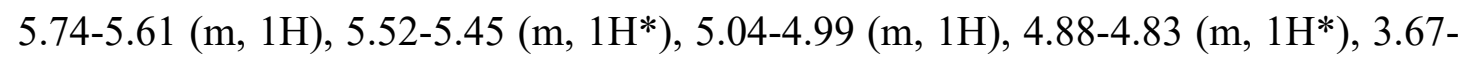
$3.65(\mathrm{~m}, 6 \mathrm{H}), 2.99-2.84(\mathrm{~m}, 2 \mathrm{H}), 2.70-2.36(\mathrm{~m}, 3 \mathrm{H}), 1.55(\mathrm{~s}, 9 \mathrm{H}), 1.52-1.48(\mathrm{~m}, 1 \mathrm{H})$, 1.44-1.41 (m, 1H). ${ }^{13} \mathrm{C}$ NMR $\left(100 \mathrm{MHz}, \mathrm{CDCl}_{3}\right) \delta 173.5,169.8,167.5,160.8,137.9$, 132.5, 132.1, 131.0, 130.7, 127.3, 127.0, 126.4, 126.0, 123.3, 122.1, 121.4, 116.9, 114.6, 114.5, 112.1, 82.4, 52.4, 52.3, 39.1, 35.3, 30.6, 28.3, 27.9, 27.3, 27.2, 26.3, 23.7, 21.6, 20.5. IR (thin film): $v_{\max }\left(\mathrm{cm}^{-1}\right)=2978,2952,1709,1536,1475,1437,1369,1313$, 1276, 1211, 1158, 1143, 1125, 1025, 1001, 964, 899, 845, 746, 705, 463. HRMS (ESI) calcd for $\mathrm{C}_{25} \mathrm{H}_{29} \mathrm{NNaO}_{7}[\mathrm{M}+\mathrm{Na}]^{+}: 478.1836$. Found: 478.1838 .

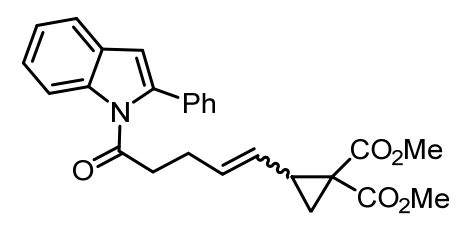

1d, yellow oil, $1.00 \mathrm{~g}, 76 \%$ yield (3 mmol scale), $E / Z=7.4 / 1 .{ }^{1} \mathrm{H}$ NMR (400 MHz, $\left.\mathrm{CDCl}_{3}\right) \delta 8.21-8.17(\mathrm{~m}, 1 \mathrm{H}), 7.47(\mathrm{~d}, J=7.6 \mathrm{~Hz}, 1 \mathrm{H}), 7.37-7.32(\mathrm{~m}, 5 \mathrm{H}), 7.28-7.24(\mathrm{~m}$, $1 \mathrm{H}), 7.19(\mathrm{t}, J=7.2 \mathrm{~Hz}, 1 \mathrm{H}), 6.55(\mathrm{~s}, 1 \mathrm{H}), 5.41-5.34(\mathrm{~m}, 1 \mathrm{H}), 5.22-5.16\left(\mathrm{~m}, 1 \mathrm{H}^{*}\right), 4.81-$ $4.68(\mathrm{~m}, 1 \mathrm{H}), 3.65-3.57(\mathrm{~m}, 6 \mathrm{H}), 2.33-2.16(\mathrm{~m}, 5 \mathrm{H}), 1.47-1.36(\mathrm{~m}, 2 \mathrm{H}) .{ }^{13} \mathrm{C}$ NMR $(100$ $\left.\mathrm{MHz}, \mathrm{CDCl}_{3}\right) \delta 173.9,170.0,167.7,139.3,137.6,134.0,132.5,128.9,128.8,128.6$, $128.5,125.9,124.9,123.5,120.4,115.4,111.3,52.6,52.4,39.2,39.1,35.4,30.7,28.0$, 26.4, 23.5, 21.7, 20.6. IR (thin film): $v_{\max }\left(\mathrm{cm}^{-1}\right)=3001,2951,1721,1603,1491,1470$, 1451, 1436, 1367, 1316, 1270, 1208, 1165, 1125, 1074, 957, 899, 864, 815, 748, 699. HRMS (ESI) calcd for $\mathrm{C}_{26} \mathrm{H}_{25} \mathrm{NNaO}_{5}[\mathrm{M}+\mathrm{Na}]^{+}$: 454.1625. Found: 454.1633.

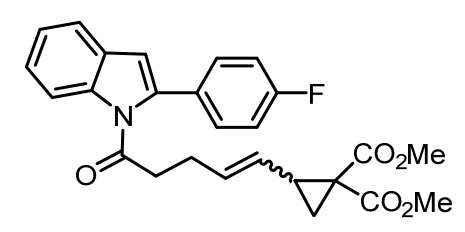

1e, colorless oil, $0.80 \mathrm{~g}, 61 \%$ yield (2.9 mmol scale), $E / Z=9 / 1 .{ }^{1} \mathrm{H}$ NMR (400 MHz, $\left.\mathrm{CDCl}_{3}\right) \delta 8.26-8.21(\mathrm{~m}, 1 \mathrm{H}), 7.51(\mathrm{~d}, J=7.6 \mathrm{~Hz}, 1 \mathrm{H}), 7.41-7.38(\mathrm{~m}, 2 \mathrm{H}), 7.33-7.29(\mathrm{~m}$, 
1H), 7.24-7.22 (m, 1H), $7.13(\mathrm{t}, J=8.8 \mathrm{~Hz}, 2 \mathrm{H}), 6.57(\mathrm{~s}, 1 \mathrm{H}), 5.52-5.45(\mathrm{~m}, 1 \mathrm{H}), 5.33-$ $5.27\left(\mathrm{~m}, 1 \mathrm{H}^{*}\right), 4.92-4.78(\mathrm{~m}, 1 \mathrm{H}), 3.71-3.64(\mathrm{~m}, 6 \mathrm{H}), 2.42-2.24(\mathrm{~m}, 5 \mathrm{H}), 1.53-1.44(\mathrm{~m}$, $2 \mathrm{H}) .{ }^{13} \mathrm{C} \mathrm{NMR}\left(100 \mathrm{MHz}, \mathrm{CDCl}_{3}\right) \delta 173.3,169.8,167.5,162.6(\mathrm{~d}, J=247.0 \mathrm{~Hz}), 138.0$, $137.3,132.3,131.9,130.2$ (d, $J=8.0 \mathrm{~Hz}), 130.0(\mathrm{~d}, J=4.0 \mathrm{~Hz}), 128.7,125.9,124.9$, 123.4, 120.3, 115.7 (d, $J=22.0 \mathrm{~Hz}), 115.4,115.3,111.4,52.4,52.2,39.1,38.9,35.3$, 30.5, 27.7, 27.2, 23.3, 21.5, 20.5. ${ }^{19} \mathrm{~F}$ NMR (376 MHz, $\left.\mathrm{CDCl}_{3}\right) \delta-112.2 \sim-112.3(\mathrm{~m}$, 1F). IR (thin film): $v_{\max }\left(\mathrm{cm}^{-1}\right)=2952,1721,1593,1561,1501,1449,1436,1367,1320$, 1270, 1209, 1157, 1126, 1015, 957, 897, 842, 814, 796, 749, 678. HRMS (ESI) calcd for $\mathrm{C}_{26} \mathrm{H}_{24} \mathrm{NNaO}_{5} \mathrm{~F}[\mathrm{M}+\mathrm{Na}]^{+}$: 472.1531. Found: 472.1542 .

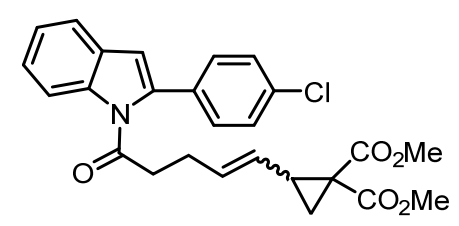

1f, brown oil, $0.80 \mathrm{~g}, 61 \%$ yield $(2.7 \mathrm{mmol} \mathrm{scale}), E / Z=6.3 / 1 .{ }^{1} \mathrm{H}$ NMR (400 MHz, $\left.\mathrm{CDCl}_{3}\right) \delta 8.23-8.19(\mathrm{~m}, 1 \mathrm{H}), 7.54(\mathrm{~d}, J=7.6 \mathrm{~Hz}, 1 \mathrm{H}), 7.43-7.31(\mathrm{~m}, 5 \mathrm{H}), 7.28-7.24(\mathrm{~m}$, $1 \mathrm{H}), 6.61(\mathrm{~s}, 1 \mathrm{H}), 5.52-5.45(\mathrm{~m}, 1 \mathrm{H}), 5.34-5.30\left(\mathrm{~m}, 1 \mathrm{H}^{*}\right), 4.92-4.78(\mathrm{~m}, 1 \mathrm{H}), 3.72-3.64$ $(\mathrm{m}, 6 \mathrm{H}), 2.45-2.19(\mathrm{~m}, 5 \mathrm{H}), 1.55-1.50(\mathrm{~m}, 1 \mathrm{H}), 1.48-1.44(\mathrm{~m}, 1 \mathrm{H}) .{ }^{13} \mathrm{C}$ NMR $(100 \mathrm{MHz}$, $\left.\mathrm{CDCl}_{3}\right) \delta 173.5,169.9,167.7,138.0,137.6,134.5,132.5,132.4,132.0,129.7,129.0$, 128.8, 126.1, 125.2, 123.6, 120.5, 115.4, 111.8, 52.6, 52.4, 39.2, 35.4, 30.7, 28.0, 27.9, 26.4, 23.5, 21.7, 20.7. IR (thin film): $v_{\max }\left(\mathrm{cm}^{-1}\right)=2951,1721,1487,1435,1402,1366$, 1317, 1274, 1208, 1167, 1126, 1090, 1013, 959, 898, 836, 812, 749, 718, 674, 667. HRMS (ESI) calcd for $\mathrm{C}_{26} \mathrm{H}_{24} \mathrm{NNaO}_{5} \mathrm{Cl}[\mathrm{M}+\mathrm{Na}]^{+}: 488.1235$. Found: 488.1246 .

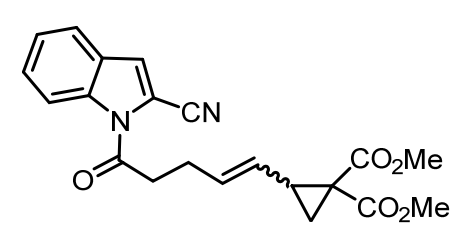

1g, colorless oil, $0.30 \mathrm{~g}, 25 \%$ yield (3.3 mmol scale), $E / Z=3.7 / 1 .{ }^{1} \mathrm{H}$ NMR (400 MHz, $\left.\mathrm{CDCl}_{3}\right) \delta 8.24-8.21(\mathrm{~m}, 1 \mathrm{H}), 7.58(\mathrm{~d}, J=8.0 \mathrm{~Hz}, 1 \mathrm{H}), 7.49-7.45(\mathrm{~m}, 1 \mathrm{H}), 7.36(\mathrm{~s}, 1 \mathrm{H})$, $7.31(\mathrm{t}, J=7.6 \mathrm{~Hz}, 1 \mathrm{H}), 5.84-5.77(\mathrm{~m}, 1 \mathrm{H}), 5.69-5.62\left(\mathrm{~m}, 1 \mathrm{H}^{*}\right), 5.21-5.15(\mathrm{~m}, 1 \mathrm{H})$, 5.00-4.95 (m, 1H*), 3.69-3.66 (m, 6H), 3.23-3.13 (m, 2H), 2.79-2.48 (m, 3H), 1.68$1.50(\mathrm{~m}, 2 \mathrm{H}) .{ }^{13} \mathrm{C} \mathrm{NMR}\left(100 \mathrm{MHz}, \mathrm{CDCl}_{3}\right) \delta 170.1,169.7,167.5,136.5,132.1,131.9$, $128.7,127.1,126.5,126.4,124.5,123.5,122.0,116.5,113.7,107.4,52.5,52.43,52.36$, 
36.9, 35.4, 35.3, 30.6, 27.0, 26.2, 22.6, 21.6, 20.5. IR (thin film): $v_{\max }\left(\mathrm{cm}^{-1}\right)=2953$, 2223, 1716, 1608, 1533, 1437, 1374, 1320, 1276, 1209, 1172, 1126, 1036, 1006, 965 , 899, 870, 830, 750, 704, 621, 416. HRMS (ESI) calcd for $\mathrm{C}_{21} \mathrm{H}_{20} \mathrm{~N}_{2} \mathrm{NaO}_{5}[\mathrm{M}+\mathrm{Na}]^{+}$: 403.1264. Found: 403.1276.

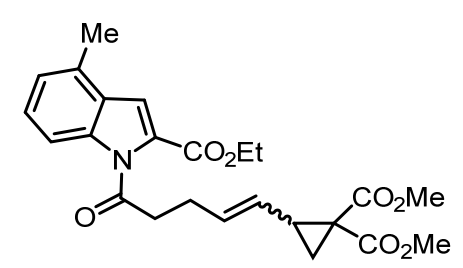

1h, yellow oil, $0.93 \mathrm{~g}, 84 \%$ yield (2.5 mmol scale), $E / Z=6 / 1 .{ }^{1} \mathrm{H}$ NMR (400 MHz, $\left.\mathrm{CDCl}_{3}\right) \delta$ 7.82-7.77 (m, 1H), $7.34(\mathrm{~s}, 1 \mathrm{H}), 7.27(\mathrm{t}, J=8.0 \mathrm{~Hz}, 1 \mathrm{H}), 7.01$ (d, $J=7.2 \mathrm{~Hz}$, $1 \mathrm{H})$, 5.69-5.62 (m, 1H), 5.54-5.47 (m, 1H*), 5.05-4.99 (m, 1H), 4.89-4.84 (m, 1H*), $4.36(\mathrm{q}, J=7.2 \mathrm{~Hz}, 2 \mathrm{H}), 3.69\left(\mathrm{~s}, 6 \mathrm{H}^{*}\right), 3.67$ (s, 6H), 2.95-2.84 (m, 2H), 2.49 (s, 3H), 2.46-2.38 (m, 3H), 1.57-1.44 (m, 2H), $1.38(\mathrm{t}, J=7.2 \mathrm{~Hz}, 3 \mathrm{H}) .{ }^{13} \mathrm{C}$ NMR (100 MHz, $\left.\mathrm{CDCl}_{3}\right) \delta 173.6,169.9,167.6,161.6,138.0,132.5,132.2,132.0,128.6,127.8,126.8$, $126.0,123.7,116.3,112.1,61.5,52.5,52.4,39.1,35.3,30.7,28.3,26.4,23.7,21.6,20.5$, 18.2, 14.1. IR (thin film): $v_{\max }\left(\mathrm{cm}^{-1}\right)=2983,2952,1713,1598,1531,1487,1436,1416$, 1376, 1329, 1280, 1249, 1192, 1154, 1124, 1032, 986, 963, 899, 871, 841, 780. HRMS (ESI) calcd for $\mathrm{C}_{24} \mathrm{H}_{27} \mathrm{NNaO}_{7}[\mathrm{M}+\mathrm{Na}]^{+}: 464.1680$. Found: 464.1673.

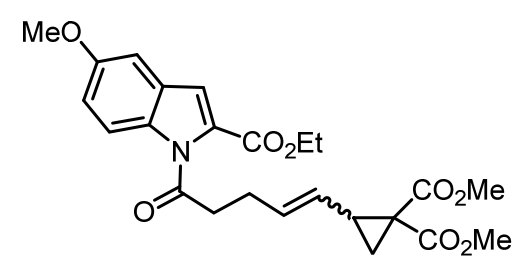

1i, yellow oil, $0.88 \mathrm{~g}, 80 \%$ yield (2.4 mmol scale), $E / Z=5.4 / 1 .{ }^{1} \mathrm{H}$ NMR (400 MHz, $\left.\mathrm{CDCl}_{3}\right) \delta$ 7.96-7.92 (m, 1H), 7.22 (s, 1H), 7.04-7.00 (m, 2H), 5.72-5.64 (m, 1H), 5.55$5.49\left(\mathrm{~m}, 1 \mathrm{H}^{*}\right), 5.08-5.02(\mathrm{~m}, 1 \mathrm{H}), 4.91-4.86\left(\mathrm{~m}, 1 \mathrm{H}^{*}\right), 4.37$ (q, J=7.2 Hz, 2H), 3.81 (s, 3H), $3.72\left(\mathrm{~s}, 6 \mathrm{H}^{*}\right), 3.69$ (s, 6H), 2.96-2.86 (m, 2H), 2.75-2.40 (m, 3H), 1.59-1.55 (m, 1H), 1.49-1.46 (m, 1H), $1.38(\mathrm{t}, J=7.2 \mathrm{~Hz}, 3 \mathrm{H}) .{ }^{13} \mathrm{C} \mathrm{NMR}\left(100 \mathrm{MHz}, \mathrm{CDCl}_{3}\right) \delta$ 173.2, 169.9, 167.6, 161.6, 156.3, 133.2, 132.6, 132.3, 129.7, 127.7, 126.0, 117.6, 117.4, $115.8,103.5,61.5,55.5,52.5,52.4,38.8,35.4,30.7,28.3,26.4,23.7,21.7,20.6,14.1$. IR (thin film): $v_{\max }\left(\mathrm{cm}^{-1}\right)=2953,1711,1614,1536,1465,1435,1375,1280,1258$, 
1213, 1191, 1163, 1126, 1107, 1031, 988, 965, 946, 899, 857, 814, 761. HRMS (ESI) calcd for $\mathrm{C}_{24} \mathrm{H}_{27} \mathrm{NNaO} 8[\mathrm{M}+\mathrm{Na}]^{+}: 480.1629$. Found: 480.1634 .

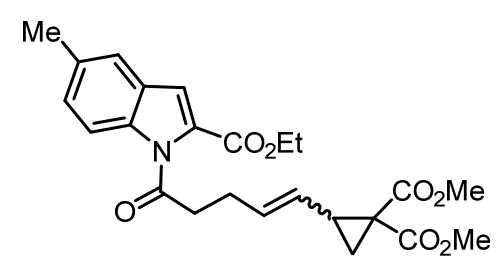

1j, yellow oil, $0.94 \mathrm{~g}, 71 \%$ yield (2.9 mmol scale), $E / Z=5.7 / 1 .{ }^{1} \mathrm{H}$ NMR (400 MHz, $\left.\mathrm{CDCl}_{3}\right) \delta 7.92-7.88(\mathrm{~m}, 1 \mathrm{H}), 7.36(\mathrm{~s}, 1 \mathrm{H}), 7.22-7.17(\mathrm{~m}, 2 \mathrm{H}), 5.72-5.65(\mathrm{~m}, 1 \mathrm{H})$, 5.56$5.49\left(\mathrm{~m}, 1 \mathrm{H}^{*}\right), 5.08-5.02(\mathrm{~m}, 1 \mathrm{H}), 4.91-4.86\left(\mathrm{~m}, 1 \mathrm{H}^{*}\right), 4.37$ (q, $\left.J=7.2 \mathrm{~Hz}, 2 \mathrm{H}\right), 3.71$ (s, 6H*), $3.69(\mathrm{~s}, 6 \mathrm{H}), 2.97-2.86(\mathrm{~m}, 2 \mathrm{H}), 2.73-2.42(\mathrm{~m}, 3 \mathrm{H}), 2.40(\mathrm{~s}, 3 \mathrm{H}), 1.59-1.54$ (m, 1H), 1.49-1.45 (m, 1H), $1.38(\mathrm{t}, J=7.2 \mathrm{~Hz}, 3 \mathrm{H}) .{ }^{13} \mathrm{C} \mathrm{NMR}\left(100 \mathrm{MHz}, \mathrm{CDCl}_{3}\right) \delta$ 173.3, 169.8, 167.6, 161.6, 136.5, 133.0, 132.6, 132.3, 129.3, 129.2, 127.2, 126.0, 121.9, $117.6,114.4,61.4,52.5,52.3,38.8,35.3,30.6,28.2,26.3,23.7,21.6,21.0,20.5,14.1$. IR (thin film): $v_{\max }\left(\mathrm{cm}^{-1}\right)=2982,2952,1712,1539,1435,1374,1276,1202,1159$, 1126, 1033, 988, 964, 878, 808, 764, 741, 705, 666, 635, 585, 434. HRMS (ESI) calcd for $\mathrm{C}_{24} \mathrm{H}_{27} \mathrm{NNaO}_{7}[\mathrm{M}+\mathrm{Na}]^{+}: 464.1680$. Found: 464.1685 .

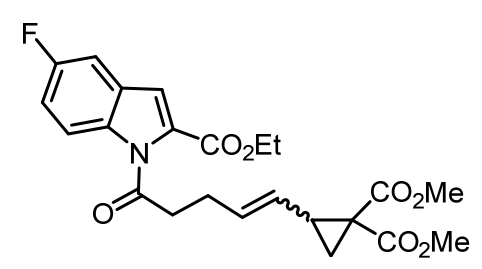

1k, yellow oil, $0.80 \mathrm{~g}$, 66\% yield (2.9 mmol scale), $E / Z=7.8 / 1 .{ }^{1} \mathrm{H}$ NMR (400 MHz, $\left.\mathrm{CDCl}_{3}\right) \delta$ 7.98-7.93 (m, 1H), 7.20-7.17 (m, 2H), 7.11-7.06 (m, 1H), 5.65-5.57 (m, 1H),

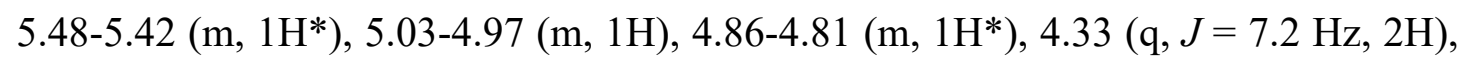
$3.67\left(\mathrm{~s}, 6 \mathrm{H}^{*}\right), 3.64(\mathrm{~s}, 6 \mathrm{H}), 2.93-2.80(\mathrm{~m}, 2 \mathrm{H}), 2.67-2.34(\mathrm{~m}, 3 \mathrm{H}), 1.54-1.49(\mathrm{~m}, 1 \mathrm{H})$, 1.44-1.41 (m, 1H), $1.34(\mathrm{t}, J=7.2 \mathrm{~Hz}, 3 \mathrm{H}) .{ }^{13} \mathrm{C} \mathrm{NMR}\left(100 \mathrm{MHz}, \mathrm{CDCl}_{3}\right) \delta 173.4,169.9$, 167.7, 161.4, 159.4 (d, $J=239.0 \mathrm{~Hz}), 134.7,132.4,130.6,127.7,127.6,126.3,117.2$ (d, $J=5.0 \mathrm{~Hz}), 116.2(\mathrm{~d}, J=9.0 \mathrm{~Hz}), 116.1,115.8,107.2$ (d, $J=23.0 \mathrm{~Hz}), 61.8,52.6$, $52.5,39.0,35.4,30.7,28.3,23.8,20.7,14.2 .{ }^{19} \mathrm{~F} \mathrm{NMR}\left(376 \mathrm{MHz}, \mathrm{CDCl}_{3}\right) \delta-119.15 \sim$ $-119.21(\mathrm{~m}, 1 \mathrm{~F})$. IR (thin film): $v_{\max }\left(\mathrm{cm}^{-1}\right)=2984,2954,1715,1617,1588,1537,1462$, 1439, 1396, 1375, 1277, 1237, 1203, 1172, 1127, 1102, 1032, 988, 956, 899, 861, 810. HRMS (ESI) calcd for $\mathrm{C}_{23} \mathrm{H}_{24} \mathrm{NFNaO}_{7}[\mathrm{M}+\mathrm{Na}]^{+}$: 468.1429. Found: 468.1432. 


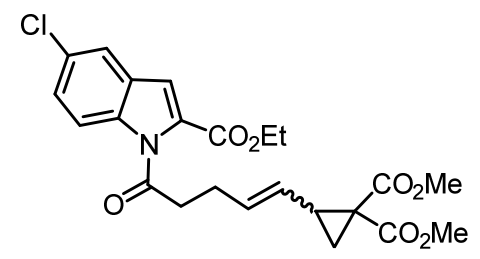

11, yellow oil, $0.74 \mathrm{~g}, 57 \%$ yield (3 mmol scale), $E / Z=5.1 / 1 .{ }^{1} \mathrm{H}$ NMR (400 MHz, $\left.\mathrm{CDCl}_{3}\right) \delta 7.91-7.86(\mathrm{~m}, 1 \mathrm{H}), 7.49(\mathrm{~d}, J=1.6 \mathrm{~Hz}, 1 \mathrm{H}), 7.29-7.26(\mathrm{~m}, 1 \mathrm{H}), 7.16(\mathrm{~s}, 1 \mathrm{H})$, 5.66-5.58 (m, 1H), 5.50-5.44 (m, 1H*), 5.04-4.98 (m, 1H), 4.88-4.83 (m, 1H*), $4.34(\mathrm{q}$, $J=7.2 \mathrm{~Hz}, 2 \mathrm{H}), 3.68$ (s, 6H*), 3.65 (s, 6H), 2.91-2.81 (m, 2H), 2.68-2.35 (m, 3H), 1.57$1.49(\mathrm{~m}, 1 \mathrm{H}), 1.45-1.41(\mathrm{~m}, 1 \mathrm{H}), 1.35(\mathrm{t}, J=7.2 \mathrm{~Hz}, 3 \mathrm{H}) .{ }^{13} \mathrm{C} \mathrm{NMR}\left(100 \mathrm{MHz}, \mathrm{CDCl}_{3}\right)$ $\delta 173.2,169.7,167.6,167.5,161.1,136.2,132.2,131.9,130.2,128.8,127.8,127.6$, $126.1,121.4,116.3,115.8,61.7,52.5,52.4,52.2,38.8,35.3,35.2,30.5,28.1,26.2,23.5$, 21.5, 20.4, 14.0. IR (thin film): $v_{\max }\left(\mathrm{cm}^{-1}\right)=2984,2953,1714,1532,1436,1395,1375$, $1274,1187,1125,1069,1031,988,965,918,898,874,810,762,737,703,658$. HRMS (ESI) calcd for $\mathrm{C}_{23} \mathrm{H}_{24} \mathrm{NClNaO}_{7}[\mathrm{M}+\mathrm{Na}]^{+}$: 484.1134. Found: 484.1122.

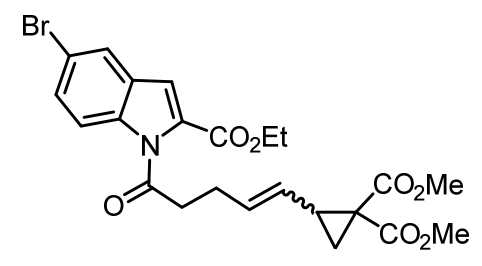

1m, yellow oil, $1.2 \mathrm{~g}, 82 \%$ yield (2.9 mmol scale), $E / Z=5.9 / 1 .{ }^{1} \mathrm{H}$ NMR (400 MHz, $\left.\mathrm{CDCl}_{3}\right) \delta$ 7.91-7.87 (m, 1H), 7.72-7.71 (m, 1H), 7.49-7.46 (m, 1H), 7.22-7.21 (m, 1H), 5.69-5.62 (m, 1H), 5.53-5.47 (m, 1H*), 5.08-5.02 (m, 1H), 4.91-4.86 (m, 1H*), $4.39(\mathrm{q}$, $J=7.2 \mathrm{~Hz}, 2 \mathrm{H}), 3.72\left(\mathrm{~s}, 6 \mathrm{H}^{*}\right), 3.69$ (s, 6H), 2.95-2.85 (m, 2H), 2.72-2.39 (m, 3H), 1.60$1.54(\mathrm{~m}, 1 \mathrm{H}), 1.49-1.46(\mathrm{~m}, 1 \mathrm{H}), 1.39(\mathrm{t}, J=7.2 \mathrm{~Hz}, 3 \mathrm{H}) .{ }^{13} \mathrm{C} \mathrm{NMR}\left(100 \mathrm{MHz}, \mathrm{CDCl}_{3}\right)$ $\delta 173.4,169.9,167.7,161.2,136.7,132.3,132.0,130.4,130.2,128.5,126.3,124.7$, 116.6, 116.4, 116.3, 61.8, 52.6, 52.4, 39.0, 35.4, 30.6, 28.3, 26.4, 23.7, 21.7, 20.6, 14.1. IR (thin film): $v_{\max }\left(\mathrm{cm}^{-1}\right)=2983,2952,1713,1530,1436,1394,1375,1270,1185$, 1125, 1058, 1029, 987, 964, 905, 874, 808, 763, 736, 704, 649. HRMS (ESI) calcd for $\mathrm{C}_{23} \mathrm{H}_{24} \mathrm{~N}^{79} \mathrm{BrNaO}_{7}[\mathrm{M}+\mathrm{Na}]^{+}: 528.0628$. Found: 528.0636 . 


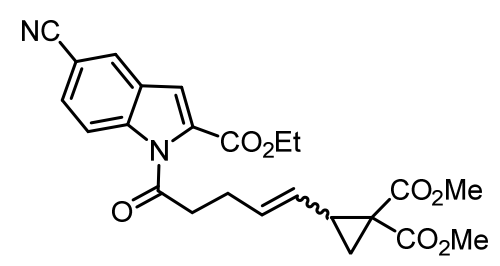

1n, yellow oil, $0.37 \mathrm{~g}, 37 \%$ yield (2.4 mmol scale), $E / Z=5.4 / 1 .{ }^{1} \mathrm{H}$ NMR (400 MHz, $\left.\mathrm{CDCl}_{3}\right) \delta$ 7.99-7.95 (m, 1H), $7.89(\mathrm{~s}, 1 \mathrm{H}), 7.54(\mathrm{dd}, J=8.8,1.2 \mathrm{~Hz}, 1 \mathrm{H}), 7.27$ (d, $J=$ $5.2 \mathrm{~Hz}, 1 \mathrm{H}), 5.63-5.55(\mathrm{~m}, 1 \mathrm{H}), 5.48-5.41\left(\mathrm{~m}, 1 \mathrm{H}^{*}\right), 5.03-4.97(\mathrm{~m}, 1 \mathrm{H}), 4.86-4.81(\mathrm{~m}$, $\left.1 \mathrm{H}^{*}\right), 4.35(\mathrm{q}, J=7.2 \mathrm{~Hz}, 2 \mathrm{H}), 3.66-3.62(\mathrm{~m}, 6 \mathrm{H}), 2.91-2.81(\mathrm{~m}, 2 \mathrm{H}), 2.65-2.32(\mathrm{~m}$, $3 \mathrm{H}), 1.53-1.46(\mathrm{~m}, 1 \mathrm{H}), 1.42-1.39(\mathrm{~m}, 1 \mathrm{H}), 1.35(\mathrm{t}, J=7.2 \mathrm{~Hz}, 3 \mathrm{H}) .{ }^{13} \mathrm{C}$ NMR $(100$ $\left.\mathrm{MHz}, \mathrm{CDCl}_{3}\right) \delta 173.3,169.6,167.4,160.7,139.1,131.8,131.5,131.0,129.7,127.3$, 126.6, 126.3, 126.2, 118.7, 116.1, 115.4, 106.7, 61.9, 52.5, 52.4, 52.2, 39.1, 35.3, 35.1, 30.3, 28.0, 27.6, 26.1, 23.4, 21.4, 20.3, 13.9. IR (thin film): $v_{\max }\left(\mathrm{cm}^{-1}\right)=2985,2954$, 2225, 1715, 1611, 1542, 1456, 1436, 1394, 1375, 1282, 1204, 1152, 1127, 1025, 987 , $965,897,862,819,765,744,704$. HRMS (ESI) calcd for $\mathrm{C}_{24} \mathrm{H}_{24} \mathrm{~N}_{2} \mathrm{NaO}_{7}[\mathrm{M}+\mathrm{Na}]^{+}$: 475.1476. Found: 475.1476.

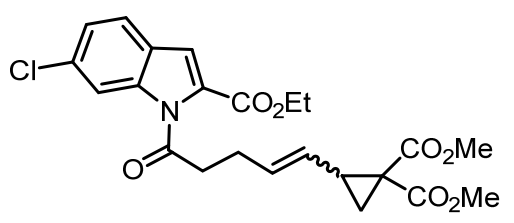

10, yellow oil, $1.30 \mathrm{~g}, 84 \%$ yield (3.3 mmol scale), $E / Z=5.6 / 1 .{ }^{1} \mathrm{H}$ NMR (400 MHz, $\left.\mathrm{CDCl}_{3}\right) \delta 8.09-8.07(\mathrm{~m}, 1 \mathrm{H}), 7.51(\mathrm{~d}, J=8.4 \mathrm{~Hz}, 1 \mathrm{H}), 7.28(\mathrm{~s}, 1 \mathrm{H}), 7.25-7.22(\mathrm{~m}, 1 \mathrm{H})$,

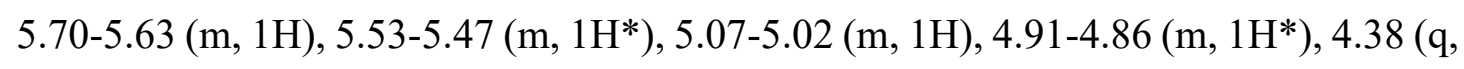
$J=7.2 \mathrm{~Hz}, 2 \mathrm{H}), 3.73-3.68$ (m, 6H), 2.98-2.85 (m, 2H), 2.62-2.40 (m, 3H), 1.59-1.54 $(\mathrm{m}, 1 \mathrm{H}), 1.50-1.46(\mathrm{~m}, 1 \mathrm{H}), 1.39(\mathrm{t}, J=7.2 \mathrm{~Hz}, 3 \mathrm{H}) .{ }^{13} \mathrm{C} \mathrm{NMR}\left(100 \mathrm{MHz}, \mathrm{CDCl}_{3}\right) \delta$ $173.5,169.8,167.6,161.2,138.4,133.7,132.3,131.9,129.7,126.3,125.3,124.3,123.0$, $117.4,114.9,61.7,52.5,52.4,39.0,35.5,35.3,30.6,28.2,26.3,23.7,21.6,20.5,14.1$. IR (thin film): $v_{\max }\left(\mathrm{cm}^{-1}\right)=2985,2953,1713,1604,1528,1436,1418,1393,1375$, 1298, 1245, 1189, 1124, 1065, 1032, 998, 965, 900, 850, 802, 762, 737, 700. HRMS (ESI) calcd for $\mathrm{C}_{23} \mathrm{H}_{24} \mathrm{NNaO}_{7} \mathrm{Cl}[\mathrm{M}+\mathrm{Na}]^{+}:$484.1134. Found: 484.1134. 


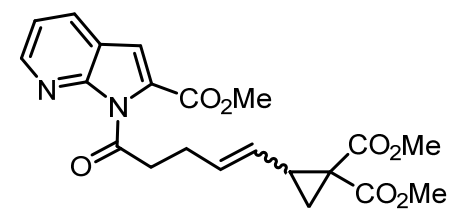

1p, colorless oil, $0.48 \mathrm{~g}, 44 \%$ yield (2.7 mmol scale), $E / Z=5 / 1 .{ }^{1} \mathrm{H}$ NMR (400 MHz, $\left.\mathrm{CDCl}_{3}\right) \delta 8.44(\mathrm{~d}, J=3.6 \mathrm{~Hz}, 1 \mathrm{H}), 7.95(\mathrm{~d}, J=7.6 \mathrm{~Hz}, 1 \mathrm{H}), 7.25-7.22(\mathrm{~m}, 1 \mathrm{H}), 7.05(\mathrm{~s}$, $1 \mathrm{H})$, 5.93-5.86 (m, 1H), 5.79-5.72 (m, $\left.1 \mathrm{H}^{*}\right), 5.26-5.20(\mathrm{~m}, 1 \mathrm{H}), 5.03-4.98\left(\mathrm{~m}, 1 \mathrm{H}^{*}\right)$, $3.93(\mathrm{~s}, 3 \mathrm{H}), 3.73(\mathrm{~s}, 6 \mathrm{H}), 3.66-3.60(\mathrm{~m}, 2 \mathrm{H}), 2.87-2.53(\mathrm{~m}, 3 \mathrm{H}), 1.73-1.56(\mathrm{~m}, 2 \mathrm{H}) .{ }^{13} \mathrm{C}$ NMR $\left(100 \mathrm{MHz}, \mathrm{CDCl}_{3}\right) \delta 171.83,171.75,169.9,167.6,162.2,148.3,146.2,146.1$, 133.0, 132.8, 130.7, 130.6, 125.7, 125.6, 120.5, 119.1, 111.5, 52.49, 52.46, 52.4, 52.3, $38.34,38.27,35.4,30.7,27.2,26.4,22.8,21.7,20.5$. IR (thin film): $v_{\max }\left(\mathrm{cm}^{-1}\right)=2952$, 1717, 1597, 1579, 1555, 1435, 1399, 1373, 1328, 1308, 1283, 1205, 1111, 966, 911, $838,793,772,752,704,668,618,524$. HRMS (ESI) calcd for $\mathrm{C}_{21} \mathrm{H}_{22} \mathrm{~N}_{2} \mathrm{NaO}_{7}[\mathrm{M}+\mathrm{Na}]^{+}$: 437.1319. Found: 437.1326 .

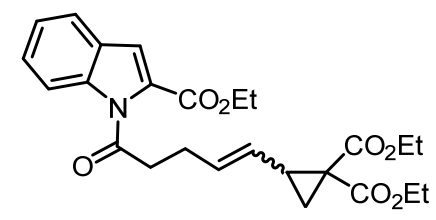

1q, yellow oil, $1.10 \mathrm{~g}, 85 \%$ yield (2.8 mmol scale), $E / Z=5.5 / 1 .{ }^{1} \mathrm{H}$ NMR $(400 \mathrm{MHz}$, $\left.\mathrm{CDCl}_{3}\right) \delta 8.05-8.00(\mathrm{~m}, 1 \mathrm{H}), 7.61(\mathrm{~d}, J=8.0 \mathrm{~Hz}, 1 \mathrm{H}), 7.41(\mathrm{t}, J=7.6 \mathrm{~Hz}, 1 \mathrm{H}), 7.32(\mathrm{~s}$, 1H), 7.27-7.24 (m, 1H), 5.72-5.65 (m, 1H), 5.58-5.49 (m, 1H*), 5.10-5.04 (m, 1H), 4.94-4.89 (m, 1H*), 4.38 (q, $J=7.2 \mathrm{~Hz}, 2 \mathrm{H}), 4.24-4.08(\mathrm{~m}, 4 \mathrm{H}), 3.03-2.88(\mathrm{~m}, 2 \mathrm{H})$, 2.74-2.39 (m, 3H), 1.57-1.51 (m, 1H), 1.46-1.41 (m, 1H), 1.27-1.19 (m, 6H). ${ }^{13} \mathrm{C}$ NMR $\left(100 \mathrm{MHz}, \mathrm{CDCl}_{3}\right) \delta 173.6,169.5,167.3,161.6,138.1,132.2,131.9,129.3,127.7$, 127.0, 126.4, 123.5, 122.3, 117.8, 114.7, 61.5, 61.4, 61.2, 39.1, 35.6, 30.2, 28.3, 27.4, 27.3, 26.0, 23.7, 21.4, 20.3, 14.1, 13.9. IR (thin film): $v_{\max }\left(\mathrm{cm}^{-1}\right)=2981,2937,1713$, 1536, 1474, 1443, 1393, 1369, 1315, 1275, 1197, 1170, 1146, 1126, 1110, 1016, 1016, 966, 861, 836, 747, 667. HRMS (ESI) calcd for $\mathrm{C}_{25} \mathrm{H}_{29} \mathrm{NNaO}_{7}[\mathrm{M}+\mathrm{Na}]^{+}$: 478.1836 . Found: 478.1843.

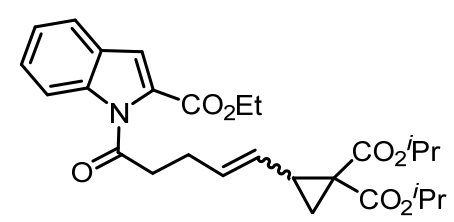


1r, yellow oil, $1.20 \mathrm{~g}, 93 \%$ yield (2.7 mmol scale), $E / Z=5.4 / 1 .{ }^{1} \mathrm{H}$ NMR (400 MHz, $\left.\mathrm{CDCl}_{3}\right) \delta 8.02-7.98(\mathrm{~m}, 1 \mathrm{H}), 7.58(\mathrm{~d}, J=8.0 \mathrm{~Hz}, 1 \mathrm{H}), 7.39(\mathrm{t}, J=8.0 \mathrm{~Hz}, 1 \mathrm{H}), 7.29(\mathrm{~s}$, $1 \mathrm{H}), 7.25-7.21(\mathrm{~m}, 1 \mathrm{H}), 5.69-5.62(\mathrm{~m}, 1 \mathrm{H}), 5.53-5.47\left(\mathrm{~m}, 1 \mathrm{H}^{*}\right), 5.06-4.86(\mathrm{~m}, 3 \mathrm{H})$, , 4.36 (q, $J=7.2 \mathrm{~Hz}, 2 \mathrm{H}), 2.99-2.86$ (m, 2H), 2.72-2.35 (m, 3H), 1.49-1.43 (m, 1H), 1.39$1.33(\mathrm{~m}, 4 \mathrm{H}), 1.23-1.12(\mathrm{~m}, 12 \mathrm{H}) .{ }^{13} \mathrm{C} \mathrm{NMR}\left(100 \mathrm{MHz}, \mathrm{CDCl}_{3}\right) \delta$ 173.6, 169.1, 166.9, $161.5,138.1,131.9,131.6,129.2,127.6,127.0,126.5,126.4,123.5,122.3,117.7,114.7$, 68.9, 68.6, 61.5, 39.1, 35.8, 29.6, 28.3, 27.3, 25.5, 23.7, 21.8, 21.7, 21.53, 21.51, 21.4, 21.1, 20.0, 14.1. IR (thin film): $v_{\max }\left(\mathrm{cm}^{-1}\right)=2980,2937,1711,1533,1468,1443,1373$, 1337, 1311, 1276, 1198, 1145, 1097, 1036, 1015, 987, 966, 918, 900, 864, 837, 802. HRMS (ESI) calcd for $\mathrm{C}_{27} \mathrm{H}_{33} \mathrm{NNaO}_{7}[\mathrm{M}+\mathrm{Na}]^{+}$: 506.2149. Found: 506.2156.

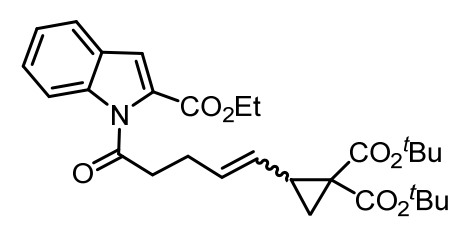

1s, yellow oil, $0.90 \mathrm{~g}, 84 \%$ yield (2.1 mmol scale), $E / Z=5 / 1 .{ }^{1} \mathrm{H}$ NMR (400 MHz, $\left.\mathrm{CDCl}_{3}\right) \delta 8.03-7.99(\mathrm{~m}, 1 \mathrm{H}), 7.60(\mathrm{~d}, J=8.0 \mathrm{~Hz}, 1 \mathrm{H}), 7.40(\mathrm{t}, J=8.0 \mathrm{~Hz}, 1 \mathrm{H}), 7.30(\mathrm{~s}$, $1 \mathrm{H}), 7.26-7.22(\mathrm{~m}, 1 \mathrm{H}), 5.69-5.61(\mathrm{~m}, 1 \mathrm{H}), 5.54-5.47\left(\mathrm{~m}, 1 \mathrm{H}^{*}\right), 5.08-5.02(\mathrm{~m}, 1 \mathrm{H})$, 4.93-4.88 (m, 1H*), 4.37 (q, $J=7.2 \mathrm{~Hz}, 2 \mathrm{H}), 3.02-2.88(\mathrm{~m}, 2 \mathrm{H}), 2.74-2.39(\mathrm{~m}, 2 \mathrm{H})$, 2.34-2.28 (m, 1H), 1.45-1.35 (m, 22H), 1.27-1.23 (m, 1H). $\left.{ }^{13} \mathrm{C} \mathrm{NMR} \mathrm{(100} \mathrm{MHz,} \mathrm{CDCl}_{3}\right)$ $\delta 173.7,168.9,166.7,161.6,138.1,131.4,131.1,129.3,127.6,127.3,127.0,126.7$, $123.5,122.3,117.7,114.7,81.4,81.0,61.5,60.7,39.2,37.3,28.8,28.4,27.9,27.4,27.3$, 24.8, 23.7, 20.7, 19.6, 14.1. IR (thin film): $v_{\max }\left(\mathrm{cm}^{-1}\right)=2977,2933,1711,1533,1475$, 1444, 1392, 1367, 1329, 1281, 1254, 1199, 1165, 1147, 1125, 1036, 1015, 966, 942, 836, 806, 745. HRMS (ESI) calcd for $\mathrm{C}_{29} \mathrm{H}_{37} \mathrm{NNaO}_{7}[\mathrm{M}+\mathrm{Na}]^{+}$: 534.2462. Found: 534.2461 .

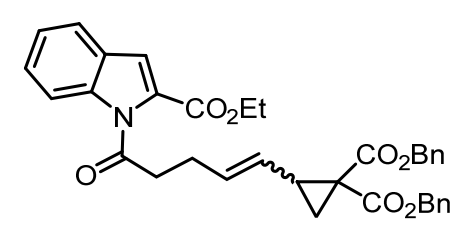

1t, yellow oil, $1.20 \mathrm{~g}, 83 \%$ yield (2.5 mmol scale), $E / Z=5.4 / 1 .{ }^{1} \mathrm{H}$ NMR (400 MHz, $\left.\mathrm{CDCl}_{3}\right) \delta 8.08-8.04(\mathrm{~m}, 1 \mathrm{H}), 7.65(\mathrm{~d}, J=8.0 \mathrm{~Hz}, 1 \mathrm{H}), 7.46(\mathrm{t}, J=7.6 \mathrm{~Hz}, 1 \mathrm{H}), 7.36-$ $7.22(\mathrm{~m}, 12 \mathrm{H}), 5.73-5.65(\mathrm{~m}, 1 \mathrm{H}), 5.54-5.48\left(\mathrm{~m}, 1 \mathrm{H}^{*}\right), 5.22-4.90(\mathrm{~m}, 5 \mathrm{H}), 4.42(\mathrm{q}, J=$ 
$7.2 \mathrm{~Hz}, 2 \mathrm{H}), 3.01-2.83(\mathrm{~m}, 2 \mathrm{H}), 2.75-2.38(\mathrm{~m}, 3 \mathrm{H}), 1.65-1.62(\mathrm{~m}, 1 \mathrm{H}), 1.56-1.52(\mathrm{~m}$, $1 \mathrm{H}), 1.43(\mathrm{t}, J=7.2 \mathrm{~Hz}, 3 \mathrm{H}) .{ }^{13} \mathrm{C} \mathrm{NMR}\left(100 \mathrm{MHz}, \mathrm{CDCl}_{3}\right) \delta 173.6,169.3,167.1,161.6$, $138.1,135.5,135.3,132.6,129.3,128.4,128.33,128.28,128.1,127.9,127.7,127.0$, 126.0, 123.5, 122.4, 117.8, 114.7, 67.1, 61.6, 39.0, 35.5, 30.9, 28.2, 27.5, 26.7, 23.7, 21.9, 20.8, 14.2. IR (thin film): $v_{\max }\left(\mathrm{cm}^{-1}\right)=3032,2980,1713,1607,1534,1497,1474$, 1443, 1376, 1310, 1273, 1192, 1146, 1117, 1030, 1014, 964, 907, 860, 744, 695, 601.

HRMS (ESI) calcd for $\mathrm{C}_{35} \mathrm{H}_{33} \mathrm{NNaO}_{7}[\mathrm{M}+\mathrm{Na}]^{+}$: 602.2149. Found: 602.2142.

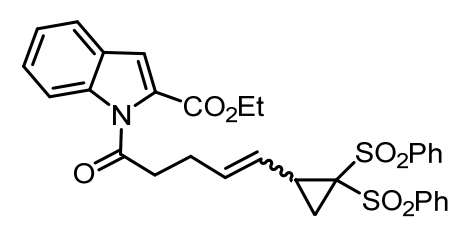

1u, white solid, $1.0 \mathrm{~g}, 71 \%$ yield $(2.5 \mathrm{mmol} \mathrm{scale}), E / Z=2.7 / 1$, m.p. $=64.5-67.2{ }^{\circ} \mathrm{C}$. ${ }^{1} \mathrm{H}$ NMR (400 MHz, $\left.\mathrm{CDCl}_{3}\right)$ 8 8.07-7.94 (m, 5H), 7.67-7.44 (m, 8H), 7.38-7.28 (m, 2H), 5.80-5.68 (m, 1H), 5.64-5.55 (m, 1H), $4.40(\mathrm{q}, J=7.2 \mathrm{~Hz}, 2 \mathrm{H}), 3.32$ (q, $J=9.2 \mathrm{~Hz}$, $\left.1 \mathrm{H}^{*}\right), 3.15(\mathrm{q}, J=9.2 \mathrm{~Hz}, 1 \mathrm{H}), 2.95-2.72(\mathrm{~m}, 2 \mathrm{H}), 2.58-2.27(\mathrm{~m}, 2 \mathrm{H}), 2.21-1.97(\mathrm{~m}$, $2 \mathrm{H}), 1.40(\mathrm{t}, J=7.2 \mathrm{~Hz}, 3 \mathrm{H}) .{ }^{13} \mathrm{C} \mathrm{NMR}\left(100 \mathrm{MHz}, \mathrm{CDCl}_{3}\right) \delta 173.4,173.0,161.6,139.3$, 138.6, 138.2, 135.8, 135.2, 134.1, 134.0, 129.52, 129.47, 129.4, 129.2, 128.8, 128.7, $128.4,128.3,127.8,127.7,127.0,123.9,123.6,123.5,122.4,118.1,117.8,114.7,64.2$, $63.5,61.6,38.5,38.3,31.7,28.3,27.4,23.3,21.3,20.3,14.1$. IR (thin film): $v_{\max }\left(\mathrm{cm}^{-1}\right)$ $=2979,1710,1534,1475,1445,1375,1311,1280,1256,1200,1156,1077,1036,999$, $941,852,799,747,725,684,633,594$. HRMS (ESI) calcd for $\mathrm{C}_{31} \mathrm{H}_{29} \mathrm{NNaO}_{7} \mathrm{~S}_{2}$ $[\mathrm{M}+\mathrm{Na}]^{+}:$614.1278. Found: 614.1280 .

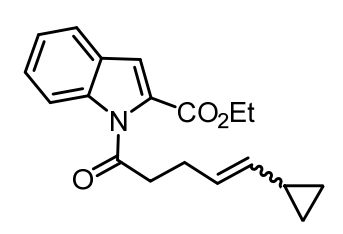

1v, colorless oil, $0.14 \mathrm{~g}, 75 \%$ yield (0.7 mmol scale), $E / Z=5.4 / 1 .{ }^{1} \mathrm{H}$ NMR (400 MHz, $\left.\mathrm{CDCl}_{3}\right) \delta 8.08-8.00(\mathrm{~m}, 1 \mathrm{H}), 7.59(\mathrm{~d}, J=8.0 \mathrm{~Hz}, 1 \mathrm{H}), 7.40(\mathrm{t}, J=7.6 \mathrm{~Hz}, 1 \mathrm{H}), 7.30(\mathrm{~s}$, $1 \mathrm{H}), 7.24(\mathrm{t}, J=7.6 \mathrm{~Hz}, 1 \mathrm{H}), 5.45-5.38\left(\mathrm{~m}, 1 \mathrm{H}^{*}\right), 5.25-5.19(\mathrm{~m}, 1 \mathrm{H}), 4.91-4.85(\mathrm{~m}$, $\left.1 \mathrm{H}^{*}\right), 4.72-4.67(\mathrm{~m}, 1 \mathrm{H}), 4.37$ (q, $\left.J=7.2 \mathrm{~Hz}, 2 \mathrm{H}\right), 2.97-2.88(\mathrm{~m}, 2 \mathrm{H}), 2.63(\mathrm{q}, J=7.2$ $\mathrm{Hz}, 2 \mathrm{H}), 2.43$ (q, $\left.J=7.2 \mathrm{~Hz}, 2 \mathrm{H}^{*}\right), 1.56-1.48(\mathrm{~m}, 1 \mathrm{H}), 1.38$ (t, $\left.J=7.2 \mathrm{~Hz}, 3 \mathrm{H}\right), 1.24-$ 1.17 (m, 1H*), 0.70-0.65 (m, 2H), 0.57-0.52 (m, 2H*), 0.27-0.23 (m, 2H), 0.17-0.13 
$\left(\mathrm{m}, 2 \mathrm{H}^{*}\right) .{ }^{13} \mathrm{C} \mathrm{NMR}\left(100 \mathrm{MHz}, \mathrm{CDCl}_{3}\right) \delta 174.1,161.7,138.1,135.7,135.5,129.4$, $127.5,127.0,126.6,125.1,125.0,123.4,122.3,122.0,117.5,114.8,114.7,61.5,39.73$, $39.70,28.5,27.8,27.7,23.8,14.1,13.3,9.5,6.8,6.2$. IR (thin film): $v_{\max }\left(\mathrm{cm}^{-1}\right)=2981$, 2934, 1713, 1607, 1533, 1474, 1443, 1394, 1375, 1322, 1279, 1256, 1196, 1166, 1146, 1109, 1071, 1036, 1015, 987, 962, 937, 765. HRMS (ESI) calcd for $\mathrm{C}_{19} \mathrm{H}_{21} \mathrm{NNaO}_{3}$ $[\mathrm{M}+\mathrm{Na}]^{+}:$334.1414. Found: 334.1410.

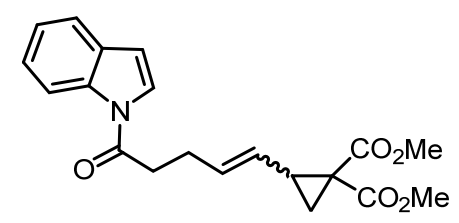

1w, yellow oil, $1.45 \mathrm{~g}, 75 \%$ yield (6 mmol scale), $E / Z=5 / 1 .{ }^{1} \mathrm{H} \mathrm{NMR}\left(400 \mathrm{MHz}, \mathrm{CDCl}_{3}\right)$ $\delta$ 8.46-8.42 (m, 1H), $7.53(\mathrm{~d}, J=7.6 \mathrm{~Hz}, 1 \mathrm{H}), 7.41-7.38(\mathrm{~m}, 1 \mathrm{H}), 7.32(\mathrm{t}, J=8.0 \mathrm{~Hz}$, $1 \mathrm{H}), 7.24(\mathrm{t}, J=7.6 \mathrm{~Hz}, 1 \mathrm{H}), 6.59(\mathrm{~s}, 1 \mathrm{H}), 5.85-5.77(\mathrm{~m}, 1 \mathrm{H}), 5.71-5.64\left(\mathrm{~m}, 1 \mathrm{H}^{*}\right), 5.23-$ $5.17(\mathrm{~m}, 1 \mathrm{H}), 5.03-4.98\left(\mathrm{~m}, 1 \mathrm{H}^{*}\right), 3.72-3.69(\mathrm{~m}, 6 \mathrm{H}), 2.95-2.87(\mathrm{~m}, 2 \mathrm{H}), 2.79-2.48(\mathrm{~m}$, $3 \mathrm{H}), 1.70-1.54(\mathrm{~m}, 2 \mathrm{H}) .{ }^{13} \mathrm{C}$ NMR $\left(100 \mathrm{MHz}, \mathrm{CDCl}_{3}\right) \delta 170.2,169.8,167.6,135.3$, $132.7,132.5,130.1,126.1,124.9,124.3,123.4,120.6,116.3,108.9,52.4,52.3,35.4$, 35.1, 30.6, 27.0, 26.3, 22.6, 20.5. IR (thin film): $v_{\max }\left(\mathrm{cm}^{-1}\right)=3002,2952,1708,1583$, 1536, 1471, 1451, 1436, 1386, 1303, 1203, 1153, 1125, 1083, 1015, 963, 931, 902, 879, 749, 717, 664, 628. HRMS (ESI) calcd for $\mathrm{C}_{20} \mathrm{H}_{21} \mathrm{NNaO}_{5}[\mathrm{M}+\mathrm{Na}]^{+}: 378.1312$. Found: 378.1319 .

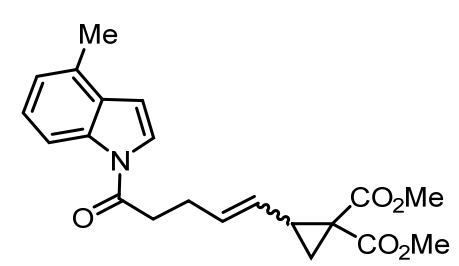

1x, yellow oil, $0.48 \mathrm{~g}, 44 \%$ yield (3.1 mmol scale), $E / Z=5.1 / 1 .{ }^{1} \mathrm{H}$ NMR (400 MHz, $\left.\mathrm{CDCl}_{3}\right) \delta 8.27-8.23(\mathrm{~m}, 1 \mathrm{H}), 7.38-7.34(\mathrm{~m}, 1 \mathrm{H}), 7.21(\mathrm{t}, J=8.0 \mathrm{~Hz}, 1 \mathrm{H}), 7.03(\mathrm{~d}, J=$ $7.2 \mathrm{~Hz}, 1 \mathrm{H}), 6.60(\mathrm{~d}, J=3.6 \mathrm{~Hz}, 1 \mathrm{H}), 5.83-5.76(\mathrm{~m}, 1 \mathrm{H}), 5.69-5.63\left(\mathrm{~m}, 1 \mathrm{H}^{*}\right), 5.22-5.16$ (m, 1H), 5.02-4.97 (m, 1H*), 3.72-3.69 (m, 6H), 2.93-2.84 (m, 2H), 2.77-2.45 (m, 3H), $2.48(\mathrm{~s}, 3 \mathrm{H}), 1.69-1.53(\mathrm{~m}, 2 \mathrm{H}) .{ }^{13} \mathrm{C} \mathrm{NMR}\left(100 \mathrm{MHz}, \mathrm{CDCl}_{3}\right) \delta 170.1,169.7,167.5$, 135.0, 132.7, 132.5, 129.9, 129.6, 125.9, 124.8, 123.7, 123.6, 113.7, 107.1, 52.4, 52.2, $35.3,35.0,30.5,26.9,26.2,22.5,21.5,20.4,18.2$. IR (thin film): $v_{\max }\left(\mathrm{cm}^{-1}\right)=2953$, $1708,1535,1486,1420,1392$, 1365, 1321, 1275, 1225, 1204, 1156, 1131, 988, 965 , 
917, 901, 789, 758, 713, 684, 664, 559. HRMS (ESI) calcd for $\mathrm{C}_{21} \mathrm{H}_{23} \mathrm{NNaO}_{5}[\mathrm{M}+\mathrm{Na}]^{+}$: 392.1468. Found: 392.1468.

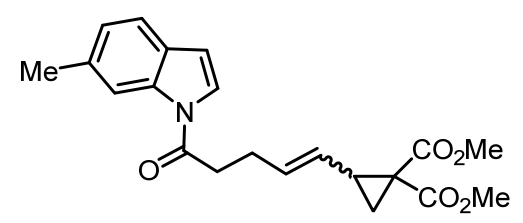

1y, yellow oil, $0.44 \mathrm{~g}, 40 \%$ yield (3.1 mmol scale), $E / Z=5.4 / 1 .{ }^{1} \mathrm{H}$ NMR (400 MHz, $\left.\mathrm{CDCl}_{3}\right) \delta 8.24(\mathrm{~s}, 1 \mathrm{H}), 7.36(\mathrm{~d}, J=8.0 \mathrm{~Hz}, 1 \mathrm{H}), 7.28-7.25(\mathrm{~m}, 1 \mathrm{H}), 7.03(\mathrm{t}, J=8.0 \mathrm{~Hz}$, $1 \mathrm{H}), 6.49(\mathrm{~d}, J=3.6 \mathrm{~Hz}, 1 \mathrm{H}), 5.80-5.73(\mathrm{~m}, 1 \mathrm{H}), 5.66-5.60\left(\mathrm{~m}, 1 \mathrm{H}^{*}\right), 5.18-5.13(\mathrm{~m}$, 1H), 4.98-4.93 (m, 1H*), 3.68-3.66 (m, 6H), 2.95-2.74 (m, 2H), 2.65-2.44 (m, 3H), 2.42 (s, 3H), 1.66-1.50 (m, 2H). $\left.{ }^{13} \mathrm{C} \mathrm{NMR} \mathrm{(100} \mathrm{MHz,} \mathrm{CDCl}_{3}\right) \delta 170.2,169.7,167.5,135.6$, 134.8, 132.8, 132.6, 127.7, 125.9, 124.7, 123.7, 123.6, 120.1, 116.5, 108.7, 52.5, 52.4, $52.3,52.2,35.4,35.3,35.0,30.6,26.9,26.3,22.5,21.6,21.5,20.5$. IR (thin film): $v_{\max }$ $\left(\mathrm{cm}^{-1}\right)=2952,2917,1719,1611,1532,1483,1433,1388,1323,1299,1203,1126,963$, 943, 918, 902, 879, 809, 760, 711, 610, 588, 572. HRMS (ESI) calcd for $\mathrm{C}_{21} \mathrm{H}_{23} \mathrm{NNaO}_{5}$ $[\mathrm{M}+\mathrm{Na}]^{+}:$392.1468. Found: 392.1476.

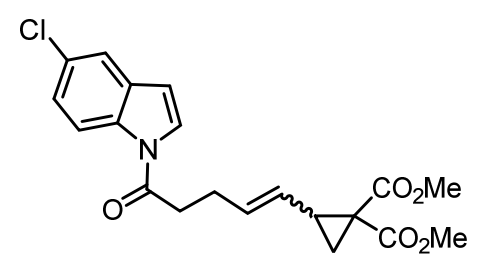

1z, white solid, $0.94 \mathrm{~g}, 73 \%$ yield (3.3 mmol scale), $E / Z=5.1 / 1$, m.p. $=70.8-73.6{ }^{\circ} \mathrm{C}$. ${ }^{1} \mathrm{H}$ NMR (400 MHz, $\left.\mathrm{CDCl}_{3}\right) \delta 8.35(\mathrm{~d}, J=7.2 \mathrm{~Hz}, 1 \mathrm{H}), 7.50-7.42(\mathrm{~m}, 2 \mathrm{H}), 7.29-7.26$ (m, 1H), $6.55(\mathrm{~s}, 1 \mathrm{H}), 5.86-5.79(\mathrm{~m}, 1 \mathrm{H}), 5.72-5.65\left(\mathrm{~m}, 1 \mathrm{H}^{*}\right), 5.24-5.18(\mathrm{~m}, 1 \mathrm{H}), 5.04-$ 4.99 (m, 1H*), 3.74-3.71 (m, 6H), 2.99-2.92 (m, 2H), 2.79-2.51 (m, 3H), 1.70-1.53 (m, 2H). ${ }^{13} \mathrm{C} \mathrm{NMR}\left(100 \mathrm{MHz}, \mathrm{CDCl}_{3}\right) \delta 170.1,169.8,167.7,133.7,132.6,132.4,131.3$, 129.0, 126.3, 125.7, 125.5, 125.0, 120.3, 117.4, 108.2, 52.7, 52.6, 52.4, 35.5, 35.4, 35.1, 30.7, 27.0, 26.4, 22.6, 21.7, 20.6. IR (thin film): $v_{\max }\left(\mathrm{cm}^{-1}\right)=2955,1711,1576,1533$, $1447,1381,1325,1274,1256,1200,1153,1129,1093,1066,988,963,932,896,878$, 821, 780, 750, 712. HRMS (ESI) calcd for $\mathrm{C}_{20} \mathrm{H}_{20} \mathrm{NCINaO}_{5}[\mathrm{M}+\mathrm{Na}]^{+}: 412.0922$. Found: 412.0919 . 


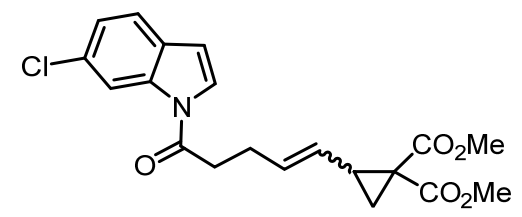

1aa, yellow oil, $0.91 \mathrm{~g}, 71 \%$ yield (3.3 mmol scale), $E / Z=3.7 / 1 .{ }^{1} \mathrm{H}$ NMR (400 MHz, $\left.\mathrm{CDCl}_{3}\right) \delta 8.46(\mathrm{~s}, 1 \mathrm{H}), 7.43-7.36(\mathrm{~m}, 2 \mathrm{H}), 7.22-7.19(\mathrm{~m}, 1 \mathrm{H}), 6.56-6.55(\mathrm{~m}, 1 \mathrm{H}), 5.85-$ $5.78(\mathrm{~m}, 1 \mathrm{H}), 5.71-5.64\left(\mathrm{~m}, 1 \mathrm{H}^{*}\right), 5.24-5.18(\mathrm{~m}, 1 \mathrm{H}), 5.04-4.99\left(\mathrm{~m}, 1 \mathrm{H}^{*}\right), 3.74-3.71$ $(\mathrm{m}, 6 \mathrm{H}), 2.96-2.76(\mathrm{~m}, 2 \mathrm{H}), 2.69-2.50(\mathrm{~m}, 3 \mathrm{H}), 1.70-1.55(\mathrm{~m}, 2 \mathrm{H}) .{ }^{13} \mathrm{C} \mathrm{NMR}(100 \mathrm{MHz}$, $\left.\mathrm{CDCl}_{3}\right) \delta 170.2,170.1,169.8,167.6,135.6,132.5,132.3,130.6,128.5,126.3,124.9$, $124.8,123.9,121.3,119.9,116.5,108.6,52.6,52.5,52.4,35.3,35.0,30.6,26.8,26.3$, 22.5, 21.6, 20.5. IR (thin film): $v_{\max }\left(\mathrm{cm}^{-1}\right)=2952,1714,1605,1531,1453,1430,1385$, 1362, 1319, 1296, 1196, 1125, 1115, 963, 938, 895, 873, 812, 791, 757, 716, 612, 586. HRMS (ESI) calcd for $\mathrm{C}_{20} \mathrm{H}_{20} \mathrm{NCINaO}_{5}[\mathrm{M}+\mathrm{Na}]^{+}: 412.0922$. Found: 412.0914.

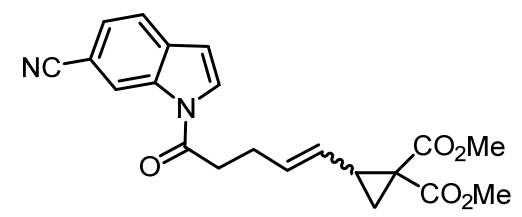

1ab, yellow oil, $0.74 \mathrm{~g}, 67 \%$ yield (3.1 mmol scale), $E / Z=5 / 1 .{ }^{1} \mathrm{H}$ NMR (400 MHz, $\left.\mathrm{CDCl}_{3}\right) \delta 8.59-8.58(\mathrm{~m}, 1 \mathrm{H}), 7.57-7.53(\mathrm{~m}, 1 \mathrm{H}), 7.47$ (d, $\left.J=8.4 \mathrm{~Hz}, 1 \mathrm{H}\right), 7.31-7.29(\mathrm{~m}$, $1 \mathrm{H}), 6.58(\mathrm{~d}, J=4.0 \mathrm{~Hz}, 1 \mathrm{H}), 5.75-5.67(\mathrm{~m}, 1 \mathrm{H}), 5.60-5.54\left(\mathrm{~m}, 1 \mathrm{H}^{*}\right), 5.14-5.08(\mathrm{~m}$, $1 \mathrm{H}), 4.93-4.88\left(\mathrm{~m}, 1 \mathrm{H}^{*}\right), 3.62-3.57(\mathrm{~m}, 6 \mathrm{H}), 2.94-2.86(\mathrm{~m}, 2 \mathrm{H}), 2.71-2.39(\mathrm{~m}, 3 \mathrm{H})$, 1.57-1.41 (m, 2H). ${ }^{13} \mathrm{C}$ NMR (100 MHz, $\left.\mathrm{CDCl}_{3}\right) \delta 170.3,170.1,169.5,167.4,134.0$, 133.1, 132.1, 131.9, 127.7, 127.6, 126.3, 126.2, 121.3, 120.4, 119.4, 108.5, 107.2, 52.4, $52.3,52.2,35.3,35.1,34.8,30.4,26.6,26.1,22.2,21.4,20.3$. IR (thin film): $v_{\max }\left(\mathrm{cm}^{-1}\right)$ $=2953,2223,1714,1609,1523,1470,1432,1390,1365,1325,1300,1282,1204,1126$, 963, 947, 919, 894, 823, 800, 758, 727, 704. HRMS (ESI) calcd for $\mathrm{C}_{21} \mathrm{H}_{20} \mathrm{~N}_{2} \mathrm{NaO}_{5}$ $[\mathrm{M}+\mathrm{Na}]^{+}:$403.1264. Found: 403.1270.

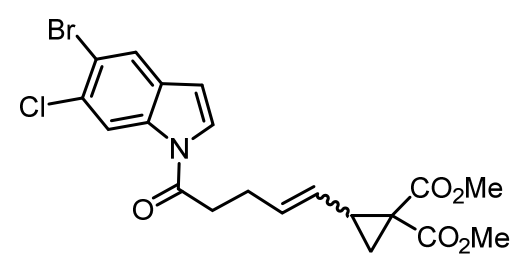


1ac, yellow oil, $0.85 \mathrm{~g}, 65 \%$ yield (2.8 mmol scale), $E / Z=5.2 / 1 .{ }^{1} \mathrm{H}$ NMR (400 MHz, $\left.\mathrm{CDCl}_{3}\right) \delta 8.55-8.53(\mathrm{~m}, 1 \mathrm{H}), 7.73(\mathrm{~s}, 1 \mathrm{H}), 7.45-7.40(\mathrm{~m}, 1 \mathrm{H}), 6.51(\mathrm{~d}, J=3.2 \mathrm{~Hz}, 1 \mathrm{H})$, 5.86-5.78 (m, 1H), 5.71-5.65 (m, $\left.1 \mathrm{H}^{*}\right), 5.25-5.19(\mathrm{~m}, 1 \mathrm{H}), 5.05-5.00\left(\mathrm{~m}, 1 \mathrm{H}^{*}\right), 3.75-$ $3.72(\mathrm{~m}, 6 \mathrm{H}), 3.00-2.91$ (m, 2H), 2.82-2.49 (m, 3H), 1.71-1.56 (m, 2H). ${ }^{13} \mathrm{C}$ NMR (100 $\left.\mathrm{MHz}, \mathrm{CDCl}_{3}\right) \delta 170.0,169.8,167.7,134.5,132.4,132.2,130.3,130.0,126.5,126.0$, $125.8,124.7,117.9,116.8,107.8,52.6,52.5,35.5,35.4,35.0,30.7,26.8,26.4,22.4$, 21.7, 20.6. IR (thin film): $\nu_{\max }\left(\mathrm{cm}^{-1}\right)=3000,2951,1714,1563,1529,1435,1407,1375$, 1318, 1293, 1239, 1197, 1126, 1090, 962, 941, 878, 800, 755, 712, 641, 598, 569. HRMS (ESI) calcd for $\mathrm{C}_{20} \mathrm{H}_{19} \mathrm{NNaO}_{5} \mathrm{Cl}^{79} \mathrm{Br}[\mathrm{M}+\mathrm{Na}]^{+}$: 490.0027. Found: 490.0024.

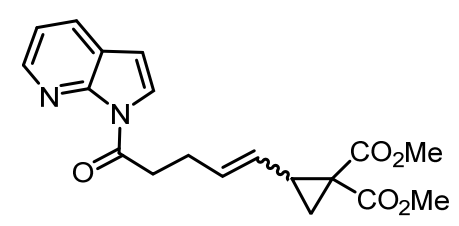

1ad, white solid, $0.89 \mathrm{~g}, 81 \%$ yield (3.1 mmol scale), $E / Z=5.1 / 1$, m.p. $=59.2-61.4{ }^{\circ} \mathrm{C}$. ${ }^{1} \mathrm{H}$ NMR $\left(400 \mathrm{MHz}, \mathrm{CDCl}_{3}\right) \delta 8.28(\mathrm{~s}, 1 \mathrm{H}), 7.90-7.89(\mathrm{~m}, 1 \mathrm{H}), 7.81-7.79(\mathrm{~m}, 1 \mathrm{H}), 7.13-$ $7.10(\mathrm{~m}, 1 \mathrm{H}), 6.52-6.51(\mathrm{~m}, 1 \mathrm{H}), 5.85-5.81(\mathrm{~m}, 1 \mathrm{H}), 5.69-5.68\left(\mathrm{~m}, 1 \mathrm{H}^{*}\right), 5.19-5.13(\mathrm{~m}$, $1 \mathrm{H}), 4.95-4.90\left(\mathrm{~m}, 1 \mathrm{H}^{*}\right), 3.67-3.66(\mathrm{~m}, 6 \mathrm{H}), 3.55-3.47(\mathrm{~m}, 2 \mathrm{H}), 2.79-2.48(\mathrm{~m}, 3 \mathrm{H})$, 1.66-1.50 (m, 2H). ${ }^{13} \mathrm{C} \mathrm{NMR}\left(100 \mathrm{MHz}, \mathrm{CDCl}_{3}\right) \delta 171.0,169.9,167.7,147.5,143.6$, 133.4, 133.2, 129.1, 125.6, 125.5, 125.3, 123.6, 118.5, 105.6, 52.5, 52.42, 52.35, 37.3, $35.4,30.8,27.1,26.5,22.8,21.7,20.6$. IR (thin film): $v_{\max }\left(\mathrm{cm}^{-1}\right)=2954,1706,1578$, 1531, 1436, 1411, 1330, 1290, 1260, 1211, 1136, 1058, 1006, 982, 943, 888, 805, 782, 759, 723, 602, 550, 489. HRMS (ESI) calcd for $\mathrm{C}_{19} \mathrm{H}_{21} \mathrm{~N}_{2} \mathrm{O}_{5}[\mathrm{M}+\mathrm{H}]^{+}: 357.1445$. Found: 357.1451 .

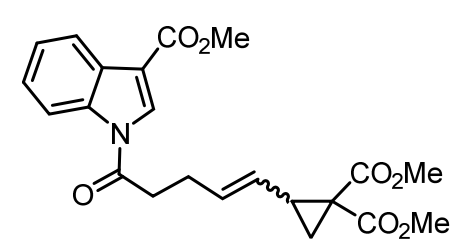

3a, yellow oil, $0.60 \mathrm{~g}, 60 \%$ yield (2.5 mmol scale), $E / Z=4.7 / 1 .{ }^{1} \mathrm{H}$ NMR (400 MHz, $\left.\mathrm{CDCl}_{3}\right) \delta 8.41-8.37(\mathrm{~m}, 1 \mathrm{H}), 8.09(\mathrm{~d}, J=8.4 \mathrm{~Hz}, 1 \mathrm{H}), 8.05(\mathrm{~s}, 1 \mathrm{H}), 7.38-7.31(\mathrm{~m}, 2 \mathrm{H})$,

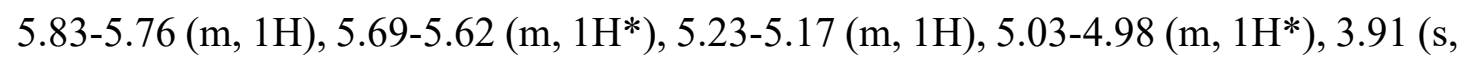
$3 \mathrm{H}), 3.70(\mathrm{~s}, 3 \mathrm{H}), 3.69$ (s, 3H), 3.03-2.93 (m, 2H), 2.79-2.48 (m, 3H), 1.68-1.65 (m, 1H), 1.60-1.53 (s, 1H). ${ }^{13} \mathrm{C} \mathrm{NMR}\left(100 \mathrm{MHz}, \mathrm{CDCl}_{3}\right) \delta 170.3,169.7,167.6,164.0,135.6$, 
$132.3,132.0,130.3,130.2,126.9,126.5,125.6,124.5,121.2,116.2,113.2,52.54,52.46$, $52.31,51.29,35.3,35.0,30.6,26.7,26.2,22.2,21.6,20.5$. IR (thin film): $v_{\max }\left(\mathrm{cm}^{-1}\right)=$ 3001, 2952, 1708, 1606, 1583, 1556, 1480, 1436, 1382, 1323, 1182, 1149, 1046, 1018, 962, 946, 899, 771, 679, 614. HRMS (ESI) calcd for $\mathrm{C}_{22} \mathrm{H}_{23} \mathrm{NO}_{7} \mathrm{Na}[\mathrm{M}+\mathrm{Na}]^{+}: 436.1367$. Found: 436.1368 .

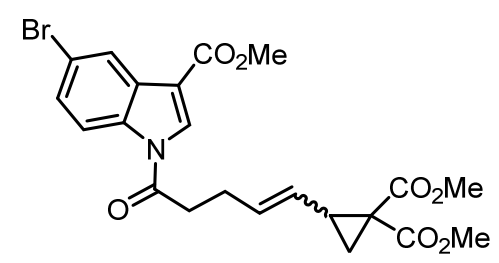

3b, yellow oil, $0.62 \mathrm{~g}, 44 \%$ yield (2.9 mmol scale), $E / Z=3.8 / 1 .{ }^{1} \mathrm{H}$ NMR (400 MHz, $\left.\mathrm{CDCl}_{3}\right) \delta 8.11(\mathrm{~d}, J=8.8 \mathrm{~Hz}, 1 \mathrm{H}), 8.05(\mathrm{~s}, 1 \mathrm{H}), 7.92\left(\mathrm{~s}, 1 \mathrm{H}^{*}\right), 7.89(\mathrm{~s}, 1 \mathrm{H}), 7.36-7.34$ $(\mathrm{m}, 1 \mathrm{H}), 5.86-5.79(\mathrm{~m}, 1 \mathrm{H}), 5.71-5.65\left(\mathrm{~m}, 1 \mathrm{H}^{*}\right), 5.26-5.20(\mathrm{~m}, 1 \mathrm{H})$, 5.05-5.00 (m, $\left.1 \mathrm{H}^{*}\right)$, $3.90(\mathrm{~s}, 3 \mathrm{H}), 3.73(\mathrm{~s}, 6 \mathrm{H}), 2.99-2.90(\mathrm{~m}, 2 \mathrm{H}), 2.71-2.50(\mathrm{~m}, 3 \mathrm{H}), 1.71-1.56(\mathrm{~m}, 2 \mathrm{H}) .{ }^{13} \mathrm{C}$ $\mathrm{NMR}\left(100 \mathrm{MHz}, \mathrm{CDCl}_{3}\right) \delta 170.1,170.0,169.6,167.5,163.2,133.9,132.1,131.8,130.7$, $128.20,128.16,126.5,123.5,117.8,117.3,112.1,52.5,52.4,52.3,51.4,35.3,35.2$, $34.7,30.5,26.5,26.2,22.0,21.5,20.4$. IR (thin film): $v_{\max }\left(\mathrm{cm}^{-1}\right)=3000,2952,1712$, 1600, 1573, 1554, 1439, 1378, 1319, 1286, 1258, 1178, 1146, 1127, 1064, 963, 947, 810, 768, 635. HRMS (ESI) calcd for $\mathrm{C}_{22} \mathrm{H}_{23} \mathrm{NO}_{7}{ }^{79} \mathrm{Br}[\mathrm{M}+\mathrm{H}]^{+}$: 492.0652. Found: 492.0656.

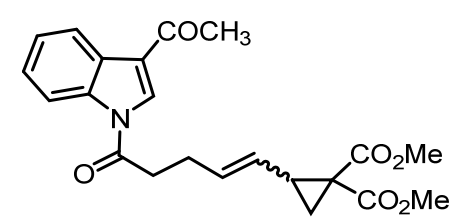

3c, yellow oil, $0.61 \mathrm{~g}, 51 \%$ yield (2.9 mmol scale), $E / Z=4.5 / 1 .{ }^{1} \mathrm{H}$ NMR (400 MHz, $\left.\mathrm{CDCl}_{3}\right) \delta 8.23-8.16(\mathrm{~m}, 2 \mathrm{H}), 7.82\left(\mathrm{~s}, 1 \mathrm{H}^{*}\right), 7.76(\mathrm{~s}, 1 \mathrm{H}), 7.28-7.22(\mathrm{~m}, 2 \mathrm{H})$, 5.76-5.69 (m, 1H), 5.64-5.57 (m, 1H*), 5.17-5.11 (m, 1H), 4.97-4.92 (m, 1H*), $3.64(\mathrm{~s}, 6 \mathrm{H}), 2.93-$ $2.70(\mathrm{~m}, 2 \mathrm{H}), 2.60-2.39(\mathrm{~m}, 3 \mathrm{H}), 2.37$ (s, 3H), 1.62-1.47 (m, 2H). ${ }^{13} \mathrm{C}$ NMR (100 MHz, $\left.\mathrm{CDCl}_{3}\right) \delta 193.4,193.3,170.3,170.1,169.6,167.5,135.5,132.2,132.0,130.6,130.4$, $126.7,126.4,126.3,125.7,124.6,122.0,120.9,115.8,52.5,52.4,52.3,35.4,35.2,34.9$, $30.5,27.4,26.5,26.2,22.1,21.5,20.4$. IR (thin film): $v_{\max }\left(\mathrm{cm}^{-1}\right)=3003,2953,1717$, 1661, 1604, 1545, 1478, 1324, 1274, 1257, 1193, 1147, 1015, 964, 945, 898, 751, 703, 666, 503. HRMS (ESI) calcd for $\mathrm{C}_{22} \mathrm{H}_{23} \mathrm{NO}_{6} \mathrm{Na}[\mathrm{M}+\mathrm{Na}]^{+}: 420.1418$. Found: 420.1422 . 


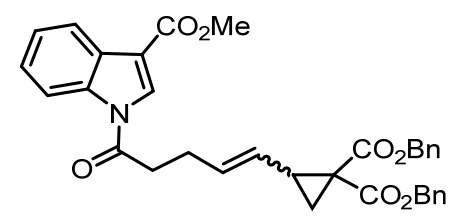

3d, colorless oil, $0.72 \mathrm{~g}, 51 \%$ yield (2.5 mmol scale), $E / Z=3.7 / 1 .{ }^{1} \mathrm{H}$ NMR (400 MHz, $\left.\mathrm{CDCl}_{3}\right) \delta 8.46-8.44(\mathrm{~m}, 1 \mathrm{H}), 8.18-8.16(\mathrm{~m}, 1 \mathrm{H}), 8.05\left(\mathrm{~s}, 1 \mathrm{H}^{*}\right), 8.03(\mathrm{~s}, 1 \mathrm{H}), 7.43-7.21$ $(\mathrm{m}, 12 \mathrm{H}), 5.83-5.76(\mathrm{~m}, 1 \mathrm{H}), 5.65-5.59\left(\mathrm{~m}, 1 \mathrm{H}^{*}\right)$, 5.24-5.02 (m, 5H), $3.96(\mathrm{~s}, 3 \mathrm{H}), 2.93-$ $2.76(\mathrm{~m}, 2 \mathrm{H}), 2.67-2.39(\mathrm{~m}, 3 \mathrm{H}), 1.78-1.61(\mathrm{~m}, 2 \mathrm{H}) .{ }^{13} \mathrm{C} \mathrm{NMR}\left(100 \mathrm{MHz}, \mathrm{CDCl}_{3}\right) \delta$ $170.4,169.2$, 167.1, 164.1, 135.7, 135.5, 135.3, 132.4, 132.2, 130.3, 128.4, 128.30, $128.29,128.25,128.1,127.9,127.0,126.33,126.25,125.8,124.6,121.4,116.3,113.4$, $67.12,67.06,51.4,35.7,35.5,34.9,30.8,26.7,26.6,22.2,21.8,20.7$. IR (thin film): $v_{\max }\left(\mathrm{cm}^{-1}\right)=3032,2950,1709,1606,1583,1556,1497,1480,1380,1310,1269,1186$, $1149,1122,1105,1051,962,946,772,695$. HRMS (ESI) calcd for $\mathrm{C}_{34} \mathrm{H}_{31} \mathrm{NO}_{7} \mathrm{Na}$ $[\mathrm{M}+\mathrm{Na}]^{+}:$588.1993. Found: 588.1988.

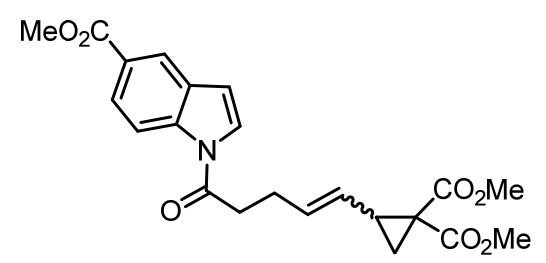

5f, white solid, $0.96 \mathrm{~g}, 83 \%$ yield $(2.8 \mathrm{mmol} \mathrm{scale}), E / Z=5.6 / 1$, m.p. $=102.5-105.6{ }^{\circ} \mathrm{C}$. ${ }^{1} \mathrm{H}$ NMR $\left(400 \mathrm{MHz}, \mathrm{CDCl}_{3}\right) \delta$ 8.46-8.44 (m, 1H), $8.26(\mathrm{~s}, 1 \mathrm{H}), 8.02-8.00(\mathrm{~m}, 1 \mathrm{H}), 7.53-$ $7.48(\mathrm{~m}, 1 \mathrm{H}), 6.68(\mathrm{~s}, 1 \mathrm{H}), 5.85-5.80(\mathrm{~m}, 1 \mathrm{H}), 5.71-5.66\left(\mathrm{~m}, 1 \mathrm{H}^{*}\right), 5.25-5.19(\mathrm{~m}, 1 \mathrm{H})$, 5.05-5.00 (m, 1H*), $3.93(\mathrm{~s}, 3 \mathrm{H}), 3.75-3.72(\mathrm{~m}, 6 \mathrm{H}), 3.02-2.94(\mathrm{~m}, 2 \mathrm{H}), 2.83-2.54(\mathrm{~m}$, $3 \mathrm{H}), 1.70-1.56(\mathrm{~m}, 2 \mathrm{H}) .{ }^{13} \mathrm{C} \mathrm{NMR}\left(100 \mathrm{MHz}, \mathrm{CDCl}_{3}\right) \delta 170.3,169.8,167.6,167.0$, $137.9,132.5,132.3,129.9,126.3,126.1,125.6,125.5,125.2,122.8,115.9,109.2,52.6$, $52.5,52.4,51.9,35.5,35.3,35.2,30.6,26.8,26.3,22.4,21.6,20.6$. IR (thin film): $v_{\max }$ $\left(\mathrm{cm}^{-1}\right)=2950,1709,1611,1537,1467,1437,1388,1321,1291,1208,1133,1087$, 1050, 1010, 957, 934, 900, 864, 752, 721, 687, 614, 498. HRMS (ESI) calcd for $\mathrm{C}_{22} \mathrm{H}_{23} \mathrm{NO}_{7} \mathrm{Na}[\mathrm{M}+\mathrm{Na}]^{+}:$436.1367. Found: 436.1368 .

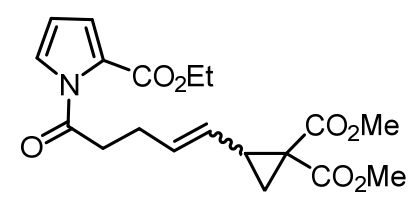


7a, colorless oil, $0.41 \mathrm{~g}, 38 \%$ yield (2.9 mmol scale), $E / Z=4.1 / 1 .{ }^{1} \mathrm{H}$ NMR (400 MHz, $\left.\mathrm{CDCl}_{3}\right) \delta$ 7.23-7.19 (m, 1H), 6.87-6.83 (m, 1H), 6.12-6.04 (m, 1H), 5.68-5.61 (m, 1H),

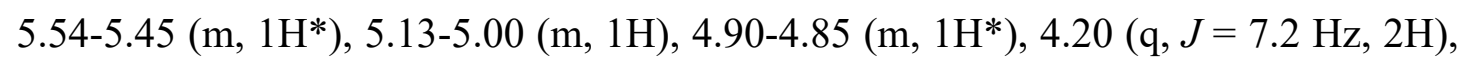
$3.64\left(\mathrm{~s}, 6 \mathrm{H}^{*}\right), 3.62(\mathrm{~s}, 6 \mathrm{H}), 2.92-2.79(\mathrm{~m}, 2 \mathrm{H}), 2.67-2.33(\mathrm{~m}, 3 \mathrm{H}), 1.58-1.53(\mathrm{~m}, 1 \mathrm{H})$, 1.46-1.43 (m, 1H), $1.24(\mathrm{t}, J=7.2 \mathrm{~Hz}, 3 \mathrm{H}) .{ }^{13} \mathrm{C} \mathrm{NMR}\left(100 \mathrm{MHz}, \mathrm{CDCl}_{3}\right) \delta 171.3,169.6$, $167.4,160.8,132.2,132.0,126.1,126.0,125.5,124.8,121.9,121.8,110.5,60.7,52.4$, 52.3, 52.21, 52.17, 36.4, 35.2, 30.4, 27.4, 27.21, 27.15, 26.1, 22.9, 21.4, 20.4, 13.9. IR (thin film): $v_{\max }\left(\mathrm{cm}^{-1}\right)=2983,2954,1717,1555,1436,1416,1368,1333,1264,1207$, 1123, 1107, 1020, 962, 899, 883, 864, 832, 754, 667. HRMS (ESI) calcd for $\mathrm{C}_{19} \mathrm{H}_{23} \mathrm{NO}_{7} \mathrm{Na}[\mathrm{M}+\mathrm{Na}]^{+}:$400.1367. Found: 400.1363 .

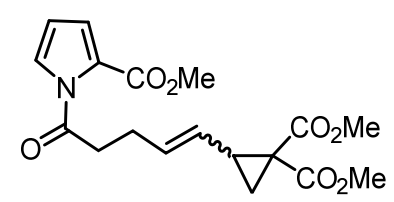

7b, colorless oil, $0.57 \mathrm{~g}, 57 \%$ yield (3.0 mmol scale), $E / Z=5.6 / 1 .{ }^{1} \mathrm{H}$ NMR (400 MHz, $\left.\mathrm{CDCl}_{3}\right) \delta$ 7.31-7.29 (m, 1H), $6.96(\mathrm{~s}, 1 \mathrm{H}), 6.23(\mathrm{~s}, 1 \mathrm{H}), 5.78-5.71(\mathrm{~m}, 1 \mathrm{H}), 5.64-5.57$ $\left(\mathrm{m}, 1 \mathrm{H}^{*}\right), 5.18-5.12(\mathrm{~m}, 1 \mathrm{H}), 5.00-4.95\left(\mathrm{~m}, 1 \mathrm{H}^{*}\right), 3.85(\mathrm{~s}, 3 \mathrm{H}), 3.73(\mathrm{~s}, 6 \mathrm{H}), 3.02-2.93$ (m, 2H), 2.78-2.44 (m, 3H), 1.69-1.66 (m, 1H), 1.58-1.54 (m, 1H). ${ }^{13} \mathrm{C}$ NMR (100 MHz, $\left.\mathrm{CDCl}_{3}\right) \delta 171.5,169.9,167.7,161.4,132.4,126.3,125.9,124.6,122.5,110.8,52.6$, $52.5,52.0,36.6,35.4,30.7,27.6,20.7$. IR (thin film): $v_{\max }\left(\mathrm{cm}^{-1}\right)=3003,2953,2847$, 1717, 1546, 1435, 1411, 1334, 1266, 1208, 1109, 966, 938, 900, 879, 801, 753, 666, 598, 496. HRMS (ESI) calcd for $\mathrm{C}_{18} \mathrm{H}_{21} \mathrm{NO}_{7} \mathrm{Na}[\mathrm{M}+\mathrm{Na}]^{+}:$386.1210. Found: 386.1205.

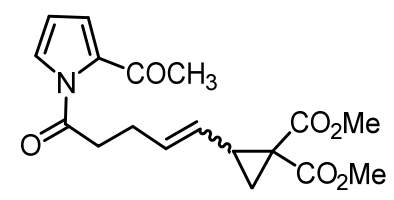

7c, colorless oil, $0.35 \mathrm{~g}, 36 \%$ yield (2.8 mmol scale), $E / Z=3.1 / 1 .{ }^{1} \mathrm{H}$ NMR (400 MHz, $\left.\mathrm{CDCl}_{3}\right) \delta 7.33(\mathrm{~s}, 1 \mathrm{H}), 7.03(\mathrm{~d}, J=2.4 \mathrm{~Hz}, 1 \mathrm{H}), 6.23(\mathrm{t}, J=3.2 \mathrm{~Hz}, 1 \mathrm{H}), 5.75-5.68(\mathrm{~m}$, $1 \mathrm{H}), 5.61-5.55\left(\mathrm{~m}, 1 \mathrm{H}^{*}\right), 5.16-5.10(\mathrm{~m}, 1 \mathrm{H}), 4.98-4.93\left(\mathrm{~m}, 1 \mathrm{H}^{*}\right), 3.724\left(\mathrm{~s}, 6 \mathrm{H}^{*}\right), 3.716$ (s, 6H), 2.94-2.85 (m, 2H), 2.75-2.41 (m, 3H), 2.46 (s, 3H), 1.68-1.60 (m, 1H), 1.56$1.52(\mathrm{~m}, 1 \mathrm{H}) .{ }^{13} \mathrm{C} \mathrm{NMR}\left(100 \mathrm{MHz}, \mathrm{CDCl}_{3}\right) \delta 188.1,173.2,173.1,169.5,167.5,167.3$, $133.1,132.2$, 131.9, 127.5, 127.4, 125.9, 125.8, 122.93, 122.86, 110.3, 52.3, 52.2, 52.1, $37.3,35.2,35.1,30.3,27.6,26.7,26.0,23.1,21.3,20.2$. IR (thin film): $v_{\max }\left(\mathrm{cm}^{-1}\right)=$ 
3004, 2953, 1720, 1657, 1537, 1434, 1409, 1362, 1331, 1263, 1208, 1124, 1084, 964 , 941, 899, 876, 832, 749, 705. HRMS (ESI) calcd for $\mathrm{C}_{18} \mathrm{H}_{21} \mathrm{NO}_{6} \mathrm{Na}[\mathrm{M}+\mathrm{Na}]^{+}: 370.1261$. Found: 370.1254.

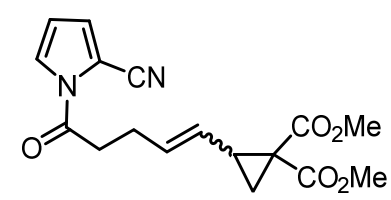

7d, colorless oil, $0.38 \mathrm{~g}$, 42\% yield (2.8 mmol scale), $E / Z=5.2 / 1 .{ }^{1} \mathrm{H}$ NMR (400 MHz, $\left.\mathrm{CDCl}_{3}\right) \delta 7.36(\mathrm{~d}, J=1.6 \mathrm{~Hz}, 1 \mathrm{H}), 6.94(\mathrm{~d}, J=2.0 \mathrm{~Hz}, 1 \mathrm{H}), 6.28(\mathrm{t}, J=3.2 \mathrm{~Hz}, 1 \mathrm{H})$,

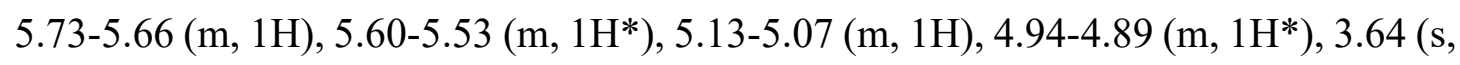
$\left.6 \mathrm{H}^{*}\right), 3.62(\mathrm{~s}, 6 \mathrm{H}), 2.98-2.90(\mathrm{~m}, 2 \mathrm{H}), 2.67-2.38(\mathrm{~m}, 3 \mathrm{H}), 1.59-1.51(\mathrm{~m}, 1 \mathrm{H}), 1.47-1.44$ $(\mathrm{m}, 1 \mathrm{H}) .{ }^{13} \mathrm{C} \mathrm{NMR}\left(100 \mathrm{MHz}, \mathrm{CDCl}_{3}\right) \delta 169.6,168.5,167.4,131.8,126.6,126.3,124.3$, $112.9,112.8,102.6,52.5,52.4,52.3,35.2,34.2,30.3,26.3,26.0,22.0,21.4,20.3$. IR (thin film): $v_{\max }\left(\mathrm{cm}^{-1}\right)=3130,3014,2954,2224,1720,1545,1436,1410,1369,1335$, 1259, 1210, 1115, 1028, 959, 900, 879, 745, 666, 619. HRMS (ESI) calcd for $\mathrm{C}_{17} \mathrm{H}_{18} \mathrm{~N}_{2} \mathrm{O}_{5} \mathrm{Na}[\mathrm{M}+\mathrm{Na}]^{+}: 353.1108$. Found: 353.1107 .

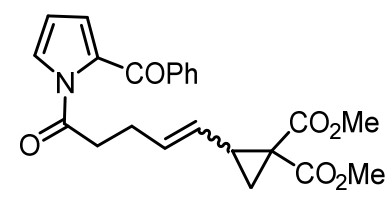

7e, colorless oil, $0.28 \mathrm{~g}, 19 \%$ yield (3.9 mmol scale), $E / Z=5.5 / 1 .{ }^{1} \mathrm{H}$ NMR (400 MHz, $\left.\mathrm{CDCl}_{3}\right) \delta 7.90(\mathrm{~d}, J=7.2 \mathrm{~Hz}, 2 \mathrm{H}), 7.59(\mathrm{t}, J=7.2 \mathrm{~Hz}, 1 \mathrm{H}), 7.47(\mathrm{t}, J=7.6 \mathrm{~Hz}, 3 \mathrm{H})$, $6.75(\mathrm{~d}, J=2.4 \mathrm{~Hz}, 1 \mathrm{H}), 6.28(\mathrm{t}, J=3.2 \mathrm{~Hz}, 1 \mathrm{H}), 5.73-5.65(\mathrm{~m}, 1 \mathrm{H}), 5.59-5.52$ (m, $\left.1 \mathrm{H}^{*}\right)$, 5.14-5.08 (m, 1H), 4.97-4.92 (m, 1H*), $3.71\left(\mathrm{~s}, 6 \mathrm{H}^{*}\right), 3.65(\mathrm{~s}, 6 \mathrm{H}), 2.98-2.88(\mathrm{~m}$, 2H), 2.74-2.40 (m, 3H), 1.68-1.58 (m, 1H), 1.55-1.52 (m, 1H). ${ }^{13} \mathrm{C}$ NMR (100 MHz, $\left.\mathrm{CDCl}_{3}\right) \delta 185.7,171.4,169.7,167.5,137.3,132.7,132.4,132.2,129.2,128.2,126.3$, 126.0, 124.1, 110.8, 57.8, 52.4, 52.2, 36.4, 36.3, 35.3, 35.2, 30.5, 27.4, 26.2, 21.8, 20.4, 18.1. IR (thin film): $v_{\max }\left(\mathrm{cm}^{-1}\right)=2953,1720,1641,1597,1577,1540,1435,1408$, 1330, 1269, 1209, 1125, 1049, 965, 891, 872, 794, 751, 723, 694. HRMS (ESI) calcd for $\mathrm{C}_{23} \mathrm{H}_{23} \mathrm{NO}_{6} \mathrm{Na}[\mathrm{M}+\mathrm{Na}]^{+}: 432.1418$. Found: 432.1418 . 


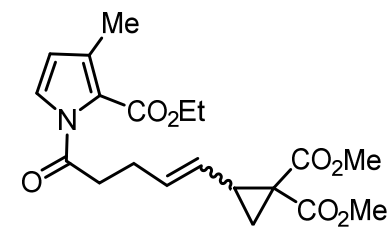

7f, colorless oil, $0.84 \mathrm{~g}, 84 \%$ yield (3.0 mmol scale), $E / Z=5.1 / 1 .{ }^{1} \mathrm{H}$ NMR (400 MHz, $\left.\mathrm{CDCl}_{3}\right) \delta$ 7.14-7.12 (m, 1H), $6.08(\mathrm{~s}, 1 \mathrm{H}), 5.78-5.71(\mathrm{~m}, 1 \mathrm{H}), 5.64-5.57\left(\mathrm{~m}, 1 \mathrm{H}^{*}\right), 5.18-$ $5.12(\mathrm{~m}, 1 \mathrm{H}), 5.00-4.95\left(\mathrm{~m}, 1 \mathrm{H}^{*}\right), 4.32(\mathrm{q}, J=7.2 \mathrm{~Hz}, 2 \mathrm{H}), 3.74\left(\mathrm{~s}, 6 \mathrm{H}^{*}\right), 3.73(\mathrm{~s}, 6 \mathrm{H})$, 2.90-2.82 (m, 2H), 2.77-2.44 (m, 3H), $2.22(\mathrm{~s}, 3 \mathrm{H}), 1.69-1.62(\mathrm{~m}, 1 \mathrm{H}), 1.57-1.55(\mathrm{~m}$, $1 \mathrm{H}), 1.35(\mathrm{t}, J=7.2 \mathrm{~Hz}, 3 \mathrm{H}) .{ }^{13} \mathrm{C} \mathrm{NMR}\left(100 \mathrm{MHz}, \mathrm{CDCl}_{3}\right) \delta 170.4,169.8,167.6,161.9$, 132.4, 132.2, 132.1, 126.2, 126.1, 123.3, 121.8, 114.0, 60.7, 52.5, 52.4, 35.7, 35.3, 30.6, 27.5, 23.0, 21.9, 20.5, 14.0, 12.2. IR (thin film): $v_{\max }\left(\mathrm{cm}^{-1}\right)=2953,1717,1578,1423$, 1385, 1365, 1284, 1255, 1207, 1124, 1102, 1059, 964, 939, 868, 836, 752, 647, 614. HRMS (ESI) calcd for $\mathrm{C}_{20} \mathrm{H}_{25} \mathrm{NO}_{7} \mathrm{Na}[\mathrm{M}+\mathrm{Na}]^{+}$: 414.1523. Found: 414.1532.

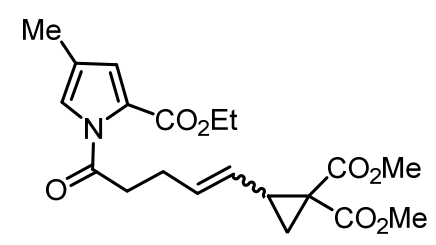

7g, colorless oil, $0.75 \mathrm{~g}$, $68 \%$ yield (2.9 mmol scale), $E / Z=5.2 / 1 .{ }^{1} \mathrm{H}$ NMR (400 MHz, $\left.\mathrm{CDCl}_{3}\right) \delta$ 7.05-7.02 (m, 1H), $6.74(\mathrm{~s}, 1 \mathrm{H}), 5.74-5.67(\mathrm{~m}, 1 \mathrm{H}), 5.59-5.53\left(\mathrm{~m}, 1 \mathrm{H}^{*}\right)$, 5.12$5.06(\mathrm{~m}, 1 \mathrm{H}), 4.95-4.90\left(\mathrm{~m}, 1 \mathrm{H}^{*}\right), 4.25(\mathrm{q}, J=7.2 \mathrm{~Hz}, 2 \mathrm{H}), 3.69\left(\mathrm{~s}, 6 \mathrm{H}^{*}\right), 3.68(\mathrm{~s}, 6 \mathrm{H})$, 2.93-2.84 (m, 2H), 2.73-2.40 (m, 3H), $2.01(\mathrm{~s}, 3 \mathrm{H}), 1.65-1.58(\mathrm{~m}, 1 \mathrm{H}), 1.53-1.49(\mathrm{~m}$, $1 \mathrm{H}), 1.30(\mathrm{t}, J=7.2 \mathrm{~Hz}, 3 \mathrm{H}) .{ }^{13} \mathrm{C} \mathrm{NMR}\left(100 \mathrm{MHz}, \mathrm{CDCl}_{3}\right) \delta 171.0,169.9,167.7,161.1$, 132.5, 132.3, 126.1, 124.8, 123.9, 123.2, 121.3, 60.8, 52.6, 52.4, 36.3, 35.4, 30.7, 27.6, 23.1, 21.7, 20.6, 14.1, 11.3. IR (thin film): $v_{\max }\left(\mathrm{cm}^{-1}\right)=2982,2953,1717,1459,1436$, 1398, 1366, 1332, 1281, 1242, 1126, 1095, 1018, 961, 900, 869, 755, 704, 601. HRMS (ESI) calcd for $\mathrm{C}_{20} \mathrm{H}_{25} \mathrm{NO}_{7} \mathrm{Na}[\mathrm{M}+\mathrm{Na}]^{+}: 414.1523$. Found: 414.1528. 


\subsection{General procedure for the dearomatized [5 +2] cycloadditions (using 1a as an}

example).

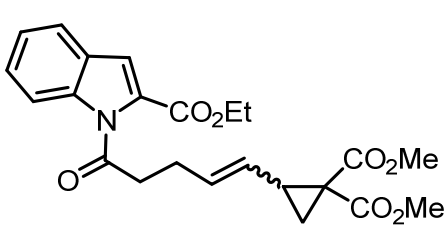

$1 \mathbf{a}(E / Z=5.5 / 1)$
$\mathrm{H}_{3} \mathrm{CN}, \mathrm{rt}$
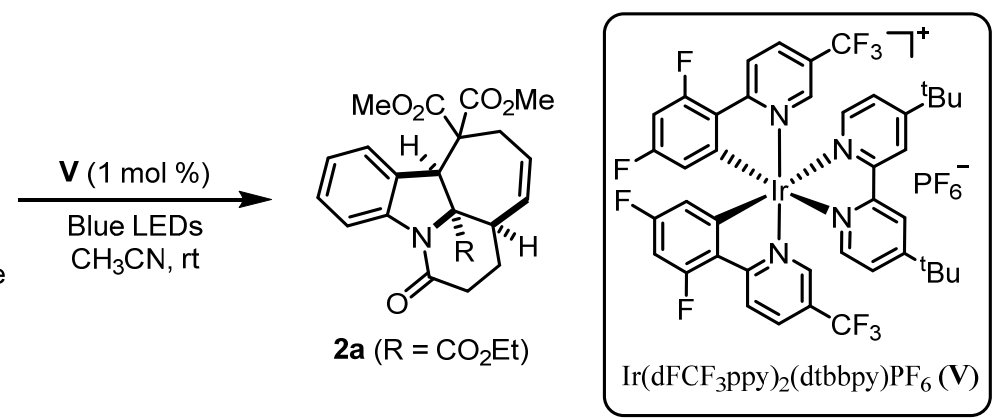

To a flame-dried sealed tube were added indole derivative $1 \mathrm{a}(85.4 \mathrm{mg}, 0.2 \mathrm{mmol})$ and photosensitizer $\mathbf{V}(2.2 \mathrm{mg}, 0.002 \mathrm{mmol})$ and anhydrous acetonitrile $(20 \mathrm{~mL})$. The reaction mixture was degassed via freeze-pump-thaw for 3 cycles. After the reaction mixture was thoroughly degassed, the vial was sealed and positioned approximately $10 \mathrm{~cm}$ from blue LEDs $\left(24 \mathrm{~W}, \lambda_{\max }=455 \mathrm{~nm}\right.$ ). Then the reaction mixture was stirred at room temperature for the indicated time (monitored by TLC) under argon atmosphere. Afterwards, the reaction mixture was concentrated by rotary evaporation. Then the residue was purified by silica gel column chromatography $(\mathrm{PE} / \mathrm{EtOAc}=2 / 1)$ to afford the desired product $\mathbf{2 a}$.

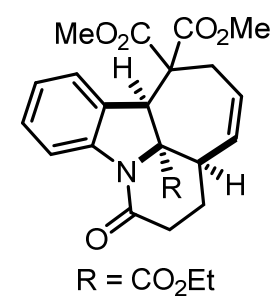

2a, yellow solid, $71.0 \mathrm{mg}, 83 \%$ yield, m.p. $=138.1-141.3{ }^{\circ} \mathrm{C} .{ }^{1} \mathrm{H}$ NMR $(400 \mathrm{MHz}$, $\left.\mathrm{CDCl}_{3}\right) \delta 8.35(\mathrm{~d}, J=8.0 \mathrm{~Hz}, 1 \mathrm{H}), 7.29(\mathrm{t}, J=7.6 \mathrm{~Hz}, 1 \mathrm{H}), 6.98(\mathrm{t}, J=7.6 \mathrm{~Hz}, 1 \mathrm{H})$, $6.78(\mathrm{~d}, J=7.6 \mathrm{~Hz}, 1 \mathrm{H}), 5.77-5.72(\mathrm{~m}, 1 \mathrm{H}), 5.24(\mathrm{~d}, J=11.6 \mathrm{~Hz}, 1 \mathrm{H}), 4.72(\mathrm{~s}, 1 \mathrm{H})$, 4.25-4.12 (m, 2H), 3.79 (s, 3H), $3.76(\mathrm{~s}, 3 \mathrm{H}), 3.69-3.67$ (m, 1H), 2.69-2.46 (m, 3H), 2.23-2.17 (m, 1H), 1.82-1.77 (m, 1H), 1.74-1.65 (m, 1H), $1.21(\mathrm{t}, J=7.2 \mathrm{~Hz}, 3 \mathrm{H}) .{ }^{13} \mathrm{C}$ NMR $\left(100 \mathrm{MHz}, \mathrm{CDCl}_{3}\right) \delta 173.2,170.7,169.1,168.9,143.8,129.7,129.6,129.4,125.7$, $124.4,123.8,117.4,63.0,62.5,52.9,52.7,50.7,37.9,28.7,26.5,26.4,13.9$. IR (thin film): $v_{\max }\left(\mathrm{cm}^{-1}\right)=2948,1734,1666,1597,1475,1384,1288,1222,1177,1138,972$, 
951, 773, 758, 678, 614, 549, 427. HRMS (ESI) calcd for $\mathrm{C}_{23} \mathrm{H}_{26} \mathrm{NO}_{7}[\mathrm{M}+\mathrm{H}]^{+}$: 428.1704. Found: 428.1703.

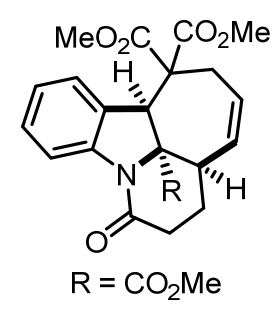

2b, colorless oil, $60.1 \mathrm{mg}, 73 \%$ yield. ${ }^{1} \mathrm{H}$ NMR (400 $\left.\mathrm{MHz}, \mathrm{CDCl}_{3}\right) \delta 8.29$ (d, $J=8.4$ $\mathrm{Hz}, 1 \mathrm{H}), 7.22$ (t, $J=7.6 \mathrm{~Hz}, 1 \mathrm{H}), 6.91(\mathrm{t}, J=7.6 \mathrm{~Hz}, 1 \mathrm{H}), 6.70(\mathrm{~d}, J=7.6 \mathrm{~Hz}, 1 \mathrm{H})$, 5.70-5.65 (m, 1H), $5.17(\mathrm{~d}, J=9.0 \mathrm{~Hz}, 1 \mathrm{H}), 4.66(\mathrm{~s}, 1 \mathrm{H}), 3.72(\mathrm{~s}, 3 \mathrm{H}), 3.69(\mathrm{~s}, 3 \mathrm{H})$, $3.68(\mathrm{~s}, 3 \mathrm{H}), 3.63-3.57(\mathrm{~m}, 1 \mathrm{H}), 2.63-2.39(\mathrm{~m}, 3 \mathrm{H}), 2.14-2.09(\mathrm{~m}, 1 \mathrm{H}), 1.75-1.70(\mathrm{~m}$, $1 \mathrm{H}), 1.62-1.53(\mathrm{~m}, 1 \mathrm{H}) .{ }^{13} \mathrm{C} \mathrm{NMR}\left(100 \mathrm{MHz}, \mathrm{CDCl}_{3}\right) \delta 173.8,170.8,169.0,168.8$, 143.7, 129.7, 129.4, 129.4, 125.6, 124.4, 123.8, 117.4, 62.9, 53.5, 52.9, 52.7, 50.6, 37.9, 28.7, 26.5, 26.5. IR (thin film): $v_{\max }\left(\mathrm{cm}^{-1}\right)=2921,1730,1659,1596,1474,1435,1385$, 1283, 1237, 1217, 1195, 1172, 1135, 1058, 1037, 982, 927, 815, 787, 773. HRMS (ESI) calcd for $\mathrm{C}_{22} \mathrm{H}_{24} \mathrm{NO}_{7}[\mathrm{M}+\mathrm{H}]^{+}:$414.1547. Found: 414.1548 .

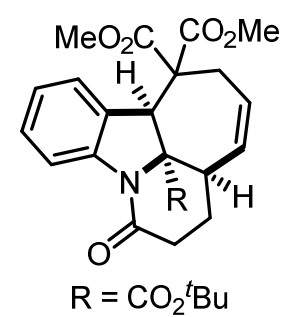

2c, yellow oil, $60.1 \mathrm{mg}, 66 \%$ yield. ${ }^{1} \mathrm{H}$ NMR (400 MHz, $\left.\mathrm{CDCl}_{3}\right) \delta 8.27$ (d, $J=8.4 \mathrm{~Hz}$, $1 \mathrm{H}), 7.21(\mathrm{t}, J=6.0 \mathrm{~Hz}, 1 \mathrm{H}), 6.90(\mathrm{t}, J=7.6 \mathrm{~Hz}, 1 \mathrm{H}), 6.72(\mathrm{~d}, J=7.6 \mathrm{~Hz}, 1 \mathrm{H}), 5.68-$ $5.63(\mathrm{~m}, 1 \mathrm{H}), 5.16(\mathrm{~d}, J=11.6 \mathrm{~Hz}, 1 \mathrm{H}), 4.62(\mathrm{~s}, 1 \mathrm{H}), 3.71(\mathrm{~s}, 3 \mathrm{H}), 3.68(\mathrm{~s}, 3 \mathrm{H}), 3.60-$ $3.49(\mathrm{~m}, 1 \mathrm{H}), 2.60-2.37(\mathrm{~m}, 3 \mathrm{H}), 2.17-2.11(\mathrm{~m}, 1 \mathrm{H}), 1.73-1.69(\mathrm{~m}, 2 \mathrm{H}), 1.27(\mathrm{~s}, 9 \mathrm{H})$. ${ }^{13} \mathrm{C}$ NMR $\left(100 \mathrm{MHz}, \mathrm{CDCl}_{3}\right) \delta 172.2,170.6,169.2,169.0,144.0,129.8,129.6,129.2$, $126.0,124.2,123.7,117.3,83.0,77.8,77.3,63.0,52.8,52.6,50.8,37.9,28.8,27.5,26.5$, 26.2. IR (thin film): $v_{\max }\left(\mathrm{cm}^{-1}\right)=2952,1732,1665,1597,1477,1462,1434,1386$, 1338, 1287, 1244, 1197, 1176, 1150, 1063, 1039, 805, 756, 700, 675. HRMS (ESI) calcd for $\mathrm{C}_{25} \mathrm{H}_{30} \mathrm{NO}_{7}[\mathrm{M}+\mathrm{H}]^{+}$: 456.2017. Found: 456.2016. 


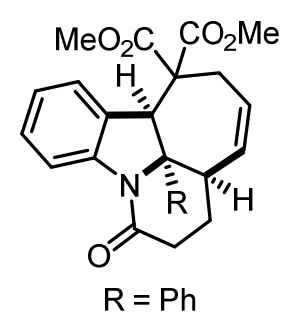

2d, white solid, $64.7 \mathrm{mg}, 76 \%$ yield, m.p. $=192.0-194.8^{\circ} \mathrm{C} .{ }^{1} \mathrm{H} \mathrm{NMR}\left(400 \mathrm{MHz}, \mathrm{CDCl}_{3}\right)$ $\delta 8.39(\mathrm{~d}, J=8.0 \mathrm{~Hz}, 1 \mathrm{H}), 8.16(\mathrm{~d}, J=8.4 \mathrm{~Hz}, 1 \mathrm{H}), 7.37(\mathrm{t}, J=7.2 \mathrm{~Hz}, 1 \mathrm{H}), 7.20(\mathrm{~d}, J$ $=9.2 \mathrm{~Hz}, 1 \mathrm{H}), 7.11(\mathrm{t}, J=7.6 \mathrm{~Hz}, 1 \mathrm{H}), 7.01(\mathrm{t}, J=7.6 \mathrm{~Hz}, 1 \mathrm{H}), 6.85(\mathrm{t}, J=7.6 \mathrm{~Hz}, 1 \mathrm{H})$, $6.71(\mathrm{~d}, J=8.0 \mathrm{~Hz}, 1 \mathrm{H}), 6.55(\mathrm{~d}, J=7.6 \mathrm{~Hz}, 1 \mathrm{H}), 5.75-5.69(\mathrm{~m}, 1 \mathrm{H}), 5.24$ (d, $J=12.0$ $\mathrm{Hz}, 1 \mathrm{H}), 4.51$ (s, 1H), 3.73 (s, 3H), 3.68 (s, 3H), 3.59-3.51 (m, 1H), 2.65-2.59 (m, 1H), 2.51-2.42 (m, 1H), 2.32-2.16 (m, 2H), 1.43-1.25 (m, 2H). $\left.{ }^{13} \mathrm{C} \mathrm{NMR} \mathrm{(100} \mathrm{MHz,} \mathrm{CDCl}_{3}\right)$ $\delta 171.9,169.7,169.4,147.7,143.6,130.4,129.4,129.4,129.1,127.8,127.1,126.7$, $125.9,124.9,124.5,124.0,117.5,76.6,63.3,56.5,52.9,52.6,42.4,28.7,26.7,25.4$. IR (thin film): $v_{\max }\left(\mathrm{cm}^{-1}\right)=2919,1755,1732,1647,1595,1478,1460,1434,1386,1334$, 1286, 1258, 1229, 1193, 1143, 1075, 1032, 1003, 978, 759, 731. HRMS (ESI) calcd for $\mathrm{C}_{26} \mathrm{H}_{26} \mathrm{NO}_{5}[\mathrm{M}+\mathrm{H}]^{+}:$432.1806. Found: 432.1805.

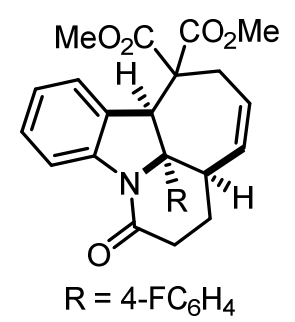

2e, colorless oil, $58.0 \mathrm{mg}, 65 \%$ yield. ${ }^{1} \mathrm{H}$ NMR (400 MHz, $\left.\mathrm{CDCl}_{3}\right) \delta 8.42(\mathrm{~d}, J=8.0$ $\mathrm{Hz}, 1 \mathrm{H}), 8.21-8.18(\mathrm{~m}, 1 \mathrm{H}), 7.27$ (t, $J=8.4 \mathrm{~Hz}, 1 \mathrm{H}), 7.11$ (t, $J=7.2 \mathrm{~Hz}, 1 \mathrm{H}), 6.92$ (t, $J$ $=7.2 \mathrm{~Hz}, 1 \mathrm{H}), 6.73-6.71(\mathrm{~m}, 2 \mathrm{H}), 6.60(\mathrm{~d}, J=7.6 \mathrm{~Hz}, 1 \mathrm{H}), 5.80-5.74(\mathrm{~m}, 1 \mathrm{H}), 5.28(\mathrm{~d}$, $J=12.0 \mathrm{~Hz}, 1 \mathrm{H}), 4.49(\mathrm{~s}, 1 \mathrm{H}), 3.77(\mathrm{~s}, 3 \mathrm{H}), 3.73(\mathrm{~s}, 3 \mathrm{H}), 3.56(\mathrm{~s}, 1 \mathrm{H}), 2.70-2.63(\mathrm{~m}$, $1 \mathrm{H}), 2.57-2.47(\mathrm{~m}, 1 \mathrm{H}), 2.37-2.31(\mathrm{~m}, 1 \mathrm{H}), 2.24-2.19(\mathrm{~m}, 1 \mathrm{H}), 1.50-1.44(\mathrm{~m}, 1 \mathrm{H}), 1.37-$ $1.28(\mathrm{~m}, 1 \mathrm{H}) .{ }^{13} \mathrm{C} \mathrm{NMR}\left(100 \mathrm{MHz}, \mathrm{CDCl}_{3}\right) \delta 171.9,169.6,169.3,161.6(\mathrm{~d}, J=245.0$ $\mathrm{Hz}), 143.5,130.2,129.5,129.2,127.7$ (d, $J=8.0 \mathrm{~Hz}), 126.5,126.3,126.2$, 124.9, 124.2, 117.5, $116.7(\mathrm{~d}, J=22.0 \mathrm{~Hz}), 114.2(\mathrm{~d}, J=22.0 \mathrm{~Hz}), 76.3,63.2,56.7,53.0,52.6,42.5$, 28.7, 26.6, 25.3. ${ }^{19} \mathrm{~F}$ NMR (376 MHz, $\left.\mathrm{CDCl}_{3}\right) \delta-115.5 \sim-115.6(\mathrm{~m}, 1 \mathrm{~F})$. IR (thin film): $v_{\max }\left(\mathrm{cm}^{-1}\right)=2916,1765,1730,1661,1598,1504,1478,1462,1437,1389,1343,1285$, 
1264, 1224, 1195, 1167, 1144, 1077, 1033, 1005, 941, 853, 757. HRMS (ESI) calcd for $\mathrm{C}_{26} \mathrm{H}_{25} \mathrm{NO}_{5} \mathrm{~F}[\mathrm{M}+\mathrm{H}]^{+}:$450.1711. Found: 450.1711 .

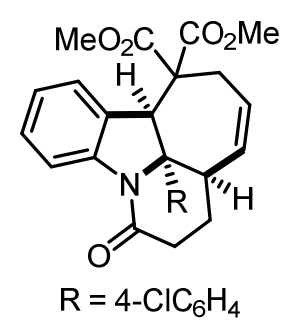

2f, yellow oil, $77.4 \mathrm{mg}, 83 \%$ yield. ${ }^{1} \mathrm{H}$ NMR (400 MHz, $\left.\mathrm{CDCl}_{3}\right) \delta 8.37(\mathrm{~d}, J=8.0 \mathrm{~Hz}$, $1 \mathrm{H}), 8.12(\mathrm{dd}, J=8.4,2.4 \mathrm{~Hz}, 1 \mathrm{H}), 7.33$ (dd, $J=8.8,2.4 \mathrm{~Hz}, 1 \mathrm{H}), 7.22(\mathrm{t}, J=7.6 \mathrm{~Hz}$, $1 \mathrm{H}), 6.97(\mathrm{dd}, J=8.4,2.4 \mathrm{~Hz}, 1 \mathrm{H}), 6.87$ (t, $J=7.6 \mathrm{~Hz}, 1 \mathrm{H}), 6.63(\mathrm{dd}, J=8.4,2.4 \mathrm{~Hz}$, $1 \mathrm{H}), 6.55(\mathrm{~d}, J=7.2 \mathrm{~Hz}, 1 \mathrm{H}), 5.75-5.70(\mathrm{~m}, 1 \mathrm{H}), 5.23$ (d, $J=12.0 \mathrm{~Hz}, 1 \mathrm{H}), 4.43$ (s, $1 \mathrm{H}), 3.72(\mathrm{~s}, 3 \mathrm{H}), 3.68(\mathrm{~s}, 3 \mathrm{H}), 3.51(\mathrm{~s}, 1 \mathrm{H}), 2.65-2.59(\mathrm{~m}, 1 \mathrm{H}), 2.52-2.42(\mathrm{~m}, 1 \mathrm{H})$, 2.32-2.26 (m, 1H), 2.19-2.14 (m, 1H), 1.46-1.40 (m, 1H), 1.33-1.24 (m, 1H). ${ }^{13} \mathrm{C} \mathrm{NMR}$ $\left(100 \mathrm{MHz}, \mathrm{CDCl}_{3}\right) \delta 171.9,169.5,169.3,146.3,143.4,132.9,130.1,129.7,129.5$, $129.2,127.8,127.5,126.4,126.0,124.9,124.2,117.5,76.3,63.2,56.6,53.0,52.6,42.3$, 28.6, 26.6, 25.3. IR (thin film): $v_{\max }\left(\mathrm{cm}^{-1}\right)=2918,1763,1732,1658,1593,1477,1460$, 1436, 1388, 1343, 1286, 1252, 1226, 1196, 1180, 1142, 1093, 1036, 972, 902, 752. HRMS (ESI) calcd for $\mathrm{C}_{26} \mathrm{H}_{25} \mathrm{NO}_{5} \mathrm{Cl}[\mathrm{M}+\mathrm{H}]^{+}:$466.1416. Found: 466.1409.

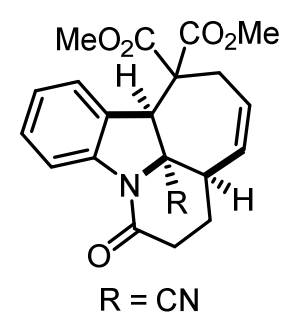

2g, white solid, $60.5 \mathrm{mg}, 79 \%$ yield, m.p. $=192.1-193.7^{\circ} \mathrm{C} .{ }^{1} \mathrm{H}$ NMR $\left(400 \mathrm{MHz}, \mathrm{CDCl}_{3}\right)$ $\delta 8.20(\mathrm{~d}, J=8.4 \mathrm{~Hz}, 1 \mathrm{H}), 7.28(\mathrm{t}, J=8.0,1 \mathrm{H}), 7.03(\mathrm{t}, J=7.6 \mathrm{~Hz}, 1 \mathrm{H}), 6.83(\mathrm{~d}, J=$ $7.6 \mathrm{~Hz}, 1 \mathrm{H}), 5.73-5.68(\mathrm{~m}, 1 \mathrm{H}), 5.19$ (d, $J=12.0 \mathrm{~Hz}, 1 \mathrm{H}), 4.79(\mathrm{~s}, 1 \mathrm{H}), 3.76(\mathrm{~s}, 3 \mathrm{H})$, $3.68(\mathrm{~s}, 3 \mathrm{H}), 3.56(\mathrm{~s}, 1 \mathrm{H}), 2.67-2.43(\mathrm{~m}, 4 \mathrm{H}), 2.07-1.93(\mathrm{~m}, 2 \mathrm{H}) .{ }^{13} \mathrm{C} \mathrm{NMR}(100 \mathrm{MHz}$, $\left.\mathrm{CDCl}_{3}\right) \delta 170.4,168.2,167.3,141.7,130.2,130.0,127.3,125.3,125.0,124.9,120.9$, 118.2, 68.9, 62.3, 53.2, 53.0, 38.9, 28.5, 27.1, 26.5. IR (thin film): $v_{\max }\left(\mathrm{cm}^{-1}\right)=2919$, $1728,1675,1463,1440,1373,1339,1311,1286,1258,1232,1205,1179,1146,1061$, 1036, 969, 934, 816, 770, 755. HRMS (ESI) calcd for $\mathrm{C}_{21} \mathrm{H}_{21} \mathrm{~N}_{2} \mathrm{O}_{5}[\mathrm{M}+\mathrm{H}]^{+}: 381.1445$. Found: 381.1454. 


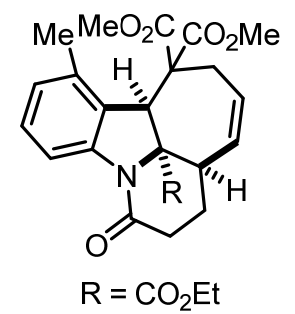

2h, yellow solid, $65.0 \mathrm{mg}, 73 \%$ yield, m.p. $=171.4-173.1{ }^{\circ} \mathrm{C} .{ }^{1} \mathrm{H}$ NMR $(400 \mathrm{MHz}$, $\left.\mathrm{CDCl}_{3}\right) \delta 8.19(\mathrm{~d}, J=8.0 \mathrm{~Hz}, 1 \mathrm{H}), 7.17(\mathrm{t}, J=8.0 \mathrm{~Hz}, 1 \mathrm{H}), 6.80(\mathrm{t}, J=8.0 \mathrm{~Hz}, 1 \mathrm{H})$, 5.76-5.71 (m, 1H), $5.20(\mathrm{~d}, J=12.0 \mathrm{~Hz}, 1 \mathrm{H}), 4.78(\mathrm{~s}, 1 \mathrm{H}), 4.14(\mathrm{q}, J=7.2 \mathrm{~Hz}, 2 \mathrm{H})$, $3.73(\mathrm{~s}, 3 \mathrm{H}), 3.62(\mathrm{~s}, 3 \mathrm{H}), 3.58(\mathrm{~s}, 1 \mathrm{H}), 2.59-2.39(\mathrm{~m}, 4 \mathrm{H}), 2.06(\mathrm{~s}, 3 \mathrm{H}), 1.77-1.71(\mathrm{~m}$, 1H), 1.67-1.58 (m, 1H), 1.13 (t, $J=7.2 \mathrm{~Hz}, 3 \mathrm{H}) .{ }^{13} \mathrm{C} \mathrm{NMR}\left(100 \mathrm{MHz}, \mathrm{CDCl}_{3}\right) \delta 173.0$, 171.5, 169.7, 168.8, 144.1, 134.7, 130.2, 129.5, 129.1, 125.7, 124.1, 114.4, 78.4, 62.4, $62.3,52.62,52.55,49.0,38.1,28.8,27.2,26.1,18.4,13.9$. IR (thin film): $v_{\max }\left(\mathrm{cm}^{-1}\right)=$ 2920, 1740, 1668, 1652, 1586, 1435, 1376, 1335, 1286, 1217, 1123, 1064, 1046, 1019 , 958, 857, 790, 743, 731. HRMS (ESI) calcd for $\mathrm{C}_{24} \mathrm{H}_{28} \mathrm{NO}_{7}[\mathrm{M}+\mathrm{H}]^{+}$: 442.1860. Found: 442.1863.

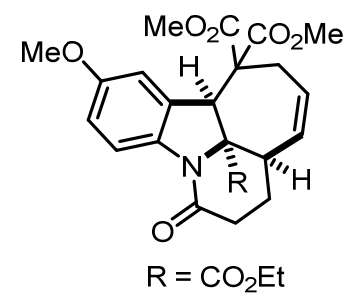

2i, white solid, $69.2 \mathrm{mg}, 76 \%$ yield, m.p. $=185.0-186.7^{\circ} \mathrm{C} .{ }^{1} \mathrm{H} \mathrm{NMR}\left(400 \mathrm{MHz}, \mathrm{CDCl}_{3}\right)$ $\delta 8.24(\mathrm{~d}, J=8.8 \mathrm{~Hz}, 1 \mathrm{H}), 6.78(\mathrm{dd}, J=8.8,2.4 \mathrm{~Hz}, 1 \mathrm{H}), 6.32(\mathrm{~d}, J=2.8 \mathrm{~Hz}, 1 \mathrm{H}), 5.74-$ $5.68(\mathrm{~m}, 1 \mathrm{H}), 5.21(\mathrm{~d}, J=11.6 \mathrm{~Hz}, 1 \mathrm{H}), 4.66(\mathrm{~s}, 1 \mathrm{H}), 4.22-4.10(\mathrm{~m}, 2 \mathrm{H}), 3.77(\mathrm{~s}, 3 \mathrm{H})$, $3.73(\mathrm{~s}, 3 \mathrm{H}), 3.70(\mathrm{~s}, 3 \mathrm{H}), 3.62(\mathrm{~s}, 1 \mathrm{H}), 2.68-2.62(\mathrm{~m}, 1 \mathrm{H}), 2.54-2.40(\mathrm{~m}, 2 \mathrm{H}), 2.22-2.15$ $(\mathrm{m}, 1 \mathrm{H}), 1.78-1.73(\mathrm{~m}, 1 \mathrm{H}), 1.70-1.61(\mathrm{~m}, 1 \mathrm{H}), 1.19(\mathrm{t}, J=7.2 \mathrm{~Hz}, 3 \mathrm{H}) .{ }^{13} \mathrm{C} \mathrm{NMR}(100$ $\left.\mathrm{MHz}_{2} \mathrm{CDCl}_{3}\right) \delta 173.2,170.6,169.0,168.2,156.1,137.5,129.6,129.2,127.2,118.0$, $113.9,111.0,77.6,62.9,62.5,55.4,52.8,52.6,50.8,37.9,28.5,26.4,26.4,13.9$. IR (thin film): $v_{\max }\left(\mathrm{cm}^{-1}\right)=2951,1731,1656,1595,1485,1434,1387,1323,1280,1265$, $1219,1126,1060,1032,971,952,879,818,784$. HRMS (ESI) calcd for $\mathrm{C}_{24} \mathrm{H}_{28} \mathrm{NO}_{8}$ $[\mathrm{M}+\mathrm{H}]^{+}:$458.1809. Found: 458.1812. 


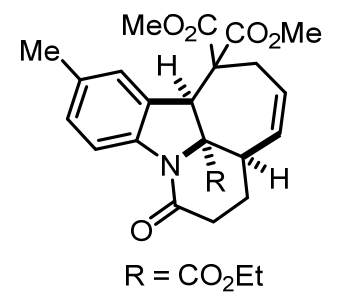

2j, white solid, $71.5 \mathrm{mg}, 81 \%$ yield, m.p. $=192.9-195.4{ }^{\circ} \mathrm{C} .{ }^{1} \mathrm{H} \mathrm{NMR}\left(400 \mathrm{MHz}, \mathrm{CDCl}_{3}\right)$ $\delta 8.17(\mathrm{~d}, J=8.4 \mathrm{~Hz}, 1 \mathrm{H}), 7.05(\mathrm{~d}, J=8.0 \mathrm{~Hz}, 1 \mathrm{H}), 6.53(\mathrm{~s}, 1 \mathrm{H}), 5.72-5.67(\mathrm{~m}, 1 \mathrm{H})$, $5.19(\mathrm{~d}, J=12.0 \mathrm{~Hz}, 1 \mathrm{H}), 4.63(\mathrm{~s}, 1 \mathrm{H}), 4.21-4.07(\mathrm{~m}, 2 \mathrm{H}), 3.74(\mathrm{~s}, 3 \mathrm{H}), 3.71(\mathrm{~s}, 3 \mathrm{H})$, $3.62(\mathrm{~s}, 1 \mathrm{H}), 2.64-2.41(\mathrm{~m}, 3 \mathrm{H}), 2.21(\mathrm{~s}, 3 \mathrm{H}), 2.19-2.13(\mathrm{~m}, 1 \mathrm{H}), 1.77-1.59(\mathrm{~m}, 2 \mathrm{H})$, $1.18(\mathrm{t}, J=7.2 \mathrm{~Hz}, 3 \mathrm{H}) .{ }^{13} \mathrm{C} \mathrm{NMR}\left(100 \mathrm{MHz}, \mathrm{CDCl}_{3}\right) \delta 173.2,170.7,169.1,168.5$, $141.5,133.2,130.2,129.6,129.3,125.7,125.0,117.0,77.5,63.0,62.5,52.8,52.5,50.7$, 37.9, 28.6, 26.4, 26.3, 21.1, 13.9. IR (thin film): $v_{\max }\left(\mathrm{cm}^{-1}\right)=2920,1733,1661,1487$, 1430, 1384, 1338, 1283, 1220, 1177, 1137, 1103, 1060, 1039, 1017, 951, 919, 830, 786. HRMS (ESI) calcd for $\mathrm{C}_{24} \mathrm{H}_{28} \mathrm{NO}_{7}[\mathrm{M}+\mathrm{H}]^{+}: 442.1860$. Found: 442.1867.

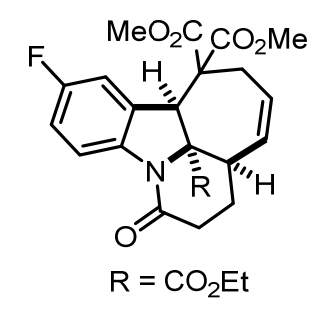

2k, yellow oil, $64.9 \mathrm{mg}, 73 \%$ yield. ${ }^{1} \mathrm{H} \mathrm{NMR}\left(400 \mathrm{MHz}, \mathrm{CDCl}_{3}\right) \delta 8.31$ (dd, $J=9.2$, $5.2 \mathrm{~Hz}, 1 \mathrm{H}), 6.97(\mathrm{td}, J=8.8,2.8 \mathrm{~Hz}, 1 \mathrm{H}), 6.47(\mathrm{dd}, J=8.4,2.4 \mathrm{~Hz}, 1 \mathrm{H}), 5.76-5.71(\mathrm{~m}$, 1H), 5.23 (d, $J=11.6 \mathrm{~Hz}, 1 \mathrm{H}), 4.68(\mathrm{~s}, 1 \mathrm{H}), 4.25-4.11(\mathrm{~m}, 2 \mathrm{H}), 3.78$ (s, 3H), 3.74 (s, $3 \mathrm{H}), 3.64(\mathrm{~s}, 1 \mathrm{H}), 2.71-2.65(\mathrm{~m}, 1 \mathrm{H}), 2.57-2.43(\mathrm{~m}, 2 \mathrm{H}), 2.19-2.14(\mathrm{~m}, 1 \mathrm{H}), 1.81-1.63$ (m, 2H), $1.21(\mathrm{t}, J=7.2 \mathrm{~Hz}, 3 \mathrm{H}) .{ }^{13} \mathrm{C} \mathrm{NMR}\left(100 \mathrm{MHz}, \mathrm{CDCl}_{3}\right) \delta 173.0,170.4,168.9$, 168.6, 159.0 (d, $J=242.0 \mathrm{~Hz}), 140.0,129.5,129.1,127.7$ (d, $J=8.0 \mathrm{~Hz}), 118.4$ (d, $J=$ $8.0 \mathrm{~Hz}), 116.1(\mathrm{~d}, J=22.0 \mathrm{~Hz}), 111.8(\mathrm{~d}, J=24.0 \mathrm{~Hz}), 77.8,62.8,62.7,53.0,52.8,50.5$, 37.9, 28.5, 26.5, 26.4, 13.9. ${ }^{19} \mathrm{~F}$ NMR $\left(376 \mathrm{MHz}, \mathrm{CDCl}_{3}\right) \delta-118.2 \sim-118.3(\mathrm{~m}, 1 \mathrm{~F})$. IR (thin film): $v_{\max }\left(\mathrm{cm}^{-1}\right)=2953,1736,1664,1610,1479,1436,1384,1313,1199,1150$, 1122, 1062, 1020, 961, 924, 870, 827, 791, 752. HRMS (ESI) calcd for $\mathrm{C}_{23} \mathrm{H}_{25} \mathrm{NO}_{7} \mathrm{~F}$ $[\mathrm{M}+\mathrm{H}]^{+}:$446.1610. Found: 446.1608 . 


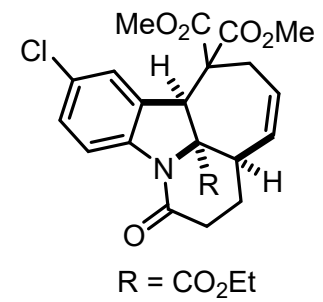

2l, white solid, $74.9 \mathrm{mg}, 82 \%$ yield, m.p. $=173.4-175.5^{\circ} \mathrm{C} .{ }^{1} \mathrm{H} \mathrm{NMR}\left(400 \mathrm{MHz}, \mathrm{CDCl}_{3}\right)$ $\delta 8.23(\mathrm{~d}, J=8.8,1 \mathrm{H}), 7.18(\mathrm{dd}, J=8.8,2.0 \mathrm{~Hz}, 1 \mathrm{H}), 6.68(\mathrm{~d}, J=1.6 \mathrm{~Hz}, 1 \mathrm{H}), 5.70$ $5.65(\mathrm{~m}, 1 \mathrm{H}), 5.17(\mathrm{~d}, J=11.6 \mathrm{~Hz}, 1 \mathrm{H}), 4.61(\mathrm{~s}, 1 \mathrm{H}), 4.19-4.07(\mathrm{~m}, 2 \mathrm{H}), 3.73(\mathrm{~s}, 3 \mathrm{H})$, $3.69(\mathrm{~s}, 3 \mathrm{H}), 3.59(\mathrm{~s}, 1 \mathrm{H}), 2.65-2.59(\mathrm{~m}, 1 \mathrm{H}), 2.51-2.38(\mathrm{~m}, 2 \mathrm{H}), 2.13-2.07(\mathrm{~m}, 1 \mathrm{H})$, 1.76-1.70 (m, 1H), 1.66-1.57 (m, 1H), 1.17 (t, $J=7.2 \mathrm{~Hz}, 3 \mathrm{H}) .{ }^{13} \mathrm{C}$ NMR (100 MHz, $\left.\mathrm{CDCl}_{3}\right) \delta 172.8,170.4,168.9,168.8,142.5,129.6,129.5,129.2,128.7,127.8,124.8$, $118.3,77.7,62.9,62.7,53.0,52.8,50.5,37.8,28.6,26.5,26.4,13.9$. IR (thin film): $v_{\max }$ $\left(\mathrm{cm}^{-1}\right)=2919,1733,1665,1472,1434,1378,1337,1285,1261,1236,1220,1172$, 1100, 1018, 970, 867, 834, 765, 743. HRMS (ESI) calcd for $\mathrm{C}_{23} \mathrm{H}_{25} \mathrm{NO}_{7} \mathrm{Cl}[\mathrm{M}+\mathrm{H}]^{+}$: 462.1314. Found: 462.1323.

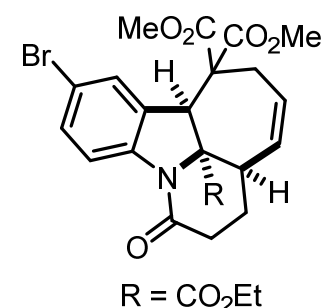

2m, colorless oil, $75.1 \mathrm{mg}, 73 \%$ yield. ${ }^{1} \mathrm{H}$ NMR (400 $\left.\mathrm{MHz} \mathrm{CDCl}_{3}\right) \delta 8.20(\mathrm{~d}, J=8.8$, $1 \mathrm{H}), 7.35(\mathrm{dd}, J=8.4,1.6 \mathrm{~Hz}, 1 \mathrm{H}), 6.86(\mathrm{~s}, 1 \mathrm{H}), 5.73-5.67(\mathrm{~m}, 1 \mathrm{H}), 5.20(\mathrm{~d}, J=12.0$ $\mathrm{Hz}, 1 \mathrm{H}), 4.64(\mathrm{~s}, 1 \mathrm{H}), 4.22-4.10(\mathrm{~m}, 2 \mathrm{H}), 3.75(\mathrm{~s}, 3 \mathrm{H}), 3.72(\mathrm{~s}, 3 \mathrm{H}), 3.62(\mathrm{~s}, 1 \mathrm{H}), 2.68-$ $2.61(\mathrm{~m}, 1 \mathrm{H}), 2.54-2.40(\mathrm{~m}, 2 \mathrm{H}), 2.15-2.10(\mathrm{~m}, 1 \mathrm{H}), 1.78-1.73(\mathrm{~m}, 1 \mathrm{H}), 1.69-1.60(\mathrm{~m}$, $1 \mathrm{H}), 1.20(\mathrm{t}, J=7.2 \mathrm{~Hz}, 3 \mathrm{H}) .{ }^{13} \mathrm{C} \mathrm{NMR}\left(100 \mathrm{MHz}, \mathrm{CDCl}_{3}\right) \delta 172.8,170.4,168.90$, $168.85,142.9,132.5,129.5,129.1,128.1,127.7,118.7,116.2,77.6,63.0,62.7,53.0$, 52.8, 50.4, 37.8, 28.6, 26.4, 26.3, 13.9. IR (thin film): $v_{\max }\left(\mathrm{cm}^{-1}\right)=2919,1732,1665$, 1470, 1432, 1376, 1336, 1284, 1218, 1171, 1133, 1099, 1060, 1037, 947, 928, 864, 763, 740. HRMS (ESI) calcd for $\mathrm{C}_{23} \mathrm{H}_{25} \mathrm{NO}_{7}{ }^{79} \mathrm{Br}[\mathrm{M}+\mathrm{H}]^{+}:$506.0809. Found: 506.0801. 


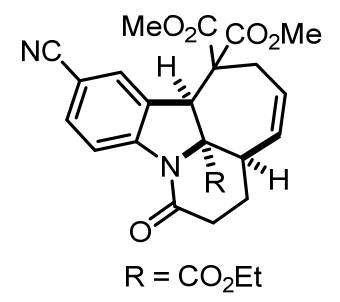

2n, colorless oil, $66.8 \mathrm{mg}, 75 \%$ yield. ${ }^{1} \mathrm{H}$ NMR (400 MHz, $\left.\mathrm{CDCl}_{3}\right) \delta 8.39(\mathrm{~d}, J=8.4$, 1H), 7.54 (dd, $J=8.4,1.2 \mathrm{~Hz}, 1 \mathrm{H}), 6.96(\mathrm{~s}, 1 \mathrm{H}), 5.72-5.66(\mathrm{~m}, 1 \mathrm{H}), 5.19$ (d, $J=11.6$ $\mathrm{Hz}, 1 \mathrm{H}), 4.65$ (s, 1H), 4.21-4.08 (m, 2H), 3.75 (s, 3H), 3.70 (s, 3H), 3.64 (s, 1H), 2.68$2.61(\mathrm{~m}, 1 \mathrm{H}), 2.55-2.41(\mathrm{~m}, 2 \mathrm{H}), 2.06-2.01(\mathrm{~m}, 1 \mathrm{H}), 1.79-1.74(\mathrm{~m}, 1 \mathrm{H}), 1.67-1.58(\mathrm{~m}$, $1 \mathrm{H}), 1.18(\mathrm{t}, J=7.2 \mathrm{~Hz}, 3 \mathrm{H}) .{ }^{13} \mathrm{C} \mathrm{NMR}\left(100 \mathrm{MHz}, \mathrm{CDCl}_{3}\right) \delta 172.4,170.1,169.4,168.6$, $147.3,134.5,129.4,129.2,128.3,127.2,118.7,117.7,106.8,77.9,63.0,62.8,53.1$, 53.0, 50.2, 37.6, 28.7, 26.6, 26.2, 13.9. IR (thin film): $v_{\max }\left(\mathrm{cm}^{-1}\right)=2954,2224,1734$, 1676, 1604, 1480, 1433, 1368, 1337, 1313, 1283, 1262, 1210, 1149, 1098, 1063, 919, 877, 781, 750, 563. HRMS (ESI) calcd for $\mathrm{C}_{24} \mathrm{H}_{25} \mathrm{~N}_{2} \mathrm{O}_{7}[\mathrm{M}+\mathrm{H}]^{+}:$453.1656. Found: 453.1658 .

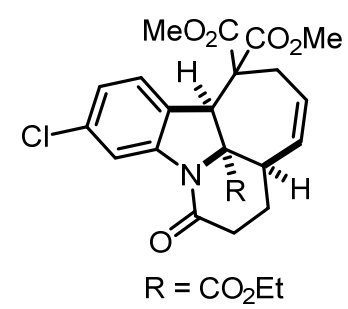

2o, colorless oil, $74.9 \mathrm{mg}, 81 \%$ yield. ${ }^{1} \mathrm{H}$ NMR (400 MHz, $\left.\mathrm{CDCl}_{3}\right) \delta 8.38(\mathrm{~d}, J=2.0$, $1 \mathrm{H}), 6.94(\mathrm{dd}, J=8.4,2.0 \mathrm{~Hz}, 1 \mathrm{H}), 6.67$ (d, $J=8.4,1 \mathrm{H}), 5.76-5.71(\mathrm{~m}, 1 \mathrm{H}), 5.22(\mathrm{~d}, J$ $=11.6 \mathrm{~Hz}, 1 \mathrm{H}), 4.66(\mathrm{~s}, 1 \mathrm{H}), 4.23-4.14(\mathrm{~m}, 2 \mathrm{H}), 3.76(\mathrm{~s}, 3 \mathrm{H}), 3.74(\mathrm{~s}, 3 \mathrm{H}), 3.65(\mathrm{~s}, 1 \mathrm{H})$, 2.70-2.63 (m, 1H), 2.58-2.44 (m, 2H), 2.18-2.13 (m, 1H), 1.81-1.76 (m, 1H), 1.72-1.62 (m, 1H), $1.22(\mathrm{t}, J=7.2 \mathrm{~Hz}, 3 \mathrm{H}) .{ }^{13} \mathrm{C} \mathrm{NMR}\left(100 \mathrm{MHz}, \mathrm{CDCl}_{3}\right) \delta 172.9,170.4,169.0$, $168.9,144.7,135.4,129.5,129.2,125.1,124.3,123.9,117.7,77.9,62.9,62.7,52.9$, 52.7, 50.3, 37.8, 28.6, 26.5, 26.3, 13.9. IR (thin film): $v_{\max }\left(\mathrm{cm}^{-1}\right)=2917,1733,1670$, 1595, 1472, 1442, 1420, 1381, 1341, 1303, 1259, 1218, 1199, 1179, 1140, 1101, 1038, 999, 931, 903, 755, 686. HRMS (ESI) calcd for $\mathrm{C}_{23} \mathrm{H}_{25} \mathrm{NO}_{7} \mathrm{Cl}[\mathrm{M}+\mathrm{H}]^{+}: 462.1314$. Found: 462.1303. 


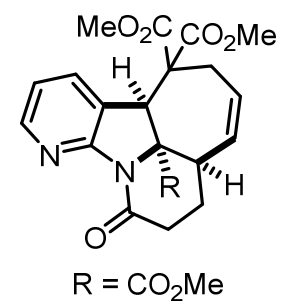

2p, yellow solid, $65.4 \mathrm{mg}, 79 \%$ yield, m.p. $=198.7-201.1{ }^{\circ} \mathrm{C} .{ }^{1} \mathrm{H}$ NMR $(400 \mathrm{MHz}$, $\left.\mathrm{CDCl}_{3}\right) \delta 8.44(\mathrm{~d}, J=3.6 \mathrm{~Hz}, 1 \mathrm{H}), 7.09$ (d, $\left.J=7.2 \mathrm{~Hz}, 1 \mathrm{H}\right), 6.95-6.92(\mathrm{~m}, 1 \mathrm{H}), 5.79$ $5.74(\mathrm{~m}, 1 \mathrm{H}), 5.26(\mathrm{~d}, J=11.6 \mathrm{~Hz}, 1 \mathrm{H}), 4.67(\mathrm{~s}, 1 \mathrm{H}), 3.80(\mathrm{~s}, 3 \mathrm{H}), 3.78(\mathrm{~s}, 3 \mathrm{H}), 3.77$ (s, $3 \mathrm{H}), 3.73(\mathrm{~s}, 1 \mathrm{H}), 2.75-2.68(\mathrm{~m}, 1 \mathrm{H}), 2.60-2.57(\mathrm{~m}, 2 \mathrm{H}), 2.22-2.18(\mathrm{~m}, 1 \mathrm{H}), 1.86-1.83$ (m, 1H), 1.70-1.65 (m, 1H). $\left.{ }^{13} \mathrm{C} \mathrm{NMR} \mathrm{(100} \mathrm{MHz,} \mathrm{CDCl}_{3}\right) \delta 173.4,170.4,168.6,167.7$, 157.3, 149.6, 133.3, 129.3, 129.2, 119.7, 118.7, 76.5, 62.7, 53.7, 53.1, 52.8, 48.4, 37.7, 29.2, 26.6, 26.4. IR (thin film): $v_{\max }\left(\mathrm{cm}^{-1}\right)=2954,1728,1682,1593,1424,1369,1336$, 1307, 1293, 1279, 1202, 1178, 1145, 1064, 1035, 992, 932, 912, 844, 804, 774, 751. HRMS (ESI) calcd for $\mathrm{C}_{21} \mathrm{H}_{23} \mathrm{~N}_{2} \mathrm{O}_{7}[\mathrm{M}+\mathrm{H}]^{+}:$415.1500. Found: 415.1507.

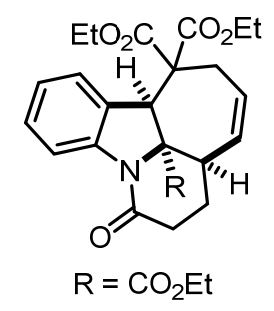

2q, yellow oil, $70.0 \mathrm{mg}, 77 \%$ yield. ${ }^{1} \mathrm{H} \mathrm{NMR}\left(400 \mathrm{MHz}, \mathrm{CDCl}_{3}\right) \delta 8.33(\mathrm{~d}, J=8.0 \mathrm{~Hz}$, $1 \mathrm{H}), 7.26$ (t, $J=8.0 \mathrm{~Hz}, 1 \mathrm{H}), 6.95$ (t, $J=7.6 \mathrm{~Hz}, 1 \mathrm{H}), 6.81(\mathrm{~d}, J=7.6 \mathrm{~Hz}, 1 \mathrm{H}), 5.76-$ $5.71(\mathrm{~m}, 1 \mathrm{H}), 5.22(\mathrm{~d}, J=12.0 \mathrm{~Hz}, 1 \mathrm{H}), 4.69(\mathrm{~s}, 1 \mathrm{H}), 4.34-4.10(\mathrm{~m}, 6 \mathrm{H}), 3.69(\mathrm{~s}, 1 \mathrm{H})$, 2.67-2.44 (m, 3H), 2.19-2.14 (m, 1H), 1.80-1.63 (m, 2H), 1.25 (q, J=7.2 Hz, 6H), 1.18 $(\mathrm{t}, J=7.2 \mathrm{~Hz}, 3 \mathrm{H}) .{ }^{13} \mathrm{C} \mathrm{NMR}\left(100 \mathrm{MHz}, \mathrm{CDCl}_{3}\right) \delta 173.3,170.1,168.8,168.7,143.8$, 129.6, 129.5, 129.4, 125.9, 124.5, 123.7, 117.3, 77.5, 63.1, 62.4, 61.7, 61.6, 50.5, 38.0, 28.7, 26.5, 26.3, 13.94, 13.90, 13.8. IR (thin film): $v_{\max }\left(\mathrm{cm}^{-1}\right)=2981,1734,1665,1597$, 1477, 1463, 1420, 1385, 1338, 1290, 1259, 1179, 1092, 1063, 1015, 906, 850, 752, 677 , 615, 549, 452. HRMS (ESI) calcd for $\mathrm{C}_{25} \mathrm{H}_{30} \mathrm{NO}_{7}[\mathrm{M}+\mathrm{H}]^{+}:$456.2017. Found: 456.2021. 


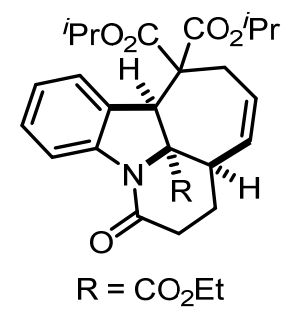

2r, yellow oil, $66.4 \mathrm{mg}, 70 \%$ yield. ${ }^{1} \mathrm{H}$ NMR (400 MHz, $\left.\mathrm{CDCl}_{3}\right) \delta 8.27(\mathrm{~d}, J=8.0 \mathrm{~Hz}$, $1 \mathrm{H}), 7.21(\mathrm{t}, J=8.8 \mathrm{~Hz}, 1 \mathrm{H}), 6.90$ (t, $J=7.6 \mathrm{~Hz}, 1 \mathrm{H}), 6.83$ (d, $J=7.6 \mathrm{~Hz}, 1 \mathrm{H}), 5.70$ $5.65(\mathrm{~m}, 1 \mathrm{H}), 5.16(\mathrm{~d}, J=11.6 \mathrm{~Hz}, 1 \mathrm{H}), 5.07-4.98(\mathrm{~m}, 2 \mathrm{H}), 4.61(\mathrm{~s}, 1 \mathrm{H}), 4.18-4.05(\mathrm{~m}$, 2H), 3.65 (s, 1H), 2.60-2.38 (m, 3H), 2.10-2.05 (m, 1H), 1.75-1.57 (m, 2H), 1.29 (d, $J$ $=6.4 \mathrm{~Hz}, 3 \mathrm{H}), 1.16(\mathrm{~d}, J=6.0 \mathrm{~Hz}, 6 \mathrm{H}), 1.11(\mathrm{~d}, J=6.0 \mathrm{~Hz}, 6 \mathrm{H}) .{ }^{13} \mathrm{C} \mathrm{NMR}(100 \mathrm{MHz}$, $\left.\mathrm{CDCl}_{3}\right) \delta 173.3,169.5,168.9,168.3,143.7,129.51,129.48,129.4,126.1,124.6,123.7$, 117.3, 77.5, 69.6, 69.1, 63.2, 62.4, 50.4, 38.0, 28.7, 26.5, 26.3, 21.6, 21.5, 21.4, 21.3, 13.9. IR (thin film): $v_{\max }\left(\mathrm{cm}^{-1}\right)=2980,1732,1666,1597,1477,1463,1419,1385$, 1337, 1287, 1261, 1218, 1194, 1144, 1059, 984, 838, 818, 801, 754, 677, 615. HRMS (ESI) calcd for $\mathrm{C}_{27} \mathrm{H}_{34} \mathrm{NO}_{7}[\mathrm{M}+\mathrm{H}]^{+}:$484.2330. Found: 484.2330 .

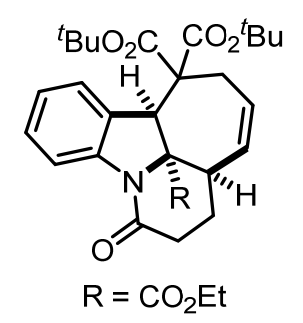

2s, yellow oil, $50.6 \mathrm{mg}, 50 \%$ yield. ${ }^{1} \mathrm{H}$ NMR (400 MHz, $\left.\mathrm{CDCl}_{3}\right) \delta 8.32(\mathrm{~d}, J=8.4 \mathrm{~Hz}$, 1H), $7.27(\mathrm{t}, J=8.8 \mathrm{~Hz}, 1 \mathrm{H}), 7.11(\mathrm{~d}, J=7.2 \mathrm{~Hz}, 1 \mathrm{H}), 6.96$ (t, $J=7.6 \mathrm{~Hz}, 1 \mathrm{H}), 5.77-$ $5.72(\mathrm{~m}, 1 \mathrm{H}), 5.21(\mathrm{~d}, J=11.6 \mathrm{~Hz}, 1 \mathrm{H}), 4.64(\mathrm{~s}, 1 \mathrm{H}), 4.15(\mathrm{q}, J=7.2 \mathrm{~Hz}, 2 \mathrm{H}), 3.70$ (s, 1H), 2.61-2.44 (m, 3H), 2.10-2.05 (m, 1H), 1.81-1.63 (m, 2H), $1.49(\mathrm{~s}, 9 \mathrm{H}), 1.47$ (s, 9H), $1.42(\mathrm{t}, J=7.2 \mathrm{~Hz}, 3 \mathrm{H}) .{ }^{13} \mathrm{C} \mathrm{NMR}\left(100 \mathrm{MHz}, \mathrm{CDCl}_{3}\right) \delta 173.6,169.1,168.9,168.2$, $143.7,129.9,129.34,129.31,126.5,125.5,123.4,117.1,82.9,81.6,77.6,64.3,62.3$, $50.5,38.1,28.8,27.8,27.7,26.9,26.4,13.9$. IR (thin film): $v_{\max }\left(\mathrm{cm}^{-1}\right)=2987,2928$, 1728, 1663, 1599, 1479, 1463, 1419, 1386, 1368, 1337, 1294, 1267, 1242, 1133, 1090, 984, 966, 811, 759, 670, 616. HRMS (ESI) calcd for $\mathrm{C}_{29} \mathrm{H}_{38} \mathrm{NO}_{7}[\mathrm{M}+\mathrm{H}]^{+}: 512.2643$. Found: 512.2647. 


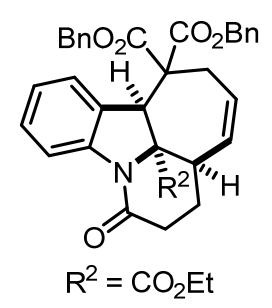

2t, yellow oil, $76.2 \mathrm{mg}, 66 \%$ yield. ${ }^{1} \mathrm{H}$ NMR (400 MHz, $\left.\mathrm{CDCl}_{3}\right) \delta 8.34(\mathrm{~d}, J=8.0 \mathrm{~Hz}$, 1H), 7.35-7.25 (m, 11H), $6.86(\mathrm{t}, J=7.6 \mathrm{~Hz}, 1 \mathrm{H}), 6.69(\mathrm{~d}, J=7.6 \mathrm{~Hz}, 1 \mathrm{H}), 5.69-5.64$ (m, 1H), 5.18-5.03 (m, 5H), $4.76(\mathrm{~s}, 1 \mathrm{H}), 4.23-4.10(\mathrm{~m}, 2 \mathrm{H}), 3.66(\mathrm{~s}, 1 \mathrm{H}), 2.72-2.66(\mathrm{~m}$, 1H), 2.58-2.45 (m, 2H), 2.24-2.19 (m, 1H), 1.79-1.65 (m, 2H), 1.18 (t, J=7.2 Hz, 3H). ${ }^{13} \mathrm{C}$ NMR $\left(100 \mathrm{MHz} \mathrm{CDCl}_{3}\right) \delta 173.2,169.7,168.8,168.3,143.7,135.0,134.5,129.59$, $129.55,129.2$, 128.6, 128.40, 128.36, 125.6, 124.6, 123.8, 117.3, 77.4, 67.4, 67.3, 63.3, $62.4,50.5,37.9,28.7,26.5,26.3,13.9$. IR (thin film): $v_{\max }\left(\mathrm{cm}^{-1}\right)=2959,1735,1665$, 1597, 1497, 1477, 1462, 1419, 1385, 1337, 1313, 1211, 1170, 1140, 845, 808, 753, 733, 696, 677, 617, 583, 549. HRMS (ESI) calcd for $\mathrm{C}_{35} \mathrm{H}_{34} \mathrm{NO}_{7}[\mathrm{M}+\mathrm{H}]^{+}: 580.2330$. Found: 580.2334 .

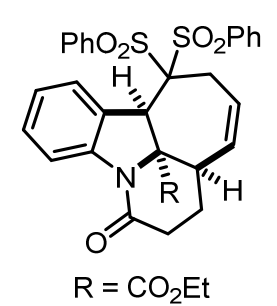

2u, yellow oil, $61.1 \mathrm{mg}, 52 \%$ yield. ${ }^{1} \mathrm{H}$ NMR $\left(400 \mathrm{MHz}, \mathrm{CDCl}_{3}\right) \delta 8.57-8.54(\mathrm{~m}, 1 \mathrm{H})$, 8.18-8.16 (m, 1H), $7.87(\mathrm{~d}, J=7.6 \mathrm{~Hz}, 2 \mathrm{H}), 7.82(\mathrm{~d}, J=7.2 \mathrm{~Hz}, 2 \mathrm{H}), 7.56(\mathrm{t}, J=7.6$ $\mathrm{Hz}, 1 \mathrm{H}), 7.48-7.35$ (m, 7H), 5.71-5.66 (m, 1H), 4.92-4.85 (m, 1H), 4.72 (s, 1H), 4.43$4.36(\mathrm{~m}, 3 \mathrm{H}), 3.02-2.87(\mathrm{~m}, 3 \mathrm{H}), 2.74-2.69(\mathrm{~m}, 1 \mathrm{H}), 2.30-2.21(\mathrm{~m}, 1 \mathrm{H}), 2.12-2.05(\mathrm{~m}$, $1 \mathrm{H}), 1.45(\mathrm{t}, J=7.2 \mathrm{~Hz}, 3 \mathrm{H}) .{ }^{13} \mathrm{C} \mathrm{NMR}\left(100 \mathrm{MHz}, \mathrm{CDCl}_{3}\right) \delta 169.4,164.3,146.1,137.7$, 137.5, 134.6, 133.5, 129.47, 129.45, 129.0, 128.9, 127.0, 125.6, 125.4, 124.9, 121.5, 116.5, 109.5, 83.3, 60.3, 34.5, 29.9, 28.8, 25.3, 14.4. IR (thin film): $v_{\max }\left(\mathrm{cm}^{-1}\right)=3060$, 2922, 1697, 1584, 1567, 1477, 1447, 1411, 1383, 1329, 1309, 1264, 1147, 1106, 1078, $998,835,792,729,685,637,597,551$. HRMS (ESI) calcd for $\mathrm{C}_{31} \mathrm{H}_{29} \mathrm{NaNO}_{7} \mathrm{~S}_{2}$ $[\mathrm{M}+\mathrm{Na}]^{+}:$614.1278. Found: 614.1269 . 


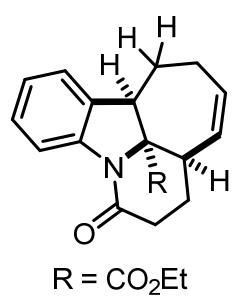

2v, colorless oil, $21.9 \mathrm{mg}, 36 \%$ yield. ${ }^{1} \mathrm{H}$ NMR (400 $\left.\mathrm{MHz}, \mathrm{CDCl}_{3}\right) \delta 8.27(\mathrm{~d}, J=8.0$ $\mathrm{Hz}, 1 \mathrm{H}), 7.25-7.22(\mathrm{~m}, 1 \mathrm{H}), 7.07-7.04(\mathrm{~m}, 2 \mathrm{H}), 5.63-5.60$ (m, 1H), 5.09 (d, $J=12.0 \mathrm{~Hz}$, $1 \mathrm{H}), 4.26-4.11(\mathrm{~m}, 2 \mathrm{H}), 3.90(\mathrm{~s}, 1 \mathrm{H}), 3.65(\mathrm{~s}, 1 \mathrm{H}), 2.64-2.51(\mathrm{~m}, 3 \mathrm{H}), 2.04-1.99$ (m, 2H), 1.87-1.81 (m, 2H), 1.77-1.68 (m, 1H), $1.24(\mathrm{t}, J=7.2 \mathrm{~Hz}, 3 \mathrm{H}) .{ }^{13} \mathrm{C}$ NMR $(100$ $\left.\mathrm{MHz}_{2} \mathrm{CDCl}_{3}\right) \delta 174.2,168.6,143.0,134.1,130.1,128.3,127.2,124.1,123.4,116.8$, 77.8, 62.1, 48.4, 36.1, 30.0, 28.4, 26.4, 25.2, 14.0. IR (thin film): $v_{\max }\left(\mathrm{cm}^{-1}\right)=2922$, 2854, 1736, 1659, 1598, 1480, 1461, 1388, 1316, 1283, 1243, 1212, 1159, 1131, 1078, 966, 860, 817, 752, 660, 605, 544, 494. HRMS (ESI) calcd for $\mathrm{C}_{19} \mathrm{H}_{22} \mathrm{NO}_{3}[\mathrm{M}+\mathrm{H}]^{+}$: 312.1594. Found: 312.1585.

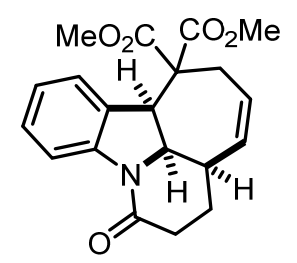

2w, yellow oil, $46.0 \mathrm{mg}, 65 \%$ yield. ${ }^{1} \mathrm{H}$ NMR $\left(400 \mathrm{MHz}, \mathrm{CDCl}_{3}\right) \delta 8.30(\mathrm{~d}, J=8.4 \mathrm{~Hz}$, 1H), 7.30-7.27 (m, 1H), 7.01 (t, $J=7.6 \mathrm{~Hz}, 1 \mathrm{H}), 6.87$ (d, $J=7.6 \mathrm{~Hz}, 1 \mathrm{H}), 5.74-5.69$ (m, $1 \mathrm{H}), 5.30(\mathrm{~d}, J=11.6 \mathrm{~Hz}, 1 \mathrm{H}), 4.59(\mathrm{dd}, J=8.4,2.4 \mathrm{~Hz}, 1 \mathrm{H}), 4.40(\mathrm{~d}, J=8.4 \mathrm{~Hz}, 1 \mathrm{H})$, $3.78(\mathrm{~s}, 3 \mathrm{H}), 3.72(\mathrm{~s}, 3 \mathrm{H}), 3.34(\mathrm{~s}, 1 \mathrm{H}), 2.71-2.64(\mathrm{~m}, 1 \mathrm{H}), 2.59-2.44(\mathrm{~m}, 2 \mathrm{H}), 2.24-2.19$ (m, 1H), 1.99-1.83 (m, 2H). ${ }^{13} \mathrm{C}$ NMR (100 MHz, $\left.\mathrm{CDCl}_{3}\right) \delta 171.3,169.5,168.3,143.4$, $129.6,129.4,129.0,128.2$, 124.7, 123.9, 117.5, 66.3, 62.4, 52.8, 52.6, 46.5, 35.6, 29.2, 28.7, 27.1. IR (thin film): $v_{\max }\left(\mathrm{cm}^{-1}\right)=3011,2918,2849,1751,1717,1644,1595,1478$, 1459, 1425, 1394, 1277, 1203, 1135, 1064, 967, 761, 738, 752, 677, 549, 516, 482. HRMS (ESI) calcd for $\mathrm{C}_{20} \mathrm{H}_{22} \mathrm{NO}_{5}[\mathrm{M}+\mathrm{H}]^{+}: 356.1493$. Found: 356.1494.

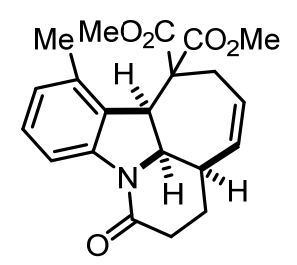


2x, colorless oil, $32.7 \mathrm{mg}, 45 \%$ yield. ${ }^{1} \mathrm{H}$ NMR (400 $\left.\mathrm{MHz}, \mathrm{CDCl}_{3}\right) \delta 8.17(\mathrm{~d}, J=8.0$ $\mathrm{Hz}, 1 \mathrm{H}), 7.19$ (t, $J=8.0 \mathrm{~Hz}, 1 \mathrm{H}), 6.87(\mathrm{~d}, J=7.6 \mathrm{~Hz}, 1 \mathrm{H}), 5.76-5.71(\mathrm{~m}, 1 \mathrm{H}), 5.30(\mathrm{~d}$, $J=11.6 \mathrm{~Hz}, 1 \mathrm{H}), 4.48-4.42(\mathrm{~m}, 2 \mathrm{H}), 3.72$ (s, 3H), 3.63 (s, 3H), 3.29 (s, 1H), 2.64-2.42 (m, 4H), $2.15(\mathrm{~s}, 3 \mathrm{H}), 1.98-1.92(\mathrm{~m}, 1 \mathrm{H}), 1.85-1.77(\mathrm{~m}, 1 \mathrm{H}) .{ }^{13} \mathrm{C}$ NMR (100 MHz, $\left.\mathrm{CDCl}_{3}\right) \delta 172.0,170.2,168.2,143.5,135.1,129.8,129.13,129.08,126.8,125.7,114.4$, $67.3,61.9,52.6,52.5,45.0,36.0,29.3,28.6,27.8,18.6$. IR (thin film): $v_{\max }\left(\mathrm{cm}^{-1}\right)=$ 2921, 1748, 1711, 1642, 1583, 1434, 1388, 1329, 1295, 1272, 1229, 1198, 1172, 1076, $1045,975,931,844,784,722,594,555,502$. HRMS (ESI) calcd for $\mathrm{C}_{21} \mathrm{H}_{24} \mathrm{NO}_{5}$ $[\mathrm{M}+\mathrm{H}]^{+}:$370.1649. Found: 370.1644 .

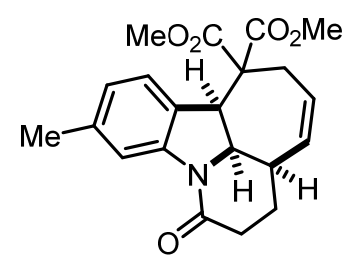

2y, yellow oil, $47.4 \mathrm{mg}, 63 \%$ yield. ${ }^{1} \mathrm{H}$ NMR $\left(400 \mathrm{MHz}, \mathrm{CDCl}_{3}\right) \delta 8.15(\mathrm{~s}, 1 \mathrm{H}), 6.83$ $(\mathrm{d}, J=7.6 \mathrm{~Hz}, 1 \mathrm{H}), 6.73(\mathrm{~d}, J=8.0 \mathrm{~Hz}, 1 \mathrm{H}), 5.74-5.69$ (m, 1H), 5.30 (d, $J=11.6 \mathrm{~Hz}$, $1 \mathrm{H}), 4.58-4.56(\mathrm{~m}, 1 \mathrm{H}), 4.36(\mathrm{~d}, J=8.0 \mathrm{~Hz}, 1 \mathrm{H}), 3.78(\mathrm{~s}, 3 \mathrm{H}), 3.72(\mathrm{~s}, 3 \mathrm{H}), 3.32(\mathrm{~s}$, 1H), 2.69-2.63 (m, 1H), 2.58-2.43 (m, 2H), 2.34 (s, 3H), 2.23-2.18 (m, 1H), 1.98-1.82 (m, 2H). ${ }^{13} \mathrm{C} \mathrm{NMR}\left(100 \mathrm{MHz}, \mathrm{CDCl}_{3}\right) \delta 171.3,169.6,168.3,143.4,139.5,129.6,129.0$, 125.2, 124.7, 124.2, 118.1, 66.5, 62.4, 52.8, 52.6, 46.1, 35.6, 29.3, 28.7, 27.0, 21.7. IR (thin film): $v_{\max }\left(\mathrm{cm}^{-1}\right)=2922,1749,1712,1651,1604,1492,1429,1393,1332,1271$, 1244, 1177, 1077, 1038, 973, 927, 882, 821, 745, 688, 561, 532, 484. HRMS (ESI) calcd for $\mathrm{C}_{21} \mathrm{H}_{24} \mathrm{NO}_{5}[\mathrm{M}+\mathrm{H}]^{+}: 370.1649$. Found: 370.1649 .

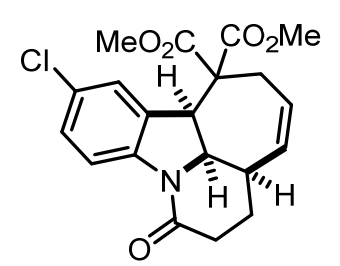

2z, yellow oil, $40.8 \mathrm{mg}, 52 \%$ yield. ${ }^{1} \mathrm{H}$ NMR (400 MHz, $\left.\mathrm{CDCl}_{3}\right) \delta 8.24(\mathrm{~d}, J=8.8 \mathrm{~Hz}$, $1 \mathrm{H}), 7.24(\mathrm{~d}, J=8.8 \mathrm{~Hz}, 1 \mathrm{H}), 6.83(\mathrm{~s}, 1 \mathrm{H}), 5.74-5.69(\mathrm{~m}, 1 \mathrm{H}), 5.30(\mathrm{~d}, J=11.6 \mathrm{~Hz}$, 1H), 4.61-4.59 (m, 1H), 4.37 (d, $J=8.4 \mathrm{~Hz}, 1 \mathrm{H}), 3.80$ (s, 3H), 3.73 (s, 3H), 3.33 (s, $1 \mathrm{H}), 2.73-2.67(\mathrm{~m}, 1 \mathrm{H}), 2.58-2.43(\mathrm{~m}, 2 \mathrm{H}), 2.20-2.16(\mathrm{~m}, 1 \mathrm{H}), 1.99-1.85(\mathrm{~m}, 2 \mathrm{H}) .{ }^{13} \mathrm{C}$ NMR $\left(100 \mathrm{MHz}, \mathrm{CDCl}_{3}\right) \delta 171.0,169.3,168.3,142.0,130.2,129.6,129.2,128.8,128.7$, 
125.0, 118.3, 66.5, 62.3, 52.9, 52.7, 46.3, 35.5, 29.1, 28.7, 27.0. IR (thin film): $v_{\max }\left(\mathrm{cm}^{-}\right.$ $\left.{ }^{1}\right)=2919,1730,1654,1591,1469,1435,1376,1329,1282,1237,1179,1075,1039$, 971, 907, 892, 830, 753, 736, 668, 588, 521, 483. HRMS (ESI) calcd for $\mathrm{C}_{20} \mathrm{H}_{21} \mathrm{NO}_{5} \mathrm{Cl}$ $[\mathrm{M}+\mathrm{H}]^{+}:$390.1103. Found: 390.1098 .

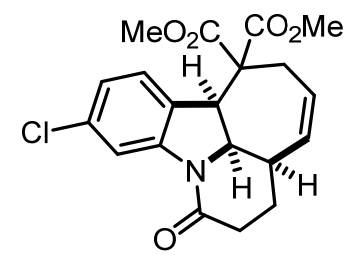

2aa, colorless oil, $60.7 \mathrm{mg}, 77 \%$ yield. ${ }^{1} \mathrm{H}$ NMR $\left(400 \mathrm{MHz}, \mathrm{CDCl}_{3}\right) \delta 8.34(\mathrm{~d}, J=1.6$ Hz, 1H), 6.98 (dd, $J=8.4,1.6 \mathrm{~Hz}, 1 \mathrm{H}), 6.76(\mathrm{~d}, J=8.0 \mathrm{~Hz}, 1 \mathrm{H}), 5.74-5.69(\mathrm{~m}, 1 \mathrm{H})$, $5.30(\mathrm{~d}, J=11.6 \mathrm{~Hz}, 1 \mathrm{H}), 4.62-4.59(\mathrm{~m}, 1 \mathrm{H}), 4.37$ (d, $J=8.0 \mathrm{~Hz}, 1 \mathrm{H}), 3.78(\mathrm{~s}, 3 \mathrm{H})$, $3.72(\mathrm{~s}, 3 \mathrm{H}), 3.33(\mathrm{~s}, 1 \mathrm{H}), 2.72-2.66(\mathrm{~m}, 1 \mathrm{H}), 2.58-2.44(\mathrm{~m}, 2 \mathrm{H}), 2.20-2.15(\mathrm{~m}, 1 \mathrm{H})$, 1.99-1.83 (m, 2H). ${ }^{13} \mathrm{C} \mathrm{NMR}\left(100 \mathrm{MHz}, \mathrm{CDCl}_{3}\right) \delta 171.1,169.3,168.5,144.3,134.9$, $129.5,128.9,126.7,125.4,123.9,117.7,66.8,62.2,52.9,52.7,46.0,35.5,29.2,28.6$, 27.0. IR (thin film): $v_{\max }\left(\mathrm{cm}^{-1}\right)=2922,1748,1713,1645,1591,1473,1389,1336$, $1275,1238,1175,1140,1038,974,948,836,810,741,671,610,558,533,489$. HRMS (ESI) calcd for $\mathrm{C}_{20} \mathrm{H}_{21} \mathrm{NO}_{5} \mathrm{Cl}[\mathrm{M}+\mathrm{H}]^{+}: 390.1103$. Found: 390.1100 .

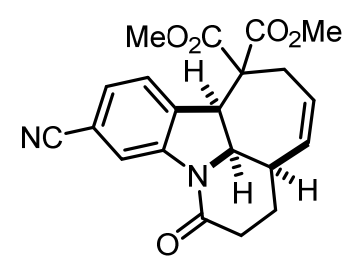

2ab, yellow oil, $70.5 \mathrm{mg}, 93 \%$ yield. ${ }^{1} \mathrm{H}$ NMR $\left(400 \mathrm{MHz}, \mathrm{CDCl}_{3}\right) \delta 8.61(\mathrm{~s}, 1 \mathrm{H}), 7.30$ (s, 1H), $6.95(\mathrm{~d}, J=8.0 \mathrm{~Hz}, 1 \mathrm{H}), 5.75-5.70(\mathrm{~m}, 1 \mathrm{H}), 5.32$ (d, $J=12.0 \mathrm{~Hz}, 1 \mathrm{H}), 4.66-$ $4.63(\mathrm{~m}, 1 \mathrm{H}), 4.46(\mathrm{~d}, J=8.4 \mathrm{~Hz}, 1 \mathrm{H}), 3.80(\mathrm{~s}, 3 \mathrm{H}), 3.74(\mathrm{~s}, 3 \mathrm{H}), 3.36(\mathrm{~s}, 1 \mathrm{H}), 2.74-$ $2.68(\mathrm{~m}, 1 \mathrm{H}), 2.60-2.47(\mathrm{~m}, 2 \mathrm{H}), 2.14-2.10(\mathrm{~m}, 1 \mathrm{H}), 2.04-1.99(\mathrm{~m}, 1 \mathrm{H}), 1.95-1.86(\mathrm{~m}$, 1H). ${ }^{13} \mathrm{C}$ NMR $\left(100 \mathrm{MHz}, \mathrm{CDCl}_{3}\right) \delta 170.7,169.0,168.7,143.8,133.6,129.4,128.7$, $127.7,125.5,120.3,118.5,112.9,66.3,62.1,53.0,52.8,46.4,35.3,29.0,28.5,27.1$. IR (thin film): $v_{\max }\left(\mathrm{cm}^{-1}\right)=2919,1748,1715,1650,1600,1485,1429,1389,1282,1243$, 1177, 1059, 1035, 995, 974, 879, 854, 840, 822, 747, 665, 635, 471. HRMS (ESI) calcd for $\mathrm{C}_{21} \mathrm{H}_{21} \mathrm{~N}_{2} \mathrm{O}_{5}[\mathrm{M}+\mathrm{H}]^{+}: 381.1445$. Found: 381.1442 . 


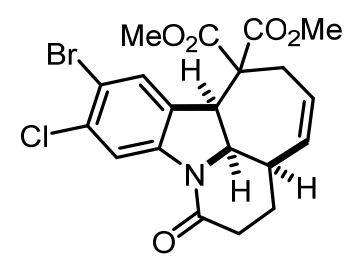

2ac, brown solid, $62.8 \mathrm{mg}$, 66\% yield, m.p. $=205.5-207.4{ }^{\circ} \mathrm{C} .{ }^{1} \mathrm{H}$ NMR $(400 \mathrm{MHz}$, $\left.\mathrm{CDCl}_{3}\right) \delta 8.44(\mathrm{~s}, 1 \mathrm{H}), 7.08(\mathrm{~s}, 1 \mathrm{H}), 5.74-5.69(\mathrm{~m}, 1 \mathrm{H}), 5.30(\mathrm{~d}, J=11.6 \mathrm{~Hz}, 1 \mathrm{H}), 4.62-$ $4.59(\mathrm{~m}, 1 \mathrm{H}), 4.36(\mathrm{~d}, J=8.4 \mathrm{~Hz}, 1 \mathrm{H}), 3.79(\mathrm{~s}, 3 \mathrm{H}), 3.73(\mathrm{~s}, 3 \mathrm{H}), 3.33(\mathrm{~s}, 1 \mathrm{H}), 2.75-$ $2.68(\mathrm{~m}, 1 \mathrm{H}), 2.57-2.43(\mathrm{~m}, 2 \mathrm{H}), 2.18-2.13(\mathrm{~m}, 1 \mathrm{H}), 2.01-1.84(\mathrm{~m}, 2 \mathrm{H}) .{ }^{13} \mathrm{C}$ NMR $(100$ $\left.\mathrm{MHz}, \mathrm{CDCl}_{3}\right) \delta 170.8,169.2,168.5,143.3,134.9,129.5,129.4,128.8,118.8,116.2$, 66.8, 62.3, 53.0, 52.7, 45.9, 35.4, 29.1, 28.6, 27.0. IR (thin film): $v_{\max }\left(\mathrm{cm}^{-1}\right)=2919$, 1748, 1714, 1655, 1590, 1467, 1390, 1374, 1274, 1238, 1102, 1057, 1040, 975, 951, 872, 822, 749, 673, 629, 578, 534, 448. HRMS (ESI) calcd for $\mathrm{C}_{20} \mathrm{H}_{20} \mathrm{NO}_{5} \mathrm{Cl}^{79} \mathrm{Br}$ $[\mathrm{M}+\mathrm{H}]^{+}:$468.0208. Found: 468.0207.

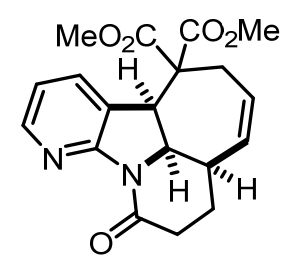

2ad, yellow solid, $60.4 \mathrm{mg}, 73 \%$ yield, m.p. $=183.7-185.4{ }^{\circ} \mathrm{C} .{ }^{1} \mathrm{H}$ NMR $(400 \mathrm{MHz}$, $\left.\mathrm{CDCl}_{3}\right) \delta 8.42(\mathrm{~d}, J=4.4 \mathrm{~Hz}, 1 \mathrm{H}), 7.16(\mathrm{~d}, J=7.6 \mathrm{~Hz}, 1 \mathrm{H}), 6.95-6.92(\mathrm{~m}, 1 \mathrm{H}), 5.76-$ $5.71(\mathrm{~m}, 1 \mathrm{H}), 5.32(\mathrm{~d}, J=11.6 \mathrm{~Hz}, 1 \mathrm{H}), 4.65-4.63(\mathrm{~m}, 1 \mathrm{H}), 4.37(\mathrm{~d}, J=8.4 \mathrm{~Hz}, 1 \mathrm{H})$, $3.79(\mathrm{~s}, 3 \mathrm{H}), 3.74(\mathrm{~s}, 3 \mathrm{H}), 3.36(\mathrm{~s}, 1 \mathrm{H}), 2.76-2.70(\mathrm{~m}, 1 \mathrm{H}), 2.59-2.56(\mathrm{~m}, 2 \mathrm{H}), 2.29-2.25$ (m, 1H), 2.01-1.89 (m, 2H). $\left.{ }^{13} \mathrm{C} \mathrm{NMR} \mathrm{(100} \mathrm{MHz,} \mathrm{CDCl}_{3}\right) \delta 171.0,169.1,167.6,157.3$, 149.3, 133.6, 129.6, 128.8, 122.3, 118.8, 65.3, 62.2, 53.0, 52.7, 44.2, 35.4, 29.8, 28.5, 27.2. IR (thin film): $v_{\max }\left(\mathrm{cm}^{-1}\right)=2912,1733,1672,1590,1415,1377,1348,1329$, 1295, 1230, 1140, 1078, 1062, 998, 949, 893, 835, 758, 675, 586, 551, 519, 477. HRMS (ESI) calcd for $\mathrm{C}_{19} \mathrm{H}_{21} \mathrm{~N}_{2} \mathrm{O}_{5}[\mathrm{M}+\mathrm{H}]^{+}: 357.1445$. Found: 357.1448 .

3.3 General procedure for the dearomatized $[2+2]$ cycloadditions (using $3 a$ as an example). 


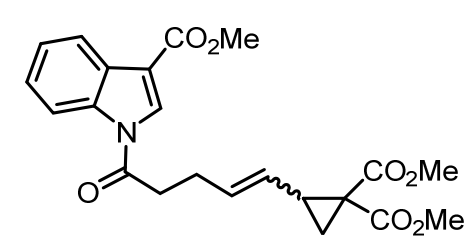

$3 \mathbf{a}(E / Z=4.7 / 1)$

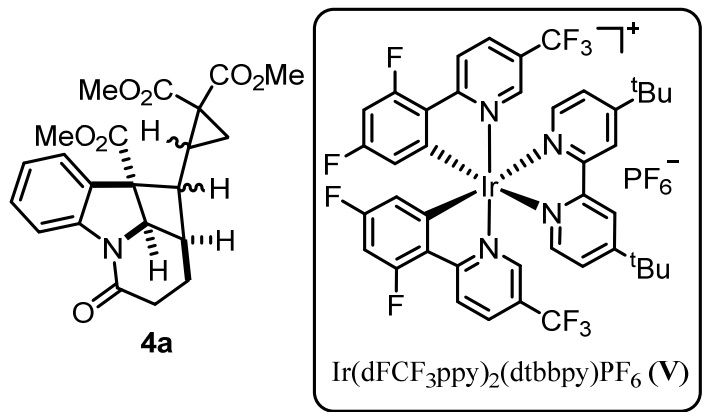

$\operatorname{Ir}\left(\mathrm{dFCF}_{3} \text { ppy }\right)_{2}(\mathrm{dtbbpy}) \mathrm{PF}_{6}(\mathbf{V})$

To a flame-dried sealed tube were added indole derivative 3a $(82.6 \mathrm{mg}, 0.2 \mathrm{mmol})$ and photosensitizer $\mathbf{V}(2.2 \mathrm{mg}, 0.002 \mathrm{mmol})$ and anhydrous acetonitrile $(20 \mathrm{~mL})$. The reaction mixture was degassed via freeze-pump-thaw for 3 cycles. After the reaction mixture was thoroughly degassed, the vial was sealed and positioned approximately $10 \mathrm{~cm}$ from blue LEDs $\left(24 \mathrm{~W}, \lambda_{\max }=455 \mathrm{~nm}\right)$. Then the reaction mixture was stirred at room temperature for the indicated time (monitored by TLC) under argon atmosphere. Afterwards, the reaction mixture was concentrated by rotary evaporation. Then the residue was purified by silica gel column chromatography $(\mathrm{PE} / \mathrm{EtOAc}=2 / 1)$ to afford the desired product 4a. The analytical data of the products are summarized below, and the peaks of minor isomers are indicated by an asterisk $\left(^{*}\right)$.

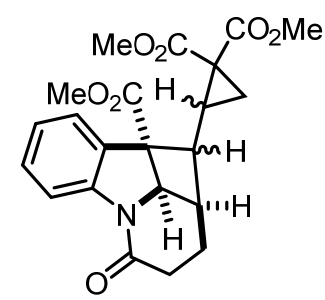

4a, yellow oil, $80.9 \mathrm{mg}, 95 \%$ yield, $\mathrm{dr}=6 / 1 .{ }^{1} \mathrm{H}$ NMR $\left(400 \mathrm{MHz}, \mathrm{CDCl}_{3}\right) \delta 7.87(\mathrm{~d}, J$ $=7.6 \mathrm{~Hz}, 1 \mathrm{H}), 7.52(\mathrm{~d}, J=7.6 \mathrm{~Hz}, 1 \mathrm{H}), 7.23$ (t, $J=8.0 \mathrm{~Hz}, 1 \mathrm{H}), 7.04$ (t, $J=7.6 \mathrm{~Hz}$, 1H), $4.82(\mathrm{~d}, J=6.8 \mathrm{~Hz}, 1 \mathrm{H}), 3.89(\mathrm{~s}, 3 \mathrm{H}), 3.86\left(\mathrm{~s}, 3 \mathrm{H}^{*}\right), 3.74\left(\mathrm{~s}, 3 \mathrm{H}^{*}\right), 3.72(\mathrm{~s}, 3 \mathrm{H})$, $3.67\left(\mathrm{~s}, 3 \mathrm{H}^{*}\right), 3.63(\mathrm{~s}, 3 \mathrm{H}), 3.07-3.01(\mathrm{~m}, 1 \mathrm{H}), 2.51-2.39(\mathrm{~m}, 1 \mathrm{H}), 2.31-2.17(\mathrm{~m}, 2 \mathrm{H})$, 2.01-1.92 (m, 2H), 1.45-1.29 (m, 3H). ${ }^{13} \mathrm{C} \mathrm{NMR}\left(100 \mathrm{MHz}, \mathrm{CDCl}_{3}\right) \delta$ 170.8, 170.01, 169.98, 167.8, 144.8, 131.5, 128.6, 124.3, 123.9, 117.2, 61.0, 56.2, 55.2, 52.8, 52.4, 36.3, 34.7, 33.4, 29.3, 25.3, 19.6. IR (thin film): $v_{\max }\left(\mathrm{cm}^{-1}\right)=2947,1725,1666,1596$, 1474, 1459, 1433, 1381, 1330, 1284, 1209, 1161, 1136, 1096, 987, 946, 749, 701, 649, 596, 539, 457. HRMS (ESI) calcd for $\mathrm{C}_{22} \mathrm{H}_{24} \mathrm{NO}_{7}[\mathrm{M}+\mathrm{H}]^{+}:$414.1547. Found: 414.1547. 


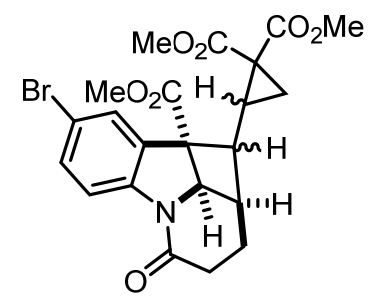

4b, yellow oil, $62.1 \mathrm{mg}, 63 \%$ yield, $\mathrm{dr}=6 / 1 .{ }^{1} \mathrm{H} \mathrm{NMR}\left(400 \mathrm{MHz}, \mathrm{CDCl}_{3}\right) \delta 7.77(\mathrm{~d}, J$ $=8.4 \mathrm{~Hz}, 1 \mathrm{H}), 7.66(\mathrm{~s}, 1 \mathrm{H}), 7.38-7.35(\mathrm{~m}, 1 \mathrm{H}), 4.84(\mathrm{~d}, J=6.8 \mathrm{~Hz}, 1 \mathrm{H}), 3.93(\mathrm{~s}, 3 \mathrm{H})$, $3.90\left(\mathrm{~s}, 3 \mathrm{H}^{*}\right), 3.77\left(\mathrm{~s}, 3 \mathrm{H}^{*}\right), 3.74(\mathrm{~s}, 3 \mathrm{H}), 3.72\left(\mathrm{~s}, 3 \mathrm{H}^{*}\right), 3.67(\mathrm{~s}, 3 \mathrm{H}), 3.11-3.04(\mathrm{~m}, 1 \mathrm{H})$, 2.48-2.45 (m, 1H), 2.33-2.18 (m, 3H), 2.01-1.95 (m, 2H), 1.47-1.44 (m, 1H), 1.38-1.35 $(\mathrm{m}, 1 \mathrm{H}) .{ }^{13} \mathrm{C} \mathrm{NMR}\left(100 \mathrm{MHz}, \mathrm{CDCl}_{3}\right) \delta$ 170.8, 169.9, 169.5, 167.9, 143.8, 133.6, 131.4, $127.1,118.5,116.9,61.2,55.9,55.3,52.9,52.7,36.3,34.6,33.3,29.1,25.2,19.6$. IR (thin film): $v_{\max }\left(\mathrm{cm}^{-1}\right)=2952,2849,1725,1591,1468,1434,1368,1324,1298,1283$, 1260, 1202, 1157, 1062, 986, 947, 913, 877, 820, 794, 733, 661. HRMS (ESI) calcd for $\mathrm{C}_{22} \mathrm{H}_{23} \mathrm{NO}_{7}{ }^{79} \mathrm{Br}[\mathrm{M}+\mathrm{H}]^{+}:$492.0652. Found: 492.0649 .

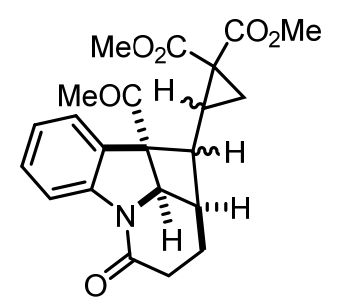

4c, colorless oil, $61.0 \mathrm{mg}, 78 \%$ yield, $\mathrm{dr}=6 / 1 .{ }^{1} \mathrm{H} \mathrm{NMR}\left(400 \mathrm{MHz}, \mathrm{CDCl}_{3}\right) \delta 7.92(\mathrm{~d}$, $J=8.0 \mathrm{~Hz}, 1 \mathrm{H}), 7.33(\mathrm{~d}, J=7.2 \mathrm{~Hz}, 1 \mathrm{H}), 7.27-7.24(\mathrm{~m}, 1 \mathrm{H}), 7.05(\mathrm{t}, J=8.0 \mathrm{~Hz}, 1 \mathrm{H})$, $4.82(\mathrm{~d}, J=6.4 \mathrm{~Hz}, 1 \mathrm{H}), 3.75\left(\mathrm{~s}, 3 \mathrm{H}^{*}\right), 3.71$ (s, 3H), 3.64 (s, 3H), 3.62 (s, 3H*), 2.96$2.88(\mathrm{~m}, 1 \mathrm{H}), 2.49\left(\mathrm{~s}, 3 \mathrm{H}^{*}\right), 2.46(\mathrm{~s}, 3 \mathrm{H}), 2.43-2.40(\mathrm{~m}, 1 \mathrm{H}), 2.30-2.17(\mathrm{~m}, 2 \mathrm{H}), 2.12-$ 1.97 (m, 2H), 1.51-1.48 (m, 1H), 1.41-1.38 (m, 1H), 1.32-1.26 (m, 1H). ${ }^{13} \mathrm{C}$ NMR (100 $\left.\mathrm{MHz}_{2} \mathrm{CDCl}_{3}\right) \delta 204.5,171.0,169.8,167.9,145.3,132.0,128.7,124.4,122.9,117.6$, $62.4,61.1,55.4,52.8,36.2,34.8,33.8,30.1,29.5,25.3,20.0$. IR (thin film): $v_{\max }\left(\mathrm{cm}^{-1}\right)$ $=2919,2850,1719,1702,1658,1593,1474,1459,1386,1284,1202,1157,1133,1069$, 988, 900, 825, 756, 711, 645, 577, 523. HRMS (ESI) calcd for $\mathrm{C}_{22} \mathrm{H}_{24} \mathrm{NO}_{6}[\mathrm{M}+\mathrm{H}]^{+}$: 398.1598. Found: 398.1601 . 


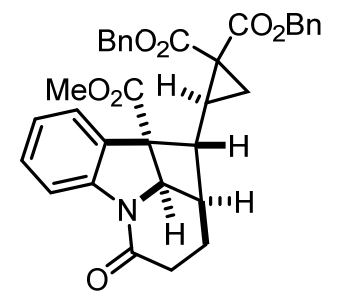

4d, colorless oil, $50.0 \mathrm{mg}, 44 \%$ yield, dr $>20 / 1 .{ }^{1} \mathrm{H}$ NMR (400 MHz, $\left.\mathrm{CDCl}_{3}\right) \delta 7.92$ (d, $J=8.0 \mathrm{~Hz}, 1 \mathrm{H}), 7.55(\mathrm{~d}, J=7.6 \mathrm{~Hz}, 1 \mathrm{H}), 7.35-7.34$ (m, 3H), 7.29-7.27 (m, 4H), 7.22 (t, $J=7.2 \mathrm{~Hz}, 2 \mathrm{H}), 7.14-7.12(\mathrm{~m}, 2 \mathrm{H}), 7.07$ (t, $J=7.6 \mathrm{~Hz}, 1 \mathrm{H}), 5.19-5.09$ (m, 3H), 4.96$4.93(\mathrm{~m}, 1 \mathrm{H}), 4.79(\mathrm{~d}, J=6.8 \mathrm{~Hz}, 1 \mathrm{H}), 3.88(\mathrm{~s}, 3 \mathrm{H}), 3.03-2.99(\mathrm{~m}, 1 \mathrm{H}), 2.35-2.31(\mathrm{~m}$, 1H), 2.19-1.99 (m, 4H), 1.49-1.40 (m, 2H), 1.13-1.05 (m, 1H). ${ }^{13} \mathrm{C}$ NMR (100 MHz, $\left.\mathrm{CDCl}_{3}\right) \delta 170.7,170.0,169.4,167.2,144.8,135.1,134.7,131.5,128.6,128.53,128.49$, $128.46,128.3,128.0,124.3,123.9,117.3,67.8,67.5,60.9,56.1,54.9,52.4,36.2,34.7$, 33.6, 29.7, 25.0, 19.9. IR (thin film): $v_{\max }\left(\mathrm{cm}^{-1}\right)=3033,2951,1724,1677,1597,1475$, 1457, 1434, 1377, 1280, 1226, 1192, 1157, 1051, 1016, 951, 750, 732, 696, 651, 597, 543. HRMS (ESI) calcd for $\mathrm{C}_{34} \mathrm{H}_{31} \mathrm{NO}_{7} \mathrm{Na}[\mathrm{M}+\mathrm{H}]^{+}$: 588.1993. Found: 588.1994.

\subsection{General procedure for the interrupted $[5+2]$ cycloadditions (using $1 \mathrm{w}$ as an example).}

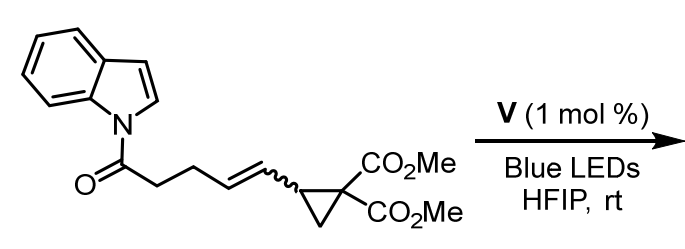

$1 \mathbf{w}(E / Z=5 / 1)$

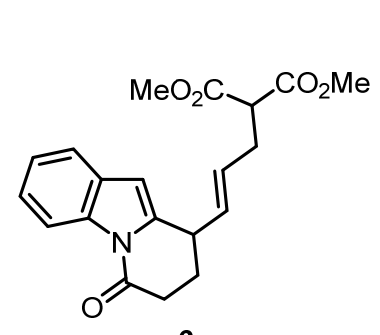

$6 \mathbf{a}$

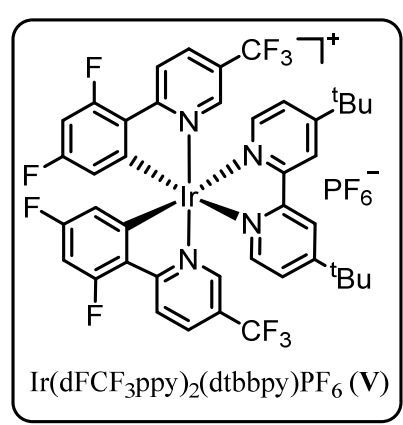

To a flame-dried sealed tube were added indole derivative $\mathbf{1 w}(71.0 \mathrm{mg}, 0.2 \mathrm{mmol})$ and photosensitizer $\mathbf{V}(2.2 \mathrm{mg}, 0.002 \mathrm{mmol})$ and anhydrous hexafluoroisopropanol $(2 \mathrm{~mL})$. The reaction mixture was degassed via freeze-pump-thaw for 3 cycles. After the reaction mixture was thoroughly degassed, the vial was sealed and positioned approximately $10 \mathrm{~cm}$ from blue LEDs $\left(24 \mathrm{~W}, \lambda_{\max }=455 \mathrm{~nm}\right)$. Then the reaction mixture was stirred at room temperature for the indicated time (monitored by TLC) under argon atmosphere. Afterwards, the reaction mixture was concentrated by rotary evaporation. 
Then the residue was purified by silica gel column chromatography $(\mathrm{PE} / \mathrm{EtOAc}=2 / 1)$ to afford the desired product $6 \mathbf{a}$. The analytical data of the products are summarized below.

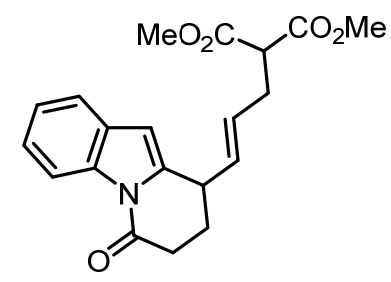

6a, brown oil, $52.0 \mathrm{mg}, 73 \%$ yield. ${ }^{1} \mathrm{H}$ NMR (400 MHz, $\left.\mathrm{CDCl}_{3}\right) \delta 8.44(\mathrm{~d}, J=8.0 \mathrm{~Hz}$, 1H), $7.46(\mathrm{~d}, J=7.6 \mathrm{~Hz}, 1 \mathrm{H}), 7.31-7.23(\mathrm{~m}, 2 \mathrm{H}), 6.27$ (s, 1H), 5.70-5.58 (m, 2H), 3.76 (s, 3H), 3.75 (s, 3H), 3.60-3.57 (m, 1H), 3.50 (t, $J=7.6 \mathrm{~Hz}, 1 \mathrm{H}), 2.88-2.70(\mathrm{~m}, 4 \mathrm{H})$, 2.14-2.08 (m, 1H), 1.99-1.90 (m, 1H). ${ }^{13} \mathrm{C}$ NMR (100 MHz, $\left.\mathrm{CDCl}_{3}\right) \delta$ 169.1, 169.0, 140.2 , 135.1, 132.7, 129.4, 128.7, 124.3, 123.9, 119.9, 116.4, 106.0, 52.5, 51.5, 38.1, 33.0, 31.5, 28.0. IR (thin film): $v_{\max }\left(\mathrm{cm}^{-1}\right)=2955,1751,1731,1589,1570,1438,1349$, $1318,1273,1178,1147,1099,1064,976,910,819,759,669,599,485,438$. HRMS (ESI) calcd for $\mathrm{C}_{20} \mathrm{H}_{22} \mathrm{NO}_{5}[\mathrm{M}+\mathrm{H}]^{+}:$356.1493. Found: 356.1491 .

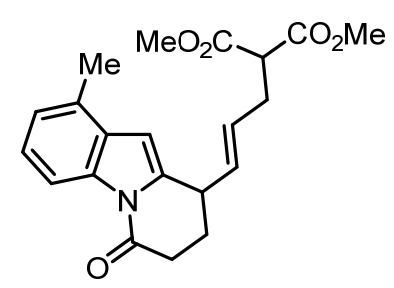

6b, yellow oil, $67.7 \mathrm{mg}, 91 \%$ yield. ${ }^{1} \mathrm{H} \mathrm{NMR}\left(400 \mathrm{MHz}, \mathrm{CDCl}_{3}\right) \delta 8.28(\mathrm{~d}, J=8.0 \mathrm{~Hz}$, $1 \mathrm{H}), 7.19$ (t, $J=8.0 \mathrm{~Hz}, 1 \mathrm{H}), 7.05(\mathrm{~d}, J=7.6 \mathrm{~Hz}, 1 \mathrm{H}), 6.30$ (s, 1H), 5.69-5.59 (m, 2H), $3.76(\mathrm{~s}, 3 \mathrm{H}), 3.75(\mathrm{~s}, 3 \mathrm{H}), 3.58-3.50(\mathrm{~m}, 2 \mathrm{H}), 2.87-2.69(\mathrm{~m}, 4 \mathrm{H}), 2.49$ (s, 3H), 2.13-2.06 $(\mathrm{m}, 1 \mathrm{H}), 1.98-1.88(\mathrm{~m}, 1 \mathrm{H}) .{ }^{13} \mathrm{C} \mathrm{NMR}\left(100 \mathrm{MHz}, \mathrm{CDCl}_{3}\right) \delta 169.1,169.0,139.6,134.8$, 132.8, 129.2, 128.9, 128.6, 124.4, 113.8, 104.4, 52.5, 51.4, 38.2, 33.0, 31.6, 28.0, 18.4. IR (thin film): $v_{\max }\left(\mathrm{cm}^{-1}\right)=2921,2850,1730,1572,1485,1434,1420,1374,1272$, 1200, 1171, 1150, 1086, 981, 940, 876, 838, 800, 748, 660, 563. HRMS (ESI) calcd for $\mathrm{C}_{21} \mathrm{H}_{23} \mathrm{NO}_{5} \mathrm{Na}[\mathrm{M}+\mathrm{Na}]^{+}:$392.1468. Found: 392.1472 . 


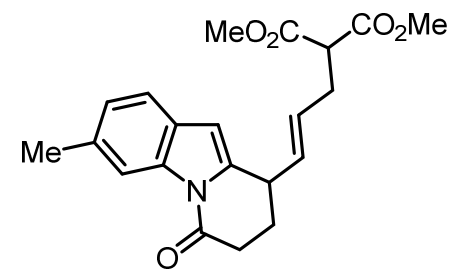

6c, brown oil, $65.7 \mathrm{mg}, 88 \%$ yield. ${ }^{1} \mathrm{H}$ NMR $\left(400 \mathrm{MHz}, \mathrm{CDCl}_{3}\right) \delta 8.29(\mathrm{~s}, 1 \mathrm{H}), 7.33(\mathrm{~d}$, $J=7.6 \mathrm{~Hz}, 1 \mathrm{H}), 7.07(\mathrm{~d}, J=8.0 \mathrm{~Hz}, 1 \mathrm{H}), 6.21(\mathrm{~s}, 1 \mathrm{H}), 5.68-5.57(\mathrm{~m}, 2 \mathrm{H}), 3.76(\mathrm{~s}, 3 \mathrm{H})$, $3.74(\mathrm{~s}, 3 \mathrm{H}), 3.57-3.47(\mathrm{~m}, 2 \mathrm{H}), 2.87-2.69(\mathrm{~m}, 4 \mathrm{H}), 2.47$ (s, 3H), 2.12-2.05 (m, 1H), 1.97-1.87 (m, 1H). ${ }^{13} \mathrm{C}$ NMR (100 MHz, $\left.\mathrm{CDCl}_{3}\right) \delta 169.1,169.0,139.5,135.4,134.3$, 132.8, 128.6, 127.0, 125.2, 119.4, 116.6, 105.8, 52.5, 51.5, 38.1, 33.0, 31.5, 28.0, 21.8. IR (thin film): $v_{\max }\left(\mathrm{cm}^{-1}\right)=2919,1731,1702,1589,1565,1438,1343,1321,1273$, 1152, 1062, 1040, 978, 883, 842, 709, 665, 600, 567, 477, 430. HRMS (ESI) calcd for $\mathrm{C}_{21} \mathrm{H}_{23} \mathrm{NO}_{5} \mathrm{Na}[\mathrm{M}+\mathrm{Na}]^{+}: 392.1468$. Found: 392.1468 .

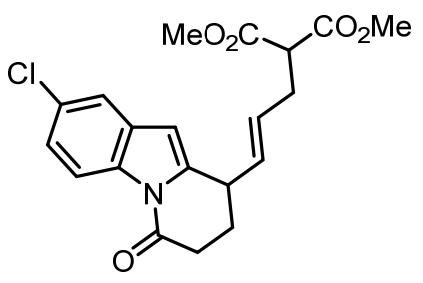

6d, colorless oil, $50.8 \mathrm{mg}, 65 \%$ yield. ${ }^{1} \mathrm{H}$ NMR (400 MHz, $\left.\mathrm{CDCl}_{3}\right) \delta 8.35$ (d, $J=8.8$ Hz, 1H), 7.42 (s, 1H), 7.23 (d, J=8.8 Hz, 1H), 6.20 (s, 1H), 5.68-5.59 (m, 2H), 3.76 (s, $3 \mathrm{H}), 3.75(\mathrm{~s}, 3 \mathrm{H}), 3.59-3.56(\mathrm{~m}, 1 \mathrm{H}), 3.50(\mathrm{t}, J=7.6 \mathrm{~Hz}, 1 \mathrm{H}), 2.89-2.70(\mathrm{~m}, 4 \mathrm{H}), 2.15-$ $2.08(\mathrm{~m}, 1 \mathrm{H}), 2.00-1.91(\mathrm{~m}, 1 \mathrm{H}) .{ }^{13} \mathrm{C} \mathrm{NMR}\left(100 \mathrm{MHz}, \mathrm{CDCl}_{3}\right) \delta$ 169.1, 168.8, 141.7, $133.4,132.3,130.7,129.4,129.0,124.3,119.5,117.3,105.2,52.6,51.4$, 38.2, 32.9, 31.5, 27.9. IR (thin film): $v_{\max }\left(\mathrm{cm}^{-1}\right)=2955,1733,1699,1585,1437,1352,1266,1232$, 1176, 1150, 1060, 1000, 983, 914, 891, 811, 729, 701, 590, 479, 456. HRMS (ESI) calcd for $\mathrm{C}_{20} \mathrm{H}_{20} \mathrm{NO}_{5} \mathrm{NaCl}[\mathrm{M}+\mathrm{Na}]^{+}: 412.0922$. Found: 412.0925 .

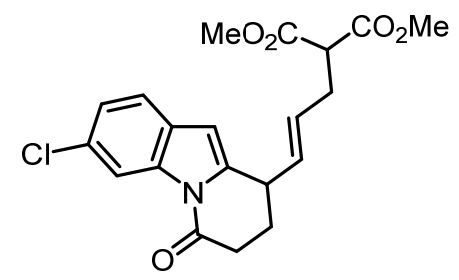

6e, green oil, $46.7 \mathrm{mg}, 59 \%$ yield. ${ }^{1} \mathrm{H}$ NMR $\left(400 \mathrm{MHz}, \mathrm{CDCl}_{3}\right) \delta 8.47(\mathrm{~s}, 1 \mathrm{H}), 7.35(\mathrm{~d}$, $J=8.4 \mathrm{~Hz}, 1 \mathrm{H}), 7.21(\mathrm{dd}, J=8.4,1.2 \mathrm{~Hz}, 1 \mathrm{H}), 6.23(\mathrm{~s}, 1 \mathrm{H}), 5.68-5.59(\mathrm{~m}, 2 \mathrm{H}), 3.76(\mathrm{~s}$, $3 \mathrm{H}), 3.75(\mathrm{~s}, 3 \mathrm{H}), 3.58-3.56(\mathrm{~m}, 1 \mathrm{H}), 3.50(\mathrm{t}, J=7.6 \mathrm{~Hz}, 1 \mathrm{H}), 2.89-2.70(\mathrm{~m}, 4 \mathrm{H}), 2.14-$ 
$2.08(\mathrm{~m}, 1 \mathrm{H}), 2.00-1.90(\mathrm{~m}, 1 \mathrm{H}) .{ }^{13} \mathrm{C} \mathrm{NMR}\left(100 \mathrm{MHz}, \mathrm{CDCl}_{3}\right) \delta 169.1,168.9,140.9$, 135.3, 132.3, 130.0, 129.0, 127.9, 124.3, 120.5, 116.5, 105.5, 52.6, 51.4, 38.1, 32.9, 31.5, 27.9. IR (thin film): $v_{\max }\left(\mathrm{cm}^{-1}\right)=2920,1749,1731,1702,1582,1455,1360,1267$, 1181, 1148, 1062, 1040, 990, 917, 880, 741, 698, 653, 601, 560, 484. HRMS (ESI) calcd for $\mathrm{C}_{20} \mathrm{H}_{20} \mathrm{NO}_{5} \mathrm{NaCl}[\mathrm{M}+\mathrm{Na}]^{+}: 412.0922$. Found: 412.0928 .

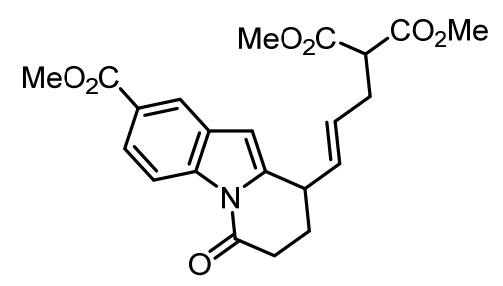

6f, viscous solid, $35.9 \mathrm{mg}, 43 \%$ yield. ${ }^{1} \mathrm{H}$ NMR (400 MHz, $\left.\mathrm{CDCl}_{3}\right) \delta 8.47$ (d, $J=8.4$ $\mathrm{Hz}, 1 \mathrm{H}), 8.19$ (s, 1H), 7.99 (dd, $J=8.4,1.2 \mathrm{~Hz}, 1 \mathrm{H}), 6.34$ (s, 1H), 5.71-5.61 (m, 2H), $3.94(\mathrm{~s}, 3 \mathrm{H}), 3.77(\mathrm{~s}, 3 \mathrm{H}), 3.76(\mathrm{~s}, 3 \mathrm{H}), 3.63-3.60(\mathrm{~m}, 1 \mathrm{H}), 3.52(\mathrm{t}, J=7.6 \mathrm{~Hz}, 1 \mathrm{H})$, 2.93-2.71 (m, 4H), 2.17-2.11 (m, 1H), 2.03-1.93 (m, 1H). $\left.{ }^{13} \mathrm{C} \mathrm{NMR} \mathrm{(100} \mathrm{MHz,} \mathrm{CDCl}_{3}\right)$ $\delta 169.1,169.0,167.4,141.7,137.7,132.3,129.2,129.1,125.7,122.0,115.9,106.1$, 52.6, 52.0, 51.4, 38.2, 33.0, 31.5, 27.8. IR (thin film): $v_{\max }\left(\mathrm{cm}^{-1}\right)=2957,1733,1701$, 1585, 1437, 1343, 1293, 1252, 1228, 1173, 1135, 1091, 1073, 970, 933, 840, 814, 770, 743, 695, 588. HRMS (ESI) calcd for $\mathrm{C}_{22} \mathrm{H}_{23} \mathrm{NO}_{7} \mathrm{Na}[\mathrm{M}+\mathrm{Na}]^{+}:$436.1367. Found: 436.1360 .

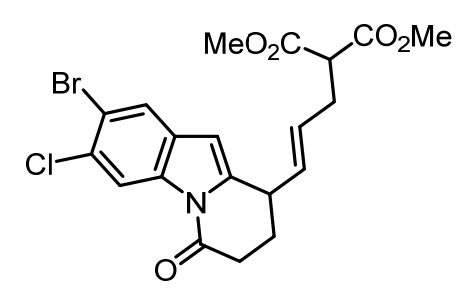

6g, colorless oil, $55.1 \mathrm{mg}, 60 \%$ yield. ${ }^{1} \mathrm{H}$ NMR (400 MHz, $\left.\mathrm{CDCl}_{3}\right) \delta 8.53(\mathrm{~s}, 1 \mathrm{H}), 7.66$ $(\mathrm{s}, 1 \mathrm{H}), 6.17(\mathrm{~s}, 1 \mathrm{H}), 5.70-5.60(\mathrm{~m}, 2 \mathrm{H}), 3.77(\mathrm{~s}, 3 \mathrm{H}), 3.76(\mathrm{~s}, 3 \mathrm{H}), 3.60-3.57(\mathrm{~m}, 1 \mathrm{H})$, $3.51(\mathrm{t}, J=7.6 \mathrm{~Hz}, 1 \mathrm{H}), 2.89-2.71(\mathrm{~m}, 4 \mathrm{H}), 2.18-2.09(\mathrm{~m}, 1 \mathrm{H}), 2.02-1.92(\mathrm{~m}, 1 \mathrm{H}) .{ }^{13} \mathrm{C}$ NMR (100 MHz, $\left.\mathrm{CDCl}_{3}\right) \delta 169.1,168.7,142.2,134.2,132.0,129.5,129.4,129.3,124.0$, 117.7, 117.2, 104.6, 52.6, 51.4, 38.2, 32.8, 31.5, 27.8. IR (thin film): $v_{\max }\left(\mathrm{cm}^{-1}\right)=2921$, 2849, 1731, 1700, 1585, 1440, 1375, 1345, 1176, 1095, 1063, 1029, 989, 963, 869, 803, 756, 692, 657, 589, 528. HRMS (ESI) calcd for $\mathrm{C}_{20} \mathrm{H}_{19} \mathrm{NO}_{5} \mathrm{NaCl}^{79} \mathrm{Br}[\mathrm{M}+\mathrm{Na}]^{+}$: 490.0027. Found: 490.0019. 
3.5 General procedure for the dearomatized [5+4] cycloadditions of pyrrole-derived VCPs (using 7 a as an example).

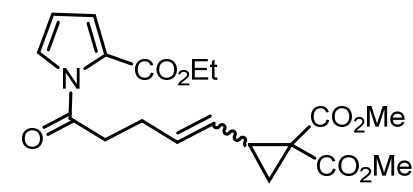

$7 \mathrm{a}(E / Z=4.1 / 1)$

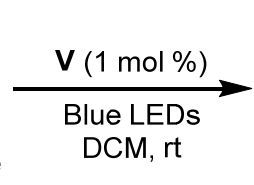

DCM, rt

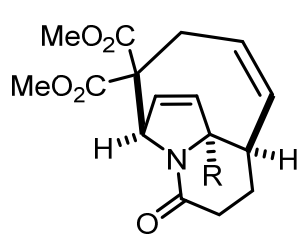

$\mathbf{8 a}\left(\mathrm{R}=\mathrm{CO}_{2} \mathrm{Et}\right)$

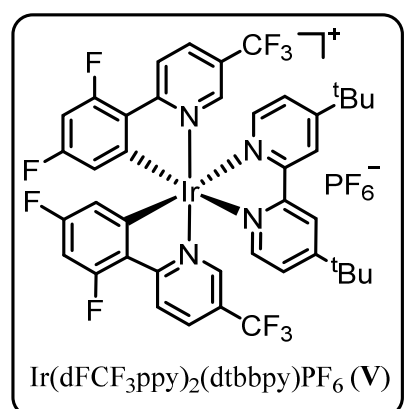

To a flame-dried sealed tube were added pyrrole derivative $7 \mathbf{a}(75.5 \mathrm{mg}, 0.2 \mathrm{mmol})$ and photosensitizer $\mathbf{V}(2.2 \mathrm{mg}, 0.002 \mathrm{mmol})$ and anhydrous DCM $(20 \mathrm{~mL})$. The reaction mixture was degassed via freeze-pump-thaw for 3 cycles. After the reaction mixture was thoroughly degassed, the vial was sealed and positioned approximately $10 \mathrm{~cm}$ from blue LEDs $\left(24 \mathrm{~W}, \lambda_{\max }=455 \mathrm{~nm}\right)$. Then the reaction mixture was stirred at room temperature for the indicated time (monitored by TLC) under argon atmosphere. Afterwards, the reaction mixture was concentrated by rotary evaporation. Then the residue was purified by silica gel column chromatography $(\mathrm{PE} / \mathrm{EtOAc}=2 / 1)$ to afford the desired product $\mathbf{8 a}$.

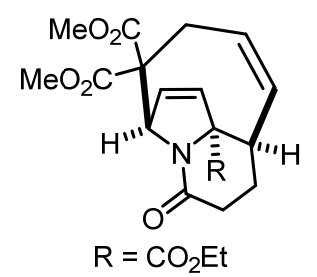

8a, viscous solid, $43.7 \mathrm{mg}, 78 \%$ yield. ${ }^{1} \mathrm{H}$ NMR (400 MHz, $\left.\mathrm{CDCl}_{3}\right) \delta$ 5.95-5.92 (m, 2H), 5.77-5.69 (m, 1H), 5.62-5.56 (m, 1H), 5.41-5.39 (m, 1H), 4.20-4.08 (m, 2H), 3.77 (s, 3H), $3.70(\mathrm{~s}, 3 \mathrm{H}), 3.68-3.61(\mathrm{~m}, 1 \mathrm{H}), 2.96-2.91(\mathrm{~m}, 1 \mathrm{H}), 2.43-2.31(\mathrm{~m}, 2 \mathrm{H}), 2.09$ $1.96(\mathrm{~m}, 2 \mathrm{H}), 1.82-1.73(\mathrm{~m}, 1 \mathrm{H}), 1.19(\mathrm{t}, J=7.2 \mathrm{~Hz}, 3 \mathrm{H}) .{ }^{13} \mathrm{C} \mathrm{NMR}\left(100 \mathrm{MHz}, \mathrm{CDCl}_{3}\right)$ $\delta 172.3,171.0,169.6,168.7,136.0,131.6,130.8,127.6,79.8,67.1,62.4,60.7,53.5$, 52.8, 39.0, 31.4, 28.7, 25.3, 13.9. IR (thin film): $v_{\max }\left(\mathrm{cm}^{-1}\right)=2920,2849,1727,1664$, $1457,1437,1403,1242,1210,1185,1118,1029,959,919,876,852,805,761,746$, 702, 603, 583. HRMS (ESI) calcd for $\mathrm{C}_{19} \mathrm{H}_{24} \mathrm{NO}_{7}[\mathrm{M}+\mathrm{H}]^{+}: 378.1547$. Found: 378.1547. 


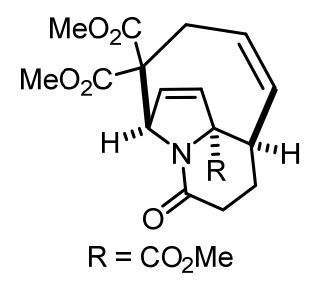

8b, colorless oil, $50.6 \mathrm{mg}, 71 \%$ yield. ${ }^{1} \mathrm{H}$ NMR (400 MHz, $\left.\mathrm{CDCl}_{3}\right) \delta 6.02-6.00(\mathrm{~m}, 2 \mathrm{H})$, 5.84-5.77 (m, 1H), 5.69-5.64 (m, 1H), 5.47 (d, $J=4.8 \mathrm{~Hz}, 1 \mathrm{H}), 3.84(\mathrm{~s}, 3 \mathrm{H}), 3.77$ (s, $3 \mathrm{H}), 3.75$ (s, 3H), 3.71-3.68 (m, 1H), 3.04-2.98 (m, 1H), 2.51-2.39 (m, 2H), 2.13-2.02 (m, 2H), 1.90-1.80 (m, 1H). ${ }^{13} \mathrm{C}$ NMR (100 MHz, $\left.\mathrm{CDCl}_{3}\right) \delta 172.4,171.5,169.5,168.6$, $135.9,131.5,130.9,127.6,79.7,67.1,60.6,53.5,53.3,52.8,39.1,31.3,28.7,25.2$. IR (thin film): $v_{\max }\left(\mathrm{cm}^{-1}\right)=2951,1748,1726,1676,1429,1398,1239,1207,1126,1070$, 1014, 960, 920, 873, 847, 797, 747, 711, 636, 603, 470, 440. HRMS (ESI) calcd for $\mathrm{C}_{18} \mathrm{H}_{21} \mathrm{NO}_{7} \mathrm{Na}[\mathrm{M}+\mathrm{Na}]^{+}: 386.1210$. Found: 386.1212 .

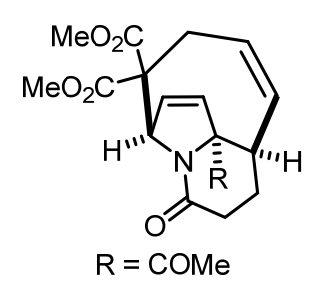

8c, colorless oil, $41.6 \mathrm{mg}, 61 \%$ yield. ${ }^{1} \mathrm{H}$ NMR (400 MHz, $\left.\mathrm{CDCl}_{3}\right) \delta$ 6.12-6.11 (m, 2H), 5.82-5.75 (m, 1H), 5.69-5.64 (m, 1H), 5.32-5.30 (m, 1H), $3.85(\mathrm{~s}, 3 \mathrm{H}), 3.82(\mathrm{~s}, 1 \mathrm{H})$, $3.78(\mathrm{~s}, 3 \mathrm{H}), 3.07-3.01(\mathrm{~m}, 1 \mathrm{H}), 2.46-2.33(\mathrm{~m}, 2 \mathrm{H}), 2.17(\mathrm{~s}, 3 \mathrm{H}), 2.10-2.04(\mathrm{~m}, 1 \mathrm{H})$, 1.88-1.76 (m, 2H). ${ }^{13} \mathrm{C}$ NMR (100 MHz, $\left.\mathrm{CDCl}_{3}\right) \delta 206.0,172.6,169.5,168.5,136.7$, $132.3,131.4,127.4,86.0,67.6,60.8,53.6,52.9,36.8,32.4,28.6,25.1,24.0$. IR (thin film): $v_{\max }\left(\mathrm{cm}^{-1}\right)=2953,1743,1714,1658,1399,1359,1261,1198,1121,1043,962$, 925, 841, 749, 702, 679, 622, 595, 556, 485, 462, 428. HRMS (ESI) calcd for $\mathrm{C}_{18} \mathrm{H}_{21} \mathrm{NO}_{6} \mathrm{Na}[\mathrm{M}+\mathrm{Na}]^{+}: 370.1261$. Found: 370.1260 .

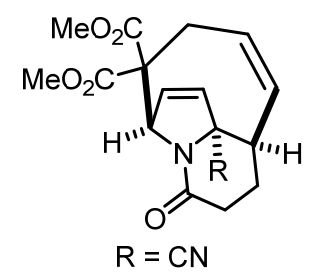

8d, colorless oil, $58.5 \mathrm{mg}, 87 \%$ yield. ${ }^{1} \mathrm{H}$ NMR (400 $\left.\mathrm{MHz}, \mathrm{CDCl}_{3}\right) \delta 6.12$ (dd, $J=6.0$, $1.2 \mathrm{~Hz}, 1 \mathrm{H}), 5.85-5.83(\mathrm{~m}, 1 \mathrm{H}), 5.76-5.68(\mathrm{~m}, 1 \mathrm{H}), 5.57-5.52(\mathrm{~m}, 1 \mathrm{H}), 4.79-4.78(\mathrm{~m}$, 1H), 3.89 (s, 3H), $3.83(\mathrm{~s}, 3 \mathrm{H}), 3.09-2.96(\mathrm{~m}, 2 \mathrm{H}), 2.52-2.46(\mathrm{~m}, 3 \mathrm{H}), 2.31-2.25(\mathrm{~m}$, 
1H), 1.94-1.84 (m, 1H), ${ }^{13} \mathrm{C}$ NMR (100 MHz, $\left.\mathrm{CDCl}_{3}\right) \delta 171.6,167.4,166.8,135.7$, $134.0,128.4,126.1,116.7,68.0,66.4,64.0,53.6,53.4,37.4,31.7,30.3,25.8$. IR (thin film): $v_{\max }\left(\mathrm{cm}^{-1}\right)=2954,1735,1673,1433,1398,1291,1204,1087,1067,1033,990$, 935, 913, 850, 822, 806, 783, 730, 702, 632, 545, 516. HRMS (ESI) calcd for $\mathrm{C}_{17} \mathrm{H}_{18} \mathrm{~N}_{2} \mathrm{O}_{5} \mathrm{Na}[\mathrm{M}+\mathrm{Na}]^{+}:$353.1108. Found: 353.1111 .

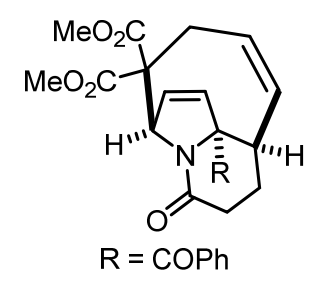

8e, colorless oil, $59.4 \mathrm{mg}, 73 \%$ yield. ${ }^{1} \mathrm{H}$ NMR (400 MHz, $\left.\mathrm{CDCl}_{3}\right) \delta 7.76(\mathrm{~d}, J=7.6$ $\mathrm{Hz}, 2 \mathrm{H}), 7.52$ (t, $J=7.2 \mathrm{~Hz}, 1 \mathrm{H}), 7.39$ (t, $J=7.6 \mathrm{~Hz}, 2 \mathrm{H}), 6.19-6.14(\mathrm{~m}, 2 \mathrm{H}), 5.89-5.82$ $(\mathrm{m}, 1 \mathrm{H}), 5.72-5.62(\mathrm{~m}, 2 \mathrm{H}), 4.20-4.11(\mathrm{~m}, 1 \mathrm{H}), 3.83(\mathrm{~s}, 3 \mathrm{H}), 3.82(\mathrm{~s}, 3 \mathrm{H}), 3.10-3.04(\mathrm{~m}$, $1 \mathrm{H}), 2.50-2.42(\mathrm{~m}, 1 \mathrm{H}), 2.36-2.27(\mathrm{~m}, 1 \mathrm{H}), 2.13-2.07(\mathrm{~m}, 1 \mathrm{H}), 1.88-1.75(\mathrm{~m}, 2 \mathrm{H}) .{ }^{13} \mathrm{C}$ NMR $\left(100 \mathrm{MHz} \mathrm{CDCl}_{3}\right) \delta 198.2,172.4,169.5,168.6,136.5,135.0,133.3,133.1,131.3$, $128.8,128.6,127.6,85.2,68.1,60.8,53.6,53.0,39.2,32.8,28.6,25.0$. IR (thin film): $v_{\max }\left(\mathrm{cm}^{-1}\right)=2952,1732,1670,1595,1432,1396,1234,1210,1179,1123,1046,960$, 916, 891, 830, 790, 714, 663, 584, 435. HRMS (ESI) calcd for $\mathrm{C}_{23} \mathrm{H}_{23} \mathrm{NO}_{6} \mathrm{Na}[\mathrm{M}+\mathrm{Na}]^{+}$: 432.1418. Found: 432.1417.

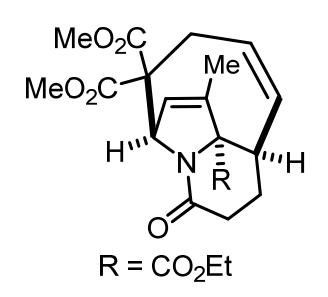

8f, colorless oil, $46.8 \mathrm{mg}, 60 \%$ yield. ${ }^{1} \mathrm{H}$ NMR (400 MHz, $\left.\mathrm{CDCl}_{3}\right) \delta 5.94$ (s, 1H), 5.84$5.73(\mathrm{~m}, 2 \mathrm{H}), 5.57(\mathrm{~s}, 1 \mathrm{H}), 4.20(\mathrm{q}, J=7.2 \mathrm{~Hz}, 2 \mathrm{H}), 3.83(\mathrm{~s}, 3 \mathrm{H}), 3.77$ (s, 3H), 3.73$3.66(\mathrm{~m}, 1 \mathrm{H}), 3.02-2.97(\mathrm{~m}, 1 \mathrm{H}), 2.52-2.40(\mathrm{~m}, 2 \mathrm{H}), 2.07-1.99(\mathrm{~m}, 2 \mathrm{H}), 1.86-1.77$ (m, $1 \mathrm{H}), 1.47(\mathrm{~s}, 3 \mathrm{H}), 1.25(\mathrm{t}, J=7.2 \mathrm{~Hz}, 3 \mathrm{H}) .{ }^{13} \mathrm{C} \mathrm{NMR}\left(100 \mathrm{MHz}, \mathrm{CDCl}_{3}\right) \delta 172.4,170.6$, 169.6, 168.8, 139.0, 133.6, 128.8, 125.2, 81.4, 66.2, 63.2, 62.3, 53.5, 52.8, 37.5, 31.3, 28.8, 25.0, 14.0, 12.6. IR (thin film): $v_{\max }\left(\mathrm{cm}^{-1}\right)=2953,1727,1676,1432,1405,1202$, 1121, 1070, 1026, 929, 913, 878, 861, 818, 794, 740, 666, 641, 619, 551, 503, 423. HRMS (ESI) calcd for $\mathrm{C}_{20} \mathrm{H}_{25} \mathrm{NO}_{7} \mathrm{Na}[\mathrm{M}+\mathrm{Na}]^{+}$: 414.1523. Found: 414.1521. 


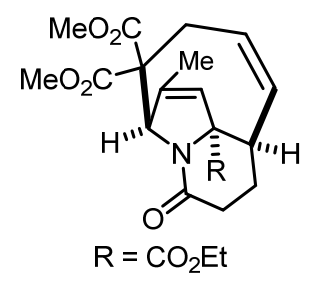

8g, colorless oil, $50.0 \mathrm{mg}, 64 \%$ yield. ${ }^{1} \mathrm{H}$ NMR (400 MHz, $\left.\mathrm{CDCl}_{3}\right) \delta 6.00-5.91(\mathrm{~m}, 2 \mathrm{H})$, 5.72-5.66 (m, 1H), $5.21(\mathrm{~s}, 1 \mathrm{H}), 4.27-4.13(\mathrm{~m}, 2 \mathrm{H}), 3.86(\mathrm{~s}, 3 \mathrm{H}), 3.72(\mathrm{~s}, 3 \mathrm{H}), 3.69-3.63$ (m, 1H), 2.90-2.84 (m, 1H), 2.47-2.39 (m, 2H), 2.15-2.03 (m, 2H), 1.86-1.77 (m, 1H), $1.70(\mathrm{~s}, 3 \mathrm{H}), 1.27$ (t, $J=7.2 \mathrm{~Hz}, 3 \mathrm{H}),{ }^{13} \mathrm{C} \mathrm{NMR}\left(100 \mathrm{MHz}, \mathrm{CDCl}_{3}\right) \delta 172.6,171.4$, 170.3, 169.1, 139.2, 135.1, 128.0, 127.3, 78.8, 69.2, 62.3, 60.2, 53.3, 52.5, 38.4, 31.5, 29.5, 25.1, 15.4, 14.0. IR (thin film): $v_{\max }\left(\mathrm{cm}^{-1}\right)=2947,1737,1712,1674,1433,1396$, 1269, 1249, 1206, 1109, 1048, 1022, 946, 914, 879, 851, 794, 772, 738, 704, 648, 600. HRMS (ESI) calcd for $\mathrm{C}_{20} \mathrm{H}_{25} \mathrm{NO}_{7} \mathrm{Na}[\mathrm{M}+\mathrm{Na}]^{+}$: 414.1523. Found: 414.1524. 


\section{Transformations of Products}
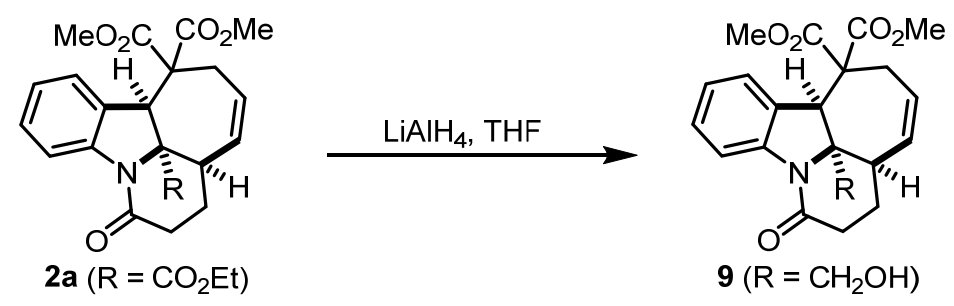

A solution of compound $\mathbf{2 a}(85.4 \mathrm{mg}, 0.2 \mathrm{mmol})$ in THF $(1 \mathrm{~mL})$ was slowly added to a flask containing $\mathrm{LiAlH}_{4}(91.2 \mathrm{mg}, 2.4 \mathrm{mmol})$ in THF $(2 \mathrm{~mL})$ at $0{ }^{\circ} \mathrm{C}$. The solution was stirred at room temperature overnight and quenched by $\mathrm{H}_{2} \mathrm{O}(1.5 \mathrm{~mL}), \mathrm{NaOH}(10 \%$ aq. $3.0 \mathrm{~mL})$ and $\mathrm{H}_{2} \mathrm{O}(4.5 \mathrm{~mL})$ carefully at $0{ }^{\circ} \mathrm{C}$. The solution was then filtered through celite and washed with EtOAc. The combined organic layers were dried over $\mathrm{Na}_{2} \mathrm{SO}_{4}$ and the solvent was removed under reduced pressure to give the crude product. Then the crude product was purified by silica gel column chromatography $(\mathrm{DCM} / \mathrm{MeOH}=$ 10/1) to afford product 9.

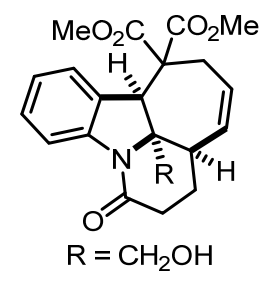

9, brown solid, $40.0 \mathrm{mg}$, 63\% yield, m.p. $=80.7-83.1{ }^{\circ} \mathrm{C} .{ }^{1} \mathrm{H}$ NMR $\left(400 \mathrm{MHz}, \mathrm{CD}_{3} \mathrm{OD}\right)$ $\delta 7.26(\mathrm{~d}, J=7.2 \mathrm{~Hz}, 1 \mathrm{H}), 6.94(\mathrm{t}, J=7.6 \mathrm{~Hz}, 1 \mathrm{H}), 6.49(\mathrm{t}, J=7.2 \mathrm{~Hz}, 1 \mathrm{H}), 6.25(\mathrm{~d}, J$ $=7.6 \mathrm{~Hz}, 1 \mathrm{H}), 5.63-5.59(\mathrm{~m}, 1 \mathrm{H}), 5.30(\mathrm{~d}, J=11.6 \mathrm{~Hz}, 1 \mathrm{H}), 3.96(\mathrm{~d}, J=10.8 \mathrm{~Hz}, 1 \mathrm{H})$, $3.80(\mathrm{~s}, 2 \mathrm{H}), 3.39-3.37(\mathrm{~m}, 2 \mathrm{H}), 3.26-3.20(\mathrm{~m}, 3 \mathrm{H}), 3.06(\mathrm{~s}, 1 \mathrm{H}), 2.94(\mathrm{~d}, J=10.8 \mathrm{~Hz}$, $1 \mathrm{H}), 2.77(\mathrm{t}, J=12.0 \mathrm{~Hz}, 1 \mathrm{H}), 2.11-2.04(\mathrm{~m}, 1 \mathrm{H}), 1.83-1.79(\mathrm{~m}, 2 \mathrm{H}), 1.58-1.50(\mathrm{~m}, 3 \mathrm{H})$. ${ }^{13} \mathrm{C} \mathrm{NMR}\left(100 \mathrm{MHz}, \mathrm{CDCl}_{3}\right) \delta 151.4,133.1,127.9,127.6,127.0,116.6,105.0,71.3$, $70.5,68.9,62.1,47.6,43.9,39.6,36.3,28.7,27.9,19.5$. IR (thin film): $v_{\max }\left(\mathrm{cm}^{-1}\right)=$ 3336, 3001, 2920, 2849, 1632, 1598, 1483, 1432, 1309, 1253, 1154, 1136, 1015, 916, 845, 749, 731, 701, 687, 649, 577. HRMS (EI) calcd for $\mathrm{C}_{19} \mathrm{H}_{26} \mathrm{NO}_{3}[\mathrm{M}+\mathrm{H}]^{+}: 316.1907$. Found: 316.1911. 


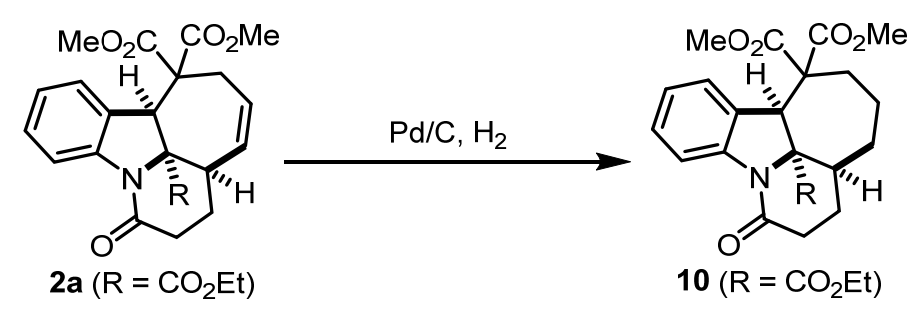

2a ( $85.4 \mathrm{mg}, 0.2 \mathrm{mmol})$ and $\mathrm{Pd} / \mathrm{C}(8.0 \mathrm{mg}, 10 \% \mathrm{wt})$ were added into a flask containing $\mathrm{MeOH}(10 \mathrm{~mL})$ under argon. The reaction mixture was stirred at room temperature under hydrogen atmosphere (1 atm). After the reaction was complete (monitored by TLC), the suspension was filtered and washed with dichloromethane. Then the filtrate was concentrated by rotary evaporation and the residue was purified by silica gel column chromatography $(\mathrm{PE} / \mathrm{EtOAc}=2 / 1)$ to afford the desired product $\mathbf{1 0}$.

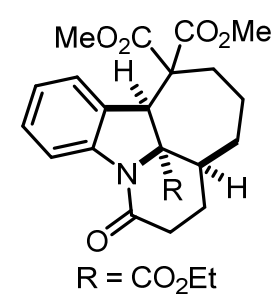

10, yellow oil, $82.4 \mathrm{mg}, 95 \%$ yield. ${ }^{1} \mathrm{H}$ NMR (400 MHz, $\left.\mathrm{CDCl}_{3}\right) \delta 8.42(\mathrm{~d}, J=8.0 \mathrm{~Hz}$, $1 \mathrm{H}), 7.28(\mathrm{t}, J=8.0 \mathrm{~Hz}, 1 \mathrm{H}), 6.97(\mathrm{t}, J=7.6 \mathrm{~Hz}, 1 \mathrm{H}), 6.71(\mathrm{~d}, J=8.0 \mathrm{~Hz}, 1 \mathrm{H}), 4.60$ (s, $1 \mathrm{H}), 4.28-4.15(\mathrm{~m}, 2 \mathrm{H}), 3.82(\mathrm{~s}, 3 \mathrm{H}), 3.81(\mathrm{~s}, 3 \mathrm{H}), 2.86-2.83(\mathrm{~m}, 1 \mathrm{H}), 2.64-2.36(\mathrm{~m}$, 2H), 2.39-2.36 (m, 1H), 1.87-1.59 (m, 5H), 1.35-1.30 (m, 2H), 1.26 (t, J=7.2 Hz, 3H). ${ }^{13} \mathrm{C}$ NMR $\left(100 \mathrm{MHz}, \mathrm{CDCl}_{3}\right) \delta 173.6,171.0,170.2,169.4,143.5,129.2,124.2,123.4$, $116.8,77.0,63.6,62.2,52.6,51.8,36.8,30.9,28.6,26.9,24.0,13.8$. IR (thin film): $v_{\max }$ $\left(\mathrm{cm}^{-1}\right)=2950,1730,1663,1594,1480,1461,1434,1312,1290,1199,1155,1112$, 1096, 1054, 1019, 979, 924, 803, 756, 683, 494. HRMS (ESI) calcd for $\mathrm{C}_{23} \mathrm{H}_{28} \mathrm{NO}_{7}$ $[\mathrm{M}+\mathrm{H}]^{+}:$430.1860. Found: 430.1860.

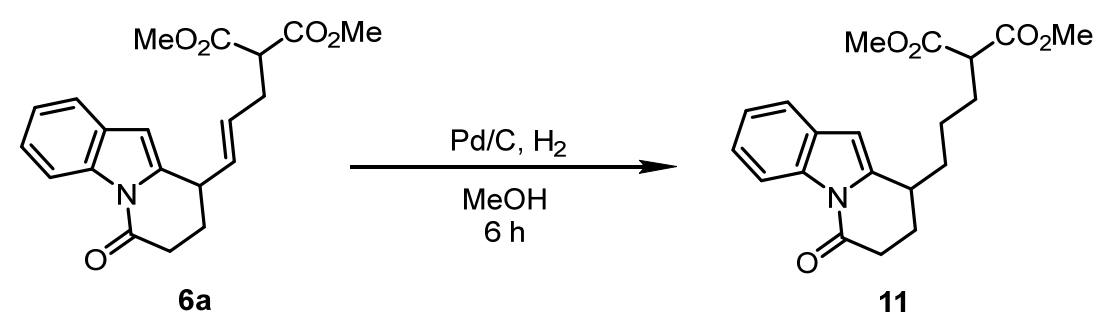


6a (71.0 mg, $0.2 \mathrm{mmol})$ and $\mathrm{Pd} / \mathrm{C}(8.0 \mathrm{mg}, 10 \% \mathrm{wt})$ were added into a flask containing $\mathrm{MeOH}(10 \mathrm{~mL})$ under argon. The reaction mixture was stirred at room temperature for $6 \mathrm{~h}$ under hydrogen atmosphere (1.0 atm). After the reaction was complete (monitored by TLC), the suspension was filtered and washed with dichloromethane. Then the filtrate was concentrated by rotary evaporation and the residue was purified by silica gel column chromatography $(\mathrm{PE} / \mathrm{EtOAc}=2 / 1)$ to afford the desired product 11.<smiles>COC(C)=O</smiles>

11, colorless oil, $60.5 \mathrm{mg}, 85 \%$ yield. ${ }^{1} \mathrm{H}$ NMR (400 $\left.\mathrm{MHz}, \mathrm{CDCl}_{3}\right) \delta 8.45(\mathrm{~d}, J=8.0$ $\mathrm{Hz}, 1 \mathrm{H}), 7.47$ (d, J=7.6 Hz, 1H), 7.30-7.23 (m, 2H), 6.34 (s, 1H), 3.75 (s, 6H), 3.42 (t, $J=7.6 \mathrm{~Hz}, 1 \mathrm{H}), 2.95-2.84(\mathrm{~m}, 2 \mathrm{H}), 2.76-2.68$ (m, 1H), 2.19-2.14 (m, 1H), 2.01-1.94 $(\mathrm{m}, 3 \mathrm{H}), 1.84-1.75(\mathrm{~m}, 1 \mathrm{H}), 1.70-1.62(\mathrm{~m}, 1 \mathrm{H}), 1.55-1.48(\mathrm{~m}, 2 \mathrm{H}) .{ }^{13} \mathrm{C}$ NMR $(100 \mathrm{MHz}$, $\left.\mathrm{CDCl}_{3}\right) \delta 169.7,169.2,141.8,134.9,129.5,124.2,123.9,119.8,116.3,104.4,52.5$, 51.4, 33.9, 33.0, 32.3, 28.7, 26.4, 24.3. IR (thin film): $v_{\max }\left(\mathrm{cm}^{-1}\right)=2944,1750,1730$, 1697, 1591, 1572, 1434, 1343, 1281, 1158, 1132, 1072, 1012, 965, 899, 828, 754, 686, $665,583,478,432$. HRMS (ESI) calcd for $\mathrm{C}_{20} \mathrm{H}_{24} \mathrm{NO}_{5}[\mathrm{M}+\mathrm{H}]^{+}:$358.1649. Found: 358.1650 .

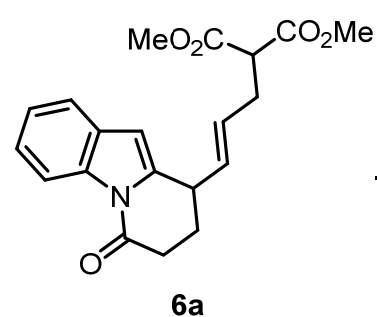

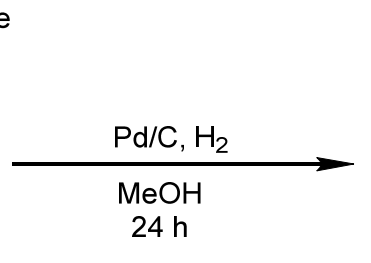

$24 \mathrm{~h}$

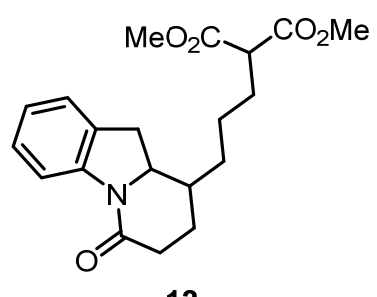

12

6a (71.0 mg, $0.2 \mathrm{mmol})$ and $\mathrm{Pd} / \mathrm{C}(8.0 \mathrm{mg}, 10 \% \mathrm{wt})$ were added into a flask containing $\mathrm{MeOH}(10 \mathrm{~mL})$ under argon. The reaction mixture was stirred at room temperature for $24 \mathrm{~h}$ under hydrogen atmosphere $(1 \mathrm{~atm})$. After the reaction was complete (monitored by TLC), the suspension was filtered and washed with dichloromethane. Then the filtrate was concentrated by rotary evaporation and the residue was purified by silica gel column chromatography $(\mathrm{PE} / \mathrm{EtOAc}=1 / 1)$ to afford the desired product $\mathbf{1 2}$. 


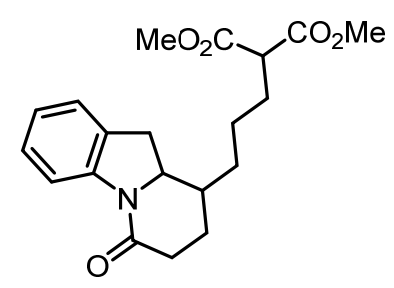

12, colorless oil, $32.2 \mathrm{mg}, 46 \%$ yield, $\mathrm{dr}=1: 1 .{ }^{1} \mathrm{H} \mathrm{NMR}\left(400 \mathrm{MHz}, \mathrm{CDCl}_{3}\right) \delta 8.17(\mathrm{~d}$, $J=8.0 \mathrm{~Hz}, 1 \mathrm{H}), 8.13\left(\mathrm{~d}, J=8.0 \mathrm{~Hz}, 1 \mathrm{H}^{*}\right), 7.22-7.19(\mathrm{~m}, 2 \mathrm{H}), 7.03(\mathrm{t}, J=7.6 \mathrm{~Hz}, 1 \mathrm{H})$,

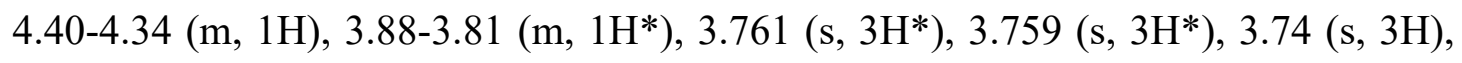
$3.73(\mathrm{~s}, 3 \mathrm{H}), 3.42-3.36(\mathrm{~m}, 1 \mathrm{H}), 3.17-3.08(\mathrm{~m}, 1 \mathrm{H}), 3.01-2.83(\mathrm{~m}, 1 \mathrm{H}), 2.67-2.41(\mathrm{~m}$, 2H), 2.13-1.61 (m, 5H), 1.57-1.29 (m, 4H). $\left.{ }^{13} \mathrm{C} \mathrm{NMR} \mathrm{(100} \mathrm{MHz,} \mathrm{CDCl}_{3}\right) \delta 169.6,168.2$, $168.0,142.8,142.5,130.1,129.7,127.4,124.4,124.3,123.94,123.89,117.2,117.0$, 66.0, 64.0, 52.53, 52.50, 52.47, 51.4, 51.3, 39.7, 35.1, 33.4, 32.2 , 31.6, 31.3, 28.9, 28.7, 28.4, 26.3, 24.8, 24.14, 24.10. IR (thin film): $v_{\max }\left(\mathrm{cm}^{-1}\right)=2919,1731,1645,1594$, 1479, 1460, 1429, 1404, 1381, 1355, 1288, 1132, 1081, 903, 759, 690, 622, 562, 536, 432. HRMS (ESI) calcd for $\mathrm{C}_{20} \mathrm{H}_{26} \mathrm{NO}_{5}[\mathrm{M}+\mathrm{H}]^{+}: 360.1806$. Found: 360.1808 . 


\section{Mechanistic Studies}

\subsection{Cyclic voltammetry}

A glassy carbon was used as the working electrode. A platinum wire was used as the auxillary electrode, and a SCE was used as the reference electrode. A solution of tetrabutylammonium hexafluorophosphate in $\mathrm{CH}_{3} \mathrm{CN}(0.1 \mathrm{M})$ was used as the supporting electrolyte. The scan rate was $100 \mathrm{mV} \mathrm{s}^{-1} .\left(\mathrm{E}_{1 / 2}=0.55 \mathrm{~V}\right.$ for the $\left[\mathrm{Cp}_{2} \mathrm{Fe}\right]^{0,+}$ couple).

Cyclic voltammogram of substrate 1a. $\left[\mathrm{E}_{1 / 2}{ }^{\mathrm{ox}}=1.65 \mathrm{~V} ; \mathrm{E}_{1 / 2}{ }^{\mathrm{red}}=-0.89 \mathrm{~V}\right]$
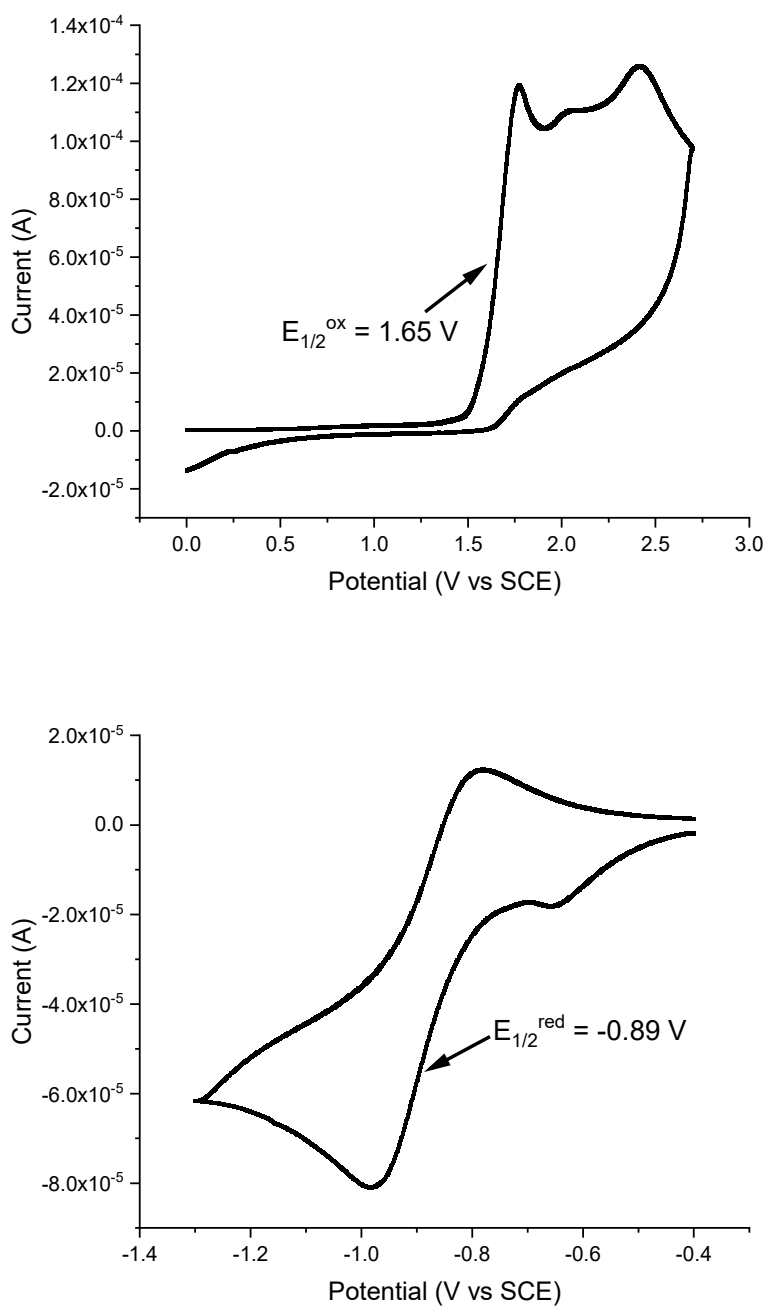


\subsection{Control experiment with triplet quencher}

2,5-Dimethylhexa-2,4-diene is known as a triplet quencher. ${ }^{3}$ The model reaction 1a $\rightarrow$ 2a is significantly inhibited in the presence of 2,5-dimethylhexa-2,4-diene (1 equiv). The NMR yield of 2 a was reduced to $34 \%$. This reaction suggested that a triplet diradical intermediate might be involved in the reaction.

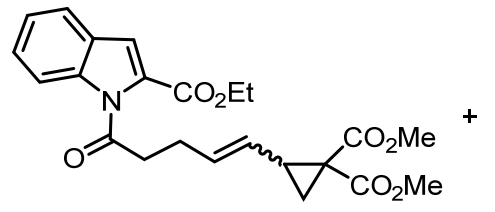

$1 \mathbf{a}(E / Z=5.5 / 1)$<smiles>CC(C)=CC=C(C)C(=O)OC(C)C</smiles>

(1.0 equiv)

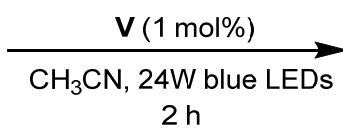

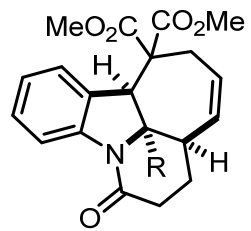

2a $\left(\mathrm{R}=\mathrm{CO}_{2} \mathrm{Et}\right)$ $34 \%$ NMR yield

\subsection{Stern-Volmer luminescence quenching studies}

Stern-Volmer experiments were conducted on a Hitachi F4600 Fluorescence Spectrophotometer. Each component was prepared in $\mathrm{CH}_{3} \mathrm{CN}$ prior to each set of experiments. The solutions were irradiated at $320 \mathrm{~nm}$ and the luminescence measured at $475 \mathrm{~nm}$. The value for $I_{0} / I$ from each run was averaged to yield a value of $I_{0} / I$ for the experiment. Linear regression of $I_{0} / I$ against concentration was performed in Origin software .

\begin{tabular}{|c|c|}
\hline species & concentration $(\mathrm{mM})$ \\
\hline $\mathrm{Ir}\left(\mathrm{dFCF}_{3} \text { ppy }\right)_{2}(\mathrm{dtbbpy}) \mathrm{PF}_{6}$ & 0.02 \\
\hline $\mathbf{1 a}$ & varied \\
\hline
\end{tabular}

\begin{tabular}{|c|c|c|c|c|c|}
\hline $\mathbf{1 a}\left(\times 10^{-5} \mathrm{M}\right)$ & 0 & 3 & 5 & 7 & 9 \\
\hline $\mathrm{I}_{1}$ & 1150 & 719.1 & 671.8 & 556.2 & 487 \\
\hline $\mathrm{I}_{2}$ & 1082 & 697.0 & 638.7 & 537.5 & 464.2 \\
\hline Average & 1116 & 703.05 & 655.25 & 546.85 & 475.6 \\
\hline $\mathrm{I}_{0} / \mathrm{I}$ & 1.00 & 1.587 & 1.703 & 2.041 & 2.347 \\
\hline
\end{tabular}




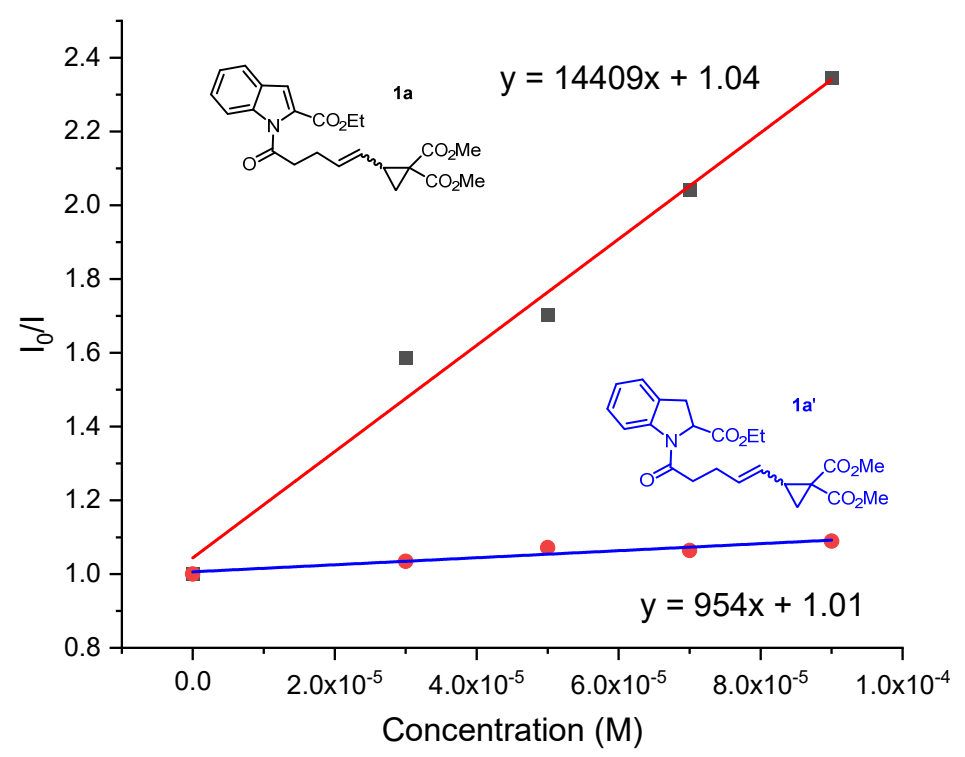

\begin{tabular}{|c|c|}
\hline species & concentration $(\mathrm{mM})$ \\
\hline $\operatorname{Ir}\left(\mathrm{dFCF}_{3} \text { ppy }\right)_{2}(\mathrm{dtbbpy}) \mathrm{PF}_{6}$ & 0.02 \\
\hline $\mathbf{1 a}^{\prime}$ & varied \\
\hline
\end{tabular}

\begin{tabular}{|c|c|c|c|c|c|}
\hline $\mathbf{1 a}^{\prime}\left(\times 10^{-5} \mathrm{M}\right)$ & 0 & 3 & 5 & 7 & 9 \\
\hline $\mathrm{I}_{1}$ & 1012 & 1004 & 985.1 & 1005 & 971.9 \\
\hline $\mathrm{I}_{2}$ & 1068 & 1006 & 954.4 & 949.6 & 936.6 \\
\hline Average & 1040 & 1005 & 969.75 & 977.3 & 954.3 \\
\hline $\mathrm{I}_{0} / \mathrm{I}$ & 1.00 & 1.035 & 1.072 & 1.064 & 1.089 \\
\hline
\end{tabular}

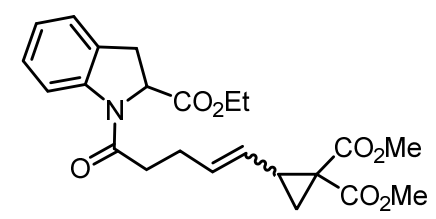

1a', yellow oil, $0.39 \mathrm{~g}, 30 \%$ yield (3.2 mmol scale), $E / Z=6 / 1$, two rotamers were observed. ${ }^{1} \mathrm{H}$ NMR $\left(400 \mathrm{MHz}, \mathrm{CDCl}_{3}\right) \delta 8.14(\mathrm{~d}, J=7.6 \mathrm{~Hz}, 1 \mathrm{H}), 7.10-7.04$ (m, 2H), 6.91 $(\mathrm{t}, J=7.2 \mathrm{~Hz}, 1 \mathrm{H}), 5.72-5.57(\mathrm{~m}, 1 \mathrm{H}), 5.05(\mathrm{~s}, 1 \mathrm{H}), 4.84-4.82(\mathrm{~m}, 1 \mathrm{H}), 4.11-4.09(\mathrm{~m}$, 2H), $3.62(\mathrm{~s}, 6 \mathrm{H}), 3.54-3.34(\mathrm{~m}, 1 \mathrm{H}), 3.16-2.95(\mathrm{~m}, 1 \mathrm{H}), 2.70-2.21(\mathrm{~m}, 5 \mathrm{H}), 1.59-1.46$ $(\mathrm{m}, 2 \mathrm{H}), 1.17(\mathrm{t}, J=6.8 \mathrm{~Hz}, 3 \mathrm{H}) .{ }^{13} \mathrm{C} \mathrm{NMR}\left(100 \mathrm{MHz}, \mathrm{CDCl}_{3}\right) \delta 170.9,170.1,169.9$, 169.6, 167.5, 167.4, 142.4, 140.6, 133.2, 133.0, 130.6, 128.2, 127.4, 125.4, 125.3, 123.9, 
$123.5,123.0,116.8,113.5,77.2,61.6,60.9,60.2,60.0,52.3,52.1,35.6,35.2,35.1,34.4$, 33.1, 31.0, 30.5, 27.1, 21.4, 20.3, 13.7. IR (thin film): $v_{\max }\left(\mathrm{cm}^{-1}\right)=2983,2952,1722$, 1661, 1600, 1480, 1462, 1436, 1397, 1264, 1192, 1125, 1023, 963, 899, 863, 751, 704, 666, 601. HRMS (ESI) calcd for $\mathrm{C}_{23} \mathrm{H}_{27} \mathrm{NO}_{7} \mathrm{Na}[\mathrm{M}+\mathrm{Na}]^{+}: 452.1680$. Found: 452.1692.

\subsection{Deuterium experiment of interrupted $[5+2]$ reaction.}

The interrupted [5+2] reaction of $\mathbf{1 w}$ was set up in HFIP- $d_{2}$ for $5 \mathrm{~h}$. The deuteration ratio was measued by ${ }^{1} \mathrm{H}$ NMR analysis of the isolated product $\mathbf{6 a}-d$. The deuteration ratio of $63 \%$ was observed.

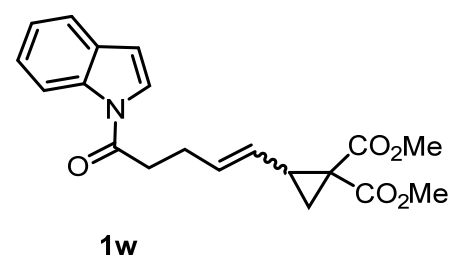

$1 w$

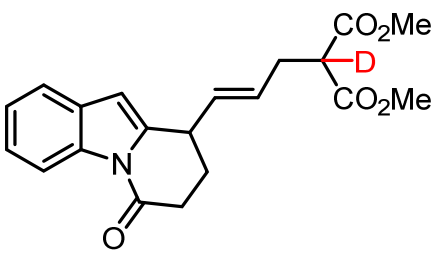

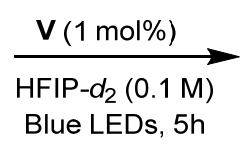

Blue LEDs, $5 \mathrm{~h}$

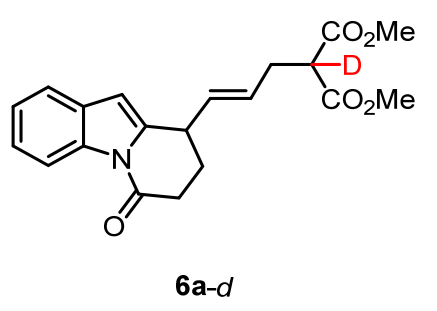

$71 \%$ yield, $>20: 1 E / Z$ Deuteration raito $63 \%$

6a- $d$, brown oil, $24.8 \mathrm{mg}, 71 \%$ yield. ${ }^{1} \mathrm{H}$ NMR $\left(400 \mathrm{MHz}, \mathrm{CDCl}_{3}\right) \delta 8.45(\mathrm{~d}, J=8.0 \mathrm{~Hz}$, 1H), 7.47 (d, $J=7.6 \mathrm{~Hz}, 1 \mathrm{H}), 7.31-7.23(\mathrm{~m}, 2 \mathrm{H}), 6.27$ (s, 1H), 5.70-5.58 (m, 2H), 3.76 $(\mathrm{s}, 3 \mathrm{H}), 3.75(\mathrm{~s}, 3 \mathrm{H}), 3.60-3.56(\mathrm{~m}, 1 \mathrm{H}), 3.50(\mathrm{t}, J=7.6 \mathrm{~Hz}, 0.37 \mathrm{H}), 2.89-2.70(\mathrm{~m}, 4 \mathrm{H})$, 2.14-2.07 (m, 1H), 2.00-1.90 (m, 1H).

Another interrupted [5+2] reaction of $\mathbf{1 x}$ was set up in $\mathrm{HFIP} / \mathrm{D}_{2} \mathrm{O}(\mathrm{v} / \mathrm{v}=5 / 1)$ for $5 \mathrm{~h}$. The deuteration ratio was measued by ${ }^{1} \mathrm{H}$ NMR analysis of the isolated product $6 \mathbf{b}-d$. The deuteration ratio of $42 \%$ was obtained. 

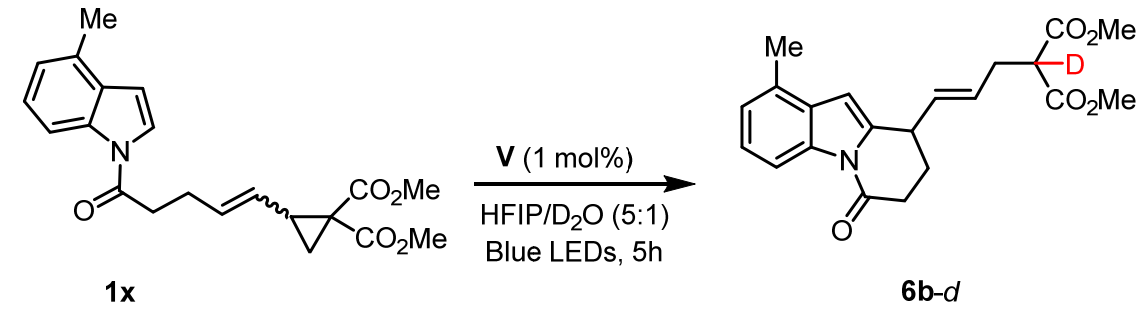

$91 \%$ yield, $>20: 1 E / Z$

Deuteration ratio $42 \%$

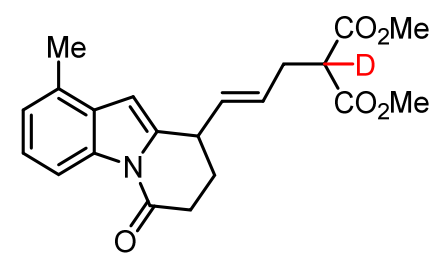

6b- $d$, yellow oil, $34.6 \mathrm{mg}, 91 \%$ yield. ${ }^{1} \mathrm{H}$ NMR $\left(400 \mathrm{MHz}, \mathrm{CDCl}_{3}\right) \delta 8.28(\mathrm{~d}, J=8.0$ $\mathrm{Hz}, 1 \mathrm{H}), 7.19$ (t, $J=8.0 \mathrm{~Hz}, 1 \mathrm{H}), 7.05$ (d, $J=7.6 \mathrm{~Hz}, 1 \mathrm{H}), 6.30$ (s, 1H), 5.69-5.59 (m, 2H), $3.76(\mathrm{~s}, 3 \mathrm{H}), 3.75(\mathrm{~s}, 3 \mathrm{H}), 3.61-3.56(\mathrm{~m}, 1 \mathrm{H}), 3.52$ (t, $J=7.6 \mathrm{~Hz}, 0.58 \mathrm{H}), 2.87-$ $2.69(\mathrm{~m}, 4 \mathrm{H}), 2.49(\mathrm{~s}, 3 \mathrm{H}), 2.13-2.06(\mathrm{~m}, 1 \mathrm{H}), 1.98-1.88(\mathrm{~m}, 1 \mathrm{H})$. 


\title{
5.5 Computational studies
}

\author{
Computational Methods
}

All the calculations in this study were performed with Gaussian $16 .{ }^{4}$ DFT studies were carried out with the (U)B3LYP functional ${ }^{5}$ including the D3 version of Grimme's empirical dispersion correction ${ }^{6}$ with Becke-Johnson damping. ${ }^{7}$ The def2-SVP basis sets of Weigend and Ahlrichs ${ }^{8}$ were employed for all atoms. Optimizations were conducted without any constraint in gas phase. Frequency analyses (at $298.15 \mathrm{~K}$ and $1 \mathrm{~atm}$ ) were carried out to confirm that each structure is a local minimum (no imaginary frequency) or a transition state (only one imaginary frequency). The energies were further estimated by single-point calculations with (U)B3LYP-D3(BJ) functional and def2-TZVP basis sets in acetonitrile (SMD model, ${ }^{9} \varepsilon=35.688$ ). The searching for minimal energy crossing points was conducted using a modified version of Harvey's $\operatorname{code}^{10}\left(\right.$ sobMECP $\left.^{11}\right)$ interfaced with Gaussian16.The relative Gibbs free energies in toluene $(\Delta G)$ including the single-point corrections were discussed throughout the paper unless otherwise specified. The optimized geometry and spin-density of the selected intermediates and transition states were visualized using VMD. ${ }^{12}$

\section{Optimized Geometry and Spin Density of Selected Intermediates and Transition States}

Listed below are the optimized structures of the intermediates and transition states shown in Figure 1 of the main text. The relative Gibbs free energies are given in $\mathrm{kcal} / \mathrm{mol}$. The spin densities are shown with isosurfaces in orange (isovalue $=0.03$ ) or blue (isovalue $=-0.03$ ). Underlined values are Mulliken spin populations at certain atoms. The forming/breaking $\mathrm{C}-\mathrm{C}$ bonds are highlighted with yellow dashes. Values in bold are the bond distances in angstrom. 


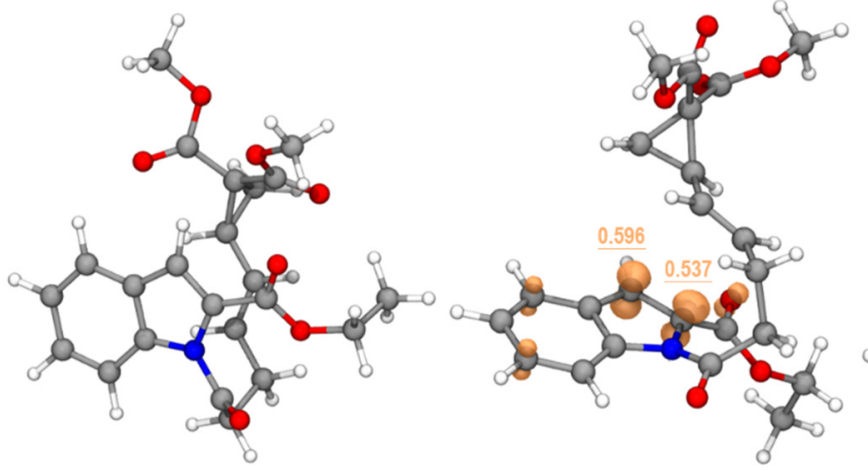

1a 0.0

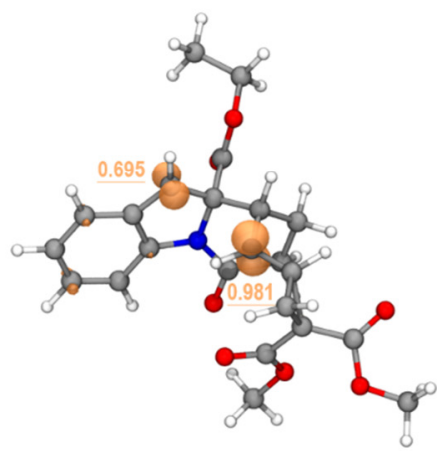

INT1a-T 33.3

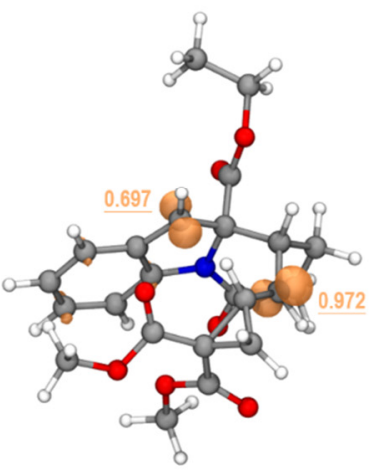

INT1b-T, 33.7

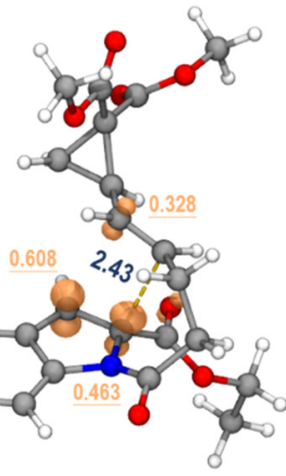

TS1a 53.3

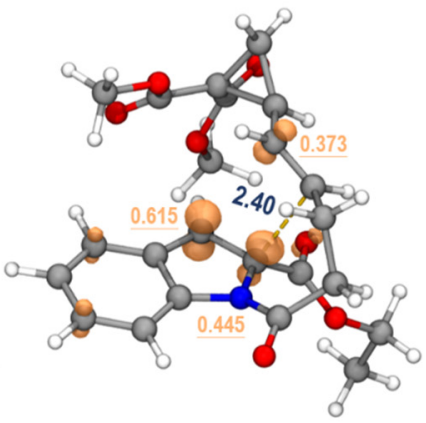

TS1b 55.1

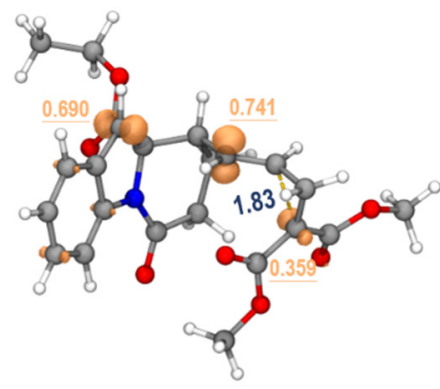

TS2a-E-T, 35.3

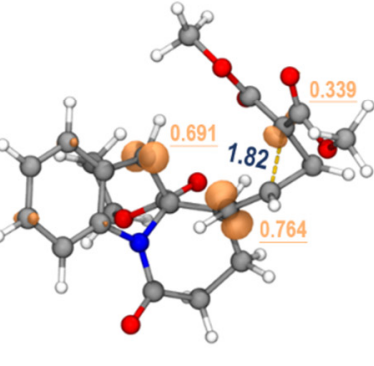

TS2a-Z-T 38.0

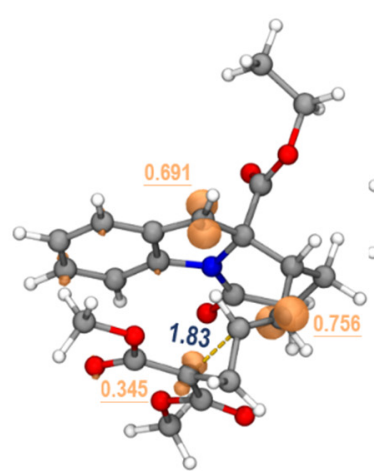

TS2b-E-T, 37.8

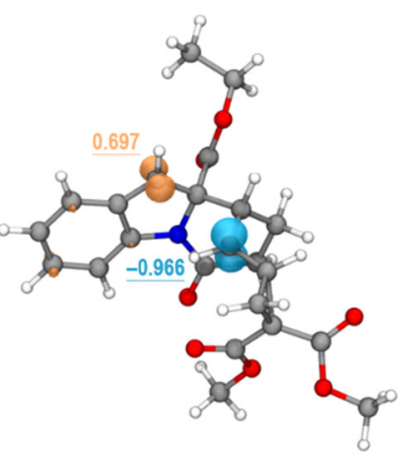

INT1a-OSS 33.6

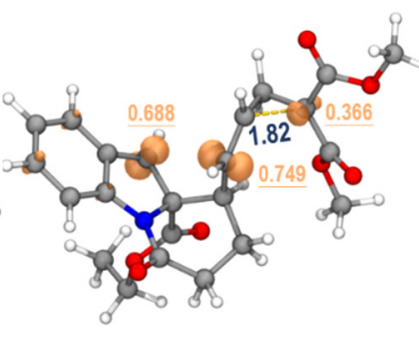

TS2b-Z-T 135.4

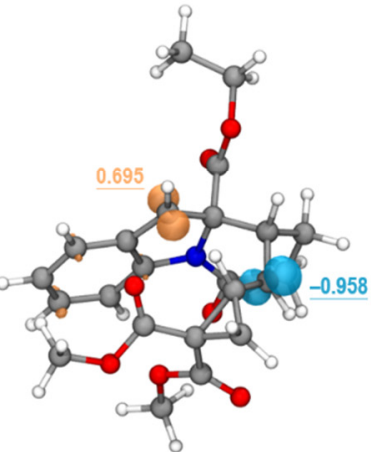

INT1b-OSS 33.7

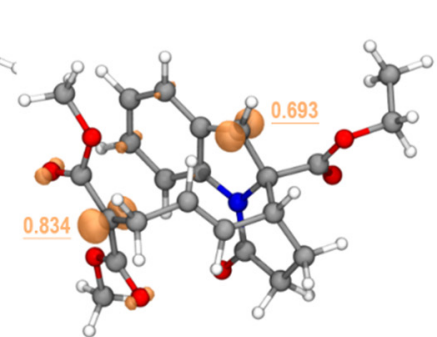

INT2-E-T, 25.0

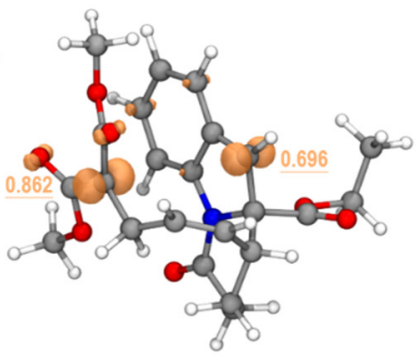

INT2-Z-T, 29.3

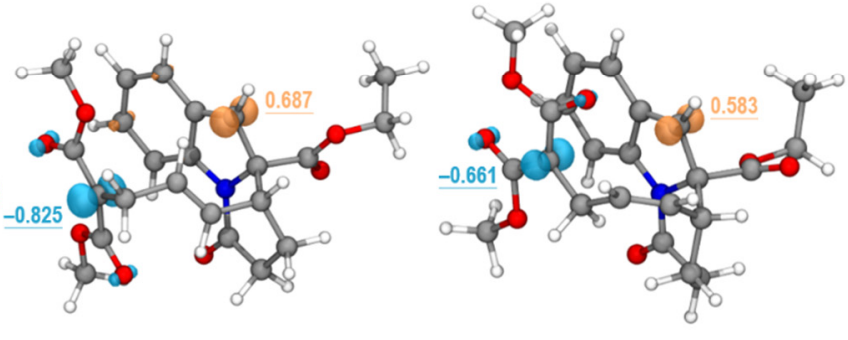

INT2-E-OSS 26.0

INT2-Z-OSS 29.2 


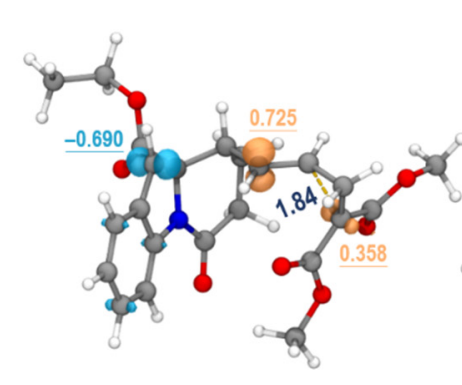

TS2a-E-OSS 35.6

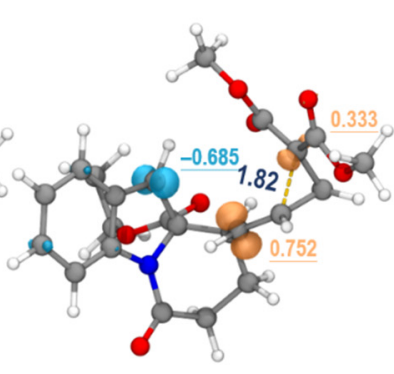

TS2a-Z-OSS 37.8

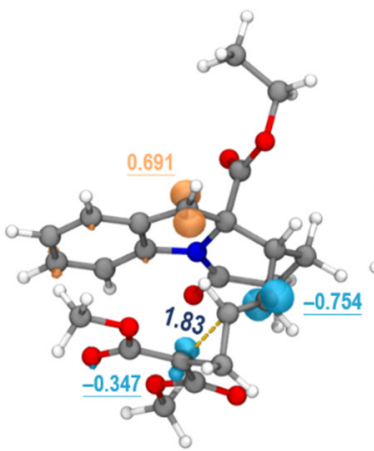

TS2b-E-OSS 38.2

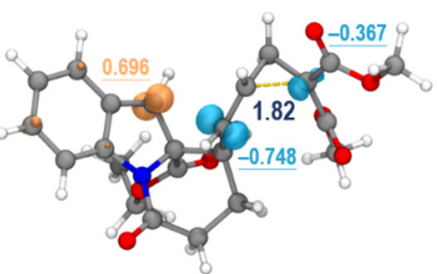

TS2b-Z-OSS 37.4

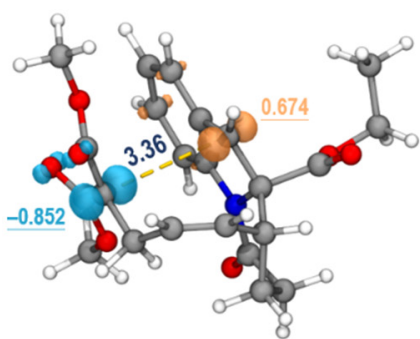

TS3 32.0

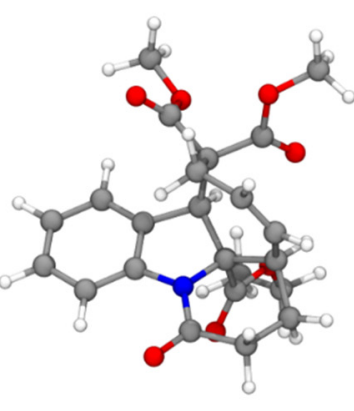

$2 a-21.2$

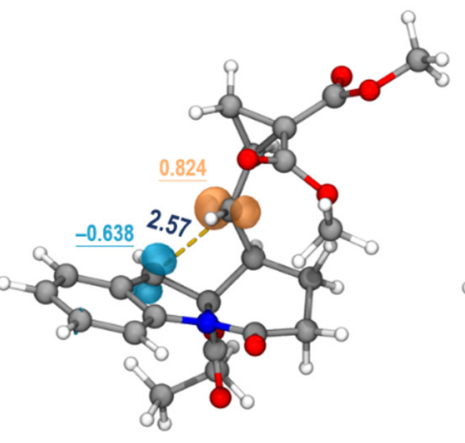

TS4a 35.9

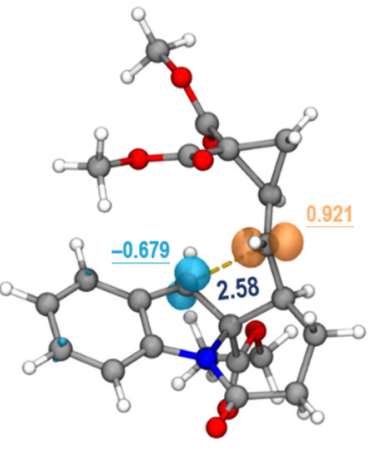

TS4b 35.5

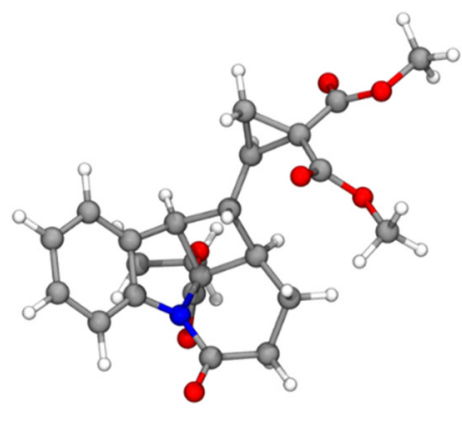

Pro-2+2a -5.6

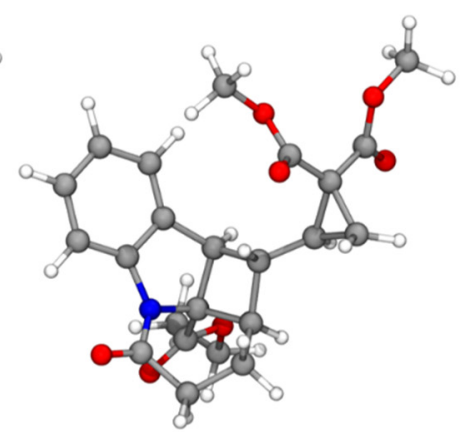

Pro- $2+2 b-5.2$

Cartesian Coordinates and Energies for All the Optimized Structures

1 a

Opt a B3LYP-D3 (BJ)/def2-SVP in gas phase

SCF Done: $\mathrm{E}(\mathrm{RB} 3 \mathrm{LYP})=-1471.87513359 \mathrm{a} \cdot \mathrm{u}$.

Zero-point correction $=0.449903$ Hartree/Particle

Sum of electronic and thermal Free Energies = -1471.487219 a.u. (@ $298.15 \mathrm{~K}$ )

SP a B3LYP-D3 (BJ)/def2-TZVP in acetonitrile (SMD model)

SCF Done: $\mathrm{E}(\mathrm{RB} 3 \mathrm{LYP})=-1473.53622702 \mathrm{a} \cdot \mathrm{u}$.

$0,0,-4.209971614,-0.6396966768,2.0382287932$

$0,0,-2.2450754893,2.1517347499,-0.4685515921$

$0,0,-4.0581297681,1.8711774134,0.8481653107$

$\mathrm{N}, 0,-2.455405308,0.6213071932,2.8212356538$ 
C, $0,-1.4719307083,0.5640343874,3.8043316006$

C, $0,-1.4374809125,-0.1244367232,5.0271422029$

C, $0,-0.2928752502,0.0021955879,5.8079575887$

$\mathrm{C}, 0,0.8088952853,0.7777963719,5.3844596093$

$\mathrm{C}, 0,0.7954811481,1.4227619375,4.1553899607$

C, $0,-0.3470533692,1.3053088851,3.340726643$

$\mathrm{C}, 0,-0.6796066296,1.796667392,2.0381230519$

C, $0,-1.9550418976,1.3693281126,1.7460020031$

$\mathrm{C}, 0,-4.5756406255,0.5257253177,4.1112711373$

C, $0,-3.7781914115,0.0656128441,2.9096519016$

$\mathrm{C}, 0,-2.7438727663,1.8168870359,0.584048148$

$\mathrm{C}, 0,-4.9569081402,2.2588235664,-0.2076642837$

$\mathrm{C}, 0,-5.3617142653,3.713134231,-0.0567557929$

$\mathrm{H}, \mathrm{O},-2.2648284851,-0.750581651,5.3611905215$

$\mathrm{H}, 0,-0.24632941,-0.5154260459,6.7690180654$

$\mathrm{H}, 0,1.6848018294,0.8602430683,6.0317232317$

$\mathrm{H}, 0,1.6382056812,2.0287403067,3.8238180987$

$\mathrm{H}, \mathrm{0},-0.0849827457,2.4475157035,1.403547684$

$\mathrm{H}, \mathrm{O},-5.5374436357,-0.0032865123,4.0721100233$

$\mathrm{H}, \mathrm{O},-4.4622806218,2.0782475384,-1.1716731878$ $\mathrm{H}, 0,-4.4847994347,4.374076507,-0.1027114968$ $\mathrm{H}, 0,-5.8677906586,3.8832109602,0.9066250081$ $\mathrm{H}, 0,-6.0603325803,3.9907972807,-0.8621870521$ $\mathrm{H}, \mathrm{O},-5.8168328551,1.5799685838,-0.1102469457$ $\mathrm{H}, \mathrm{O},-4.0679985263,0.2357439535,5.0430086653$ $\mathrm{C}, 0,-4.7987928566,2.0593175548,4.1192877842$ $\mathrm{H}, 0,-5.5253514022,2.2640027782,4.9261847013$ $\mathrm{H}, \mathrm{O},-5.2726940795,2.3653251203,3.1751718758$ $\mathrm{C}, 0,-3.5547872867,2.8681952992,4.3554866572$ C, $0,-3.1116492596,3.8418095897,3.5503018792$ $\mathrm{H}, \mathrm{O},-2.9785258425,2.6198729789,5.2550615586$ $\mathrm{H}, 0,-3.6702845453,4.100918185,2.6471710711$ $\mathrm{C}, 0,-1.8936747487,4.6331967936,3.8348783197$ $\mathrm{C}, 0,-1.0625861436,5.3082339291,2.7356053617$ C, $0,-1.8856683914,6.1291357509,3.7061889917$ $\mathrm{H}, \mathrm{O},-1.2593845759,4.2190363999,4.6238671896$ $\mathrm{H}, \mathrm{0},-1.3418331656,6.7181808491,4.4486531856$ $\mathrm{H}, 0,-2.7705463211,6.6037205995,3.2754199953$ C, $0,-1.5374370676,5.2609163885,1.3148373836$ $0,0,-2.6692439225,5.5026603084,0.9600959265$ $0,0,-0.5518419023,4.9105326555,0.4823552277$ $\mathrm{C}, 0,0.4328862135,5.3228133836,2.9202257566$ $0,0,1.0841502513,4.4818891124,3.4909691493$ $0,0,0.9635429792,6.4330793582,2.3874831713$ C, $0,-0.8504182566,4.9111769951,-0.9217088949$ $\mathrm{H}, \mathrm{O},-1.2890488091,5.8764176903,-1.2126714113$ $\mathrm{H}, 0,0.1100508067,4.7571469005,-1.4275302067$ $\mathrm{H}, \mathrm{0},-1.5408068469,4.0921856214,-1.1568436156$ C, $0,2.3859513801,6.534645052,2.4299115298$ $\mathrm{H}, 0,2.6381626057,7.4960357895,1.9666519817$ $\mathrm{H}, 0,2.7503213365,6.5003766184,3.4675004275$ $\mathrm{H}, 0,2.8488293256,5.7079656391,1.8698272719$

\section{$1 \mathrm{a}-\mathrm{T}_{1}$}

Opt a B3LYP-D3 (BJ)/def2-SVP in gas phase SCF Done: $\mathrm{E}(\mathrm{UB} 3 \mathrm{LYP})=-1471.77278437 \mathrm{a} \cdot \mathrm{u}$. 
Zero-point correction $=0.445997$ Hartree/Particle

Sum of electronic and thermal Free Energies = -1471.393894 a.u. (@ $298.15 \mathrm{~K}$ ) $\mathrm{SP}$ a B3LYP-D3 (BJ)/def2-TZVP in acetonitrile (SMD model)

SCF Done: $\mathrm{E}(\mathrm{UB} 3 \mathrm{LYP})=-1473.44375768 \mathrm{a} \cdot \mathrm{u}$.

$0,0,4.0569241816,-0.6289390045,2.1041705115$

$0,0,0.7281602761,2.5227134285,-1.08253338$

$0,0,2.5195457802,2.6610160064,0.314907926$

$\mathrm{N}, 0,2.6839805403,-0.2108071103,0.3406439056$

C, $0,3.0782882234,-0.0450614286,1.6829002694$

$\mathrm{C}, 0,2.1434268652,0.7283705709,2.5907558443$

$\mathrm{C}, 0,0.7234462985,0.1113723233,2.6463457475$

C, $0,-0.1840124278,0.481202305,1.5127336892$

$C, 0,1.7464611937,0.544569065,-0.3739569178$

C, $0,1.1815482698,-0.3371161721,-1.4262555353$

C, $0,1.9205203637,-1.5202801272,-1.419388131$

$\mathrm{C}, 0,2.8902689468,-1.4463965402,-0.3578905872$

$\mathrm{C}, 0,-2.3330311474,-0.8458950488,-1.3748223847$

$\mathrm{C}, 0,-3.3150389705,-0.3495765266,-0.3367684638$

$\mathrm{C}, 0,-1.8089567481,0.0533908385,-0.3068207227$

C, $0,-0.879400537,-0.3911476282,0.7501540563$

C, 0,1.6110013259,1.977403312,-0.4279466033

C, 0,2.4550593043,4.0948010787,0.2557153862

C, $0,3.1586748382,4.6355480185,-0.9766051496$

$\mathrm{H}, 0,2.6106330244,0.6748263562,3.5826839723$

$\mathrm{H}, 0,2.0902485019,1.7847484901,2.2974684125$

$\mathrm{H}, 0,0.7925079218,-0.9849017322,2.7397992432$

$\mathrm{H}, 0,0.2593904354,0.4710762954,3.5826823793$

$\mathrm{H}, 0,-0.349104129,1.5531550445,1.3557492978$

$\mathrm{H}, 0,-1.6581604256,1.095822388,-0.6052729134$

$\mathrm{H}, \mathrm{O},-0.7630229149,-1.4650412455,0.9068902365$

$\mathrm{H}, \mathrm{O}, 1.4003282899,4.4072801465,0.2719353575$

$\mathrm{H}, 0,2.9470826708,4.4338947641,1.1788446497$

$\mathrm{H}, \mathrm{0}, 4.2011731056,4.2840198047,-1.0162231424$

$\mathrm{H}, 0,2.637843543,4.312528117,-1.8894292938$

$\mathrm{H}, \mathrm{0}, 3.1640395348,5.7369604648,-0.9537985387$

$\mathrm{H}, 0,0.4065537407,-0.0212701549,-2.1167975211$

C, $0,1.878078691,-2.6862814781,-2.2292441164$

$\mathrm{C}, 0,2.781357738,-3.7179156359,-1.975618356$

$\mathrm{C}, 0,3.7226902244,-3.6109071479,-0.9426137265$

C, $0,3.7931884717,-2.4559272926,-0.1175343373$

$\mathrm{H}, 0,1.1477647478,-2.7612830954,-3.0374795174$

$\mathrm{H}, \mathrm{0}, 2.7603819756,-4.6187201461,-2.5931023757$

$\mathrm{H}, 0,4.4222439676,-4.4296929753,-0.7614656747$

$\mathrm{H}, 0,4.5262563131,-2.3659099987,0.6801174294$

C, $0,-3.9174359125,-1.328855274,0.6219501203$

$0,0,-5.0672175193,-1.3218749542,0.9836231723$

$0,0,-3.0380551269,-2.2799690368,1.0030565548$

$C, 0,-4.2016039489,0.7930099835,-0.757858855$

$0,0,-4.3304296151,1.1688190808,-1.8985996121$

$0,0,-4.7619996245,1.3983384718,0.2935047406$

$\mathrm{C}, 0,-3.5456189135,-3.2845001025,1.8801676551$

$\mathrm{H}, \mathrm{O},-3.9159832095,-2.835342338,2.8135244739$

$\mathrm{H}, \mathrm{O},-4.3732743307,-3.833205423,1.4066558994$

$\mathrm{H}, \mathrm{O},-2.7071046254,-3.960543426,2.0869618943$

$\mathrm{C}, 0,-5.6333739117,2.4886776727,0.0008790405$

$\mathrm{H}, \mathrm{O},-6.4691695399,2.1577347996,-0.6334993763$ 
$\mathrm{H}, 0,-6.0084550574,2.8466333153,0.9670843825$

$\mathrm{H}, \mathrm{O},-5.0928583336,3.2907631349,-0.5243369923$

$\mathrm{H}, \mathrm{O},-2.0671086554,-1.9026086732,-1.3146026614$

$\mathrm{H}, \mathrm{O},-2.4680852204,-0.43355297,-2.3767536916$

\section{TS1a}

Opt a B3LYP-D3 (BJ)/def2-SVP in gas phase

SCF Done: $\mathrm{E}(\mathrm{UB} 3 \mathrm{LYP})=-1471.77242354 \mathrm{a} \cdot \mathrm{u}$.

Zero-point correction $=0.445706$ Hartree/Particle

Sum of electronic and thermal Free Energies = -1471.392348 a.u. (@ $298.15 \mathrm{~K}$ )

Imaginary Frequency $=-160.8316 \mathrm{~cm}^{-1}$

SP \& B3LYP-D3 (BJ)/def2-TZVP in acetonitrile (SMD model)

SCF Done: $\mathrm{E}(\mathrm{UB} 3 \mathrm{LYP})=-1473.44340864 \mathrm{a} \cdot \mathrm{u}$.

$0,0,4.0808912899,1.4984155821,-0.8147696168$

$0,0,0.4898872301,-2.5758540844,0.4169617256$

$0,0,2.6854743722,-2.1360211412,0.0408171869$

$\mathrm{N}, 0,2.1675571903,0.6486001703,0.081940909$

$\mathrm{C}, 0,3.1507342741,0.7236595509,-0.9166036637$

C, $0,2.8771637583,-0.0701263982,-2.1781963948$

$\mathrm{C}, 0,1.4830364055,0.2543253984,-2.7619766074$

$\mathrm{C}, 0,0.3304579162,-0.4356978807,-2.0956158954$

C, $0,1.1670451956,-0.3397827975,0.1874478828$

$\mathrm{C}, 0,0.0328258166,0.273847109,0.911679212$

C, $0,0.4394024721,1.5482717387,1.3334598348$

$\mathrm{C}, 0,1.7743297946,1.7801678458,0.8566885291$

$C, 0,-3.2243282976,0.0077379576,-0.6846081828$

$\mathrm{C}, 0,-3.4087890562,-0.5786098503,-2.0652883636$

$C, 0,-2.0265973792,-0.6041564896,-1.3300057794$

C, $0,-0.8628159171,0.1666533622,-1.7935669845$

C, $0,1.3963708678,-1.7740148187,0.24426851$

C, $0,2.9833395243,-3.5411196421,0.0996156312$

$\mathrm{C}, 0,3.1626052825,-4.012579503,1.5316713642$

$\mathrm{H}, 0,3.6640673815,0.2279109862,-2.8831284026$

$\mathrm{H}, 0,2.9744136105,-1.1492593559,-1.9974371311$

$\mathrm{H}, 0,1.3223665279,1.3450810044,-2.7660630374$

$\mathrm{H}, 0,1.4909626936,-0.0572704793,-3.8225109801$

$\mathrm{H}, \mathrm{O}, \mathrm{0} .3658418288,-1.5297016224,-2.0789385497$

$\mathrm{H}, 0,-1.7707716139,-1.6114841435,-0.9857507595$

$\mathrm{H}, 0,-0.9678706576,1.2472972992,-1.8981980658$

$\mathrm{H}, 0,2.1761432339,-4.0985113282,-0.3983538217$

$\mathrm{H}, 0,3.9117419523,-3.6528410248,-0.4787402696$

$\mathrm{H}, 0,3.9378132617,-3.4212526364,2.0427591104$

$\mathrm{H}, 0,2.2187299395,-3.9207431221,2.0880787631$

$\mathrm{H}, 0,3.4685818477,-5.0707852526,1.543541904$

$\mathrm{H}, 0,-0.8706214612,-0.266903929,1.1734810168$

$\mathrm{C}, 0,-0.200332684,2.5559728597,2.0963707681$

C, $0,0.4856626947,3.7404901562,2.3602572975$

C, $0,1.7914911389,3.9369175634,1.8854701188$

C, $0,2.460172377,2.9471868038,1.1274635382$

$\mathrm{H}, \mathrm{0},-1.2143868782,2.3968630556,2.469056386$

$\mathrm{H}, 0,0.0041952119,4.5244041945,2.9492944749$

$\mathrm{H}, 0,2.3109453037,4.87183823,2.1068324693$

$\mathrm{H}, 0,3.4757880167,3.0877197094,0.7654084732$

$C, 0,-3.7027461074,0.3370388584,-3.2125246264$

$0,0,-4.4792310755,0.0997199357,-4.1032368254$ 
$0,0,-3.0399294652,1.50940419,-3.1144758973$

C, $0,-4.0401249161,-1.9445027562,-2.1255335154$

$0,0,-4.5869792555,-2.4806773016,-1.1912724776$

$0,0,-3.8445446004,-2.5299582362,-3.3105841041$

$\mathrm{C}, 0,-3.3033716387,2.4638234855,-4.1423313313$

$\mathrm{H}, \mathrm{0},-3.0366081868,2.0569707146,-5.1289142707$

$\mathrm{H}, \mathrm{O},-4.3688690768,2.7371964505,-4.1559885191$

$\mathrm{H}, \mathrm{O},-2.6863982248,3.3402331973,-3.9097471486$

C, $0,-4.4145986186,-3.8272536546,-3.4730044725$

$\mathrm{H}, \mathrm{O},-5.5053385264,-3.7917609108,-3.3334548033$

$\mathrm{H}, \mathrm{O},-4.1723861855,-4.1403924408,-4.4955448897$

$\mathrm{H}, \mathrm{O},-3.991955263,-4.5337835204,-2.7425929966$

$\mathrm{H}, \mathrm{O},-3.2552653131,1.096639349,-0.6196971606$

$\mathrm{H}, \mathrm{O},-3.7105252418,-0.5431505581,0.1229813787$

\section{TS1b}

Opt a B3LYP-D3 (BJ)/def2-SVP in gas phase

SCF Done: $\mathrm{E}(\mathrm{UB} 3 \mathrm{LYP})=-1471.78344623 \mathrm{a} \cdot \mathrm{u}$.

Zero-point correction $=0.446541$ Hartree/Particle

Sum of electronic and thermal Free Energies $=-1471.398987$ a.u. (@ $298.15 \mathrm{~K}$ )

Imaginary Frequency $=-229.5675 \mathrm{~cm}^{-1}$

SP @ B3LYP-D3 (BJ)/def2-TZVP in acetonitrile (SMD model)

$\mathrm{SCF}$ Done: $\mathrm{E}(\mathrm{UB} 3 \mathrm{LYP})=-1473.44500505 \mathrm{a} \cdot \mathrm{u}$.

$0,0,-2.0732541247,0.4183603355,2.6194134045$

$0,0,-1.9680865691,-2.182368293,-2.3177403543$

$0,0,-3.2553614937,-1.7856906643,-0.4914517627$

$0,0,1.4765752833,-3.3042400061,-4.7906265392$

$0,0,0.690895522,-1.2240353207,-4.4688458758$

$0,0,2.7025777316,0.2611525415,-3.2951846234$

$0,0,3.5171673279,-0.7125696333,-1.4447889008$

$\mathrm{N}, 0,-1.3310294521,0.0559407379,0.4971448522$

C, $0,-1.7444098228,-0.3792358138,1.7635456553$

C, $0,-1.5772443093,-1.8589225431,2.0415416867$

C, $0,-0.1516381202,-2.3414657588,1.6969601859$

$\mathrm{C}, 0,0.0892915773,-2.6148219164,0.242107615$

$\mathrm{C}, 0,-1.1383129398,-0.7540380841,-0.6450977669$

C, $0,-0.1802122132,-0.0408669785,-1.5186226329$

$\mathrm{C}, 0,0.0697308806,1.2104173186,-0.9399042721$

$\mathrm{C}, 0,-0.6622845365,1.3004582265,0.292189536$

$\mathrm{C}, 0,2.2270393663,-2.064269905,-2.8841989812$

$\mathrm{C}, 0,2.8691600037,-3.2919498015,-2.289210126$

C, $0,1.528216862,-2.8970674879,-1.7511124$

$\mathrm{C}, 0,1.2727961306,-2.362991041,-0.4089949348$

C, $0,-2.1442498555,-1.6195670644,-1.2463969802$

C, $0,-4.2948657957,-2.616232082,-1.0354930724$

C, $0,-5.1712780659,-1.8451944202,-2.0063692104$

C, $0,1.4712935619,-2.2668424217,-4.1710086499$

C, $0,-0.2597399526,-1.411298372,-5.5186995916$

$C, 0,2.809866064,-0.7145904066,-2.5939232034$

C, 0,4.0996413036,0.5345571487,-1.0589341504

$\mathrm{H}, 0,-1.7740205385,-1.975421602,3.1151378961$

$\mathrm{H}, \mathrm{O},-2.3229822221,-2.4511647919,1.4932379312$

$\mathrm{H}, 0,0.5941688171,-1.6250927907,2.0798707198$

$\mathrm{H}, 0,0.0212396637,-3.2842079541,2.2475331011$

$\mathrm{H}, \mathrm{O},-0.6019380194,-3.3220979885,-0.2275216375$ 
$\mathrm{H}, 0,3.7397635447,-3.113102973,-1.6578903314$

$\mathrm{H}, \mathrm{0}, 2.9069231316,-4.1650800434,-2.9431098133$

$\mathrm{H}, \mathrm{O}, 0.6854373889,-3.4650687214,-2.1549852952$

$\mathrm{H}, \mathrm{0}, 2.042441985,-1.7524455801,0.065281101$

$\mathrm{H}, \mathrm{O},-3.8357145315,-3.4866500171,-1.5270786552$

$\mathrm{H}, 0,-4.8676487707,-2.9544562317,-0.1600831842$

$\mathrm{H}, 0,-5.5915025264,-0.9488394225,-1.5249993898$

$\mathrm{H}, \mathrm{O},-4.5905783813,-1.5370665899,-2.8877552948$

$\mathrm{H}, \mathrm{O},-6.0047058484,-2.4800600241,-2.3467616738$

$\mathrm{H}, 0,0.2242465366,-1.8222376798,-6.4154432186$

$\mathrm{H}, 0,-1.0517367728,-2.0956917226,-5.1795583645$

$\mathrm{H}, \mathrm{O},-0.6804523514,-0.4199277725,-5.724483694$

$\mathrm{H}, 0,4.7074829318,0.9480560099,-1.8761767301$

$\mathrm{H}, 0,3.3152590768,1.2590614812,-0.7955552456$

$\mathrm{H}, 0,4.7254991536,0.318064546,-0.1848026987$

$\mathrm{H}, 0,0.0969462315,-0.3855662135,-2.5084511329$

C, $0,0.8550148104,2.3142311473,-1.3600364646$

$\mathrm{C}, 0,0.8939398045,3.4523308075,-0.5554271149$

C, $0,0.1668790943,3.513729811,0.6441174579$

C, $0,-0.6348619699,2.4324749731,1.081675481$

$\mathrm{H}, 0,1.4180013537,2.2471001263,-2.2922604954$

$\mathrm{H}, 0,1.492772723,4.3122583024,-0.8646980612$

$\mathrm{H}, 0,0.2112842647,4.417320999,1.2562009455$

$\mathrm{H}, \mathrm{O},-1.2132947733,2.4804734111,2.0011079547$

\section{INT1a-T}

Opt a B3LYP-D3 (BJ)/def2-SVP in gas phase

SCF Done: $\mathrm{E}(\mathrm{UB} 3 \mathrm{LYP})=-1471.81532888 \mathrm{a} \cdot \mathrm{u}$.

Zero-point correction $=0.447744$ Hartree/Particle

Sum of electronic and thermal Free Energies = -1471.432512 a.u. (@ $298.15 \mathrm{~K}$ ) $\mathrm{SP}$ a B3LYP-D3 (BJ)/def2-TZVP in acetonitrile (SMD model)

SCF Done: E (UB3LYP) $=-1473.47808608 \mathrm{a} \cdot \mathrm{u}$.

$0,0,2.9542119316,-0.7948654526,-2.0303977811$

$\mathrm{N}, 0,1.9863214972,-0.5749000323,0.0122936622$

$\mathrm{C}, 0,2.3093141975,0.7647340381,0.2714816639$

$\mathrm{C}, 0,3.0997820318,1.6475902208,-0.4531793868$

$\mathrm{C}, 0,3.213935872,2.9639270778,0.0243036285$

C, $0,2.5561900548,3.3843120212,1.1957255149$

C, 0,1.7672149983,2.5022852014,1.925226848

C, $0,1.631595694,1.1675263605,1.4718846165$

C, $0,0.9188581004,0.0726394919,1.9787467937$

C, $0,1.2090544358,-1.1371147875,1.1289083861$

C, $0,2.246772294,-1.2616331761,-1.1520879996$

C, $0,1.5397463062,-2.5968667478,-1.3545151716$

$\mathrm{C}, 0,0.5741611248,-3.0887459341,-0.2658464881$

$\mathrm{C}, 0,0.0011195999,-1.9236218123,0.5705371697$

C, $0,-0.9043943213,-1.0184413015,-0.2059630662$

C, $0,-1.9696538666,-1.5650236603,-1.0345877153$

C, $0,-1.9894987482,-1.3522225167,-2.5917100754$

$\mathrm{H}, 0,3.6033081147,1.3147352795,-1.3566716061$

$\mathrm{H}, 0,3.830589506,3.6755054887,-0.5293849028$

$\mathrm{H}, 0,2.6705222466,4.4164182006,1.535043676$

$\mathrm{H}, 0,1.2561022955,2.8233675265,2.8353458516$

$\mathrm{H}, 0,1.0100195174,-2.4680162167,-2.3104588755$

$\mathrm{H}, \mathrm{0}, 2.3280618139,-3.339394972,-1.5505611229$ 
$\mathrm{H}, \mathrm{O}, 1.0852020184,-3.7882979987,0.4136242691$

$\mathrm{H}, \mathrm{O},-0.2421773821,-3.6589187849,-0.7320526813$

$\mathrm{H}, 0,-0.5383116182,-2.3598052885,1.4318438494$

$\mathrm{H}, 0,-0.6979734183,0.0521691015,-0.2464131148$

$\mathrm{H}, \mathrm{O},-2.2713796417,-2.5925089726,-0.8120191751$

$\mathrm{C}, 0,-3.019568061,-0.7219450109,-1.6830435161$

$\mathrm{H}, 0,0.3252578953,0.0246544045,2.8884349753$

C, $0,2.1187366439,-2.0828384789,1.9470779827$

$0,0,3.2553507531,-2.3608091518,1.6668904312$

$0,0,1.4583656452,-2.5463883595,3.0188604148$

C, $0,2.1865634995,-3.4052726231,3.9199785951$

$\mathrm{C}, 0,3.0327679002,-2.5988700412,4.8876035358$

$\mathrm{H}, 0,2.8086567428,-4.0917589667,3.327097531$

$\mathrm{H}, \mathrm{O}, 1.4093356737,-3.9805945526,4.4418556243$

$\mathrm{H}, 0,3.5215118923,-3.272609791,5.6089823094$

$\mathrm{H}, 0,3.8159466598,-2.0458320713,4.3492981722$

$\mathrm{H}, 0,2.4123320237,-1.8829553851,5.4482823168$

$\mathrm{C}, 0,-2.4200151036,-2.5503496298,-3.3881407541$

$0,0,-2.6366135884,-3.646393951,-2.9248132565$

$0,0,-2.599683365,-2.2449285034,-4.6789463742$

$\mathrm{C}, 0,-0.9333758653,-0.4467881108,-3.1492640217$

$0,0,-0.7842758844,0.7067184046,-2.8222529152$

$0,0,-0.1268310285,-1.0981993031,-3.9979930911$

$\mathrm{H}, \mathrm{O},-2.9303410361,0.3623626583,-1.5815677496$

$\mathrm{H}, \mathrm{O},-4.0363427716,-1.1180890928,-1.7349614216$

C, $0,0.942876351,-0.3468587051,-4.592933045$

$\mathrm{H}, 0,1.2619421371,-0.9242899404,-5.4692550215$

$\mathrm{H}, 0,0.5878076257,0.648781638,-4.8916209426$

$\mathrm{H}, \mathrm{O}, 1.772551127,-0.2527385343,-3.8782227496$

$\mathrm{C}, 0,-2.9861395906,-3.3198571315,-5.5345672591$

$\mathrm{H}, \mathrm{O},-3.946802275,-3.749231115,-5.2129052554$

$\mathrm{H}, \mathrm{O},-3.0749532924,-2.8919912457,-6.5402342484$

$\mathrm{H}, 0,-2.2267918825,-4.1160777841,-5.5231074342$

\section{INT $1 \mathrm{~b}-\mathrm{T}_{1}$}

Opt a B3LYP-D3 (BJ)/def2-SVP in gas phase

$\mathrm{SCF}$ Done: $\mathrm{E}(\mathrm{UB} 3 \mathrm{LYP})=-1471.82033855 \mathrm{a} \cdot \mathrm{u}$.

Zero-point correction $=0.448328$ Hartree/Particle

Sum of electronic and thermal Free Energies = -1471.434290 a.u. (@ $298.15 \mathrm{~K}$ )

$\mathrm{SP}$ a B3LYP-D3(BJ)/def2-TZVP in acetonitrile (SMD model)

$\mathrm{SCF}$ Done: $\mathrm{E}(\mathrm{UB} 3 \mathrm{LYP})=-1473.48062747 \mathrm{a} \cdot \mathrm{u}$.

$0,0,2.9542119316,-0.7948654526,-2.0303977811$

$\mathrm{N}, 0,1.9863214972,-0.5749000323,0.0122936622$

C, $0,2.3093141975,0.7647340381,0.2714816639$

$\mathrm{C}, 0,3.0997820318,1.6475902208,-0.4531793868$

C, $0,3.213935872,2.9639270778,0.0243036285$

C, $0,2.5561900548,3.3843120212,1.1957255149$

C, $0,1.7672149983,2.5022852014,1.925226848$

C, $0,1.631595694,1.1675263605,1.4718846165$

C, $0,0.9188581004,0.0726394919,1.9787467937$

C, $0,1.2090544358,-1.1371147875,1.1289083861$

C, $0,2.246772294,-1.2616331761,-1.1520879996$

C, $0,1.5397463062,-2.5968667478,-1.3545151716$

$C, 0,0.5741611248,-3.0887459341,-0.2658464881$

C, $0,0.0011195999,-1.9236218123,0.5705371697$ 
$C, 0,-0.9043943213,-1.0184413015,-0.2059630662$

C, $0,-1.9696538666,-1.5650236603,-1.0345877153$

C, $0,-1.9894987482,-1.3522225167,-2.5917100754$

$\mathrm{H}, 0,3.6033081147,1.3147352795,-1.3566716061$

$\mathrm{H}, 0,3.830589506,3.6755054887,-0.5293849028$

$\mathrm{H}, 0,2.6705222466,4.4164182006,1.535043676$

$\mathrm{H}, 0,1.2561022955,2.8233675265,2.8353458516$

$\mathrm{H}, 0,1.0100195174,-2.4680162167,-2.3104588755$

$\mathrm{H}, 0,2.3280618139,-3.339394972,-1.5505611229$

$\mathrm{H}, 0,1.0852020184,-3.7882979987,0.4136242691$

$\mathrm{H}, 0,-0.2421773821,-3.6589187849,-0.7320526813$

$\mathrm{H}, \mathrm{O},-0.5383116182,-2.3598052885,1.4318438494$

$\mathrm{H}, 0,-0.6979734183,0.0521691015,-0.2464131148$

$\mathrm{H}, \mathrm{O},-2.2713796417,-2.5925089726,-0.8120191751$

C, $0,-3.019568061,-0.7219450109,-1.6830435161$

$\mathrm{H}, 0,0.3252578953,0.0246544045,2.8884349753$

$\mathrm{C}, 0,2.1187366439,-2.0828384789,1.9470779827$

$0,0,3.2553507531,-2.3608091518,1.6668904312$

$0,0,1.4583656452,-2.5463883595,3.0188604148$

C, $0,2.1865634995,-3.4052726231,3.9199785951$ $\mathrm{C}, 0,3.0327679002,-2.5988700412,4.8876035358$ $\mathrm{H}, 0,2.8086567428,-4.0917589667,3.327097531$

$\mathrm{H}, 0,1.4093356737,-3.9805945526,4.4418556243$

$\mathrm{H}, 0,3.5215118923,-3.272609791,5.6089823094$

$\mathrm{H}, 0,3.8159466598,-2.0458320713,4.3492981722$ $\mathrm{H}, \mathrm{O}, 2.4123320237,-1.8829553851,5.4482823168$ $\mathrm{C}, 0,-2.4200151036,-2.5503496298,-3.3881407541$ $0,0,-2.6366135884,-3.646393951,-2.9248132565$ $0,0,-2.599683365,-2.2449285034,-4.6789463742$ $C, 0,-0.9333758653,-0.4467881108,-3.1492640217$ $0,0,-0.7842758844,0.7067184046,-2.8222529152$ $0,0,-0.1268310285,-1.0981993031,-3.9979930911$ $\mathrm{H}, 0,-2.9303410361,0.3623626583,-1.5815677496$ $\mathrm{H}, \mathrm{O},-4.0363427716,-1.1180890928,-1.7349614216$ C, $0,0.942876351,-0.3468587051,-4.592933045$ $\mathrm{H}, 0,1.2619421371,-0.9242899404,-5.4692550215$ $\mathrm{H}, 0,0.5878076257,0.648781638,-4.8916209426$ $\mathrm{H}, 0,1.772551127,-0.2527385343,-3.8782227496$ C, $0,-2.9861395906,-3.3198571315,-5.5345672591$ $\mathrm{H}, \mathrm{O},-3.946802275,-3.749231115,-5.2129052554$ $\mathrm{H}, \mathrm{O},-3.0749532924,-2.8919912457,-6.5402342484$ $\mathrm{H}, 0,-2.2267918825,-4.1160777841,-5.5231074342$

\section{TS2a-E-T}

Opt a B3LYP-D3 (BJ)/def2-SVP in gas phase $\mathrm{SCF}$ Done: $\mathrm{E}(\mathrm{UB} 3 \mathrm{LYP})=-1471.81180205 \mathrm{a} \cdot \mathrm{u}$. Zero-point correction $=0.447218$ Hartree/Particle

Sum of electronic and thermal Free Energies = -1471.427815 a.u. (@ $298.15 \mathrm{~K}$ ) Imaginary Frequency $=-415.7555 \mathrm{~cm}^{-1}$

SP a B3LYP-D3 (BJ)/def2-TZVP in acetonitrile (SMD model)

SCF Done: E (UB3LYP) $=-1473.47600221 \mathrm{a} \cdot \mathrm{u}$.

$0,0,-0.3467392149,-0.8819493593,2.5142764702$

$0,0,-3.4598058348,-0.6497334036,0.0491395179$

$0,0,-3.3766445412,0.5008428013,-1.8903640398$

$\mathrm{N}, 0,-1.2199826175,0.6031080371,1.0339235892$ 
C, $0,-1.4974592032,1.6623839421,1.9078268255$

$\mathrm{C}, 0,-1.3480615828,1.7441236791,3.2863373344$

C, $0,-1.6624315375,2.9647307996,3.9054317148$

C, $0,-2.1182702594,4.0729285726,3.1662373138$ C, $0,-2.2745722444,3.9930287856,1.7874544482$ C, $0,-1.9651984227,2.7765322004,1.131877156$ $\mathrm{C}, 0,-2.0152482965,2.3996615603,-0.2170458077$ C, $0,3.2706715344,1.6350845829,-1.1006017279$ $\mathrm{C}, 0,3.205205978,0.3934176824,-0.2646715215$ C, $0,1.9844499245,1.001139368,-1.4894543643$ C, $0,0.7171004965,1.3602815031,-1.0130408042$ $\mathrm{C}, 0,-0.5219772456,0.5714282313,-1.3276019934$ $C, 0,-1.6460660834,0.943511356,-0.3322864718$ $C, 0,-0.3306661361,-0.9568024624,-1.23212501$ C, $0,-0.0667976667,-1.4306066033,0.2064591015$ $\mathrm{C}, 0,-0.573697377,-0.5714199381,1.3576627773$ $C, 0,-2.931271885,0.1546576508,-0.6724229399$ $C, 0,-4.6064820238,-0.1056735052,-2.3375465701$ $\mathrm{C}, 0,-5.8155553223,0.6155605521,-1.7713720008$ $\mathrm{H}, \mathrm{O},-0.9987206162,0.8835354703,3.849769282$ $\mathrm{H}, 0,-1.5514584707,3.0520568121,4.988614467$ $\mathrm{H}, \mathrm{O},-2.3538515921,5.0048953261,3.6852630254$ $\mathrm{H}, \mathrm{0},-2.6297206505,4.8488492228,1.2094022445$ $\mathrm{H}, \mathrm{O},-2.3577504689,2.9896651756,-1.0637772762$ $\mathrm{H}, 0,2.0440871985,0.2775451367,-2.3064801089$ $\mathrm{H}, 0,0.6469151579,2.163595926,-0.2784713287$ $\mathrm{H}, \mathrm{O},-0.885935044,0.8125295621,-2.3424019369$ $\mathrm{H}, \mathrm{O},-1.2340393057,-1.4411919513,-1.6340290373$ $\mathrm{H}, 0,0.4957813634,-1.2822038555,-1.8800024034$ $\mathrm{H}, 0,-0.4779683612,-2.4384108983,0.3678596027$ $\mathrm{H}, 0,1.0142149975,-1.5317853728,0.3932699045$ $\mathrm{H}, \mathrm{O},-4.5671898833,-0.0343209133,-3.4333897392$ $\mathrm{H}, \mathrm{0},-5.8486204505,0.516045305,-0.6768641447$ $\mathrm{H}, \mathrm{O},-6.7388882335,0.1795153061,-2.1842797931$ $\mathrm{H}, \mathrm{O},-5.7914477765,1.6849623907,-2.0314264669$ $\mathrm{H}, \mathrm{O},-4.6041383182,-1.1654968586,-2.0432574577$ $\mathrm{C}, 0,2.7714942997,0.5648581565,1.1485390668$ $0,0,2.1866061604,1.5523586075,1.547428373$ $0,0,3.1283606183,-0.4529813988,1.9313710351$ $\mathrm{C}, 0,3.7250505977,-0.8959489306,-0.7700244279$ $0,0,3.4820446552,-1.9940898027,-0.3230960306$ $0,0,4.4705400609,-0.7190974938,-1.8913063392$ $\mathrm{H}, 0,3.179500549,2.5562737147,-0.5187330944$ $\mathrm{H}, 0,4.053673015,1.6503967586,-1.8610912336$ $\mathrm{C}, 0,2.7501768687,-0.3715278416,3.3108697782$ $\mathrm{H}, 0,3.2961708681,-1.1781078255,3.8147487312$ $\mathrm{H}, 0,3.0256201571,0.6069214545,3.7299214707$ $\mathrm{H}, 0,1.6663573289,-0.5243668749,3.4046202737$ C, $0,4.9372200622,-1.9102226798,-2.5185205106$ $\mathrm{H}, 0,5.6010671833,-2.4727240664,-1.8449944205$ $\mathrm{H}, 0,4.0960593771,-2.5642317652,-2.7954432616$ $\mathrm{H}, 0,5.485028184,-1.5911752087,-3.4137859307$

\section{TS2a- $z-\mathrm{T}_{1}$}

Opt a B3LYP-D3 (BJ)/def2-SVP in gas phase SCF Done: $\mathrm{E}(\mathrm{UB} 3 \mathrm{LYP})=-1471.80431347 \mathrm{a} \cdot \mathrm{u}$. 
Zero-point correction $=0.447174$ Hartree/Particle

Sum of electronic and thermal Free Energies = -1471.420974 a.u. (@ $298.15 \mathrm{~K}$ ) Imaginary Frequency $=-410.9934 \mathrm{~cm}^{-1}$

SP a B3LYP-D3 (BJ)/def2-TZVP in acetonitrile (SMD model)

$\mathrm{SCF}$ Done: $\mathrm{E}(\mathrm{UB} 3 \mathrm{LYP})=-1473.47113589 \mathrm{a} \cdot \mathrm{u}$.

$0,0,-2.7964809815,0.2492703838,3.19059729$

$0,0,-2.040254545,-2.4028389381,-1.4017326076$

$0,0,-3.6089111142,-0.9428107427,-0.6979118561$

$0,0,5.2392783521,0.7336036803,-0.5555301087$

$0,0,5.060016634,-0.4321323535,1.3580579103$

$0,0,1.7142590311,-1.0113773679,-1.9175671518$

$0,0,2.884403073,0.9055243767,-1.8894989123$

$\mathrm{N}, 0,-1.9815254181,0.2410017278,1.05780678$

C, $0,-1.9962893727,1.6068637127,0.7579999126$

$\mathrm{C}, 0,-2.4976460068,2.6713505562,1.4958866023$

$\mathrm{C}, 0,-2.3607200675,3.9606399809,0.953531557$

C, $0,-1.7372404763,4.1744047566,-0.2904411642$

C, $0,-1.231610481,3.1095633582,-1.0288357479$

$\mathrm{C}, 0,-1.3530159834,1.796559986,-0.5121820846$

C, $0,-0.9363029805,0.550328059,-0.9998079733$

$\mathrm{C}, 0,3.3098539664,-0.6641587971,-0.1849484001$

$\mathrm{C}, 0,2.9085798605,-1.9252438414,0.5227881366$

$\mathrm{C}, 0,2.2112154347,-0.8381520982,1.2531630737$

$C, 0,0.8332523929,-0.5386400415,1.1970763465$

$\mathrm{C}, 0,-0.213946655,-1.3928813673,0.5503355746$

$\mathrm{C}, 0,-1.3541692151,-0.5242087627,-0.0313402658$

$\mathrm{C}, 0,-0.834632515,-2.3858087437,1.5846055163$

$\mathrm{C}, 0,-2.1444156727,-1.8737991226,2.2330174268$

C, $0,-2.3406068842,-0.3652648408,2.2447841848$

$\mathrm{C}, 0,-2.3642475188,-1.4212591257,-0.779793767$

C, $0,-4.6229111595,-1.6007589754,-1.4835673127$

$C, 0,-4.6085525234,-1.1058420742,-2.9179392784$

$C, 0,4.6096822251,-0.0253070464,0.1406740226$

$\mathrm{C}, 0,6.317876154,0.1005680287,1.7626495456$

C, $0,2.5655092789,-0.3019218462,-1.4157643679$

C, $0,2.2465938223,1.3227255222,-3.0908722649$

$\mathrm{H}, \mathrm{O},-2.9711179129,2.4938735995,2.4586877935$

$\mathrm{H}, \mathrm{O},-2.7482841028,4.8148473045,1.5132749857$

$\mathrm{H}, 0,-1.6504122499,5.1916085391,-0.6792628154$

$\mathrm{H}, \mathrm{O},-0.7470024284,3.2747898676,-1.9935012714$

$\mathrm{H}, 0,-0.4060781442,0.3111593199,-1.9171987925$

$\mathrm{H}, 0,2.7891724689,-0.3460659265,2.0367277302$

$\mathrm{H}, 0,0.4962615709,0.3261420364,1.7755068784$

$\mathrm{H}, \mathrm{O}, \mathrm{O} .212859111,-1.9594985745,-0.2843295688$

$\mathrm{H}, \mathrm{O},-1.0359985695,-3.3394064294,1.077367969$

$\mathrm{H}, \mathrm{O},-0.0900171892,-2.5912718618,2.3666132734$

$\mathrm{H}, \mathrm{O},-3.0109927003,-2.2829030489,1.6860440952$

$\mathrm{H}, \mathrm{O},-2.2463241669,-2.2109829223,3.2722762071$

$\mathrm{H}, \mathrm{O},-4.4586489338,-2.6873471827,-1.4356899107$

$\mathrm{H}, 0,-5.5662681457,-1.3532106241,-0.9779024246$

$\mathrm{H}, \mathrm{O},-3.6675488323,-1.3834040793,-3.4148319101$

$\mathrm{H}, 0,-4.7210217291,-0.0115114339,-2.9532196009$

$\mathrm{H}, \mathrm{O},-5.4403287473,-1.5594654385,-3.4795992235$

$\mathrm{H}, 0,7.1102055512,-0.1855128075,1.0545897928$

$\mathrm{H}, 0,6.5230966586,-0.3195276614,2.7550021209$

$\mathrm{H}, 0,6.2807450264,1.1993023317,1.8120440281$ 
$\mathrm{H}, 0,2.8201840515,2.183015905,-3.4568107679$

$\mathrm{H}, 0,1.2079162277,1.6285397019,-2.8913220678$

$\mathrm{H}, 0,2.2397977317,0.5151642053,-3.8369692938$

$\mathrm{H}, 0,2.2795205722,-2.5875681288,-0.0769476327$

$\mathrm{H}, \mathrm{0}, 3.7137832275,-2.4312837349,1.0592987886$

\section{TS2b-E-T}

Opt a B3LYP-D3 (BJ)/def2-SVP in gas phase

SCF Done: E (UB3LYP) $=-1471.81569830 \mathrm{a} \cdot \mathrm{u}$.

Zero-point correction $=0.447687$ Hartree/Particle

Sum of electronic and thermal Free Energies = -1471.428745 a.u. (@ $298.15 \mathrm{~K}$ )

Imaginary Frequency $=-426.0458 \mathrm{~cm}^{-1}$

$\mathrm{SP}$ a B3LYP-D3 (BJ)/def2-TZVP in acetonitrile (SMD model)

$\mathrm{SCF}$ Done: $\mathrm{E}(\mathrm{UB} 3 \mathrm{LYP})=-1473.47504903 \mathrm{a} \cdot \mathrm{u}$.

$0,0,0.6024862135,1.9808486445,1.9051061246$

$0,0,3.7455857529,0.36964632,0.2610392741$

$0,0,3.8767799548,-0.1351295041,-1.9345846326$

$\mathrm{N}, 0,1.0122380003,0.5356113648,0.1973270974$

C, $0,0.4667926955,-0.6361447745,0.7400684481$

C, $0,-0.1636355838,-0.8445159843,1.9595347534$

C, $0,-0.6591528343,-2.1293041025,2.225206069$

C, $0,-0.5155858883,-3.1820795678,1.3030830704$

C, $0,0.117336992,-2.9781593545,0.0825327928$

C, $0,0.6176625975,-1.690163482,-0.2218369799$

C, $0,1.2784406473,-1.1836382389,-1.3513945515$

$C, 0,-2.3992676368,0.501402137,-1.3385863548$

C, $0,-2.450640341,0.8120859503,-2.8080079934$

C, $0,-1.0219500452,0.5210009402,-2.5436563153$

$C, 0,-0.045733196,1.5270294767,-2.4468862462$

C, $0,1.4066831037,1.3526454376,-2.1073598752$

$C, 0,1.6950800795,0.2370171469,-1.0701269183$

C, 0,1.9678840542,2.655273127,-1.5057949096

C, $0,1.3165015246,2.9648327689,-0.1524022229$

C, $0,0.9872294134,1.7936998844,0.7641370125$

C, $0,3.223159712,0.1786522201,-0.8053617611$

$\mathrm{C}, 0,5.3068247728,-0.3011397887,-1.8419283197$

C, $0,5.6694677236,-1.6840572961,-1.3335443971$

$\mathrm{H}, 0,-0.2644774921,-0.0284911267,2.6690580851$

$\mathrm{H}, \mathrm{O},-1.1718018077,-2.3127652432,3.1715317567$

$\mathrm{H}, \mathrm{O},-0.9110259375,-4.1697088625,1.5512913913$

$\mathrm{H}, 0,0.2335731761,-3.7911930147,-0.6373654532$

$\mathrm{H}, 0,1.6010961125,-1.7292850986,-2.2350099121$

$\mathrm{H}, \mathrm{O},-2.9929592355,0.0847985773,-3.4149704024$

$\mathrm{H}, \mathrm{O},-2.6812797881,1.8580421726,-3.0291062847$

$\mathrm{H}, 0,-0.7159190636,-0.5233298192,-2.5920585137$

$\mathrm{H}, \mathrm{O},-0.3897185542,2.5575062541,-2.5568183196$

$\mathrm{H}, \mathrm{O}, 1.9844503489,1.1002018079,-3.0146194976$

$\mathrm{H}, 0,3.0597740166,2.5714761519,-1.3966552186$

$\mathrm{H}, 0,1.791337211,3.49093753,-2.2003207878$

$\mathrm{H}, 0,1.9142270007,3.6751720585,0.436306316$

$\mathrm{H}, 0,0.3327504145,3.4411560503,-0.3073249146$

$\mathrm{H}, 0,5.6708926477,-0.1355892374,-2.86537825$

$\mathrm{H}, 0,5.3127902958,-1.8238280863,-0.3029052254$

$\mathrm{H}, 0,6.7636886046,-1.8097116253,-1.3386545768$

$\mathrm{H}, 0,5.2287374273,-2.4648240465,-1.972333443$ 
$\mathrm{H}, 0,5.7104863252,0.4814413035,-1.1828734327$

C, $0,-2.7603207343,-0.8650408667,-0.8809834974$

$0,0,-3.1231709222,-1.1949231591,0.2208832519$

C, $0,-2.3431844023,1.6644278638,-0.4136380056$

$0,0,-2.1375008251,2.8019825093,-0.7994605966$

$0,0,-2.6710251192,-1.754892484,-1.9086385208$

$0,0,-2.5230284657,1.3476788899,0.8658074847$

$C, 0,-3.0724794764,-3.0877290555,-1.6075178147$

$\mathrm{H}, \mathrm{O},-2.4635880778,-3.501913708,-0.7924302663$

$\mathrm{H}, \mathrm{O},-4.1298599282,-3.1170968772,-1.3031233145$

$\mathrm{H}, \mathrm{O},-2.9266435276,-3.6656608243,-2.5288275126$

C, $0,-2.5284477405,2.4228798262,1.8088778932$

$\mathrm{H}, 0,-1.5051797074,2.7883800149,1.9703110316$

$\mathrm{H}, \mathrm{O},-3.1705057924,3.2426958132,1.4564450812$

$\mathrm{H}, \mathrm{O},-2.9212920154,1.9949773268,2.7391758344$

\section{TS2b-Z-T}

Opt a B3LYP-D3 (BJ)/def2-SVP in gas phase

SCF Done: $\mathrm{E}(\mathrm{UB} 3 \mathrm{LYP})=-1471.80412009 \mathrm{a} \cdot \mathrm{u}$.

Zero-point correction $=0.447153$ Hartree/Particle

Sum of electronic and thermal Free Energies = -1471.422561 a.u. (@ $298.15 \mathrm{~K}$ )

Imaginary Frequency $=-441.2670 \mathrm{~cm}^{-1}$

$\mathrm{SP}$ a B3LYP-D3 (BJ)/def2-TZVP in acetonitrile (SMD model)

$\mathrm{SCF}$ Done: $\mathrm{E}(\mathrm{UB} 3 \mathrm{LYP})=-1473.47342683 \mathrm{a} \cdot \mathrm{u}$.

$0,0,-2.5928916796,0.0116060582,3.2053810199$

$0,0,-2.0068326582,-2.0716899136,-1.7535965894$

$0,0,-3.635558268,-0.9339076102,-0.6854178123$

$0,0,2.9981767474,-3.3819820158,0.9061893387$

$0,0,2.0610678809,-3.0667983384,-1.1168696128$

$0,0,5.1001060841,0.2282047933,0.1580222622$

$0,0,5.3931863919,-1.9386430704,0.6845253916$

$\mathrm{N}, 0,-1.9579911976,0.2563351312,1.0265870557$

$\mathrm{C}, 0,-2.0700392154,1.6422229506,0.8756390217$

C, $0,-2.5773720811,2.5917637698,1.7534900172$

$\mathrm{C}, 0,-2.5533212545,3.9351735358,1.342953899$

C, $0,-2.034002232,4.3149081221,0.0905918556$

C, $0,-1.5223928091,3.3658617149,-0.787813898$

$\mathrm{C}, 0,-1.5314597557,2.0028208406,-0.4050322791$

C, $0,-1.0823436362,0.8411807744,-1.0487502605$

$\mathrm{C}, 0,3.3207530183,-1.2930082761,-0.241543371$

C, $0,2.6138840991,-0.3235074104,-1.1328166527$

$\mathrm{C}, 0,2.1436018178,0.0099435874,0.2394425359$

C, $0,0.9191998329,-0.3394382672,0.8349504236$

$\mathrm{C}, 0,-0.1400332225,-1.2071456111,0.2319658434$

C, $0,-1.3712929291,-0.352601161,-0.1751822497$

$\mathrm{C}, 0,-0.6047577488,-2.3187739153,1.2126335158$

$\mathrm{C}, 0,-1.8869488602,-1.9638055352,2.0045214308$

$\mathrm{C}, 0,-2.1781243515,-0.4812399321,2.1738350824$

$\mathrm{C}, 0,-2.3658522506,-1.2381512175,-0.9555948199$

$C, 0,-4.6566638535,-1.5905178246,-1.4655232428$

C, $0,-4.8585511613,-0.8917388933,-2.7969436237$

C, $0,2.813887233,-2.6798135726,-0.057235459$

$\mathrm{C}, 0,1.4710363886,-4.3633297678,-1.0482035765$

$\mathrm{C}, 0,4.6739188421,-0.9081980605,0.2206867626$

C, $0,6.7041059103,-1.6282439041,1.1484192836$ 
$\mathrm{H}, 0,-2.9690614995,2.2877093023,2.7212119668$ $\mathrm{H}, \mathrm{0},-2.9475718743,4.7011127586,2.0145918442$ $\mathrm{H}, \mathrm{O},-2.033256766,5.3700167682,-0.1923987535$ $\mathrm{H}, 0,-1.115616875,3.6577523968,-1.7583085672$ $\mathrm{H}, 0,-0.6281095126,0.7524341124,-2.0332447762$ $\mathrm{H}, 0,2.7634315284,0.7349891935,0.7700662797$ $\mathrm{H}, 0,0.7378251798,0.0427971275,1.8437164479$ $\mathrm{H}, 0,0.2249716924,-1.6731779729,-0.6882326167$ $\mathrm{H}, \mathrm{0},-0.7876635903,-3.2396801033,0.642987028$ $\mathrm{H}, 0,0.2192945251,-2.540557585,1.9054630077$ $\mathrm{H}, 0,-2.7682470935,-2.3824984731,1.4894298232$ $\mathrm{H}, \mathrm{0},-1.879098231,-2.3991559698,3.0116163971$ $\mathrm{H}, \mathrm{O},-4.3716445204,-2.6434833685,-1.6060168726$ $\mathrm{H}, 0,-5.556830021,-1.5378647268,-0.8382268377$ $\mathrm{H}, \mathrm{O},-3.9565330217,-0.9752341009,-3.4205067688$ $\mathrm{H}, 0,-5.0903374406,0.1735852646,-2.6464295972$ $\mathrm{H}, 0,-5.6959592052,-1.3565135742,-3.3407126152$ $\mathrm{H}, 0,2.2158127681,-5.1340807391,-1.3010585429$ $\mathrm{H}, 0,0.6542158027,-4.3691133397,-1.7807122613$ $\mathrm{H}, 0,1.0900946879,-4.5667660343,-0.0381663946$ $\mathrm{H}, 0,7.1329161526,-2.5743411955,1.4999096714$ $\mathrm{H}, 0,6.664530987,-0.8971312652,1.9700653772$ $\mathrm{H}, 0,7.3175380028,-1.2049822545,0.3384100559$ $\mathrm{H}, \mathrm{0}, 3.2625386742,0.4280934382,-1.5909274633$ $\mathrm{H}, 0,1.8862640986,-0.7662157801,-1.8155575543$

\section{INT2-E-T}

Opt a B3LYP-D3 (BJ)/def2-SVP in gas phase SCF Done: $\mathrm{E}(\mathrm{UB} 3 \mathrm{LYP})=-1471.83117155 \mathrm{a} \cdot \mathrm{u}$. Zero-point correction $=0.448821$ Hartree/Particle Sum of electronic and thermal Free Energies = -1471.445324 a.u. (@ $298.15 \mathrm{~K}$ ) $\mathrm{SP}$ a B3LYP-D3 (BJ)/def2-TZVP in acetonitrile (SMD model)

SCF Done: $\mathrm{E}(\mathrm{UB} 3 \mathrm{LYP})=-1473.49428344 \mathrm{a} \cdot \mathrm{u}$.

$0,0,-0.2461429781,-2.2966619531,2.0585574094$

$0,0,-3.6380134305,-0.3278922244,1.2131417233$

$0,0,-4.0034114254,0.5752785549,-0.8227225637$

$\mathrm{N}, 0,-0.9336201286,-0.5714762592,0.7499496568$

$\mathrm{C}, 0,-0.1125040025,0.4453631037,1.2556868804$

C, $0,0.789225485,0.4210815606,2.3117284029$

C, $0,1.5299068564,1.5855964047,2.5658742977$

C, 0,1.359005291,2.7500201013,1.7955470174

C, $0,0.4483952564,2.7829206345,0.7454672947$

C, $0,-0.3043406199,1.6205880255,0.4548536396$

C, $0,-1.2652716863,1.3462251236,-0.528010735$

C, $0,2.725787689,-0.3654017976,-1.1707499904$

C, $0,2.1018346018,-0.5722515976,-2.5203374737$

$C, 0,0.6184644221,-0.2946695734,-2.3572224027$

C, $0,-0.2426733079,-1.2405205312,-1.9647758087$

C, $0,-1.6813212324,-1.0374761749,-1.552746686$

$\mathrm{C}, 0,-1.7727258937,-0.0570794646,-0.3427051709$

C, $0,-2.303841655,-2.3603928884,-1.081113882$

$\mathrm{C}, 0,-1.6308168313,-2.8887226874,0.2025417479$

$\mathrm{C}, 0,-0.8959767383,-1.9019043047,1.1057591201$

$\mathrm{C}, 0,-3.2381281873,0.0340594874,0.1375266618$

C, $0,-5.3990491635,0.7741856711,-0.5185004036$ 
$C, 0,-5.6163916222,2.0550059268,0.265608019$ $\mathrm{H}, 0,0.9086645031,-0.4787147605,2.907552847$ $\mathrm{H}, \mathrm{O}, 2.2608694515,1.5820370723,3.3764770263$ $\mathrm{H}, 0,1.9543334093,3.6359362078,2.0272825851$ $\mathrm{H}, 0,0.311578141,3.6839982162,0.1439738559$ $\mathrm{H}, 0,-1.6780707696,2.0294961756,-1.2660949064$ $\mathrm{H}, 0,2.5469809024,0.1072812982,-3.2571117963$ $\mathrm{H}, 0,2.257555122,-1.6151214772,-2.8260433299$ $\mathrm{H}, 0,0.312860112,0.7509059823,-2.4465470098$ $\mathrm{H}, \mathrm{O}, \mathrm{0} .1556629288,-2.2519258492,-1.8368667455$ $\mathrm{H}, \mathrm{O},-2.2823414979,-0.6103159057,-2.3697346287$ $\mathrm{H}, 0,-3.3813640101,-2.2162969714,-0.9133788299$ $\mathrm{H}, \mathrm{0},-2.2175564899,-3.1143016399,-1.8783173461$ $\mathrm{H}, \mathrm{O},-2.3564202514,-3.414360854,0.8414243966$ $\mathrm{H}, \mathrm{O},-0.8536326341,-3.6316519347,-0.035663228$ $\mathrm{H}, \mathrm{O},-5.8932678442,0.8094317028,-1.499294218$ $\mathrm{H}, 0,-5.132253071,1.9931395125,1.2508769116$ $\mathrm{H}, 0,-6.6941228847,2.2200485557,0.421852202$ $\mathrm{H}, \mathrm{O},-5.209125885,2.9213799668,-0.2779162791$ $\mathrm{H}, \mathrm{O},-5.7658239352,-0.0991684973,0.0405887716$ C, $0,3.227816778,0.9612968847,-0.7720054249$ $0,0,3.9518551053,1.2119563815,0.1645890943$ C, $0,2.6994887803,-1.5311150443,-0.2587342859$ $0,0,2.2675098029,-2.6154510935,-0.6126427345$ $0,0,2.8069513143,1.9139700219,-1.6445693278$ $0,0,3.1601857735,-1.2932600177,0.9677014559$ C, $0,3.304534209,3.2274826915,-1.4126605202$ $\mathrm{H}, 0,3.0193963373,3.5805631632,-0.412011115$ $\mathrm{H}, \mathrm{O}, 4.4026725919,3.2476459151,-1.4892244877$ $\mathrm{H}, 0,2.8575615409,3.8647817934,-2.1857094046$ $C, 0,3.0871808522,-2.3851767909,1.888697049$ $\mathrm{H}, 0,2.0403981396,-2.6836965546,2.0426928327$ $\mathrm{H}, 0,3.6603393457,-3.2455917787,1.512006277$ $\mathrm{H}, 0,3.525076575,-2.0129738486,2.8225456049$

\section{INT2- $Z-T_{1}$}

Opt \& B3LYP-D3 (BJ)/def2-SVP in gas phase SCF Done: E (UB3LYP) $=-1471.82379071$ a.u. Zero-point correction $=0.448951$ Hartree/Particle Sum of electronic and thermal Free Energies $=-1471.437967 \mathrm{a} \cdot \mathrm{u}$. (e $298.15 \mathrm{~K}$ ) SP \& B3LYP-D3(BJ)/def2-TZVP in acetonitrile (SMD model) SCF Done: E (UB3LYP) $=-1473.48744439$ a.u.

$0,0,-1.1401025947,2.8569716859,-0.7924782281$ $0,0,-3.6075057088,-1.5013997965,0.9644666673$ $0,0,-3.3457783565,-0.2705931951,-0.9049503281$ $0,0,3.6692394416,-1.6618152744,1.1546607059$ $0,0,3.5132650626,-1.3397301862,-1.0633219915$ $0,0,3.6330670982,1.3930909443,-1.5140792662$ $0,0,1.9674154127,2.4145721274,-0.3892086879$ $\mathrm{N}, 0,-0.9736566949,0.6290901337,-0.3061166679$ $\mathrm{C}, 0,-1.3795309652,1.9217725985,-0.0502230764$ C, $0,-2.1175778683,2.047189468,1.2716662765$ C, $0,-1.4169722096,1.2579344739,2.3878126881$ C $, 0,-1.2393053561,-0.2495401619,2.0561953292$ C, $0,-1.4340395139,-0.4957123713,0.5250948601$ 
$C, 0,-0.6987926136,-1.6677214546,-0.0785659238$

C, $0,-0.0675944919,-1.2532824938,-1.2608159849$

$\mathrm{C}, 0,-0.2663629128,0.1587831833,-1.418410323$

$C, 0,2.6045474961,0.3019073728,0.3586491694$

$\mathrm{C}, 0,1.8327909993,0.6063724564,1.6093109853$

C, $0,1.3068697721,-0.5480608123,2.4165091146$

$\mathrm{C}, 0,0.0234982834,-0.881331037,2.6104648577$

$\mathrm{C}, 0,-2.9321249941,-0.8053372925,0.2457313557$

$C, 0,-4.657502962,-0.6390034195,-1.3767681168$

$C, 0,-4.6163624179,-1.9690222975,-2.1056052679$

$\mathrm{C}, 0,3.3151875763,-0.9825855425,0.2100153044$

$\mathrm{C}, 0,4.1900279624,-2.5722592545,-1.2756859249$

C, $0,2.8091186864,1.3850449294,-0.6262924814$

C, $0,2.0413100338,3.5312664012,-1.2702659465$

$\mathrm{H}, \mathrm{O},-2.1761462044,3.1177533283,1.5079805252$

$\mathrm{H}, \mathrm{0},-3.1532313693,1.6904573253,1.1322117939$

$\mathrm{H}, \mathrm{O},-0.4395599879,1.7202674437,2.5792828026$

$\mathrm{H}, \mathrm{O},-1.9854787726,1.3564571454,3.3241825956$

$\mathrm{H}, \mathrm{O},-2.0759080546,-0.7927460213,2.5118457623$

$\mathrm{H}, \mathrm{O},-0.7625140293,-2.6733077299,0.3297107388$

$\mathrm{H}, 0,1.0354295128,1.2973315863,1.3202662102$

$\mathrm{H}, 0,2.5045844272,1.2069730878,2.2610806733$

$\mathrm{H}, 0,2.068970709,-1.1685504946,2.8915001757$

$\mathrm{H}, \mathrm{O},-0.1535765644,-1.7587646929,3.2418515714$

$\mathrm{H}, \mathrm{O},-5.3452984241,-0.6734186283,-0.5192354681$

$\mathrm{H}, \mathrm{O},-4.9493810759,0.1823186608,-2.0452246976$

$\mathrm{H}, 0,-3.885116307,-1.93966673,-2.9276216431$

$\mathrm{H}, \mathrm{O},-4.3436824877,-2.7813078506,-1.4160918219$

$\mathrm{H}, \mathrm{O},-5.6076703711,-2.1961378753,-2.5283657968$

$\mathrm{H}, 0,5.2070369327,-2.5374858284,-0.856562284$

$\mathrm{H}, 0,3.6472951746,-3.4039693064,-0.8004523488$

$\mathrm{H}, 0,4.2300045551,-2.7114957488,-2.3625559112$

$\mathrm{H}, 0,2.9317273983,4.1391822703,-1.0445449574$

$\mathrm{H}, \mathrm{O}, 2.1044204467,3.1939246257,-2.3141539553$

$\mathrm{C}, 0,0.6601669462,-1.9589648605,-2.2474464515$

C, $0,1.1566513961,-1.2633226047,-3.3437256851$

C, $0,0.9332324484,0.1178675037,-3.4842507977$

$\mathrm{C}, 0,0.2076686951,0.8455877595,-2.5265793553$

$\mathrm{H}, \mathrm{O}, \mathrm{0} .828865176,-3.0320899936,-2.1367539184$

$\mathrm{H}, 0,1.7309448956,-1.7932609726,-4.1067442885$

$\mathrm{H}, 0,1.3344411398,0.640561651,-4.3548590114$

$\mathrm{H}, 0,0.017883852,1.9101910631,-2.6301850087$

$\mathrm{H}, 0,1.119658368,4.1004421216,-1.1063412071$

\section{INT1a-OSS}

Opt a B3LYP-D3 (BJ)/def2-SVP in gas phase

SCF Done: E (UB3LYP) $=-1471.81600128 \mathrm{a} \cdot \mathrm{u}$.

Zero-point correction $=0.447806$ Hartree/Particle

Sum of electronic and thermal Free Energies = -1471.431931 a.u. (@ 298.15 K)

$\mathrm{SP}$ a B3LYP-D3 (BJ)/def2-TZVP in acetonitrile (SMD model)

$\mathrm{SCF}$ Done: $\mathrm{E}(\mathrm{UB} 3 \mathrm{LYP})=-1473.47889563 \mathrm{a} \cdot \mathrm{u}$.

$0,0,2.9795537908,-0.8010876772,-2.0246776026$

$\mathrm{N}, 0,2.005300901,-0.5819142945,0.01484235$

$\mathrm{C}, 0,2.307867709,0.764873484,0.266987113$

C, $0,3.0909523788,1.6529870559,-0.4584956806$ 
C, $0,3.1842109982,2.9744368063,0.0108704429$

C, $0,2.5137953049,3.393525651,1.1745689093$

C, $0,1.7315644237,2.504819238,1.9048497968$

$C, 0,1.6167763484,1.1666801132,1.4591859129$

C, 0,0.9111384395,0.0649752116,1.9665617329

C, $0,1.2222273954,-1.144641797,1.1265588228$

$\mathrm{C}, 0,2.2698812404,-1.2681662534,-1.1481643614$

$\mathrm{C}, 0,1.5594992078,-2.6016265089,-1.3565306802$

$C, 0,0.5879055388,-3.0966669352,-0.2738653471$

$\mathrm{C}, 0,0.0169600537,-1.9332011509,0.5655641961$

$\mathrm{C}, 0,-0.8871874784,-1.0207425539,-0.2026497097$

$\mathrm{C}, 0,-1.9609471288,-1.5586441485,-1.0259761747$

C, $0,-1.9852853158,-1.3430333924,-2.583006317$

$\mathrm{H}, 0,3.6052833221,1.3206217393,-1.356109335$

$\mathrm{H}, 0,3.7951003057,3.6903112733,-0.5437010736$

$\mathrm{H}, 0,2.6119118131,4.4291494944,1.5081135165$

$\mathrm{H}, 0,1.2099855073,2.8249324399,2.8093763666$

$\mathrm{H}, 0,1.0316953054,-2.4657130794,-2.3126268806$

$\mathrm{H}, \mathrm{O}, 2.3456434867,-3.3455380431,-1.5556839978$

$\mathrm{H}, \mathrm{O}, 1.0945707838,-3.8010850183,0.4039663826$

$\mathrm{H}, \mathrm{O},-0.2287370811,-3.6618852258,-0.745820753$

$\mathrm{H}, 0,-0.5176881217,-2.3671813403,1.4302713482$

$\mathrm{H}, \mathrm{O},-0.6556093249,0.0432590279,-0.2689326625$

$\mathrm{H}, \mathrm{O},-2.2723312369,-2.5829281329,-0.8021675889$

$C, 0,-3.0053521708,-0.7044422498,-1.669422269$

$\mathrm{H}, 0,0.3159755906,0.0135631362,2.8750169152$

$\mathrm{C}, 0,2.127746824,-2.0883490582,1.9502560861$

$0,0,3.2651139426,-2.3682609973,1.6752120448$

$0,0,1.4618389929,-2.5504194079,3.0192644046$

C, $0,2.1835944325,-3.4125226543,3.9224575033$

$\mathrm{C}, 0,3.0275955627,-2.6097786916,4.8950244762$

$\mathrm{H}, \mathrm{0}, 2.8065475968,-4.0997954421,3.331401644$

$\mathrm{H}, \mathrm{0}, 1.4024867734,-3.9864952209,4.4400202175$

$\mathrm{H}, 0,3.5112153013,-3.2858887174,5.6176374212$

$\mathrm{H}, 0,3.8147297811,-2.0581867722,4.3610215639$

$\mathrm{H}, 0,2.4065941063,-1.8929720391,5.4539335675$

C, $0,-2.4315574502,-2.5358268939,-3.378688074$

$0,0,-2.6689388551,-3.6267027827,-2.9133862201$

$0,0,-2.5999943264,-2.2309779435,-4.6711268874$

C, $0,-0.922662899,-0.4467741897,-3.1435535468$

$0,0,-0.765950695,0.7069298167,-2.8206712435$

$0,0,-0.1196922483,-1.1065473471,-3.9891361623$

$\mathrm{H}, \mathrm{O},-2.9054793467,0.3787650081,-1.5664352935$

$\mathrm{H}, \mathrm{O},-4.0258942125,-1.09122915,-1.7170927634$

C, $0,0.9544448626,-0.3639077872,-4.5873718053$

$\mathrm{H}, 0,1.2686798668,-0.9458524057,-5.4624453439$

$\mathrm{H}, \mathrm{0}, 0.6056241541,0.6332287386,-4.8883642199$

$\mathrm{H}, \mathrm{O}, 1.7856588613,-0.2729704439,-3.8739682845$

C, $0,-3.0019662939,-3.3012851008,-5.525424275$

$\mathrm{H}, \mathrm{O},-3.9716698461,-3.712255901,-5.2068730623$

$\mathrm{H}, \mathrm{O},-3.0785333393,-2.8748003381,-6.5326791492$

$\mathrm{H}, 0,-2.2572300526,-4.1110791682,-5.50826637$

\section{INT1b-OSS}

Opt a B3LYP-D3 (BJ)/def2-SVP in gas phase

SCF Done: $\mathrm{E}(\mathrm{UB} 3 \mathrm{LYP})=-1471.82090512 \mathrm{a} \cdot \mathrm{u}$. 
Zero-point correction $=0.448248$ Hartree/Particle

Sum of electronic and thermal Free Energies = -1471.433746 a.u. (@ $298.15 \mathrm{~K}$ ) $\mathrm{SP}$ a B3LYP-D3 (BJ)/def2-TZVP in acetonitrile (SMD model)

SCF Done: $\mathrm{E}(\mathrm{UB} 3 \mathrm{LYP})=-1473.48140704 \mathrm{a} \cdot \mathrm{u}$.

$0,0,3.1331224886,0.8011605385,-1.0562415956$

$\mathrm{N}, 0,1.7647986643,-0.4394291052,0.2671860846$

C, $0,1.0300131843,0.599731445,0.8599734386$

$\mathrm{C}, 0,1.2274013368,1.9728860645,0.8135020102$

C, $0,0.2821678168,2.7898156384,1.4557919025$

C, $0,-0.8244497521,2.244619181,2.132526516$

$\mathrm{C}, 0,-1.0081961721,0.867462473,2.2053201162$

$\mathrm{C}, 0,-0.0674622283,0.0196235177,1.5758754534$

$\mathrm{C}, 0,0.0283098425,-1.3774002527,1.4978547203$

C, $0,1.2883168272,-1.7388039477,0.7617640193$

C, $0,2.7298257323,-0.2946804489,-0.7043472976$

C, $0,3.2061163389,-1.5589809278,-1.4134852309$

C, $0,2.6466670429,-2.9131572099,-0.9507891125$

C, $0,1.2151304262,-2.779009276,-0.411948011$

$C, 0,0.254281512,-2.4000367166,-1.4867061052$

C, $0,-1.153590135,-2.1469877614,-1.2222200739$

C, $0,-2.1848995239,-2.0393598605,-2.2973459347$

$\mathrm{H}, 0,2.0805058992,2.3847387152,0.2814680382$

$\mathrm{H}, 0,0.4143029583,3.8739816727,1.4295511826$

$\mathrm{H}, \mathrm{0},-1.539955362,2.9134533721,2.6170189481$

$\mathrm{H}, \mathrm{O},-1.8613101634,0.4333888909,2.7292084816$

$\mathrm{H}, 0,2.9519565641,-1.370757373,-2.4694345398$

$\mathrm{H}, \mathrm{0}, 4.305571776,-1.5446516074,-1.3715657639$

$\mathrm{H}, 0,3.2924466956,-3.3419143452,-0.1700302642$

$\mathrm{H}, 0,2.6638388611,-3.6257368787,-1.7892583626$

$\mathrm{H}, 0,0.9045866568,-3.726191749,0.0631961947$

$\mathrm{H}, 0,0.6350296889,-2.1649581781,-2.4820940834$

$\mathrm{H}, 0,-1.5374975729,-2.4752455531,-0.2551790521$

$0,0,-3.2030756892,-0.8937138419,0.3384411069$

$0,0,-3.1818150977,1.0057337488,-0.856724223$

$0,0,-0.4265014306,-0.1158919904,-3.4520153482$

$0,0,-0.6199259898,1.2881981463,-1.7126168528$

C, $0,-1.7968602019,-0.7551373563,-1.5975615265$

$C, 0,-2.7743765975,-0.2368859274,-0.5843704538$

C, $0,-4.105979298,1.5869052006,0.0614391868$

C, $0,-0.901349079,0.1673461677,-2.3737617284$

$\mathrm{C}, 0,0.2638812095,2.2116451604,-2.3574598081$

$\mathrm{H}, \mathrm{O},-4.3730704905,2.565016579,-0.3561402746$

$\mathrm{H}, 0,-4.9997404854,0.9552601978,0.1713074898$

$\mathrm{H}, \mathrm{O},-3.6347680588,1.7123816198,1.0469205124$

$\mathrm{H}, \mathrm{O},-0.0580216634,2.3899450601,-3.3930484121$

$\mathrm{H}, 0,0.211089728,3.1326478151,-1.7666646758$

$\mathrm{H}, 0,1.2920356345,1.8235673938,-2.34842503$

$\mathrm{H}, \mathrm{O},-1.8483206434,-2.1103649005,-3.3338132932$

$\mathrm{H}, \mathrm{O},-3.1798507865,-2.4325503421,-2.0786094899$

$\mathrm{H}, 0,-0.6113903907,-2.1106009194,1.9815606947$

C, $0,2.2866386774,-2.2627550063,1.8223012987$

$0,0,3.3057138549,-1.7086654524,2.1423965938$

$0,0,1.8463731599,-3.4187140191,2.3420489388$

C, $0,2.6318000328,-4.0067619819,3.399107399$

$C, 0,2.3166322957,-3.369300058,4.7395734477$

$\mathrm{H}, 0,3.6973853268,-3.8975130711,3.1493035496$ 
$\mathrm{H}, 0,2.3635682758,-5.0722031945,3.3825504707$

$\mathrm{H}, 0,2.8748879104,-3.8822223557,5.5385945211$

$\mathrm{H}, 0,2.6087877507,-2.3092790986,4.7426542015$

$\mathrm{H}, 0,1.2418710226,-3.4433634718,4.9658024859$

\section{INT2-E-OSS}

Opt a B3LYP-D3 (BJ)/def2-SVP in gas phase

SCF Done: $\mathrm{E}(\mathrm{UB} 3 \mathrm{LYP})=-1471.83125378 \mathrm{a} \cdot \mathrm{u}$.

Zero-point correction $=0.448748$ Hartree/Particle

Sum of electronic and thermal Free Energies = -1471.444286 a.u. (@ $298.15 \mathrm{~K}$ )

SP a B3LYP-D3 (BJ)/def2-TZVP in acetonitrile (SMD model)

SCF Done: $\mathrm{E}(\mathrm{UB} 3 \mathrm{LYP})=-1473.49377339 \mathrm{a} \cdot \mathrm{u}$.

$0,0,-0.2356212509,-2.2758750187,2.0425728128$

$0,0,-3.6344177298,-0.3381109746,1.2171759411$

$0,0,-4.0242027949,0.5669639118,-0.8133141529$

$\mathrm{N}, 0,-0.931197156,-0.5567426391,0.7301716941$

C, $0,-0.1062046458,0.4616702493,1.2222706327$

C, $0,0.8125219749,0.4402302373,2.2643056997$

$\mathrm{C}, 0,1.5490388911,1.6081878891,2.5102861107$

C, $0,1.3567992652,2.7753298934,1.7474962518$

$\mathrm{C}, 0,0.4318506768,2.8056758471,0.711072374$

$\mathrm{C}, 0,-0.3180237126,1.6387205198,0.427323844$

C, $0,-1.2908818844,1.3612626763,-0.5406882906$

$\mathrm{C}, 0,2.717570682,-0.3653298893,-1.1363576009$

C, $0,2.1021004256,-0.565374374,-2.4925964591$

$C, 0,0.6204424902,-0.2790255073,-2.3429005753$

$\mathrm{C}, 0,-0.2510633624,-1.225654955,-1.9763378366$

C, $0,-1.6914395116,-1.0258224486,-1.5640148023$

$\mathrm{C}, 0,-1.7848782588,-0.0474516743,-0.3539336123$

C, $0,-2.3046846875,-2.3525892861,-1.0898212888$

$\mathrm{C}, 0,-1.6244513043,-2.877138403,0.1918976932$

$C, 0,-0.889173557,-1.8866622124,1.0904064963$

$\mathrm{C}, 0,-3.2465490438,0.0301089504,0.1392762492$

C, $0,-5.4193738428,0.7516911335,-0.4980228019$

C, $0,-5.6430773039,2.0284825902,0.2908300411$

$\mathrm{H}, 0,0.9466973374,-0.4602045095,2.8557614941$

$\mathrm{H}, 0,2.2940067223,1.606489688,3.3078198166$

$\mathrm{H}, 0,1.949299542,3.6643117926,1.9744348659$

$\mathrm{H}, 0,0.2801494563,3.707853607,0.1147911456$

$\mathrm{H}, \mathrm{O},-1.7144757275,2.0416249215,-1.2753549628$

$\mathrm{H}, 0,2.5596122881,0.1131954777,-3.22269445$

$\mathrm{H}, 0,2.254463621,-1.6082906193,-2.7997799498$

$\mathrm{H}, 0,0.323609267,0.7702099139,-2.4155882174$

$\mathrm{H}, 0,0.1399026604,-2.2421637692,-1.8683614255$

$\mathrm{H}, \mathrm{O},-2.296378122,-0.6046682204,-2.3814336183$

$\mathrm{H}, \mathrm{O},-3.3826238395,-2.2152986353,-0.919108238$

$\mathrm{H}, \mathrm{O},-2.2158455962,-3.1058977595,-1.8873267404$

$\mathrm{H}, \mathrm{O},-2.3453900185,-3.4049455128,0.8342145578$

$\mathrm{H}, \mathrm{O},-0.8448377931,-3.616619757,-0.0489585382$

$\mathrm{H}, 0,-5.9213201102,0.7845000469,-1.4749643106$

$\mathrm{H}, \mathrm{O},-5.1510515929,1.9689289902,1.2723284163$

$\mathrm{H}, \mathrm{O},-6.721144534,2.1828061068,0.455470255$

$\mathrm{H}, \mathrm{O},-5.2482050792,2.9000088053,-0.253599779$

$\mathrm{H}, \mathrm{O},-5.7734056165,-0.1265218397,0.0616681271$

C, $0,3.2229267732,0.9567420785,-0.7312295623$ 
$0,0,3.9090231035,1.2100193705,0.2337025326$

C, $0,2.6855616669,-1.5373161734,-0.2329481641$

$0,0,2.2281151857,-2.6107247281,-0.5889141654$

$0,0,2.8536316724,1.9063485039,-1.6316009969$

$0,0,3.1756548698,-1.3221516535,0.9866813672$

$C, 0,3.3604646945,3.214652924,-1.3936137277$

$\mathrm{H}, 0,3.0199765815,3.5929753212,-0.4197569584$

$\mathrm{H}, 0,4.4613844419,3.2145722273,-1.4011158483$

$\mathrm{H}, 0,2.9743389811,3.8445251171,-2.2046855961$

C, $0,3.0920154411,-2.4213039123,1.8977564586$

$\mathrm{H}, 0,2.041121939,-2.6999484582,2.0617090919$

$\mathrm{H}, 0,3.6415102116,-3.2901549552,1.5053269197$

$\mathrm{H}, \mathrm{O}, 3.5500103555,-2.0679972436,2.8293638268$

\section{INT2- $Z$-OSS}

Opt a B3LYP-D3 (BJ)/def2-SVP in gas phase

SCF Done: E (UB3LYP) $=-1471.82690216$ a.u.

Zero-point correction $=0.449130$ Hartree/Particle

Sum of electronic and thermal Free Energies $=-1471.438167 \mathrm{a} \cdot \mathrm{u}$. (e $298.15 \mathrm{~K}$ ) SP a B3LYP-D3(BJ)/def2-TZVP in acetonitrile (SMD model)

SCF Done: $\mathrm{E}(\mathrm{UB} 3 \mathrm{LYP})=-1473.49055778 \mathrm{a} \cdot \mathrm{u}$.

$0,0,1.5496152259,2.4601347081,1.6661980956$

$0,0,3.8139997804,-1.3028241506,-1.3052704222$

$0,0,3.3763635871,-0.8141072096,0.850060059$

$0,0,-2.2252932525,-1.4662795899,-2.0128792303$

$0,0,-3.8804789153,-1.2020727053,-0.5190741888$

$0,0,-3.4998550119,0.7179727095,1.3454363188$

$0,0,-2.1537774124,2.3891778136,0.659408016$

$\mathrm{N}, 0,1.2436923749,0.5979636556,0.375533336$

C, $0,1.815142419,1.8290569609,0.6635170999$

$C, 0,2.7382899919,2.3060719193,-0.4419703872$

C, $0,2.0873904081,2.0793071472,-1.8140538977$

$C, 0,1.776901302,0.5860385688,-2.1054830729$

C, $0,1.7037692096,-0.217780215,-0.7620548625$

C, $0,0.7665672689,-1.3988998916,-0.7156473847$

$C, 0,-0.0237996303,-1.3094781455,0.4242554784$

C, $0,0.2920049686,-0.0887957125,1.1169542143$

C, $0,-2.2702926384,0.5189205935,-0.7172298938$

C, $0,-1.2712769724,1.273488334,-1.5530565388$

C, $0,-0.7015615803,0.6368189821,-2.7881765081$

$C, 0,0.5920031757,0.3869268822,-3.030404384$

C, $0,3.1019561988,-0.8279600684,-0.4555412739$

C, $0,4.5633194447,-1.5081626074,1.2873964725$

C, $0,4.2972410179,-2.9937164152,1.4412864398$

C, $0,-2.7640842224,-0.7916899725,-1.1487519885$

$C, 0,-4.3579793081,-2.4890662312,-0.8771659958$

C, $0,-2.7368883975,1.164664971,0.5111468857$

C, $0,-2.4675955851,3.0923578907,1.8522051117$

$\mathrm{H}, \mathrm{O}, 2.9413926833,3.3687714095,-0.2557720387$

$\mathrm{H}, 0,3.7019322647,1.7720523844,-0.3685020186$

$\mathrm{H}, 0,1.1581682961,2.6655371787,-1.8571514065$

$\mathrm{H}, 0,2.7353957274,2.4744506029,-2.6101275694$

$\mathrm{H}, 0,2.6458420537,0.1545323795,-2.6169954856$

$\mathrm{H}, 0,0.7555389083,-2.175127245,-1.4757439528$

$\mathrm{H}, \mathrm{O},-0.4709985741,1.6188249778,-0.8853795234$ 
$\mathrm{H}, \mathrm{0},-1.7702044423,2.2170730233,-1.8552019581$ $\mathrm{H}, 0,-1.4168612135,0.4006711671,-3.5797016393$ $\mathrm{H}, 0,0.8400889548,-0.0389599657,-4.0077769666$ $\mathrm{H}, 0,5.369811079,-1.318537816,0.5641547628$ $\mathrm{H}, 0,4.8168662463,-1.0381794852,2.2472505844$ $\mathrm{H}, 0,3.4545661422,-3.1697389268,2.1270115038$ $\mathrm{H}, 0,4.0653000709,-3.450110856,0.4679257333$ $\mathrm{H}, 0,5.1892665089,-3.4934940838,1.8504564785$ $\mathrm{H}, 0,-4.5579108198,-2.5558236652,-1.9577939642$ $\mathrm{H}, \mathrm{O},-3.6235465478,-3.2691082779,-0.6189679197$ $\mathrm{H}, 0,-5.2823228936,-2.6406963239,-0.3060418411$ $\mathrm{H}, 0,-3.5394704722,3.3417909364,1.8939259841$ $\mathrm{H}, \mathrm{O},-2.2194412494,2.491743223,2.7395678527$ $\mathrm{C}, 0,-1.0339431542,-2.1560828701,0.9572805363$ $\mathrm{C}, 0,-1.6502338858,-1.8016230628,2.1429405834$ $\mathrm{C}, 0,-1.2896084425,-0.6158471851,2.8226495919$ $\mathrm{C}, 0,-0.3120023928,0.2510004798,2.3244746102$ $\mathrm{H}, \mathrm{O},-1.3099877493,-3.0652300866,0.4216081722$ $\mathrm{H}, \mathrm{O},-2.4411224114,-2.4307506029,2.5545749687$ $\mathrm{H}, \mathrm{O},-1.8001897893,-0.3617786285,3.7529799377$ $\mathrm{H}, \mathrm{O},-0.035489379,1.1716192067,2.8314957408$ $\mathrm{H}, 0,-1.8608746297,4.00616811,1.8366679308$

\section{TS2a-E-OSS}

Opt a B3LYP-D3 (BJ)/def2-SVP in gas phase SCF Done: $\mathrm{E}(\mathrm{UB} 3 \mathrm{LYP})=-1471.81237556 \mathrm{a} \cdot \mathrm{u}$. Zero-point correction $=0.447230$ Hartree/Particle Sum of electronic and thermal Free Energies $=-1471.427337$ a.u. (@ $298.15 \mathrm{~K}$ ) Imaginary Frequency $=-414.9206 \mathrm{~cm}^{-1}$ SP @ B3LYP-D3 (BJ)/def2-TZVP in acetonitrile (SMD model) SCF Done: $\mathrm{E}(\mathrm{UB} 3 \mathrm{LYP})=-1473.47662680 \mathrm{a} \cdot \mathrm{u}$.

$0,0,-0.3586937997,-0.8892941989,2.5204231068$ $0,0,-3.4678090152,-0.6590290743,0.0442371447$ $0,0,-3.3773341988,0.495787008,-1.8924452868$ $\mathrm{N}, 0,-1.2333906838,0.59449771,1.0401674621$ $\mathrm{C}, 0,-1.4919202773,1.6630970314,1.9095751419$ $\mathrm{C}, 0,-1.3318986087,1.7513915857,3.2862100153$ C, $0,-1.6291005571,2.9793012573,3.9000793397$ C, $0,-2.0786383839,4.0873342337,3.1578867739$ $\mathrm{C}, 0,-2.2452767645,4.0002652769,1.7801564907$ $\mathrm{C}, 0,-1.9524717095,2.777378834,1.1306247558$ C, $0,-2.0122654643,2.3931625609,-0.2172938287$ C, $0,3.2583981722,1.6453195248,-1.0889079255$ C, $0,3.1980906974,0.4004233335,-0.2582265948$ C, $0,1.974418758,1.0092348395,-1.4834635133$ $\mathrm{C}, 0,0.7059317571,1.3585878264,-1.0039285358$ C, $0,-0.5274674969,0.5639782055,-1.3215544981$ C, $0,-1.6539553197,0.9356773754,-0.3267907638$ $\mathrm{C}, 0,-0.3321255694,-0.9635810437,-1.2278211598$ $\mathrm{C}, 0,-0.076687287,-1.4381436795,0.2127427122$ $\mathrm{C}, 0,-0.586666694,-0.5793887901,1.3637870305$ $\mathrm{C}, 0,-2.9372244272,0.1476507014,-0.6731701134$ $C, 0,-4.6026420154,-0.1136504108,-2.348013894$ $\mathrm{C}, 0,-5.8172547249,0.6027427674,-1.7876083301$ $\mathrm{H}, \mathrm{0},-0.9882842882,0.8903411373,3.8524913536$ 
$\mathrm{H}, \mathrm{0},-1.5096333033,3.0717386077,4.9819496037$ $\mathrm{H}, \mathrm{O},-2.3010804803,5.0248483908,3.6726382943$ $\mathrm{H}, 0,-2.5953575069,4.8562085906,1.1991776597$ $\mathrm{H}, \mathrm{O},-2.3582999437,2.9800917714,-1.0647166193$ $\mathrm{H}, 0,2.0385466121,0.2906492804,-2.3045691981$ $\mathrm{H}, 0,0.636090725,2.1466808476,-0.2532668405$ $\mathrm{H}, \mathrm{0},-0.8969996026,0.8079197847,-2.3332062917$ $\mathrm{H}, 0,-1.2311544876,-1.450512186,-1.6364105639$ $\mathrm{H}, 0,0.5001366497,-1.285786502,-1.8698901216$ $\mathrm{H}, \mathrm{O},-0.4885596237,-2.4460265059,0.3717033788$ $\mathrm{H}, 0,1.0035358392,-1.5393910574,0.4043355094$ $\mathrm{H}, 0,-4.5571279765,-0.0400936237,-3.4434708403$ $\mathrm{H}, 0,-5.8564730918,0.5009926355,-0.6935088443$ $\mathrm{H}, \mathrm{O},-6.7367467054,0.1645805186,-2.2067940475$ $\mathrm{H}, 0,-5.7950198792,1.6727006889,-2.0455397652$ $\mathrm{H}, 0,-4.5987086943,-1.1740001493,-2.0556734126$ $\mathrm{C}, 0,2.763519618,0.5643947716,1.1555262712$ $0,0,2.1813224135,1.5513369183,1.5599825992$ $0,0,3.1168732303,-0.4591296146,1.9326232849$ $\mathrm{C}, 0,3.7185281182,-0.8858681534,-0.770325431$ $0,0,3.475657982,-1.9865382035,-0.32951271$ $0,0,4.4643188136,-0.7026189203,-1.8904288737$ $\mathrm{H}, 0,3.1632497209,2.5635686535,-0.5030822381$ $\mathrm{H}, 0,4.0416001151,1.6673674726,-1.8490453968$ C, $0,2.7414344982,-0.3833409616,3.3132979049$ $\mathrm{H}, 0,3.2877418311,-1.1926266345,3.8124974807$ $\mathrm{H}, 0,3.0187646861,0.5929973701,3.7360017365$ $\mathrm{H}, 0,1.657806363,-0.5357537138,3.408717079$ $\mathrm{C}, 0,4.9315260564,-1.8901250267,-2.5240592224$ $\mathrm{H}, 0,5.59512056,-2.4562892224,-1.8533546842$ $\mathrm{H}, 0,4.0906453215,-2.5427296086,-2.8051227192$ $\mathrm{H}, 0,5.4797510418,-1.5659722304,-3.4172338653$

\section{TS2a-z-OSS}

Opt a B3LYP-D3 (BJ)/def2-SVP in gas phase SCF Done: $\mathrm{E}(\mathrm{UB} 3 \mathrm{LYP})=-1471.80488071 \mathrm{a} \cdot \mathrm{u}$. Zero-point correction $=0.447054$ Hartree/Particle Sum of electronic and thermal Free Energies $=-1471.421048$ a.u. (@ $298.15 \mathrm{~K}$ ) Imaginary Frequency $=-410.3305 \mathrm{~cm}^{-1}$ $\mathrm{SP}$ a B3LYP-D3 (BJ)/def2-TZVP in acetonitrile (SMD model) SCF Done: $\mathrm{E}(\mathrm{UB} 3 \mathrm{LYP})=-1473.47185941 \mathrm{a} \cdot \mathrm{u}$.

$0,0,2.8043800854,-0.4558523892,-3.2871546146$ $0,0,2.063364717,-2.1356936587,1.6966565741$ $0,0,3.607725182,-0.7748311743,0.7748031978$ $0,0,-5.2215730619,0.8360484723,0.6847268147$ $0,0,-5.1150480178,-0.5965791546,-1.0439899327$ $0,0,-1.6103705472,-0.687618122,2.1146567945$ $0,0,-2.8269762106,1.1867650935,1.8995773194$ $\mathrm{N}, 0,1.980116226,-0.0085174988,-1.2048216415$ C, $0,2.0402870372,1.3885731267,-1.1778078317$ C, $0,2.5759770169,2.2708283899,-2.1066067599$ $\mathrm{C}, 0,2.4782014888,3.6454928294,-1.8293031707$ $\mathrm{C}, 0,1.858706195,4.1188247526,-0.6578476081$ C, $0,1.3180047982,3.2349992972,0.2713837684$ C, $0,1.4009242103,1.8443605822,0.0239751937$ 
$C, 0,0.9385496075,0.7300668637,0.741581814$

$C, 0,-3.3024088717,-0.6044881711,0.4430187852$

C, $0,-2.9229517947,-1.9520713628,-0.0985487631$

C, $0,-2.2604735098,-0.9803252651,-1.0032590421$

$C, 0,-0.8815364705,-0.6830123126,-1.0409005395$

C, $0,0.1922766514,-1.4665330256,-0.3419292032$

$C, 0,1.3558793362,-0.528220555,0.0235350471$

C, $0,0.7247660366,-2.6291450443,-1.2404593665$

C, $0,2.0786457185,-2.3177917797,-1.9199229874$

C, $0,2.3280101807,-0.8517116646,-2.2400799505$

C, $0,2.3732046843,-1.2629376135,0.9237498614$

C, $0,4.6279669208,-1.2721572763,1.6631352694$

C, $0,4.5875604826,-0.5518917709,2.9981897925$

C, $0,-4.6173940579,-0.0175685867,0.0819917096$

C, $0,-6.3898909811,-0.1253452468,-1.4709201783$

C, $0,-2.505722912,-0.0680952899,1.5716897243$

$C, 0,-2.1358922443,1.7669742431,2.9995224409$

$\mathrm{H}, 0,3.0452950366,1.8928953439,-3.0120095662$

$\mathrm{H}, 0,2.8940269934,4.3605615907,-2.542764961$

$\mathrm{H}, 0,1.8027244355,5.1947557369,-0.4772980852$

$\mathrm{H}, 0,0.8347901415,3.6006023884,1.1800491957$

$\mathrm{H}, 0,0.4144749536,0.6912596526,1.6923184446$

$\mathrm{H}, 0,-2.8723982845,-0.5967056645,-1.820887363$

$\mathrm{H}, \mathrm{O},-0.5638767506,0.0892386219,-1.7469439013$

$\mathrm{H}, \mathrm{O},-0.1863463002,-1.8743142435,0.6015968303$

$\mathrm{H}, \mathrm{O}, 0.8353864496,-3.5352404788,-0.6285839323$

$\mathrm{H}, 0,-0.0276121463,-2.8535361857,-2.0104539239$

$\mathrm{H}, 0,2.9047171188,-2.6331600568,-1.2595457458$

$\mathrm{H}, 0,2.2005167556,-2.8731231006,-2.8586079804$

$\mathrm{H}, 0,4.4887548612,-2.3557630939,1.7912567888$

$\mathrm{H}, 0,5.5708160122,-1.0875924575,1.1303476643$

$\mathrm{H}, 0,3.6475990831,-0.7673644061,3.5268817134$

$\mathrm{H}, 0,4.675547448,0.536044985,2.8561485435$

$\mathrm{H}, 0,5.4230783964,-0.8887287531,3.6318547877$

$\mathrm{H}, 0,-7.152179447,-0.3031524349,-0.6974365827$

$\mathrm{H}, 0,-6.6334149423,-0.6850494785,-2.3823991014$

$\mathrm{H}, \mathrm{O},-6.3585374914,0.9543760408,-1.6813439414$

$\mathrm{H}, \mathrm{O},-2.7005754471,2.6667194189,3.2726371923$

$\mathrm{H}, \mathrm{O},-1.110671055,2.0474475381,2.7109574295$

$\mathrm{H}, \mathrm{O},-2.0838887924,1.0691322628,3.8476395645$

$\mathrm{H}, 0,-2.2654040713,-2.5243410838,0.5603479661$

$\mathrm{H}, 0,-3.7475308535,-2.5278698306,-0.5236315531$

\section{TS2b-E-OSS}

Opt a B3LYP-D3 (BJ)/def2-SVP in gas phase

SCF Done: $\mathrm{E}(\mathrm{UB} 3 \mathrm{LYP})=-1471.81578327 \mathrm{a} \cdot \mathrm{u}$.

Zero-point correction $=0.447578$ Hartree/Particle

Sum of electronic and thermal Free Energies = -1471.427997 a.u. (@ $298.15 \mathrm{~K}$ )

Imaginary Frequency $=-426.1785 \mathrm{~cm}^{-1}$

$\mathrm{SP}$ a B3LYP-D3 (BJ)/def2-TZVP in acetonitrile (SMD model)

SCF Done: $\mathrm{E}(\mathrm{UB} 3 \mathrm{LYP})=-1473.47519857 \mathrm{a} \cdot \mathrm{u}$.

$0,0,0.603054961,1.9783657399,1.9075174792$

$0,0,3.7468202671,0.3676848369,0.2617337531$

$0,0,3.8761986814,-0.1332221253,-1.9349577891$

$\mathrm{N}, 0,1.0134579053,0.5321686894,0.2007462746$ 
$C, 0,0.4665713972,-0.6393340552,0.742943629$

C, $0,-0.1647400969,-0.8475059153,1.9619093803$

$C, 0,-0.6600762725,-2.1325226989,2.2275977982$

$\mathrm{C}, 0,-0.51617047,-3.1851592074,1.3056540506$

C, $0,0.1172923314,-2.9810334832,0.0850918188$

$\mathrm{C}, 0,0.6178642513,-1.6932898629,-0.218793456$

$\mathrm{C}, 0,1.2805323632,-1.1866179763,-1.3477516356$

$\mathrm{C}, 0,-2.4028449613,0.5040341289,-1.3427024834$

$C, 0,-2.4503437292,0.8119606263,-2.8124362405$

C, $0,-1.0220128564,0.5201151224,-2.5446986937$

$C, 0,-0.0461282895,1.5261254148,-2.442713384$

$\mathrm{C}, 0,1.40581543,1.3503990805,-2.1055597373$

C, $0,1.695141958,0.2337904378,-1.0670453897$

C, $0,1.9688570115,2.6522769632,-1.5042697896$

C, $0,1.3186961291,2.9613290068,-0.1499438638$

C, $0,0.988499219,1.7903908488,0.7669076469$

$\mathrm{C}, 0,3.2234824897,0.1776041102,-0.8043865311$

$\mathrm{C}, 0,5.3067036668,-0.2963660091,-1.8444613123$

C, $0,5.672972475,-1.679282889,-1.3386916412$

$\mathrm{H}, \mathrm{O},-0.2658592862,-0.0314195572,2.6713525555$

$\mathrm{H}, \mathrm{O},-1.1728104736,-2.3159895895,3.1738858559$

$\mathrm{H}, 0,-0.9114820389,-4.1728933337,1.5536507297$

$\mathrm{H}, 0,0.2337605961,-3.7940760621,-0.6347728237$

$\mathrm{H}, 0,1.6049499772,-1.7325770791,-2.230538567$

$\mathrm{H}, \mathrm{O},-2.9915328338,0.0839891184,-3.4195752871$ $\mathrm{H}, \mathrm{O},-2.6795492289,1.8576523827,-3.0361485615$ $\mathrm{H}, 0,-0.7171430609,-0.5244246369,-2.5925841726$ $\mathrm{H}, \mathrm{O},-0.3926966826,2.5569547894,-2.5404861876$ $\mathrm{H}, 0,1.9832267755,1.0946414122,-3.0119685604$ $\mathrm{H}, 0,3.0607794393,2.5680228218,-1.3961958852$ $\mathrm{H}, \mathrm{0}, 1.7920139252,3.4885429499,-2.1979793273$ $\mathrm{H}, 0,1.9172700919,3.6710227148,0.4386819445$ $\mathrm{H}, 0,0.335406286,3.4389663443,-0.3040306225$ $\mathrm{H}, 0,5.6690480577,-0.1284645209,-2.8681403088$ $\mathrm{H}, 0,5.317997101,-1.8213866201,-0.3077853122$ $\mathrm{H}, 0,6.7674525433,-1.8025799629,-1.3454766658$ $\mathrm{H}, 0,5.233066542,-2.4600141607,-1.9780931225$ $\mathrm{H}, 0,5.7095937144,0.4860535263,-1.1847430466$ $\mathrm{C}, 0,-2.7641540469,-0.8618876621,-0.8833899373$ $0,0,-3.1290714664,-1.189551321,0.2183715172$ C, $0,-2.3475255749,1.6680305978,-0.4189326206$ $0,0,-2.1468396716,2.8059461134,-0.8062994272$ $0,0,-2.6722400826,-1.7537206064,-1.9089036668$ $0,0,-2.5206770615,1.3512983249,0.8614445658$ $C, 0,-3.0738814611,-3.0861617578,-1.6058254408$ $\mathrm{H}, \mathrm{O},-2.4685151516,-3.4973595081,-0.7866388841$ $\mathrm{H}, \mathrm{O},-4.1327142828,-3.1155395683,-1.306497315$ $\mathrm{H}, 0,-2.9231531097,-3.6665511049,-2.5247897454$ C, $0,-2.5269539306,2.426827481,1.8041569003$ $\mathrm{H}, \mathrm{O},-1.5041228326,2.7939533942,1.9645924527$ $\mathrm{H}, 0,-3.1707792307,3.2454023915,1.4521277081$ $\mathrm{H}, 0,-2.917925403,1.9985659057,2.7350793745$

\section{TS2b-z-OSS}

Opt a B3LYP-D3 (BJ)/def2-SVP in gas phase SCF Done: $\mathrm{E}(\mathrm{UB} 3 \mathrm{LYP})=-1471.80403435 \mathrm{a} \cdot \mathrm{u}$. 
Zero-point correction $=0.447032$ Hartree/Particle

Sum of electronic and thermal Free Energies = -1471.421230 a.u. (@ $298.15 \mathrm{~K}$ ) Imaginary Frequency $=-455.8847 \mathrm{~cm}^{-1}$

$\mathrm{SP}$ a B3LYP-D3 (BJ)/def2-TZVP in acetonitrile (SMD model)

$\mathrm{SCF}$ Done: $\mathrm{E}(\mathrm{UB} 3 \mathrm{LYP})=-1473.47152229 \mathrm{a} \cdot \mathrm{u}$.

$0,0,-2.5130856167,0.6849471328,3.1660640423$

$0,0,-1.3517317448,-2.5243456131,-0.9920124395$

$0,0,-3.2619613557,-1.6797745343,-0.1427806492$

$0,0,3.582898481,-1.4322675861,1.1075964664$

$0,0,2.6932013935,-1.9932112696,-0.8937024311$

$0,0,4.6875821077,2.1892136083,-0.8429011329$

$0,0,5.5519016681,0.4158164554,0.2400560942$

$\mathrm{N}, 0,-2.0580869627,0.454993564,0.9406729957$

$\mathrm{C}, 0,-2.6841621132,1.5923063405,0.4214806294$

$\mathrm{C}, 0,-3.4830219049,2.5332809506,1.0583944689$

$\mathrm{C}, 0,-3.9741122335,3.598845588,0.285424677$

$\mathrm{C}, 0,-3.6705898804,3.7182117827,-1.08420919$

$\mathrm{C}, 0,-2.8676761184,2.778794538,-1.7222896977$

C, $0,-2.3571454153,1.6917825919,-0.972606443$

$\mathrm{C}, 0,-1.5252803523,0.6174746479,-1.31848633$

$\mathrm{C}, 0,3.363884187,0.2305770978,-0.6137102617$

$\mathrm{C}, 0,2.3836799486,0.6865950668,-1.6426006123$

C, $0,1.893819249,1.2810324488,-0.3669088313$

$\mathrm{C}, 0,0.8534714153,0.8203645788,0.4570918771$

$\mathrm{C}, 0,0.1371864341,-0.4882326958,0.3269718267$

$\mathrm{C}, 0,-1.3317031977,-0.2677635176,-0.1125005235$

$\mathrm{C}, 0,0.157615609,-1.2854051686,1.6636918365$

C, $0,-1.1597190128,-1.1956102256,2.4691168919$

C, $0,-1.9703245669,0.0736625072,2.2657513594$

$\mathrm{C}, 0,-1.9674333788,-1.6328136944,-0.4549284732$

C, $0,-4.0031798475,-2.8498635068,-0.5475441391$

$\mathrm{C}, 0,-4.4411944503,-2.7430550757,-1.9961360534$

C, $0,3.2574280114,-1.128886579,-0.0134234754$

C, $0,2.398383794,-3.3210880528,-0.4580342271$

C, $0,4.5763991682,1.05497964,-0.4211095085$

$\mathrm{C}, 0,6.7464024283,1.1590130685,0.4650986047$

$\mathrm{H}, \mathrm{O},-3.704380653,2.4346746066,2.1184755521$

$\mathrm{H}, \mathrm{O},-4.6060047694,4.3515661842,0.7622092219$

$\mathrm{H}, \mathrm{0},-4.071816025,4.5613370411,-1.6512040285$

$\mathrm{H}, \mathrm{O},-2.6278162709,2.8706738133,-2.7836887944$

$\mathrm{H}, \mathrm{O},-1.0976554985,0.3868611471,-2.2919100839$

$\mathrm{H}, 0,2.2984018697,2.2703804461,-0.1451570295$

$\mathrm{H}, 0,0.5907773112,1.4480680971,1.3135876536$

$\mathrm{H}, 0,0.5939835873,-1.0929535082,-0.461605241$

$\mathrm{H}, 0,0.3682698832,-2.3415230478,1.4503893903$

$\mathrm{H}, 0,0.9983299895,-0.9256146109,2.2725368264$

$\mathrm{H}, \mathrm{O},-1.8265600548,-2.0283496014,2.1868432219$

$\mathrm{H}, \mathrm{O},-0.9829877029,-1.2899127786,3.5479257752$

$\mathrm{H}, \mathrm{O},-3.3767859875,-3.7389646895,-0.3837451504$

$\mathrm{H}, \mathrm{O},-4.8624759781,-2.8794904509,0.1359844623$

$\mathrm{H}, \mathrm{0},-3.5695386385,-2.73933704,-2.6667006134$

$\mathrm{H}, \mathrm{O},-5.0213076502,-1.8223431551,-2.160583597$

$\mathrm{H}, 0,-5.0739832007,-3.6047883159,-2.2605484131$

$\mathrm{H}, \mathrm{O}, 3.0358712224,-4.0241125555,-1.0147043071$

$\mathrm{H}, 0,1.3411918619,-3.5180605395,-0.6894387615$

$\mathrm{H}, \mathrm{0}, 2.5899263363,-3.4214482528,0.6182954697$ 
$\mathrm{H}, 0,7.4167998865,0.4939580693,1.0226191368$

$\mathrm{H}, 0,6.5365062658,2.0679889344,1.0490205194$

$\mathrm{H}, 0,7.20768006,1.4593778522,-0.4880843525$

$\mathrm{H}, 0,2.7781317874,1.4103303416,-2.3611659576$

$\mathrm{H}, 0,1.7792946254,-0.1055350755,-2.088907251$

\section{TS3}

Opt a B3LYP-D3 (BJ)/def2-SVP in gas phase

SCF Done: E (UB3LYP) $=-1471.82232288$ a.u.

Zero-point correction $=0.448900$ Hartree/Particle

Sum of electronic and thermal Free Energies = -1471.433808 a.u. (@ $298.15 \mathrm{~K}$ )

Imaginary Frequency $=-51.6557 \mathrm{~cm}^{-1}$

$\mathrm{SP}$ a B3LYP-D3 (BJ)/def2-TZVP in acetonitrile (SMD model)

$\mathrm{SCF}$ Done: $\mathrm{E}(\mathrm{UB} 3 \mathrm{LYP})=-1473.48588534 \mathrm{a} \cdot \mathrm{u}$.

$0,0,1.424447112,2.620218895,1.6859482308$

$0,0,3.4426078258,-1.3987743055,-1.2929613878$

$0,0,3.1688934077,-0.7407663638,0.844710485$

$0,0,-3.3201048437,-0.7102367163,-2.7460179656$

$0,0,-3.2983542736,-1.5131112008,-0.6474663987$

$0,0,-3.4789241411,0.68460181,1.053252033$

$0,0,-1.8551387991,2.1856244347,0.6014352548$

$\mathrm{N}, 0,1.0882816862,0.7399021157,0.4354952268$

C, $0,1.6470384688,1.9758019136,0.6761981455$

C, $0,2.5197083233,2.4589566707,-0.4670734743$

C, $0,1.834769451,2.2036274597,-1.8140700363$

C, $0,1.602324091,0.6967202996,-2.0757993356$

C, $0,1.4668043515,-0.0886833606,-0.7235705621$

C, $0,0.4650101478,-1.2180175349,-0.6410148789$

$\mathrm{C}, 0,-0.1289534764,-1.1977902777,0.6323474774$

$C, 0,0.2772400324,-0.0004275054,1.3063267985$

$\mathrm{C}, 0,-2.3287828477,0.6040511791,-1.0462431069$

C, $0,-1.5083257892,1.4386317693,-1.9845140189$

$\mathrm{C}, 0,-0.7790640136,0.6978624841,-3.0727695675$

$\mathrm{C}, 0,0.5255877264,0.3972026252,-3.0990839499$

$\mathrm{C}, 0,2.8239353961,-0.8070629428,-0.4421263013$

C, 0,4.2798924769,-1.5510343202,1.2792373997

C, $0,3.8292151077,-2.9723999467,1.5592390037$

$C, 0,-3.0296420402,-0.5835098865,-1.5721113342$

$C, 0,-4.0090221807,-2.6612489254,-1.0975673225$

C, $0,-2.6298001623,1.1171366204,0.3052606617$

$\mathrm{C}, 0,-2.0695243153,2.8214293953,1.8593026907$

$\mathrm{H}, 0,2.7131438578,3.5262961277,-0.298333135$

$\mathrm{H}, 0,3.4919691166,1.9358697538,-0.4194570515$

$\mathrm{H}, 0,0.883735225,2.7532052788,-1.8310364678$

$\mathrm{H}, 0,2.4394531629,2.6160183211,-2.6352948619$

$\mathrm{H}, \mathrm{0}, 2.5297179185,0.3034928905,-2.5084047566$

$\mathrm{H}, 0,0.4013798715,-1.9985288433,-1.3950736799$

$\mathrm{H}, \mathrm{O},-0.8258694296,2.0295895258,-1.3672657984$

$\mathrm{H}, \mathrm{O},-2.1936501234,2.1733661606,-2.4576314033$

$\mathrm{H}, \mathrm{O},-1.3972952692,0.3630373965,-3.9082613973$

$\mathrm{H}, 0,0.8775718669,-0.1593237928,-3.9743391892$

$\mathrm{H}, 0,5.0624752454,-1.5221328682,0.5071829013$

$\mathrm{H}, 0,4.6446859681,-1.0530040773,2.1877579486$

$\mathrm{H}, 0,3.0084054001,-2.9829031751,2.2922881203$

$\mathrm{H}, \mathrm{0}, 3.4852119386,-3.4593809768,0.6349337004$ 
$\mathrm{H}, \mathrm{0}, 4.6681342342,-3.559751662,1.9644836098$

$\mathrm{H}, \mathrm{O},-4.9929929568,-2.3779686473,-1.500667946$

$\mathrm{H}, \mathrm{O},-3.4495912472,-3.1856373732,-1.8876716645$

$\mathrm{H}, \mathrm{O},-4.1299750877,-3.3060395167,-0.2187292837$

$\mathrm{H}, \mathrm{O},-2.9444545929,3.4886126504,1.8004339687$

$\mathrm{H}, \mathrm{0},-2.2446868596,2.0736310946,2.6431382277$

$\mathrm{C}, 0,-0.9827302886,-2.1078224224,1.2967804984$

C, $0,-1.3884135665,-1.8236407861,2.595087717$

C, $0,-0.9548098762,-0.6544265017,3.2456962418$

C, $0,-0.112324371,0.2724279104,2.6098158592$

$\mathrm{H}, 0,-1.3115430998,-3.0153402192,0.7889794112$

$\mathrm{H}, \mathrm{O},-2.0520886525,-2.5146934858,3.1188799237$

$\mathrm{H}, \mathrm{0},-1.2823504466,-0.4576181257,4.2689495241$

$\mathrm{H}, 0,0.2278665618,1.1789718013,3.1028110383$

$\mathrm{H}, \mathrm{O},-1.1559602211,3.3902241762,2.0673531776$

\section{$2 a$}

Opt a B3LYP-D3 (BJ)/def2-SVP in gas phase

SCF Done: $E($ RB3LYP $)=-1471.91680779$ a.u.

Zero-point correction $=0.454425$ Hartree/Particle

Sum of electronic and thermal Free Energies = -1471.520928 a.u. (@ $298.15 \mathrm{~K}$ )

SP a B3LYP-D3 (BJ)/def2-TZVP in acetonitrile (SMD model)

$\mathrm{SCF}$ Done: $\mathrm{E}(\mathrm{RB} 3 \mathrm{LYP})=-1473.57790384 \mathrm{a} \cdot \mathrm{u}$.

$0,0,-0.8706363176,6.4375947686,9.6784927434$

$0,0,-0.5449119227,5.226927247,6.0219538707$

$0,0,0.857377457,3.6297872154,5.2825704015$

$0,0,3.5764496693,2.2210981629,6.77923701$

$0,0,5.1722458118,2.4109901851,8.347160449$

$0,0,5.7730038844,5.781419849,8.4042916852$

$0,0,5.455157151,4.9298312067,6.3497640332$

$\mathrm{N}, 0,0.749441352,5.5215094269,8.3667282731$

$\mathrm{C}, 0,1.4357556685,6.7027739103,8.0203949347$

C, $0,1.132107852,8.0277815576,8.3438653383$

C, $0,1.9519583671,9.0311451197,7.8136772292$

$\mathrm{C}, 0,3.0334893316,8.7271363824,6.9814128955$

$\mathrm{C}, 0,3.3337646891,7.3924147859,6.680864291$

$\mathrm{C}, 0,2.5415387162,6.3810013046,7.2151462595$

C, $0,2.6817978436,4.8740108561,7.1082773245$

$\mathrm{C}, 0,3.8458707983,4.3341202184,7.9887395657$

$\mathrm{C}, 0,3.5865352446,4.4588981299,9.5015073489$

$\mathrm{C}, 0,2.7050637402,3.3736277298,10.0513624843$

C, $0,1.6621275789,2.7775469511,9.4634100363$

C, $0,0.9660458533,3.056644223,8.1519838024$

C, $0,1.2325002207,4.4294167828,7.5014730074$

C, $0,-0.5557461156,2.967325562,8.3916548982$

$\mathrm{C}, 0,-1.0176917329,4.0802765734,9.3326057554$

C, $0,-0.4016251955,5.4595371001,9.126761067$

C, $0,0.4000159645,4.4993851821,6.1898341551$

$C, 0,0.2076375094,3.6103695845,3.9967775629$

C, 0,0.7471915979,4.7058876498,3.0957289025

$\mathrm{C}, 0,4.1454518346,2.8730625808,7.6165482556$

C, $0,5.5504121537,1.0536633503,8.1196955281$

$\mathrm{C}, 0,5.1277553998,5.11220477,7.6434142567$

$\mathrm{C}, 0,6.6527933246,5.5700242479,5.9071689131$

$\mathrm{H}, 0,0.2761937329,8.2501554164,8.974803164$ 
$\mathrm{H}, 0,1.7277139821,10.0747070445,8.047714503$

$\mathrm{H}, 0,3.646004128,9.5294823845,6.5646063746$

$\mathrm{H}, \mathrm{O}, 4.1715444445,7.1490471359,6.0268351031$

$\mathrm{H}, 0,2.8869534511,4.5416669495,6.0864605391$

$\mathrm{H}, 0,4.5543751967,4.4276816643,10.0159847065$

$\mathrm{H}, 0,3.175032413,5.4606952272,9.7077159462$

$\mathrm{H}, 0,2.987455693,3.0035318822,11.0423711624$

$\mathrm{H}, 0,1.1915693759,1.9586907096,10.0204339045$

$\mathrm{H}, \mathrm{0}, 1.2638593102,2.2916342918,7.4199813844$

$\mathrm{H}, \mathrm{O},-1.093772239,3.028998395,7.4345550826$

$\mathrm{H}, 0,-0.8048651157,1.9846981485,8.8211853853$

$\mathrm{H}, \mathrm{0},-2.1071763106,4.2229016016,9.3000934426$

$\mathrm{H}, \mathrm{O},-0.7755133651,3.8207528039,10.3771352573$

$\mathrm{H}, 0,0.4215937772,2.6107351613,3.5943530225$

$\mathrm{H}, 0,0.5204290392,5.6975080135,3.5135433549$

$\mathrm{H}, 0,0.2806784156,4.6372875116,2.1003003014$

$\mathrm{H}, 0,1.8371973343,4.6106777779,2.9738263472$

$\mathrm{H}, \mathrm{O},-0.8766336715,3.7191640698,4.1465989694$

$\mathrm{H}, 0,5.8279341594,0.8987548843,7.066617107$

$\mathrm{H}, 0,4.7182501474,0.3777107261,8.3670727625$

$\mathrm{H}, 0,6.4070423521,0.8610397778,8.7763207464$

$\mathrm{H}, 0,6.7693279115,5.302604027,4.8503444547$

$\mathrm{H}, 0,7.5157795874,5.2201227508,6.4924856954$

$\mathrm{H}, 0,6.5731485205,6.6613390309,6.0221590046$

\section{TS4a}

Opt a B3LYP-D3 (BJ)/def2-SVP in gas phase

SCF Done: $\mathrm{E}(\mathrm{UB} 3 \mathrm{LYP})=-1471.81322829 \mathrm{a} \cdot \mathrm{u}$.

Zero-point correction $=0.448462$ Hartree/Particle

Sum of electronic and thermal Free Energies = -1471.426330 a.u. (@ $298.15 \mathrm{~K}$ )

Imaginary Frequency $=-147.7196 \mathrm{~cm}^{-1}$

SP a B3LYP-D3 (BJ)/def2-TZVP in acetonitrile (SMD model)

SCF Done: $\mathrm{E}(\mathrm{UB} 3 \mathrm{LYP})=-1473.47805246 \mathrm{a} \cdot \mathrm{u}$.

$0,0,3.0767047117,-0.825523149,-2.2179088438$

$\mathrm{N}, 0,2.2050166639,-0.7000599447,-0.1133593133$

C, 0,2.2647213903,0.6897992694,0.0906944316

$\mathrm{C}, 0,2.9581102196,1.6567780542,-0.6249258932$

$\mathrm{C}, 0,2.8241533078,2.9933671423,-0.2113627965$

C, $0,2.0170180702,3.3473817468,0.8820990166$

C, $0,1.3140245992,2.3757057773,1.5928810882$

C, $0,1.4288155255,1.0266204338,1.2007525795$

$C, 0,0.8120839651,-0.154889564,1.6771936526$

$\mathrm{C}, 0,1.404270083,-1.3401216429,0.9448766891$

C, $0,2.5453223511,-1.3741594143,-1.2669716359$

C, $0,2.1885660602,-2.8494276943,-1.240081534$

$\mathrm{C}, 0,0.7523339692,-3.1520368327,-0.7576407484$

$\mathrm{C}, 0,0.252406427,-2.1340258245,0.3065107922$

C, $0,-0.6673691634,-1.0641921544,-0.2156910558$

C, $0,-2.0498509377,-1.370770335,-0.5585564186$

C, $0,-2.4751577985,-1.3020105593,-2.0773574174$

$\mathrm{H}, 0,3.5695261406,1.3741050527,-1.4783049657$

$\mathrm{H}, \mathrm{O}, 3.3616384659,3.7721337053,-0.7570997571$

$\mathrm{H}, 0,1.9381940642,4.3964297962,1.1761580476$

$\mathrm{H}, 0,0.6783614242,2.6475208583,2.4383416949$

$\mathrm{H}, 0,2.3682632085,-3.2390947496,-2.2499519683$ 
$\mathrm{H}, 0,2.9189678252,-3.3280919816,-0.5684965762$ $\mathrm{H}, 0,0.7216227636,-4.1720321538,-0.3460889987$ $\mathrm{H}, 0,0.0667261319,-3.1315179583,-1.6154018299$ $\mathrm{H}, \mathrm{O},-0.2574954551,-2.6729327247,1.1187917641$ $\mathrm{H}, 0,-0.21600653,-0.2135113364,-0.7311324502$ $\mathrm{H}, \mathrm{O},-2.5054730133,-2.2346303119,-0.0656960402$ $\mathrm{C}, 0,-3.0067671222,-0.3337277683,-1.0545294586$ $\mathrm{H}, 0,0.2003713787,-0.2715814301,2.5684648251$ $\mathrm{C}, 0,2.3004020891,-2.1841003131,1.8652718069$ $0,0,3.485102413,-2.350972779,1.7281189433$ $0,0,1.5669300926,-2.7103105681,2.8582557886$ $\mathrm{C}, 0,2.262841626,-3.4903209111,3.8518371516$ $\mathrm{C}, 0,2.8945142383,-2.6015861252,4.9072262499$ $\mathrm{H}, 0,3.0215118018,-4.1066992176,3.347823008$ $\mathrm{H}, 0,1.4908612235,-4.144257722,4.2805549431$ $\mathrm{H}, 0,3.3568816866,-3.2220960579,5.6909918175$ $\mathrm{H}, 0,3.6774531278,-1.9694437092,4.4639453121$ $\mathrm{H}, 0,2.1387309457,-1.9551027169,5.3791292823$ C, $0,-3.3992060967,-2.3890258431,-2.5197178061$ $0,0,-4.1215904617,-3.0106136204,-1.7744399227$ $0,0,-3.3726564492,-2.5673639877,-3.8479512373$ $\mathrm{C}, 0,-1.4160620536,-0.7872404938,-3.0147230291$ $0,0,-1.2025033897,0.3801497107,-3.2328707043$ $0,0,-0.6719441504,-1.7859426378,-3.5106032753$ $\mathrm{H}, 0,-2.6401975512,0.6901385137,-1.1563910008$ $\mathrm{H}, \mathrm{O},-4.0556660342,-0.4486880208,-0.7750757023$ $\mathrm{C}, 0,0.3997390774,-1.4114139403,-4.3873256133$ $\mathrm{H}, \mathrm{0}, \mathrm{0} .7646892904,-2.3463383419,-4.8293885723$ $\mathrm{H}, \mathrm{O}, 0.0352830488,-0.7310299575,-5.1694365027$ $\mathrm{H}, 0,1.2079737066,-0.9223267375,-3.8241142549$ $\mathrm{C}, 0,-4.2357258923,-3.579415843,-4.3655939233$ $\mathrm{H}, 0,-5.2866061173,-3.3523977919,-4.1318221158$ $\mathrm{H}, 0,-4.0775766472,-3.5880891358,-5.4505562175$ $\mathrm{H}, 0,-3.9868189808,-4.5603096991,-3.9337791553$

\section{TS4b}

Opt a B3LYP-D3 (BJ)/def2-SVP in gas phase

SCF Done: $\mathrm{E}(\mathrm{UB} 3 \mathrm{LYP})=-1471.81093121 \mathrm{a} \cdot \mathrm{u}$.

Zero-point correction $=0.447697$ Hartree/Particle

Sum of electronic and thermal Free Energies = -1471.426441 a.u. (@ $298.15 \mathrm{~K}$ ) Imaginary Frequency $=-257.0557 \mathrm{~cm}^{-1}$

SP @ B3LYP-D3 (BJ)/def2-TZVP in acetonitrile (SMD model)

SCF Done: $\mathrm{E}(\mathrm{UB} 3 \mathrm{LYP})=-1473.47626311 \mathrm{a} \cdot \mathrm{u}$.

$0,0,5.1080301009,-0.3256181657,-0.6678613108$

$\mathrm{N}, 0,2.9916957955,-0.456511607,0.1820764324$

$\mathrm{C}, 0,2.7497480038,0.8810950509,0.5171741745$

$\mathrm{C}, 0,3.5863162565,1.9857316803,0.4198683206$

$\mathrm{C}, 0,3.0643417999,3.2326488032,0.8059683476$

$\mathrm{C}, 0,1.7468588207,3.3658974131,1.2792284946$

C, $0,0.9078901596,2.2577633368,1.3735998592$

C, $0,1.401824467,0.9924160253,0.989335054$

$\mathrm{C}, 0,0.8038699972,-0.2835670814,0.9597151146$

$\mathrm{C}, 0,1.8711976571,-1.3059089949,0.6086087028$

C, $0,4.1192935403,-0.9860780546,-0.4156911648$

C, $0,3.9446814655,-2.4477399181,-0.7910096818$ 
C, $0,2.5725932746,-2.6715781699,-1.4444234832$

C, $0,1.3895025375,-2.2279238287,-0.546560797$

C, $0,0.3325853502,-1.4504463772,-1.2876066634$

C, $0,-1.0928216622,-1.6570558665,-1.0368945361$

$C, 0,-2.1610315527,-1.6400881583,-2.1006045963$

$\mathrm{H}, 0,4.6044483079,1.8689860183,0.0558967809$

$\mathrm{H}, 0,3.7031745633,4.1164208226,0.7405592018$

$\mathrm{H}, 0,1.3802109964,4.3507914343,1.578252674$

$\mathrm{H}, \mathrm{O},-0.1179696594,2.3510011407,1.7342936475$

$\mathrm{H}, 0,4.7646777767,-2.7044379102,-1.4742437118$

$\mathrm{H}, 0,4.0621724101,-3.0652205222,0.114283905$

$\mathrm{H}, 0,2.4532755621,-3.7284348983,-1.7260680047$

$\mathrm{H}, 0,2.5423194599,-2.0960487607,-2.3831357783$

$\mathrm{H}, 0,0.9243456913,-3.1077466971,-0.078697087$

$\mathrm{H}, 0,0.6401916037,-0.7575458072,-2.0735596646$

$\mathrm{H}, \mathrm{O},-1.3188713039,-2.2938685895,-0.1766327923$

$0,0,-3.0718381375,-1.0540184869,1.0043220757$

$0,0,-4.1163734277,0.4146372375,-0.3367462818$

$0,0,-1.0630114804,0.9445154062,-2.7501589109$

$0,0,-1.6335940178,1.7877444172,-0.7471167442$

$C, 0,-2.0979593612,-0.4867974851,-1.1262596593$

$\mathrm{C}, 0,-3.1162363023,-0.4185859508,-0.0232048948$

$\mathrm{C}, 0,-5.1219801311,0.5988191877,0.6594639769$

$C, 0,-1.5599657649,0.8069189451,-1.6574616852$

C, $0,-1.1190508415,3.0578965089,-1.156368381$

$\mathrm{H}, \mathrm{O},-5.8423005609,1.3101978269,0.2383232899$

$\mathrm{H}, 0,-5.6168931773,-0.3550081646,0.8956587853$

$\mathrm{H}, 0,-4.6812881043,1.001693263,1.5837459128$

$\mathrm{H}, \mathrm{O},-1.6243491469,3.3992209355,-2.0715091621$

$\mathrm{H}, \mathrm{0},-1.3139431597,3.7463104715,-0.3266001937$

$\mathrm{H}, \mathrm{O},-0.0383644583,2.9916511929,-1.3426995928$

$\mathrm{H}, 0,-1.8602573132,-1.3900999006,-3.1205797639$

$\mathrm{H}, \mathrm{O},-2.9943953389,-2.3395330579,-1.9993993185$

$\mathrm{H}, \mathrm{O},-0.1732636717,-0.5625574963,1.3458410806$

$\mathrm{C}, 0,2.2660533725,-2.0816451582,1.8837387635$

$0,0,3.3471319411,-2.0518934516,2.4140760824$

$0,0,1.215214193,-2.7837500302,2.3335330506$

C, $0,1.3751393791,-3.4943826099,3.5777827273$

C, $0,1.1576486647,-2.5748727768,4.7651221544$

$\mathrm{H}, 0,2.3791491013,-3.9428195135,3.6014951583$

$\mathrm{H}, 0,0.6213501089,-4.2928871016,3.5395354041$

$\mathrm{H}, \mathrm{0}, 1.2138875301,-3.1523527623,5.7013613949$

$\mathrm{H}, 0,1.9309640762,-1.7936549423,4.7971583581$

$\mathrm{H}, 0,0.1681898889,-2.0955122822,4.712071066$

\section{Pro-2+2a}

Opt a B3LYP-D3 (BJ)/def2-SVP in gas phase

SCF Done: $\mathrm{E}(\mathrm{RB} 3 \mathrm{LYP})=-1471.88203950 \mathrm{a} \cdot \mathrm{u}$.

Zero-point correction $=0.453284$ Hartree/Particle

Sum of electronic and thermal Free Energies = -1471.491244 a.u. (@ $298.15 \mathrm{~K}$ ) $\mathrm{SP}$ a B3LYP-D3 (BJ)/def2-TZVP in acetonitrile (SMD model)

SCF Done: $\mathrm{E}(\mathrm{RB} 3 \mathrm{LYP})=-1473.54808041 \mathrm{a} \cdot \mathrm{u}$.

$0,0,-2.9978505701,-2.2551902644,-2.2091546384$

$\mathrm{N}, 0,-2.4053785179,-0.7165596186,-0.6272680569$

C, $0,-2.8171452834,-1.3687011297,0.5580715825$ 
$C, 0,-3.7030822467,-2.4343453136,0.6997469676$ C, $0,-3.9196036219,-2.9279862413,1.9949172473$ C, $0,-3.2746864607,-2.3738014831,3.1044963043$ C, $0,-2.3781215261,-1.3056902711,2.941861062$ $\mathrm{C}, 0,-2.1482766537,-0.8091737857,1.6650276274$ C, $0,-1.2245974141,0.2707141116,1.1747545003$ $\mathrm{C}, 0,-1.6521418098,0.4912399239,-0.2995079563$ C, $0,-2.353141019,-1.2754620539,-1.8911798302$ C, $0,-1.3134108467,-0.6091608034,-2.7816285622$ $\mathrm{C}, 0,0.0167630001,-0.479142371,-2.0115634431$ $\mathrm{C}, 0,-0.170482339,0.3897222921,-0.7605262168$ $\mathrm{C}, 0,0.1976906763,-0.1453642331,0.6567094492$ C, $0,1.3673707913,0.4989163602,1.34194467$ C, 0,2.7928929738,0.0346714647,1.0390905895 $\mathrm{H}, \mathrm{O},-4.1925887558,-2.8584043865,-0.1744071223$ $\mathrm{H}, 0,-4.6126848638,-3.7610895527,2.1348530033$ $\mathrm{H}, \mathrm{0},-3.4676208326,-2.7738219972,4.102349952$ $\mathrm{H}, \mathrm{0},-1.8633092073,-0.8753197749,3.8044533173$ $\mathrm{H}, \mathrm{O},-1.2118287859,-1.2333343943,-3.6787806662$ $\mathrm{H}, \mathrm{O},-1.6769001775,0.3827361542,-3.0956062483$ $\mathrm{H}, 0,0.8007944428,-0.0642750542,-2.6613352098$ $\mathrm{H}, 0,0.3506217233,-1.4872385849,-1.7186158118$ $\mathrm{H}, 0,0.2427229136,1.3984708423,-0.9025362605$ $\mathrm{H}, 0,0.303162444,-1.2394314456,0.6549010669$ $\mathrm{H}, 0,1.3062915173,1.5881560678,1.4352786377$ C, $0,2.1506006041,-0.1997829764,2.3987460459$ $\mathrm{H}, \mathrm{O},-1.2051895864,1.1845814676,1.7799494724$ C, $0,-2.4030362763,1.7497486219,-0.6918946273$ $0,0,-3.2975369282,1.7993797119,-1.4988656371$ $0,0,-1.8929591511,2.8170972819,-0.0559375676$ $\mathrm{C}, 0,-2.4717323953,4.1037212147,-0.3523888403$ $\mathrm{C}, 0,-3.7250050656,4.3482076999,0.4676222133$ $\mathrm{H}, \mathrm{O},-2.6890421087,4.1521448718,-1.4295218178$ $\mathrm{H}, 0,-1.6803060717,4.826147812,-0.1087878573$ $\mathrm{H}, \mathrm{O},-4.1056256243,5.3637899554,0.2755016424$ $\mathrm{H}, 0,-4.5119931005,3.6297563022,0.1964831103$ $\mathrm{H}, \mathrm{O},-3.5136675338,4.256678584,1.5441678784$ $\mathrm{C}, 0,3.8356149679,1.1106396314,0.9902895771$ $0,0,3.6610547677,2.2476194661,1.3606586539$ $0,0,5.0137896978,0.6454884718,0.5520118725$ C, $0,2.9399692922,-1.1542025581,0.1338030643$ $0,0,2.7433453862,-2.3017272341,0.4522340722$ $0,0,3.2416097096,-0.7742982966,-1.1188494741$ $\mathrm{H}, 0,1.912234772,-1.2440341757,2.6140163319$ $\mathrm{H}, 0,2.5420497861,0.3887277962,3.2307258135$ C, $0,3.4468912624,-1.825853594,-2.0621153048$ $\mathrm{H}, 0,3.6833514276,-1.3384720852,-3.0154246991$ $\mathrm{H}, 0,4.2792732397,-2.4713964024,-1.7449817595$ $\mathrm{H}, 0,2.5429941093,-2.4442707453,-2.1613906038$ C, $0,6.082525736,1.5898012259,0.4934468357$ $\mathrm{H}, 0,6.2940224259,2.001119876,1.4917496571$ $\mathrm{H}, 0,6.952616007,1.0418797831,0.1126707462$ $\mathrm{H}, 0,5.8288201298,2.4221025867,-0.1798979528$

\section{Pro $-2+2 b$}

Opt a B3LYP-D3 (BJ)/def2-SVP in gas phase 
SCF Done: $\mathrm{E}(\mathrm{RB} 3 \mathrm{LYP})=-1471.88386768 \mathrm{a} \cdot \mathrm{u}$. Zero-point correction $=0.453525$ Hartree/Particle Sum of electronic and thermal Free Energies = -1471.491882 a.u. (@ $298.15 \mathrm{~K}$ ) $\mathrm{SP}$ a B3LYP-D3 (BJ)/def2-TZVP in acetonitrile (SMD model) $\mathrm{SCF}$ Done: $\mathrm{E}(\mathrm{RB} 3 \mathrm{LYP})=-1473.54858777 \mathrm{a} \cdot \mathrm{u}$.

$0,0,4.9684507215,-0.3841931929,-0.9546507768$

$\mathrm{N}, 0,3.1173499495,-0.5800359399,0.3693035002$

$\mathrm{C}, 0,2.8322506515,0.7766847106,0.6457969635$ $\mathrm{C}, 0,3.6650435428,1.8858758971,0.5172546173$ C, $0,3.1262886079,3.1401883955,0.8422852343$ C, $0,1.8060590598,3.2761231266,1.2819098984$ C, $0,0.9760757049,2.1484771164,1.3923121844$ C, $0,1.4922833568,0.9022282161,1.0643469502$ $\mathrm{C}, 0,0.8511781136,-0.4555934549,1.0222188719$ $\mathrm{C}, 0,2.0460665813,-1.4322967017,0.8817798549$ $C, 0,4.0458850533,-1.0562062918,-0.5377362813$ $\mathrm{C}, 0,3.7159120773,-2.4588138923,-1.0284778415$ C, $0,2.232057383,-2.5438438794,-1.4446111664$ C, $0,1.3156374598,-2.1833116455,-0.2656765713$ $\mathrm{C}, 0,0.3232183641,-0.9839315891,-0.3575017385$ C, $0,-1.1402716319,-1.3006896841,-0.4432195696$ C, $0,-1.8437165909,-1.474465475,-1.7443029544$ $\mathrm{H}, 0,4.689374718,1.7641010353,0.1714906823$ $\mathrm{H}, 0,3.7596890901,4.0264112628,0.7560940264$ $\mathrm{H}, 0,1.4175371509,4.2641842549,1.5392501664$ $\mathrm{H}, 0,-0.0630108045,2.2451345946,1.7134327932$ $\mathrm{H}, \mathrm{O}, 4.3903035478,-2.6731545043,-1.8674663181$ $\mathrm{H}, \mathrm{0}, 3.9311032803,-3.1827983803,-0.2256721191$ $\mathrm{H}, \mathrm{0}, 2.0070858122,-3.5512683584,-1.8255331632$ $\mathrm{H}, \mathrm{0}, 2.058390222,-1.8455413458,-2.2792392826$ $\mathrm{H}, 0,0.8065242312,-3.0669552366,0.1449364563$ $\mathrm{H}, 0,0.6209332317,-0.3070979019,-1.1703471138$ $\mathrm{H}, 0,-1.5338709529,-1.894638264,0.3880664506$ $0,0,-3.8238858658,-1.092010993,0.5826505354$ $0,0,-4.2495752251,0.7700073025,-0.5999297498$ $0,0,-0.9727263214,1.1940035332,-2.4374088611$ $0,0,-1.7563021882,2.0504787798,-0.5155423699$ $\mathrm{C}, 0,-2.1439673809,-0.2401281738,-0.9040561973$ $C, 0,-3.4685651379,-0.2425964491,-0.2003614491$ C, $0,-5.5446076965,0.8404330811,-0.0044521405$ $\mathrm{C}, 0,-1.578788855,1.0605097315,-1.4006996964$ $\mathrm{C}, 0,-1.2561577345,3.3323390057,-0.9047607546$ $\mathrm{H}, 0,-6.0312655097,1.7225767385,-0.4372295832$ $\mathrm{H}, \mathrm{O},-6.1258177698,-0.0670465268,-0.2266075201$ $\mathrm{H}, 0,-5.4647290537,0.9441401093,1.087970103$ $\mathrm{H}, \mathrm{O},-1.7298011668,3.6599819935,-1.8417548845$ $\mathrm{H}, \mathrm{0},-1.5095385717,4.0172016824,-0.0869662314$ $\mathrm{H}, 0,-0.1670155468,3.2952433861,-1.0452006769$ $\mathrm{H}, \mathrm{O},-1.2924725871,-1.2675349746,-2.6644676$ $\mathrm{H}, \mathrm{O},-2.6329522668,-2.2259528345,-1.8108186459$ $\mathrm{H}, 0,0.1721372147,-0.6872335609,1.8510258407$ C, $0,2.5009126146,-2.2576995408,2.070815096$ $0,0,3.6495077671,-2.4906807501,2.3540007073$ $0,0,1.4402677182,-2.7392941809,2.7392657027$ C, $0,1.7031323355,-3.5724589947,3.8859514767$ C, $0,1.9729172611,-2.7381644183,5.1245404891$ 
$\mathrm{H}, 0,2.5567432845,-4.2273141889,3.6572645373$

$\mathrm{H}, 0,0.7965774703,-4.1833107844,3.998353339$

$\mathrm{H}, \mathrm{0}, 2.0992392686,-3.3961979293,5.9987375275$

$\mathrm{H}, 0,2.8935037381,-2.1493866055,5.0023567607$

$\mathrm{H}, 0,1.1350848236,-2.05306153,5.3260892517$

\section{MECP1}

Opt @ UB3LYP-D3 (BJ)/def2-SVP in gas phase

For open-shell singlet state

SCF Done: E (UB3LYP) $=-1471.81267604 \mathrm{a} \cdot \mathrm{u}$.

For triplet state

SCF Done: E (UB3LYP) $=-1471.81267718 \mathrm{a} \cdot \mathrm{u}$.

\begin{tabular}{|c|c|c|c|}
\hline 8 & 3.07423 & -0.33421 & -2.02005 \\
\hline 7 & 2.18879 & -0.36696 & 0.07913 \\
\hline 6 & 2.28002 & 0.99522 & 0.38961 \\
\hline 6 & 2.91343 & 2.02286 & -0.30051 \\
\hline 6 & 2.82486 & 3.31683 & 0.23545 \\
\hline 6 & 2.12151 & 3.57619 & 1.42879 \\
\hline 6 & 1.48556 & 2.5528 & 2.11875 \\
\hline 6 & 1.55497 & 1.23255 & 1.60674 \\
\hline 6 & 1.04281 & 0.01763 & 2.07384 \\
\hline 6 & 1.38175 & -1.06722 & 1.09142 \\
\hline 6 & 2.50747 & -0.9502 & -1.134 \\
\hline 6 & 2.12659 & -2.41433 & -1.26936 \\
\hline 6 & 0.83134 & -2.8622 & -0.55359 \\
\hline 6 & 0.19759 & -1.75055 & 0.33562 \\
\hline 6 & -0.59477 & -0.78311 & -0.47374 \\
\hline 6 & -1.86968 & -1.19643 & -1.04527 \\
\hline 6 & -2.04331 & -1.20052 & -2.61125 \\
\hline 1 & 3.44551 & 1.81446 & -1.22506 \\
\hline 1 & 3.31462 & 4.14131 & -0.28744 \\
\hline 1 & 2.0752 & 4.59791 & 1.81323 \\
\hline 1 & 0.93516 & 2.75127 & 3.04055 \\
\hline 1 & 2.09108 & -2.62036 & -2.34514 \\
\hline 1 & 2.98556 & -2.97245 & -0.86488 \\
\hline 1 & 1.03967 & -3.73737 & 0.08019 \\
\hline 1 & 0.08593 & -3.18023 & -1.29484 \\
\hline 1 & -0.45941 & -2.24967 & 1.0674 \\
\hline 1 & -0.15067 & 0.15762 & -0.80626 \\
\hline 1 & -2.33046 & -2.09046 & -0.61383 \\
\hline 6 & -2.81683 & -0.25923 & -1.72156 \\
\hline 1 & 0.44511 & -0.16147 & 2.96392 \\
\hline 6 & 2.22163 & -2.12763 & 1.83076 \\
\hline 8 & 3.37902 & -2.37571 & 1.61158 \\
\hline 8 & 1.47598 & -2.72893 & 2.76973 \\
\hline 6 & 2.13048 & -3.70446 & 3.60754 \\
\hline 6 & 2.87596 & -3.0429 & 4.75185 \\
\hline 1 & 2.81267 & -4.30081 & 2.9848 \\
\hline 1 & 1.31665 & -4.3479 & 3.96928 \\
\hline 1 & 3.31436 & -3.81348 & 5.40606 \\
\hline 1 & 3.69164 & -2.41228 & 4.36912 \\
\hline 1 & 2.19749 & -2.42072 & 5.35545 \\
\hline 6 & -2.79211 & -2.36728 & -3.16848 \\
\hline 8 & -3.51616 & -3.08754 & -2.51927 \\
\hline 0 & -2.61471 & -2.49363 & -4.49146 \\
\hline
\end{tabular}




$\begin{array}{llll}6 & -0.89915 & -0.6087 & -3.38563 \\ 8 & -0.71711 & 0.57476 & -3.54039 \\ 8 & -0.05711 & -1.55573 & -3.81836 \\ 1 & -2.52786 & 0.79067 & -1.807 \\ 1 & -3.88375 & -0.4621 & -1.61189 \\ 6 & 1.05591 & -1.10381 & -4.60289 \\ 1 & 1.49299 & -2.00751 & -5.04451 \\ 1 & 0.71484 & -0.41556 & -5.38932 \\ 1 & 1.79335 & -0.59732 & -3.96363 \\ 6 & -3.31459 & -3.56382 & -5.12377 \\ 1 & -4.4021 & -3.41653 & -5.0444 \\ 1 & -3.00403 & -3.55175 & -6.17531 \\ 1 & -3.06086 & -4.52729 & -4.65813 \\ -------------------------------------------------------------\end{array}$

\section{MECP2}

Opt a UB3LYP-D3 (BJ)/def2-SVP in gas phase

For open-shell singlet state

SCF Done: E (UB3LYP) $=-1471.81912949$ a.u.

For triplet state

$\mathrm{SCF}$ Done: $\mathrm{E}(\mathrm{UB} 3 \mathrm{LYP})=-1471.81909516 \mathrm{a} \cdot \mathrm{u}$.

\begin{tabular}{|c|c|c|c|}
\hline 8 & 3.107 & 0.80243 & -1.08962 \\
\hline 7 & 1.77191 & -0.45602 & 0.25142 \\
\hline 6 & 1.04435 & 0.57416 & 0.86044 \\
\hline 6 & 1.25056 & 1.94825 & 0.83354 \\
\hline 6 & 0.30331 & 2.76197 & 1.47109 \\
\hline 6 & -0.81748 & 2.21465 & 2.12841 \\
\hline 6 & -1.01321 & 0.84148 & 2.18118 \\
\hline 6 & -0.06922 & -0.00907 & 1.55229 \\
\hline 6 & 0.00439 & -1.40073 & 1.44449 \\
\hline 6 & 1.30795 & -1.76174 & 0.75355 \\
\hline 6 & 2.72198 & -0.29876 & -0.73498 \\
\hline 6 & 3.21418 & -1.55785 & -1.43801 \\
\hline 6 & 2.65797 & -2.90911 & -0.96536 \\
\hline 6 & 1.23985 & -2.77284 & -0.39483 \\
\hline 6 & 0.25423 & -2.36133 & -1.46394 \\
\hline 6 & -1.15504 & -2.10802 & -1.21476 \\
\hline 6 & -2.18068 & -2.0323 & -2.29773 \\
\hline 1 & 2.10863 & 2.36178 & 0.31053 \\
\hline 1 & 0.43759 & 3.84614 & 1.45405 \\
\hline 1 & -1.53521 & 2.88385 & 2.60907 \\
\hline 1 & -1.8768 & 0.40724 & 2.68699 \\
\hline 1 & 2.97004 & -1.37609 & -2.49711 \\
\hline 1 & 4.31303 & -1.53721 & -1.38186 \\
\hline 1 & 3.31595 & -3.33939 & -0.19493 \\
\hline 1 & 2.65512 & -3.62354 & -1.80289 \\
\hline 1 & 0.93239 & -3.74482 & 0.0339 \\
\hline 1 & 0.61055 & -2.28686 & -2.49368 \\
\hline 1 & -1.53514 & -2.41737 & -0.23878 \\
\hline 8 & -3.24645 & -0.88925 & 0.30517 \\
\hline 8 & -3.20284 & 1.03079 & -0.85716 \\
\hline 8 & -0.44269 & -0.09471 & -3.45848 \\
\hline 8 & -0.63942 & 1.31042 & -1.71993 \\
\hline 6 & -1.82069 & -0.73091 & -1.60951 \\
\hline 6 & -2.80549 & -0.22013 & -0.60415 \\
\hline 6 & -4.12234 & 1.60306 & 0.07006 \\
\hline
\end{tabular}




$\begin{array}{cccc}6 & -0.92241 & 0.18943 & -2.3828 \\ 6 & 0.25922 & 2.22377 & -2.35704 \\ 1 & -4.39232 & 2.5848 & -0.33697 \\ 1 & -5.01487 & 0.96989 & 0.18133 \\ 1 & -3.64522 & 1.72046 & 1.05409 \\ 1 & -0.05002 & 2.40483 & -3.39606 \\ 1 & 0.21093 & 3.14593 & -1.7673 \\ 1 & 1.2833 & 1.82519 & -2.33756 \\ 1 & -1.84211 & -2.10521 & -3.33384 \\ 1 & -3.17047 & -2.4379 & -2.0774 \\ 1 & -0.64241 & -2.13643 & 1.91467 \\ 6 & 2.29481 & -2.25196 & 1.84688 \\ 8 & 3.3096 & -1.69136 & 2.16747 \\ 8 & 1.85173 & -3.39966 & 2.38188 \\ 6 & 2.64237 & -3.99095 & 3.43368 \\ 6 & 2.32784 & -3.3658 & 4.77978 \\ 1 & 3.70684 & -3.87499 & 3.18141 \\ 1 & 2.37982 & -5.05773 & 3.4097 \\ 1 & 2.88269 & -3.88865 & 5.57484 \\ 1 & 2.62253 & -2.30659 & 4.7934 \\ 1 & 1.25253 & -3.43893 & 5.00376\end{array}$

\section{MECP3}

Opt \& UB3LYP-D3 (BJ)/def2-SVP in gas phase

For open-shell singlet state

$\mathrm{SCF}$ Done: E (UB3LYP) $=-1471.83118481 \mathrm{a} \cdot \mathrm{u}$.

For triplet state

SCF Done: $\mathrm{E}(\mathrm{UB} 3 \mathrm{LYP})=-1471.83114701 \mathrm{a} \cdot \mathrm{u}$.

$\begin{array}{cccc}8 & 0.28497 & -2.3799 & -1.91447 \\ 8 & 3.64404 & -0.32323 & -1.19884 \\ 8 & 4.00681 & 0.71341 & 0.77245 \\ 7 & 0.94649 & -0.57182 & -0.70901 \\ 6 & 0.10636 & 0.4001 & -1.26899 \\ 6 & -0.80323 & 0.29765 & -2.31338 \\ 6 & -1.56359 & 1.43345 & -2.63179 \\ 6 & -1.40434 & 2.64518 & -1.93599 \\ 6 & -0.4865 & 2.75538 & -0.89694 \\ 6 & 0.28517 & 1.62403 & -0.54182 \\ 6 & 1.25493 & 1.4228 & 0.45097 \\ 6 & -2.71804 & -0.36178 & 1.19686 \\ 6 & -2.08305 & -0.45859 & 2.55317 \\ 6 & -0.60932 & -0.14907 & 2.36044 \\ 6 & 0.28183 & -1.09727 & 2.04831 \\ 6 & 1.71345 & -0.89105 & 1.61277 \\ 6 & 1.78221 & 0.01863 & 0.34685 \\ 6 & 2.35604 & -2.22881 & 1.2137 \\ 6 & 1.68843 & -2.84081 & -0.03532 \\ 6 & 0.93117 & -1.92054 & -0.98843 \\ 6 & 3.24408 & 0.10132 & -0.14634 \\ 6 & 5.39863 & 0.9067 & 0.44727 \\ 6 & 5.59817 & 2.1339 & -0.42248 \\ 1 & -0.91375 & -0.63797 & -2.85288 \\ 1 & -2.30019 & 1.3684 & -3.43473 \\ 1 & -2.01331 & 3.50699 & -2.21771 \\ 1 & -0.35734 & 3.69392 & -0.35365\end{array}$




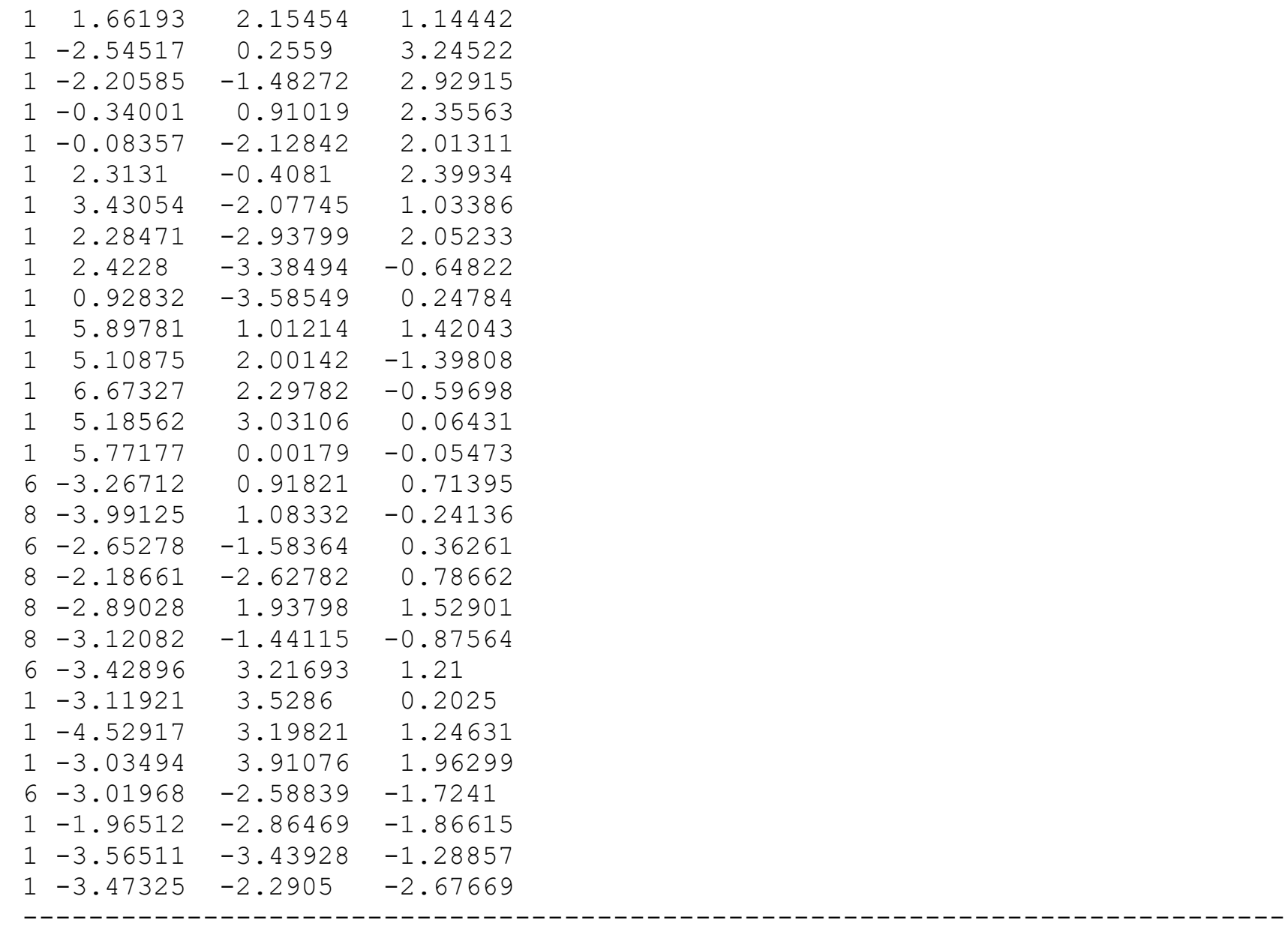

\section{MECP4}

Opt a UB3LYP-D3 (BJ)/def2-SVP in gas phase

For open-shell singlet state

SCF Done: $\mathrm{E}$ (UB3LYP) $=-1471.82357018 \mathrm{a} \cdot \mathrm{u}$.

For triplet state

$\mathrm{SCF}$ Done: $\mathrm{E}(\mathrm{UB} 3 \mathrm{LYP})=-1471.82357834 \mathrm{a} \cdot \mathrm{u}$.

$\begin{array}{cccc}-1.35118 & 2.53269 & 1.64818 \\ 8 & 3.75697 & -1.2749 & -1.21098 \\ 8 & 3.27315 & -0.73814 & 0.92377 \\ 8 & -3.22227 & -0.76116 & -2.61911 \\ 8 & -3.39804 & -1.40692 & -0.47524 \\ 8 & -3.55598 & 0.8845 & 1.08395 \\ 8 & -1.83553 & 2.2745 & 0.6407 \\ 7 & 1.13749 & 0.63676 & 0.39005 \\ 6 & 1.65034 & 1.88787 & 0.65919 \\ 6 & 2.58179 & 2.38114 & -0.43451 \\ 6 & 1.98693 & 2.11088 & -1.82336 \\ 6 & 1.73753 & 0.60243 & -2.08584 \\ 6 & 1.63307 & -0.17802 & -0.73355 \\ 6 & 0.71647 & -1.37758 & -0.68892 \\ 6 & -0.0189 & -1.34409 & 0.50648 \\ 6 & 0.27048 & -0.11711 & 1.19051 \\ 6 & -2.29108 & 0.62475 & -0.9389 \\ 6 & -1.3499 & 1.36882 & -1.83953 \\ 6 & -0.69155 & 0.61118 & -2.95829\end{array}$




$\begin{array}{cccc}6 & 0.61052 & 0.31273 & -3.05811 \\ 6 & 3.02836 & -0.7769 & -0.38746 \\ 6 & 4.41473 & -1.47105 & 1.41103 \\ 6 & 4.07331 & -2.93887 & 1.58935 \\ 6 & -3.01249 & -0.56208 & -1.43768 \\ 6 & -4.11481 & -2.56014 & -0.90001 \\ 6 & -2.64681 & 1.22968 & 0.36166 \\ 6 & -2.06092 & 2.96815 & 1.86407 \\ 1 & 2.74697 & 3.4525 & -0.26094 \\ 1 & 3.55935 & 1.87844 & -0.32539 \\ 1 & 1.04837 & 2.67478 & -1.91511 \\ 1 & 2.6542 & 2.50504 & -2.60396 \\ 1 & 2.64463 & 0.19399 & -2.54716 \\ 1 & 0.75333 & -2.17212 & -1.43007 \\ 1 & -0.61225 & 1.84714 & -1.18841 \\ 1 & -1.9258 & 2.21209 & -2.27919 \\ 1 & -1.3558 & 0.28365 & -3.76004 \\ 1 & 0.91482 & -0.23814 & -3.9545 \\ 1 & 5.24939 & -1.33586 & 0.7078 \\ 1 & 4.66149 & -0.99091 & 2.36773 \\ 1 & 3.19851 & -3.05757 & 2.2467 \\ 1 & 3.85338 & -3.4066 & 0.61842 \\ 1 & 4.92506 & -3.4712 & 2.04105 \\ 1 & -5.05868 & -2.27507 & -1.389 \\ 1 & -3.52045 & -3.15122 & -1.61395 \\ 1 & -4.31692 & -3.14182 & 0.00746 \\ 1 & -2.93531 & 3.63198 & 1.77168 \\ 1 & -2.24424 & 2.25811 & 2.68141 \\ 6 & -0.90602 & -2.27099 & 1.10179 \\ 6 & -1.46466 & -1.96959 & 2.33772 \\ 6 & -1.1462 & -0.77 & 2.99968 \\ 6 & -0.26648 & 0.16962 & 2.43698 \\ 1 & -1.14415 & -3.20369 & 0.58728 \\ 1 & -2.16011 & -2.67095 & 2.80362 \\ 1 & -1.59485 & -0.55997 & 3.97281 \\ 1 & -0.00867 & 1.09669 & 2.94157 \\ 1 & -1.14761 & 3.54378 & 2.05302 \\ -1 & -1-19 & -1919 & \\ 1 & \end{array}$




\section{Data of X-Ray Crystalloraphic Analyses}

Figure S1. X-Ray crystal structure of 2a (The crystal was obtained by slow evaporation of the solution of DCM and PE) (CCDC 2092621). Displacement ellipsoids are drawn at the $30 \%$ probability level.
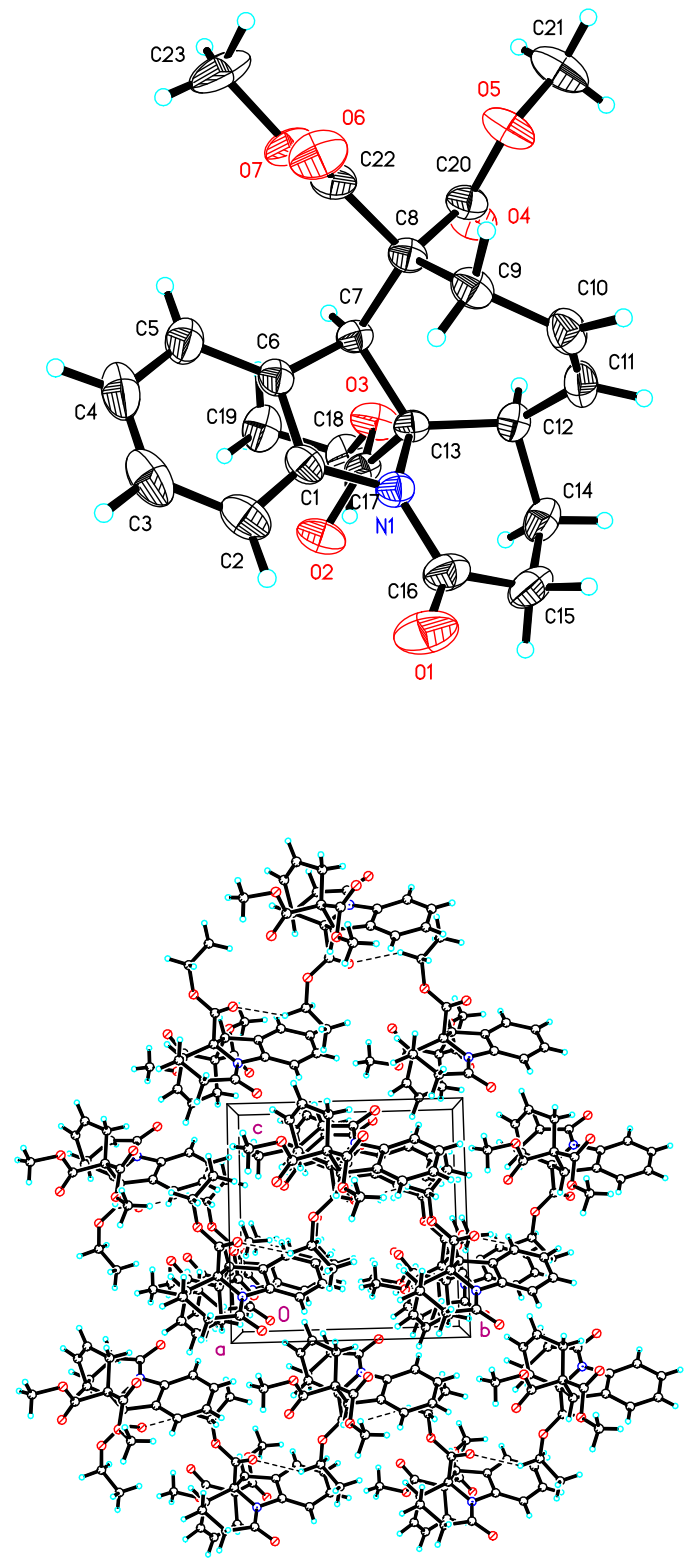

Crystal data and structure refinement for mo_d8v20412_0m.

Identification code

mo_d8v20412_0m 
Empirical formula

Formula weight

Temperature

Wavelength

Crystal system

Space group

Unit cell dimensions

Volume

Z

Density (calculated)

Absorption coefficient

$\mathrm{F}(000)$

Crystal size

Theta range for data collection

Index ranges

Reflections collected

Independent reflections

Completeness to theta $=25.242^{\circ}$

Absorption correction

Max. and min. transmission

Refinement method

Data / restraints / parameters

Goodness-of-fit on $\mathrm{F}^{2}$

Final $\mathrm{R}$ indices [I $>2 \operatorname{sigma}(\mathrm{I})]$

$\mathrm{R}$ indices (all data)

Absolute structure parameter

Extinction coefficient

Largest diff. peak and hole
$\mathrm{C} 23 \mathrm{H} 25 \mathrm{~N} \mathrm{O} 7$

427.44

293(2) K

$0.71073 \AA$

Monoclinic

P 21

$\mathrm{a}=10.2924(8) \AA \quad \alpha=90^{\circ}$.

$\mathrm{b}=10.2096(6) \AA \quad \beta=97.697(2)^{\circ}$.

$\mathrm{c}=10.3749(5) \AA \quad \gamma=90^{\circ}$.

$1080.39(12) \AA^{3}$

2

$1.314 \mathrm{Mg} / \mathrm{m}^{3}$

$0.098 \mathrm{~mm}^{-1}$

452

$0.100 \times 0.080 \times 0.050 \mathrm{~mm}^{3}$

2.823 to $25.499^{\circ}$.

$-12<=\mathrm{h}<=12,-12<=\mathrm{k}<=12,-12<=1<=11$

11452

$4008[\mathrm{R}($ int $)=0.0338]$

$99.5 \%$

Semi-empirical from equivalents

0.7456 and 0.6963

Full-matrix least-squares on $\mathrm{F}^{2}$

$4008 / 41 / 303$

1.078

$\mathrm{R} 1=0.0409, \mathrm{wR} 2=0.0834$

$\mathrm{R} 1=0.0575, \mathrm{wR} 2=0.0935$

$0.4(5)$

$0.060(7)$

0.148 and -0.123 e. $\AA^{-3}$ 
Figure S2. X-Ray crystal structure of 4a (The crystal was obtained by slow evaporation of the solution of DCM and PE) (CCDC 2092622). Displacement ellipsoids are drawn at the $30 \%$ probability level.
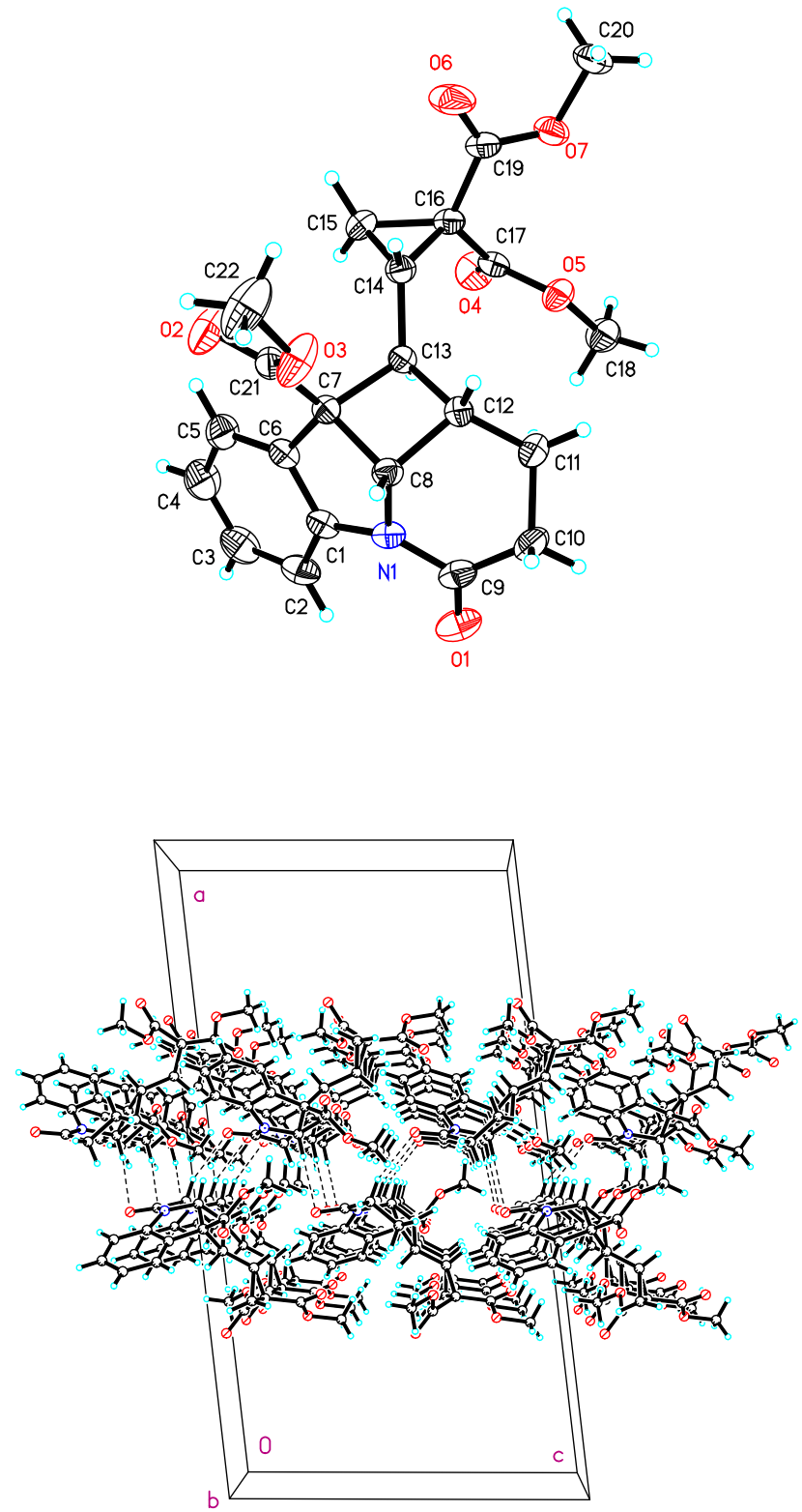

Crystal data and structure refinement for mo_d8v20736_0m.

Identification code

Empirical formula

Formula weight

Temperature mo_d8v20736_0m

$\mathrm{C} 22 \mathrm{H} 23 \mathrm{~N} \mathrm{O} 7$

413.41

293(2) K 
Wavelength

Crystal system

Space group

Unit cell dimensions

Volume

Z

Density (calculated)

Absorption coefficient

$\mathrm{F}(000)$

Crystal size

Theta range for data collection

Index ranges

Reflections collected

Independent reflections

Completeness to theta $=25.242^{\circ}$

Absorption correction

Max. and min. transmission

Refinement method

Data / restraints / parameters

Goodness-of-fit on $\mathrm{F}^{2}$

Final $\mathrm{R}$ indices [I $>2 \operatorname{sigma}(\mathrm{I})]$

$\mathrm{R}$ indices (all data)

Absolute structure parameter

Extinction coefficient

Largest diff. peak and hole

\section{$0.71073 \AA$}

Monoclinic

C c

$\mathrm{a}=28.8461(9) \AA$

$\alpha=90^{\circ}$.

$\mathrm{b}=9.0356(3) \AA$

$\beta=96.6640(10)^{\circ}$.

$\mathrm{c}=15.6373(5) \AA$

$\gamma=90^{\circ}$.

4048.2(2) $\AA^{3}$

8

$1.357 \mathrm{Mg} / \mathrm{m}^{3}$

$0.102 \mathrm{~mm}^{-1}$

1744

$0.170 \times 0.140 \times 0.110 \mathrm{~mm}^{3}$

2.623 to $26.000^{\circ}$.

$-35<=\mathrm{h}<=33,-11<=\mathrm{k}<=11,-18<=\mathrm{l}<=19$

30034

$7414[\mathrm{R}(\mathrm{int})=0.0388]$

$99.6 \%$

Semi-empirical from equivalents

0.7456 and 0.5796

Full-matrix least-squares on $\mathrm{F}^{2}$

7414 / 2 / 547

1.050

$\mathrm{R} 1=0.0393, \mathrm{wR} 2=0.0864$

$\mathrm{R} 1=0.0513, \mathrm{wR} 2=0.0947$

$-1.1(4)$

$\mathrm{n} / \mathrm{a}$

0.125 and -0.142 e. $\AA^{-3}$ 
Figure S3. X-Ray crystal structure of $\mathbf{6 f}$ (The crystal was obtained by slow evaporation of the solution of DCM and PE) (CCDC 2092623). Displacement ellipsoids are drawn at the $30 \%$ probability level.
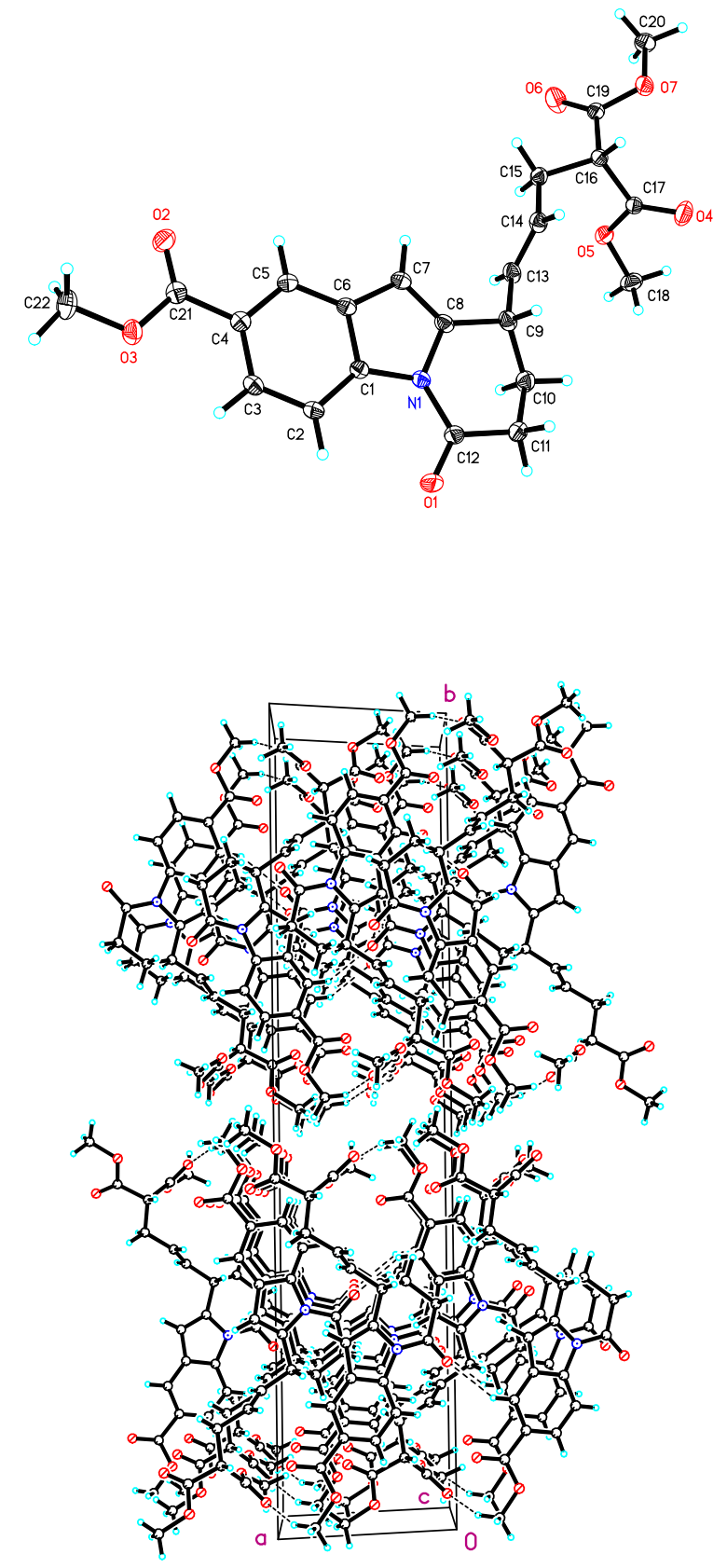

Crystal data and structure refinement for mo_d8v21063_0m.

Identification code

mo_d8v21063_0m

Empirical formula

C22 H23 N O7 
Formula weight

Temperature

Wavelength

Crystal system

Space group

Unit cell dimensions

Volume

Z

Density (calculated)

Absorption coefficient

$\mathrm{F}(000)$

Crystal size

Theta range for data collection

Index ranges

Reflections collected

Independent reflections

Completeness to theta $=25.242^{\circ}$

Absorption correction

Max. and min. transmission

Refinement method

Data / restraints / parameters

Goodness-of-fit on $\mathrm{F}^{2}$

Final $\mathrm{R}$ indices [I $>2 \operatorname{sigma}(\mathrm{I})]$

$\mathrm{R}$ indices (all data)

Extinction coefficient

Largest diff. peak and hole
413.41

193(2) K

$0.71073 \AA$

Monoclinic

P 21/n

$$
\begin{array}{ll}
\mathrm{a}=7.8498(2) \AA & \alpha=90^{\circ} . \\
\mathrm{b}=34.4434(10) \AA & \beta=108.6570(10)^{\circ} . \\
\mathrm{c}=7.8857(2) \AA & \gamma=90^{\circ} .
\end{array}
$$

$2020.05(9) \AA^{3}$

4

$1.359 \mathrm{Mg} / \mathrm{m}^{3}$

$0.102 \mathrm{~mm}^{-1}$

872

$0.180 \times 0.150 \times 0.110 \mathrm{~mm}^{3}$

2.365 to $25.998^{\circ}$.

$-9<=\mathrm{h}<=9,-42<=\mathrm{k}<=42,-9<=\mathrm{l}<=9$

20255

$3979[\mathrm{R}(\mathrm{int})=0.0352]$

$99.7 \%$

Semi-empirical from equivalents

0.7456 and 0.6915

Full-matrix least-squares on $\mathrm{F}^{2}$

3979 / 0 / 275

1.033

$\mathrm{R} 1=0.0407, \mathrm{wR} 2=0.0933$

$\mathrm{R} 1=0.0525, \mathrm{wR} 2=0.1013$

$0.013(2)$

0.298 and -0.242 e. $\AA^{-3}$ 
Figure S4. X-Ray crystal structure of 8a (The crystal was obtained by slow evaporation of the solution of DCM and PE) (CCDC 2092624). Displacement ellipsoids are drawn at the $30 \%$ probability level.
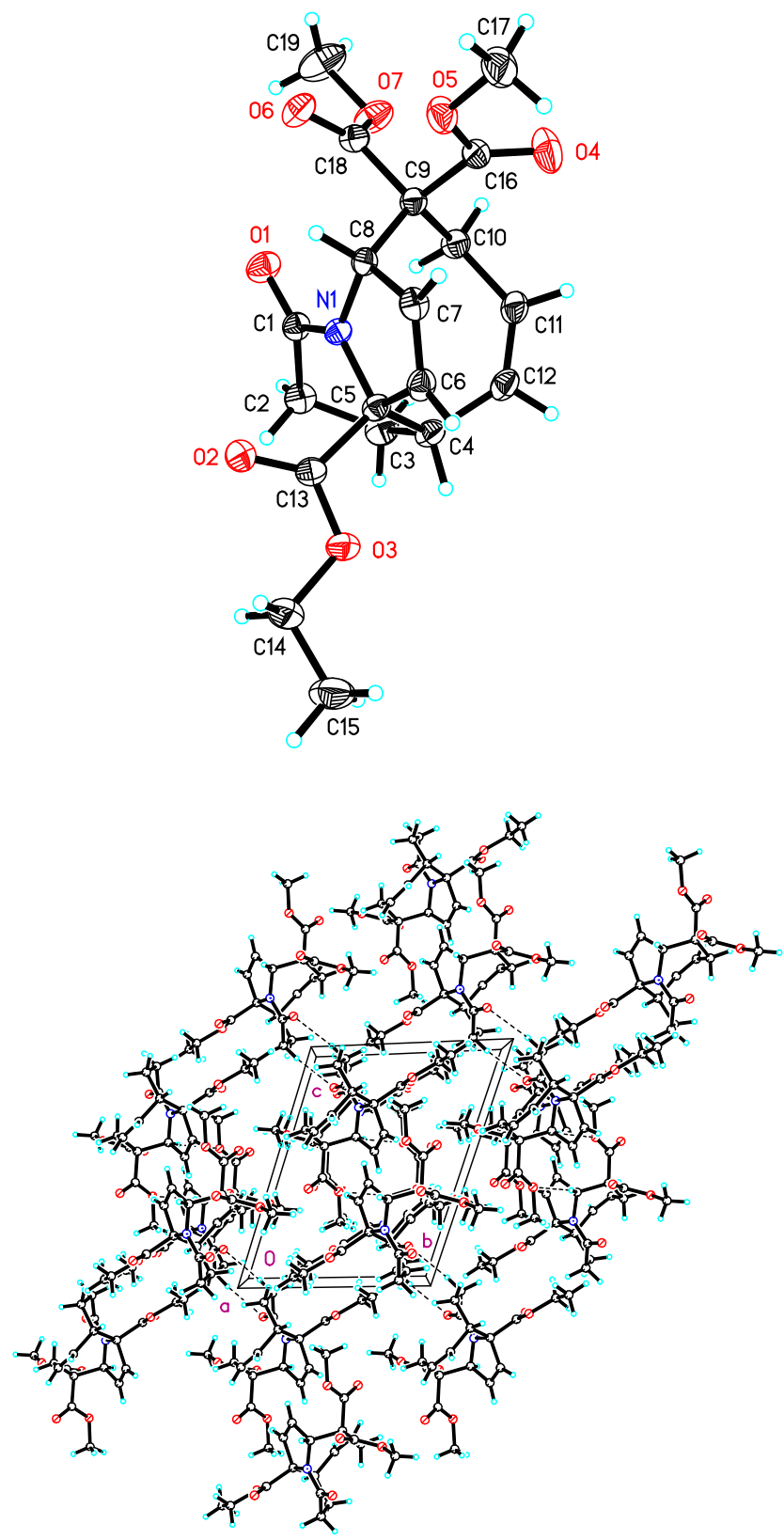

Crystal data and structure refinement for mo_d8v20730_0m.

Identification code

Empirical formula

Formula weight

Temperature

Wavelength mo_d8v20730_0m

C19 H23 N O7

377.38

293(2) K

$0.71073 \AA$ 
Crystal system

Space group

Unit cell dimensions

Volume

Z

Density (calculated)

Absorption coefficient

$\mathrm{F}(000)$

Crystal size

Theta range for data collection

Index ranges

Reflections collected

Independent reflections

Completeness to theta $=25.242^{\circ}$

Absorption correction

Max. and min. transmission

Refinement method

Data / restraints / parameters

Goodness-of-fit on $\mathrm{F}^{2}$

Final $\mathrm{R}$ indices [I $>2 \operatorname{sigma}(\mathrm{I})]$

$\mathrm{R}$ indices (all data)

Extinction coefficient

Largest diff. peak and hole
Triclinic

$\mathrm{P}-1$

$$
\begin{array}{ll}
\mathrm{a}=8.9395(8) \AA & \alpha=67.843(3)^{\circ} . \\
\mathrm{b}=9.4269(10) \AA & \beta=72.587(3)^{\circ} . \\
\mathrm{c}=12.4377(13) \AA & \gamma=75.996(3)^{\circ} .
\end{array}
$$

916.32(16) $\AA^{3}$

2

$1.368 \mathrm{Mg} / \mathrm{m}^{3}$

$0.105 \mathrm{~mm}^{-1}$

400

$0.170 \times 0.120 \times 0.060 \mathrm{~mm}^{3}$

2.358 to $25.998^{\circ}$

$-11<=\mathrm{h}<=11,-11<=\mathrm{k}<=11,-15<=\mathrm{l}<=15$

22555

$3583[\mathrm{R}($ int $)=0.0503]$

$99.6 \%$

Semi-empirical from equivalents

0.7456 and 0.6187

Full-matrix least-squares on $\mathrm{F}^{2}$

$3583 / 0 / 248$

1.042

$\mathrm{R} 1=0.0448, \mathrm{wR} 2=0.1141$

$\mathrm{R} 1=0.0577, \mathrm{wR} 2=0.1264$

$0.052(7)$

0.250 and -0.184 e. $\AA^{-3}$ 


\section{References}

1. Feng, Y.; Yang, C.; Deng, Q.; Xiong, R.; Zhang, X.; Xiong, Yan. Synthesis of Antitricyclic Morpholine Derivatives through Iodine(III)-Mediated Intramolecular Umpolung Cycloaddition of Olefins. J. Org. Chem. 2020, 85, 4500.

2. Umehara, A.; Ueda, H.; Tokuyama, H. Condensation of Carboxylic Acids with Non-Nucleophilic N-Heterocycles and Anilides Using Boc 2 O. J. Org. Chem. 2016, 81,11444 .

3. Handbook of Photochemistry, $3^{\text {rd }}$ ed, Montalti, M.; Credi, A.; Prodi, L.; Gandolfi, M. T., Ed.; CRC, Taylor \& Francis Group, Boca Raton, FL, 2006.

4. Frisch, M. J.; Trucks, G. W.; Schlegel, H. B.; Scuseria, G. E.; Robb, M. A.; Cheeseman, J. R.; Scalmani, G.; Barone, V.; Pe-Tersson, G. A.; Nakatsuji, H.; Li, X.; Caricato, M.; Marenich, A. V.; Bloino, J.; Janesko, B. G.; Gomperts, R.; Mennucci, B.; Hratchian, H. P.; Ortiz, J. V.; Iz-Maylov, A. F.; Sonnenberg, J. L.; Williams-Young, D.; Ding, F.; Lipparini, F.; Egidi, F.; Goings, J.; Peng, B.; Petrone, A.; Henderson, T.; Ranasinghe, D.; Zakrzewski, V. G.; Gao, J.; Rega, N.; Zheng, G.; Liang, W.; Hada, M.; Ehara, M.; Toyota, K.; Fukuda, R.; Hasegawa, J.; Ishida, M.; Nakajima, T.; Honda, Y.; Kitao, O.; Nakai, H.; Vreven, T.; Throssell, K.; Montgomery, J. A., Jr.; Peralta, J. E.; Ogliaro, F.; Bearpark, M. J.; Heyd, J. J.; Brothers, E. N.; Kudin, K. N.; Staroverov, V. N.; Keith, T. A.; Kobayashi, R.; Normand, J.; Raghavachari, K.; Rendell, A. P.; Burant, J. C.; Iyengar, S. S.; Tomasi, J.; Cossi, M.; Millam, J. M.; Klene, M.; Adamo, C.; Cammi, R.; Ochterski, J. W.; Martin, R. L.; Morokuma, K.; Farkas, O.; Foresman, J. B.; Fox, D. J. Gaussian 16, revision A.03; Gaussian, Inc.: Wallingford, CT, 2016.

5. (a) Becke, A. D. J. Chem. Phys. 1993, 98, 5648. (b) Lee, C.; Yang, W.; Parr, R. G. Phys. Rev. B: Condens. Matter Mater. Phys. 1988, 37, 785. (c) Stephens, P. J.; Devlin, F. J.; Chabalowski, C. F.; Frisch, M. J. J. Phys. Chem. 1994, 98, 11623.

6. Grimme, S.; Antony, J.; Ehrlich, S.; Krieg, H. J. Chem. Phys. 2010, 132, 154104.

7. Grimme, S.; Ehrlich, S.; Goerigk, L. J. Comp. Chem. 2011, 32, 1456.

8. (a) Weigend, F.; Ahlrichs, R.; Phys. Chem. Chem. Phys. 2005, 7, 3297. (b) Weigend, F. Phys. Chem. Chem. Phys. 2006, 8, 1057.

9. Marenich, A. V.; Cramer, C. J.; Truhlar, D. G. J. Phys. Chem. B 2009, 113, 6378. 
10. Harvey, J. N.; Aschi, M.; Schwarz, H.; Koch, W. Theor. Chem. Acc. 1998, 99, 95.

11. Lu, T. sobMECP program; http://sobereva.com/286.

12. Humphrey, W.; Dalke, A.; Schulten, K. J. Molec. Graphics 1996, 14, 33. 
8. Copies of NMR Spectra

${ }^{1} \mathrm{H}$ NMR Spectrum of $\mathbf{1 a}$

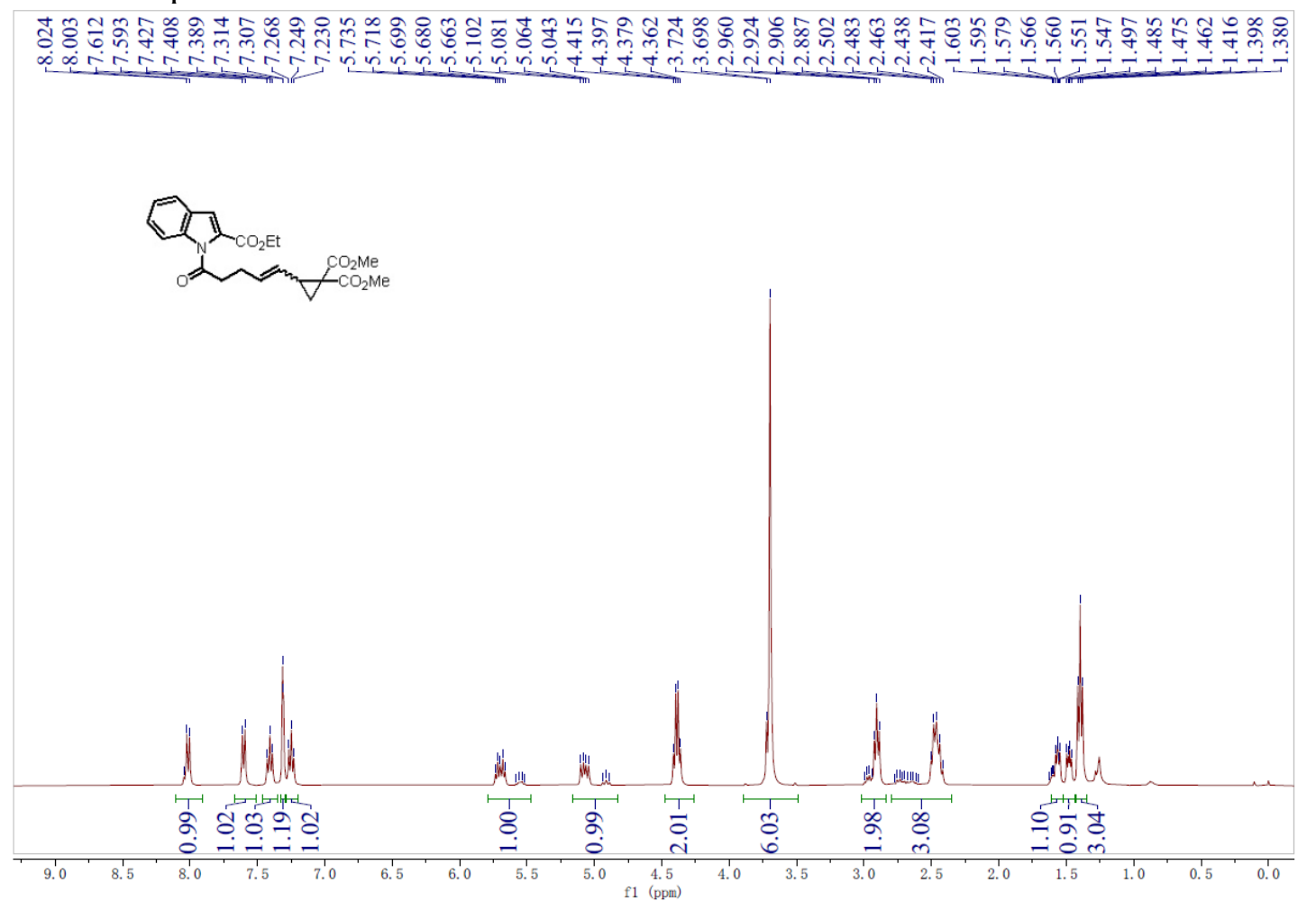

${ }^{13} \mathrm{C}$ NMR Spectrum of $\mathbf{1 a}$

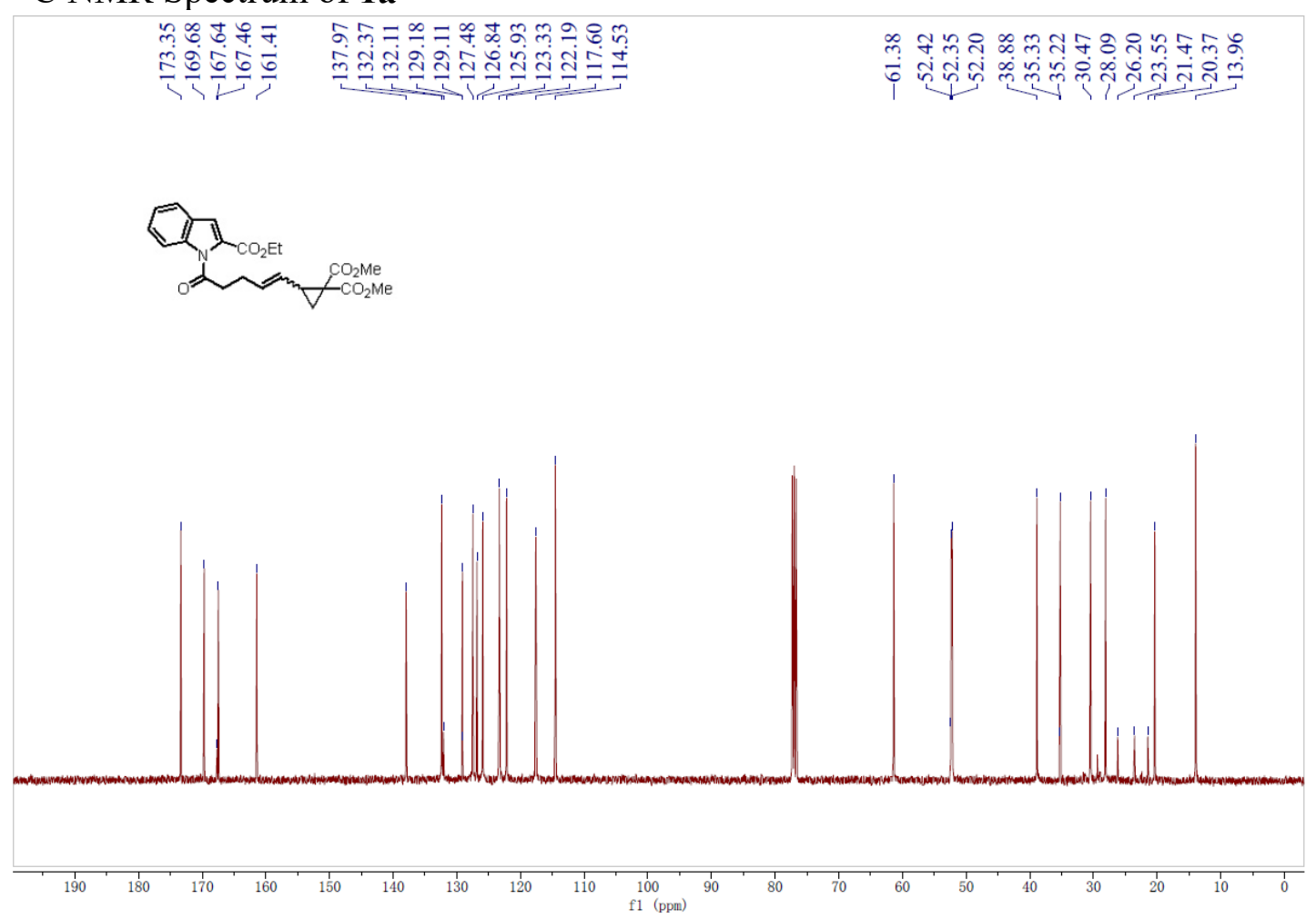

S106 
${ }^{1} \mathrm{H}$ NMR Spectrum of $\mathbf{1 b}$

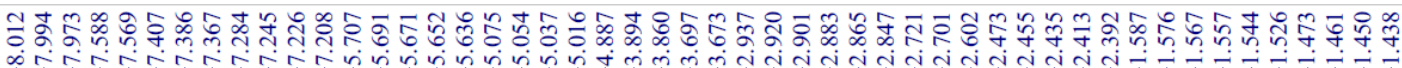

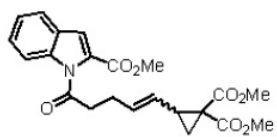

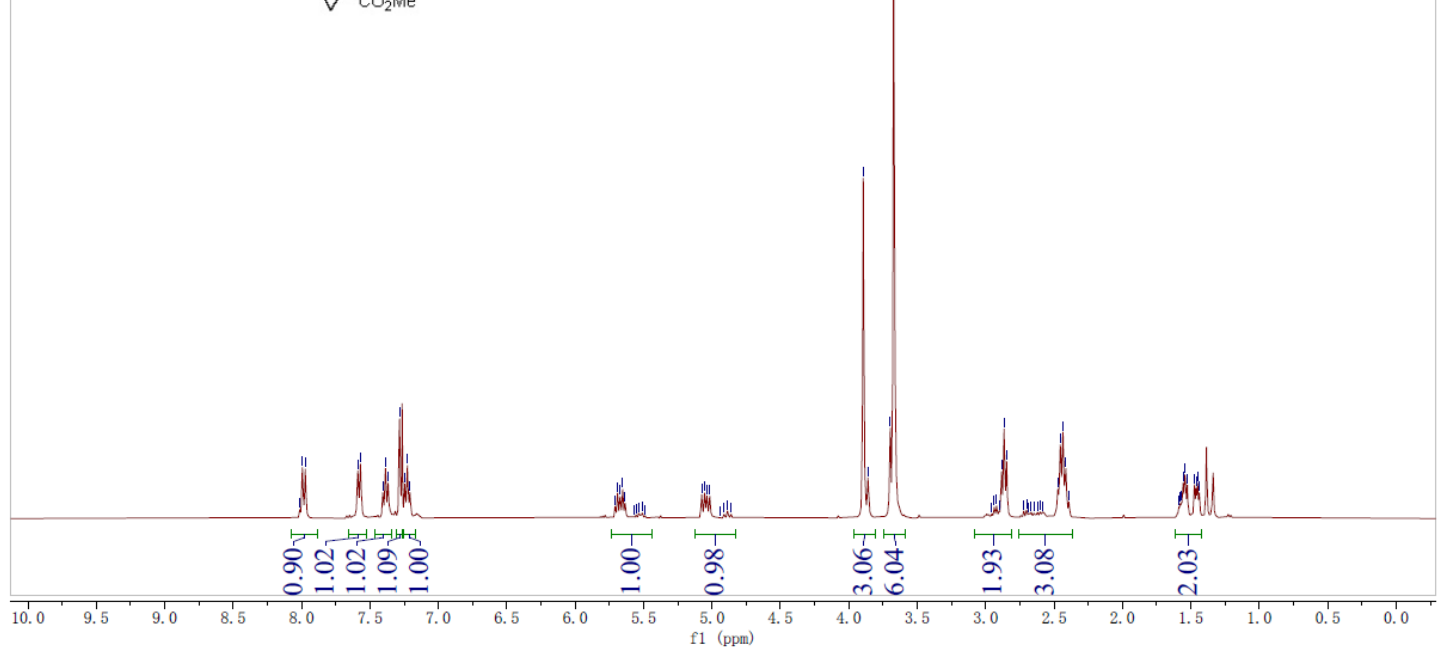

${ }^{13} \mathrm{C}$ NMR Spectrum of $\mathbf{1 b}$

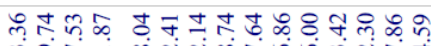

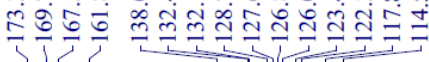

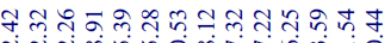

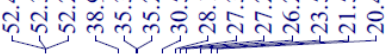
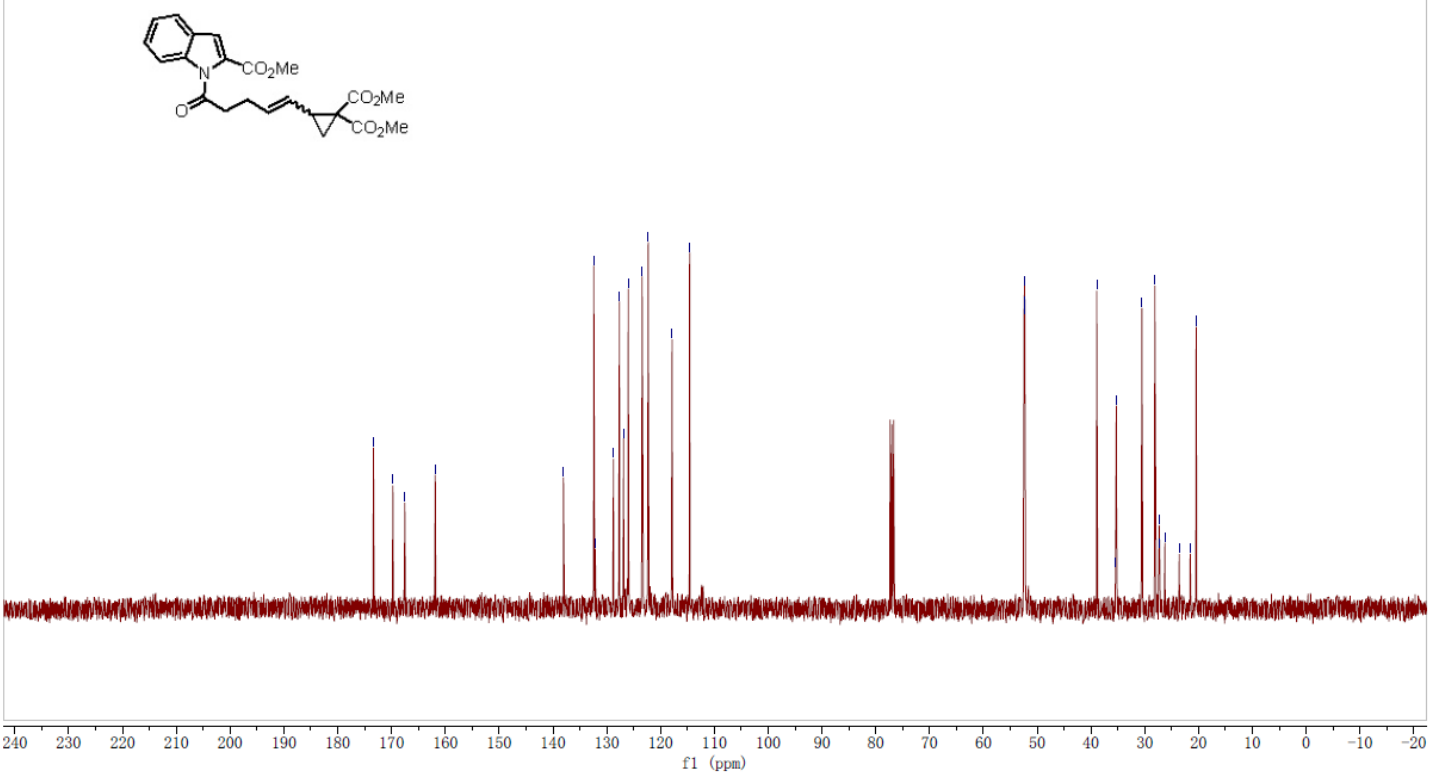


\section{${ }^{1} \mathrm{H}$ NMR Spectrum of $\mathbf{1 c}$}

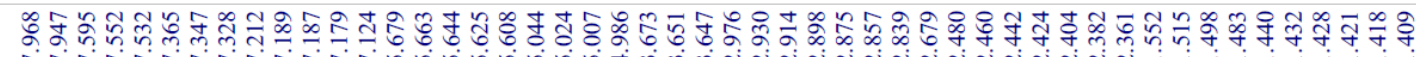

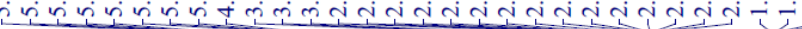
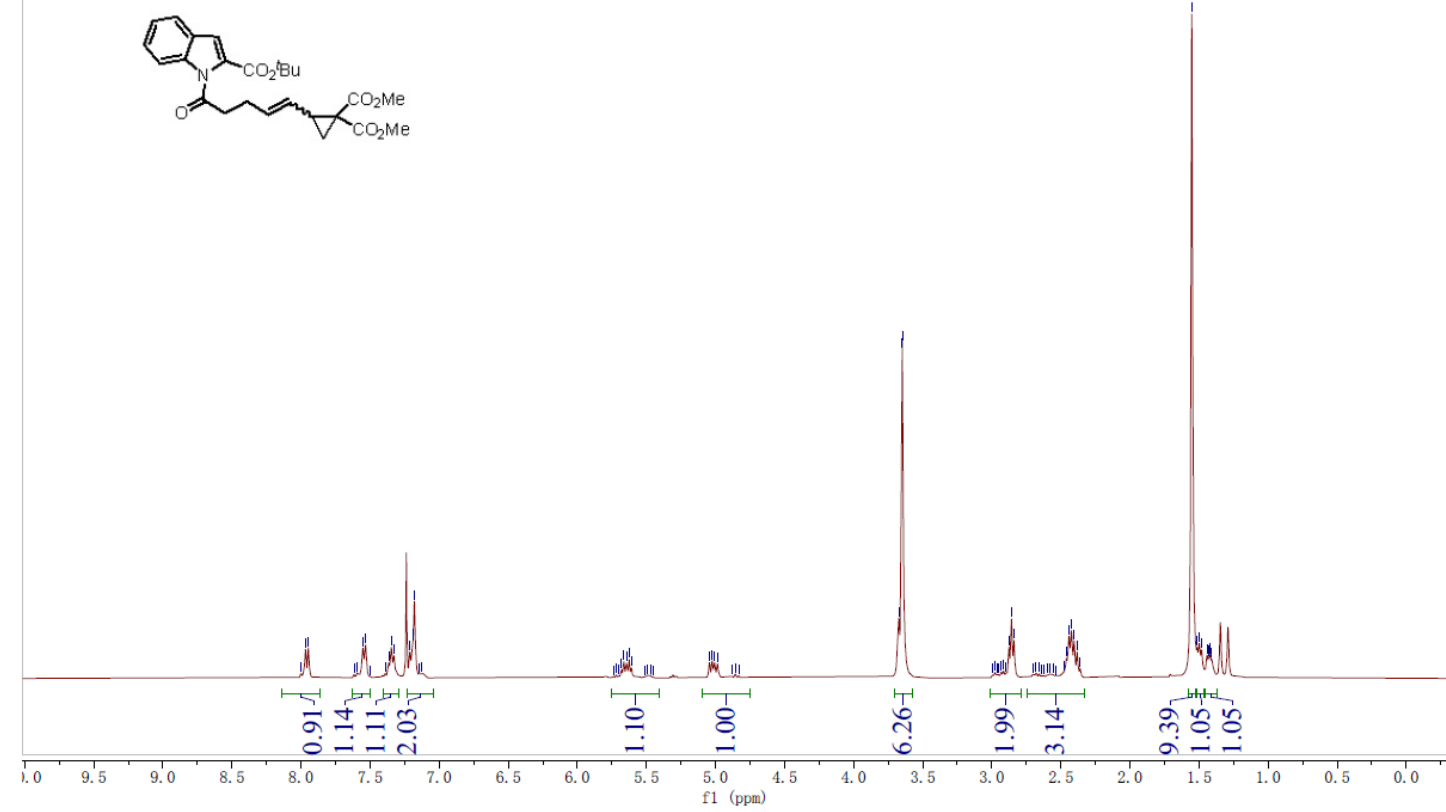

\section{${ }^{13} \mathrm{C}$ NMR Spectrum of $\mathbf{1 c}$}

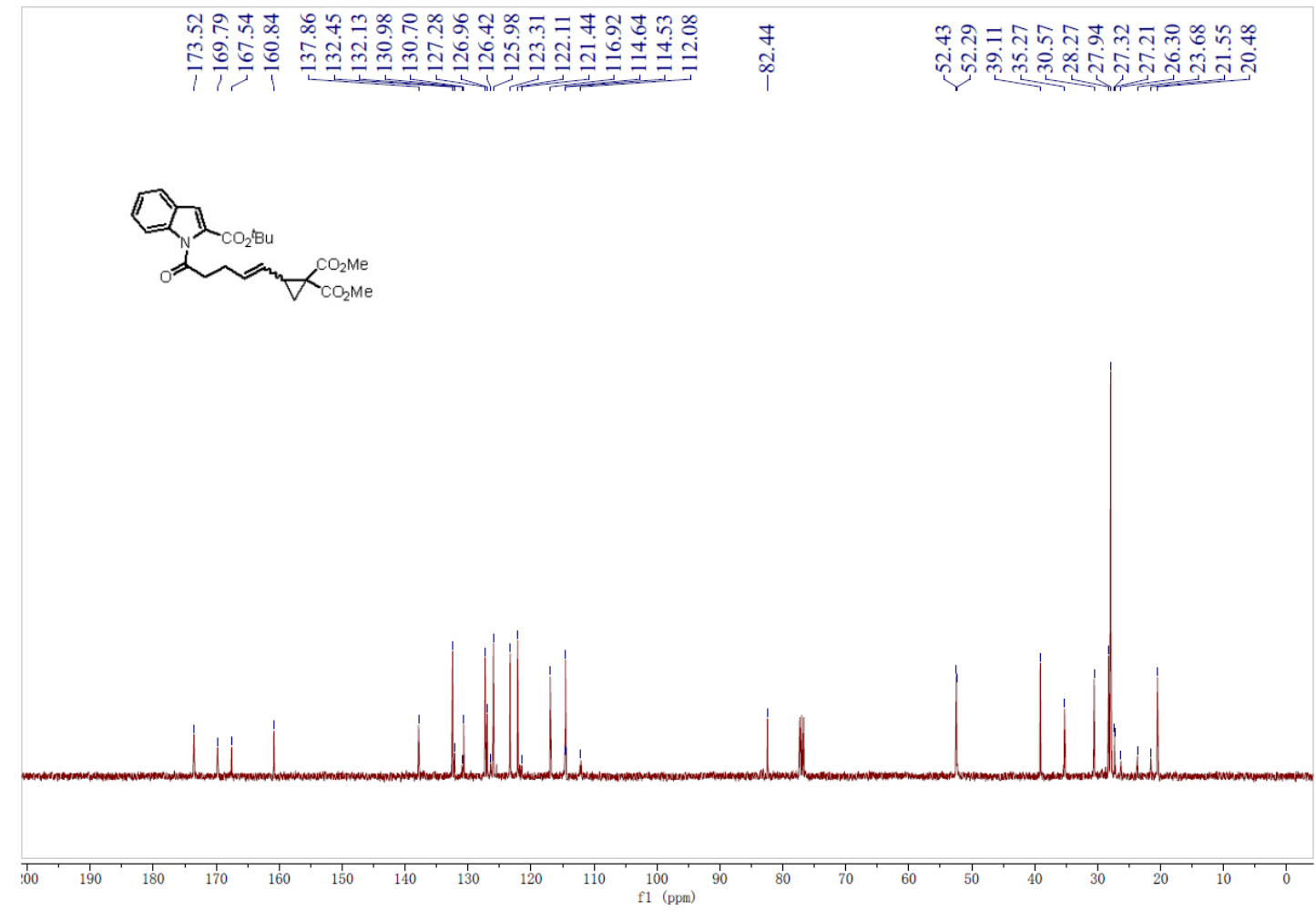


${ }^{1} \mathrm{H}$ NMR Spectrum of $\mathbf{1 d}$

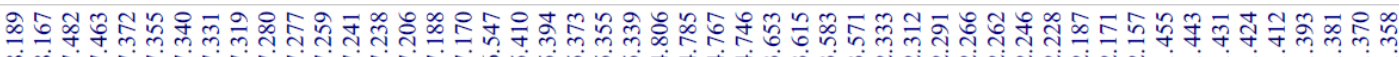

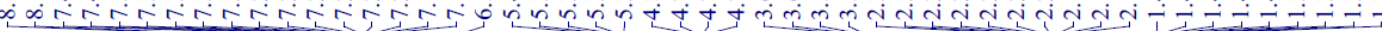
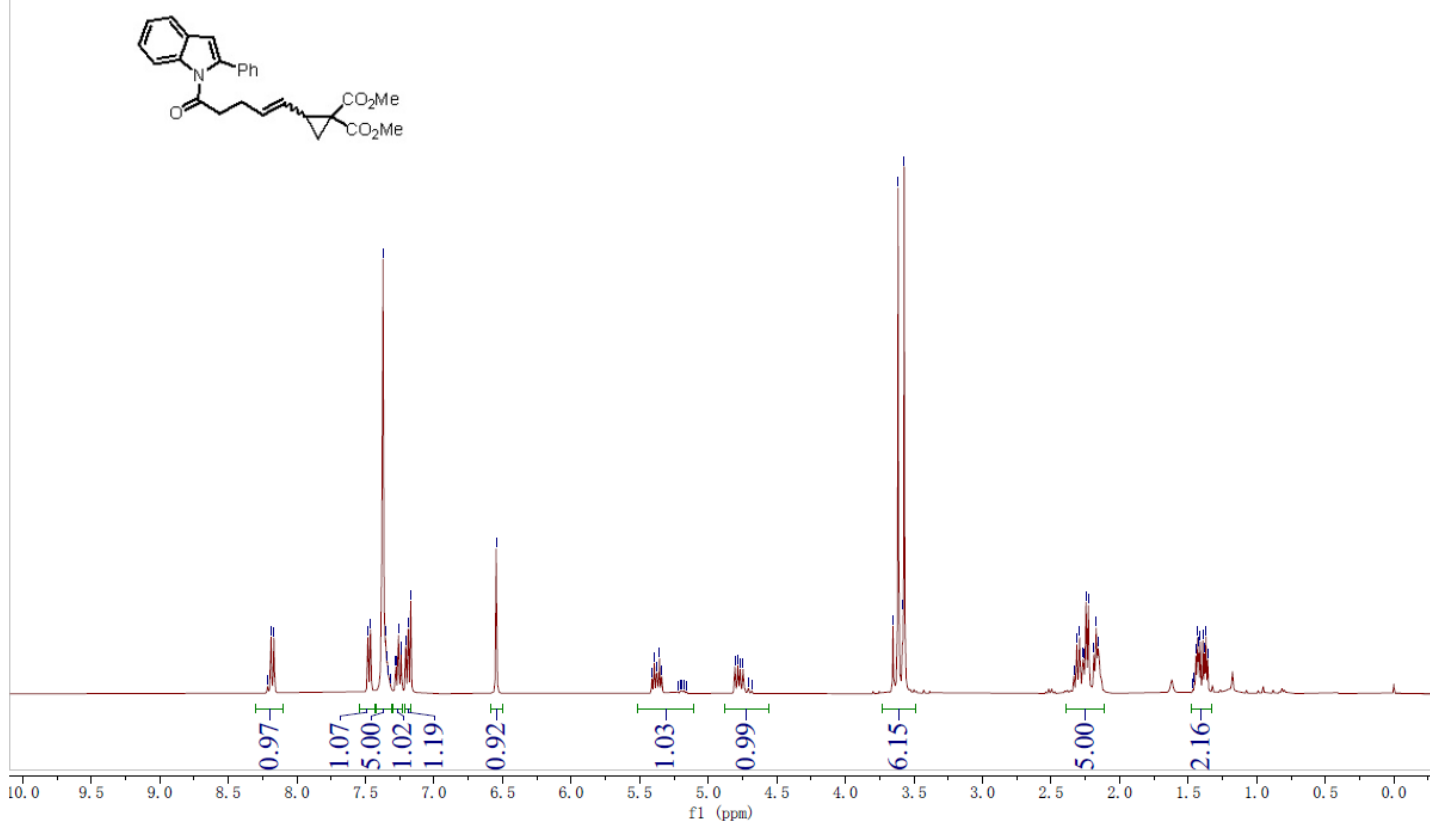

${ }^{13} \mathrm{C}$ NMR Spectrum of $\mathbf{1 d}$

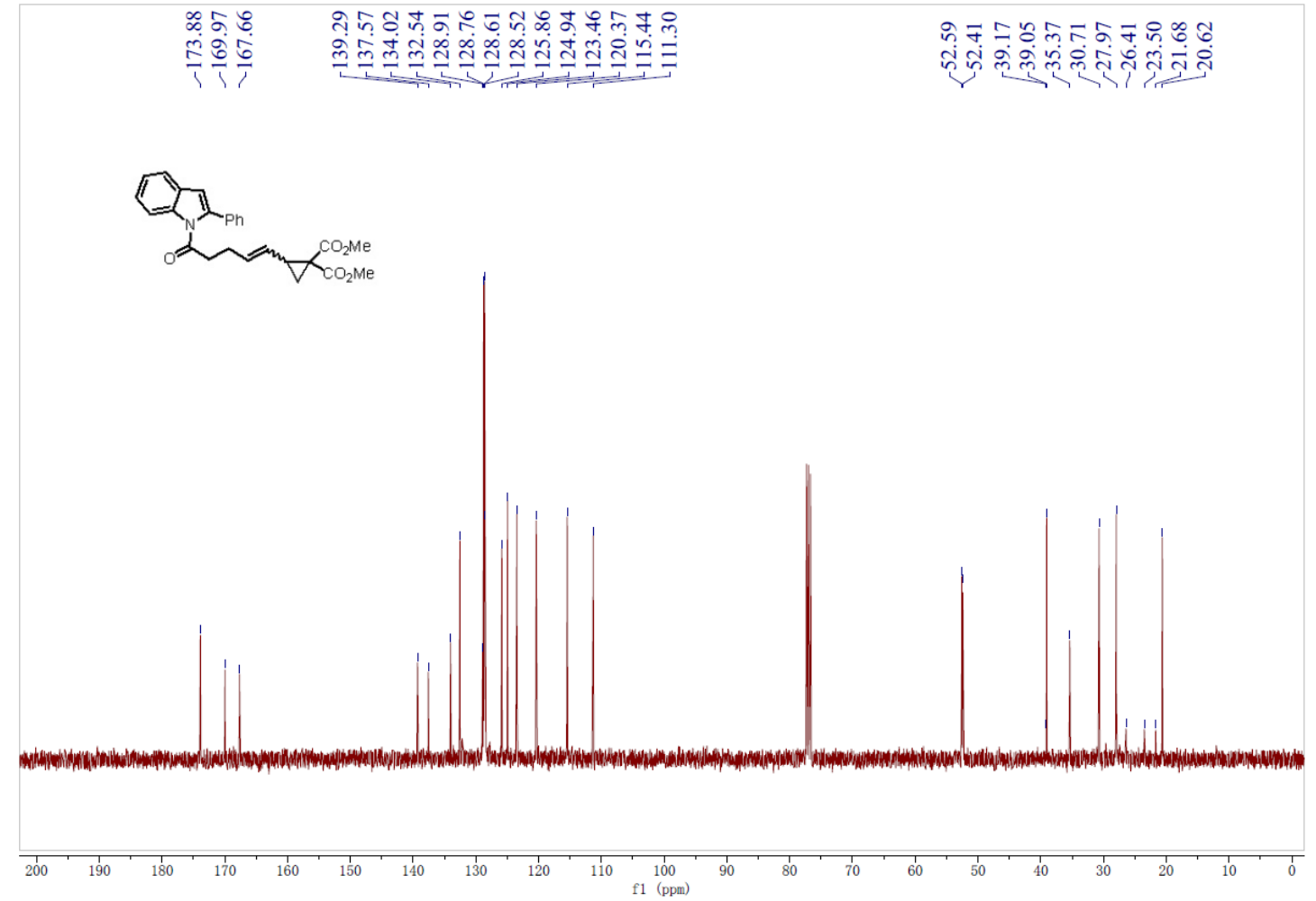


${ }^{1} \mathrm{H}$ NMR Spectrum of $\mathbf{1 e}$

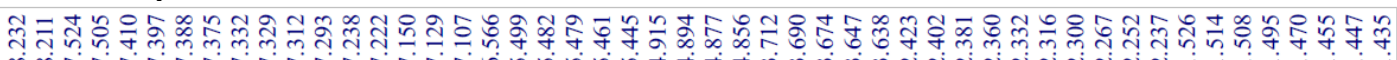

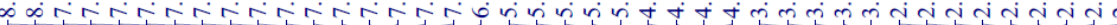
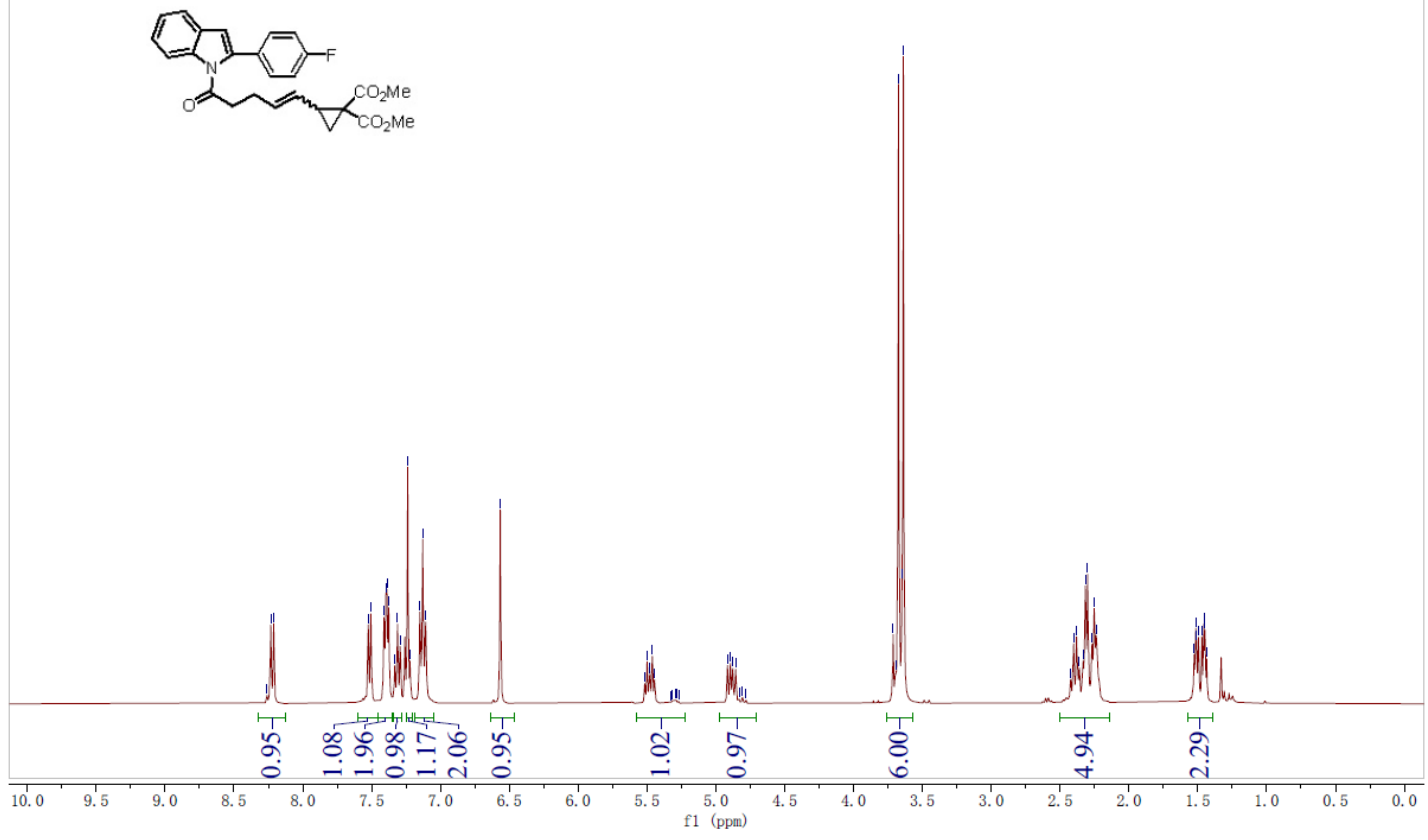

${ }^{13} \mathrm{C}$ NMR Spectrum of $\mathbf{1 e}$

닷

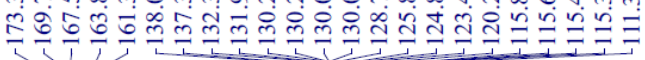

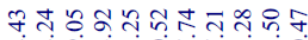

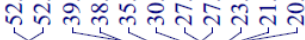
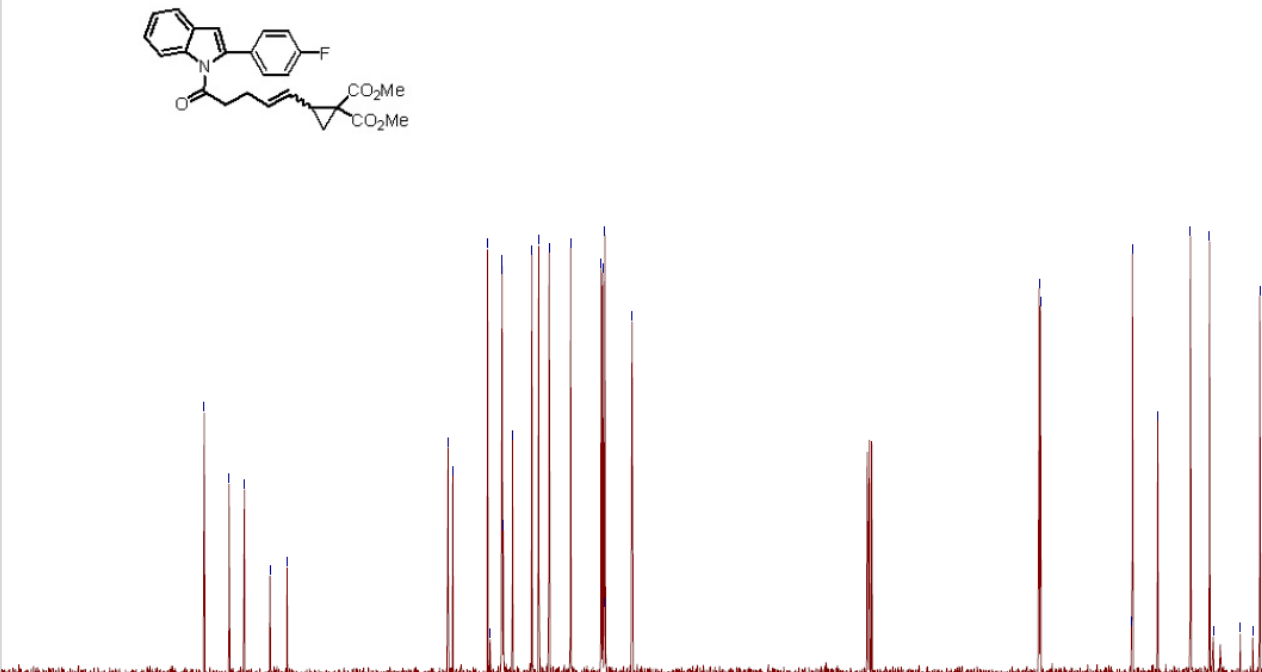

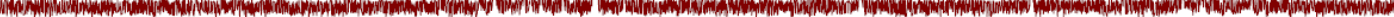

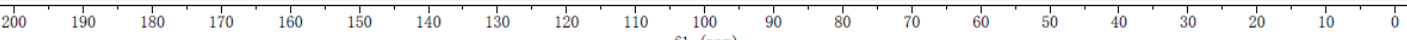


${ }^{19}$ F NMR Spectrum of $\mathbf{1 e}$

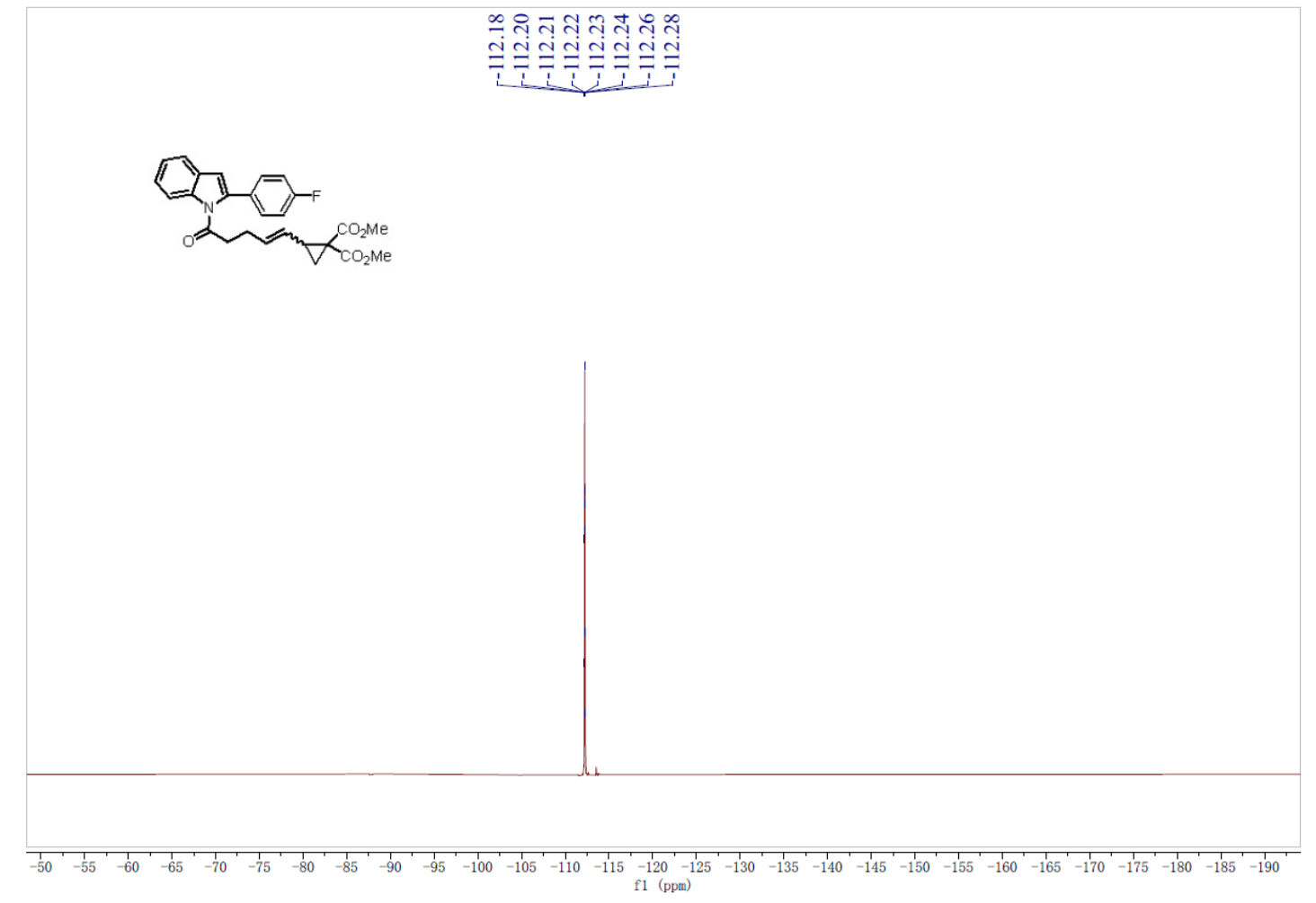


${ }^{1} \mathrm{H}$ NMR Spectrum of $\mathbf{1 f}$

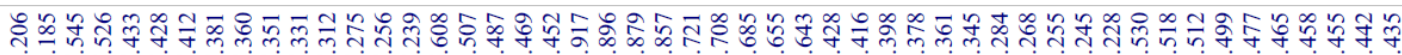

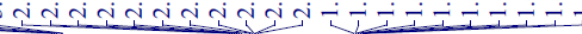
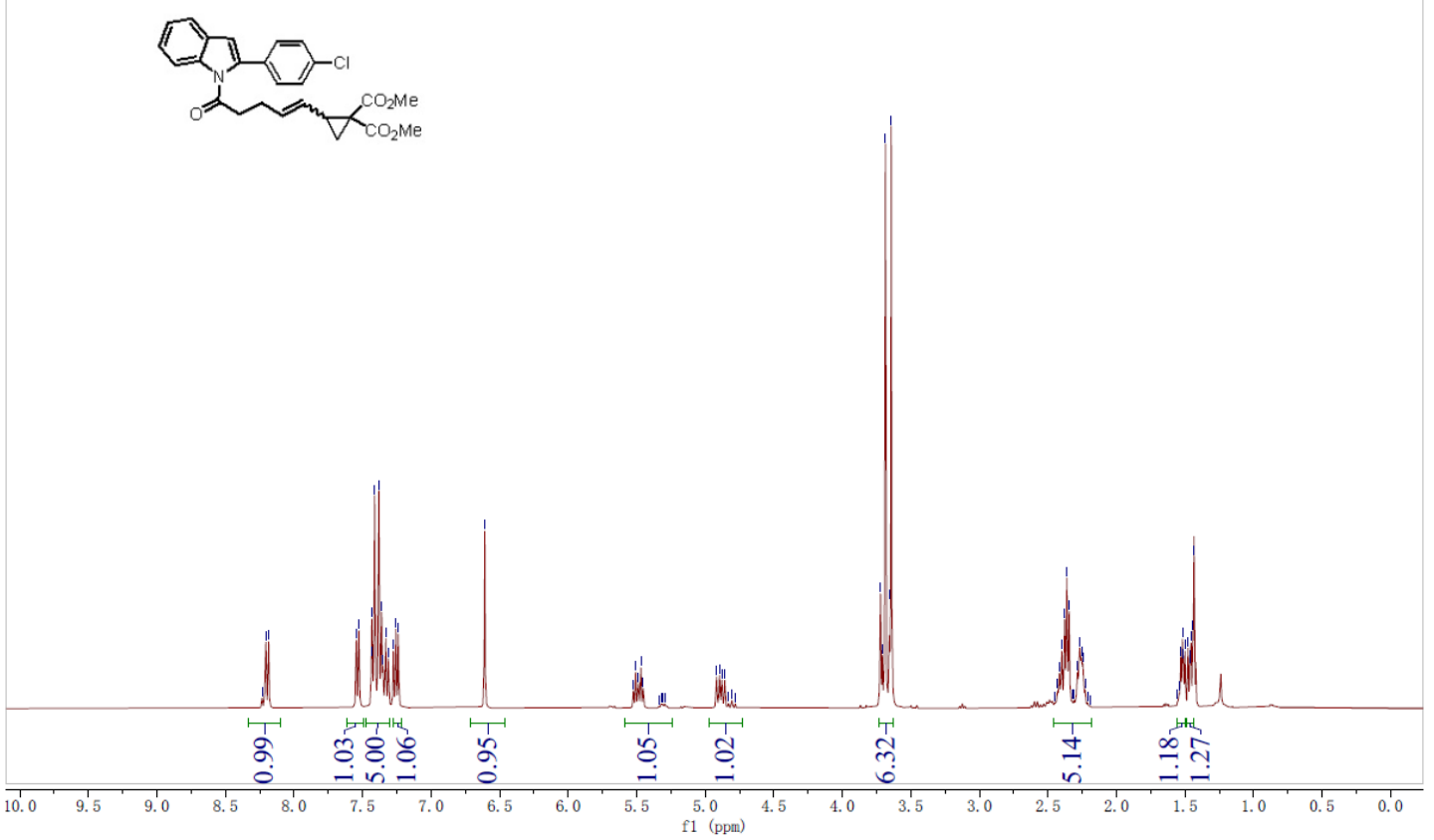

${ }^{13} \mathrm{C}$ NMR Spectrum of $\mathbf{1 f}$
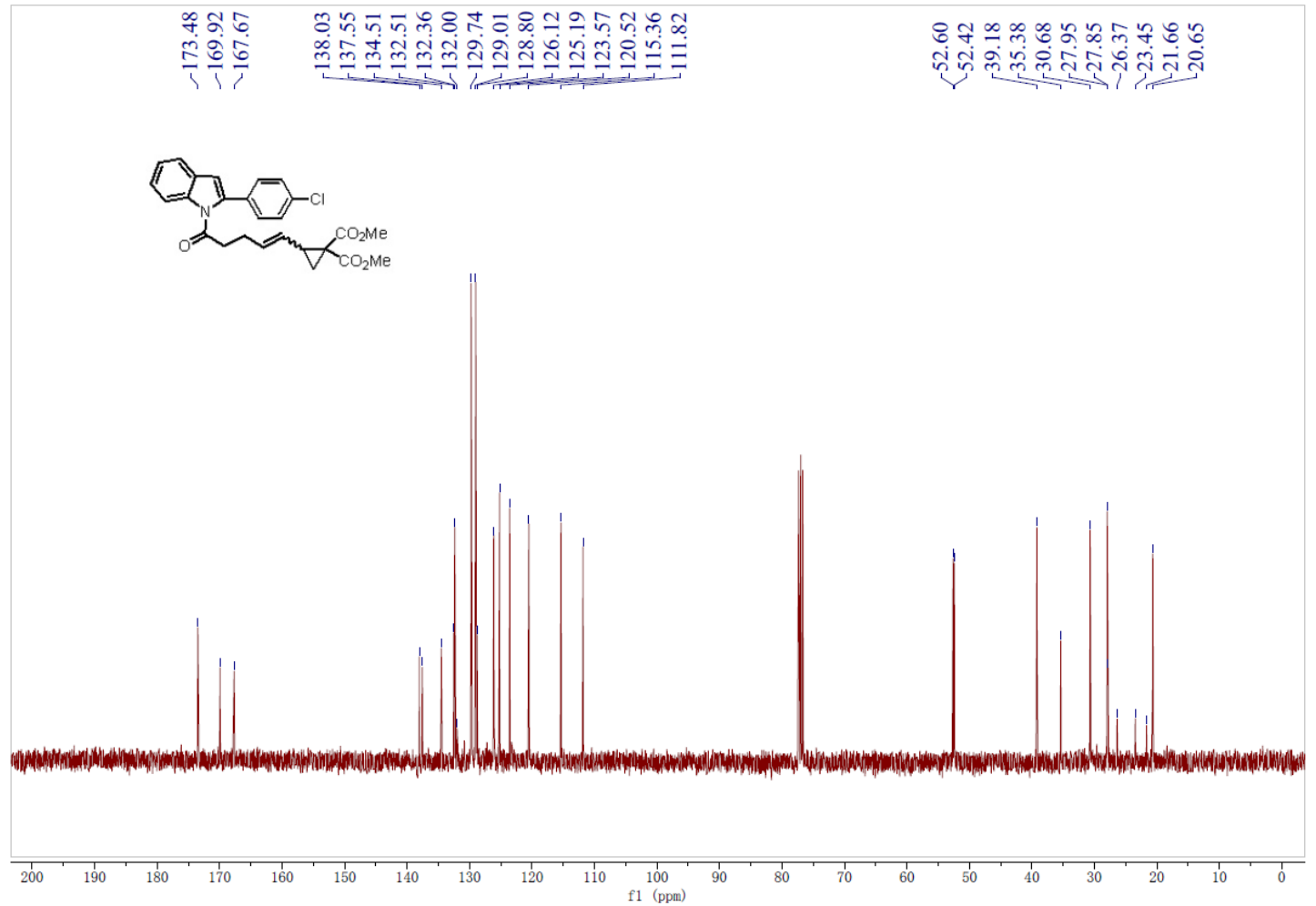
${ }^{1} \mathrm{H}$ NMR Spectrum of $\mathbf{1 g}$

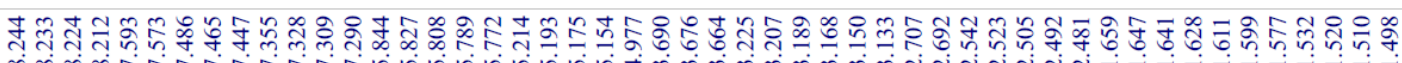

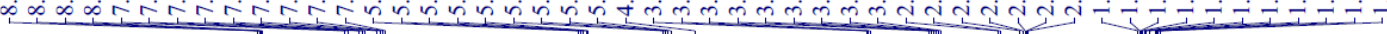
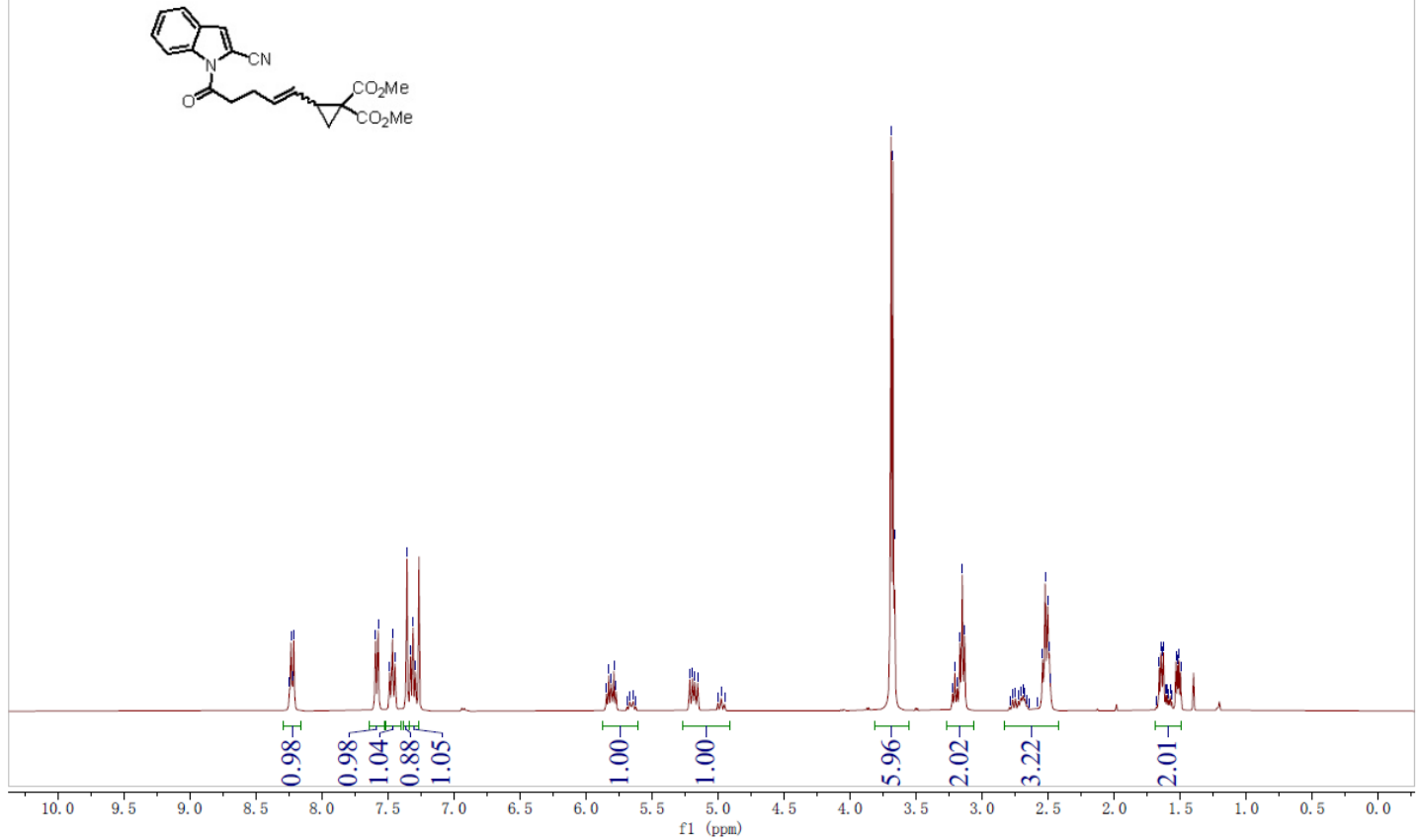

${ }^{13} \mathrm{C}$ NMR Spectrum of $\mathbf{1 g}$

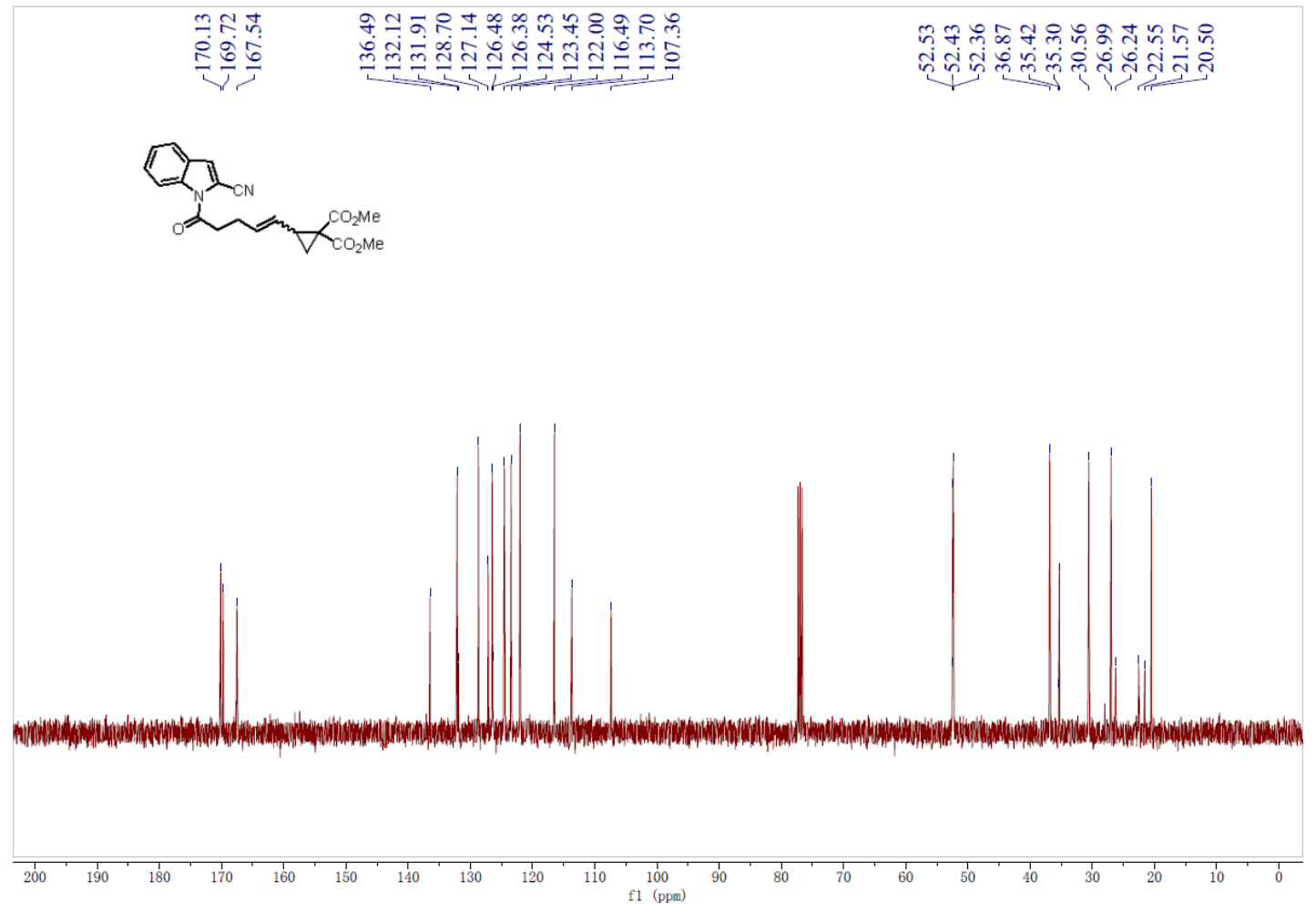


${ }^{1} \mathrm{H}$ NMR Spectrum of $\mathbf{1 h}$

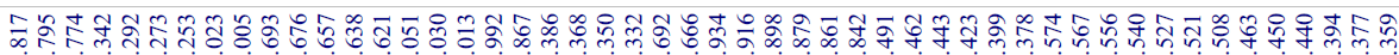

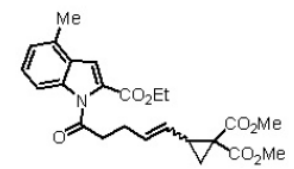

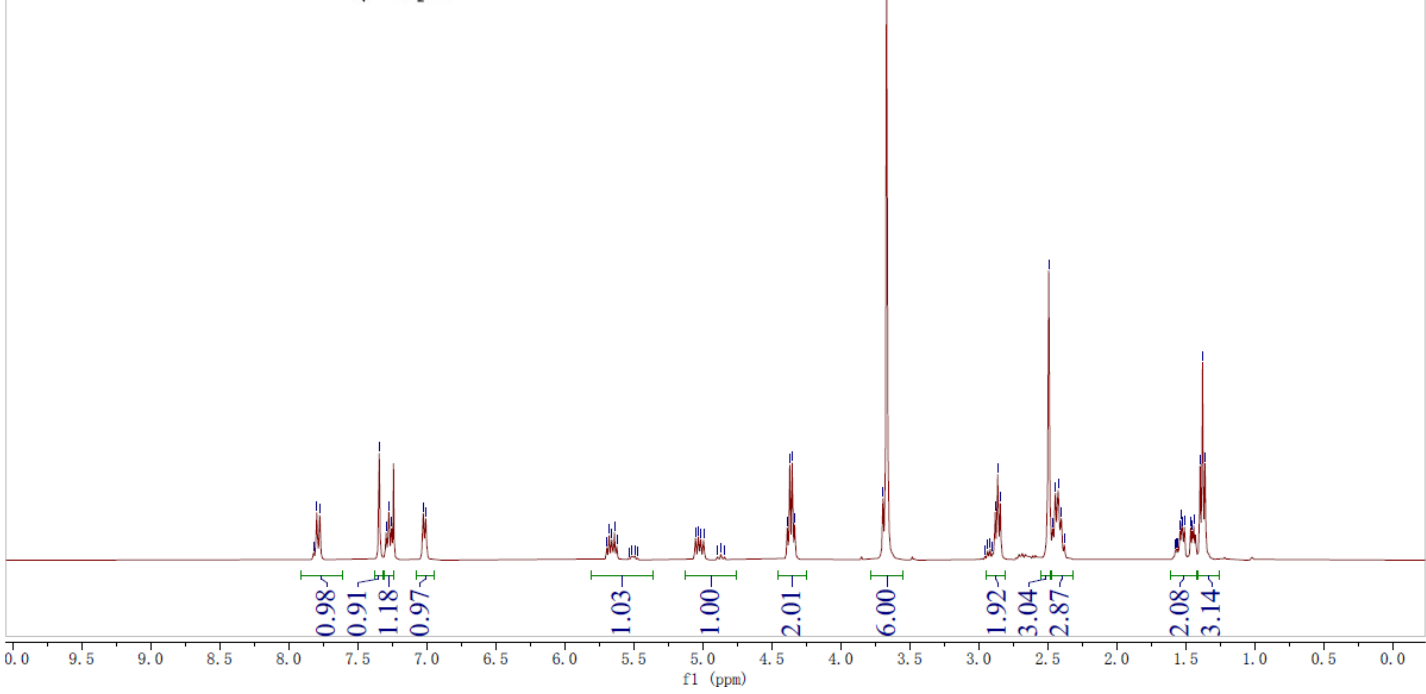

${ }^{13} \mathrm{C}$ NMR Spectrum of $\mathbf{1 h}$

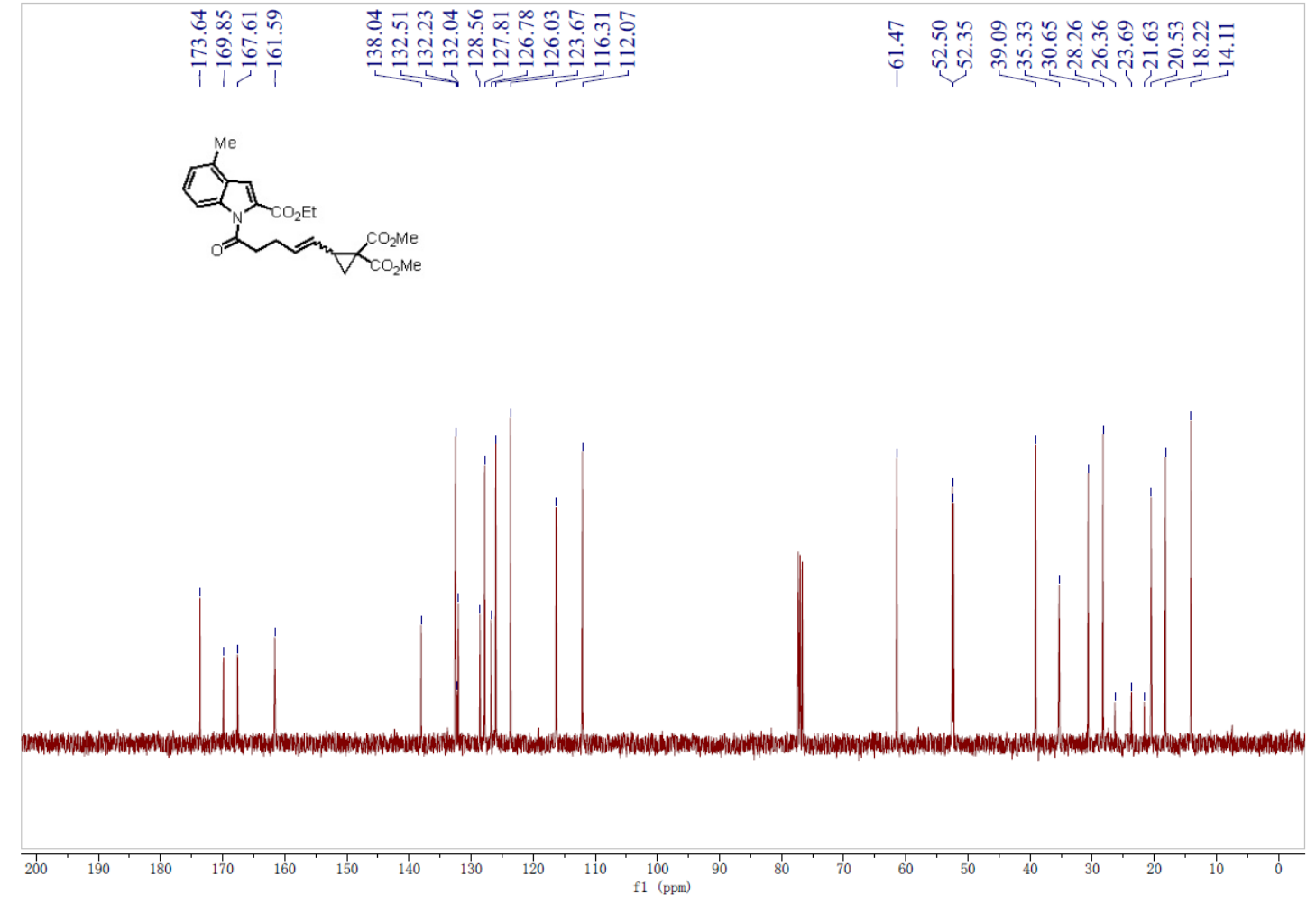


${ }^{1} \mathrm{H}$ NMR Spectrum of $\mathbf{1 i}$

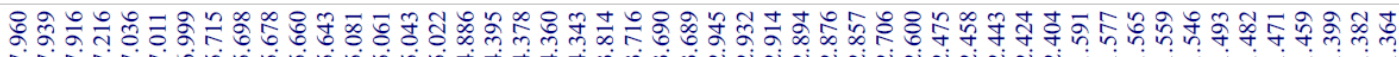

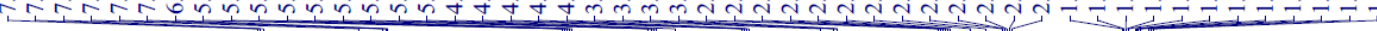

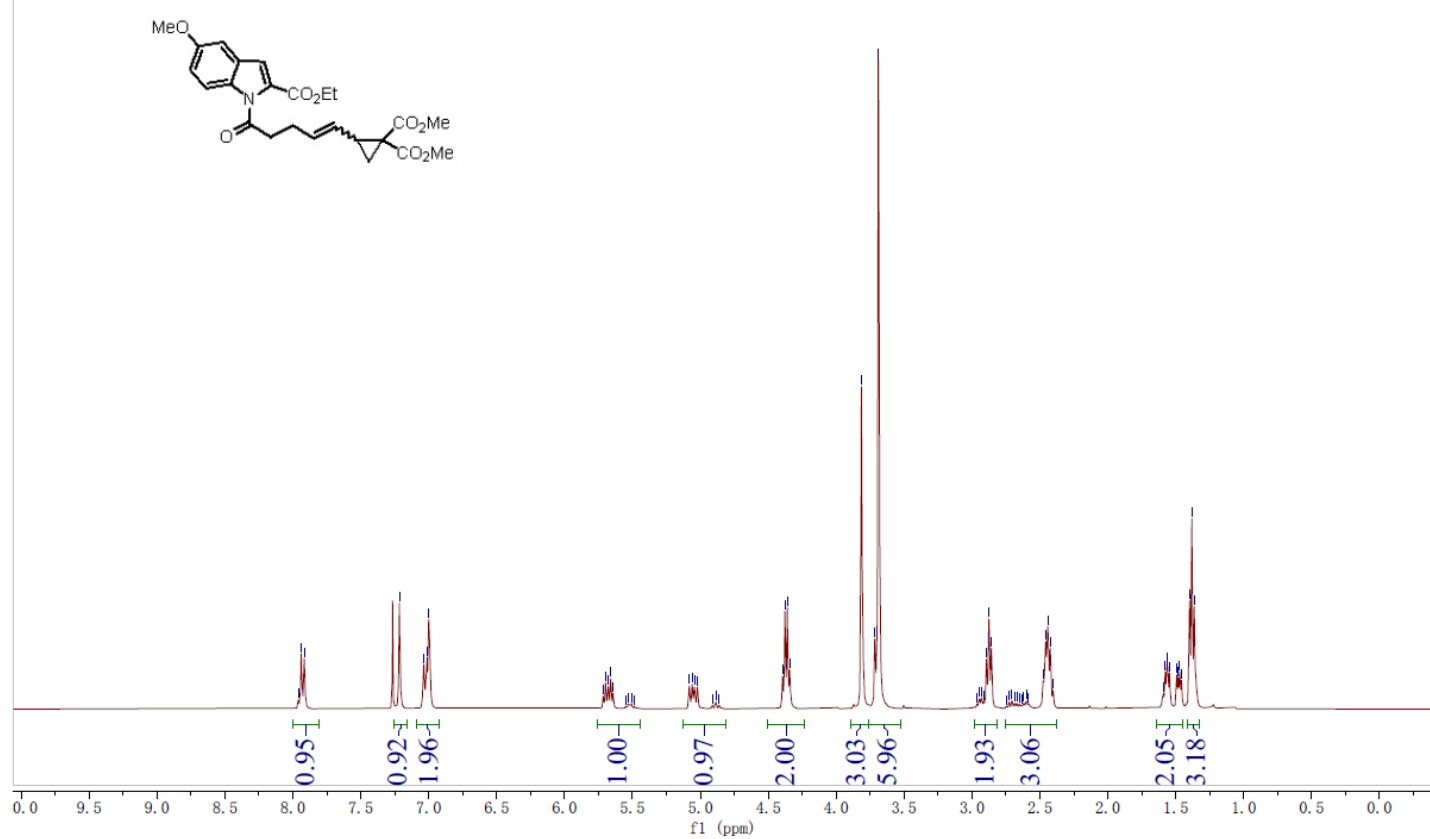

${ }^{13} \mathrm{C}$ NMR Spectrum of $\mathbf{1 i}$

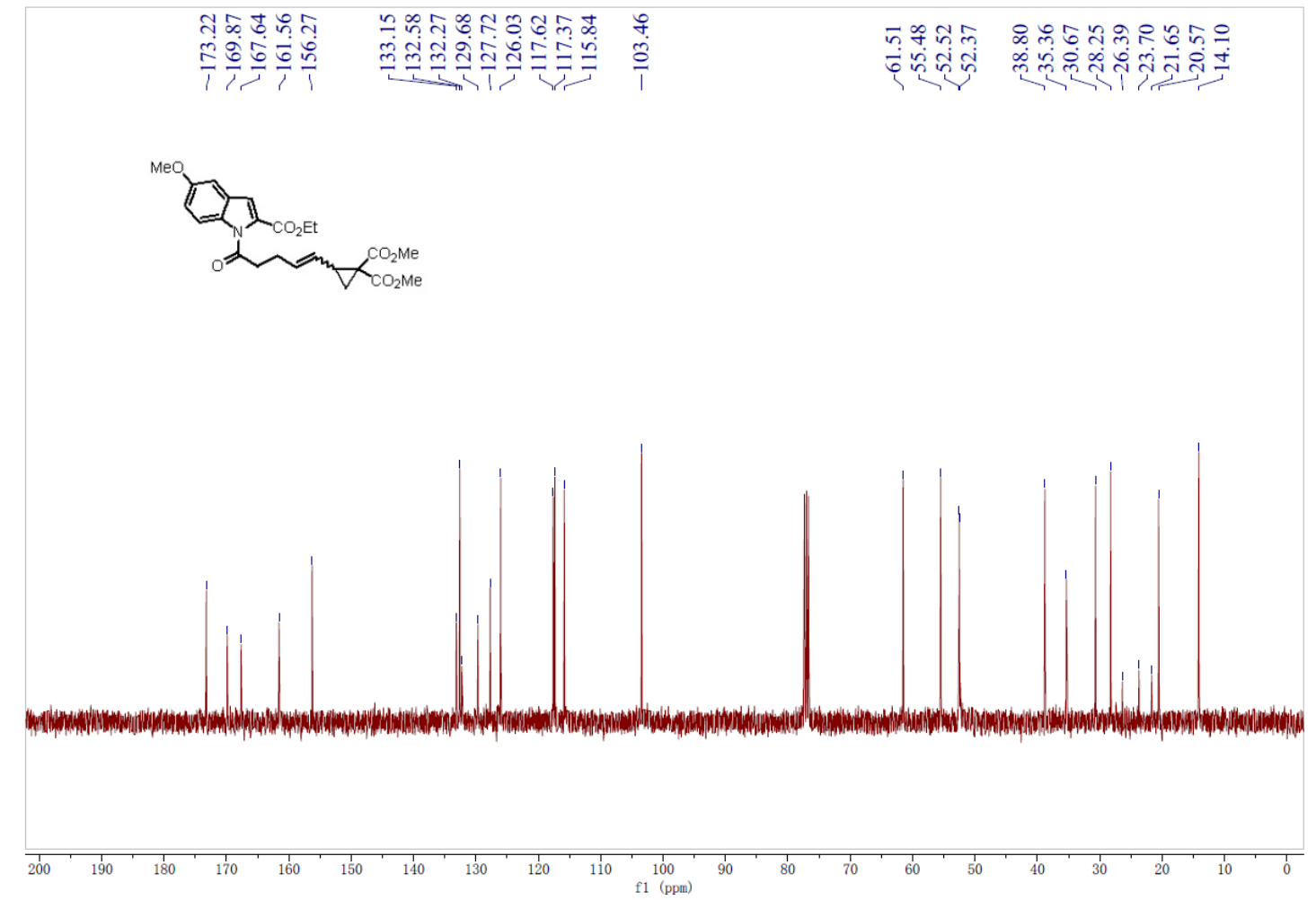


${ }^{1} \mathrm{H}$ NMR Spectrum of $\mathbf{1 j}$

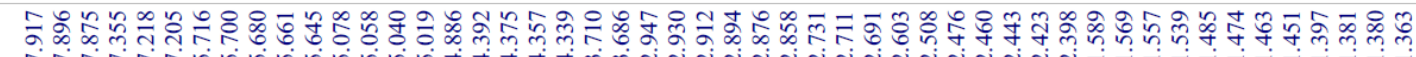

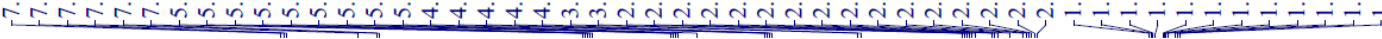
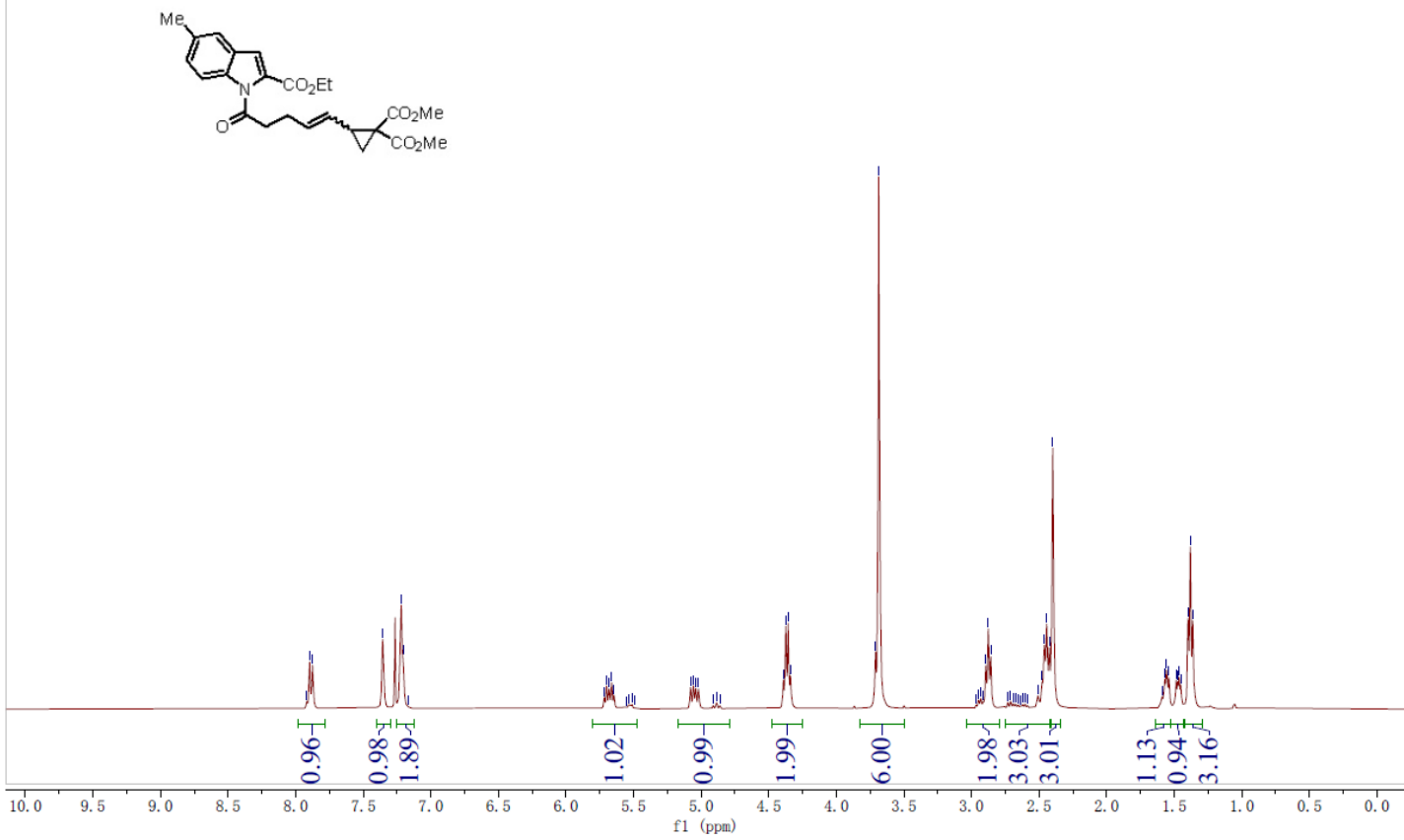

${ }^{13} \mathrm{C}$ NMR Spectrum of $\mathbf{1} \mathbf{j}$
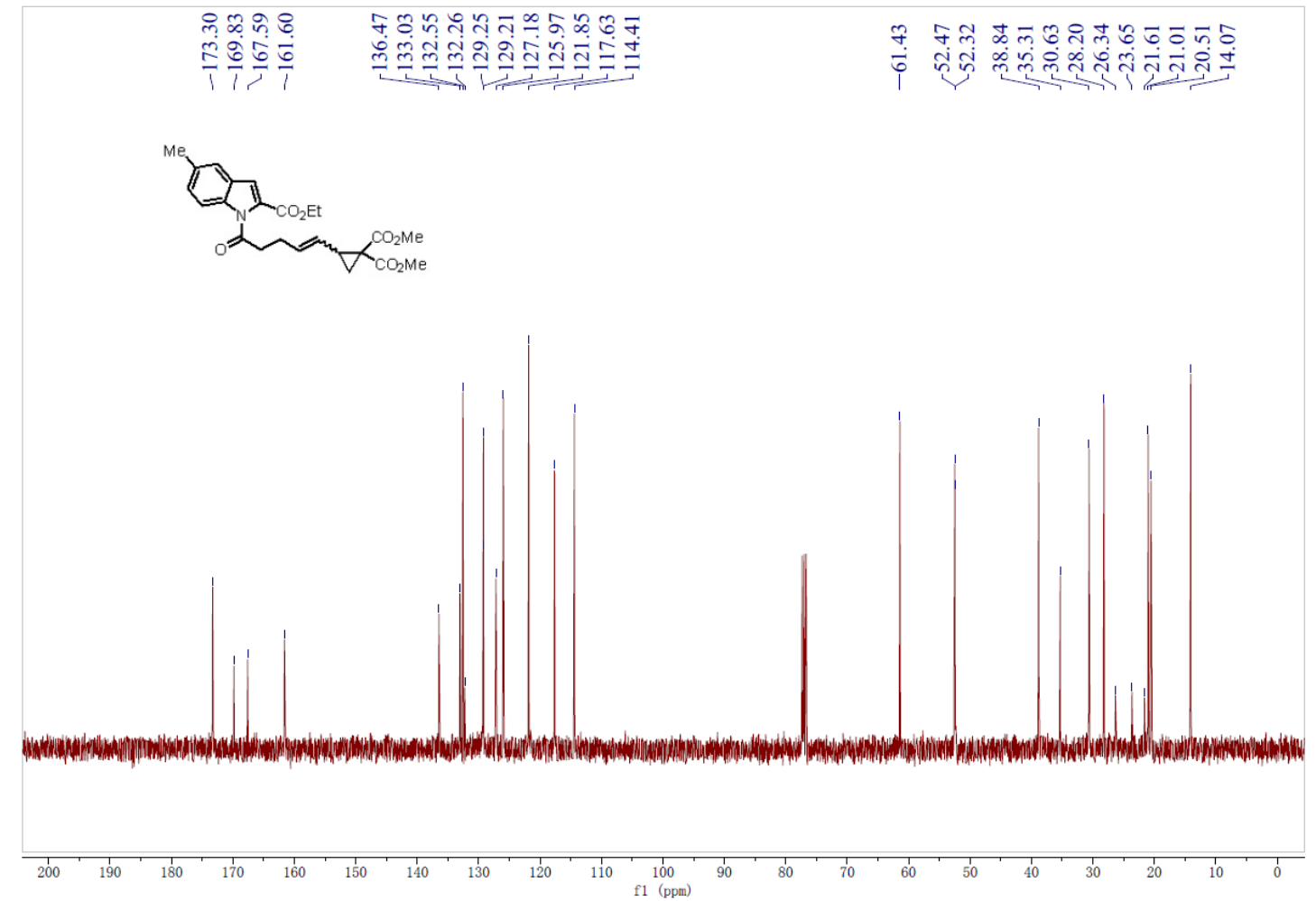
${ }^{1} \mathrm{H}$ NMR Spectrum of $\mathbf{1 k}$

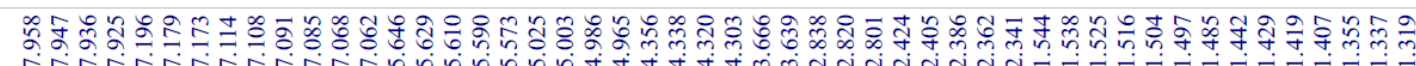

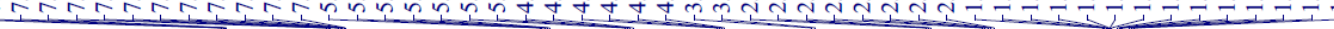
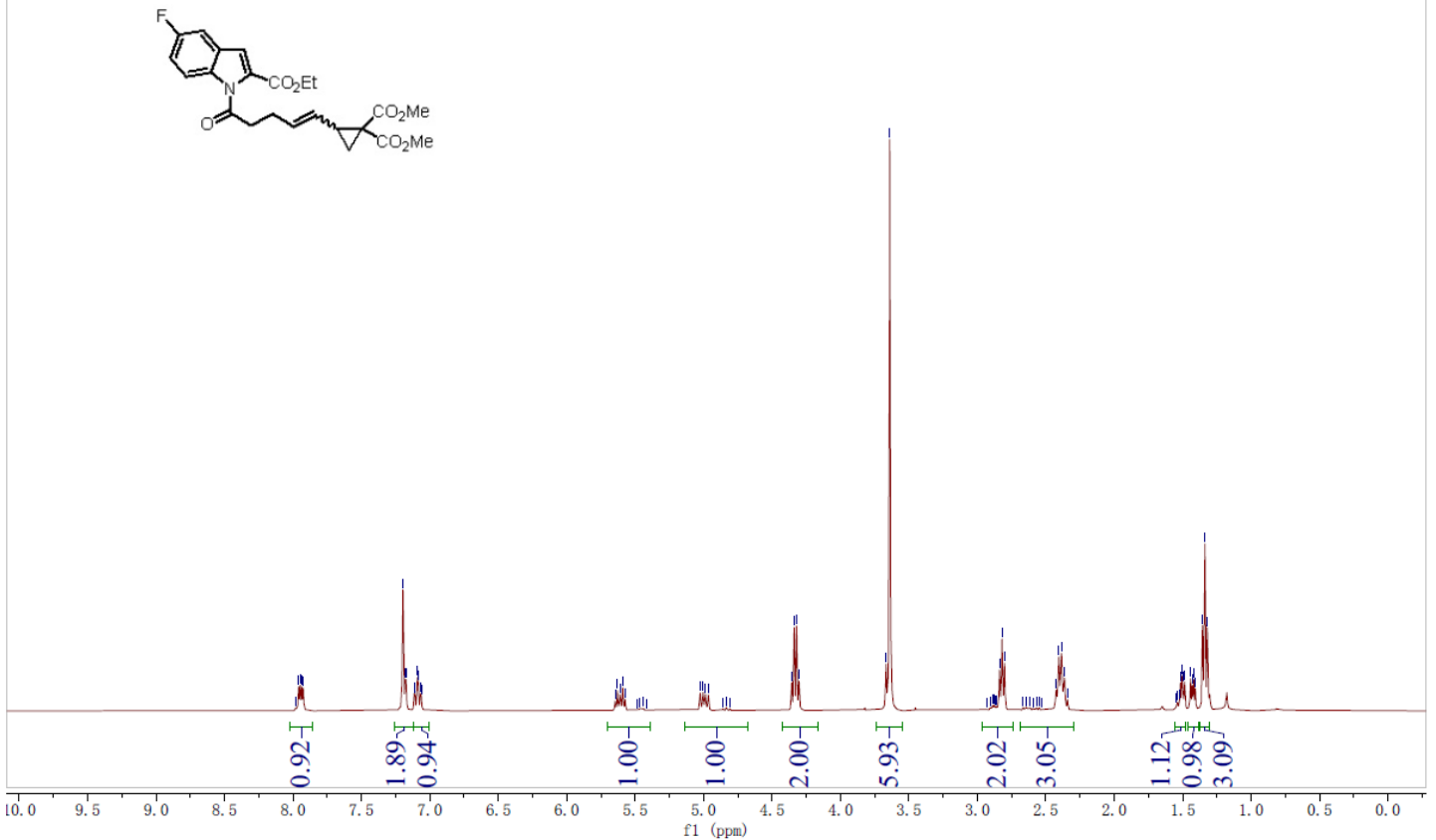

${ }^{13} \mathrm{C}$ NMR Spectrum of $\mathbf{1 k}$
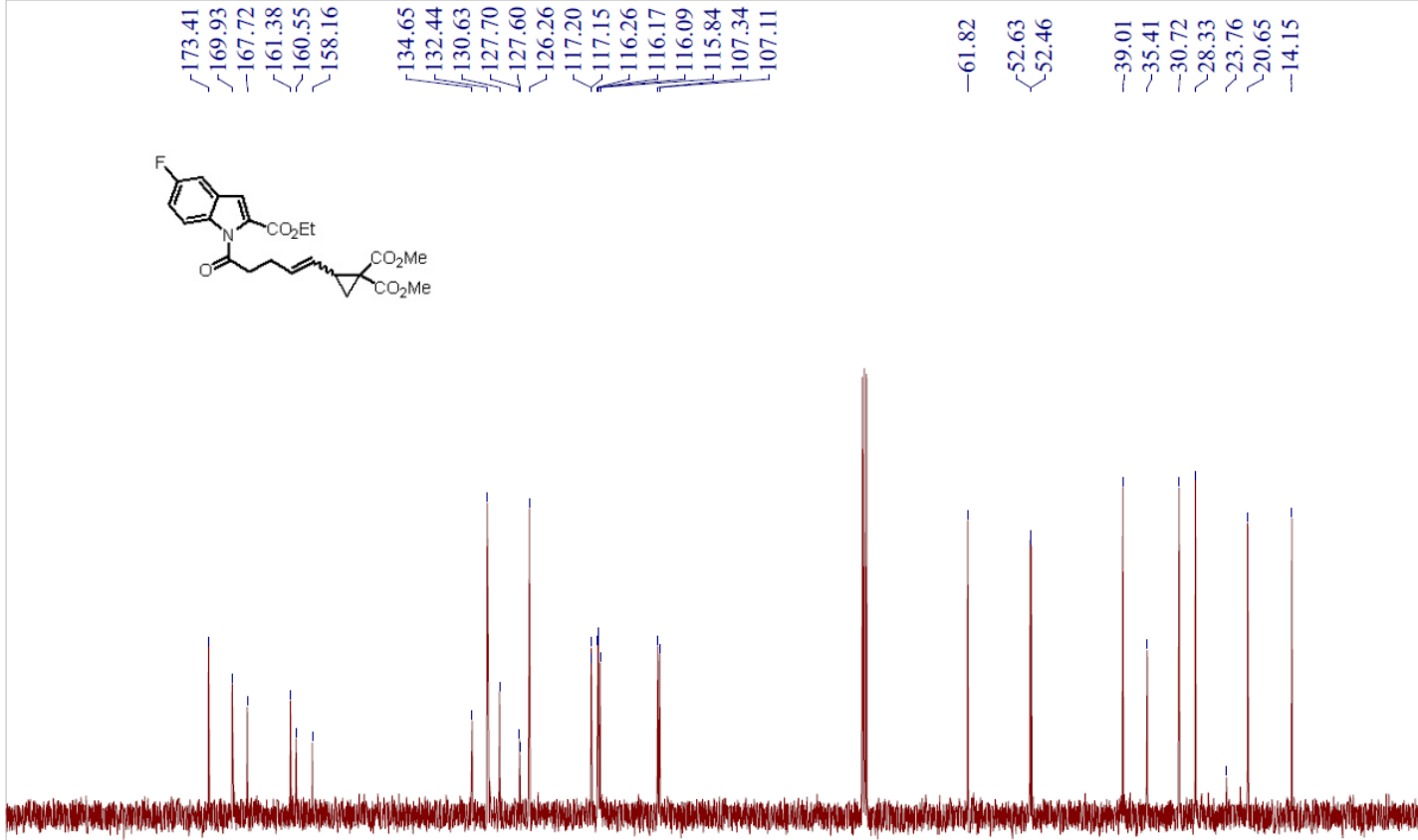

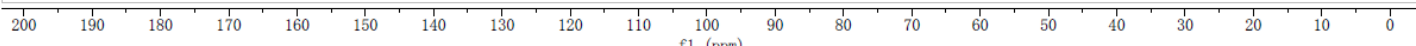


${ }^{19}$ F NMR Spectrum of $\mathbf{1 k}$

$$
\begin{aligned}
& \text { 느느두우ำ } \\
& \text { 국ำ }
\end{aligned}
$$

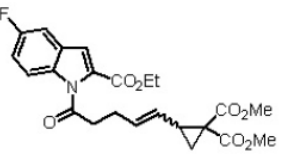

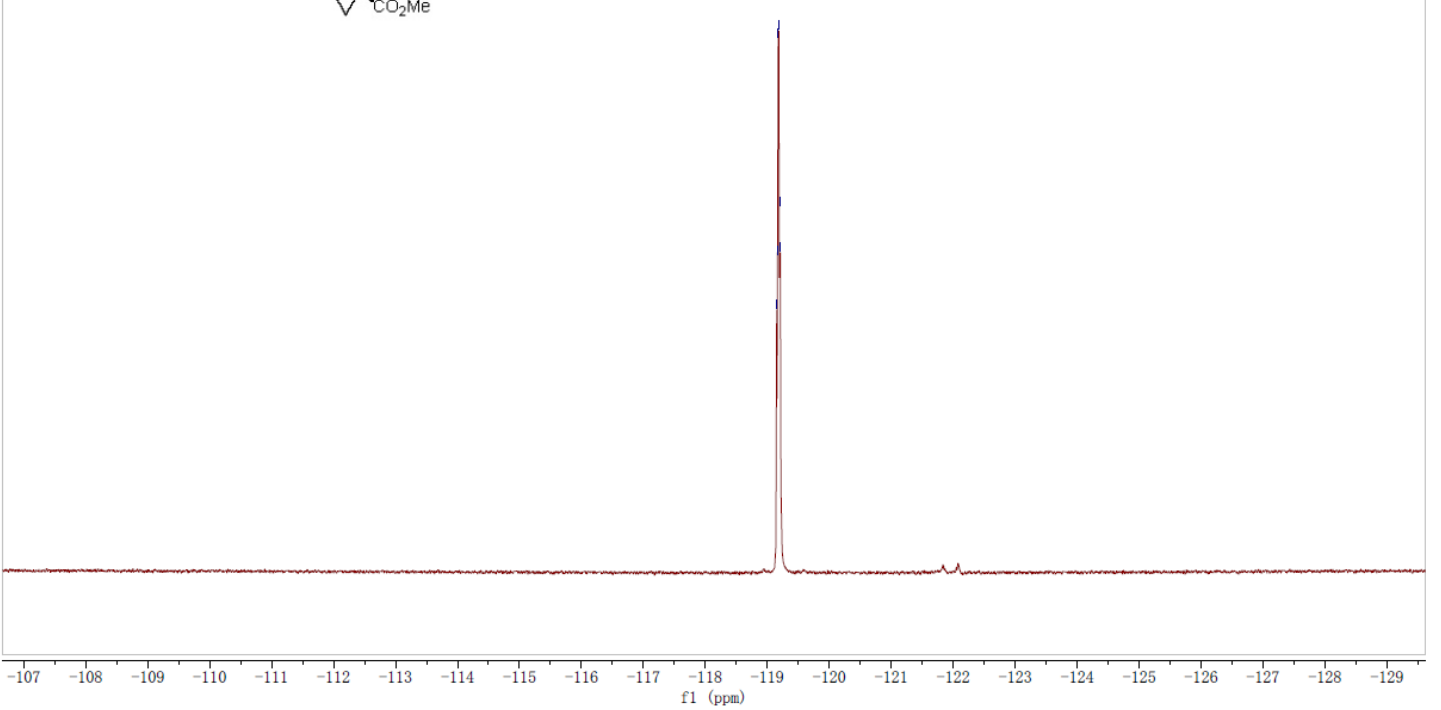


${ }^{1} \mathrm{H}$ NMR Spectrum of $\mathbf{1 l}$

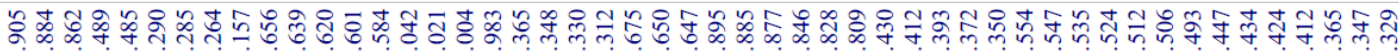

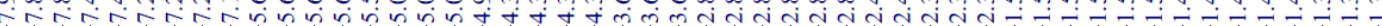
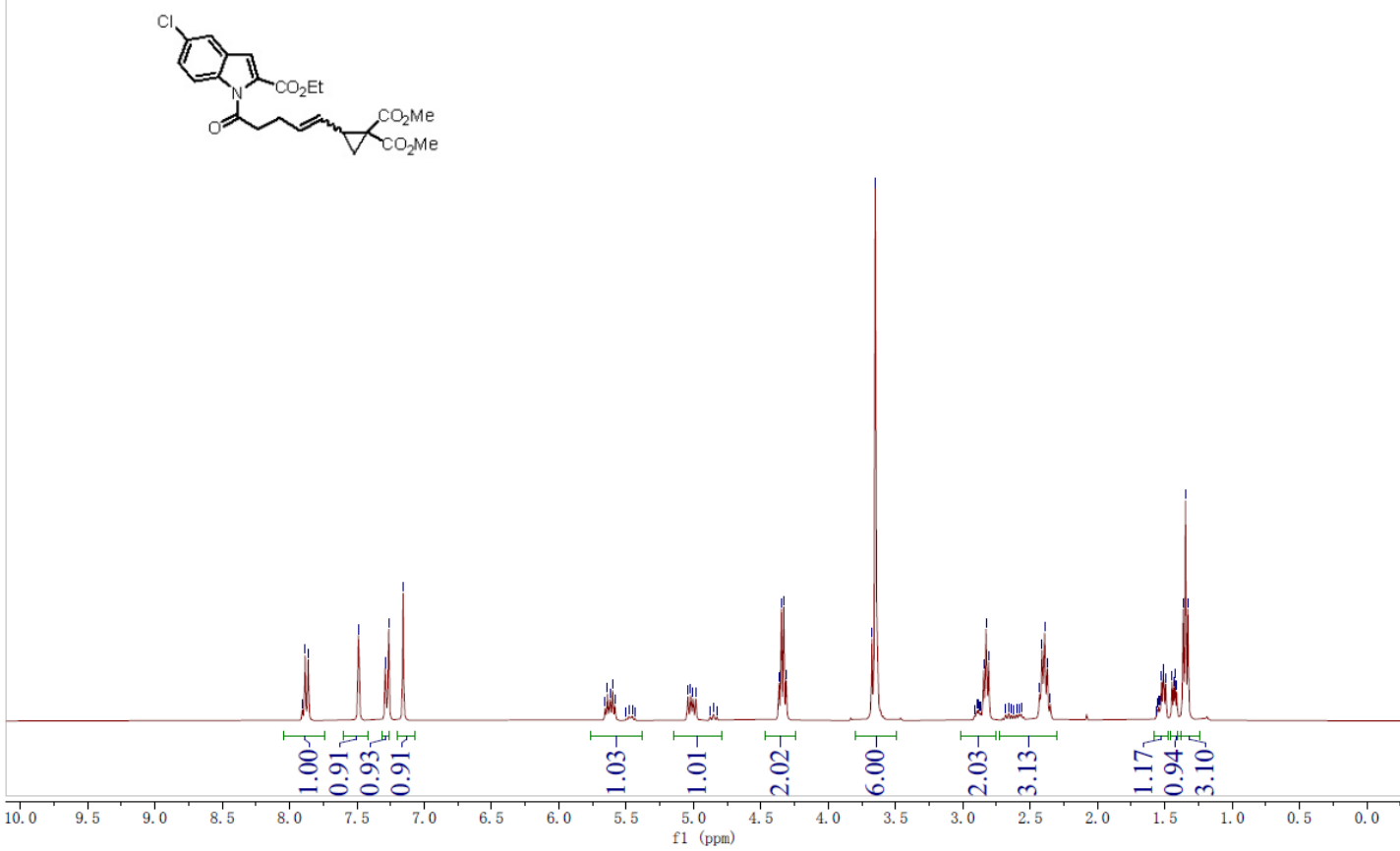

${ }^{13} \mathrm{C}$ NMR Spectrum of $\mathbf{1 1}$

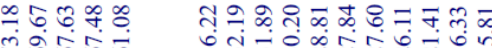

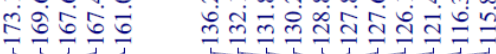

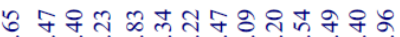

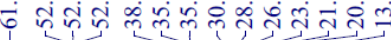
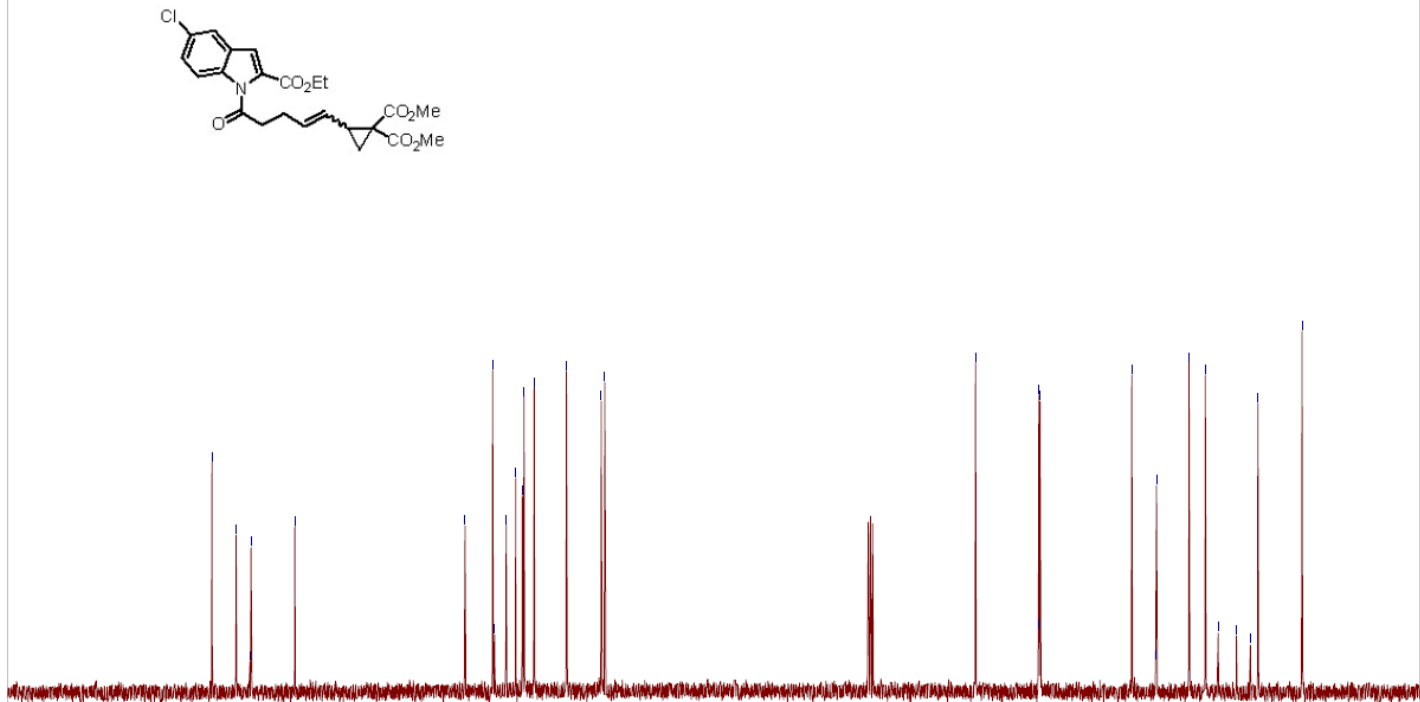

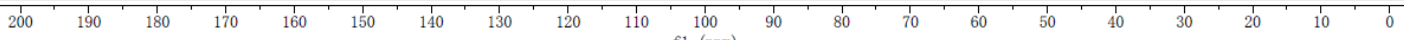


${ }^{1} \mathrm{H}$ NMR Spectrum of $\mathbf{1 m}$

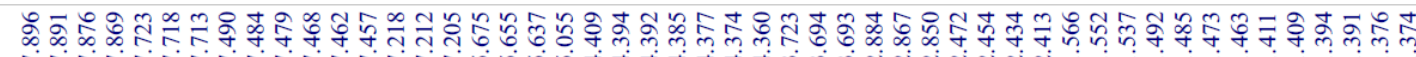
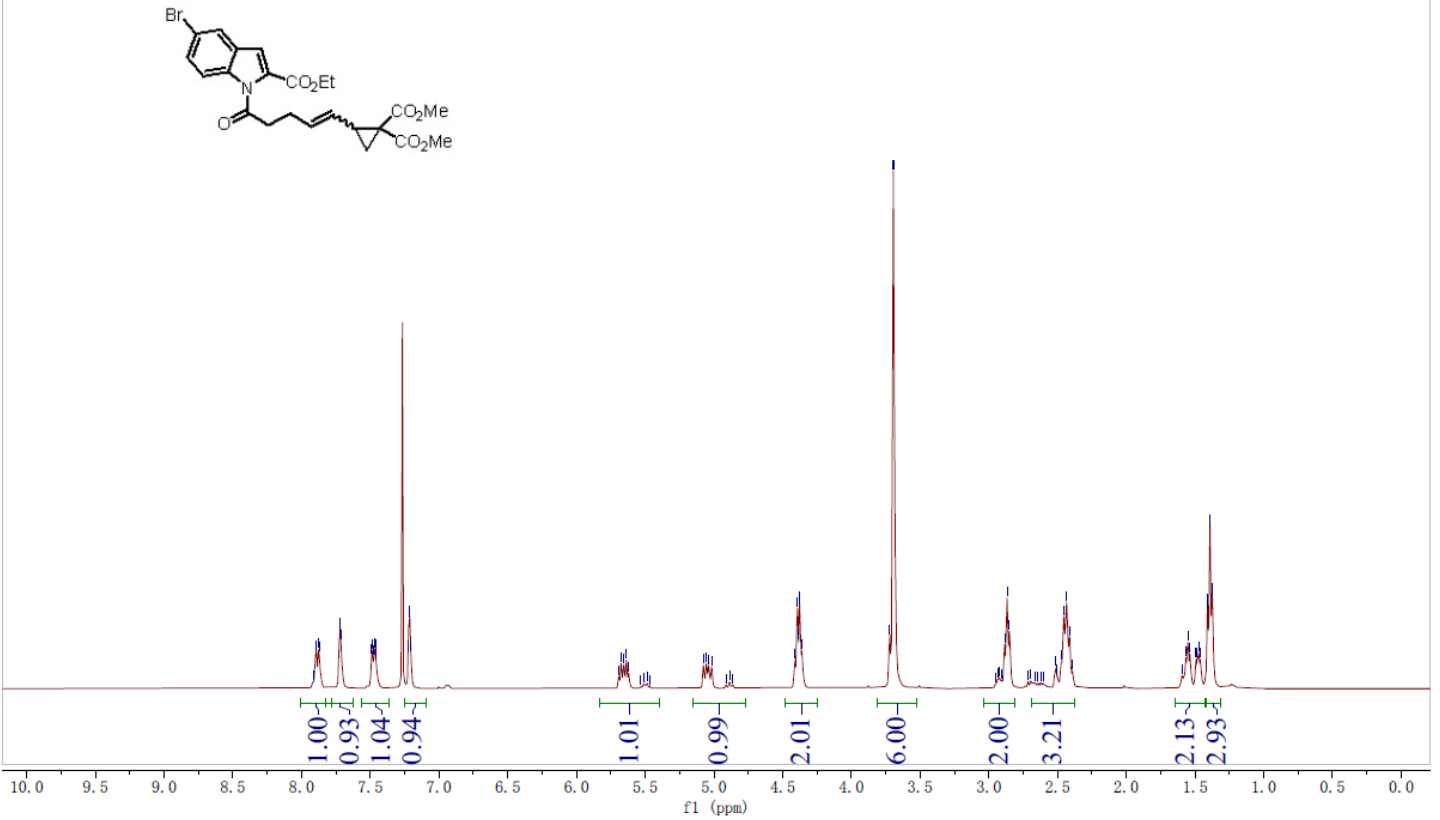

${ }^{13} \mathrm{C}$ NMR Spectrum of $\mathbf{1} \mathbf{m}$

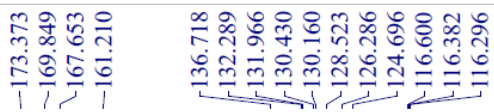

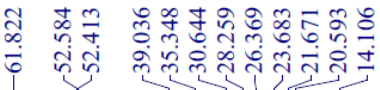
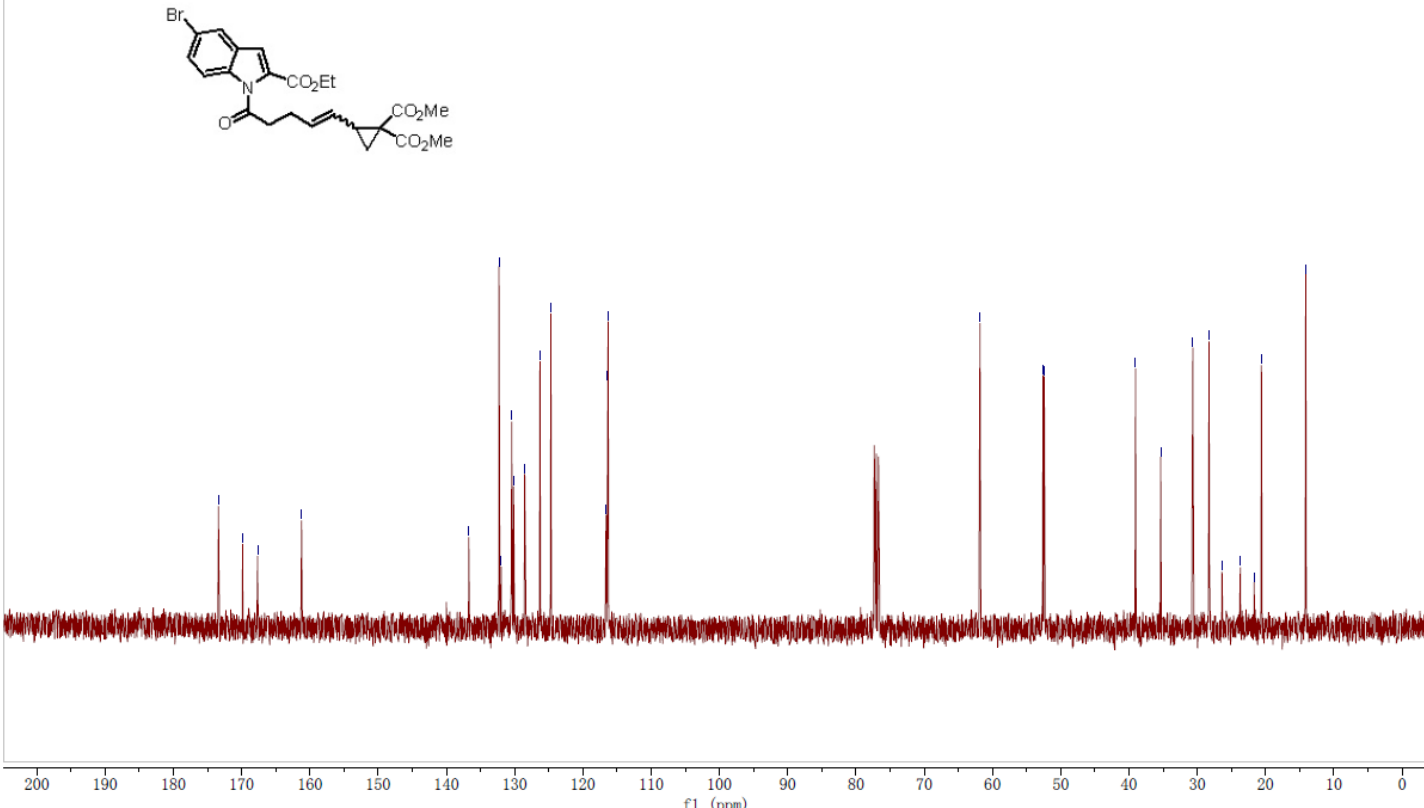
${ }^{1} \mathrm{H}$ NMR Spectrum of $\mathbf{1 n}$

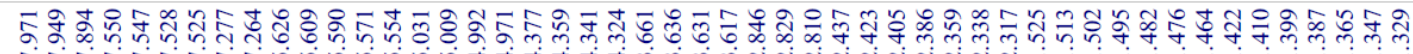

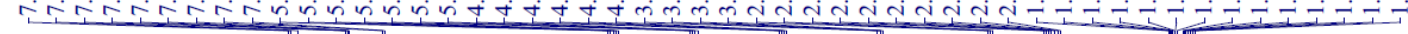
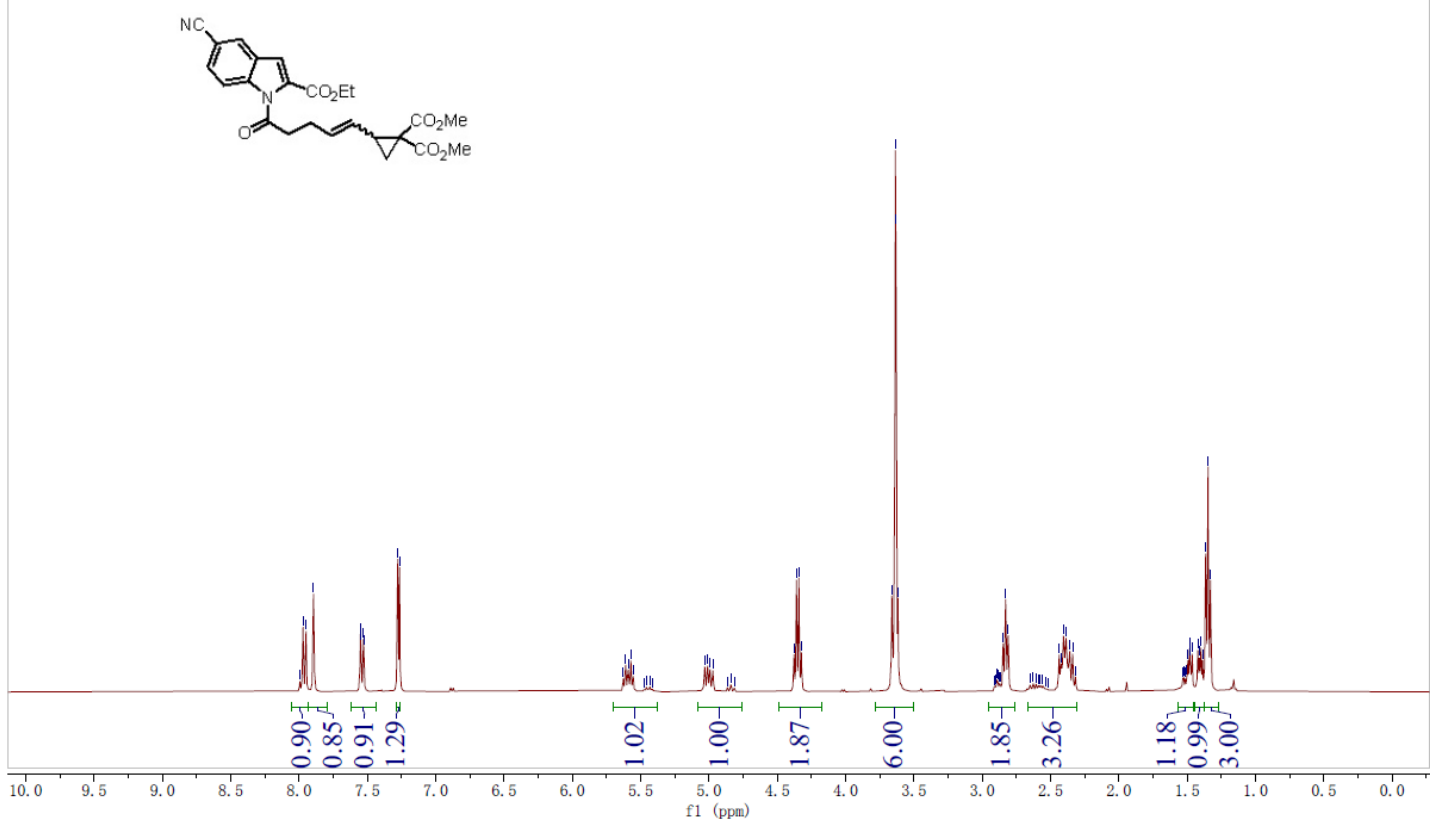

${ }^{13} \mathrm{C}$ NMR Spectrum of $\mathbf{1 n}$

त约守

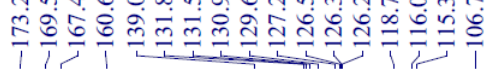

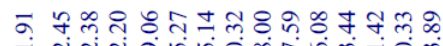

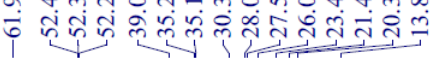
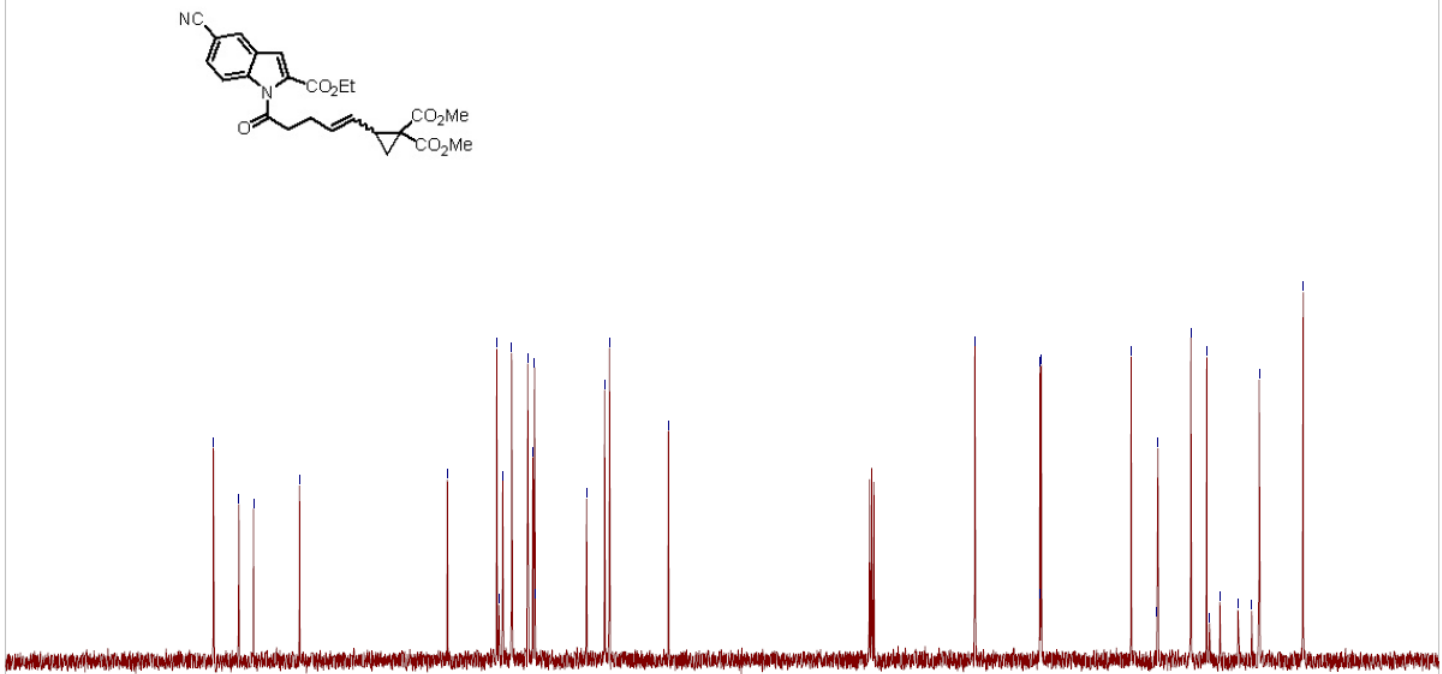

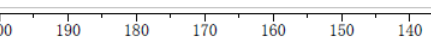

100 
${ }^{1} \mathrm{H}$ NMR Spectrum of $\mathbf{1 0}$

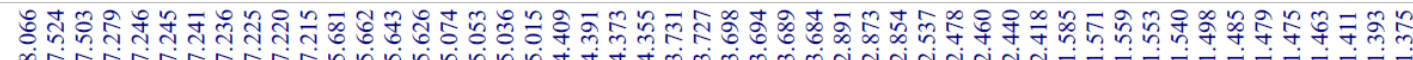
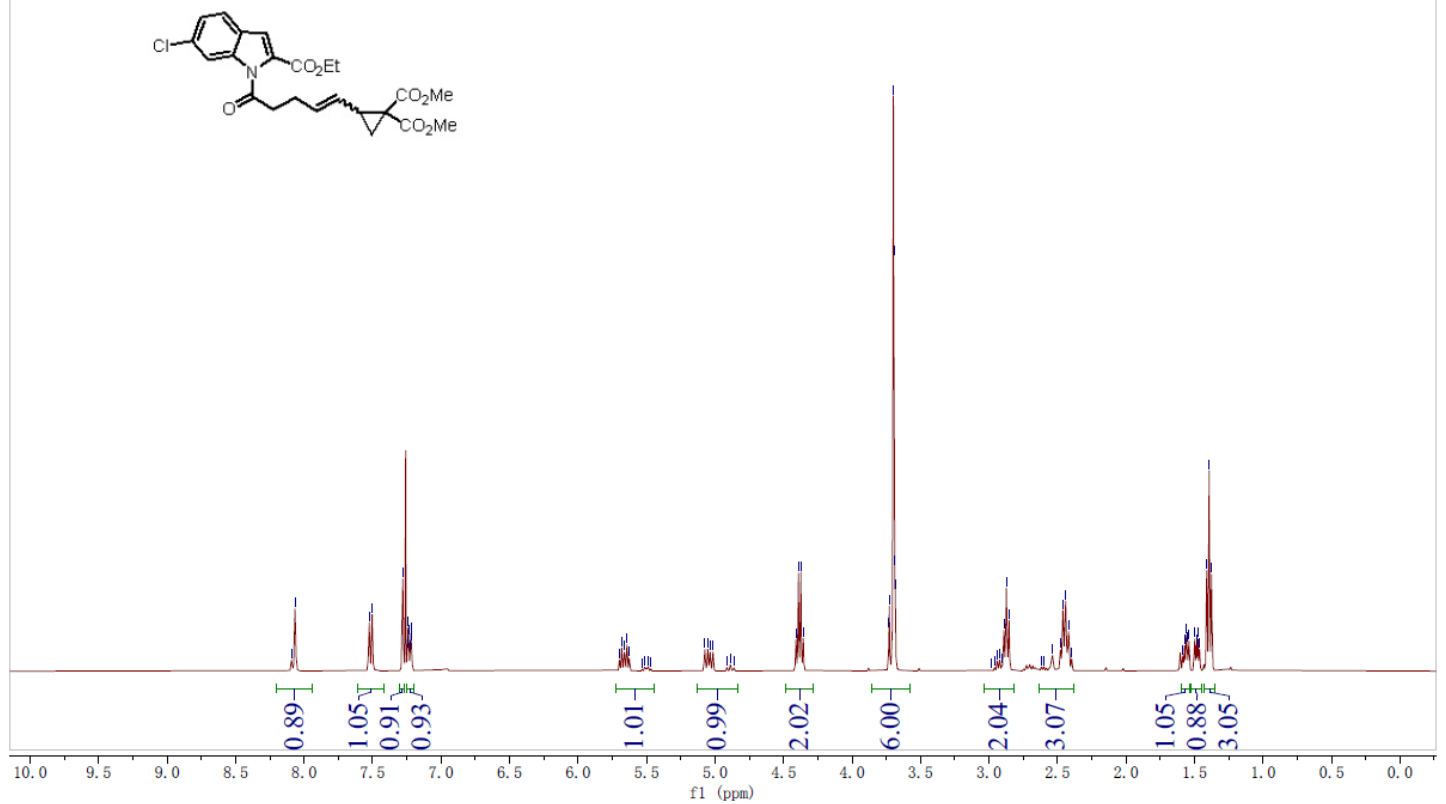

${ }^{13} \mathrm{C}$ NMR Spectrum of $\mathbf{1 0}$

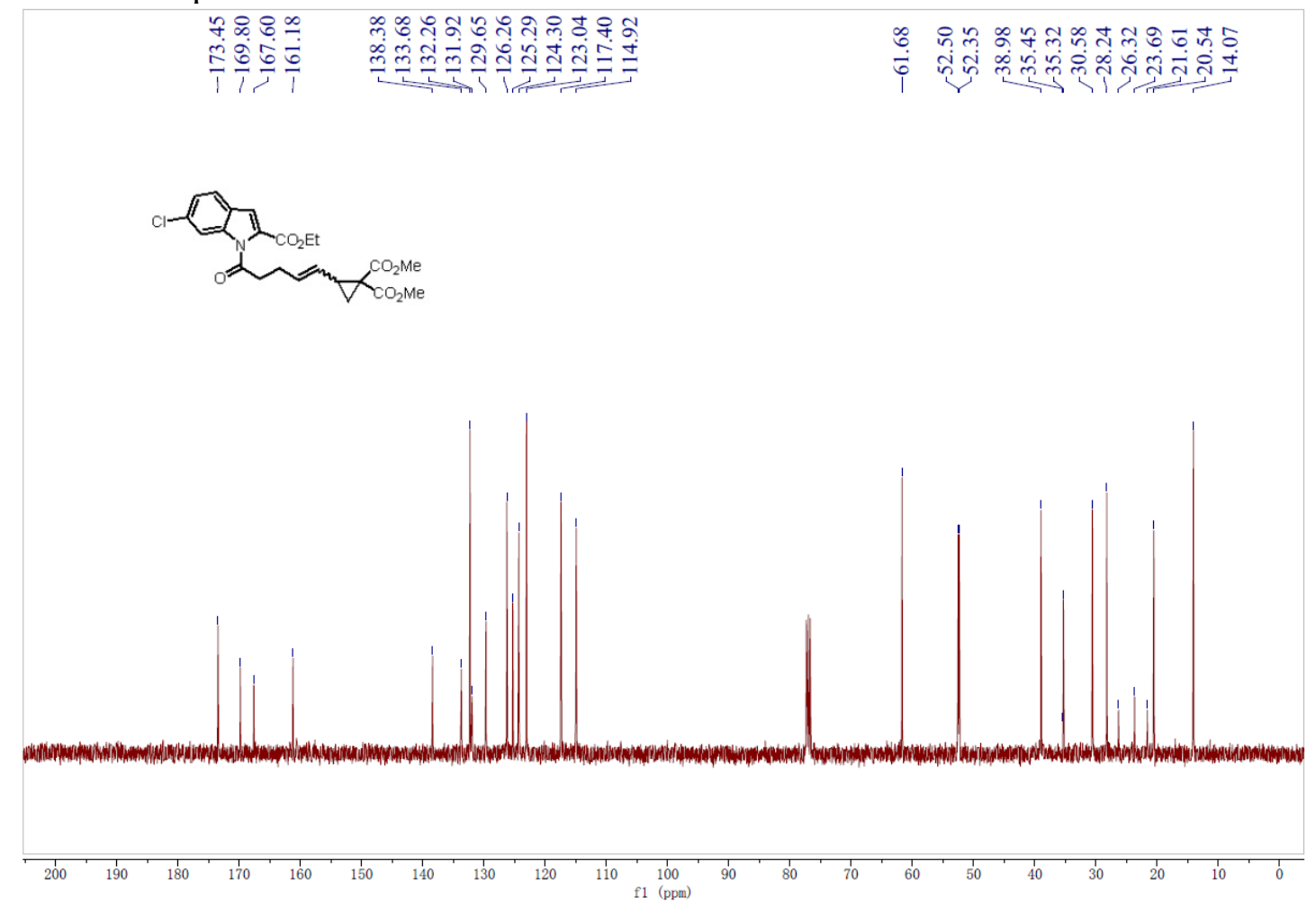


${ }^{1} \mathrm{H}$ NMR Spectrum of $\mathbf{1 p}$

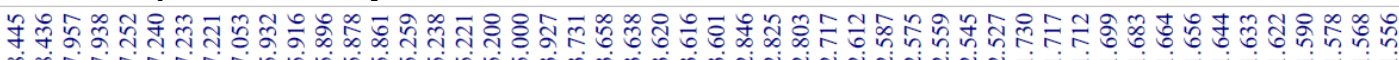

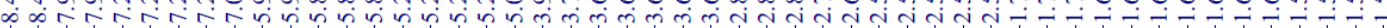

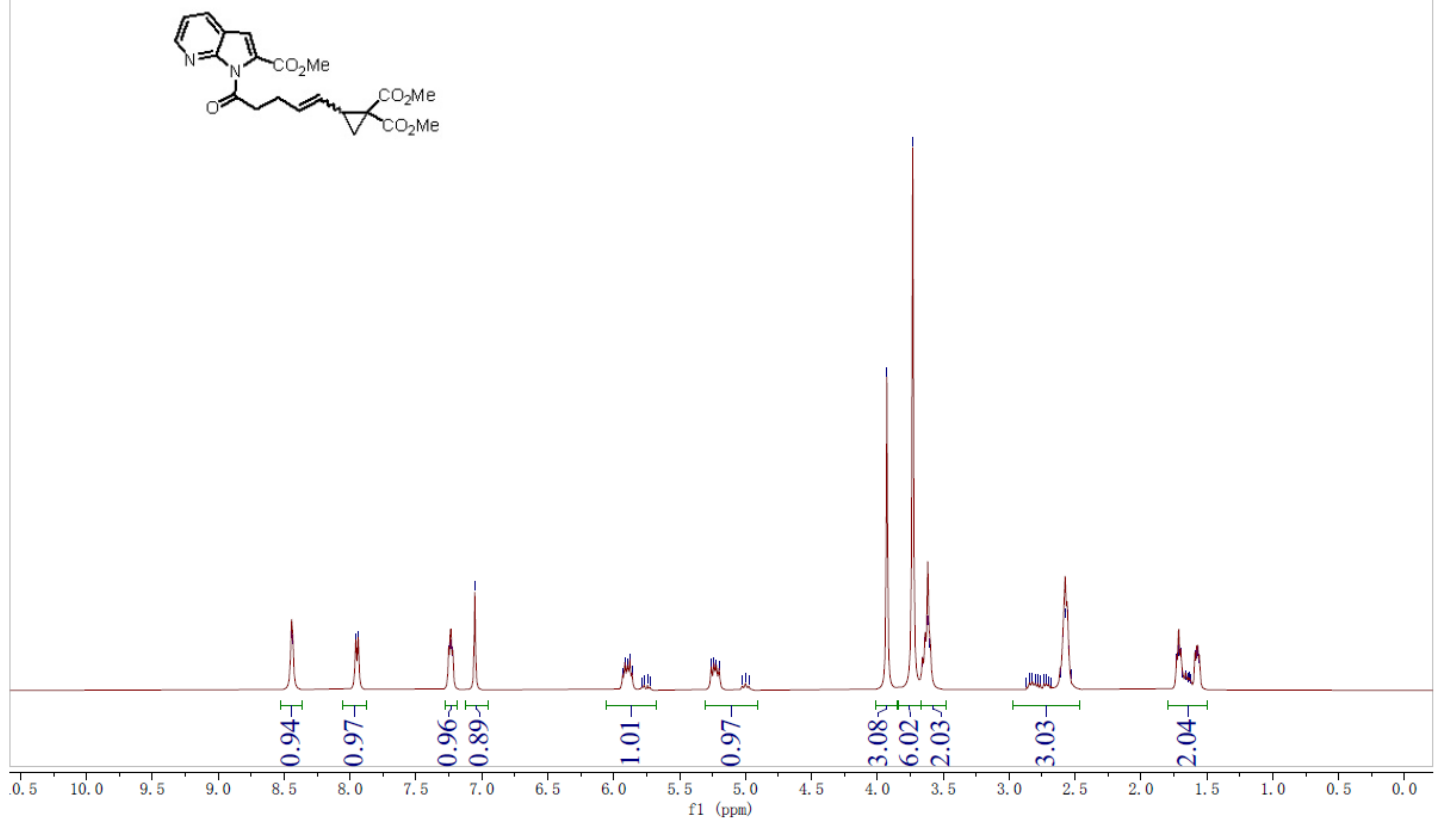

${ }^{13} \mathrm{C}$ NMR Spectrum of $\mathbf{1 p}$

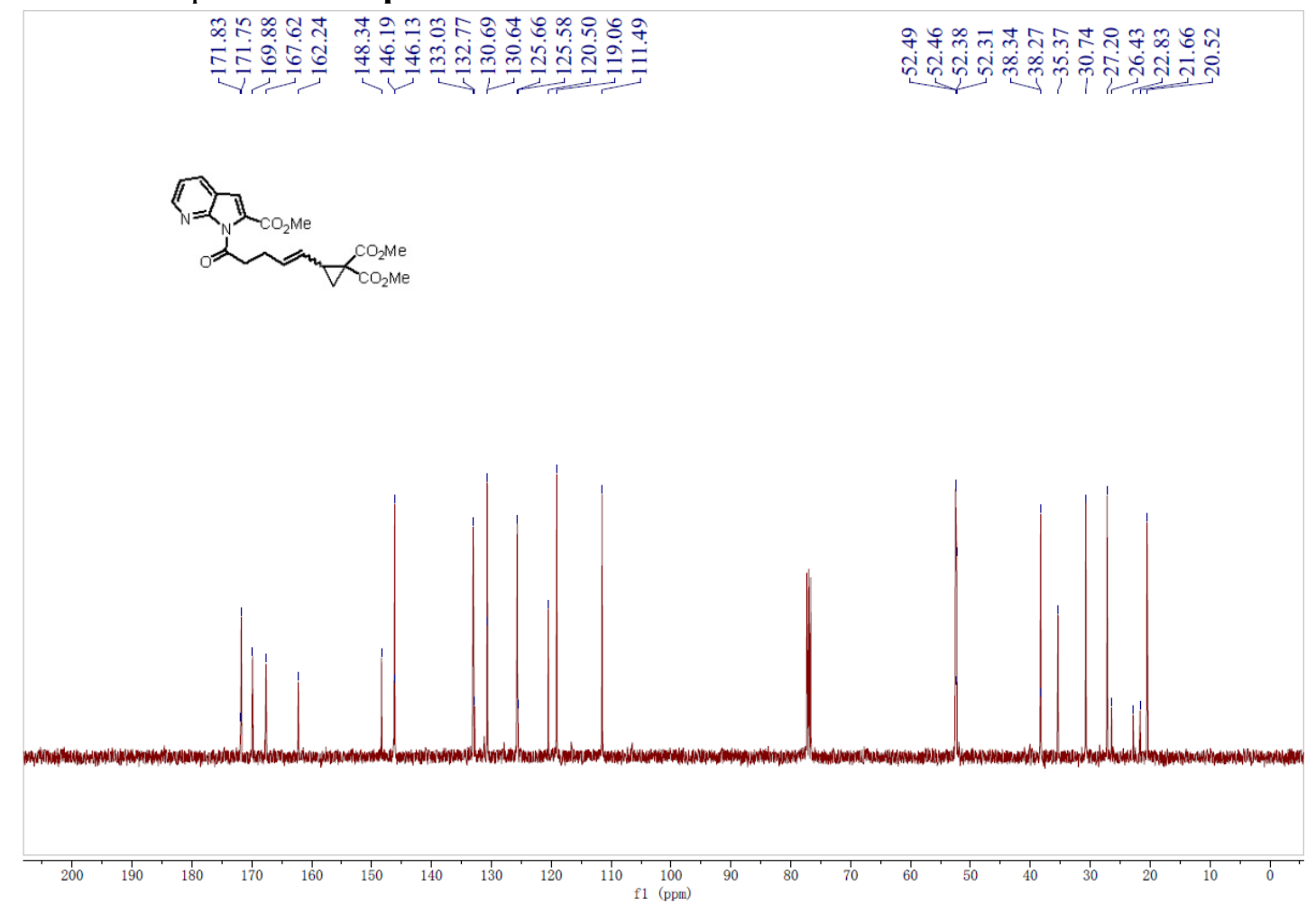


${ }^{1} \mathrm{H}$ NMR Spectrum of $\mathbf{1 q}$

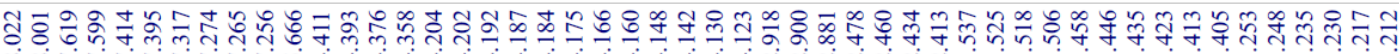

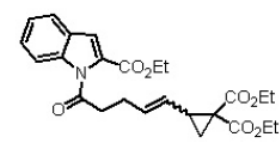

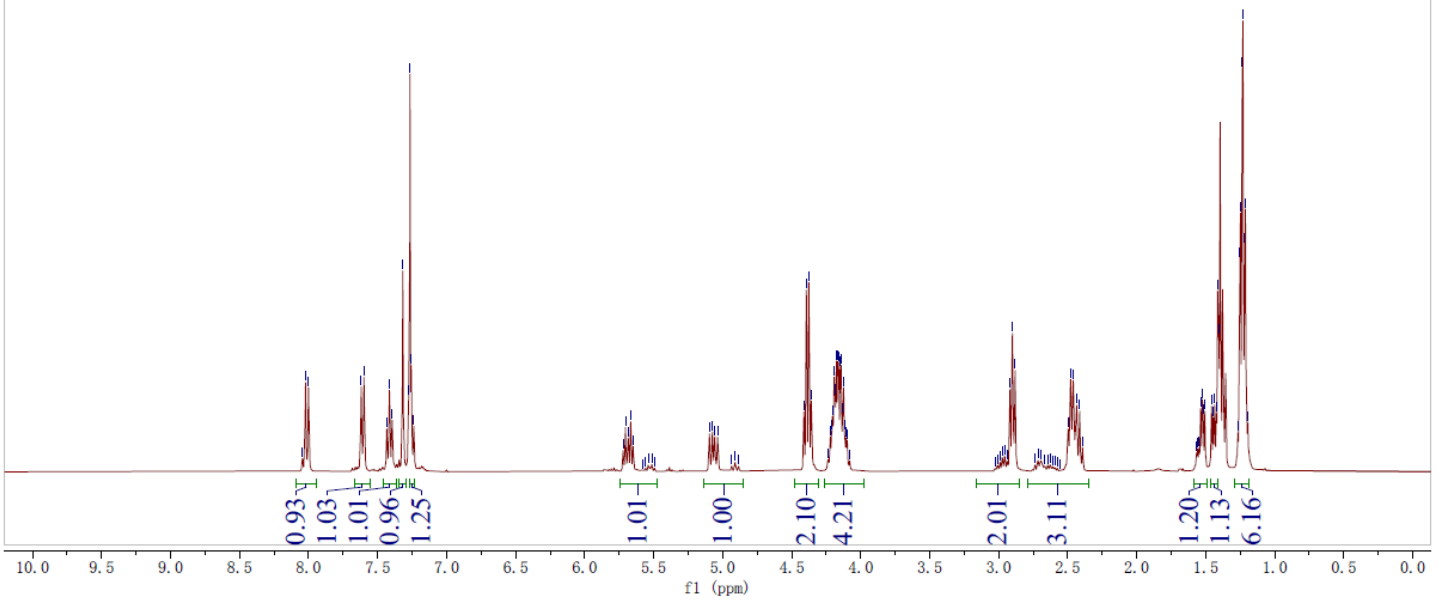

${ }^{13} \mathrm{C}$ NMR Spectrum of $\mathbf{1 q}$

\begin{tabular}{|c|c|c|c|}
\hline 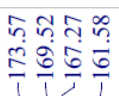 & 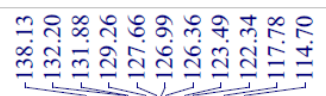 & 華告 & 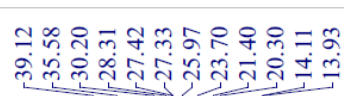 \\
\hline
\end{tabular}

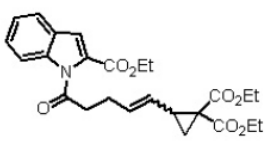

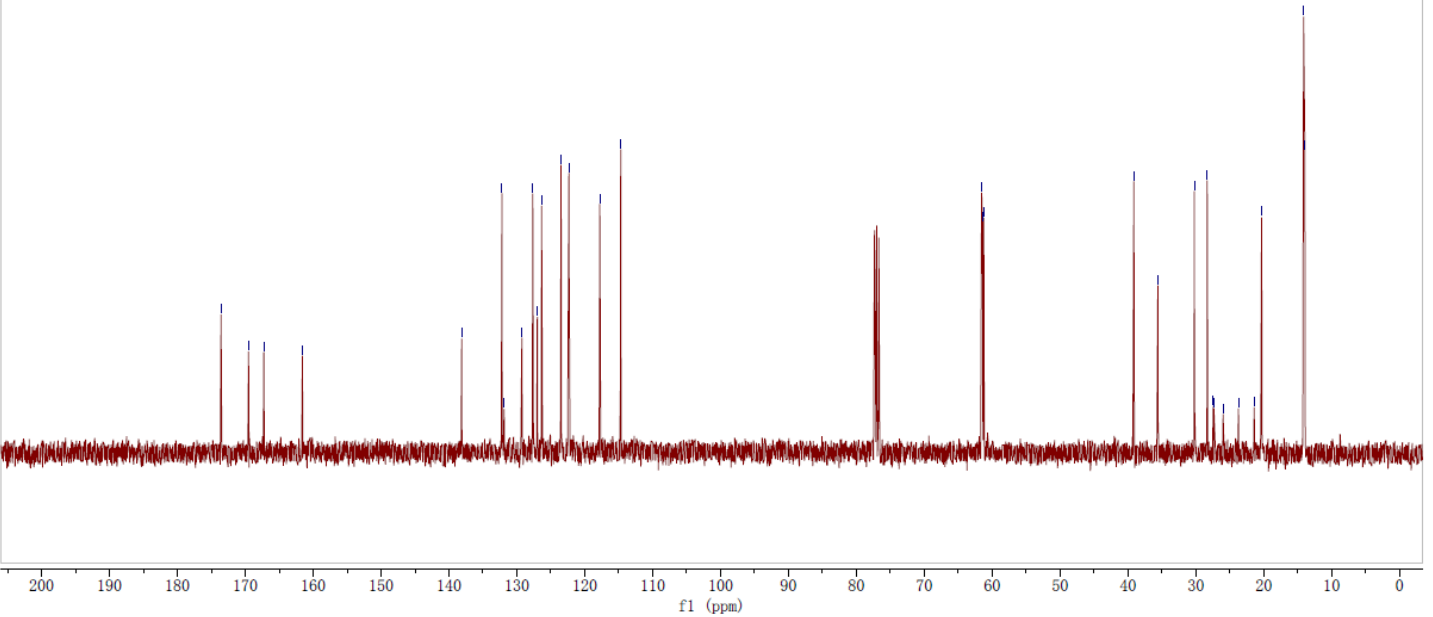


${ }^{1} \mathrm{H}$ NMR Spectrum of $\mathbf{1 r}$

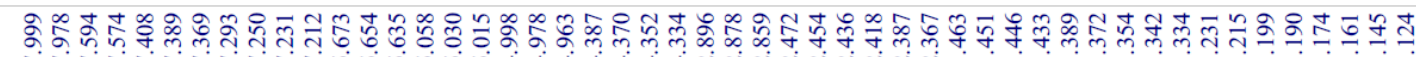

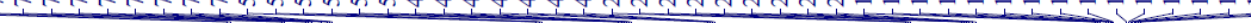
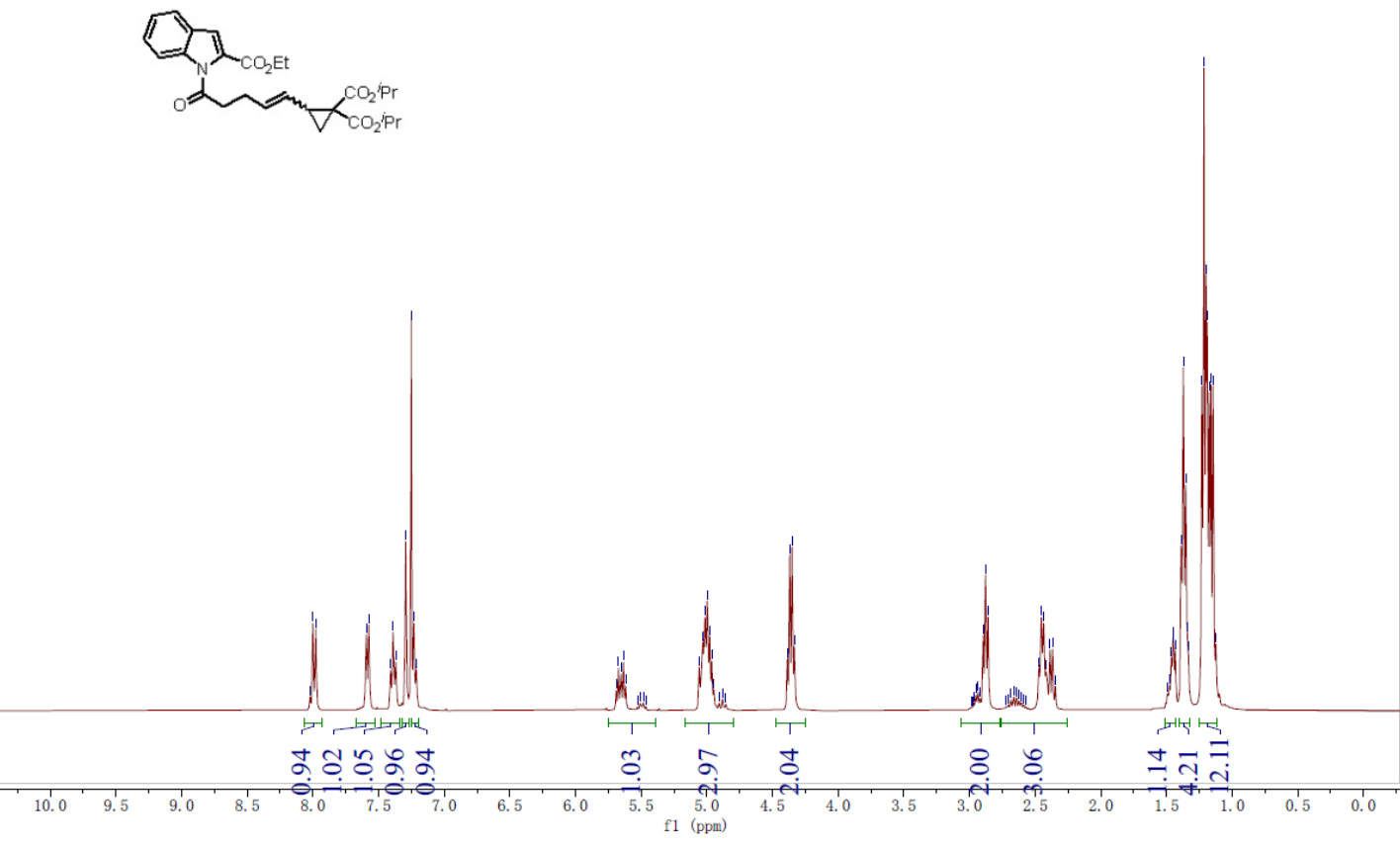

${ }^{13} \mathrm{C}$ NMR Spectrum of 1r

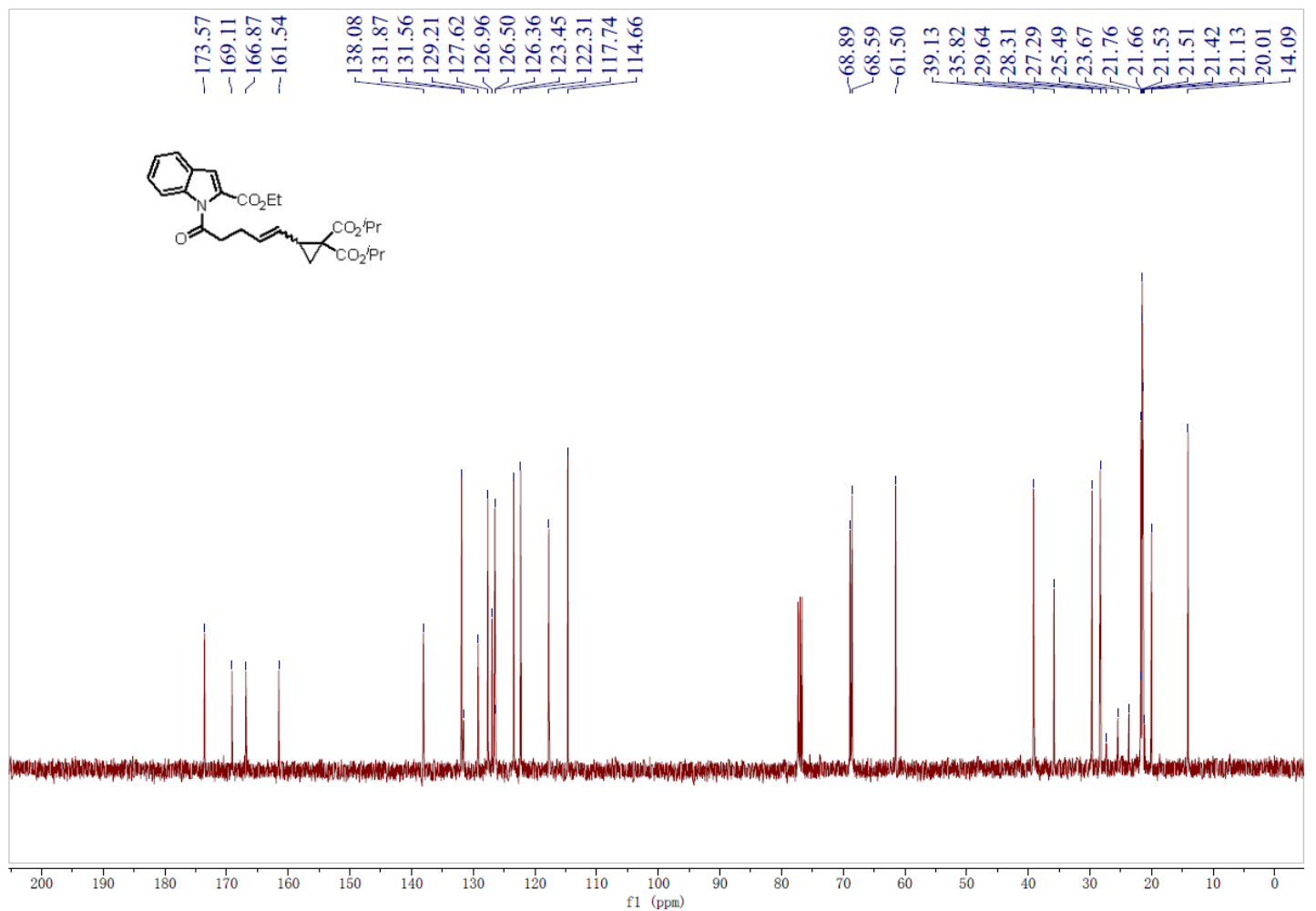




\section{${ }^{1} \mathrm{H}$ NMR Spectrum of $\mathbf{1 s}$}

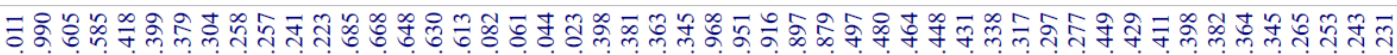
夜
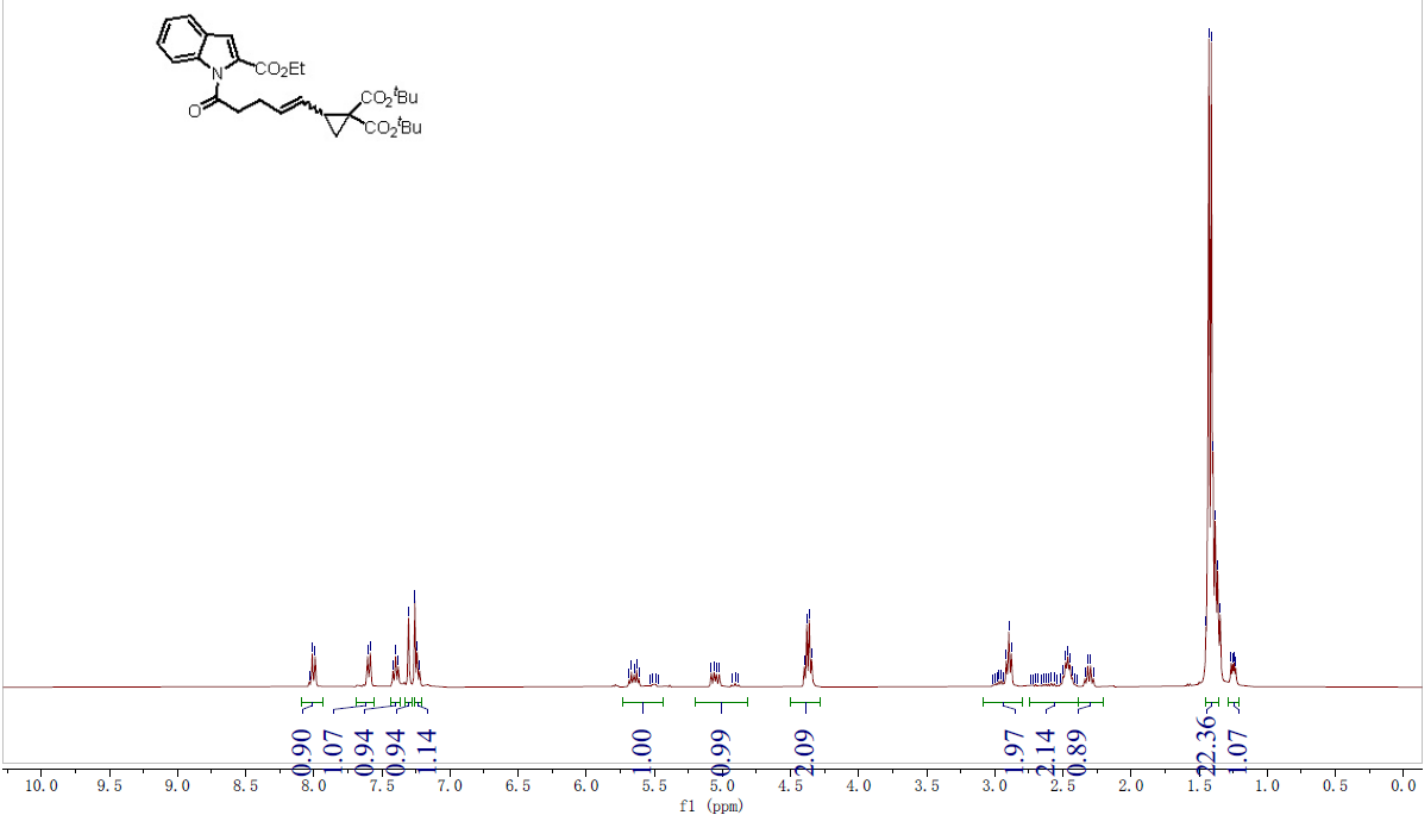

${ }^{13} \mathrm{C}$ NMR Spectrum of $\mathbf{1 s}$

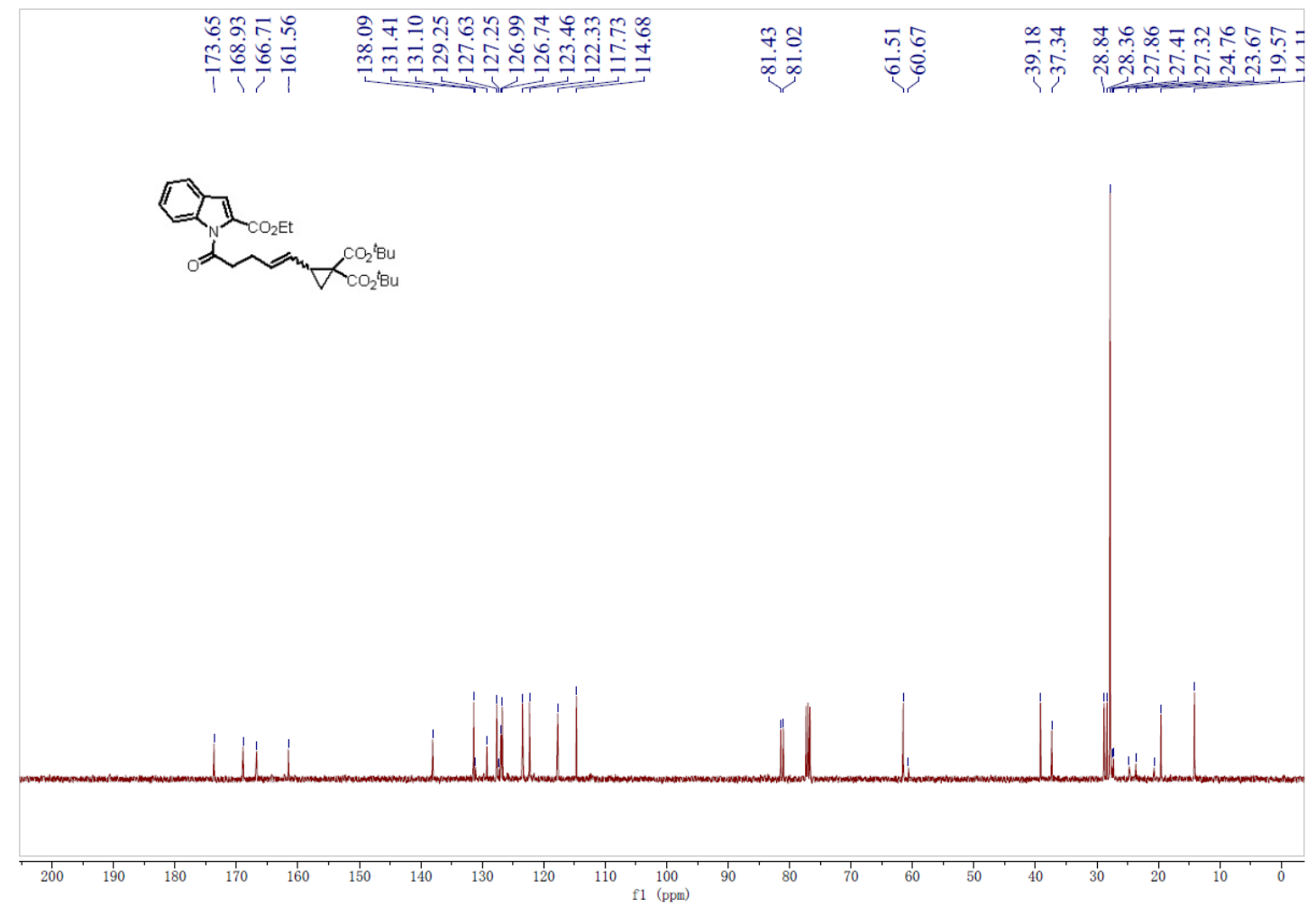


${ }^{1} \mathrm{H}$ NMR Spectrum of $\mathbf{1 t}$

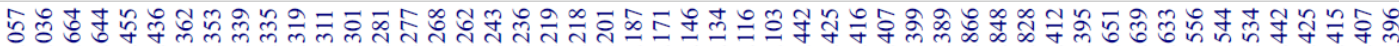

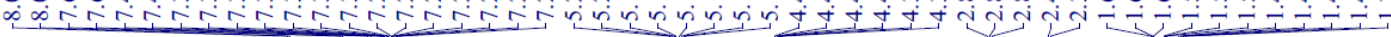

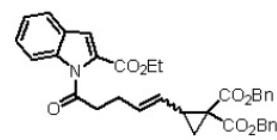

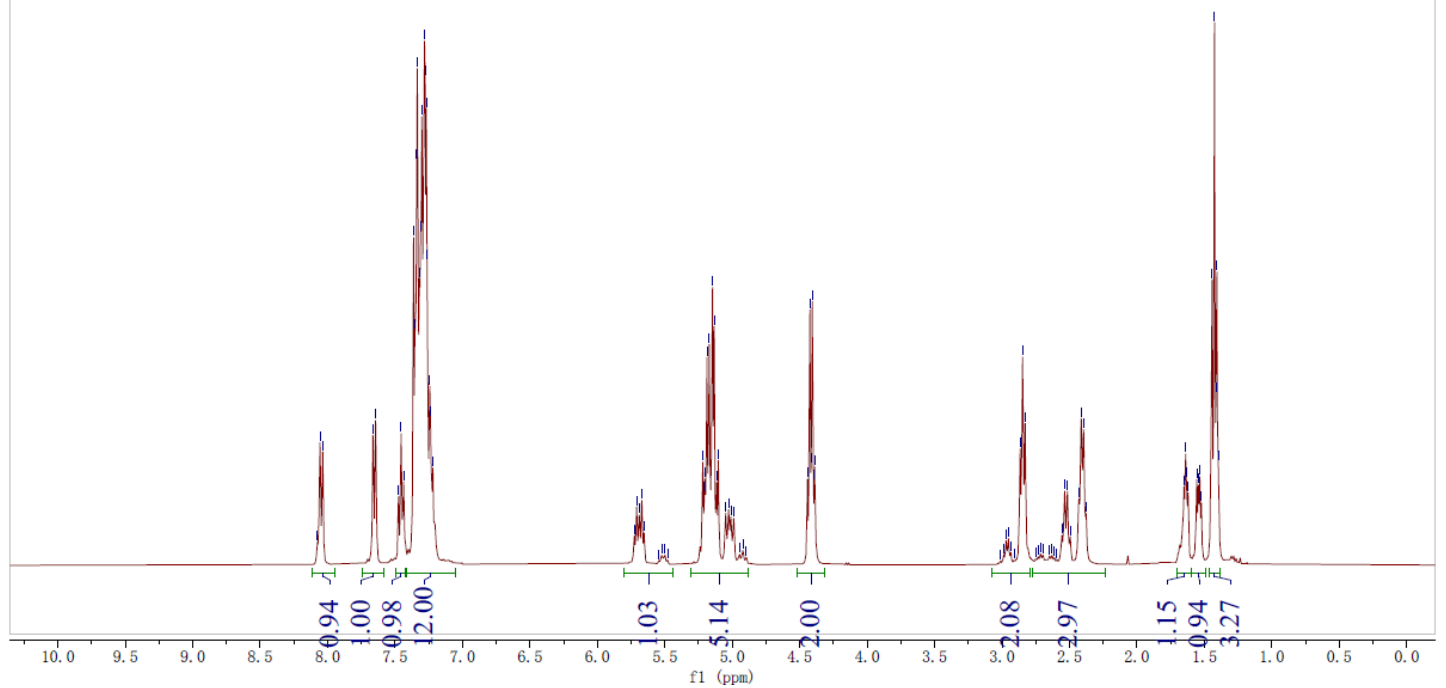

${ }^{13} \mathrm{C}$ NMR Spectrum of $\mathbf{1 t}$
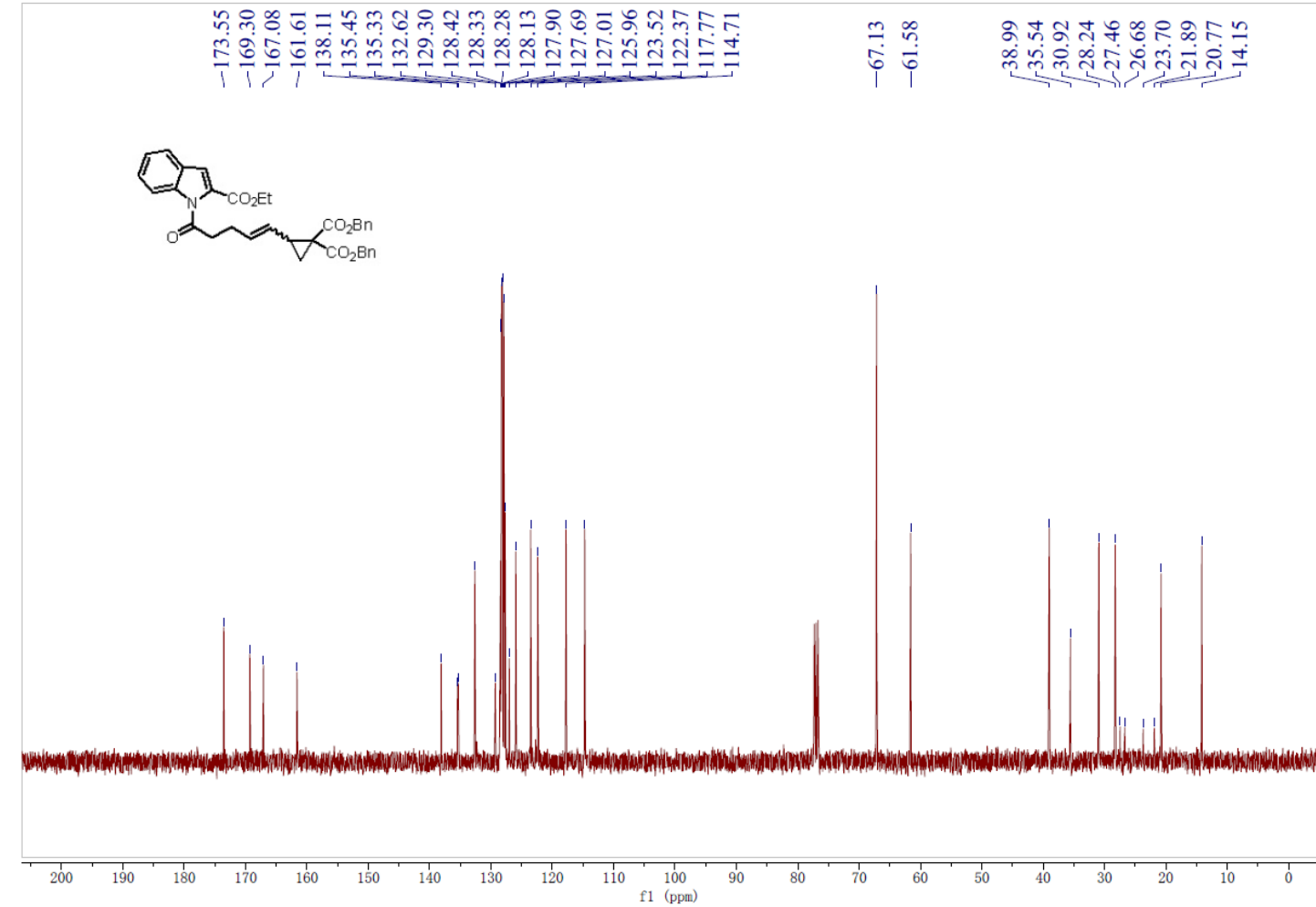
${ }^{1} \mathrm{H}$ NMR Spectrum of $\mathbf{1 u}$

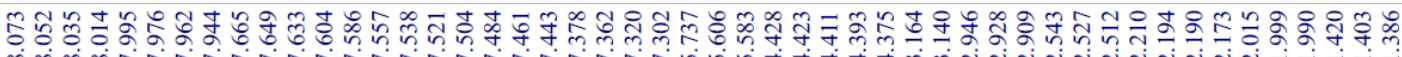

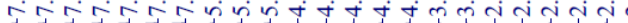

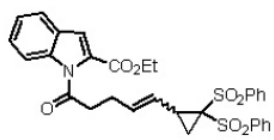

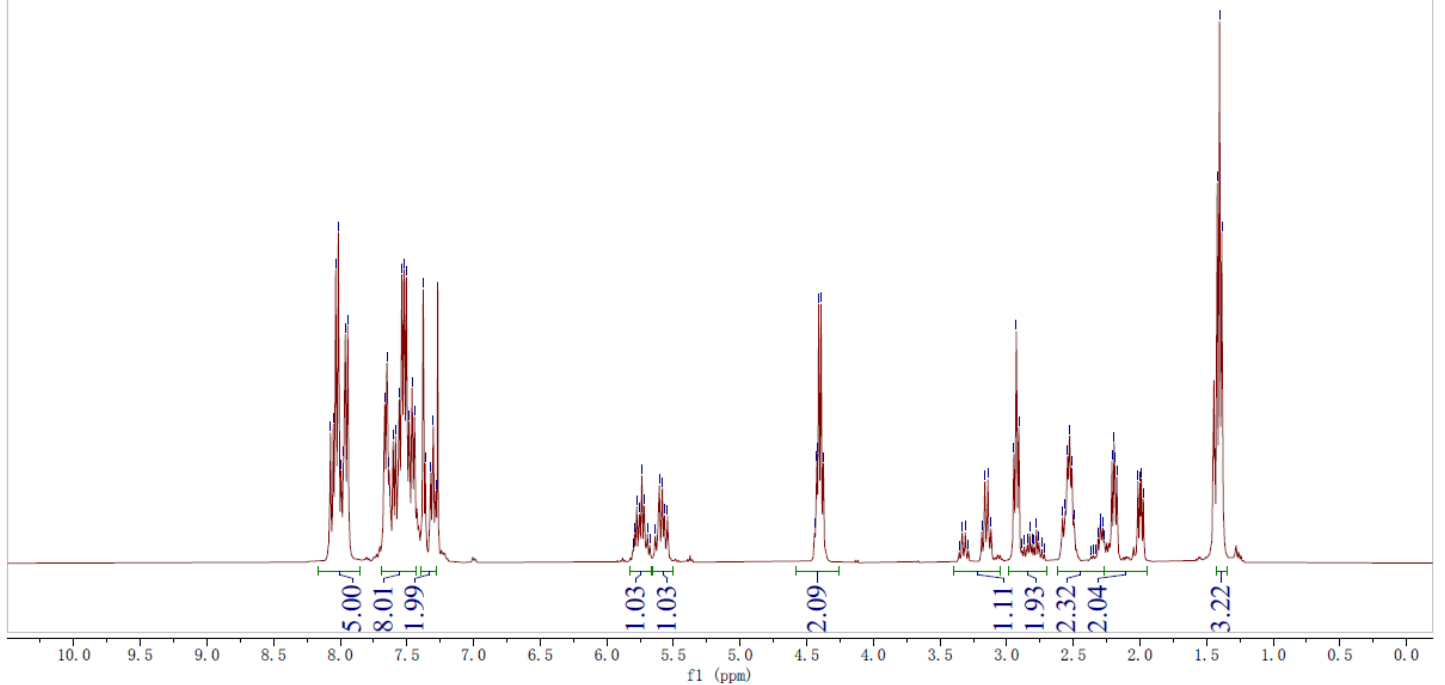

${ }^{13} \mathrm{C}$ NMR Spectrum of $\mathbf{1} \mathbf{u}$

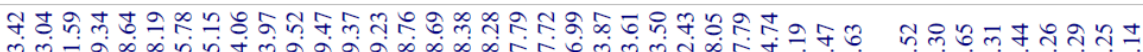
mo
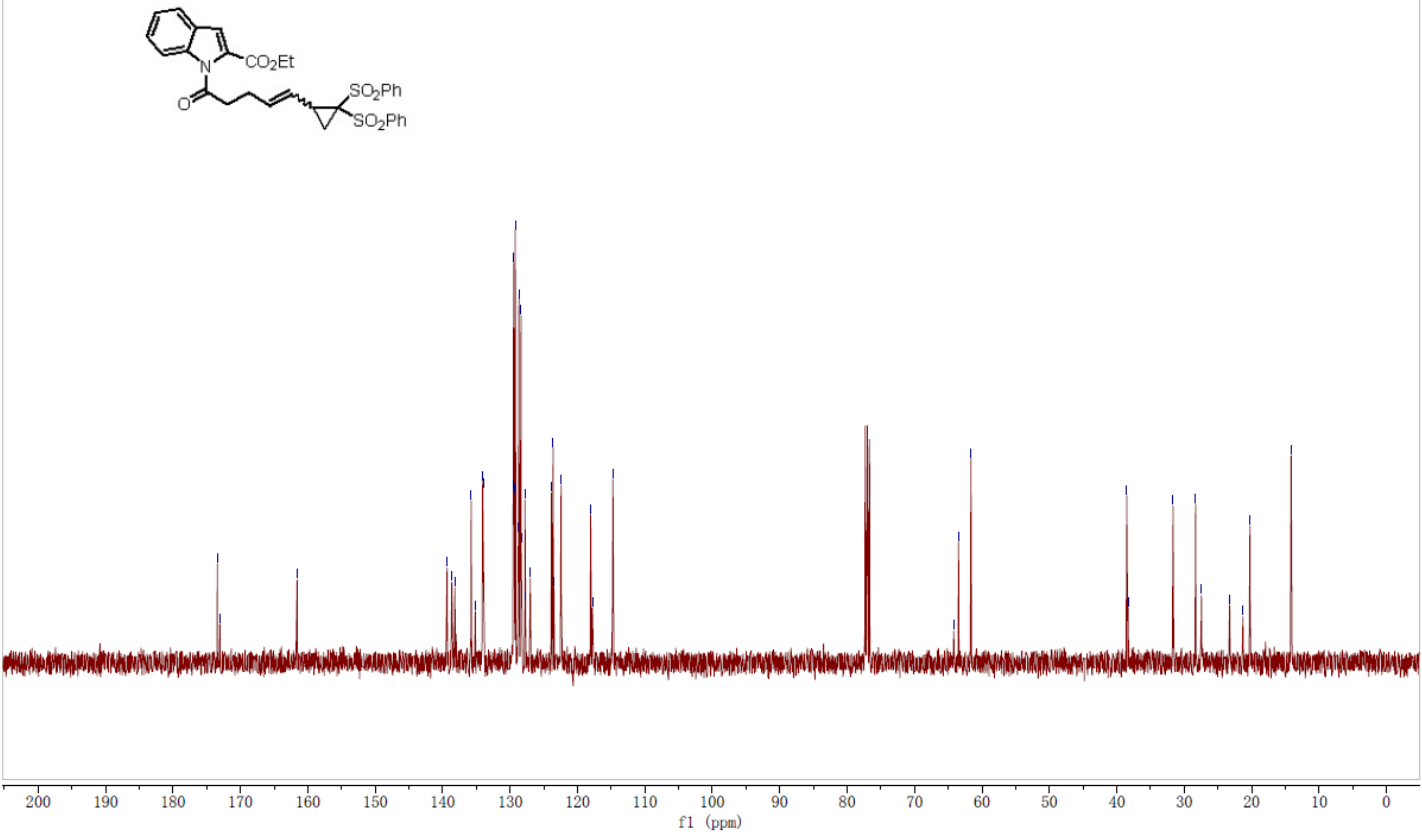
${ }^{1} \mathrm{H}$ NMR Spectrum of $\mathbf{1 v}$

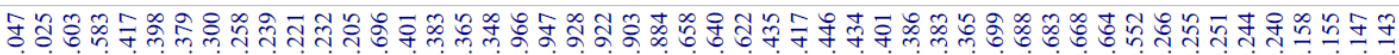

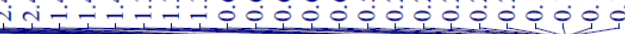
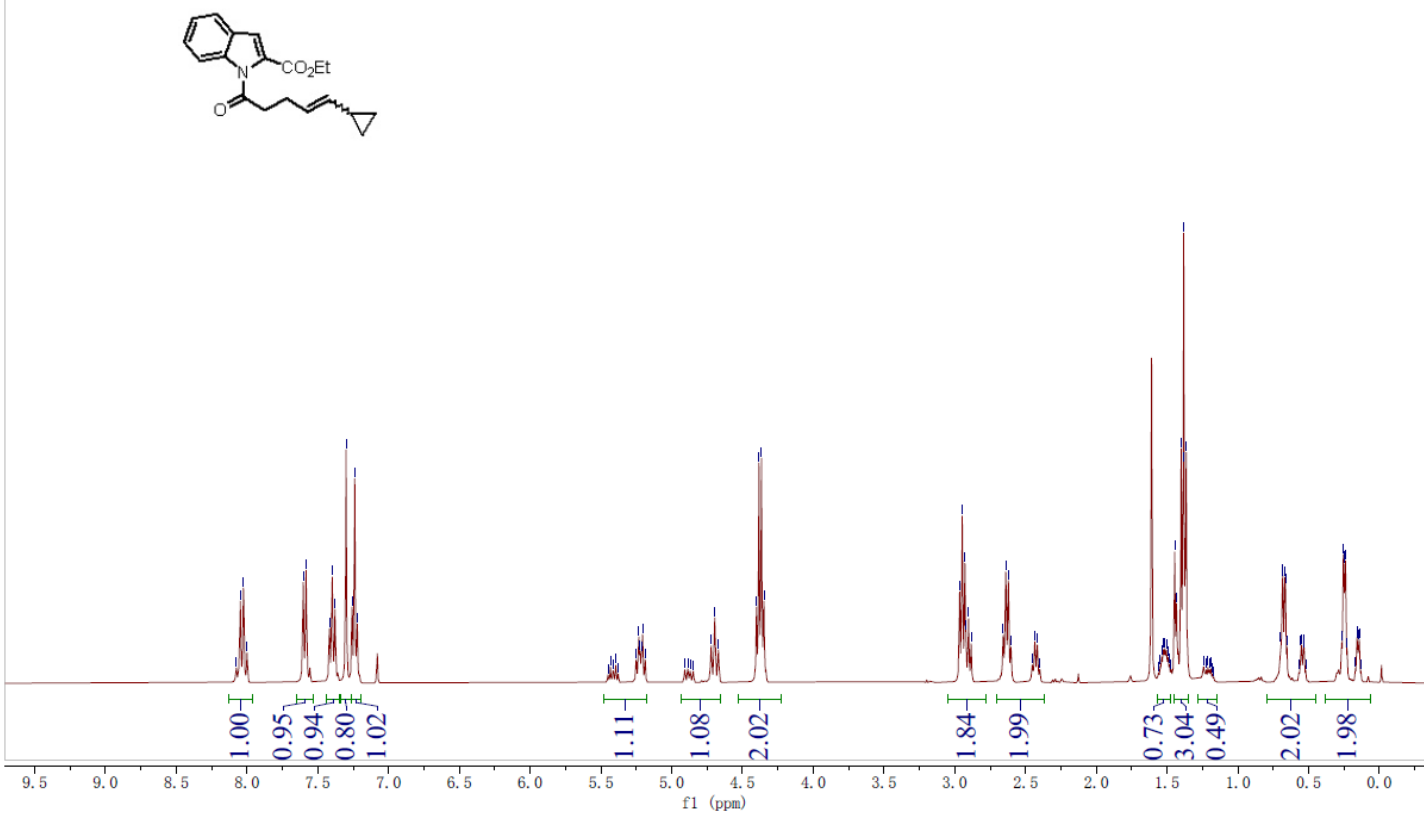

${ }^{13} \mathrm{C}$ NMR Spectrum of $\mathbf{1 v}$

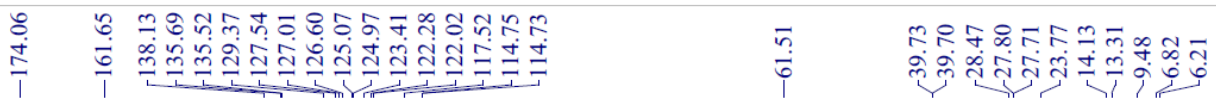<smiles>CCCC=CCC(=O)c1ccccc1OCC</smiles>

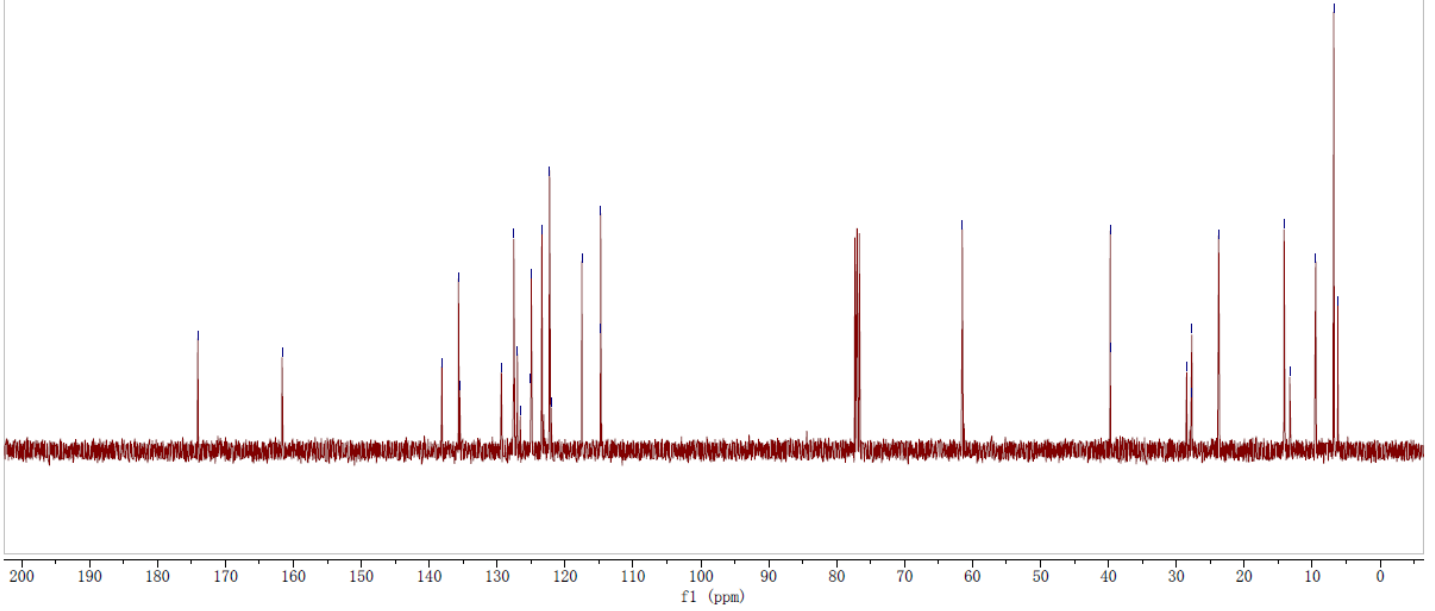


${ }^{1} \mathrm{H}$ NMR Spectrum of $\mathbf{1 w}$

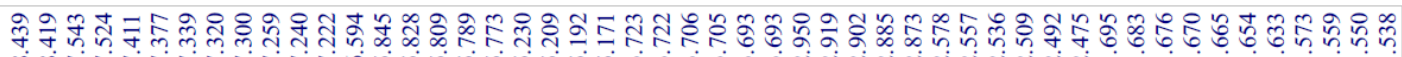

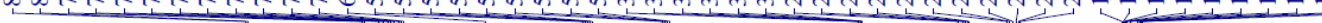
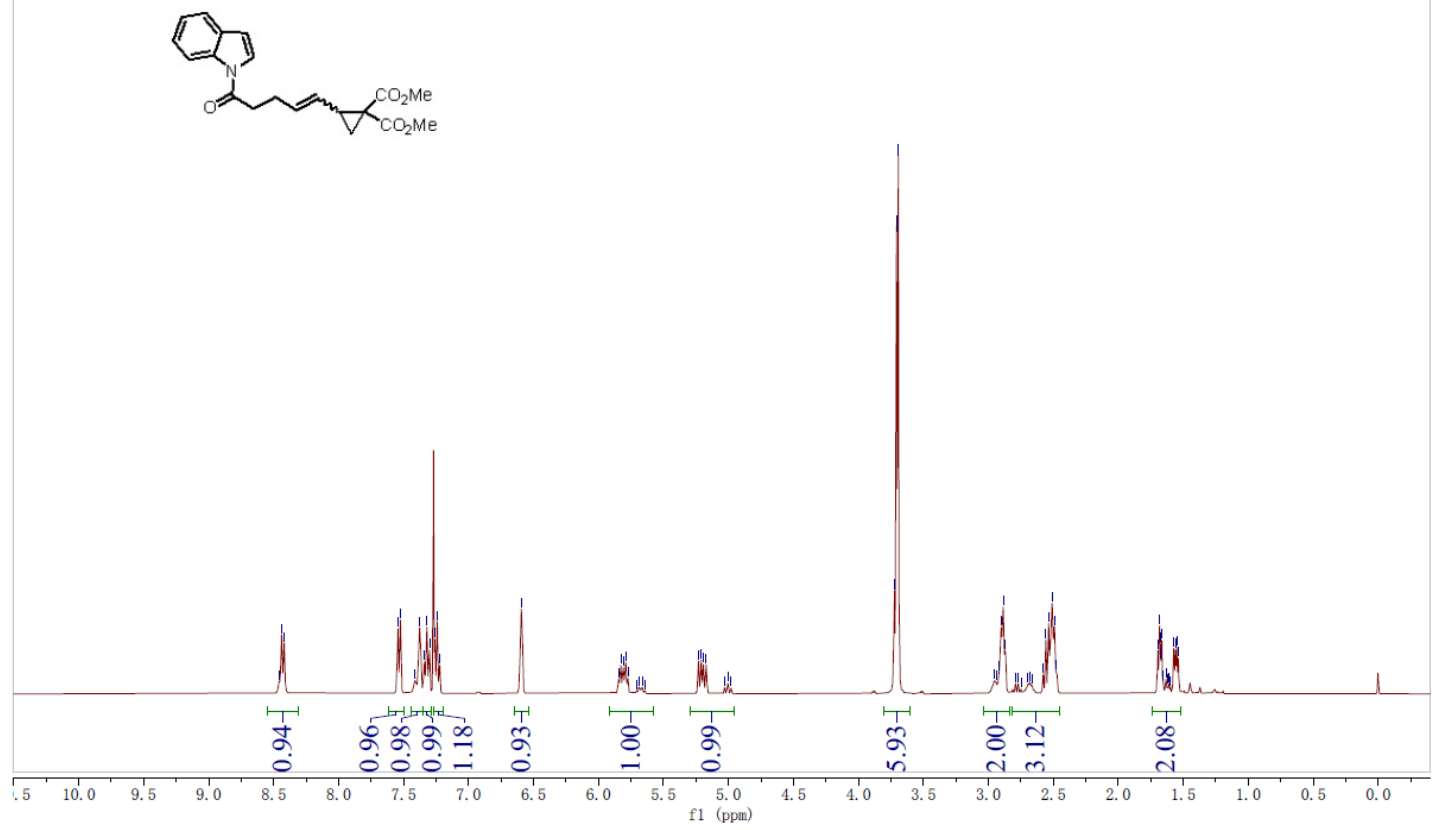

${ }^{13} \mathrm{C}$ NMR Spectrum of $\mathbf{1 w}$

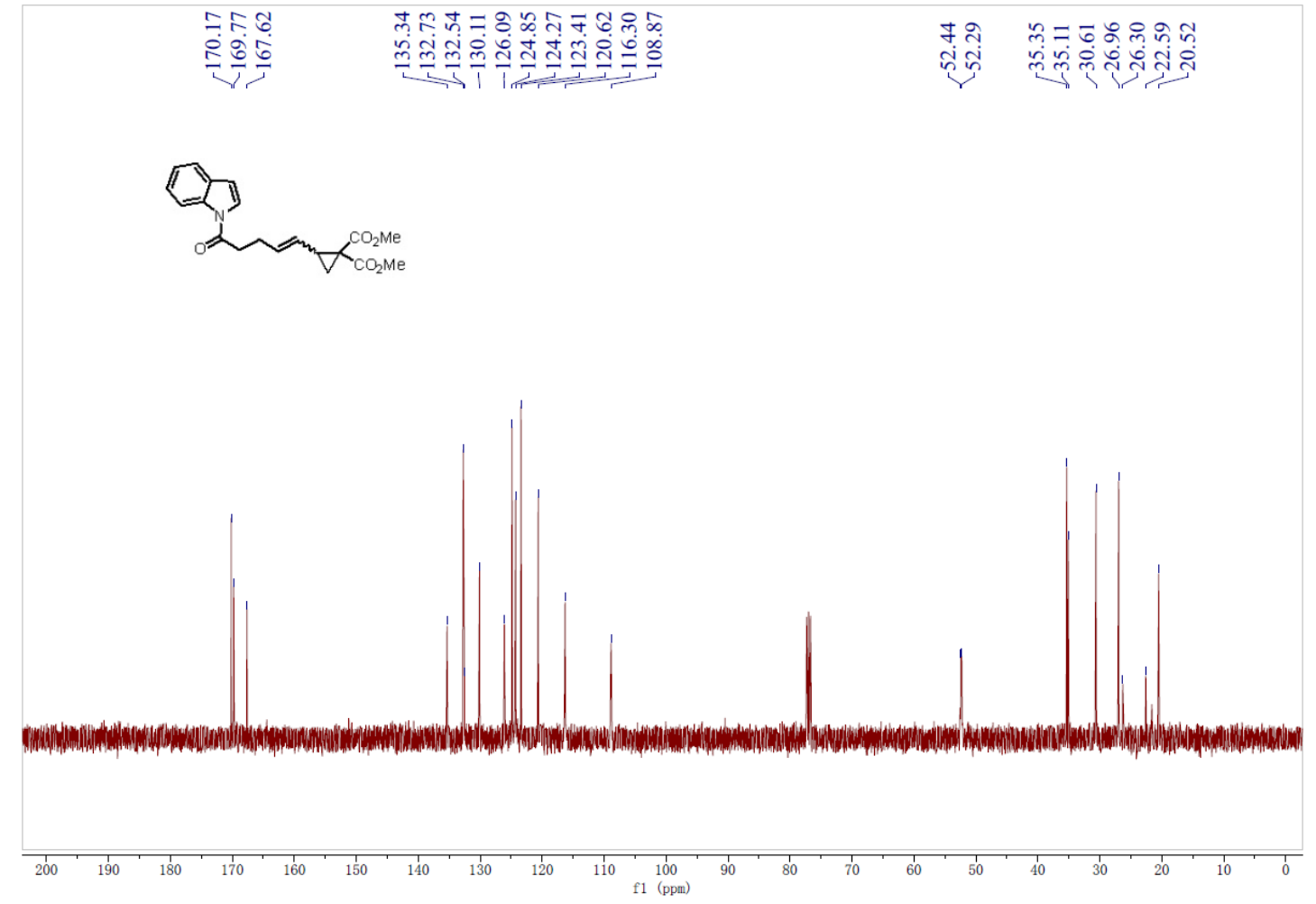


${ }^{1} \mathrm{H}$ NMR Spectrum of $\mathbf{1 x}$

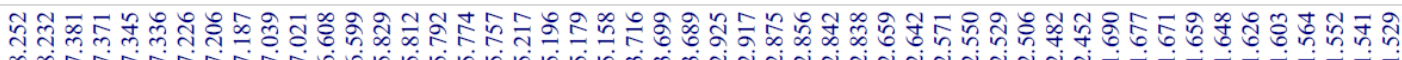

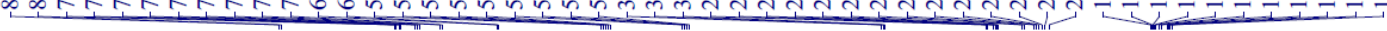<smiles>COC(C)(C)CC=CCC(=O)n1cccc2cccc1-2</smiles>

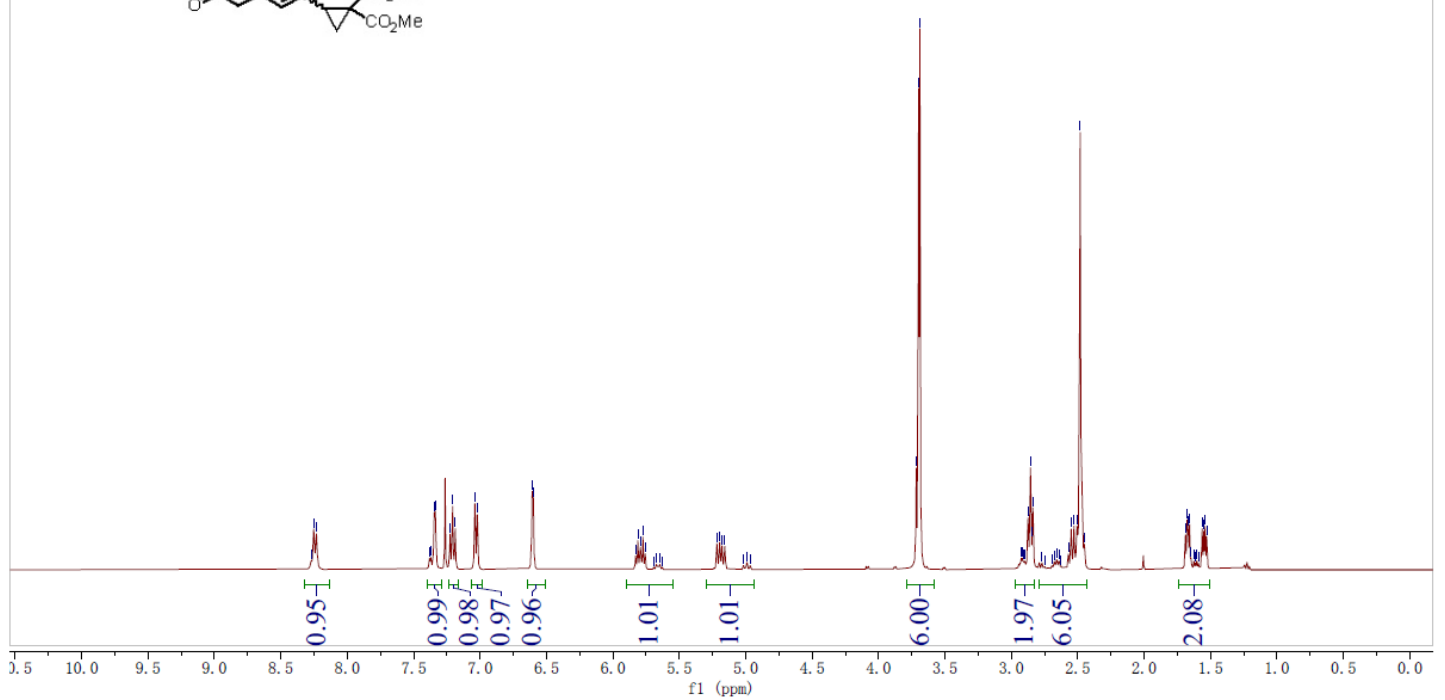

${ }^{13} \mathrm{C}$ NMR Spectrum of $\mathbf{1 x}$

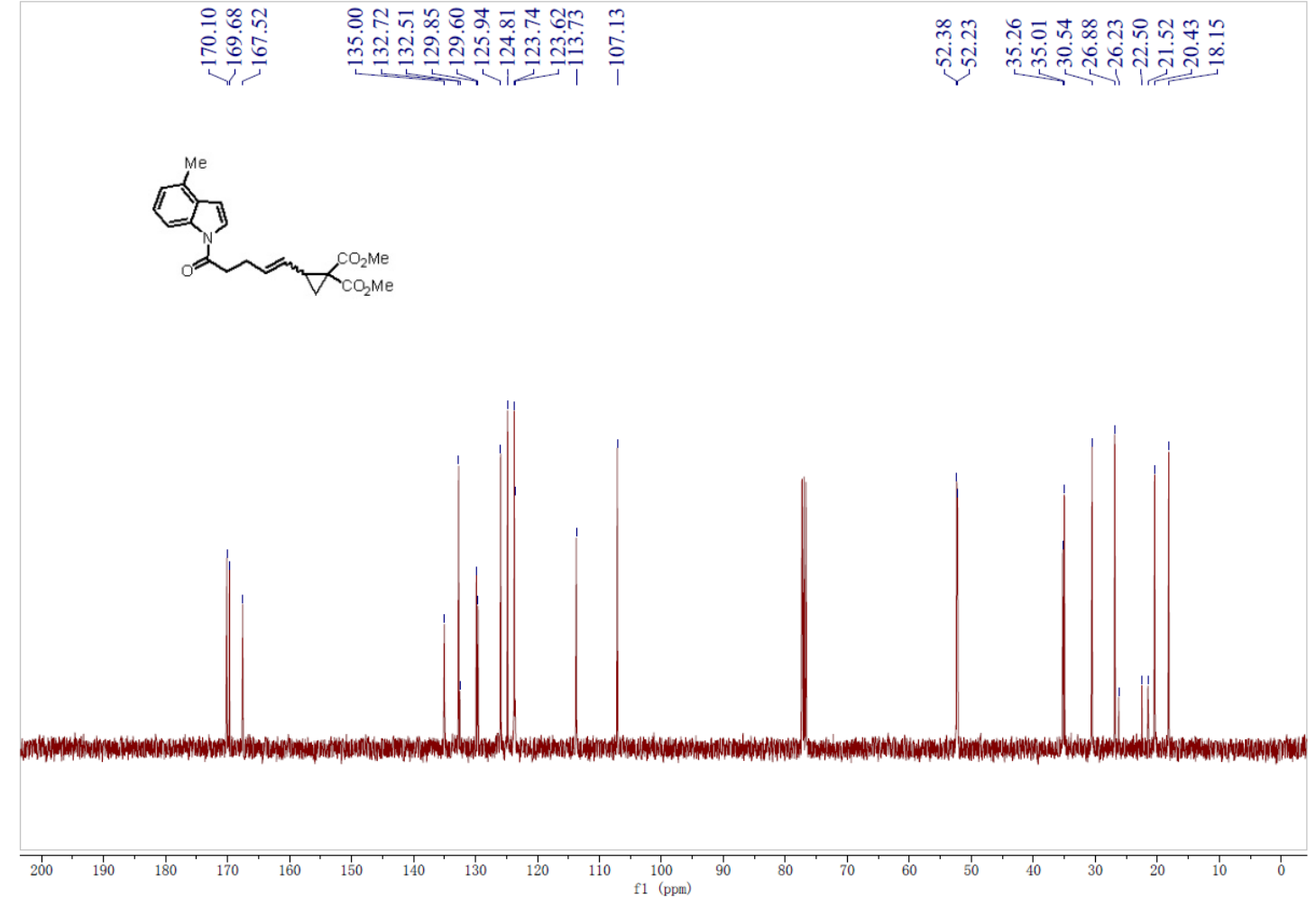


${ }^{1} \mathrm{H}$ NMR Spectrum of $\mathbf{1 y}$

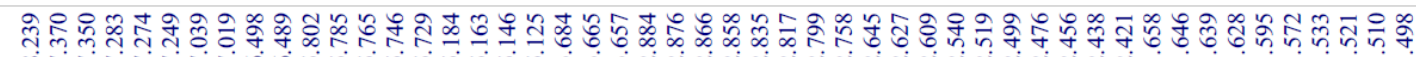
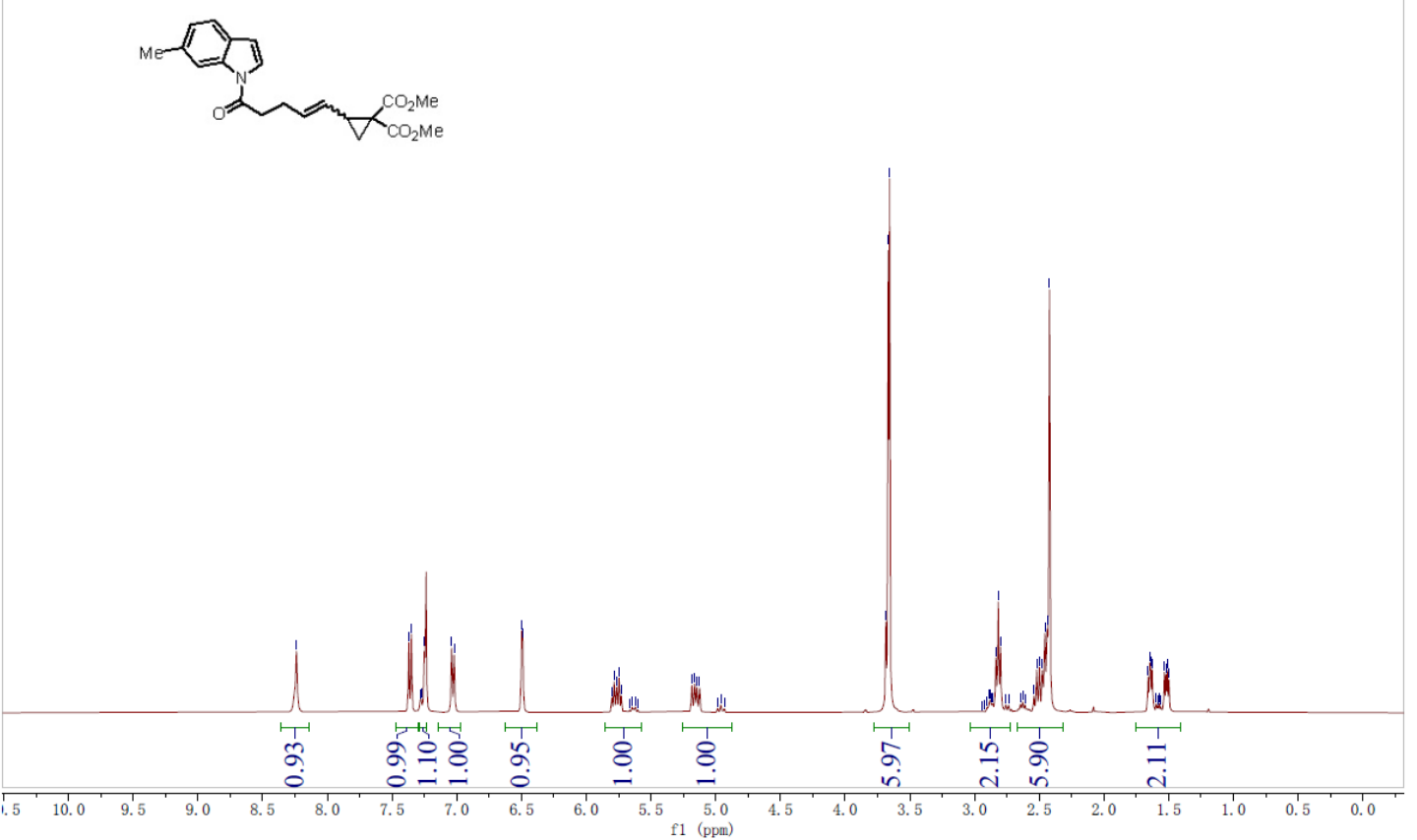

${ }^{13} \mathrm{C}$ NMR Spectrum of $\mathbf{1 y}$

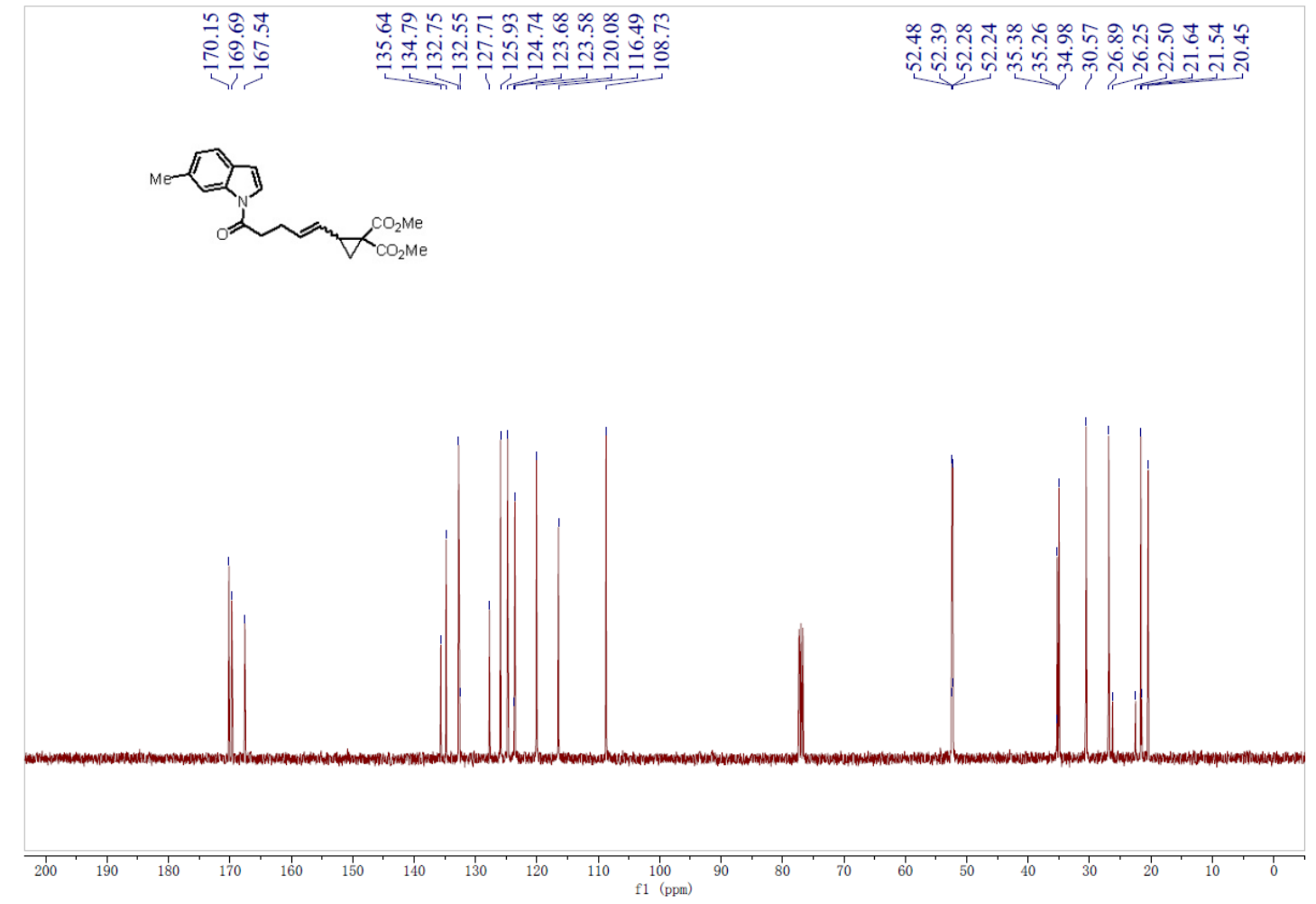


${ }^{1} \mathrm{H}$ NMR Spectrum of $\mathbf{1 z}$

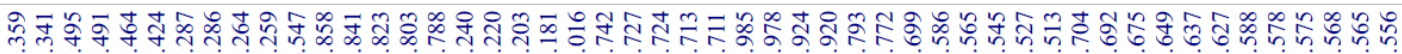

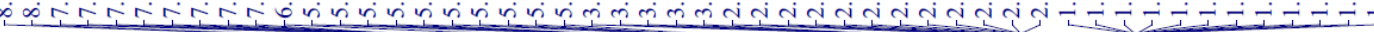

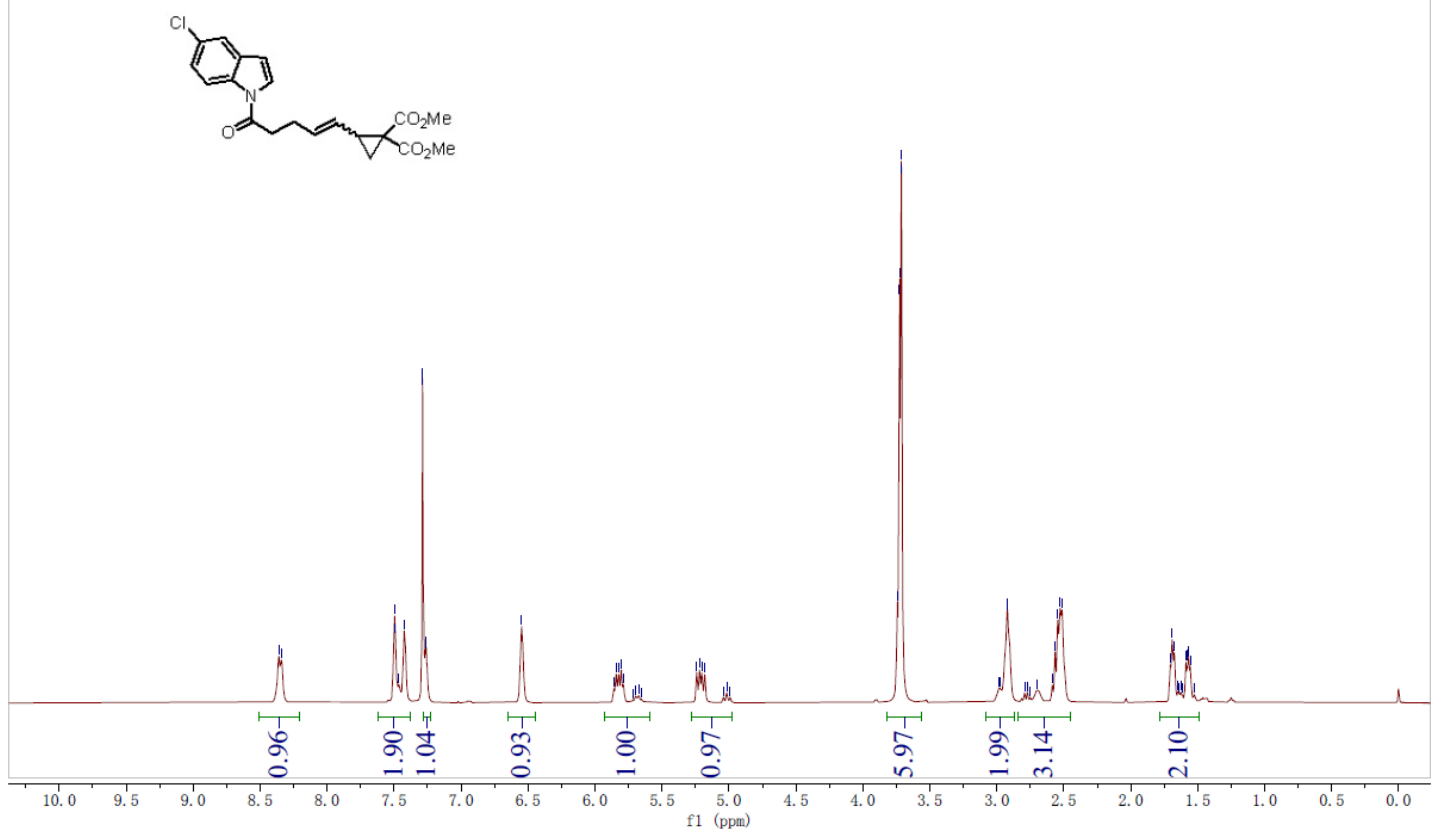

${ }^{13} \mathrm{C}$ NMR Spectrum of $\mathbf{1 z}$

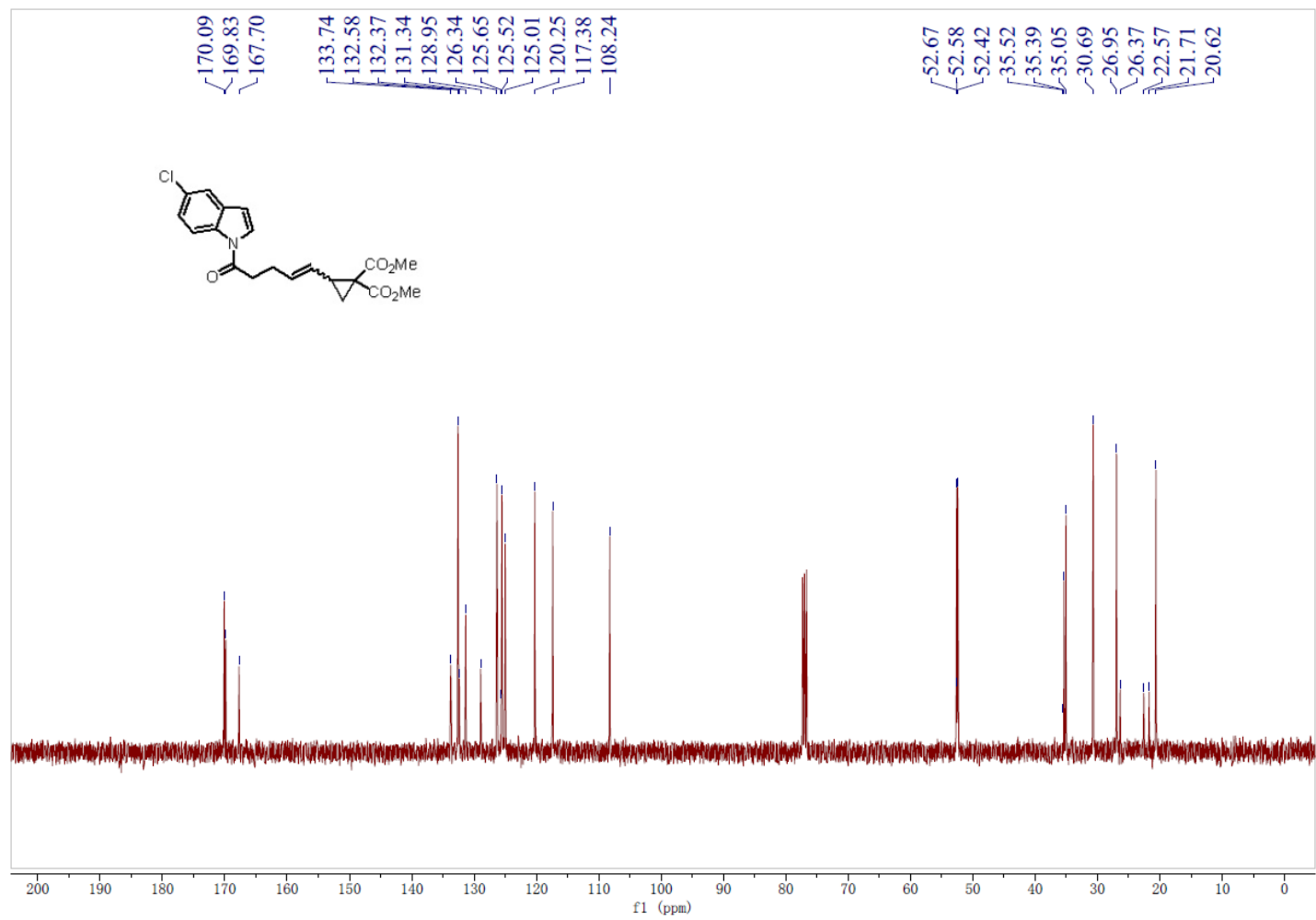


${ }^{1} \mathrm{H}$ NMR Spectrum of $\mathbf{1 a a}$

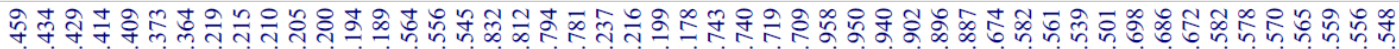

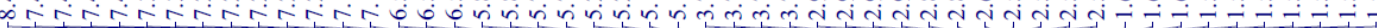

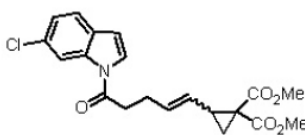

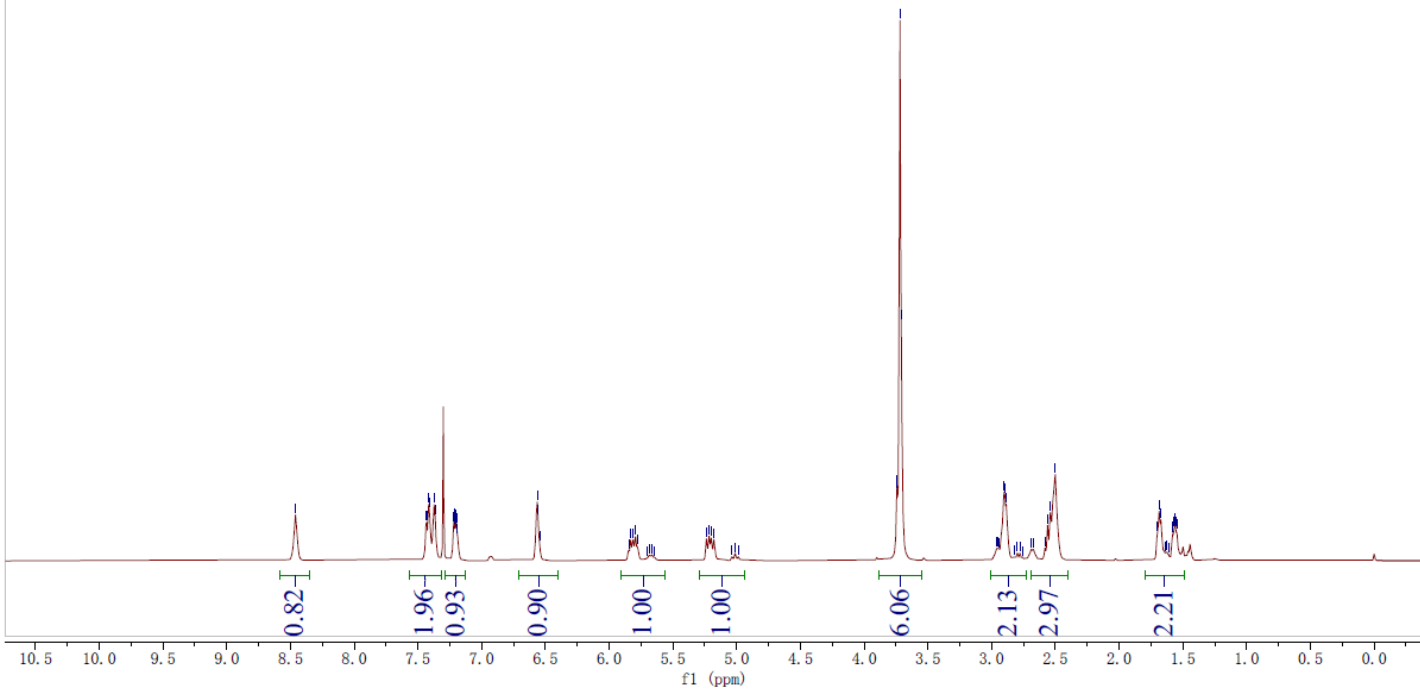

${ }^{13} \mathrm{C}$ NMR Spectrum of $\mathbf{1 a a}$
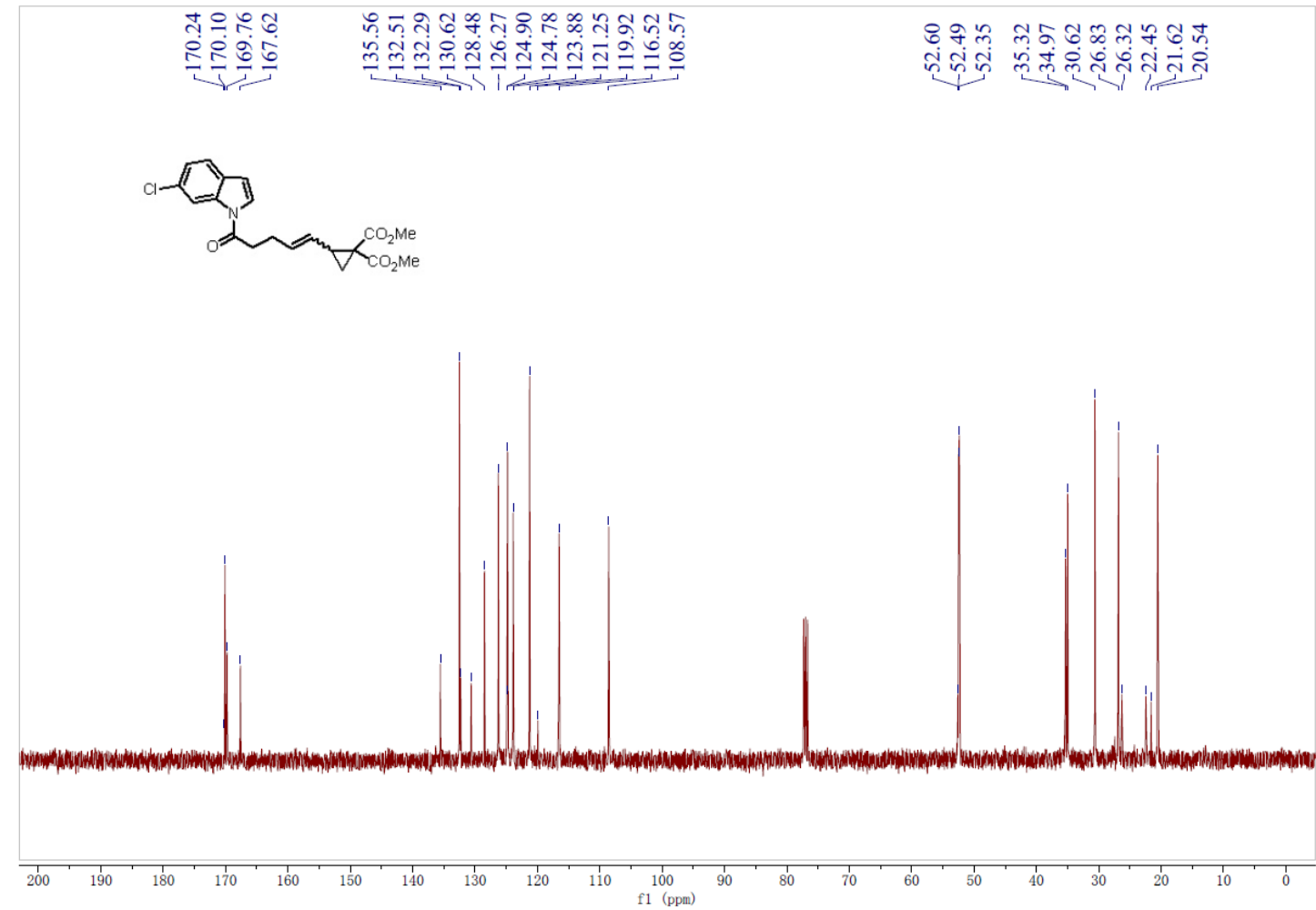
${ }^{1} \mathrm{H}$ NMR Spectrum of $\mathbf{1 a b}$

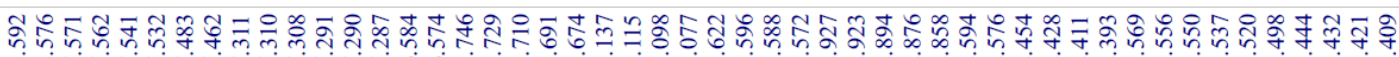

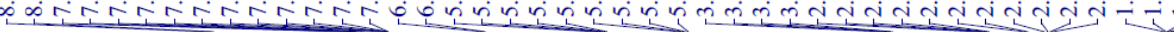
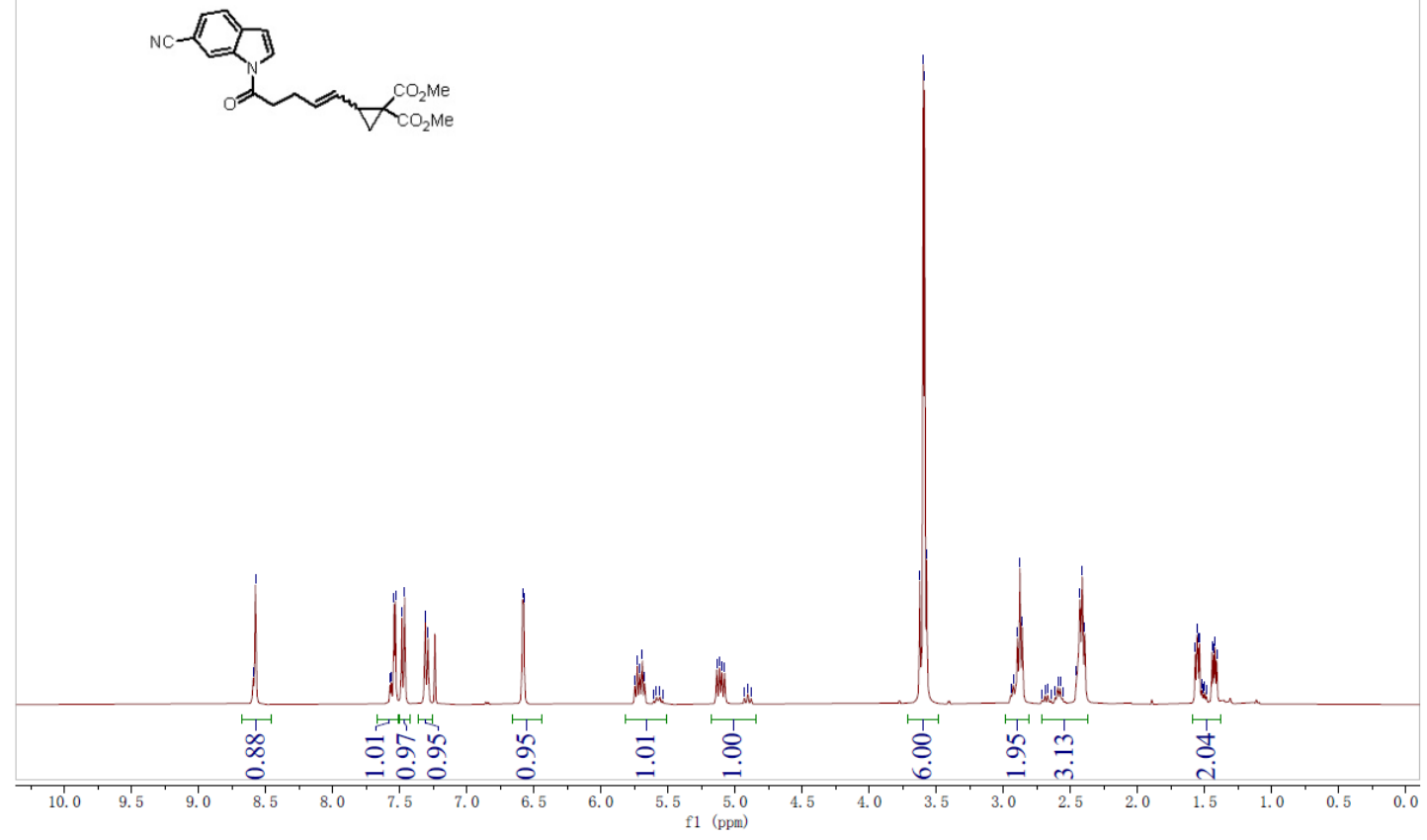

${ }^{13} \mathrm{C}$ NMR Spectrum of $\mathbf{1 a b}$

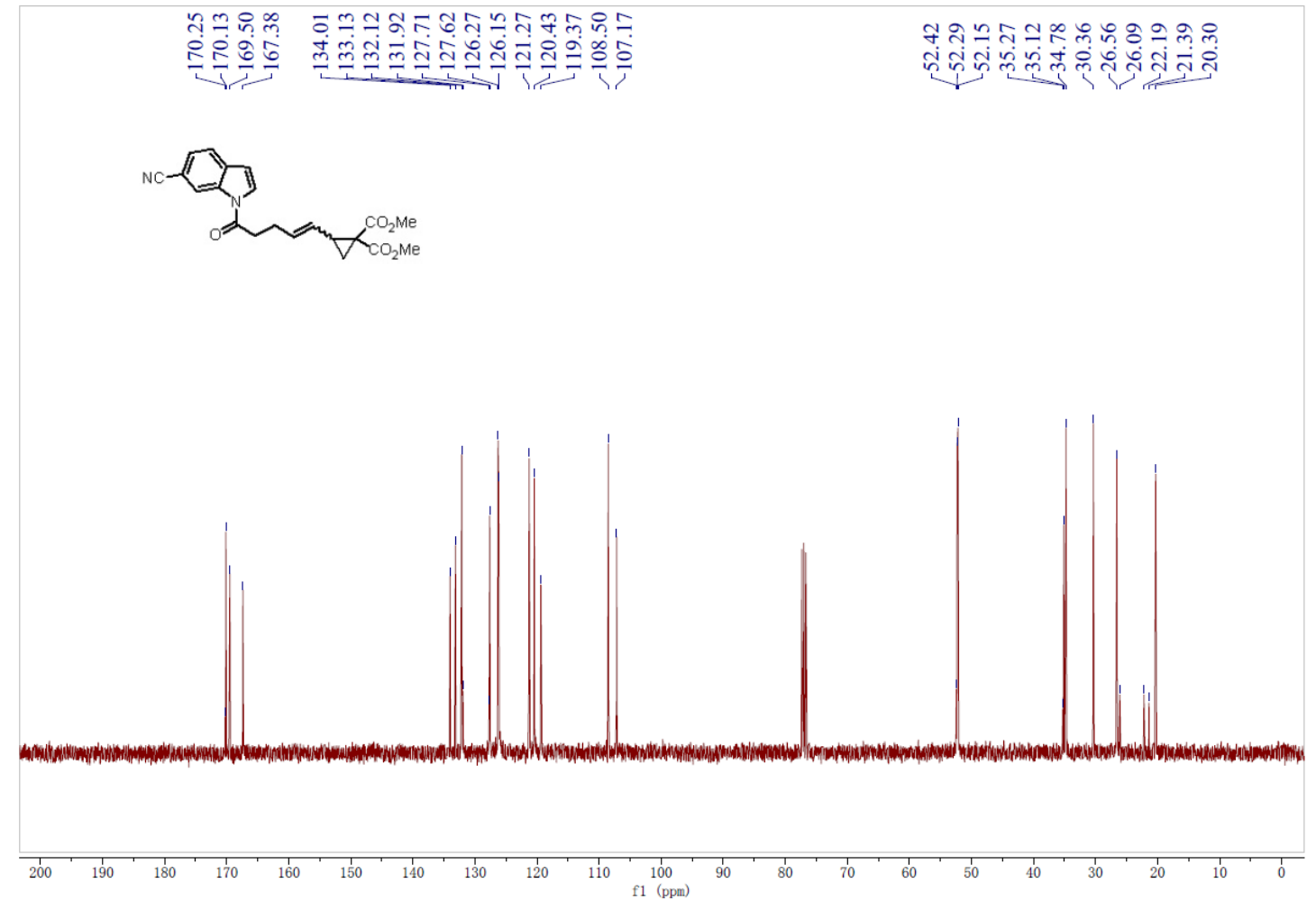


${ }^{1} \mathrm{H}$ NMR Spectrum of $\mathbf{1 a c}$

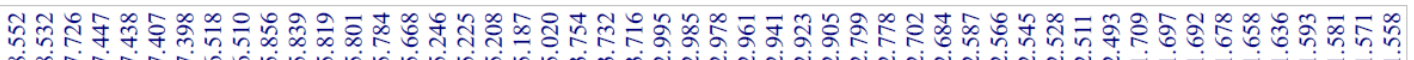
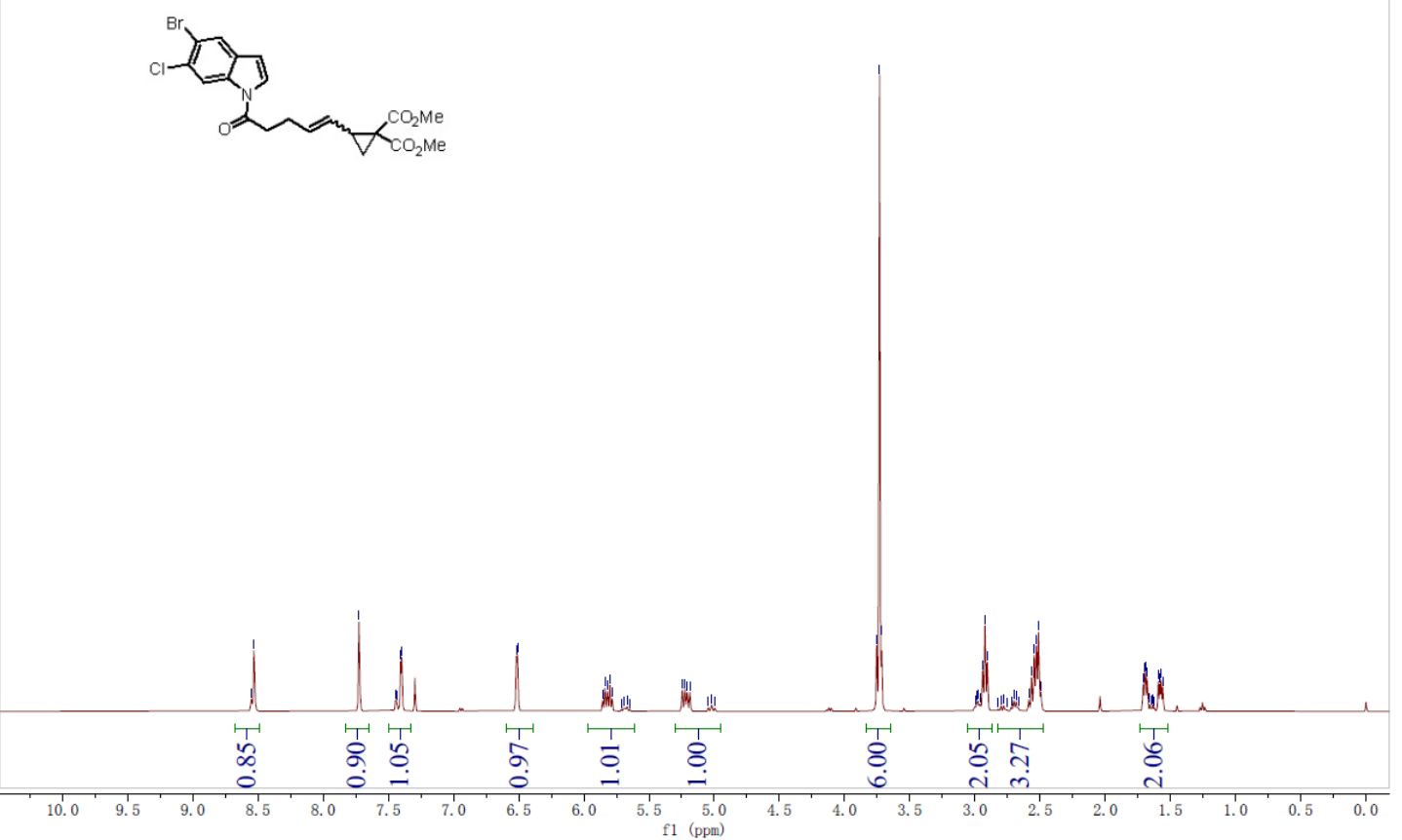

${ }^{13} \mathrm{C}$ NMR Spectrum of $\mathbf{1 a c}$

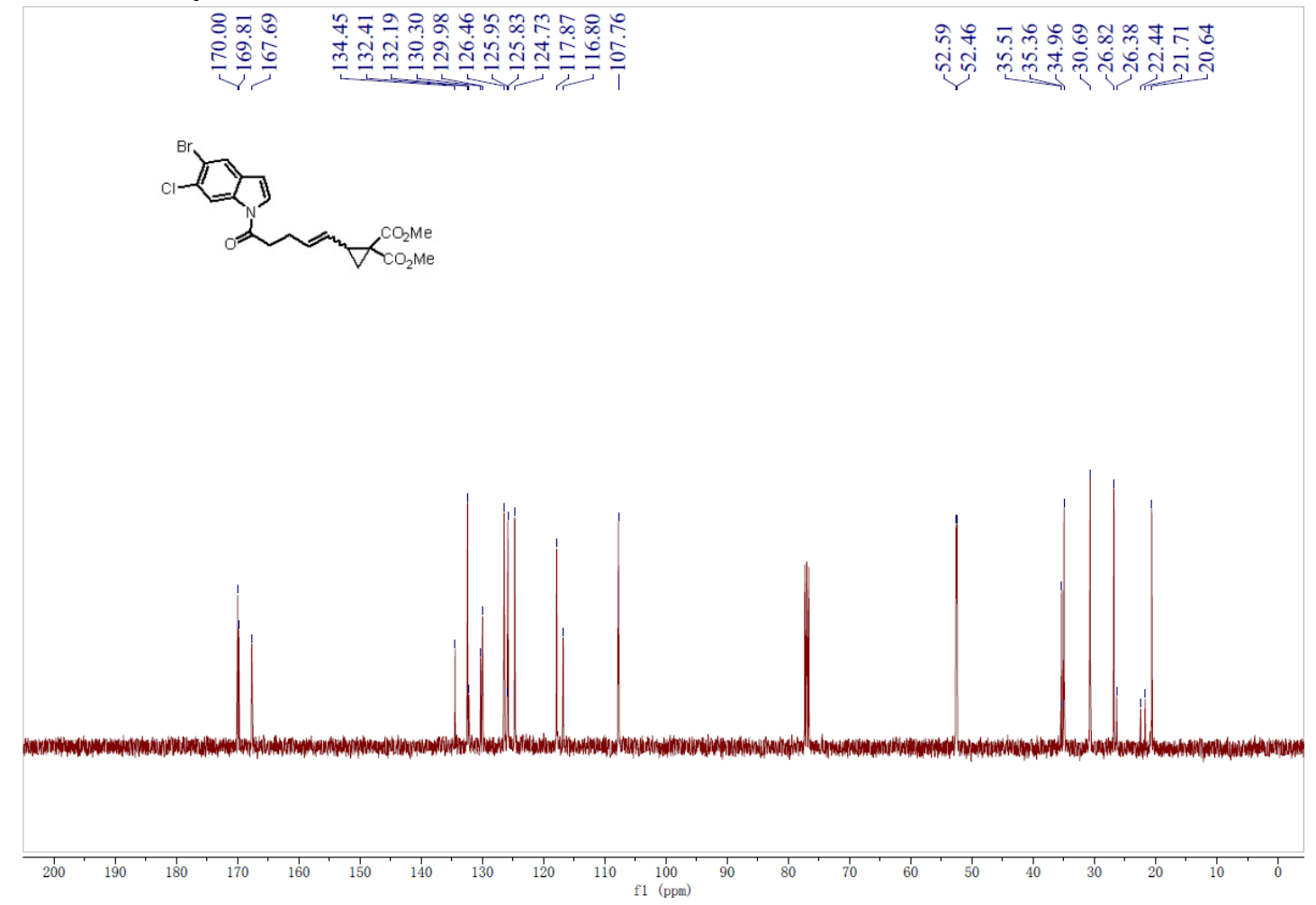


${ }^{1} \mathrm{H}$ NMR Spectrum of $\mathbf{1 a d}$

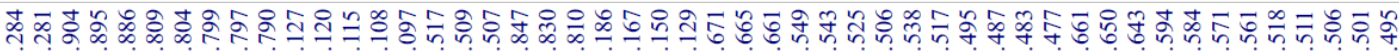

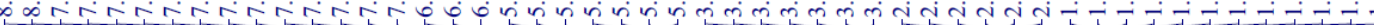
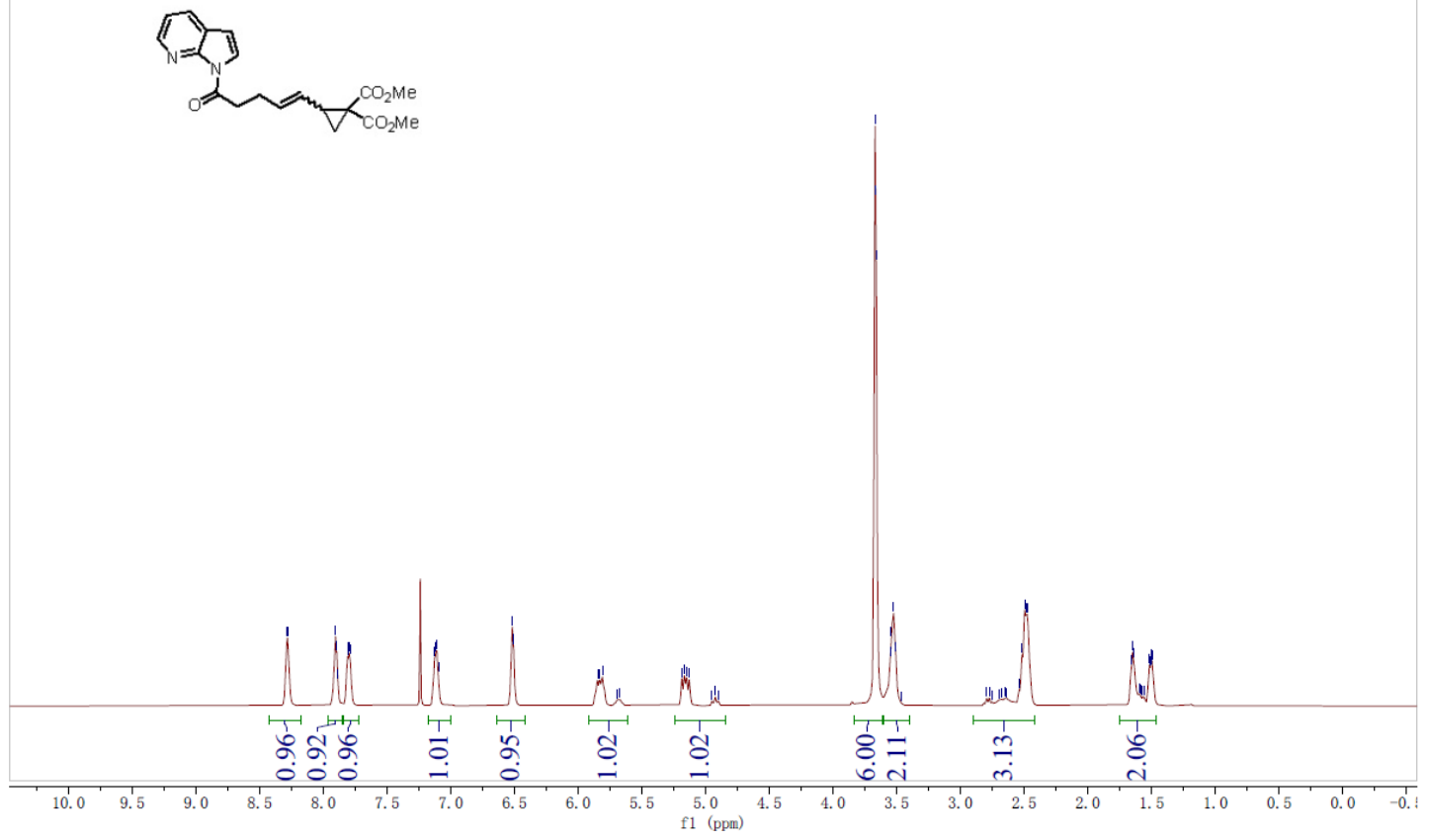

${ }^{13} \mathrm{C}$ NMR Spectrum of $\mathbf{1 a d}$

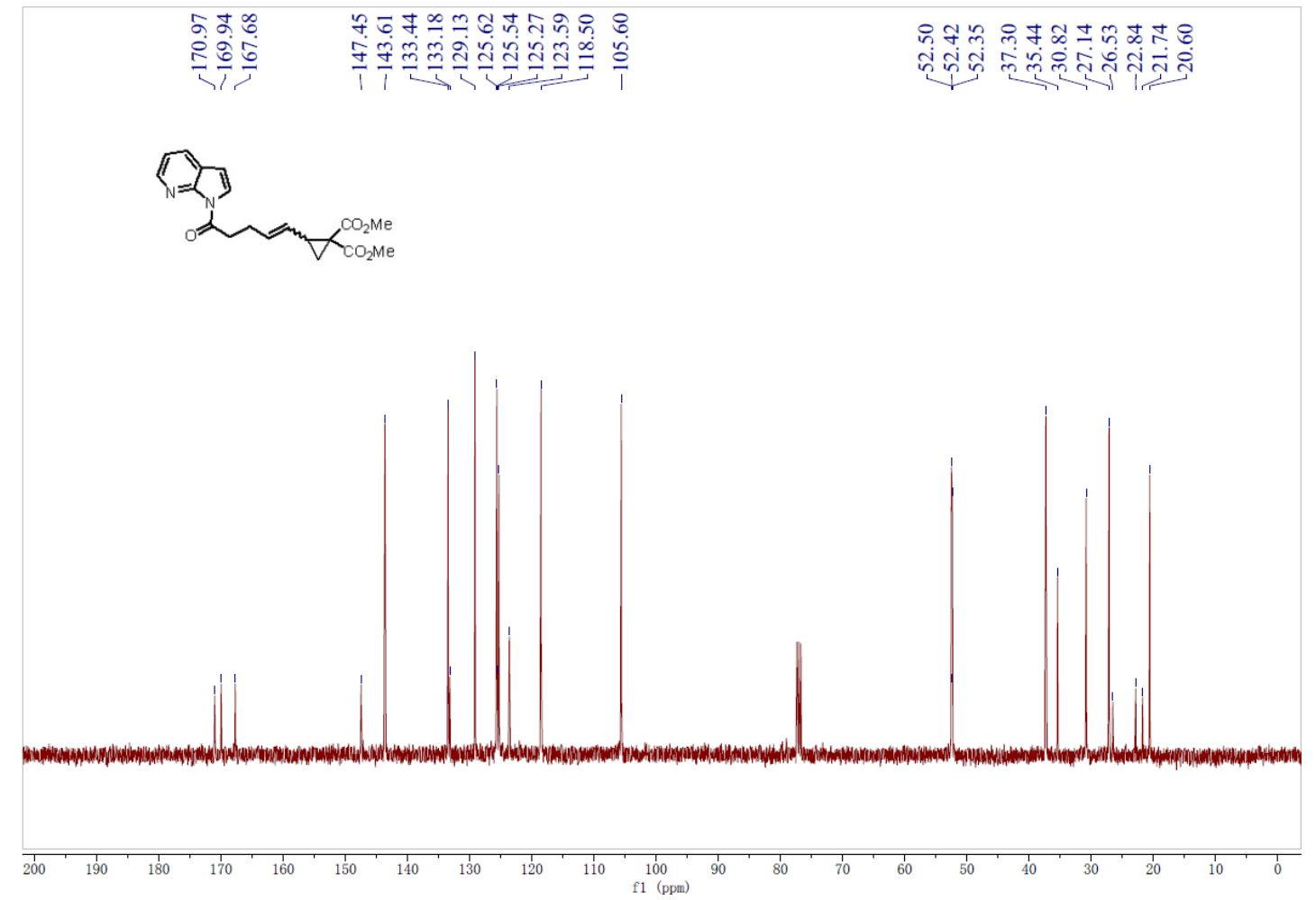


${ }^{1} \mathrm{H}$ NMR Spectrum of $\mathbf{3 a}$

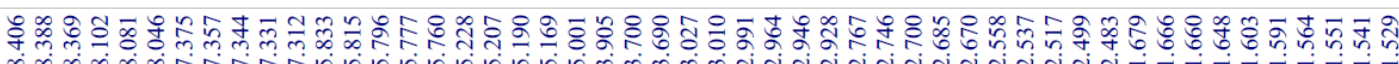

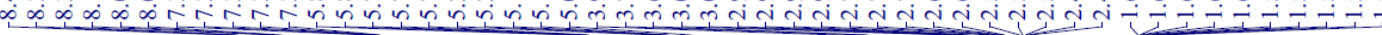

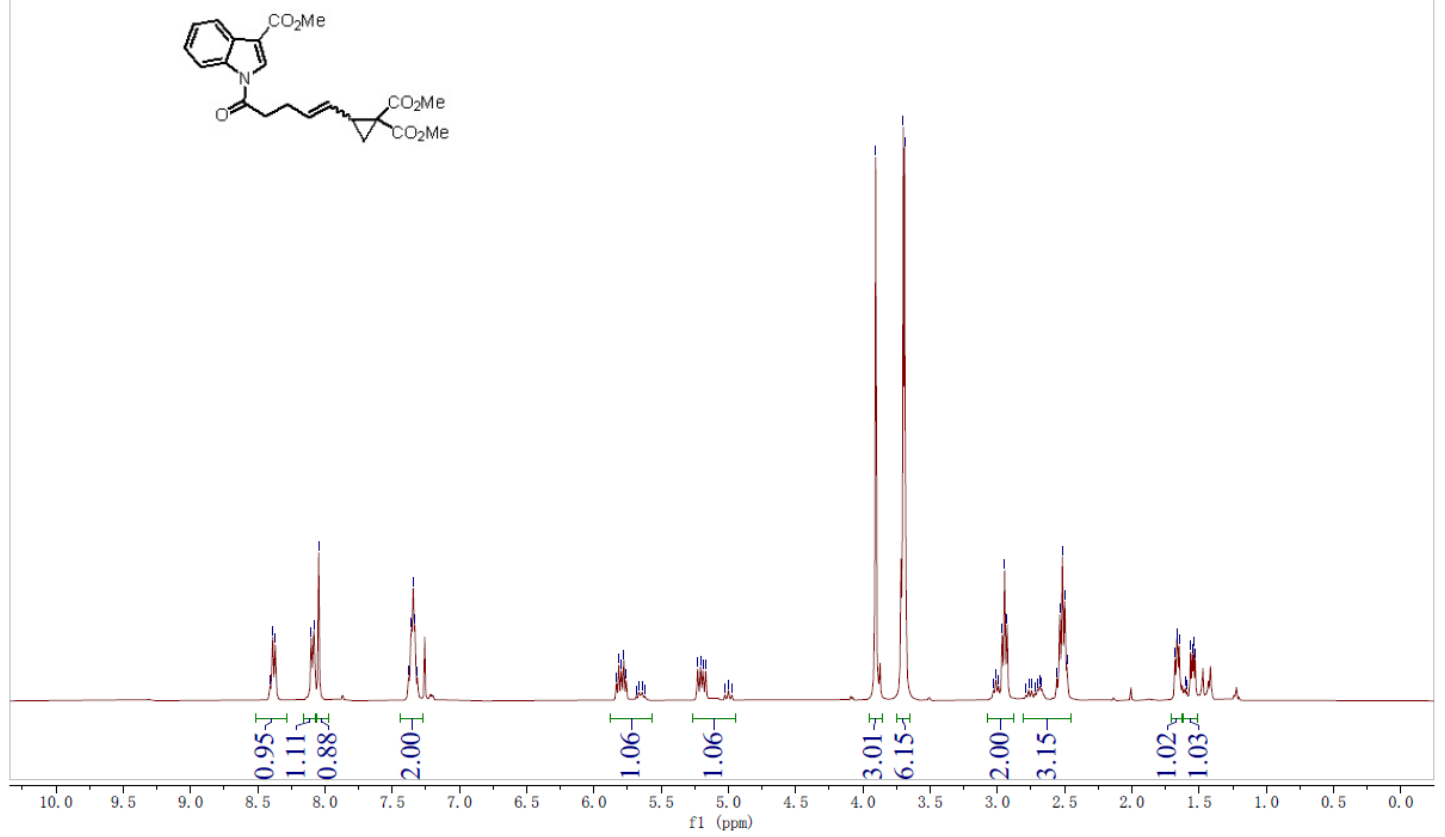

${ }^{13} \mathrm{C}$ NMR Spectrum of $\mathbf{3 a}$

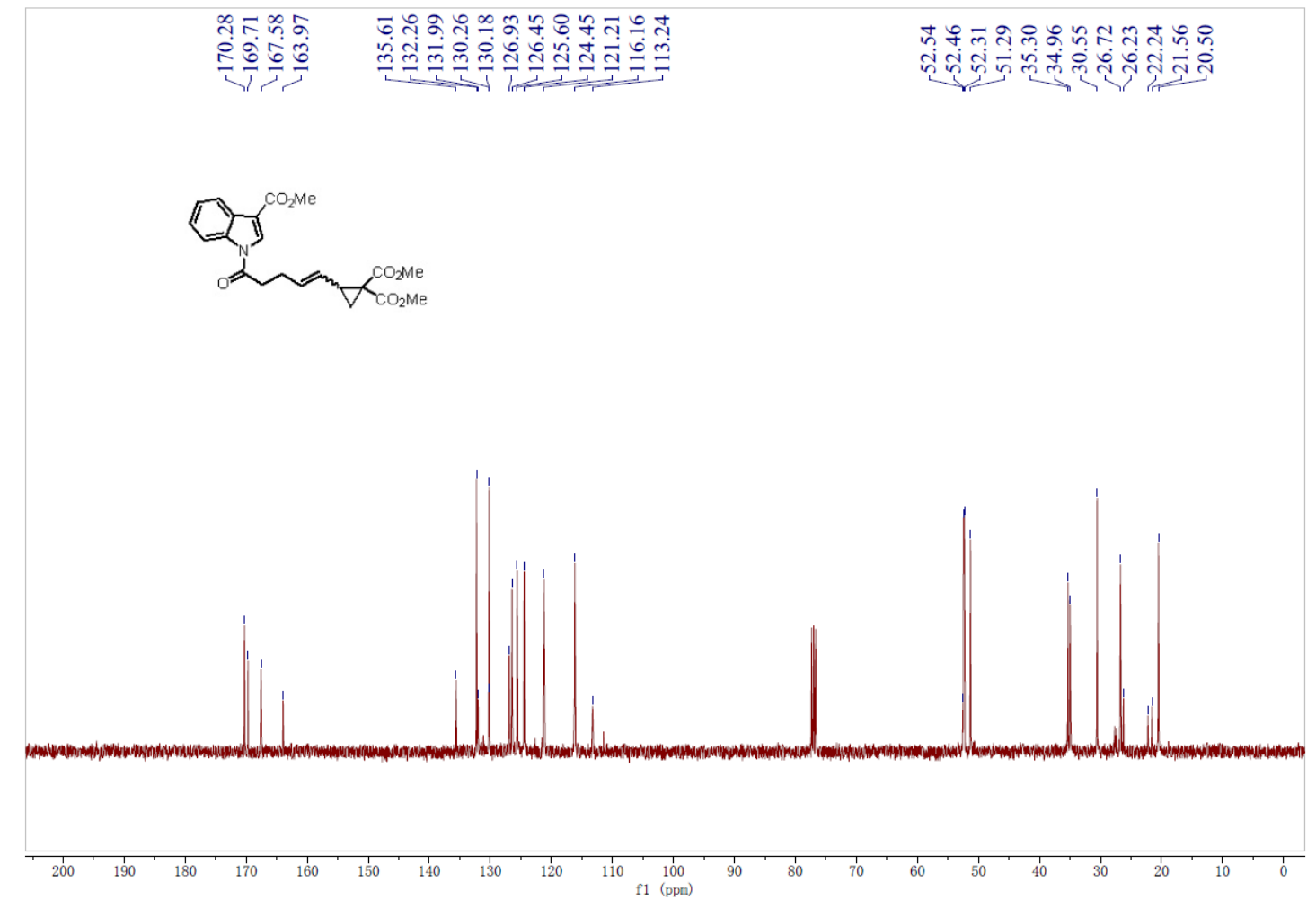


${ }^{1} \mathrm{H}$ NMR Spectrum of $\mathbf{3 b}$

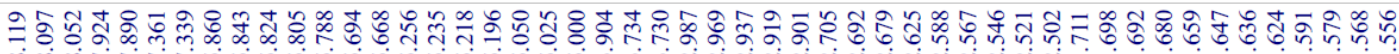

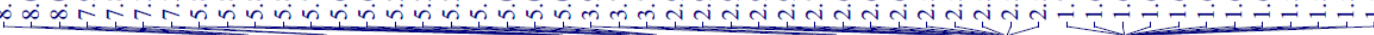
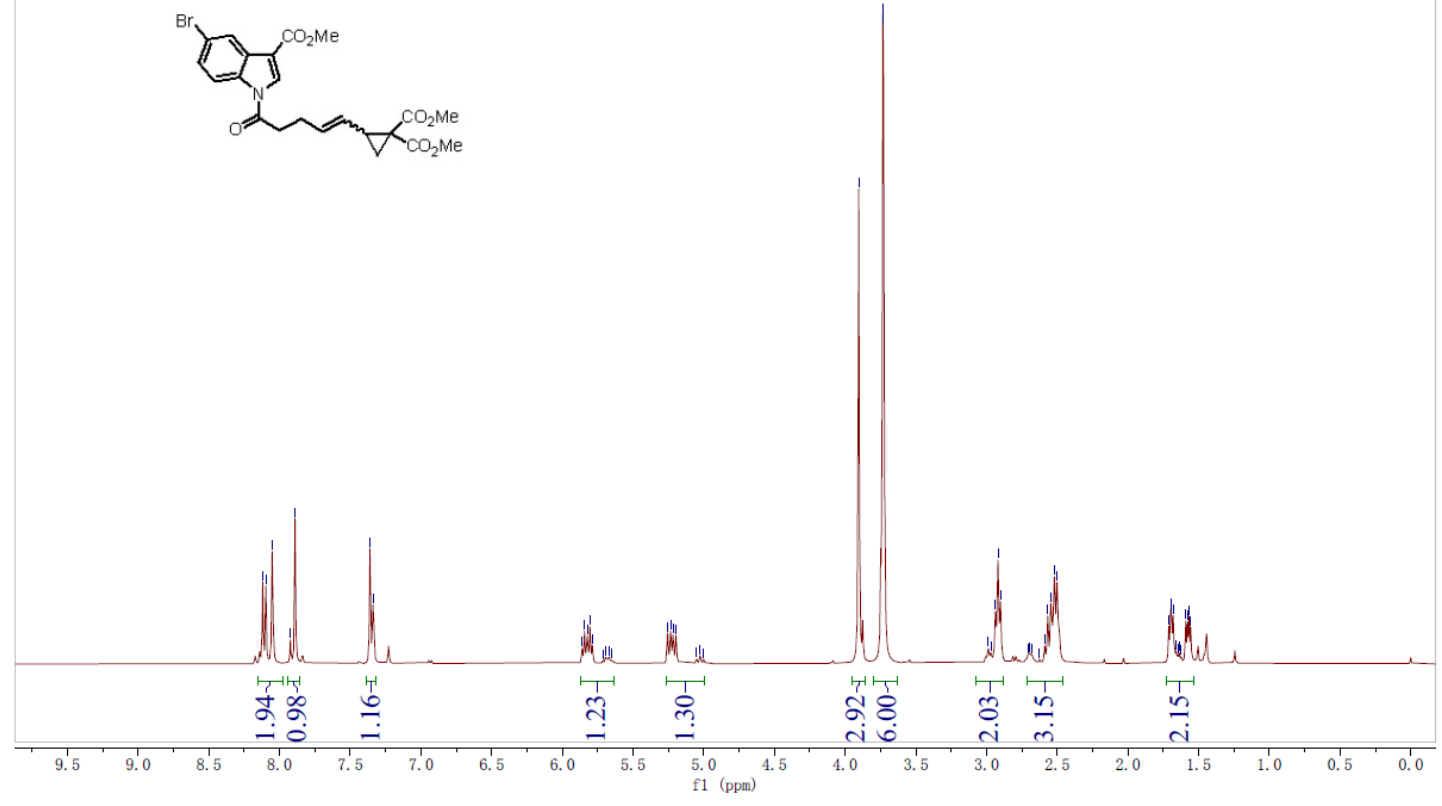

${ }^{13}$ C NMR Spectrum of $\mathbf{3 b}$

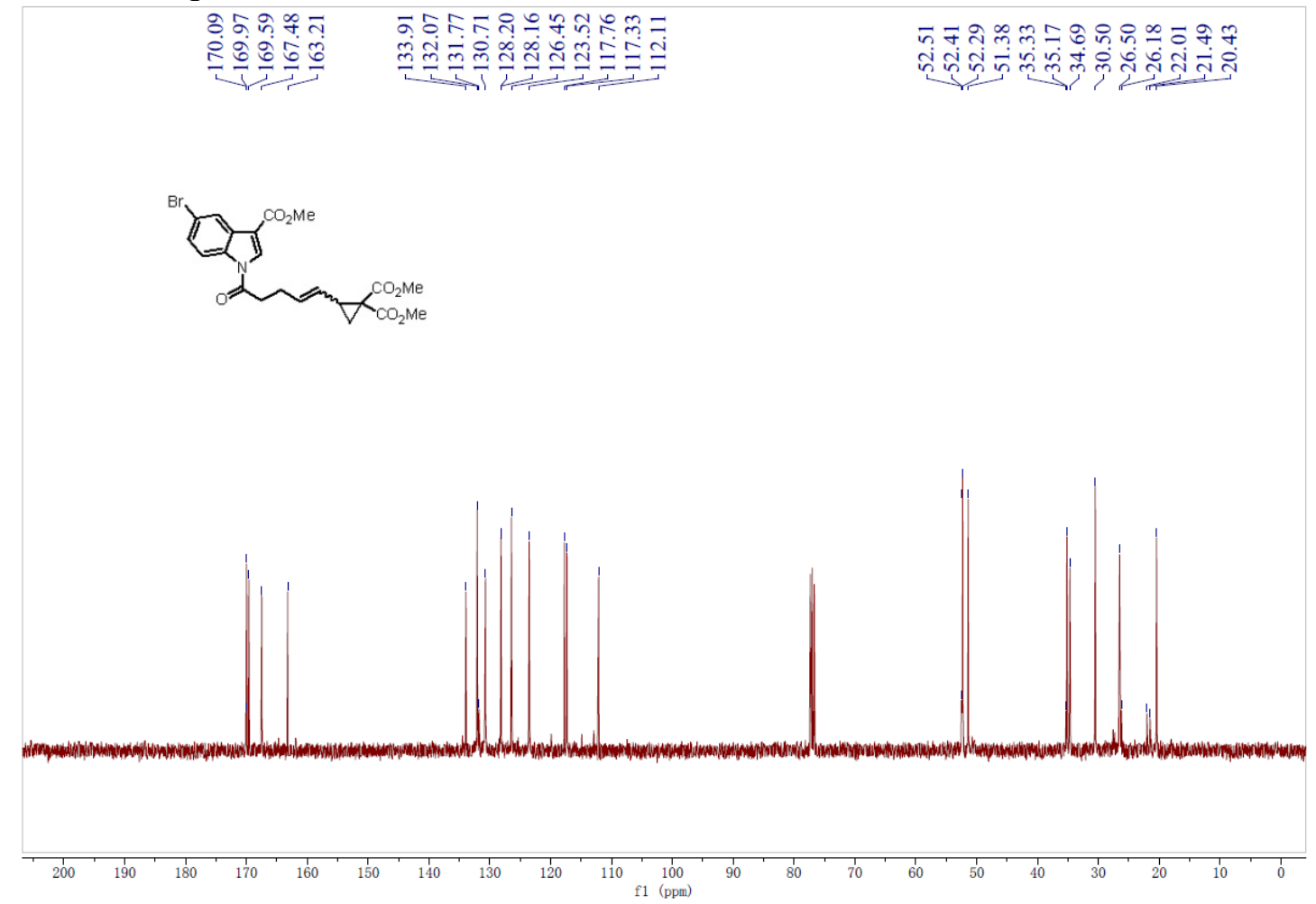


${ }^{1} \mathrm{H}$ NMR Spectrum of $\mathbf{3 c}$

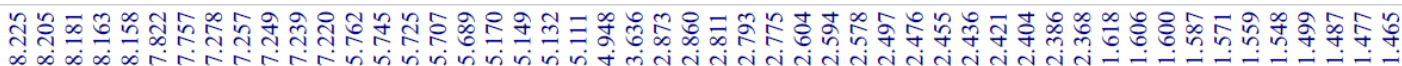<smiles>CCCCCCC(=O)C1(O)CC2C=CCC21CO</smiles>

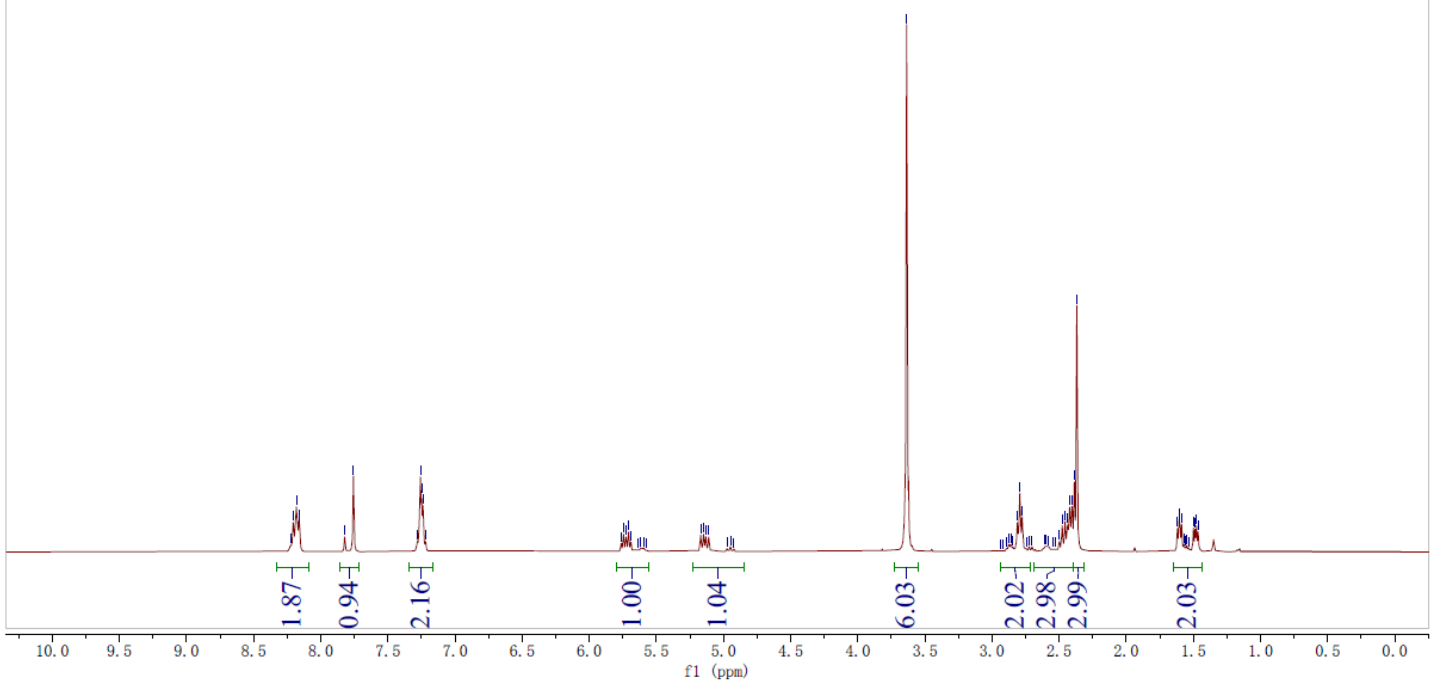

${ }^{13} \mathrm{C}$ NMR Spectrum of 3c

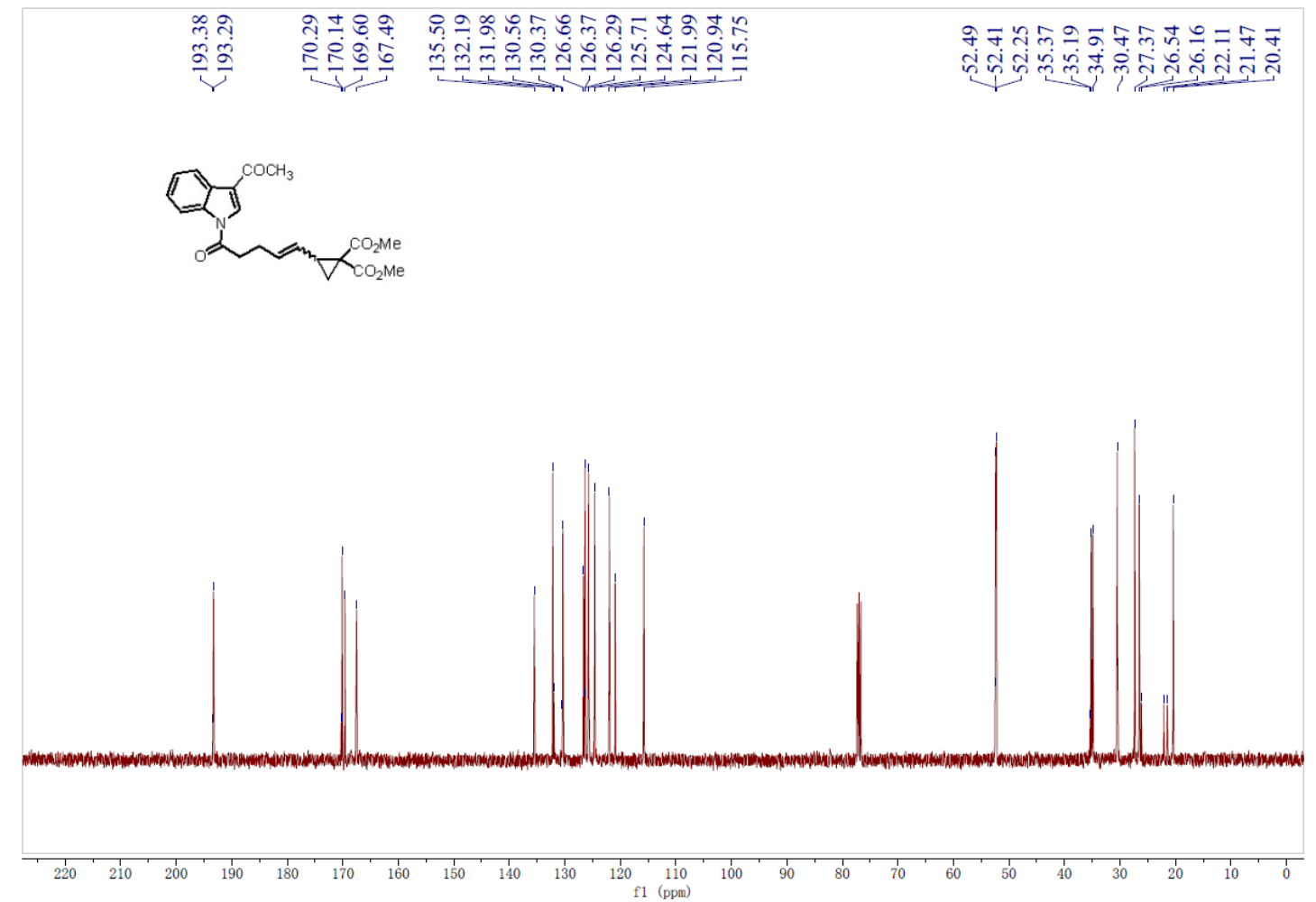


${ }^{1} \mathrm{H}$ NMR Spectrum of $\mathbf{3 d}$

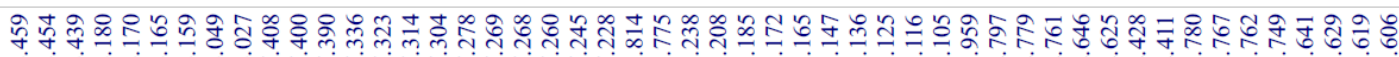

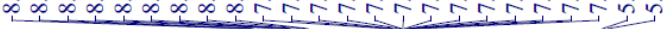
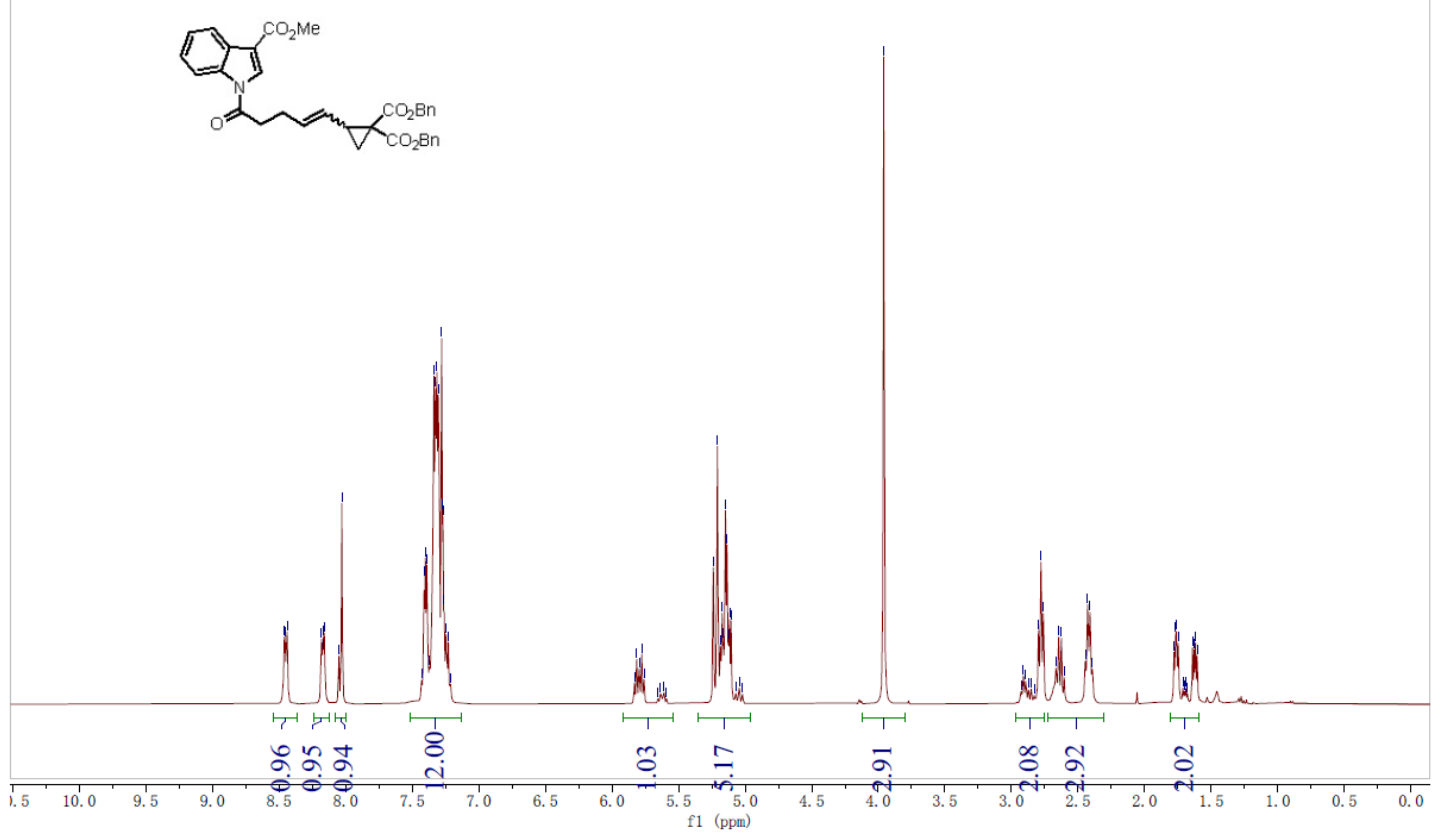

${ }^{13} \mathrm{C}$ NMR Spectrum of $\mathbf{3 d}$

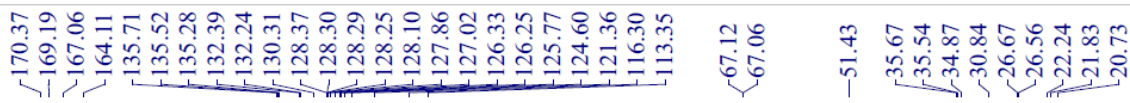
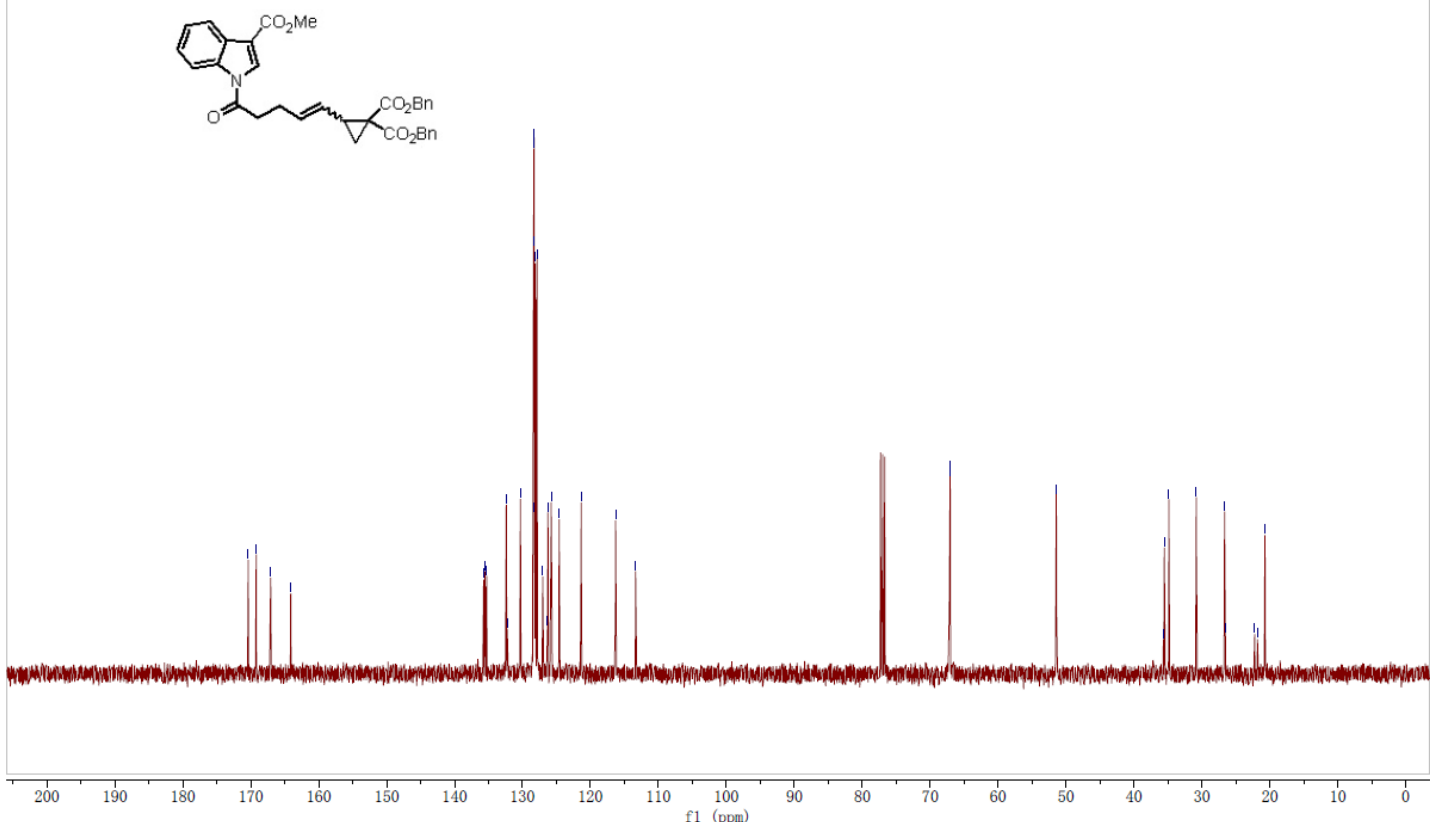
${ }^{1} \mathrm{H}$ NMR Spectrum of $\mathbf{5 f}$

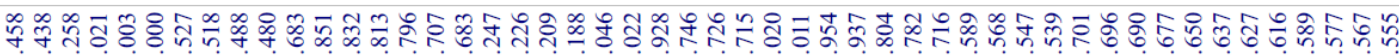
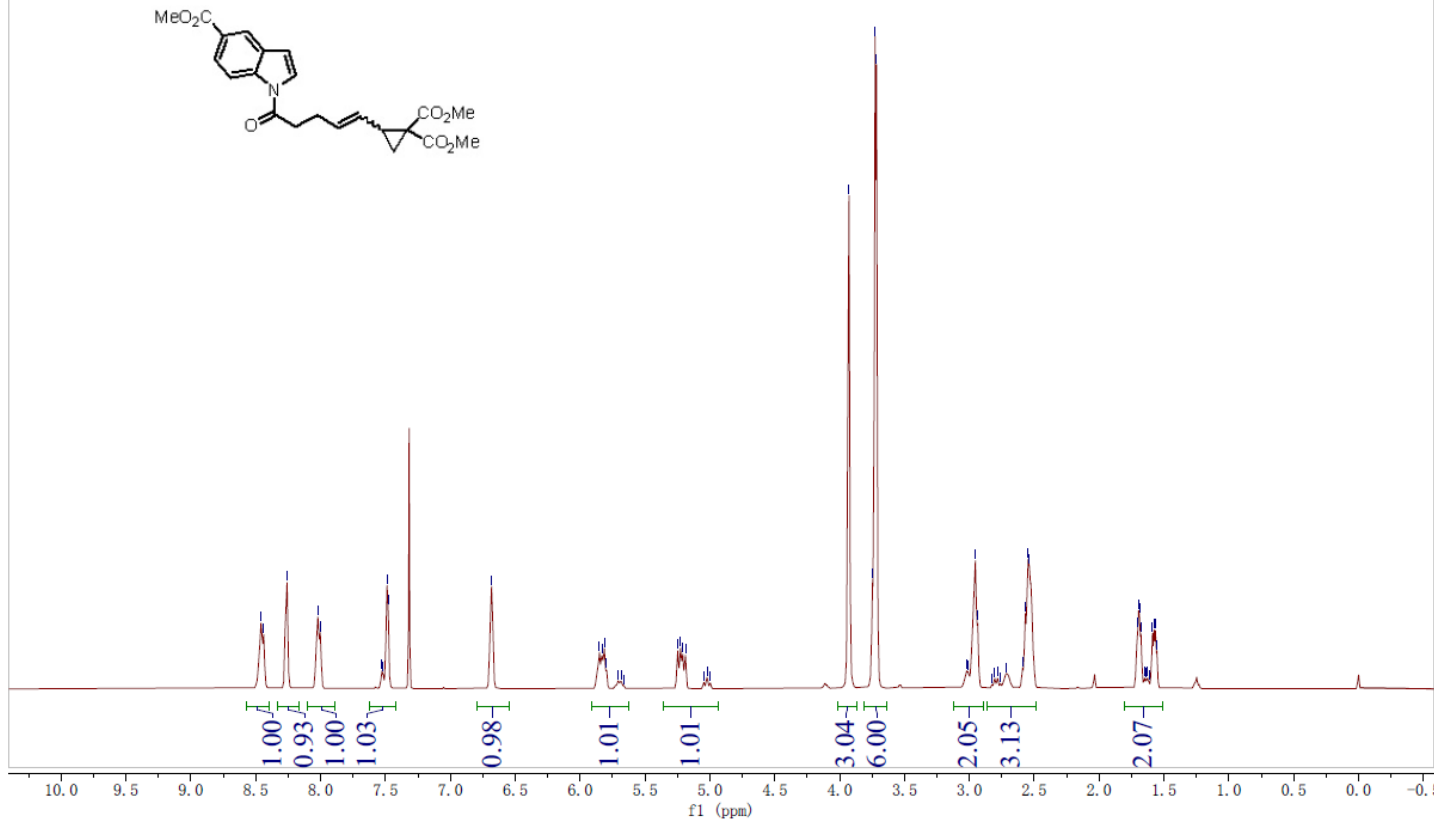

${ }^{13} \mathrm{C}$ NMR Spectrum of $\mathbf{5 f}$

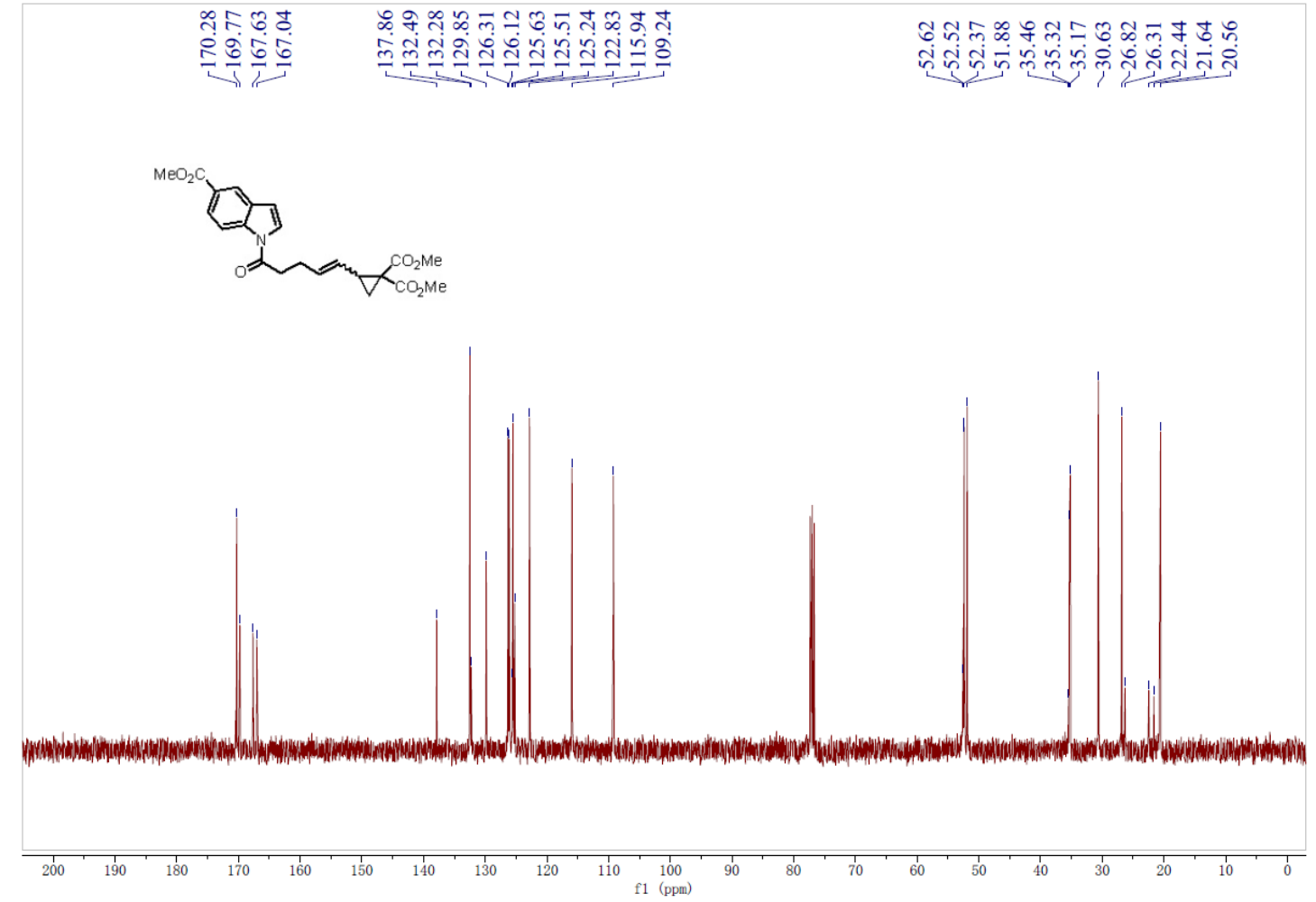


${ }^{1} \mathrm{H}$ NMR Spectrum of $7 \mathbf{a}$

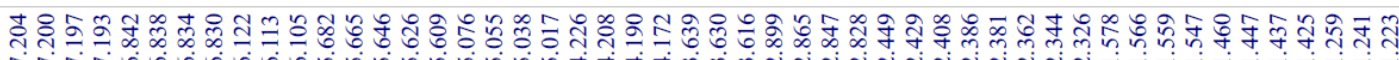

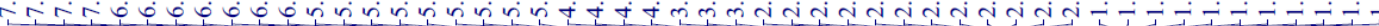<smiles></smiles>

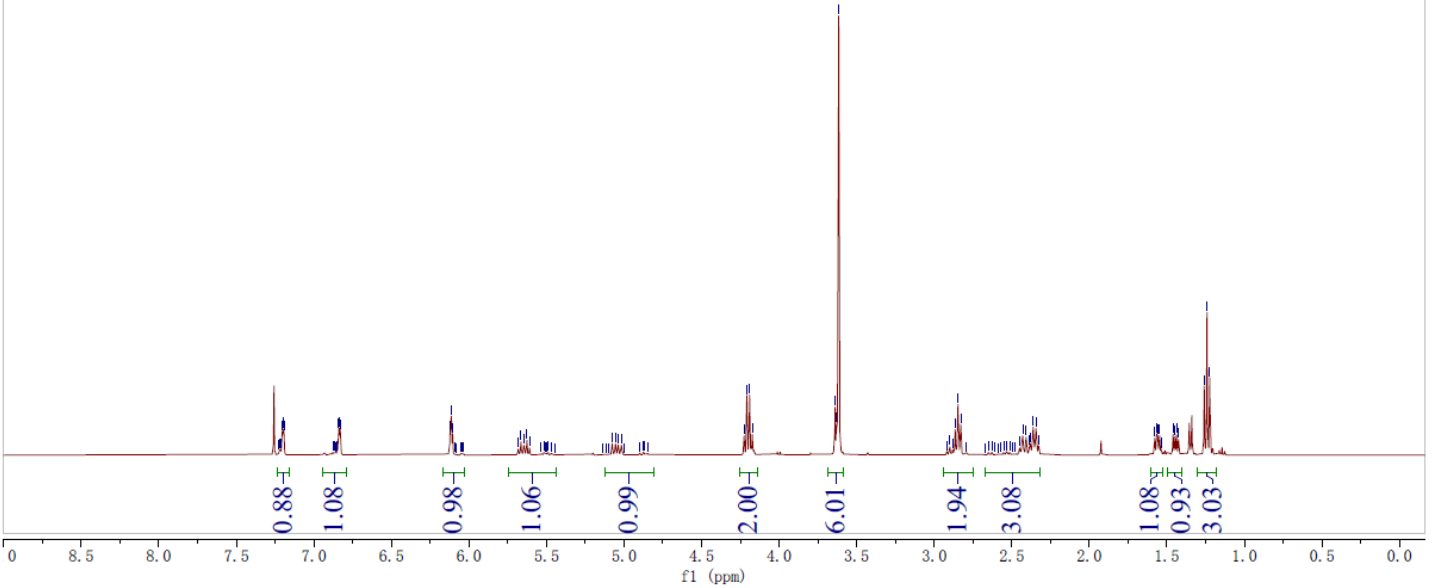

${ }^{13} \mathrm{C}$ NMR Spectrum of 7a

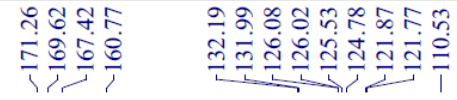

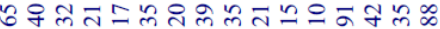

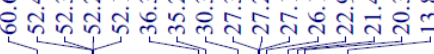
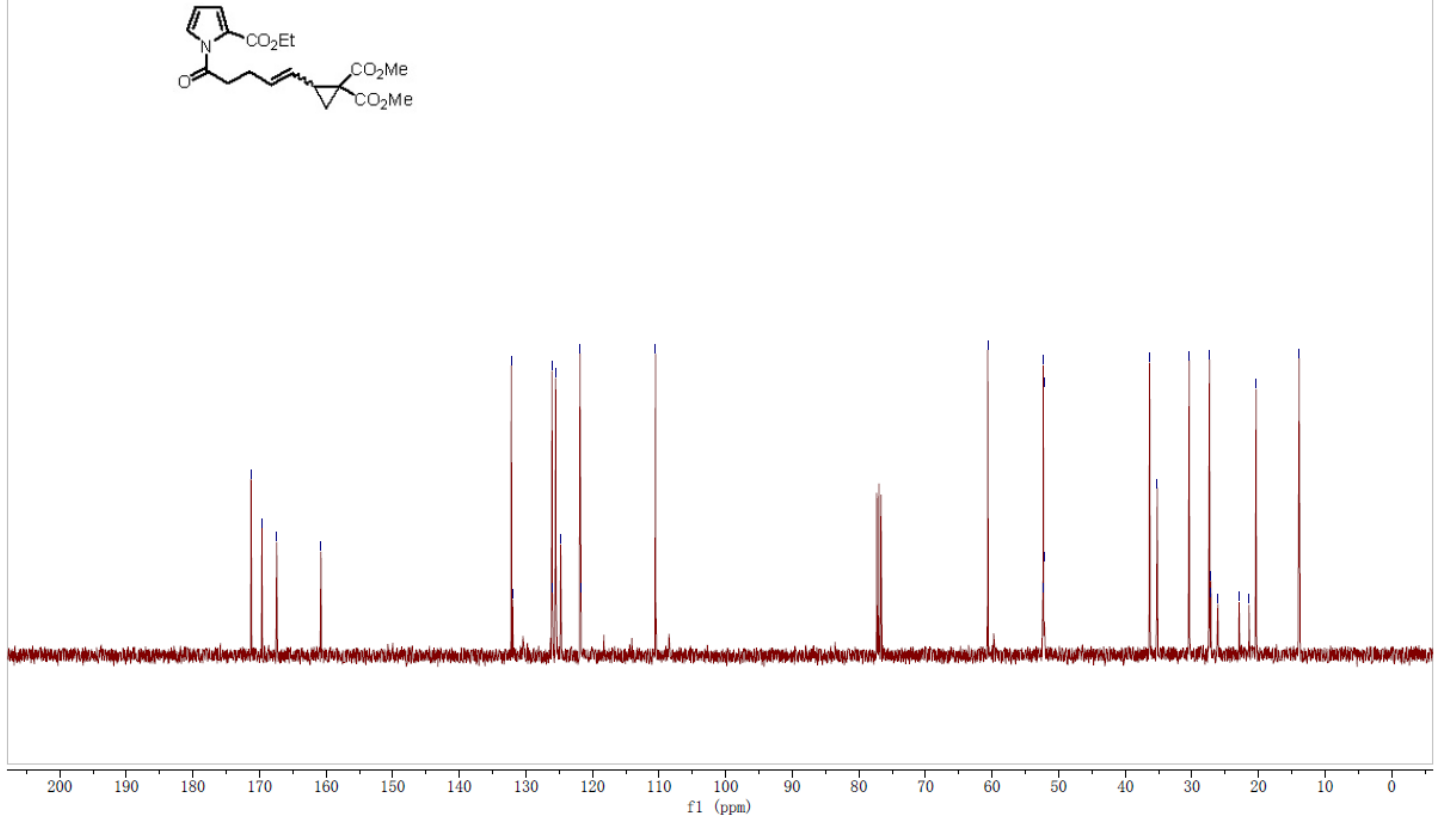
${ }^{1} \mathrm{H}$ NMR Spectrum of $7 \mathbf{b}$

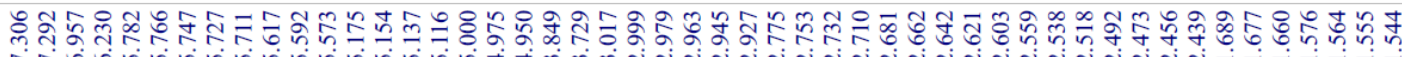

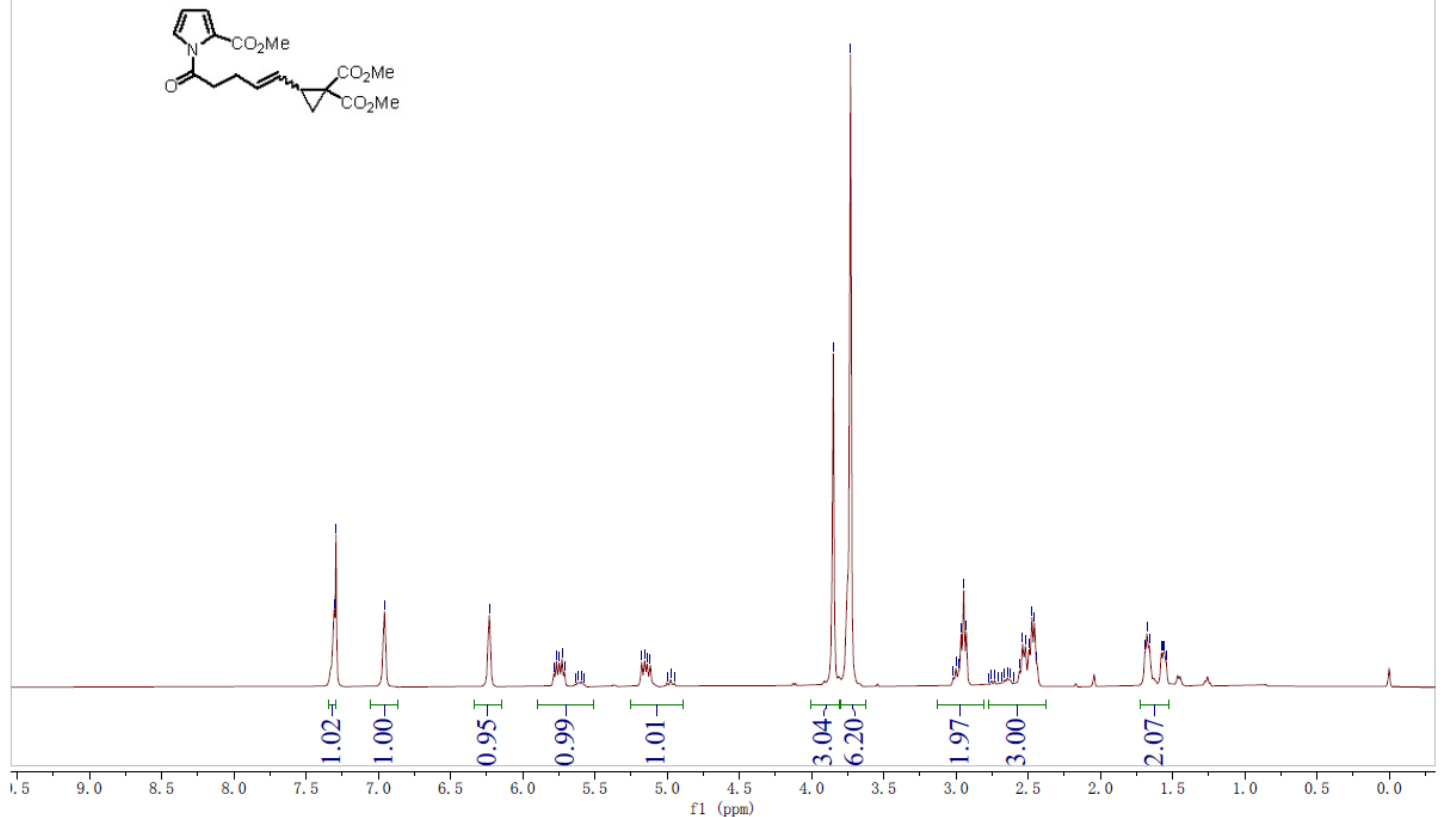

${ }^{13} \mathrm{C}$ NMR Spectrum of $\mathbf{7 b}$

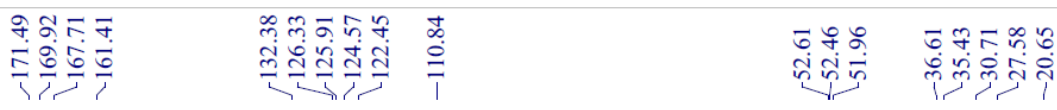
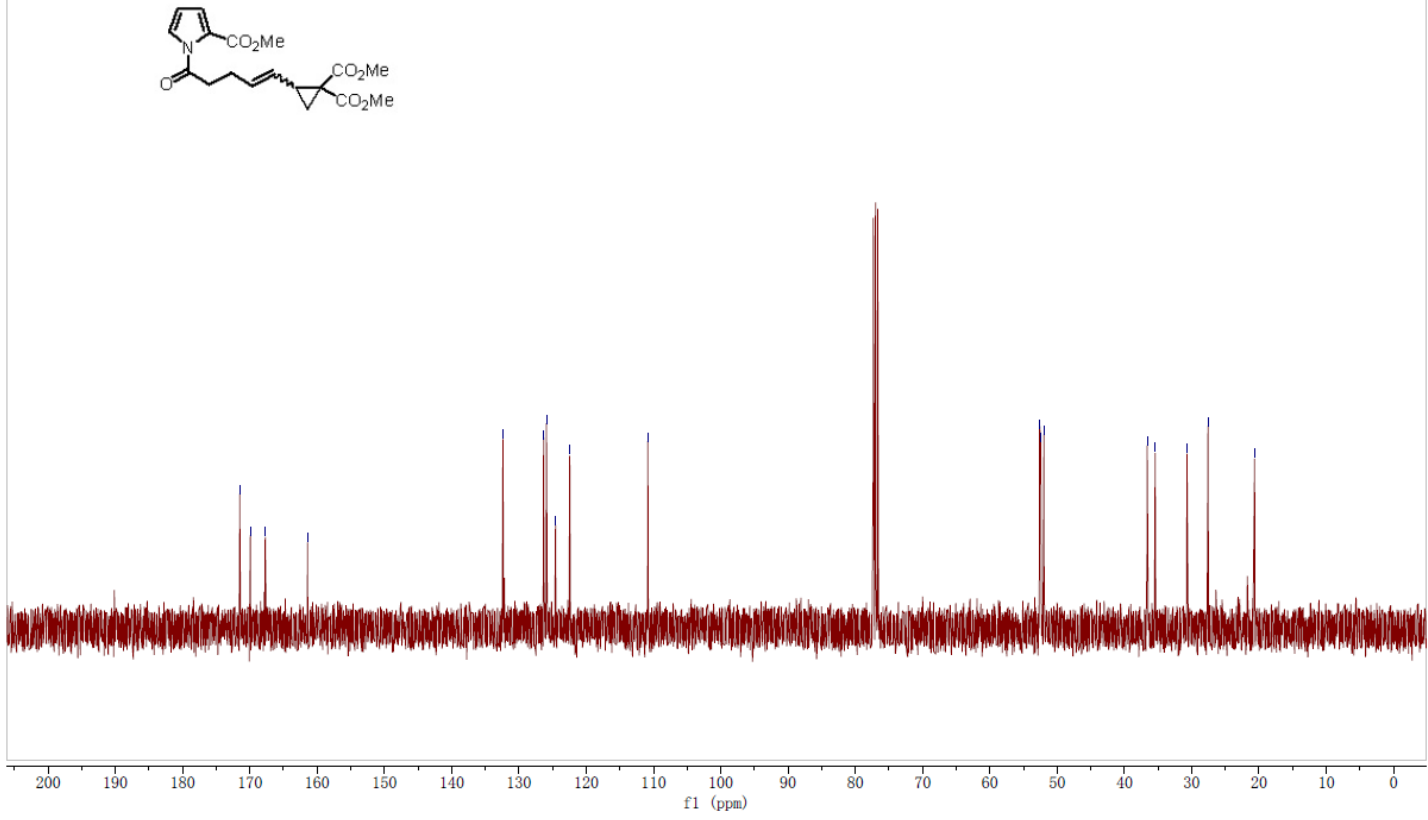
${ }^{1} \mathrm{H}$ NMR Spectrum of $\mathbf{7} \mathbf{c}$

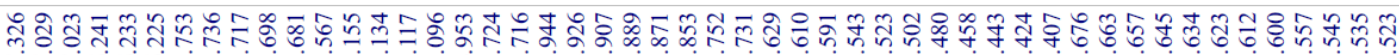

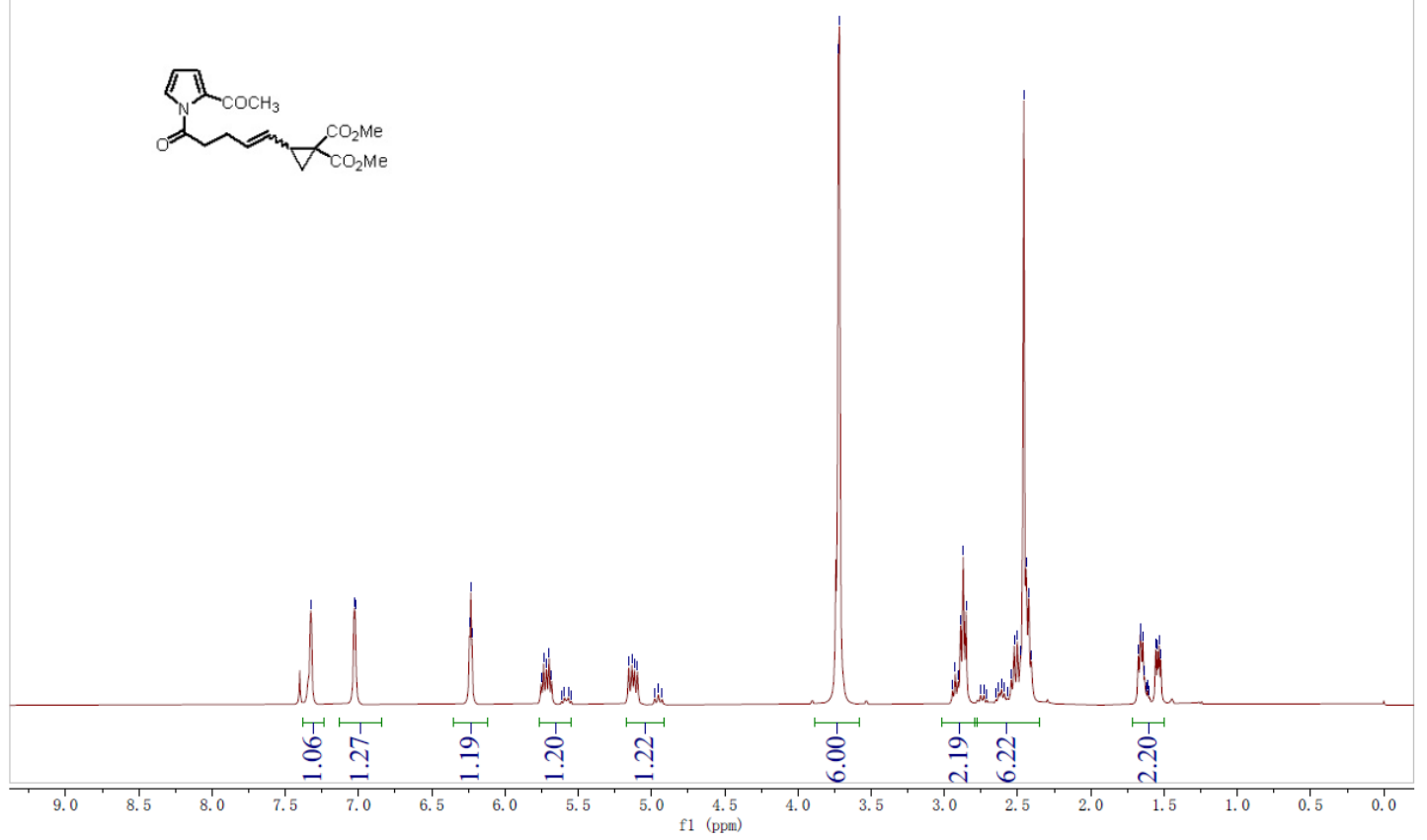

${ }^{13} \mathrm{C}$ NMR Spectrum of 7c

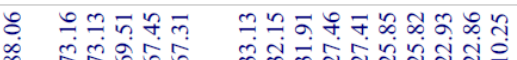

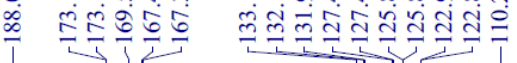

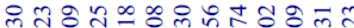

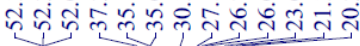<smiles>COc1cccn1C(=O)CC=CC1CC1(O)OC</smiles>
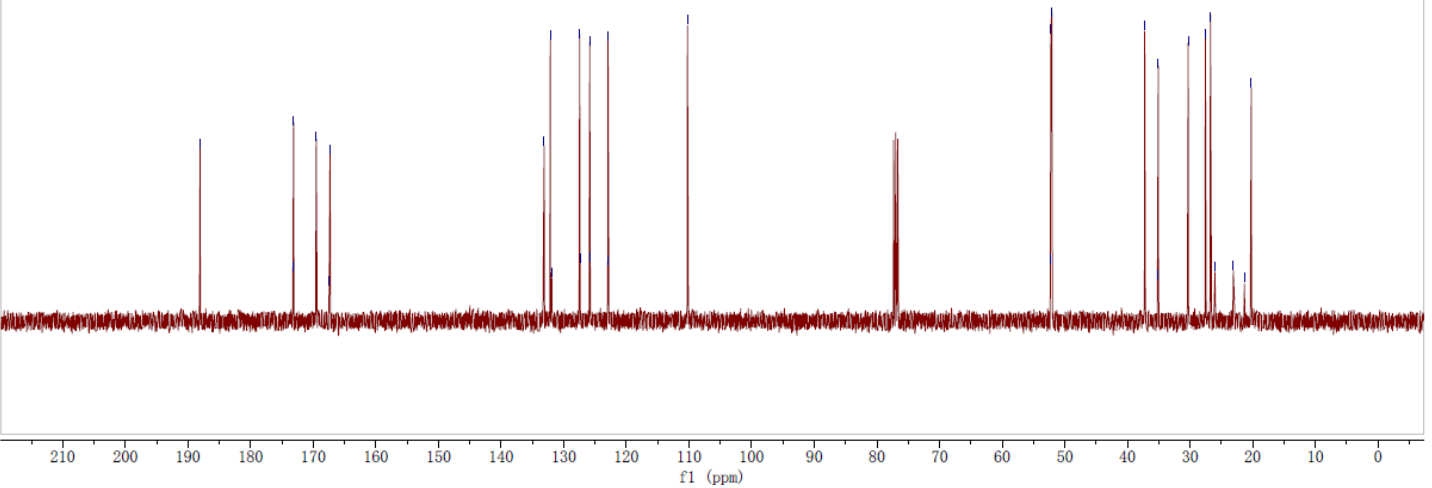
${ }^{1} \mathrm{H}$ NMR Spectrum of $\mathbf{7 d}$

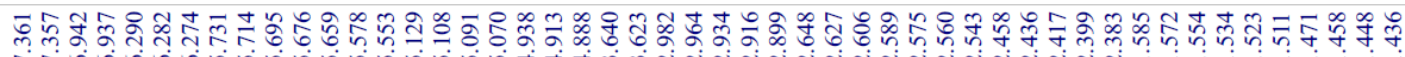

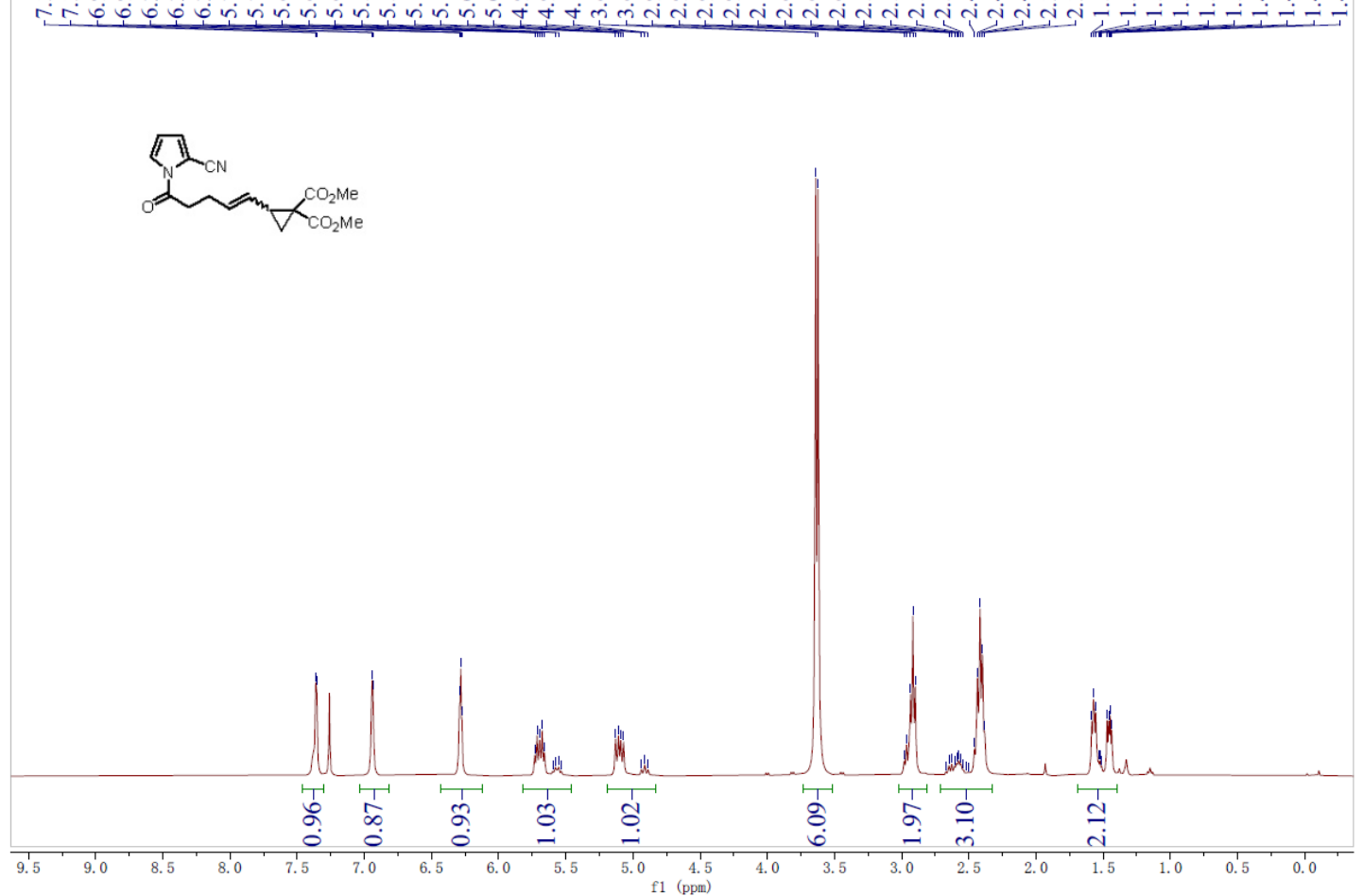

${ }^{13} \mathrm{C}$ NMR Spectrum of 7d

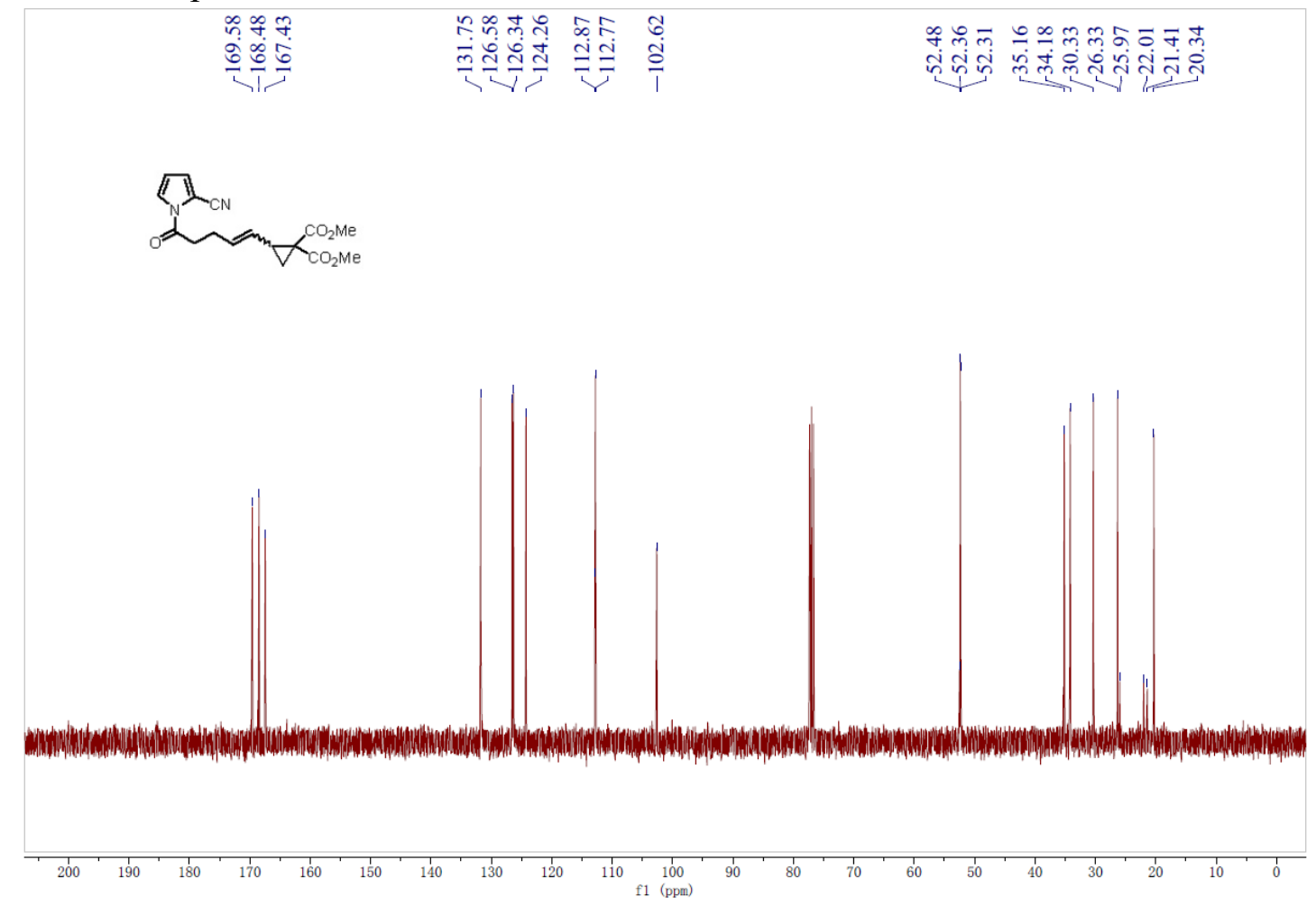


${ }^{1} \mathrm{H}$ NMR Spectrum of $7 \mathbf{e}$

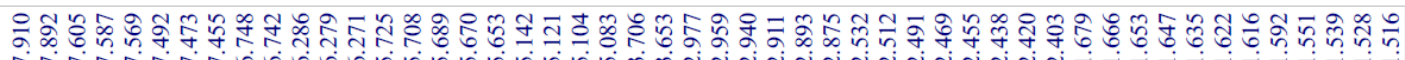

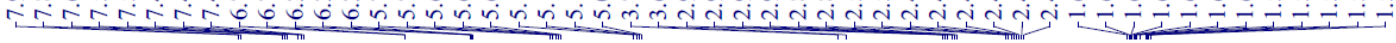

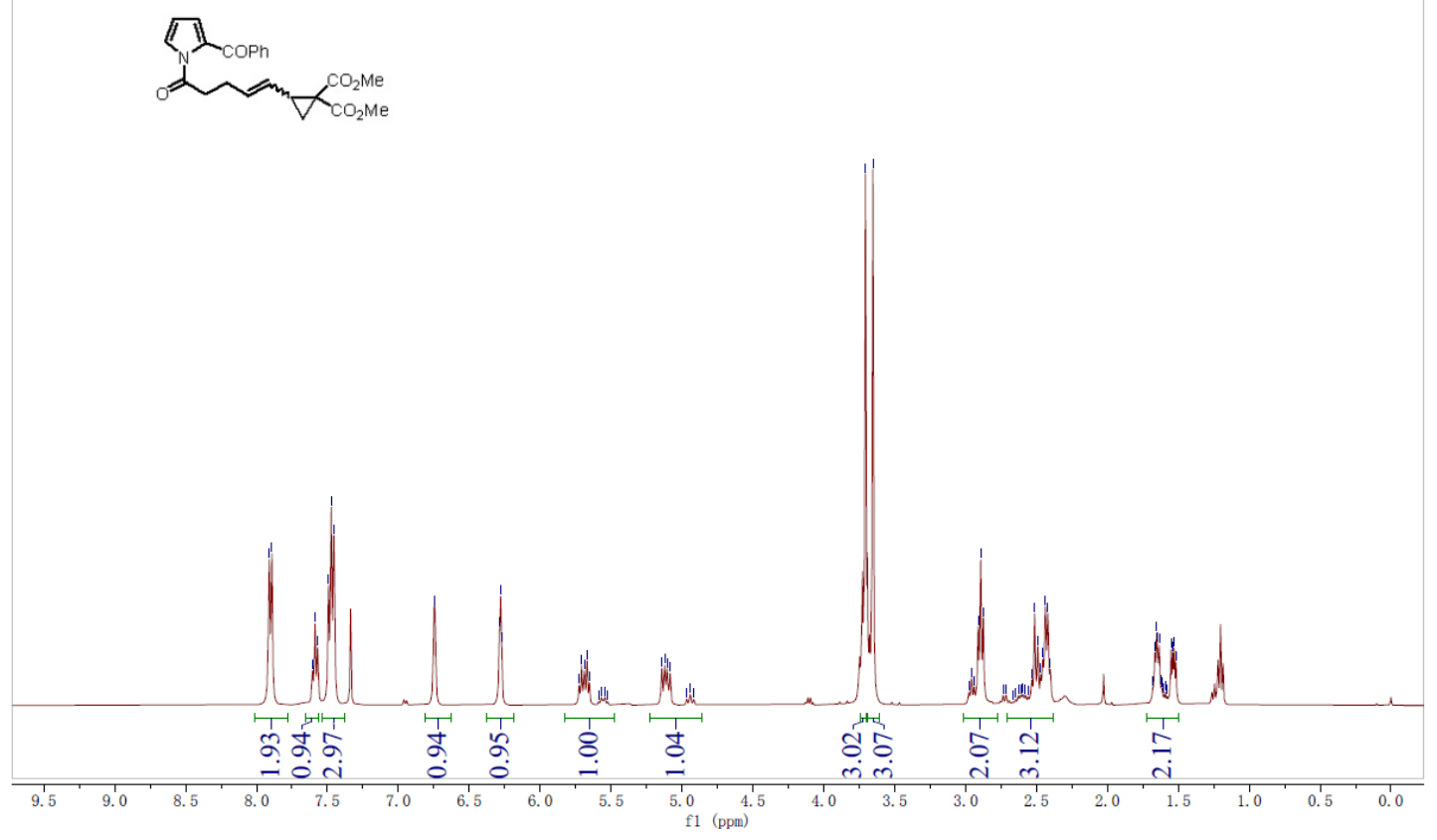

${ }^{13} \mathrm{C}$ NMR Spectrum of $7 \mathbf{e}$

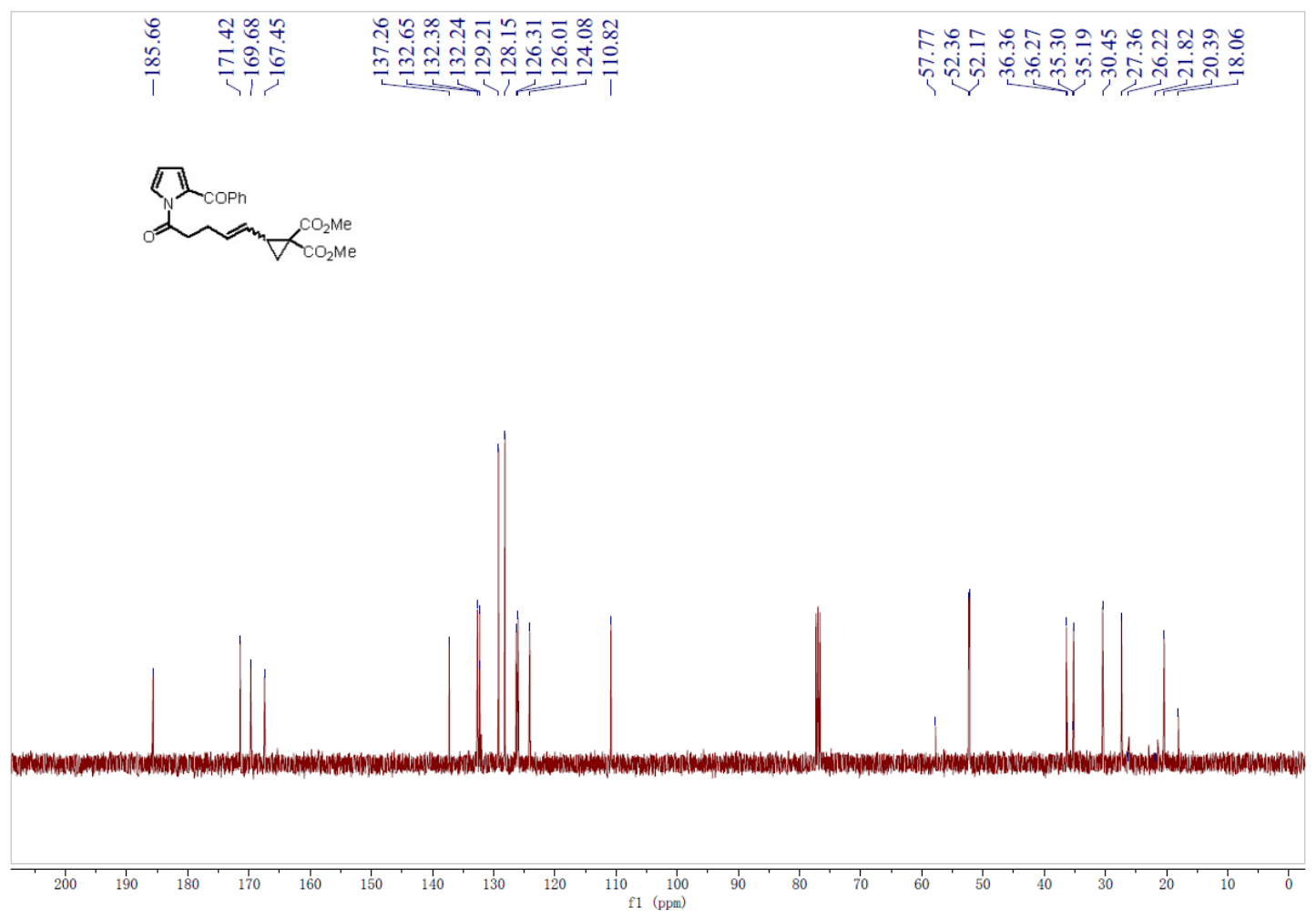


${ }^{1} \mathrm{H}$ NMR Spectrum of $7 \mathbf{f}$

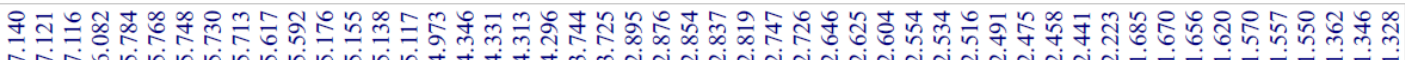
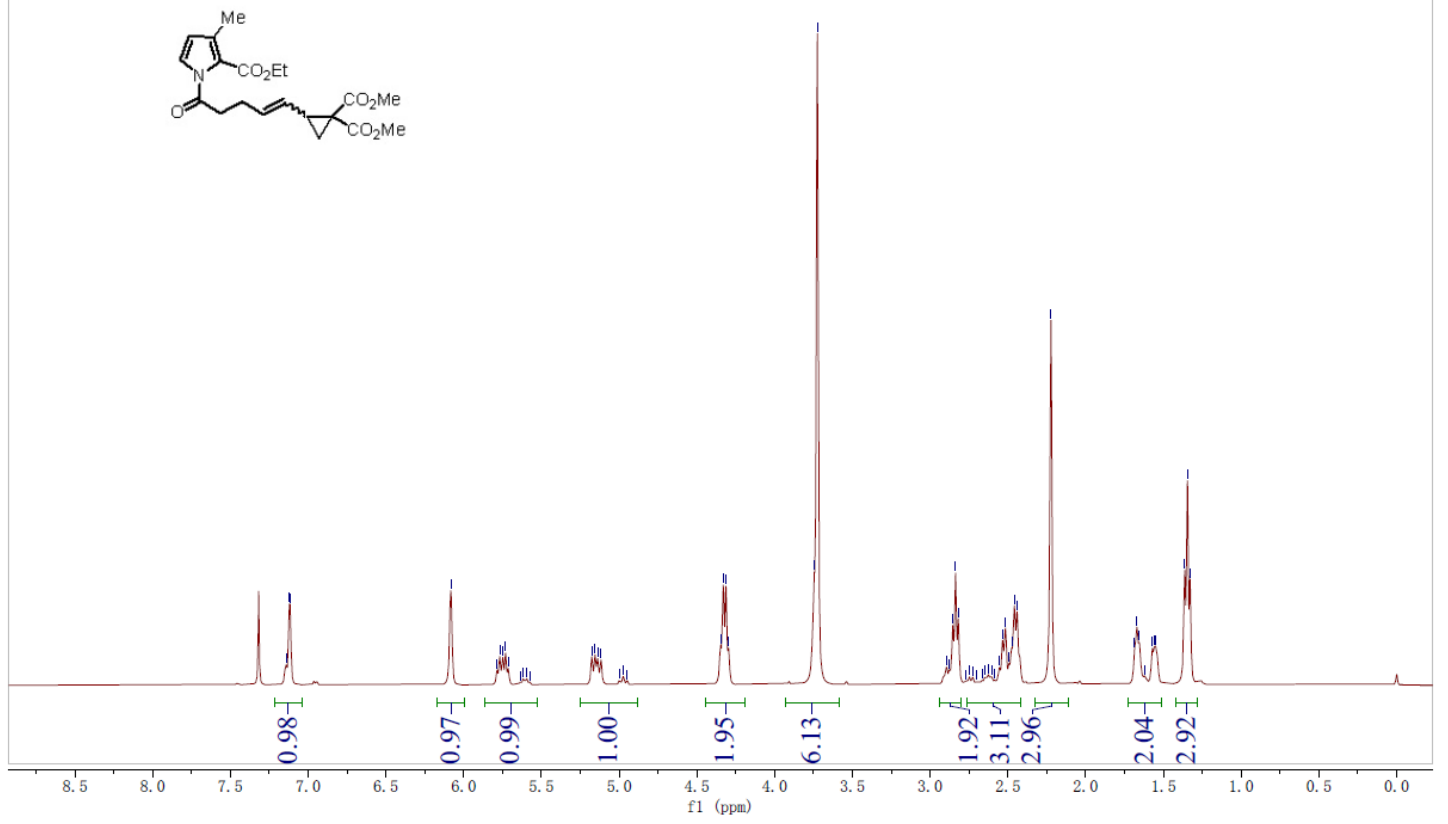

${ }^{13} \mathrm{C}$ NMR Spectrum of $7 \mathbf{f}$

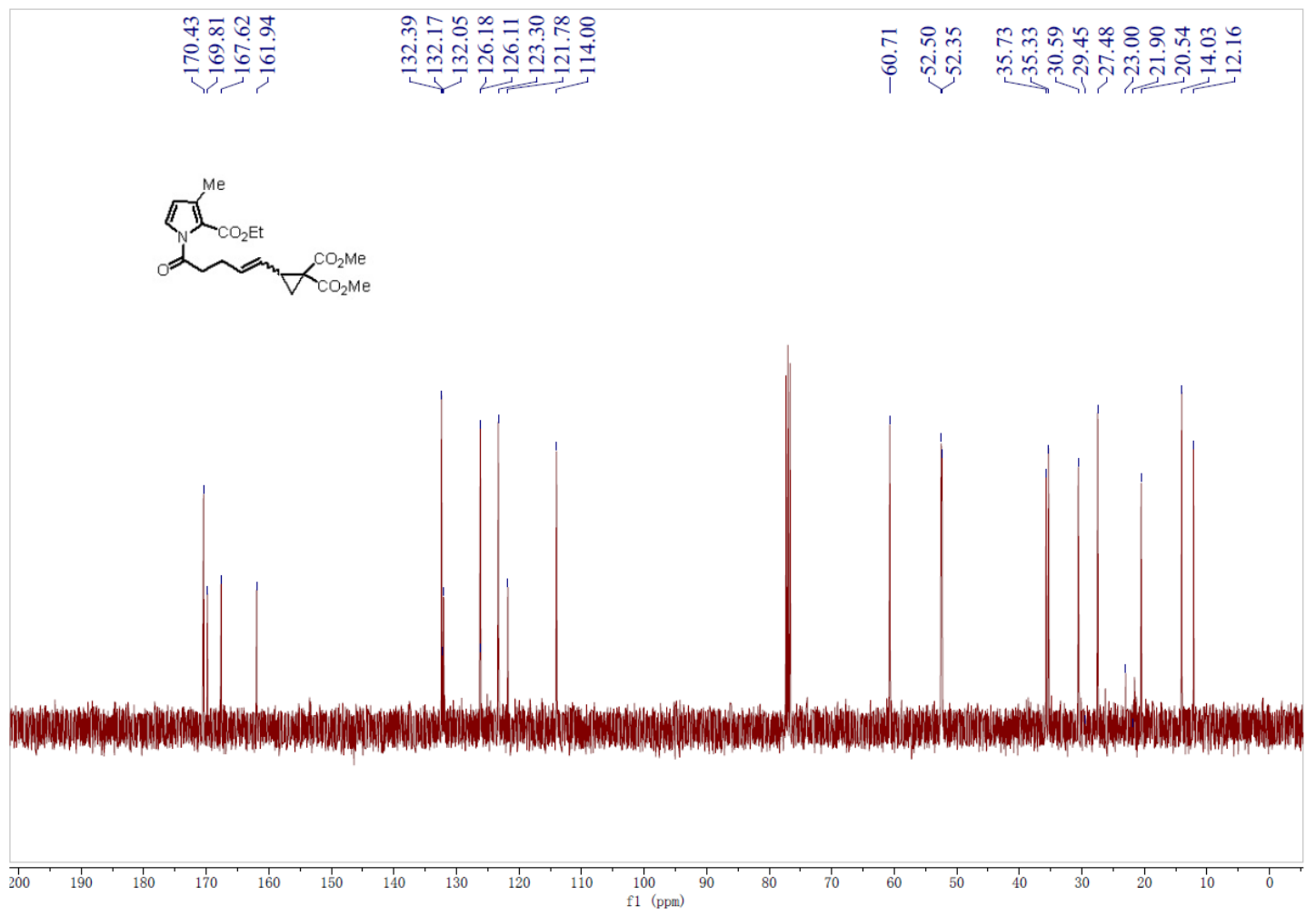


${ }^{1} \mathrm{H}$ NMR Spectrum of $\mathbf{7 g}$

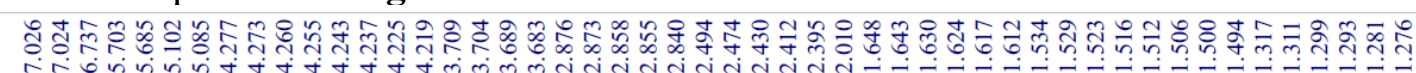

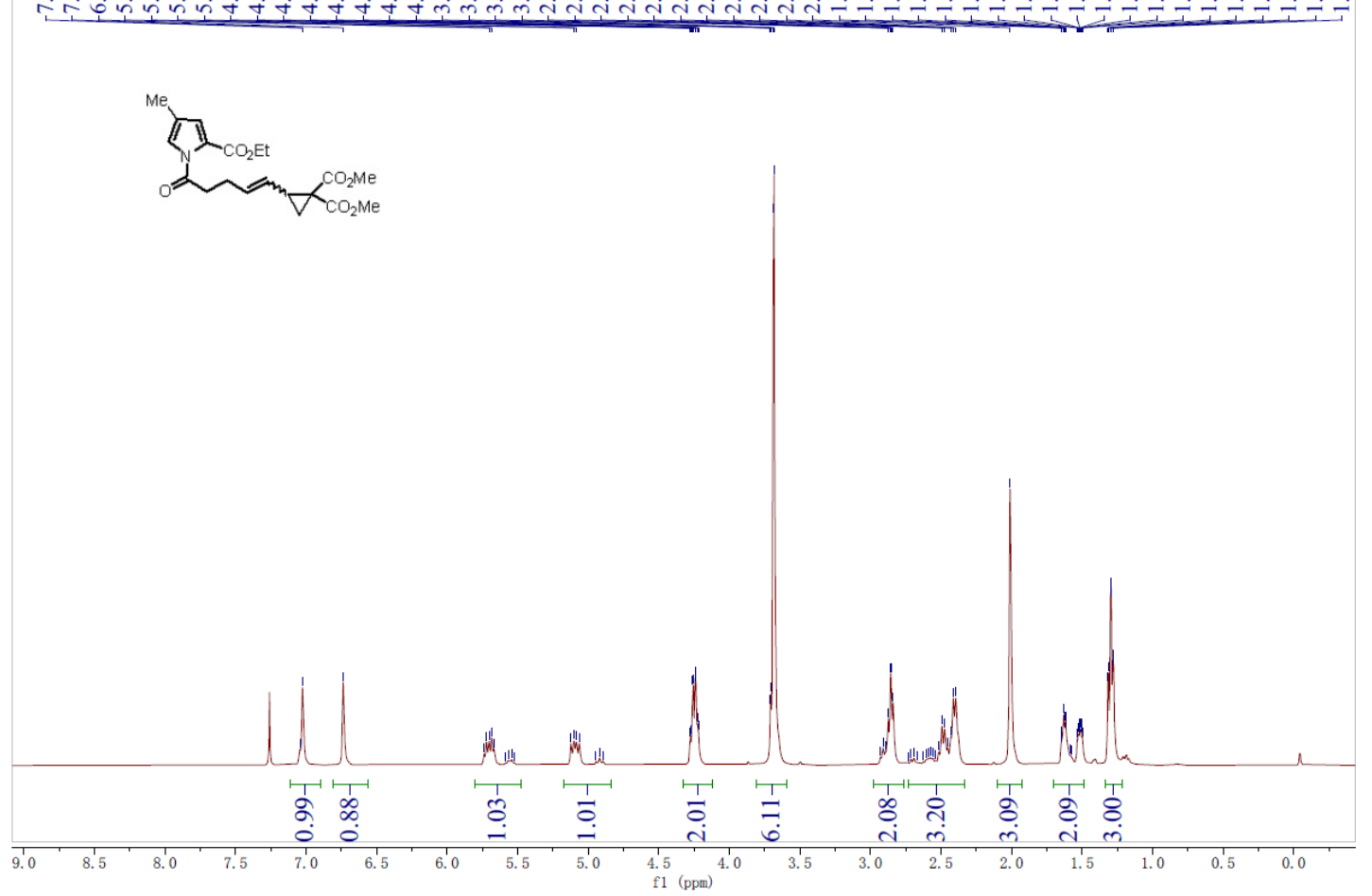

${ }^{13} \mathrm{C}$ NMR Spectrum of $\mathbf{7 g}$

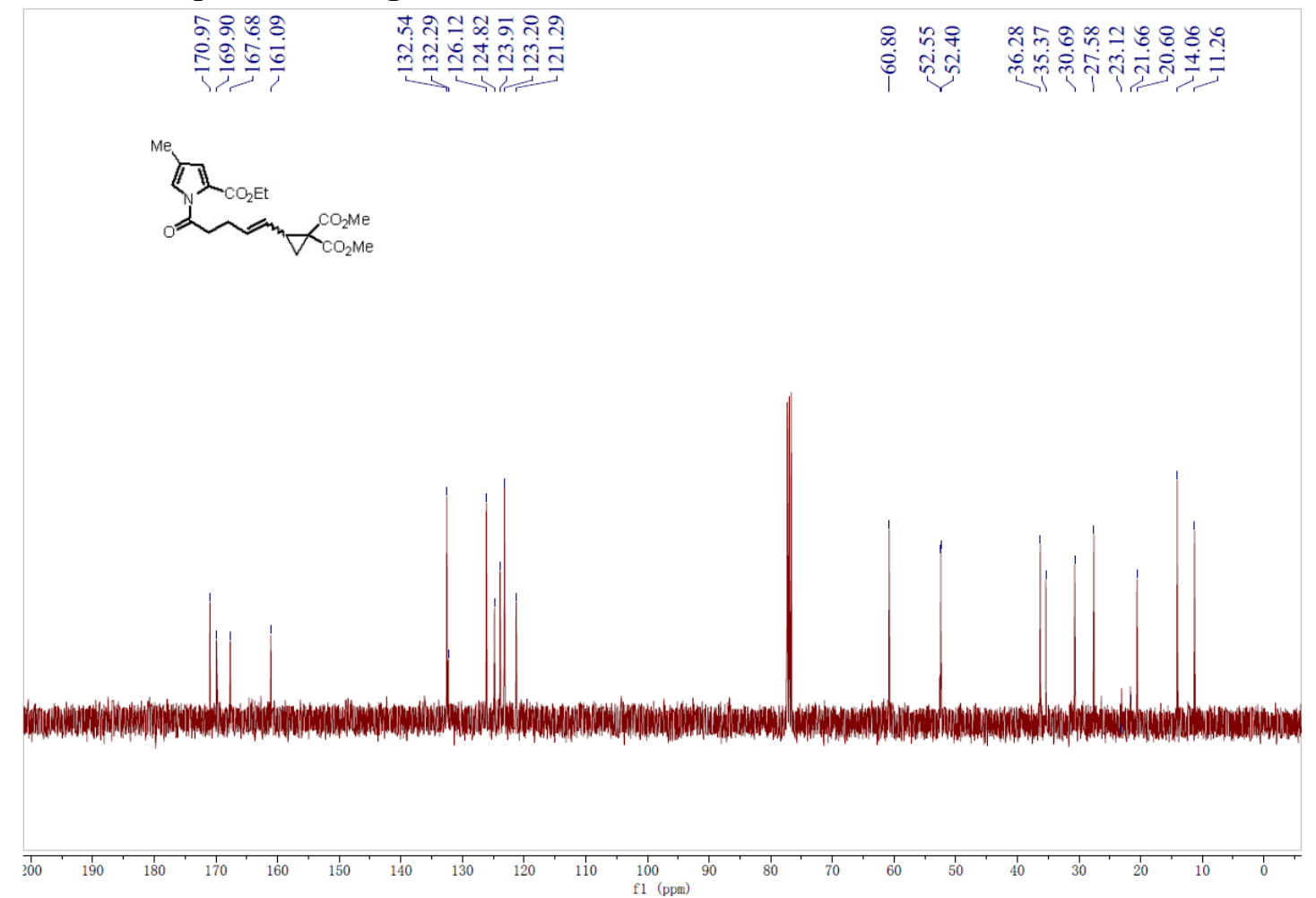


${ }^{1} \mathrm{H}$ NMR Spectrum of $\mathbf{1 a}$

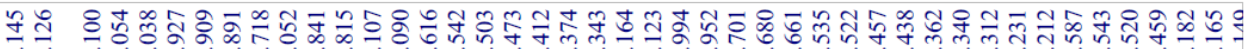

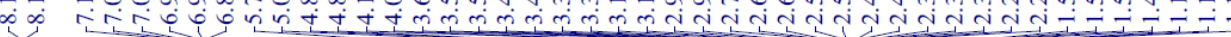
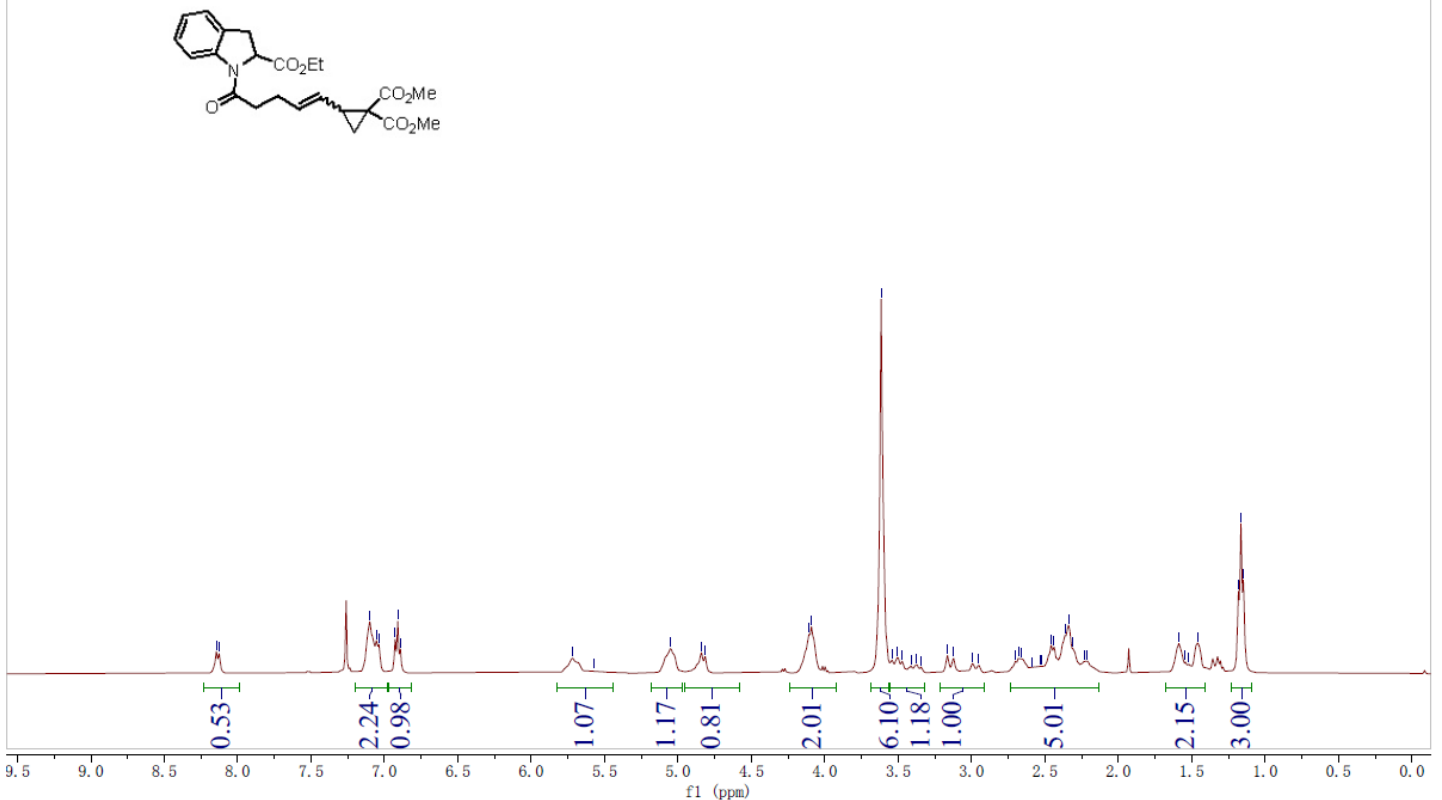

${ }^{13} \mathrm{C}$ NMR Spectrum of $\mathbf{1} \mathbf{a}^{\prime}$

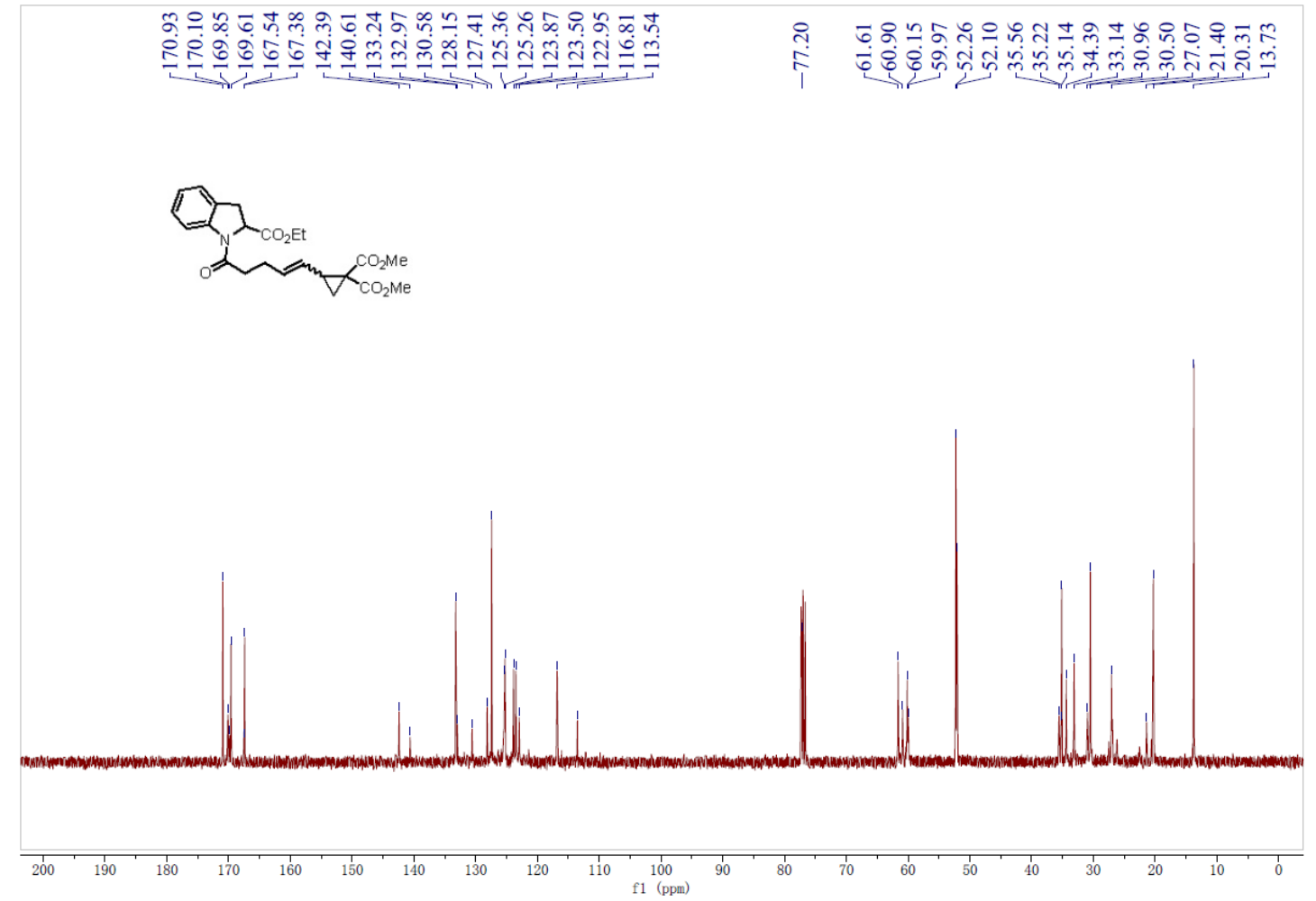


${ }^{1} \mathrm{H}$ NMR Spectrum of $\mathbf{2 a}$

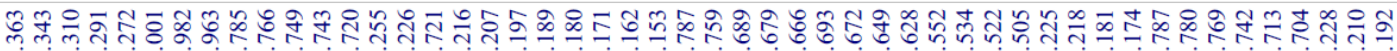

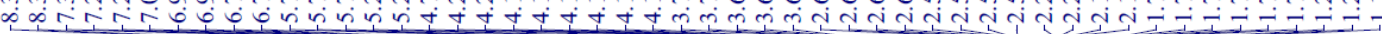

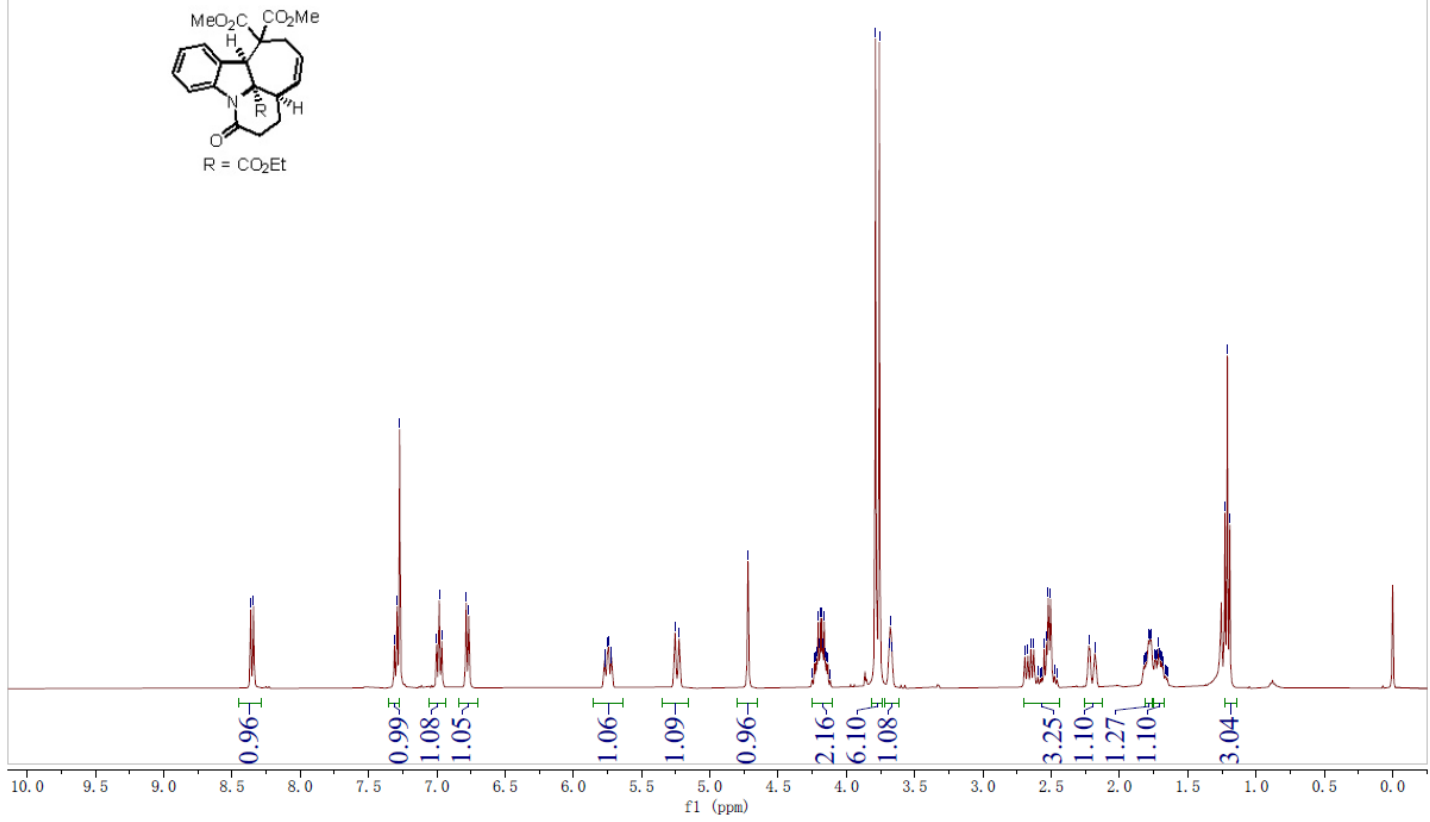

${ }^{13} \mathrm{C}$ NMR Spectrum of $\mathbf{2 a}$

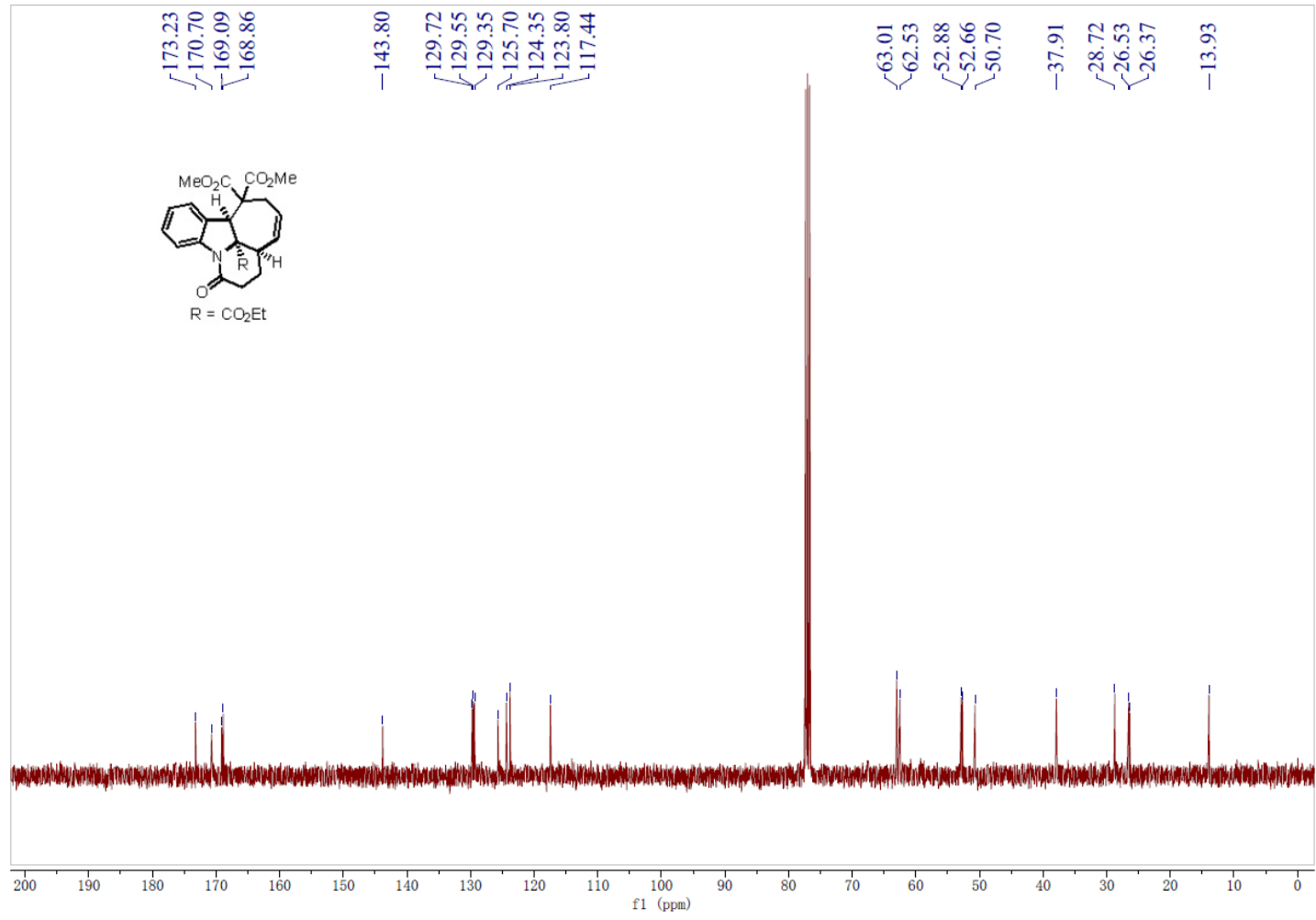


${ }^{1} \mathrm{H}$ NMR Spectrum of $\mathbf{2 b}$

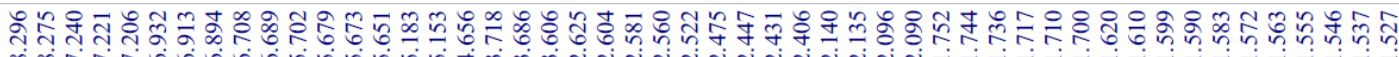

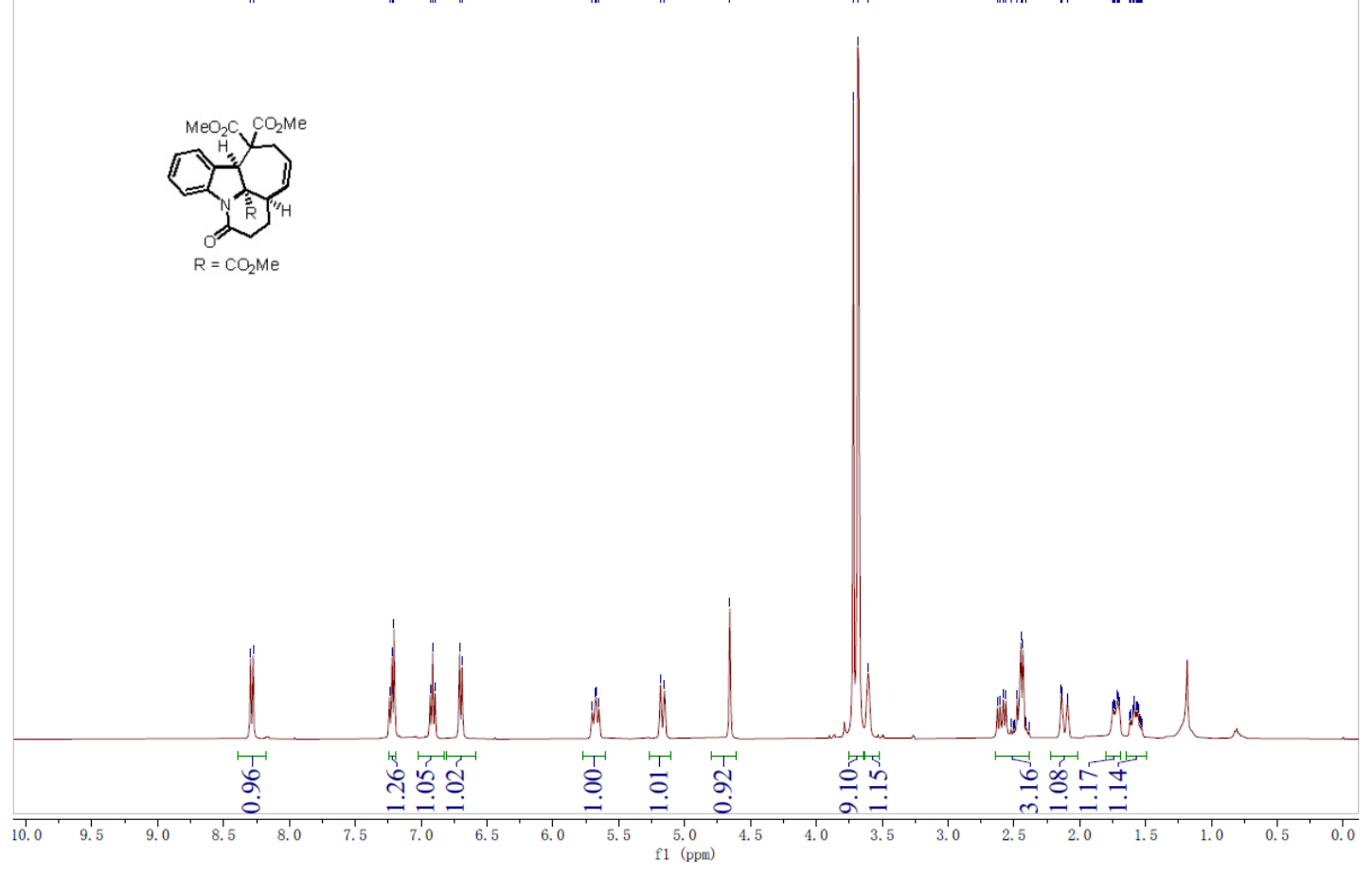

${ }^{13} \mathrm{C}$ NMR Spectrum of $\mathbf{2 b}$

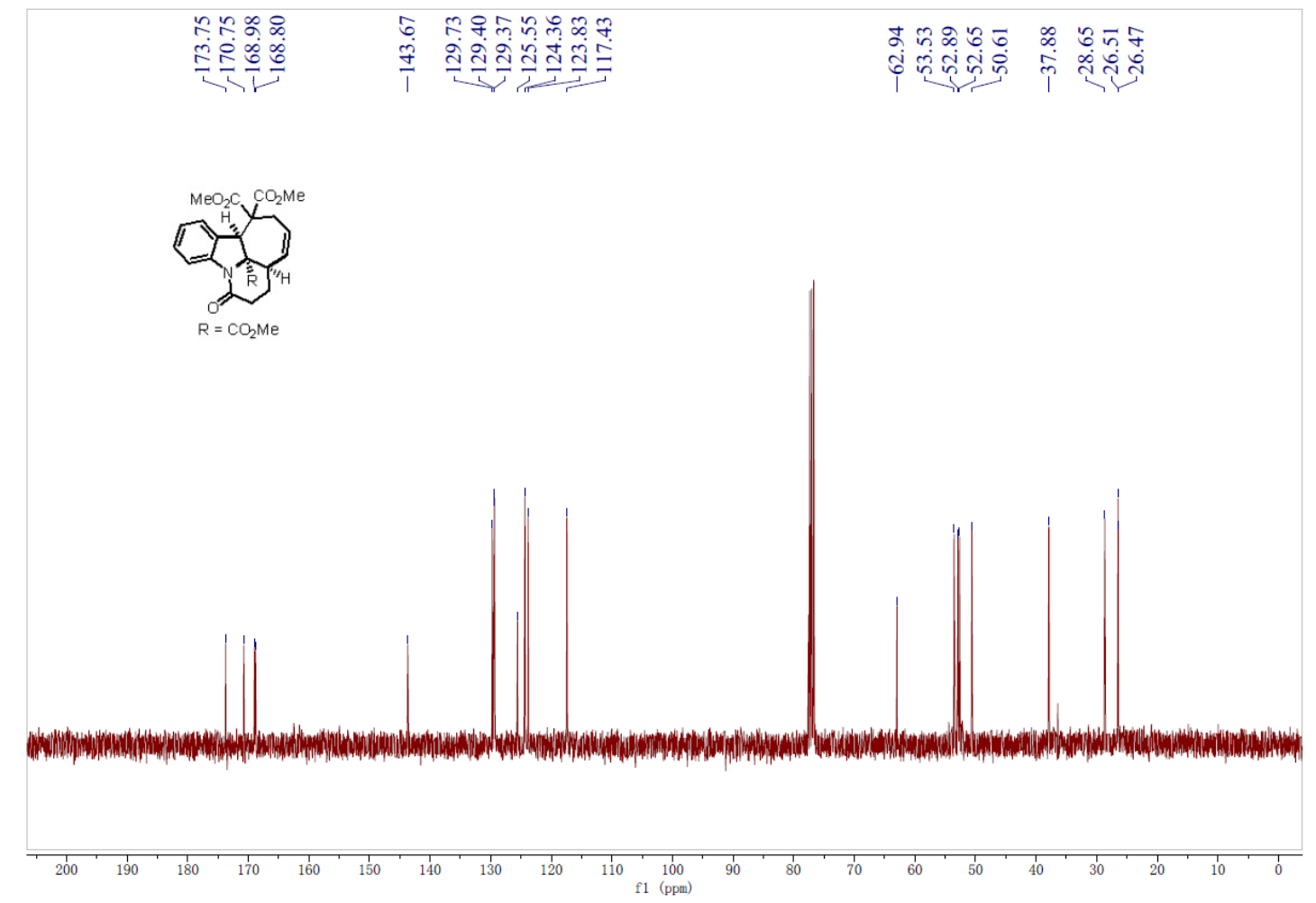


${ }^{1} \mathrm{H}$ NMR Spectrum of 2c

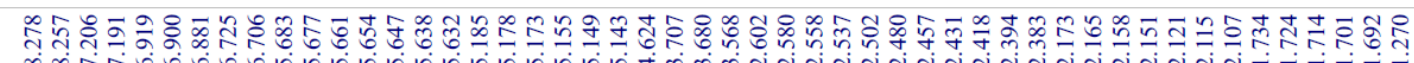

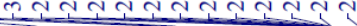
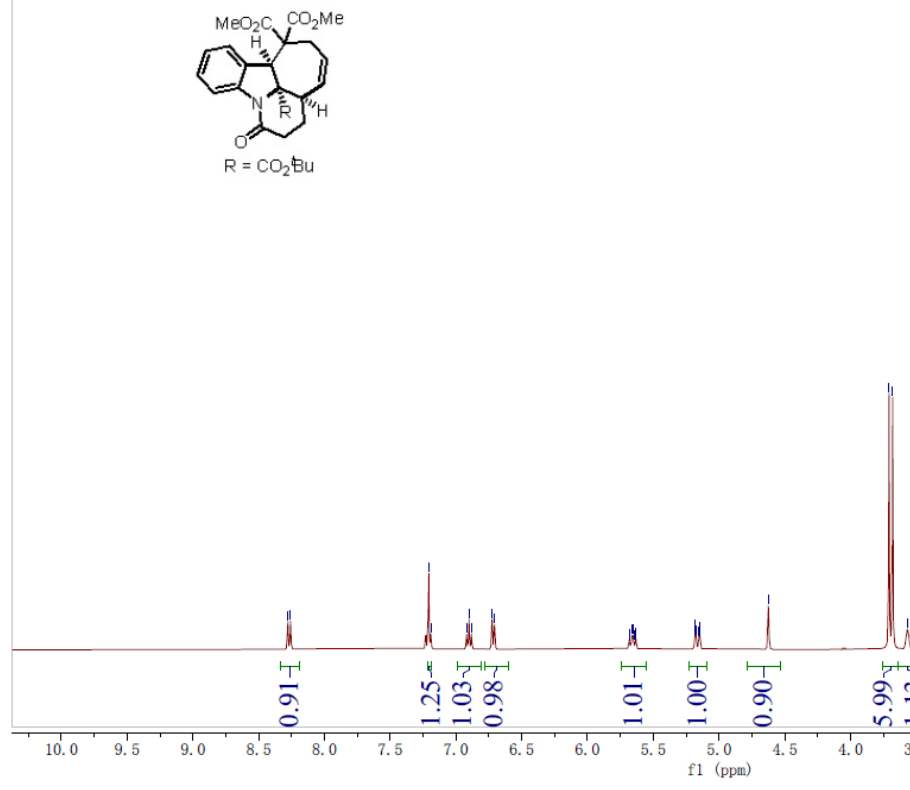

w

가에 - ले

${ }^{13} \mathrm{C}$ NMR Spectrum of $\mathbf{2 c}$
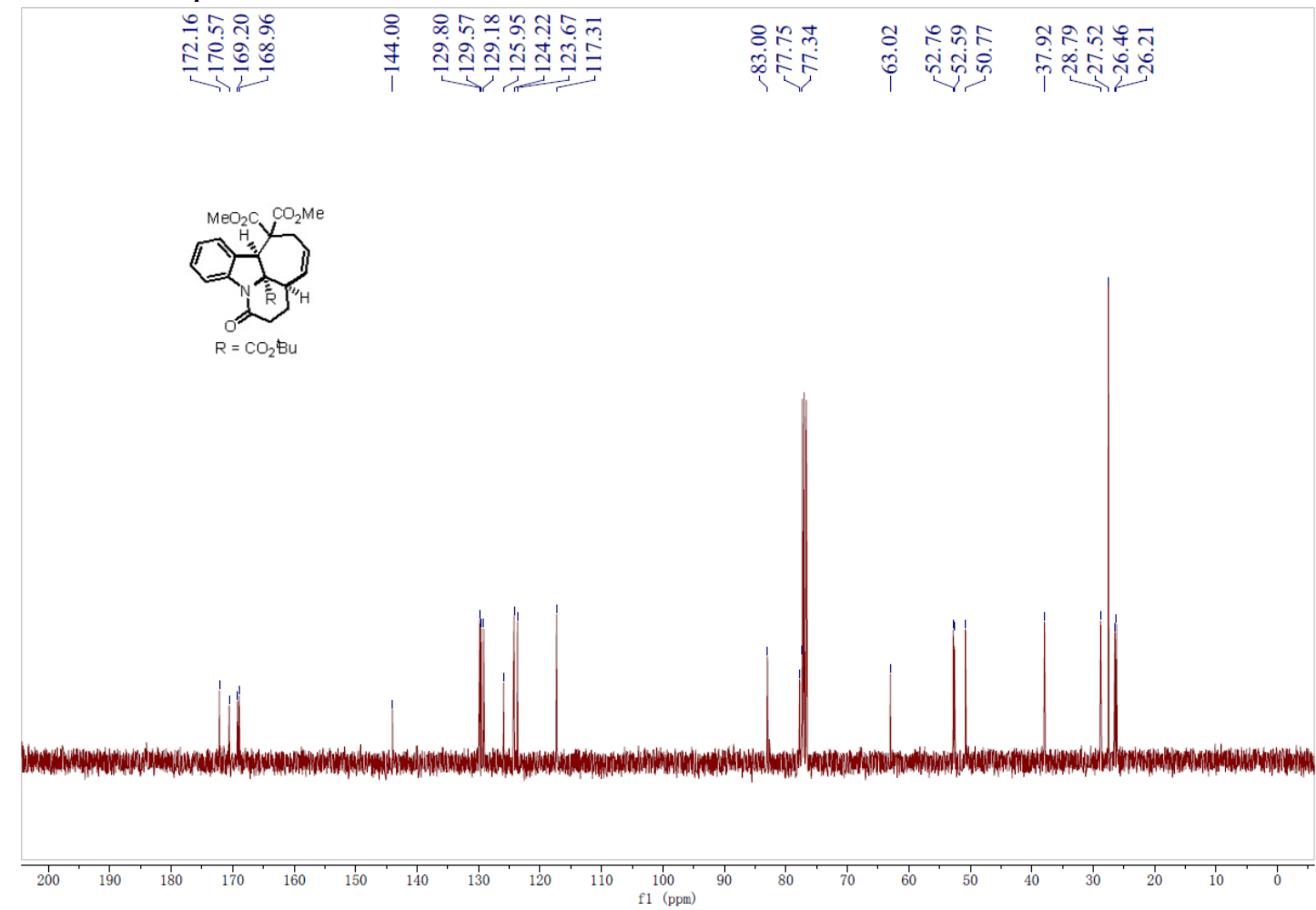
${ }^{1} \mathrm{H}$ NMR Spectrum of $\mathbf{2 d}$

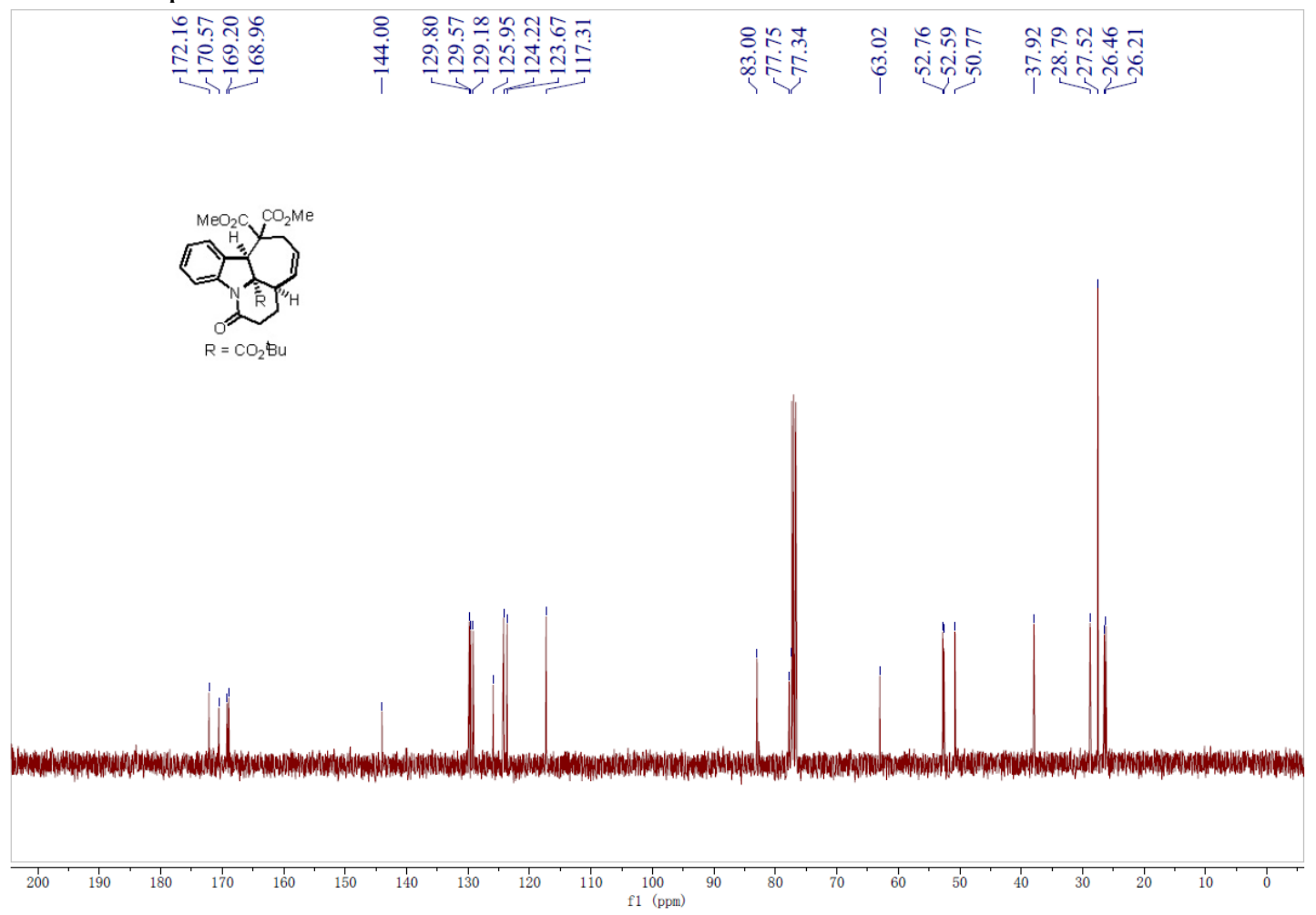

${ }^{13} \mathrm{C}$ NMR Spectrum of $\mathbf{2 d}$

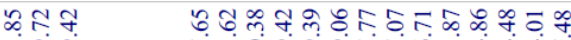

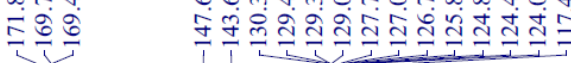

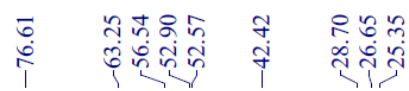
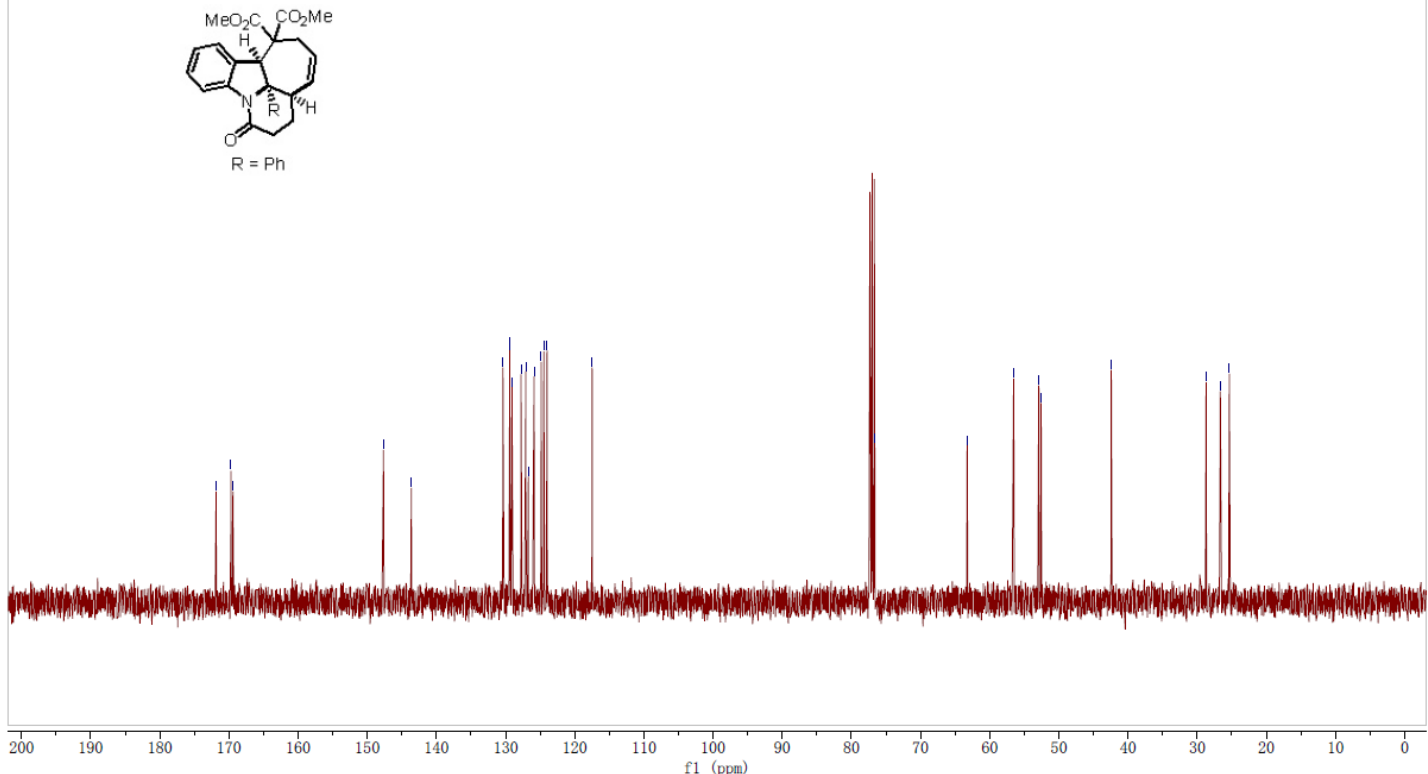
${ }^{1} \mathrm{H}$ NMR Spectrum of $\mathbf{2 e}$

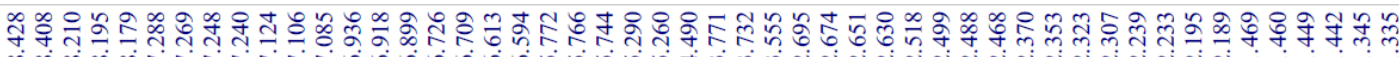

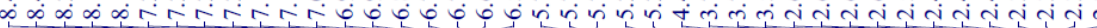
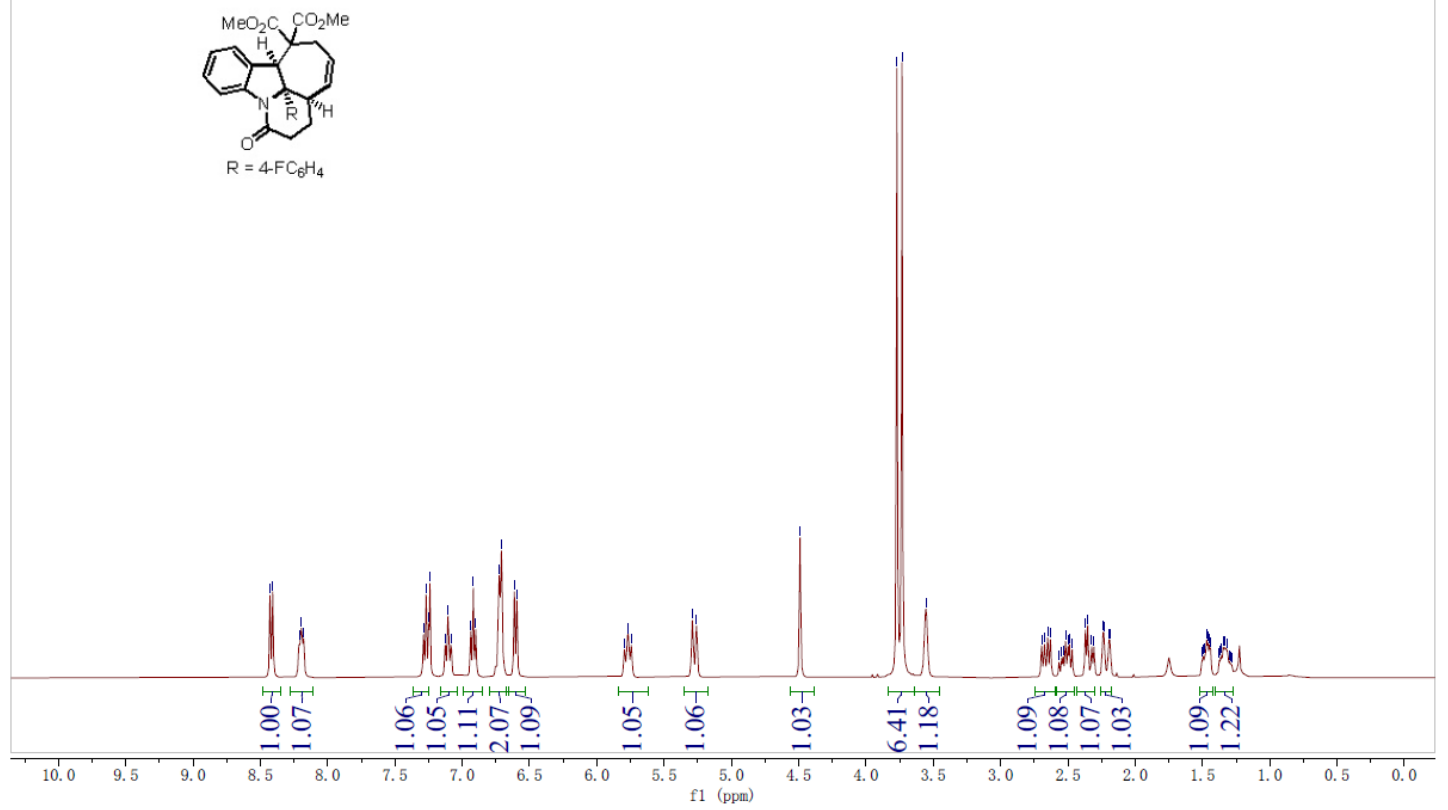

${ }^{13} \mathrm{C}$ NMR Spectrum of $\mathbf{2 e}$

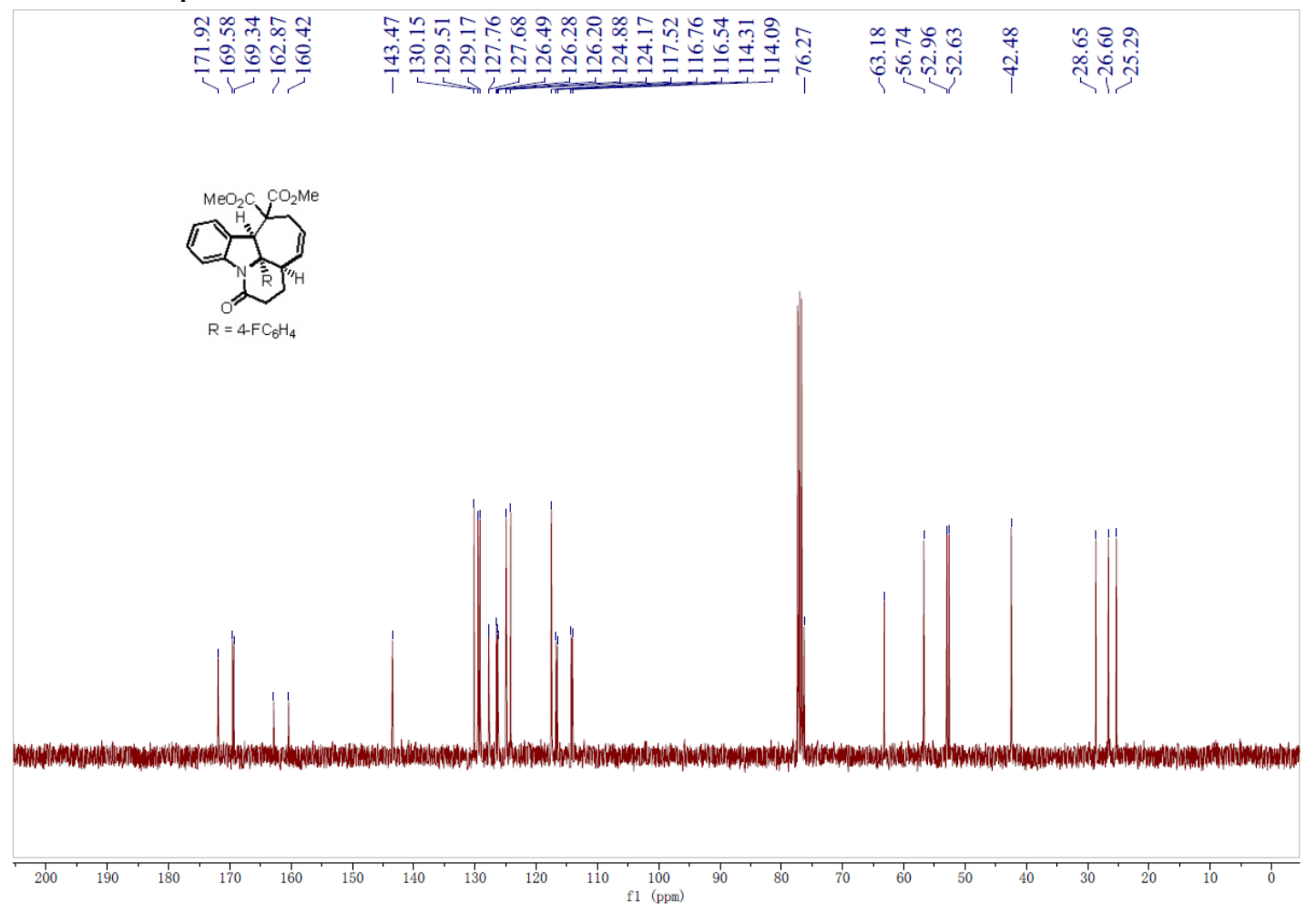


${ }^{19} \mathrm{~F}$ NMR Spectrum of $\mathbf{2 e}$

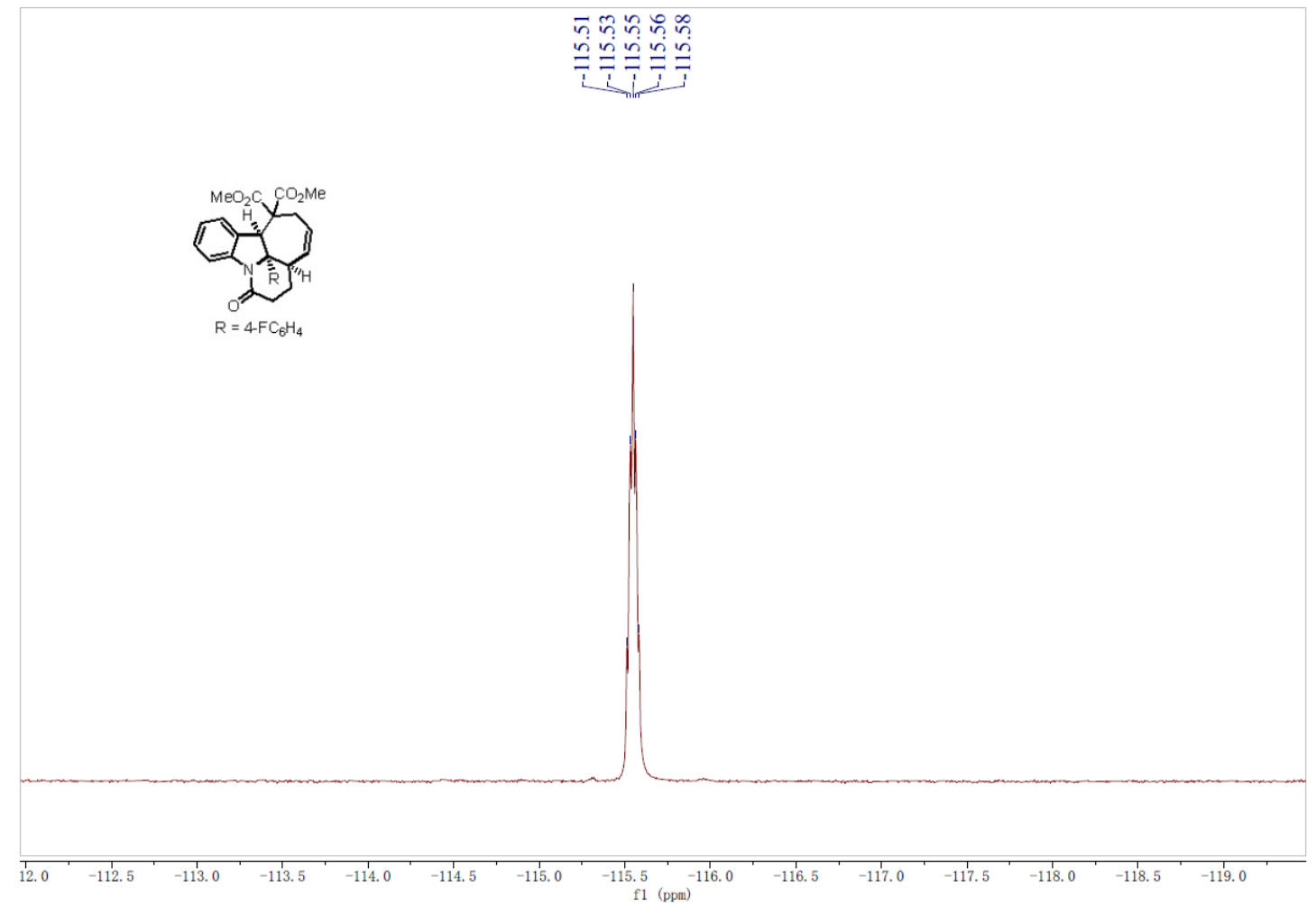


${ }^{1} \mathrm{H}$ NMR Spectrum of $\mathbf{2 f}$

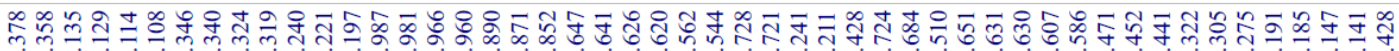

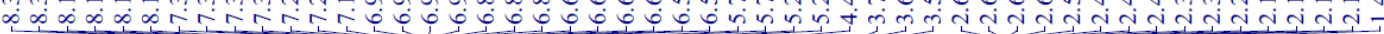

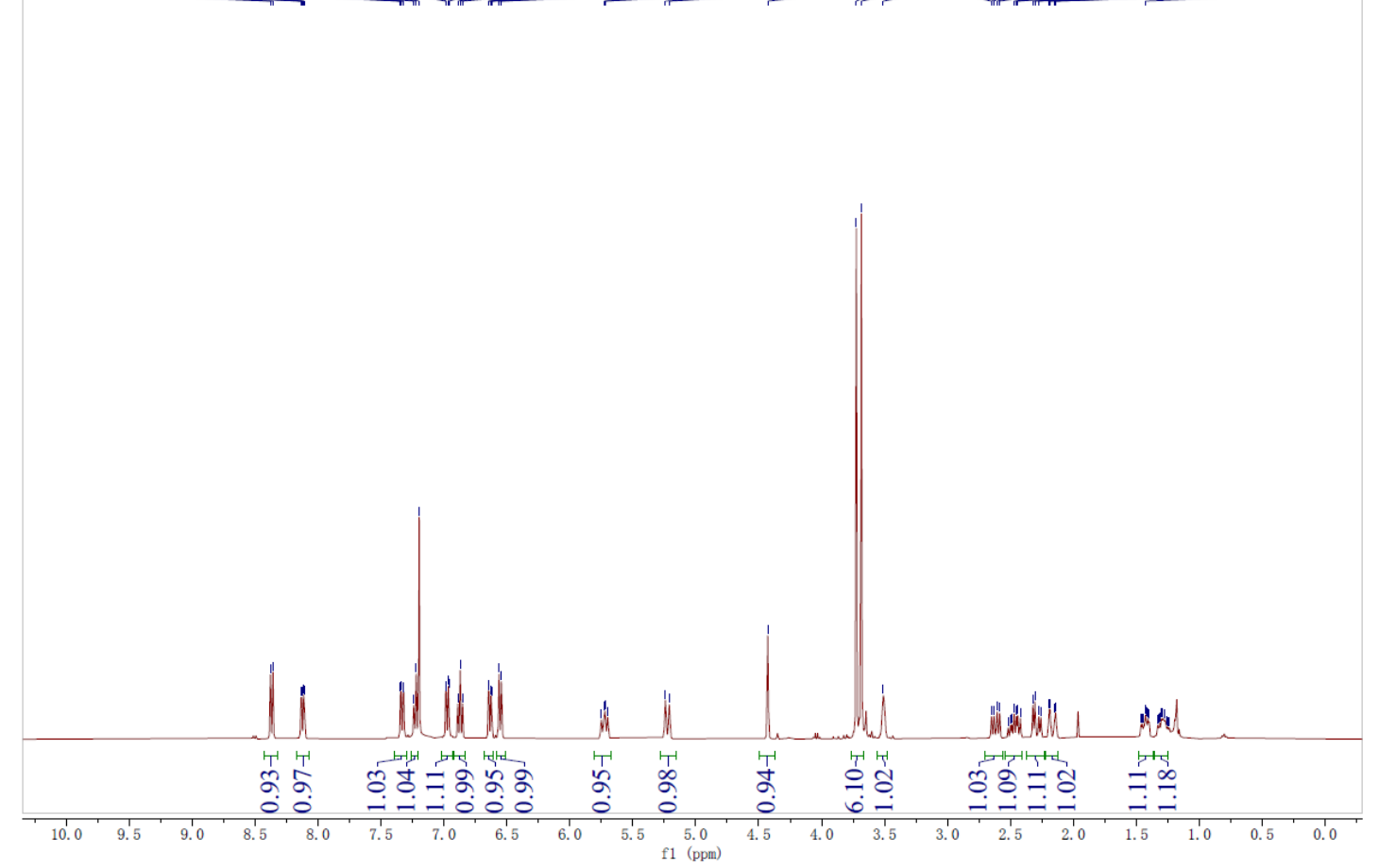

${ }^{13} \mathrm{C}$ NMR Spectrum of $\mathbf{2 f}$

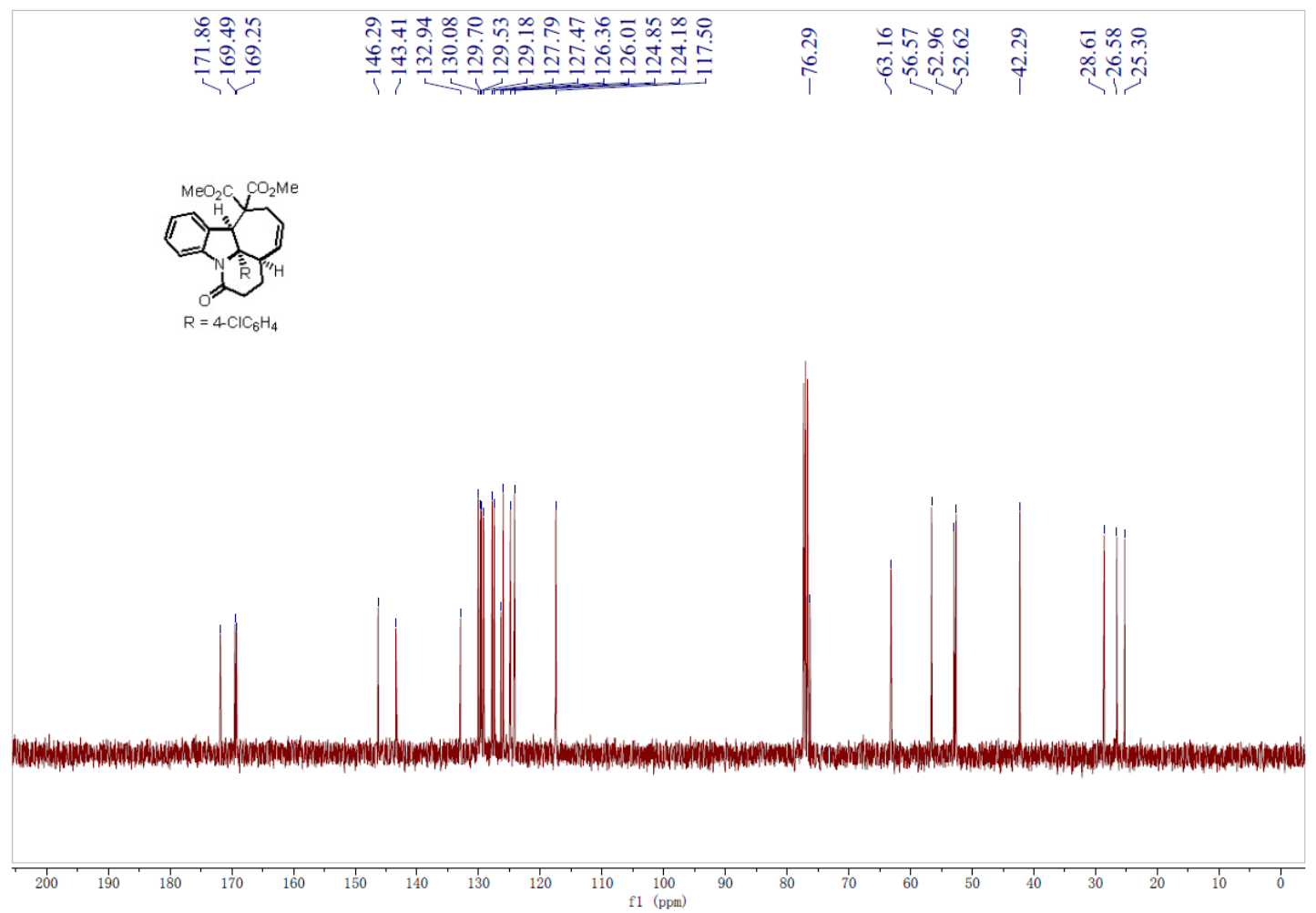


${ }^{1} \mathrm{H}$ NMR Spectrum of $\mathbf{2 g}$

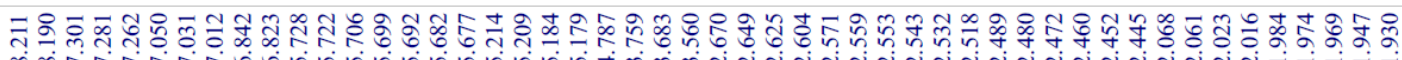

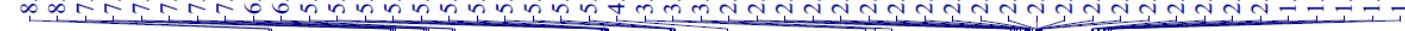

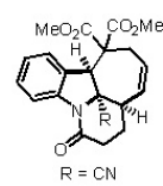

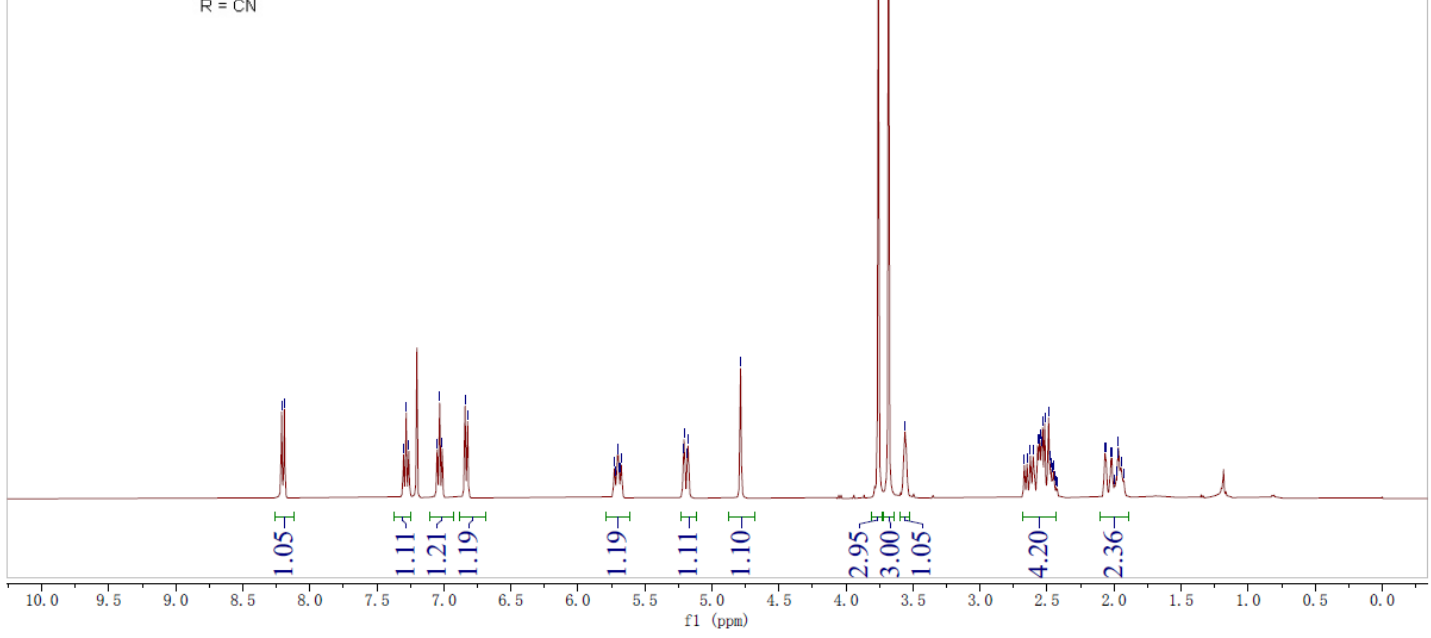

${ }^{13} \mathrm{C}$ NMR Spectrum of $\mathbf{2 g}$

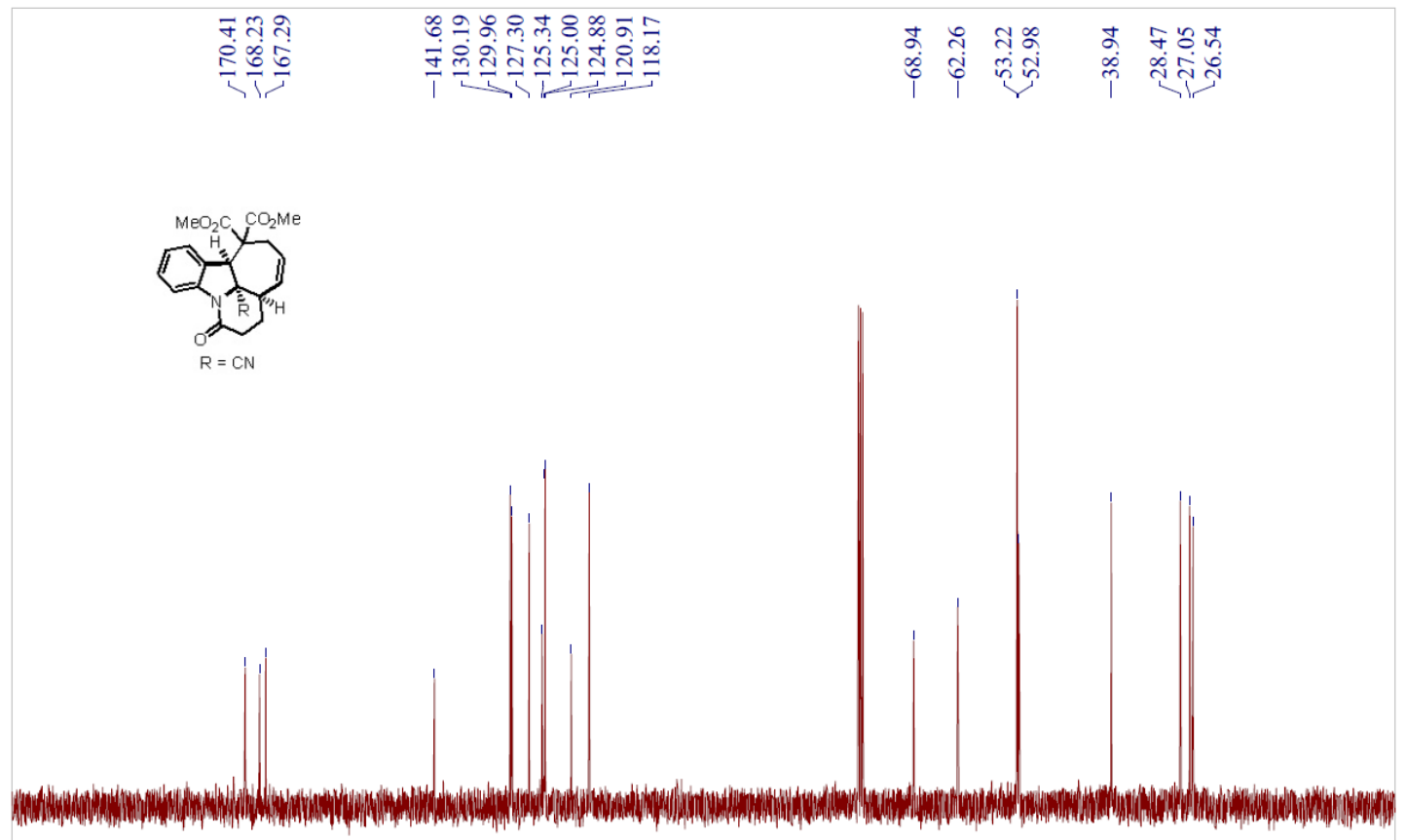


${ }^{1} \mathrm{H}$ NMR Spectrum of $\mathbf{2 h}$

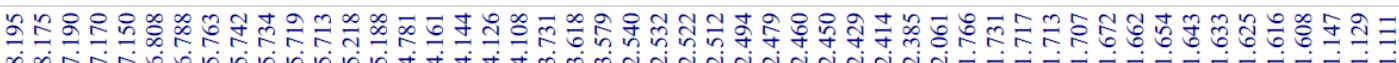
管
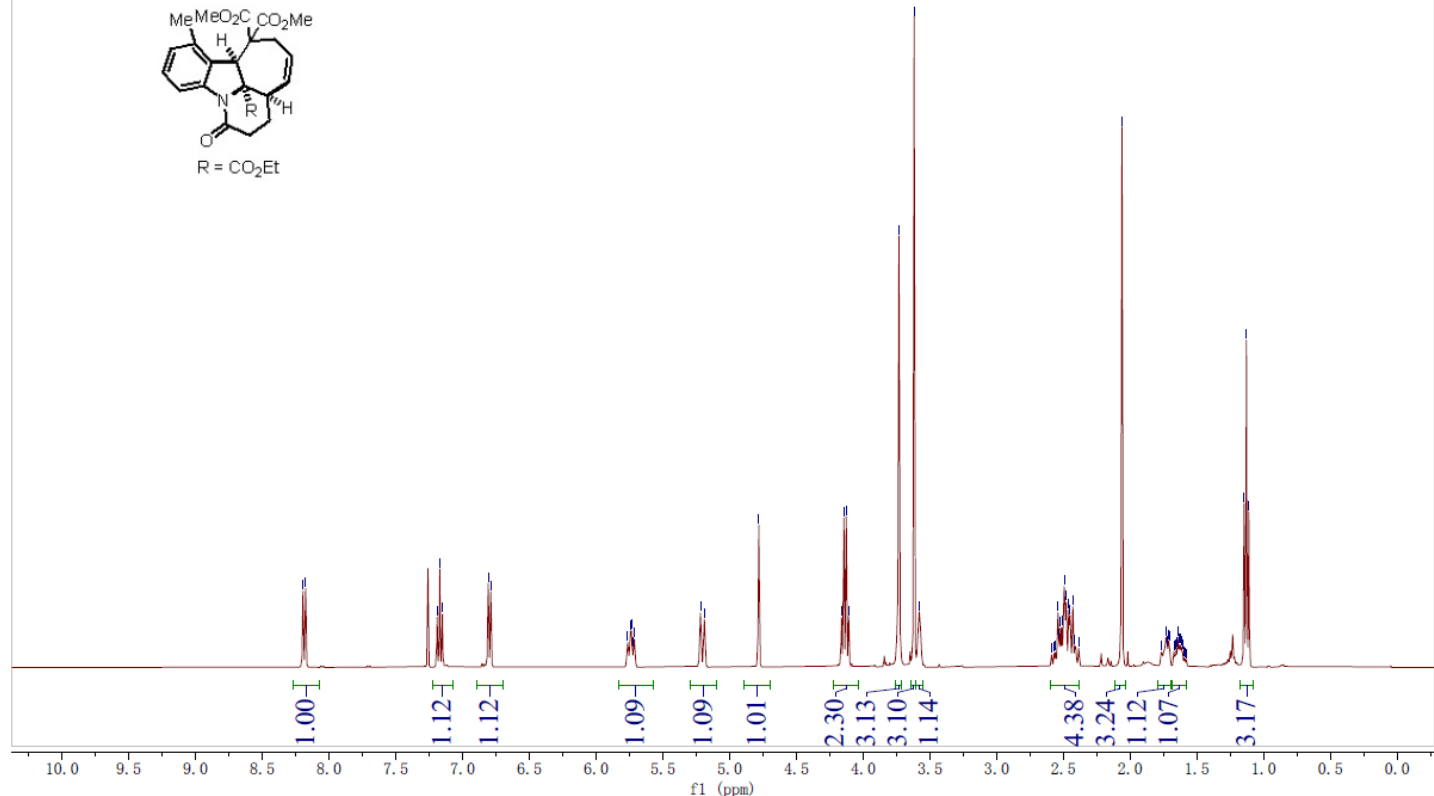

${ }^{13} \mathrm{C}$ NMR Spectrum of $\mathbf{2 h}$

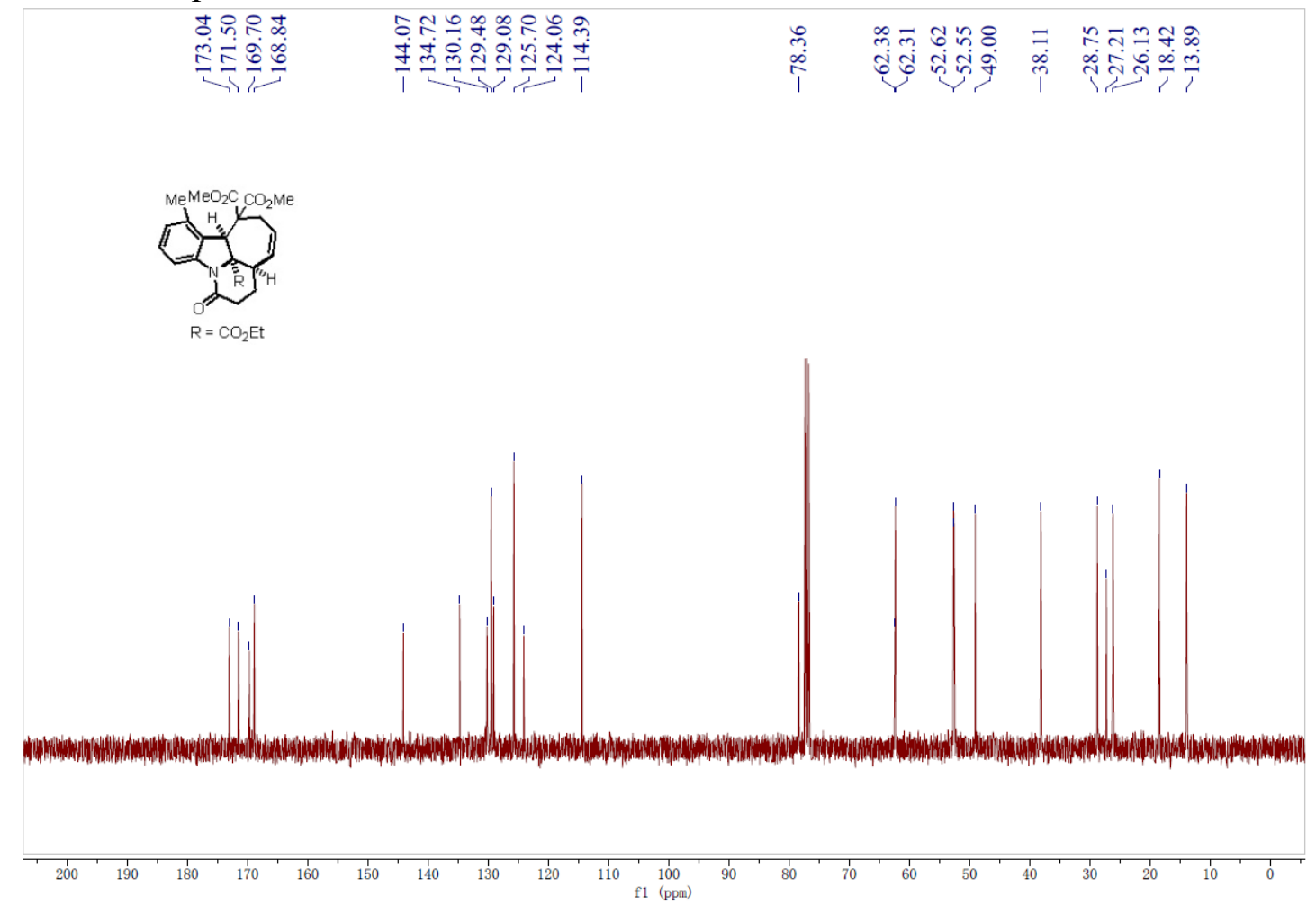


${ }^{1} \mathrm{H}$ NMR Spectrum of $\mathbf{2 i}$

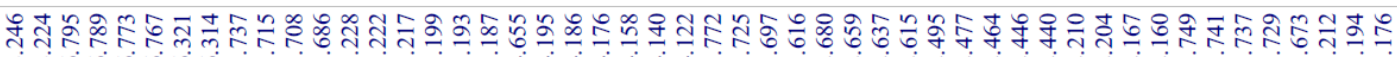

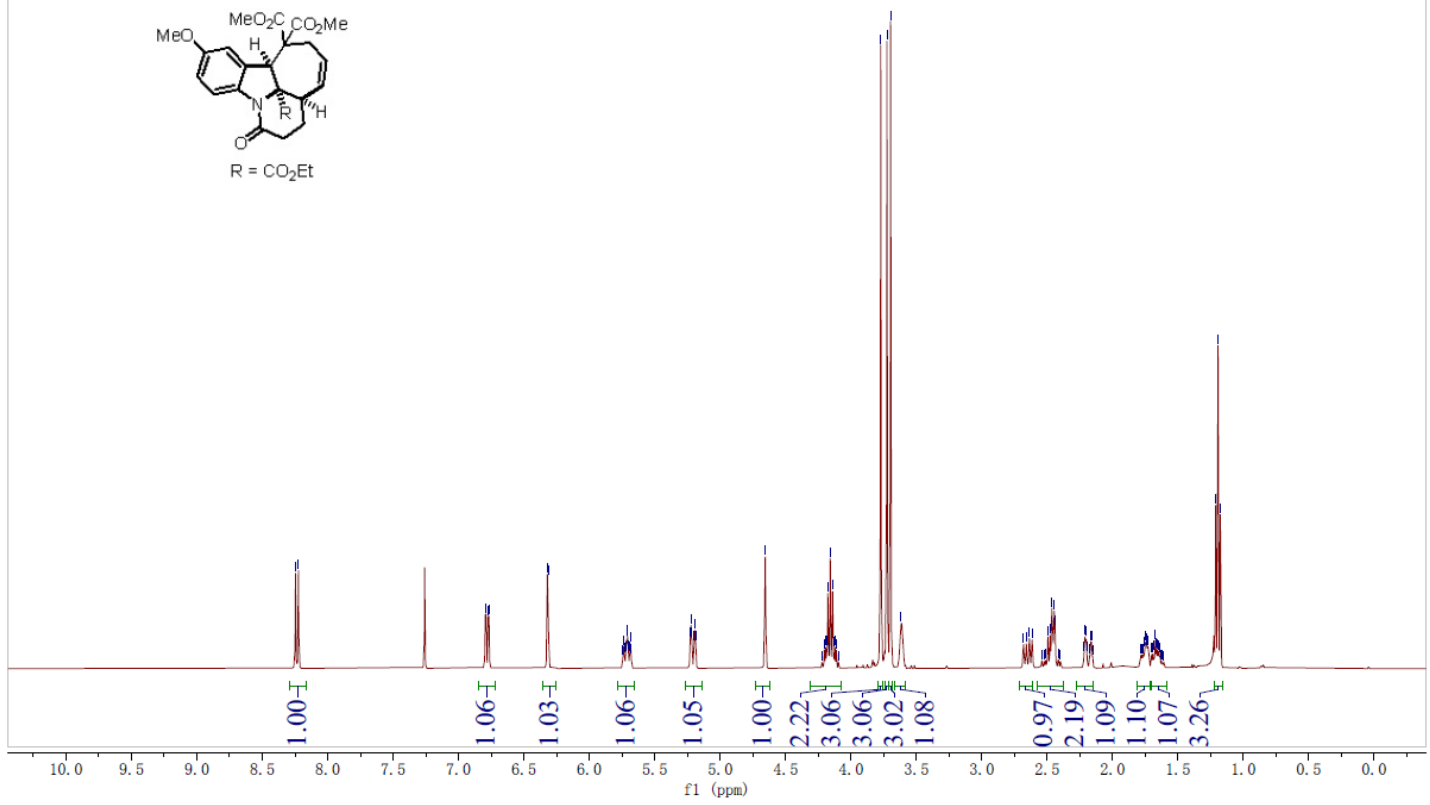

${ }^{13} \mathrm{C}$ NMR Spectrum of $\mathbf{2 i}$

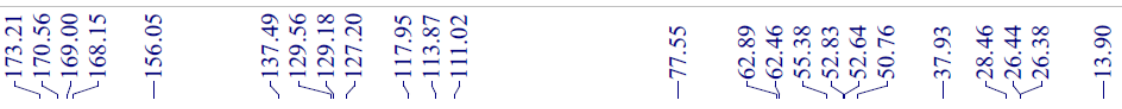
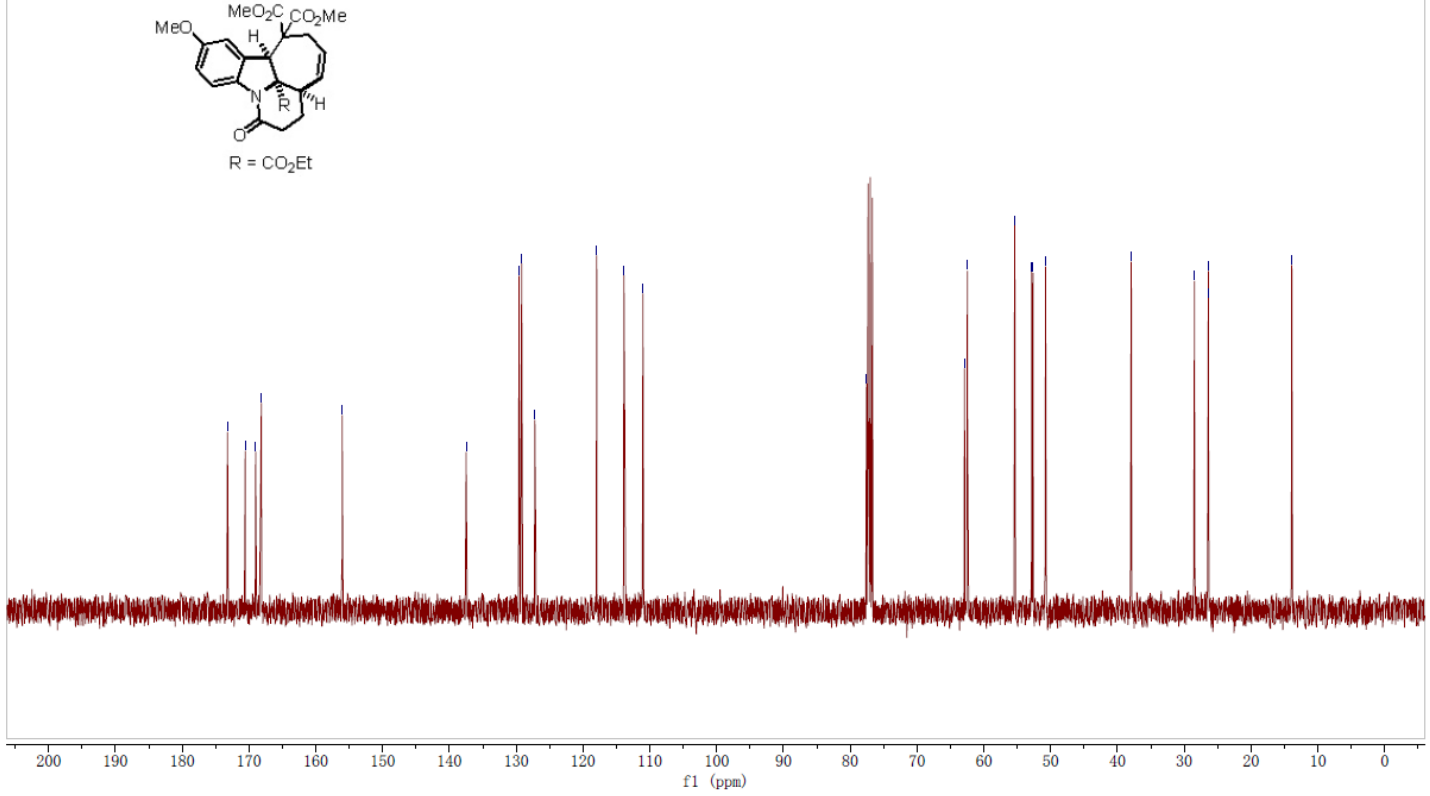
${ }^{1} \mathrm{H}$ NMR Spectrum of $\mathbf{2 j}$

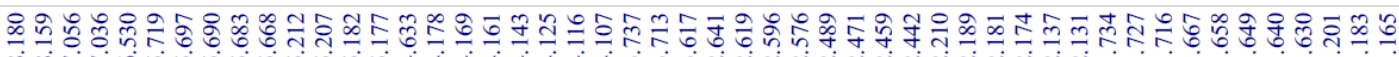
年
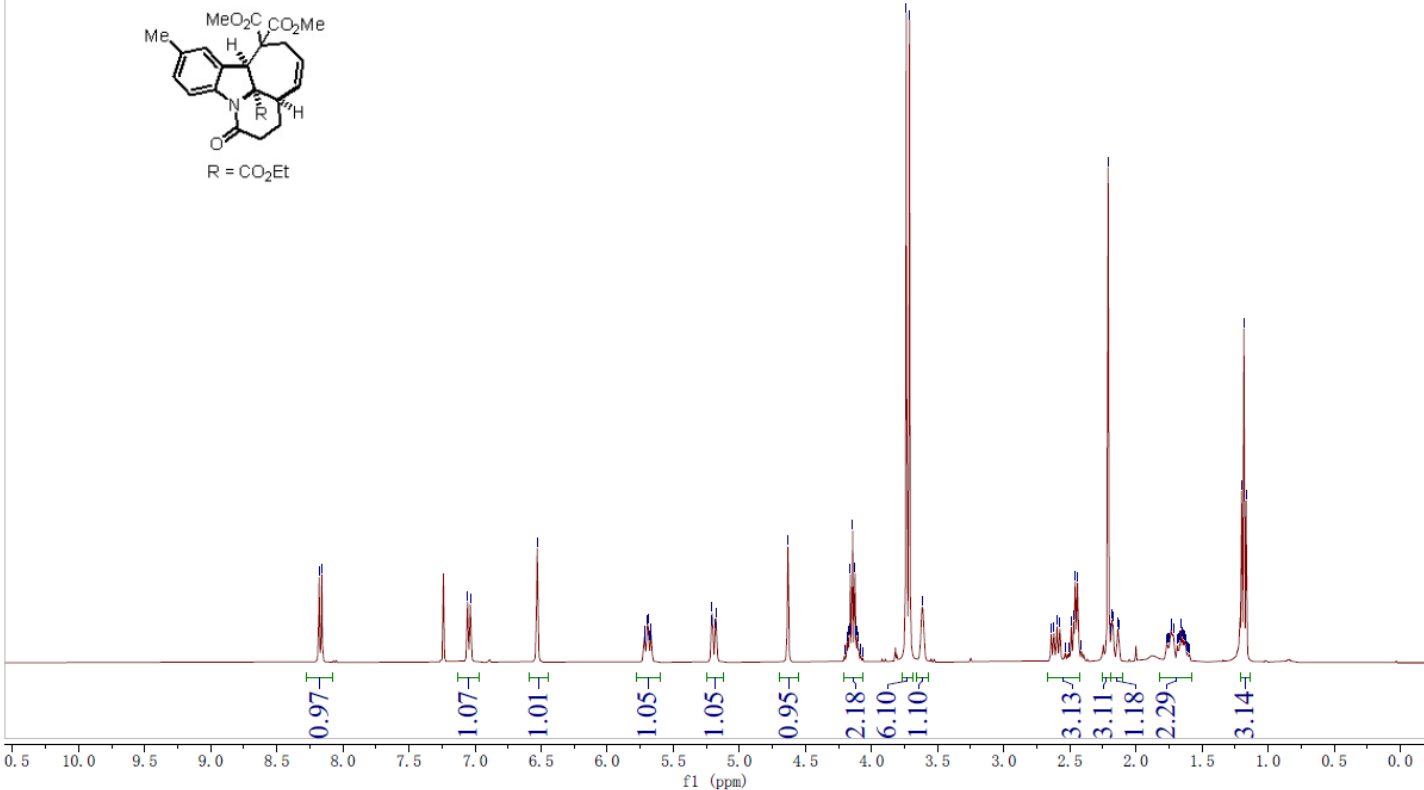

${ }^{13} \mathrm{C}$ NMR Spectrum of $\mathbf{2} \mathbf{j}$

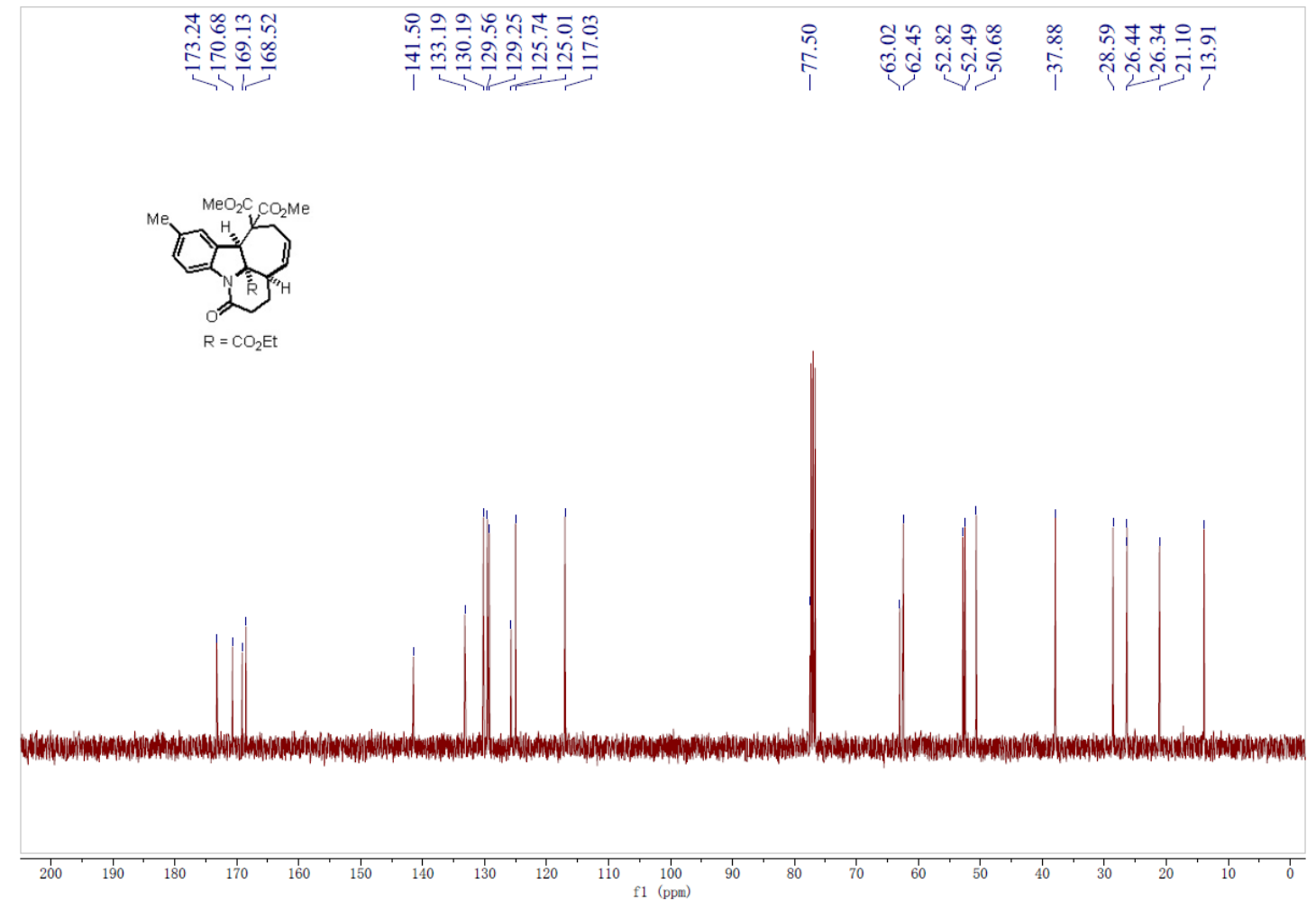


${ }^{1} \mathrm{H}$ NMR Spectrum of $\mathbf{2 k}$

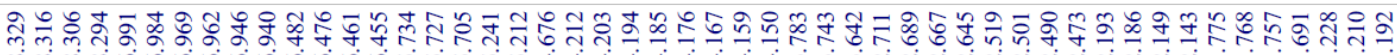

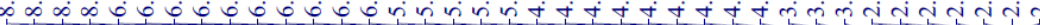

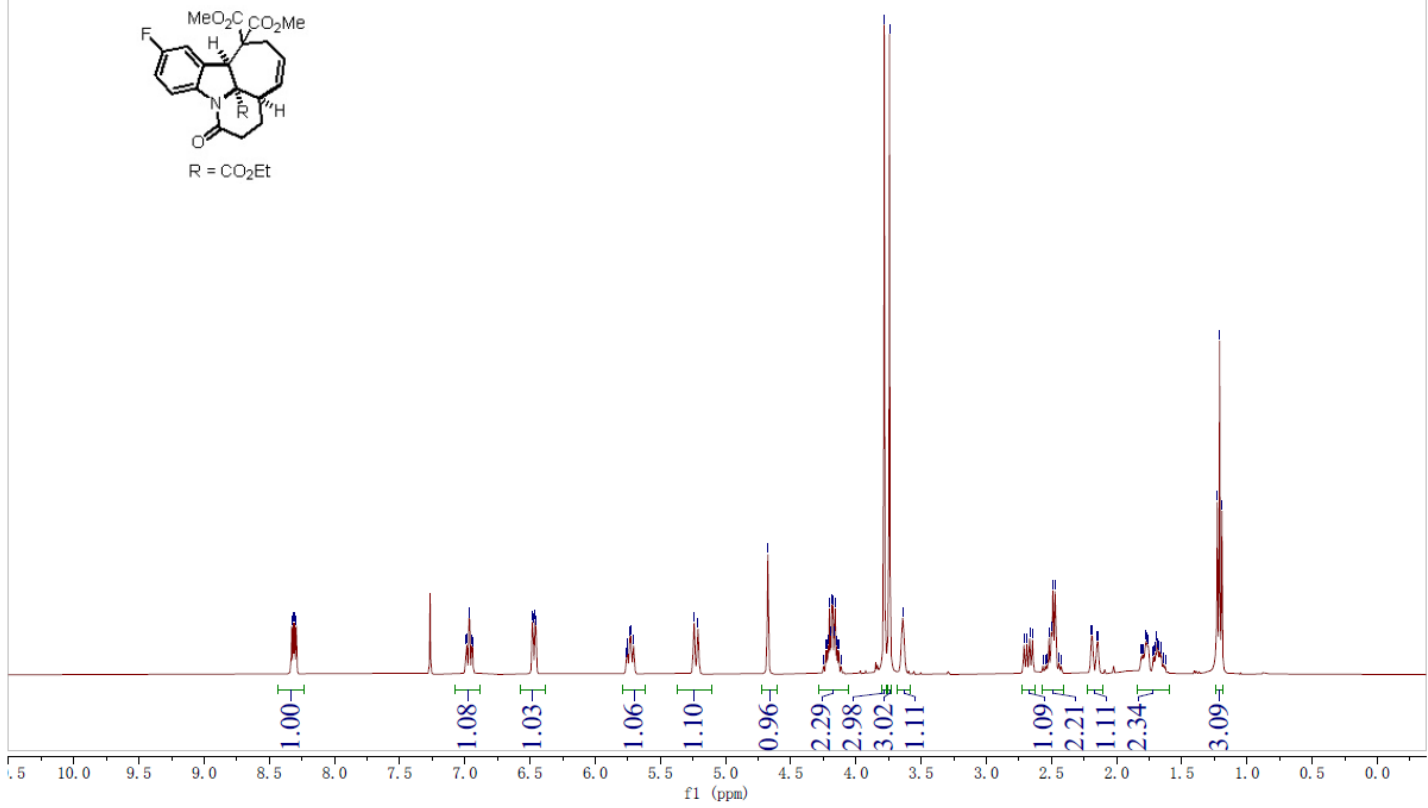

${ }^{13} \mathrm{C}$ NMR Spectrum of $\mathbf{2 k}$

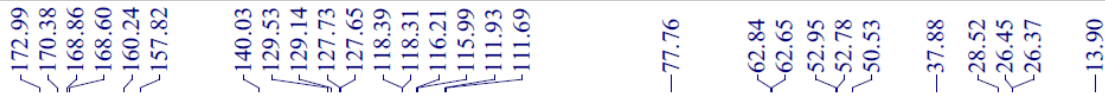
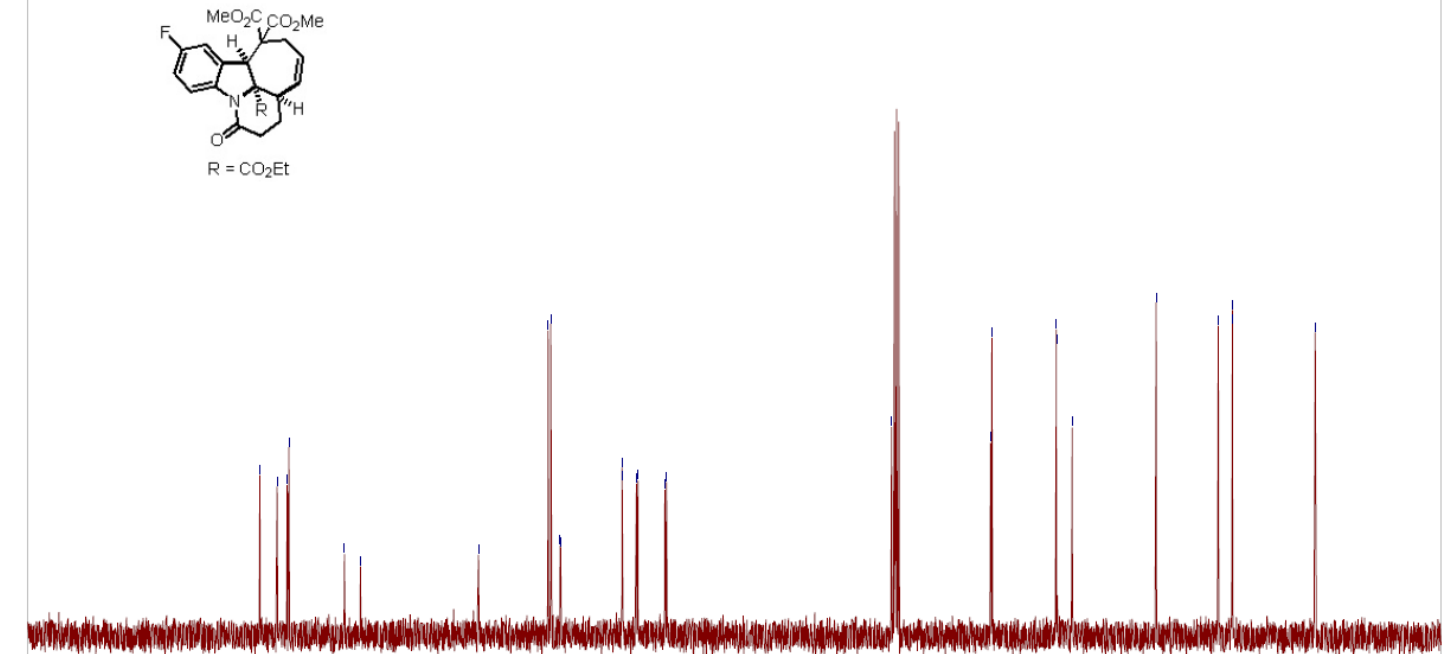

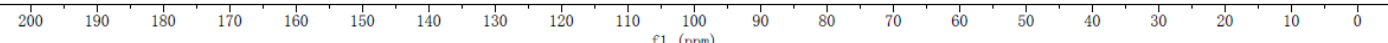


${ }^{19}$ F NMR Spectrum of $\mathbf{2 k}$

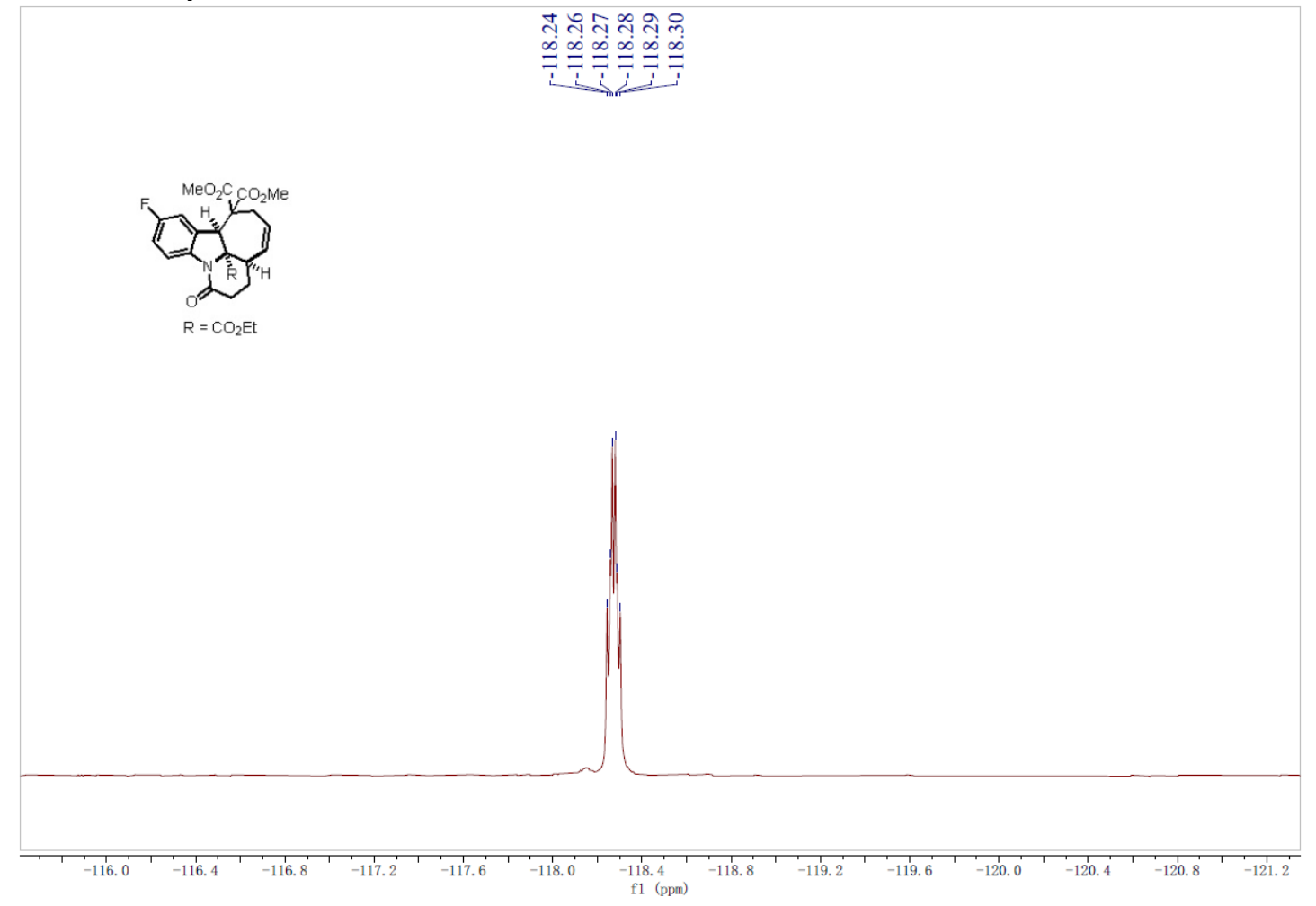


${ }^{1} \mathrm{H}$ NMR Spectrum of $\mathbf{2 l}$

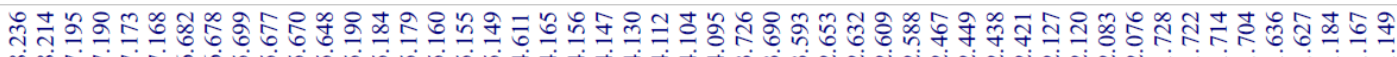

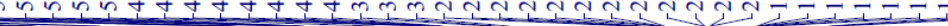
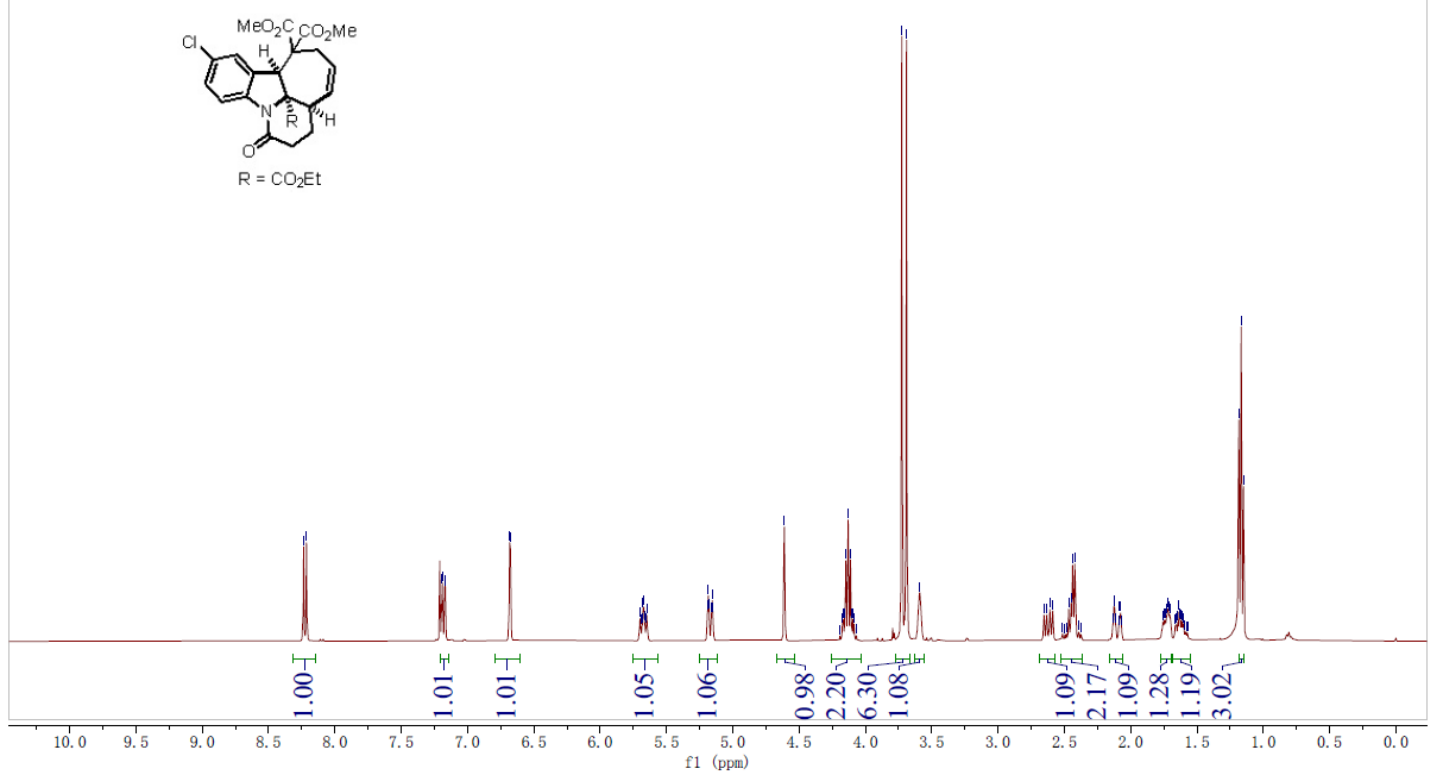

${ }^{13} \mathrm{C}$ NMR Spectrum of $\mathbf{2 l}$
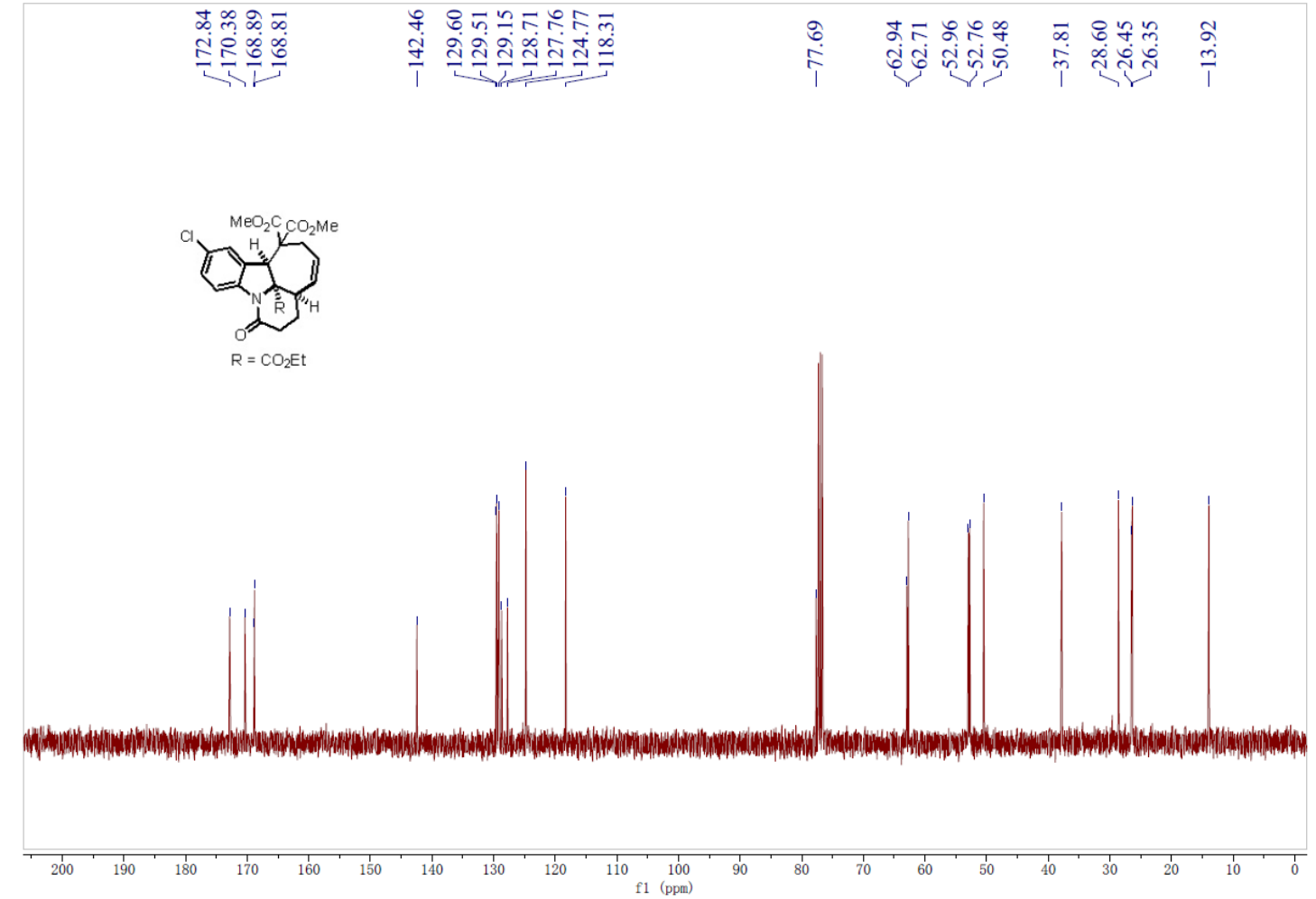
${ }^{1} \mathrm{H}$ NMR Spectrum of $\mathbf{2 m}$

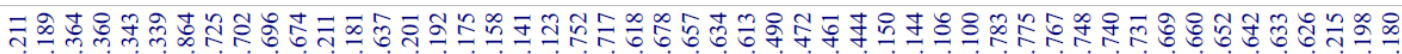

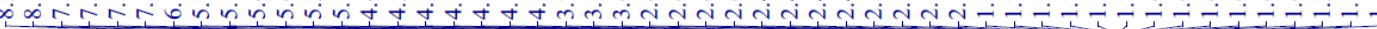

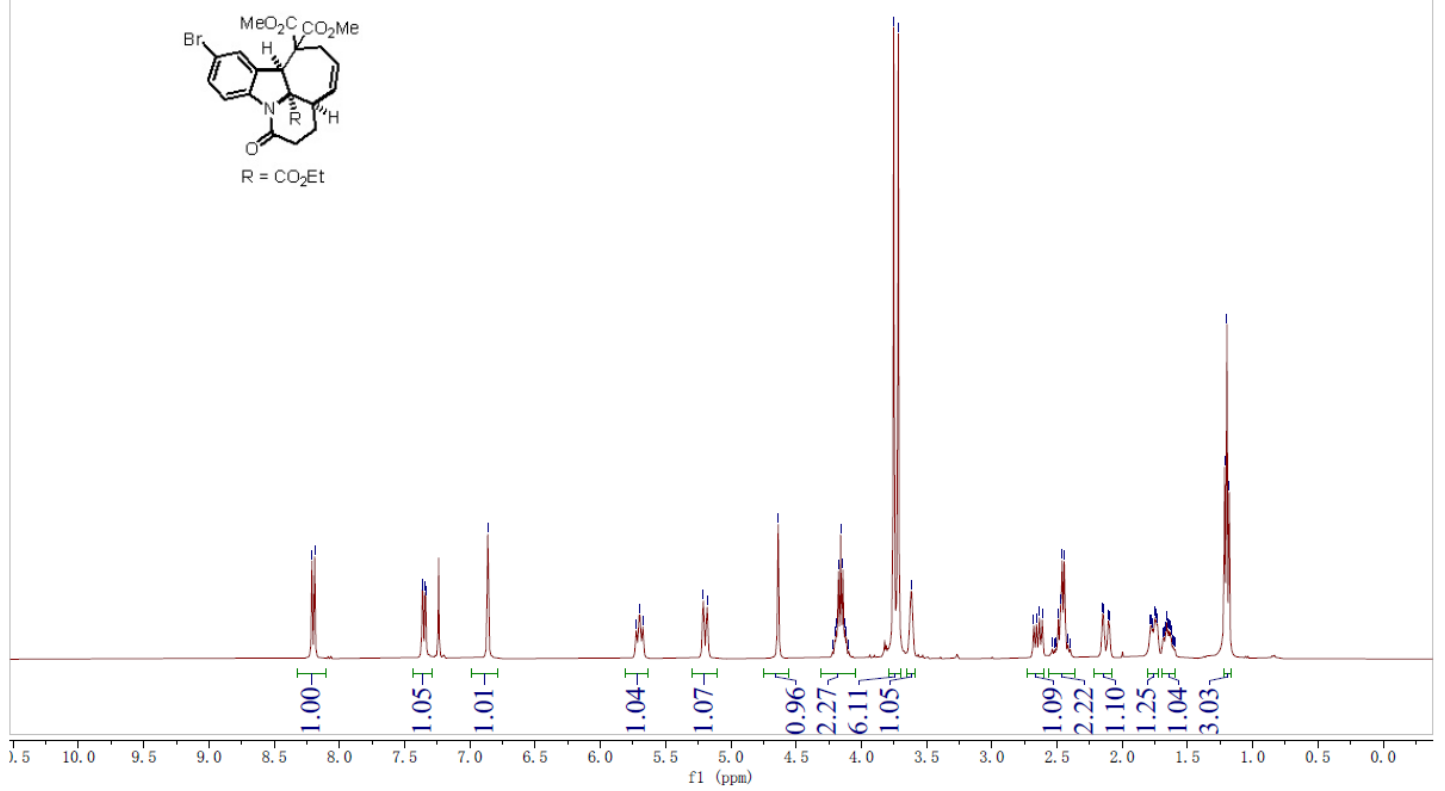

${ }^{13} \mathrm{C}$ NMR Spectrum of $\mathbf{2} \mathbf{m}$

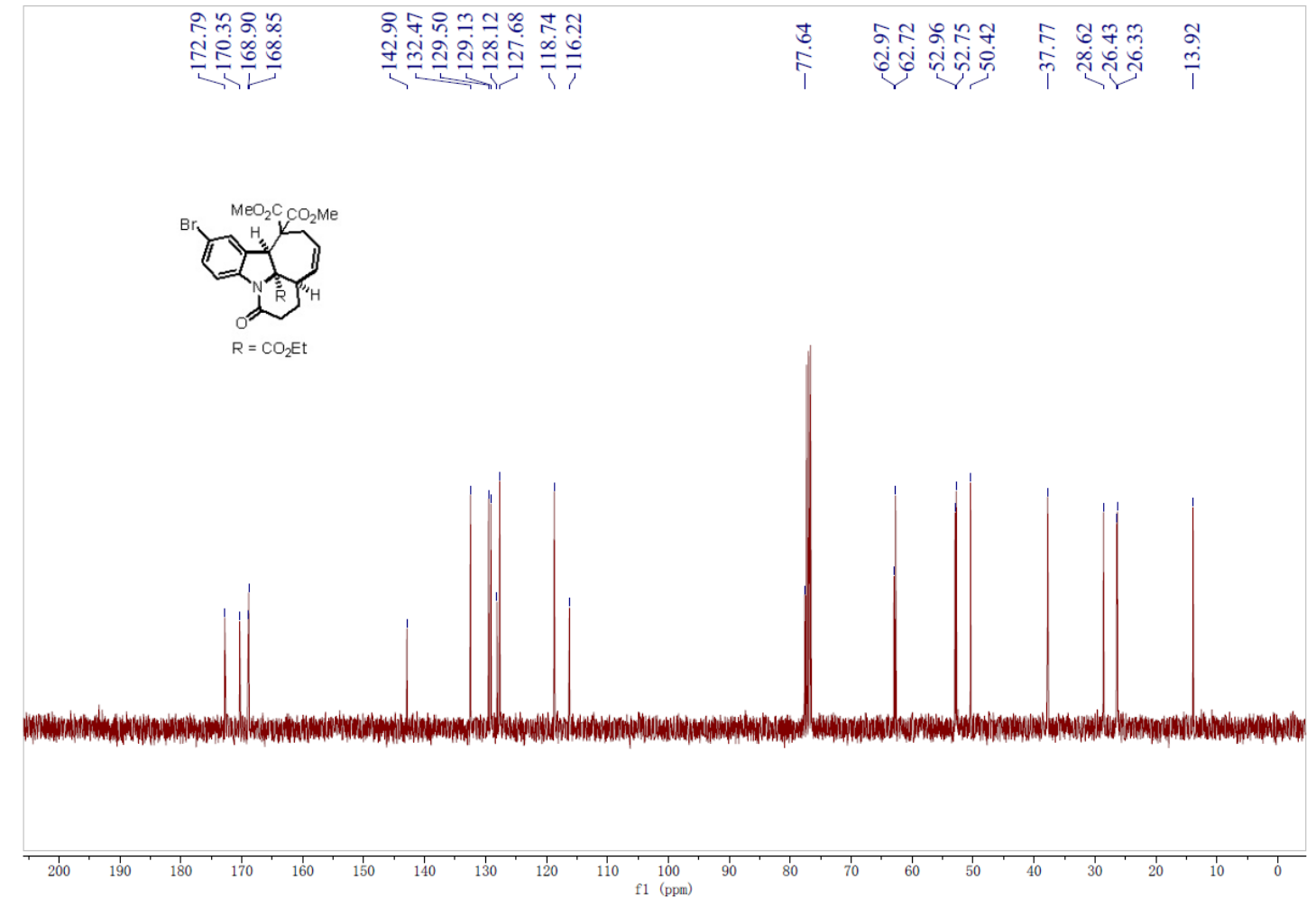


${ }^{1} \mathrm{H}$ NMR Spectrum of $\mathbf{2 n}$

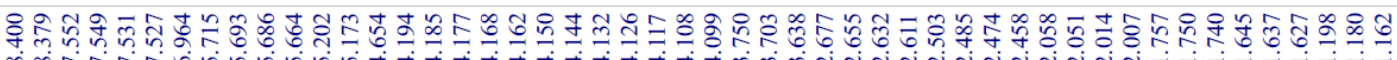

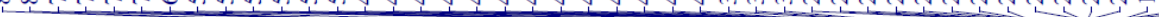

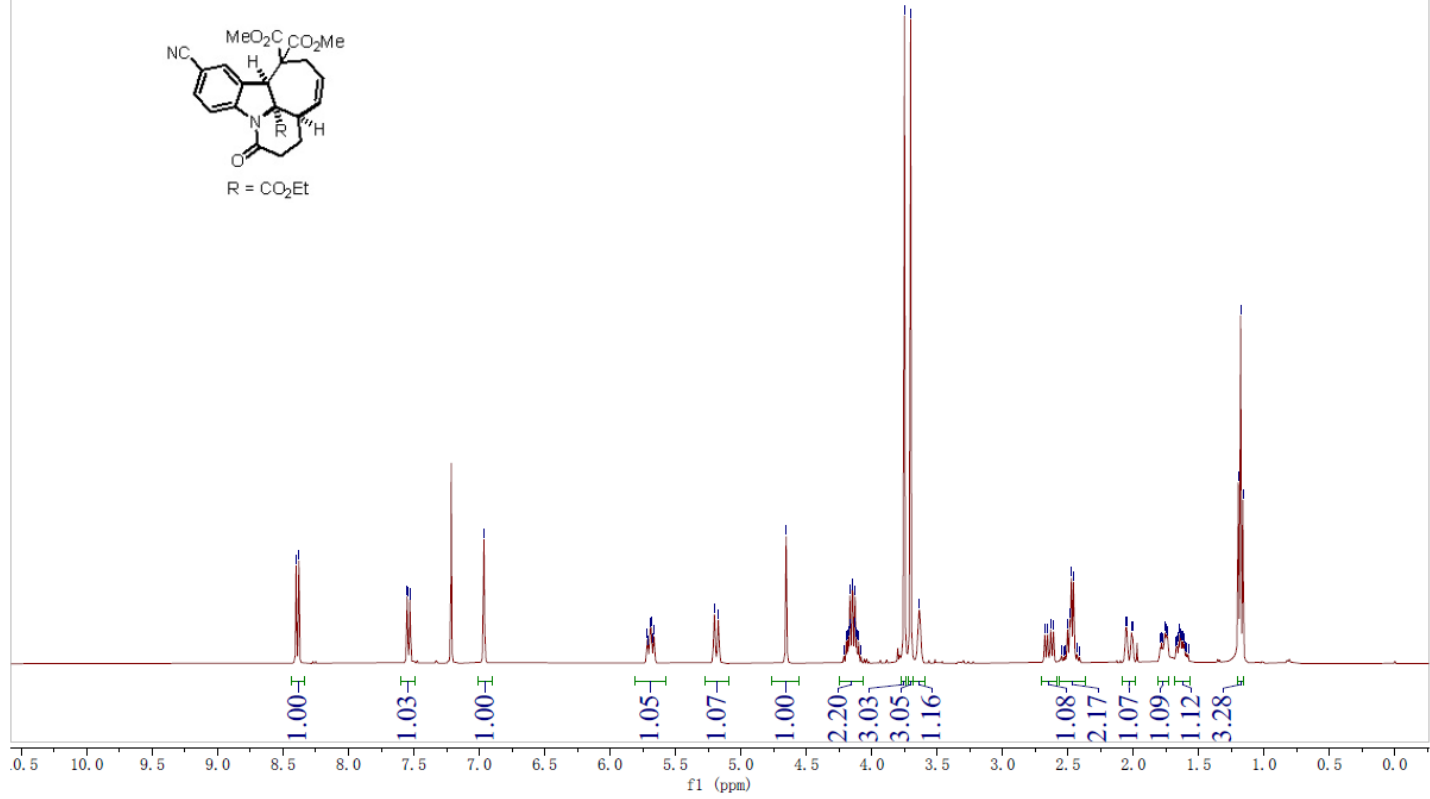

${ }^{13} \mathrm{C}$ NMR Spectrum of $\mathbf{2 n}$

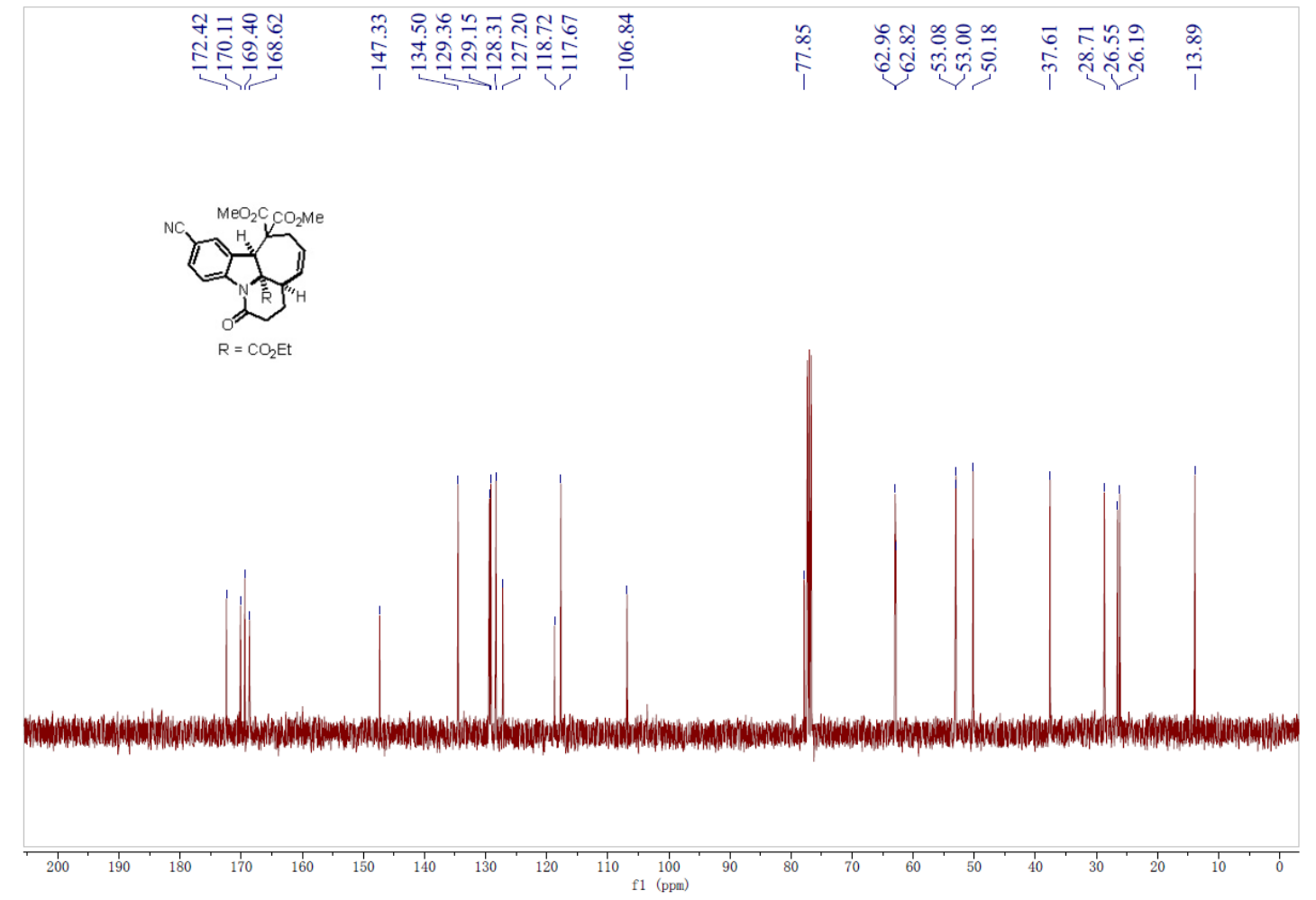


${ }^{1} \mathrm{H}$ NMR Spectrum of $\mathbf{2 0}$

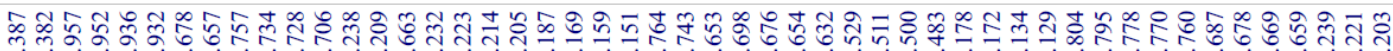

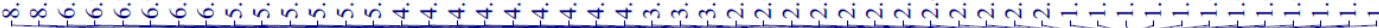

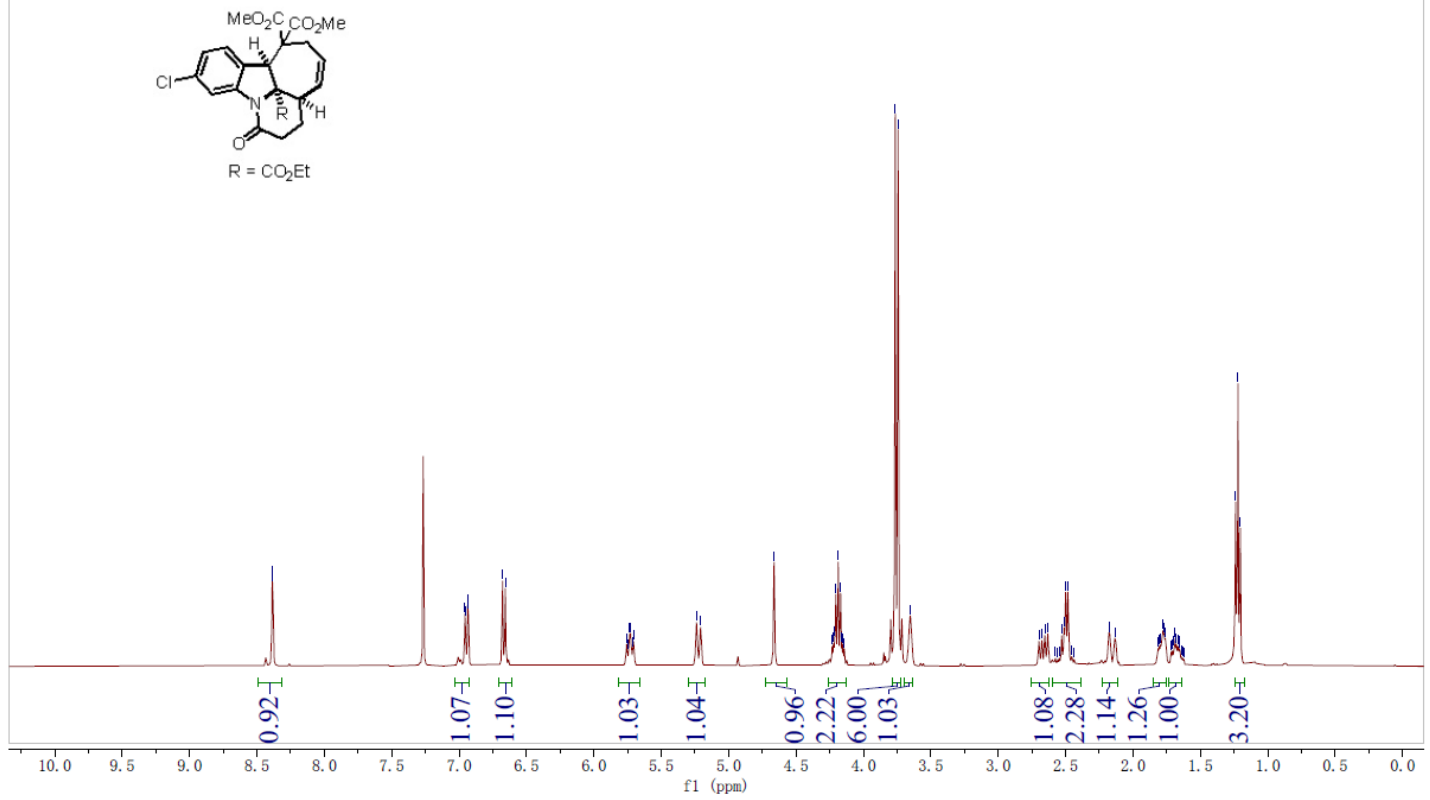

${ }^{13} \mathrm{C}$ NMR Spectrum of 20

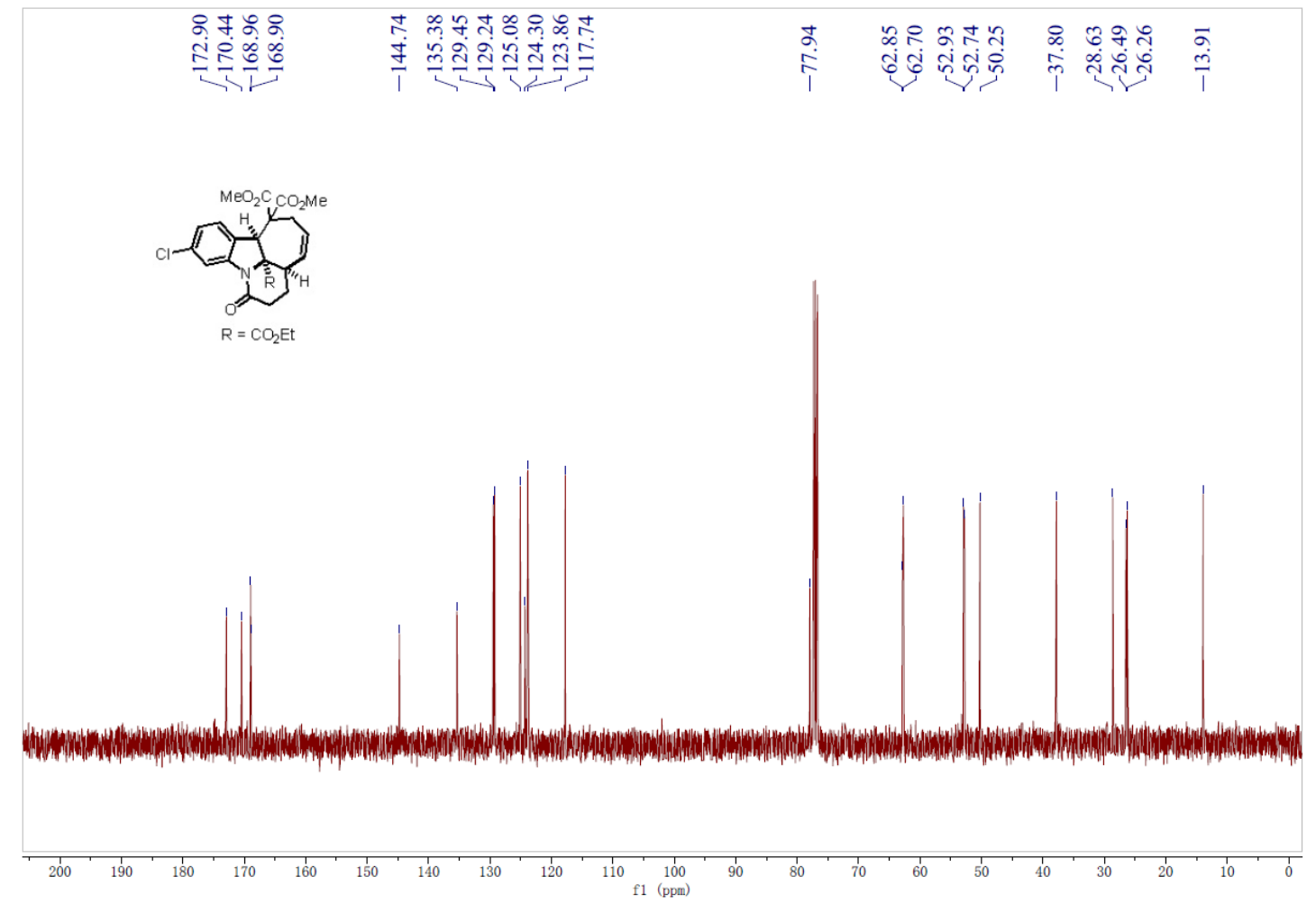


${ }^{1} \mathrm{H}$ NMR Spectrum of $\mathbf{2 p}$

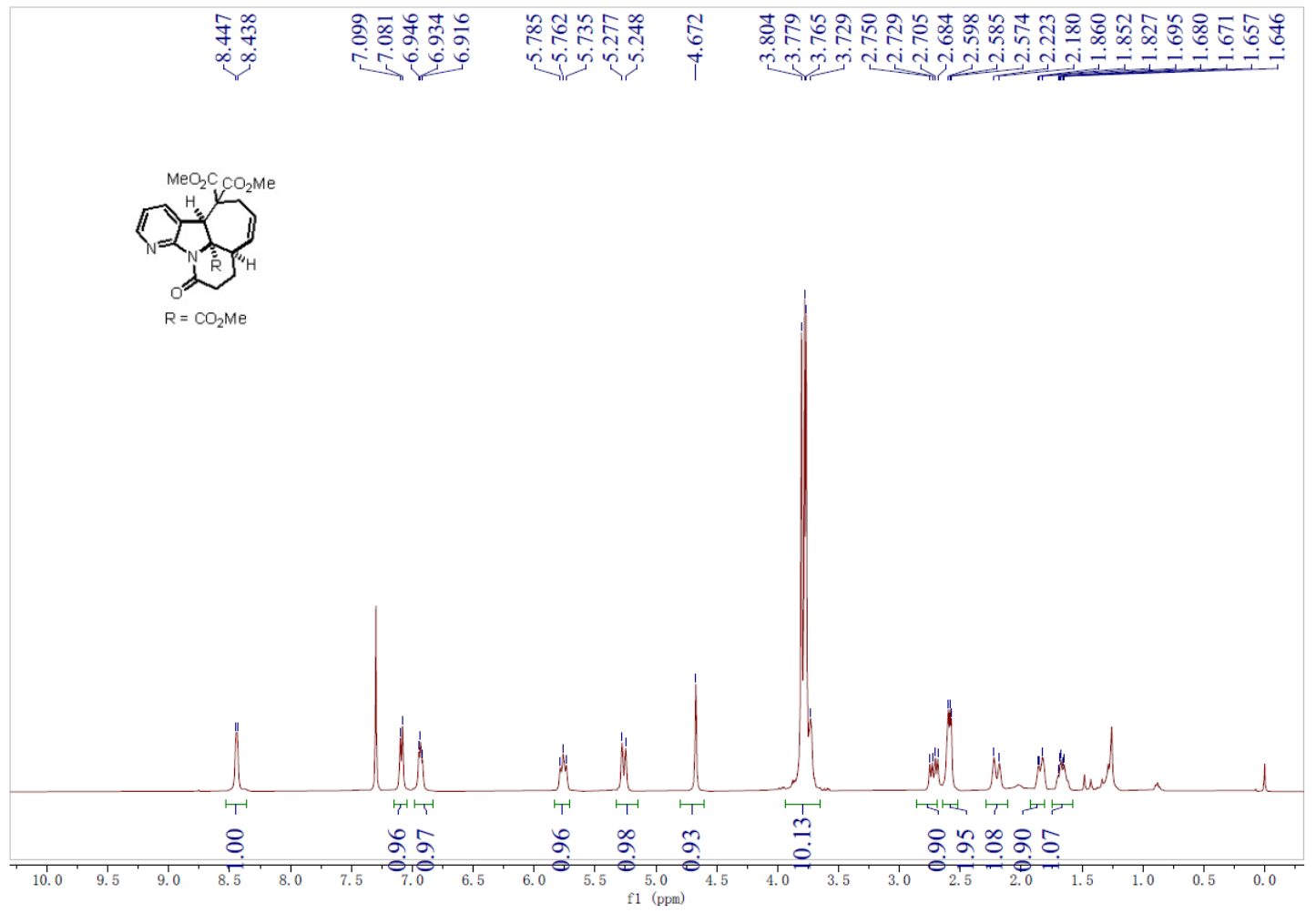

${ }^{13} \mathrm{C}$ NMR Spectrum of $\mathbf{2 p}$

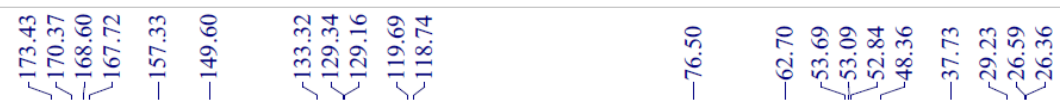
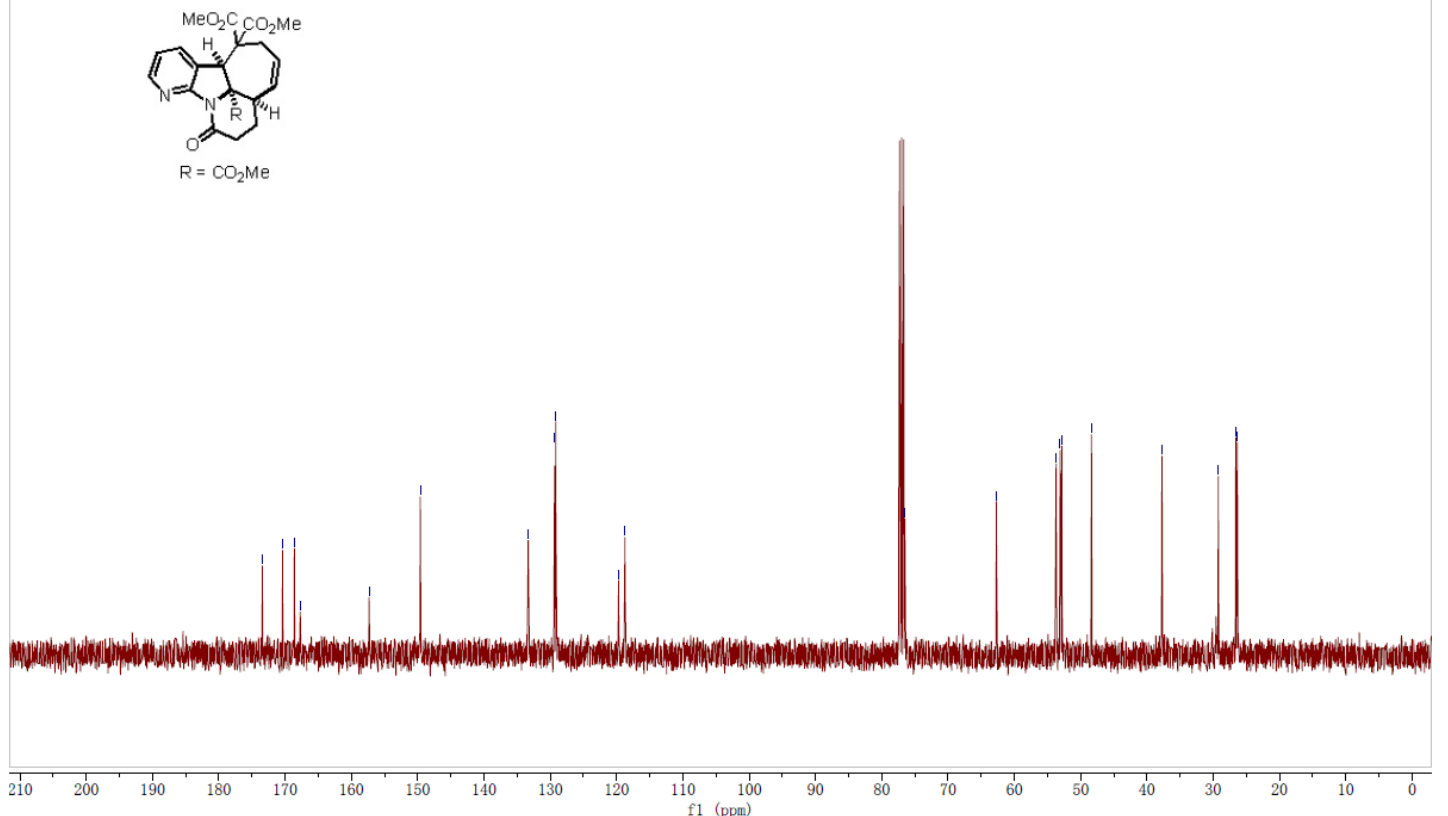
${ }^{1} \mathrm{H}$ NMR Spectrum of $\mathbf{2 q}$

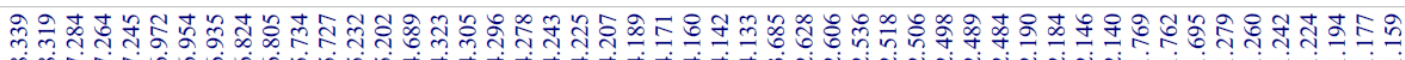

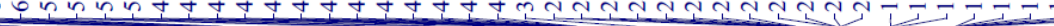
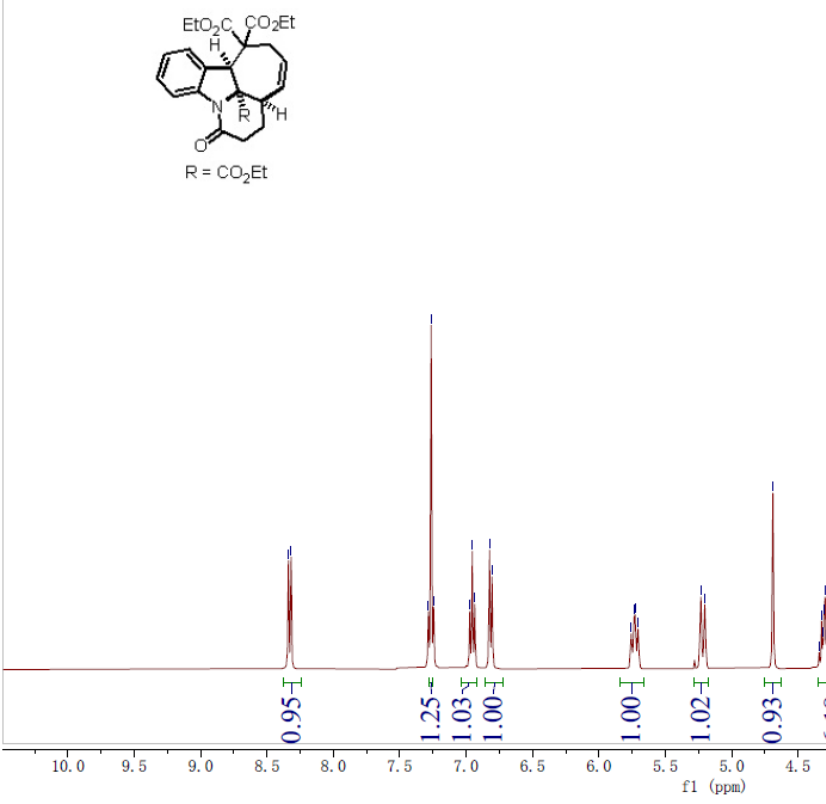

${ }^{13} \mathrm{C}$ NMR Spectrum of $\mathbf{2 q}$

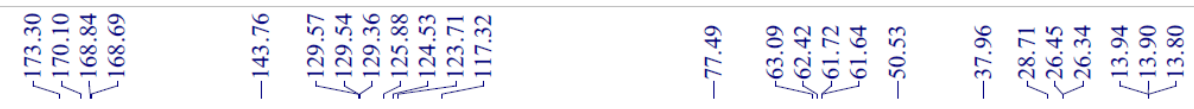
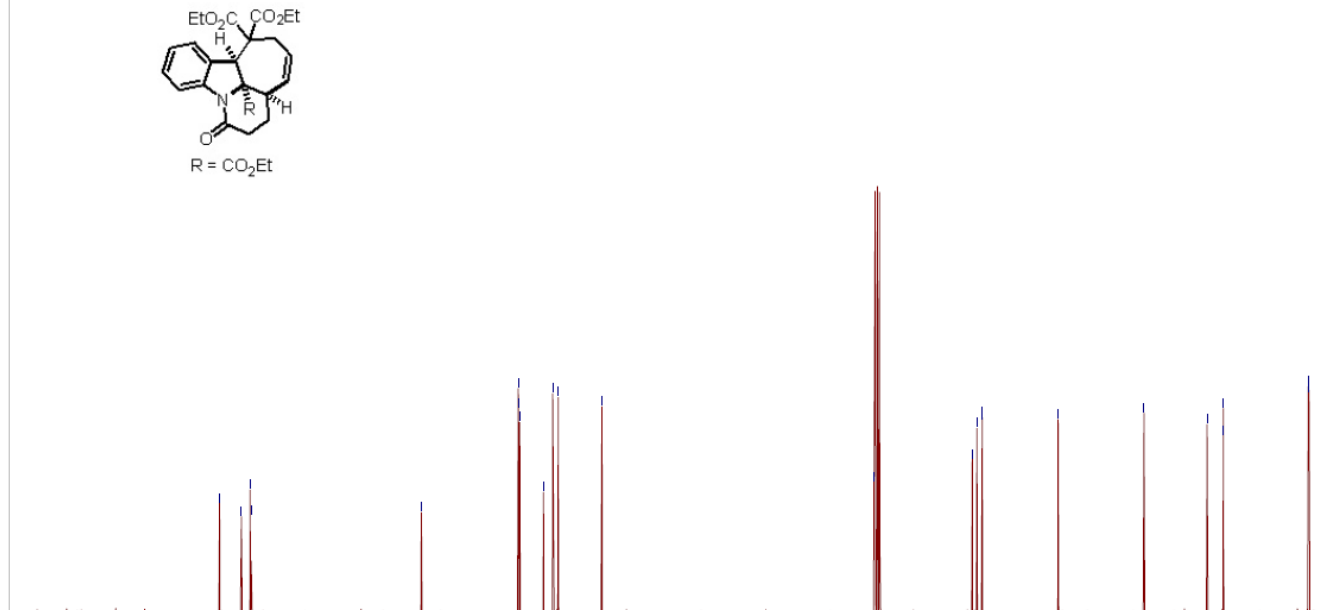

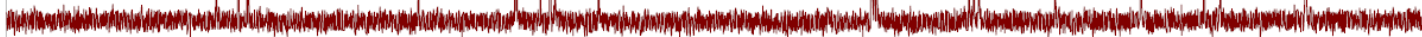

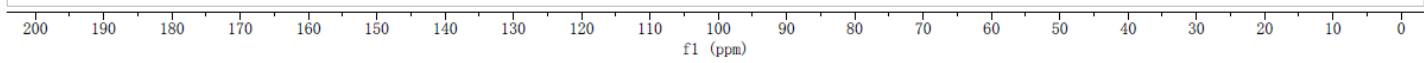


${ }^{1} \mathrm{H}$ NMR Spectrum of $\mathbf{2 r}$

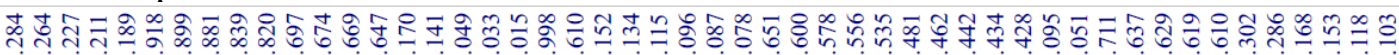

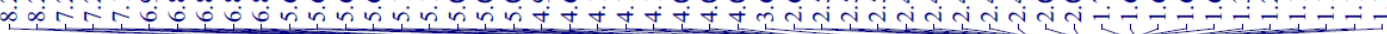
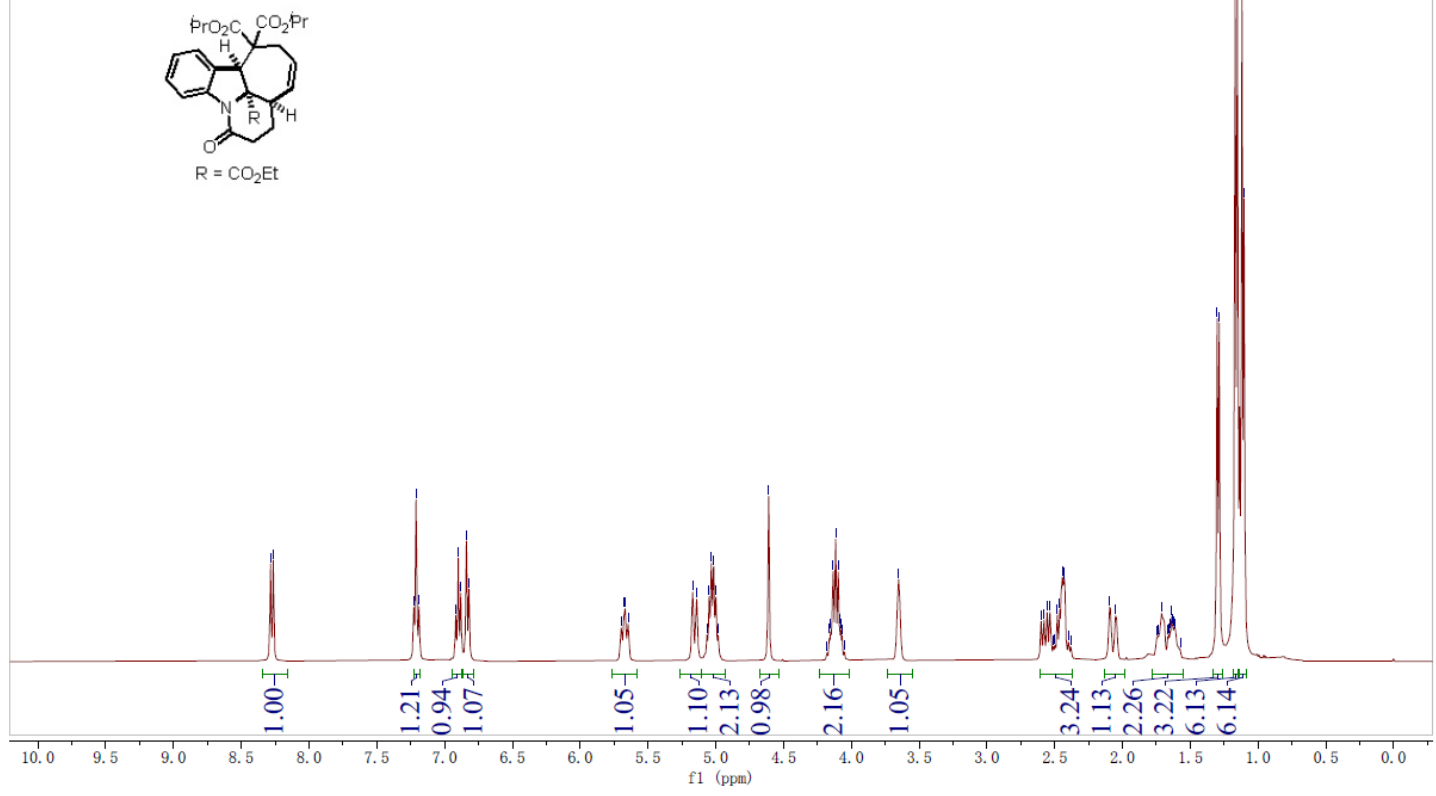

${ }^{13} \mathrm{C}$ NMR Spectrum of $\mathbf{2 r}$

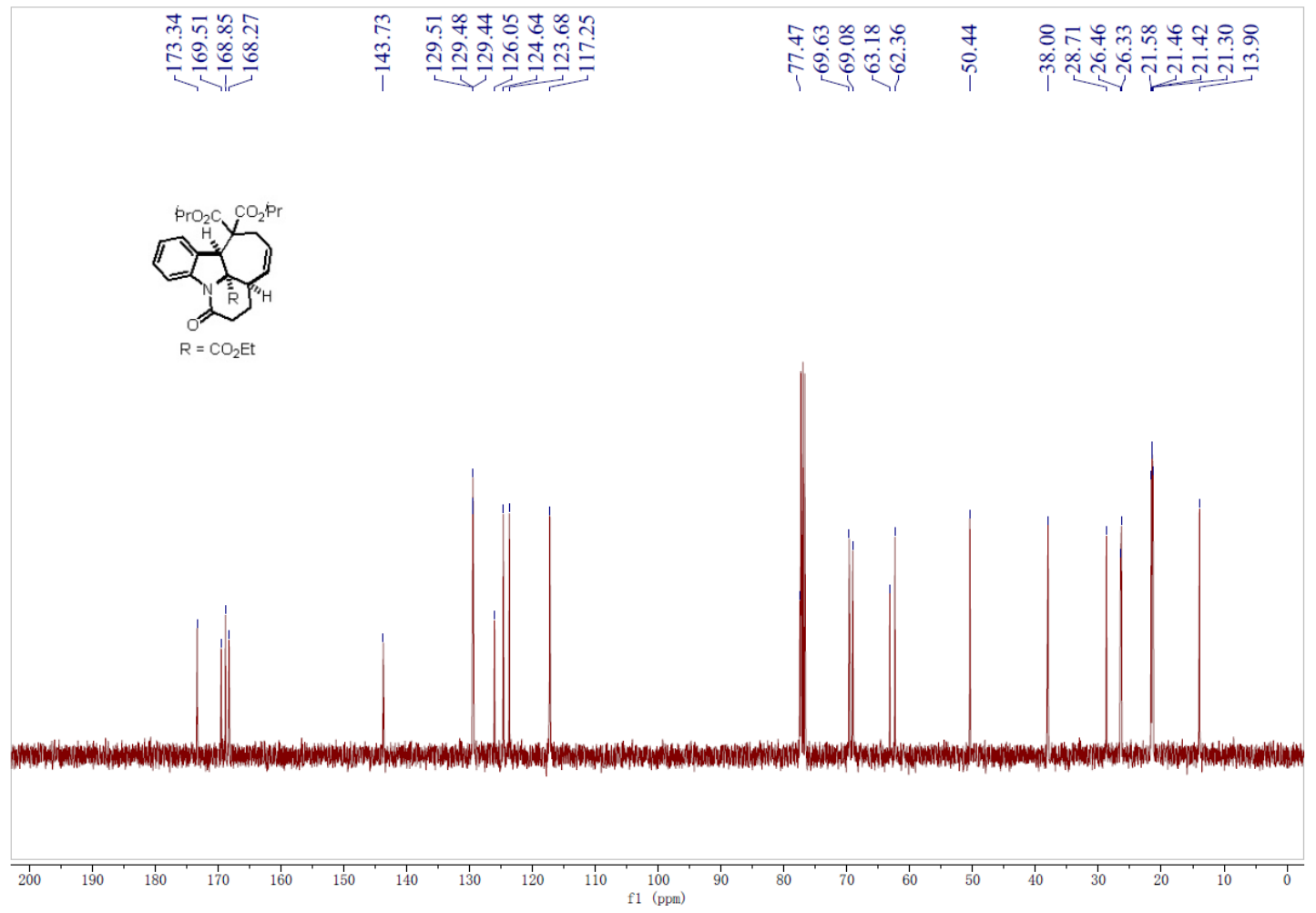


${ }^{1} \mathrm{H}$ NMR Spectrum of $\mathbf{2 s}$

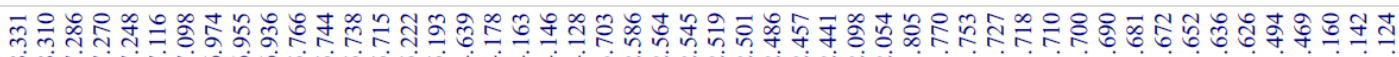
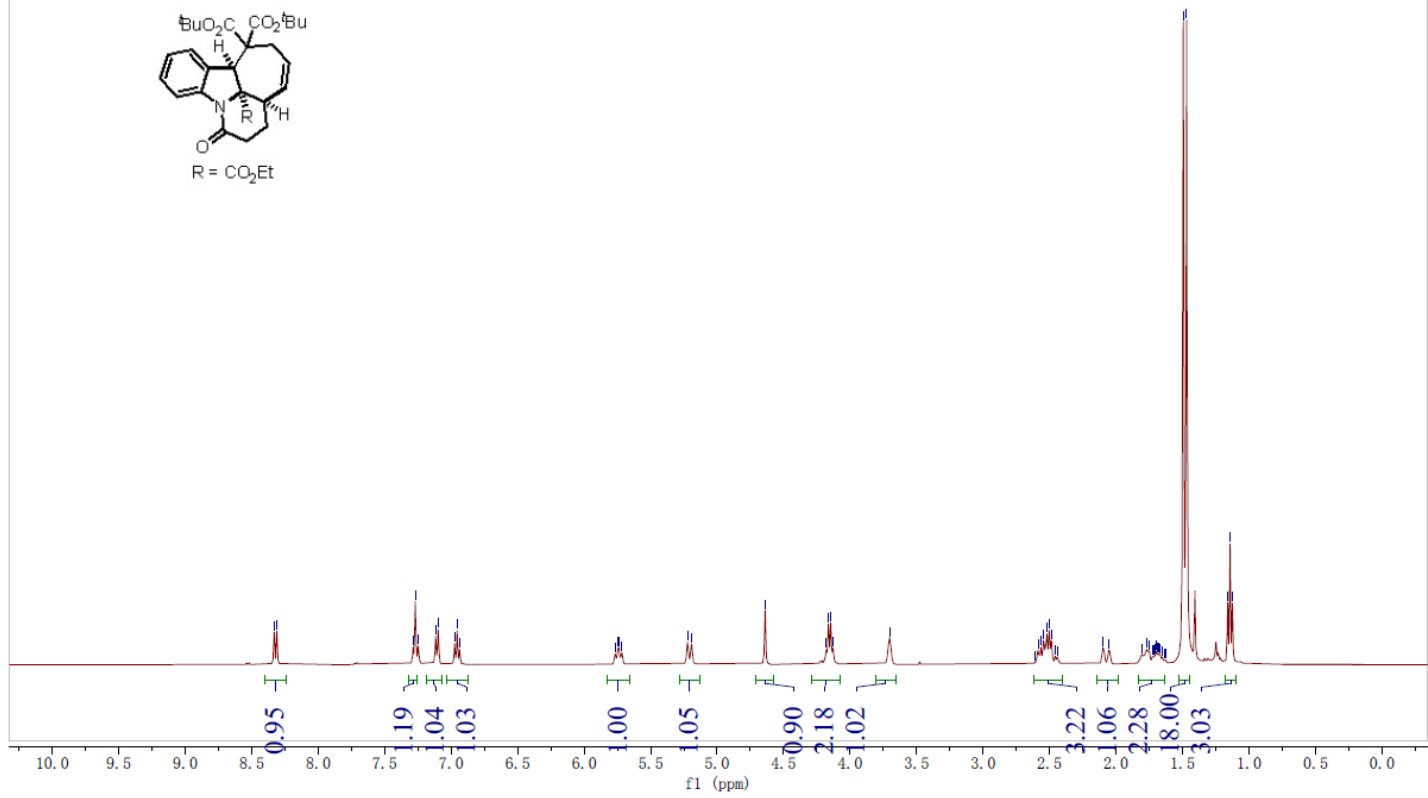

${ }^{13} \mathrm{C}$ NMR Spectrum of $\mathbf{2 s}$

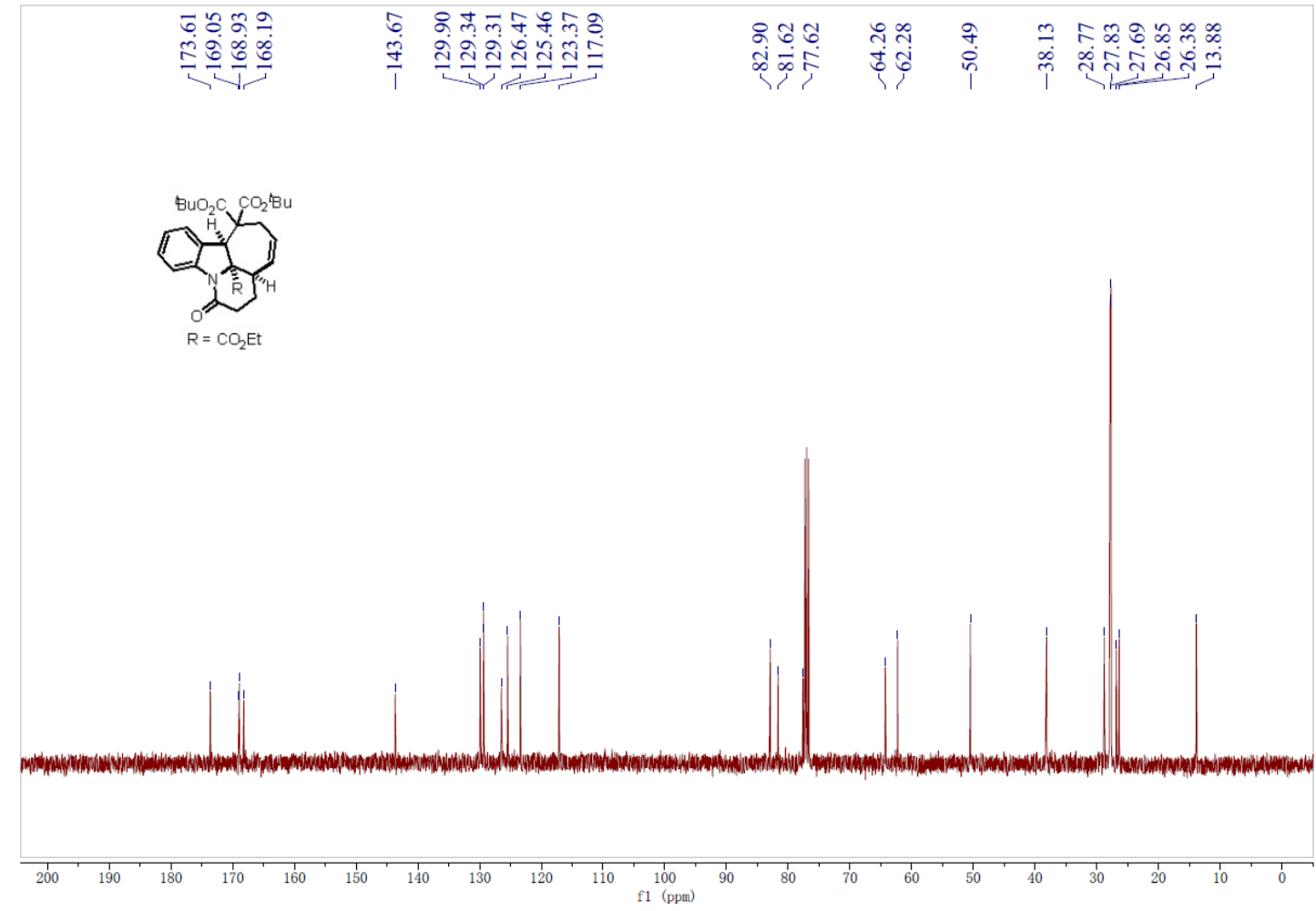


${ }^{1} \mathrm{H}$ NMR Spectrum of $\mathbf{2 t}$

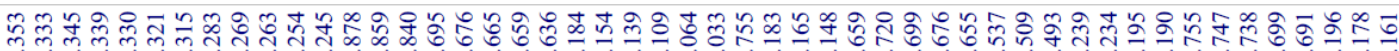

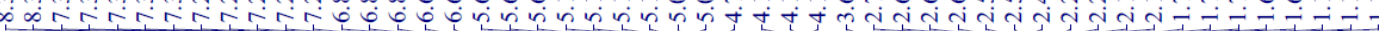

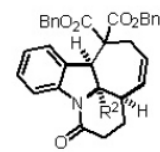

$\mathrm{R}^{2}=\mathrm{CO}_{2} \mathrm{Et}$

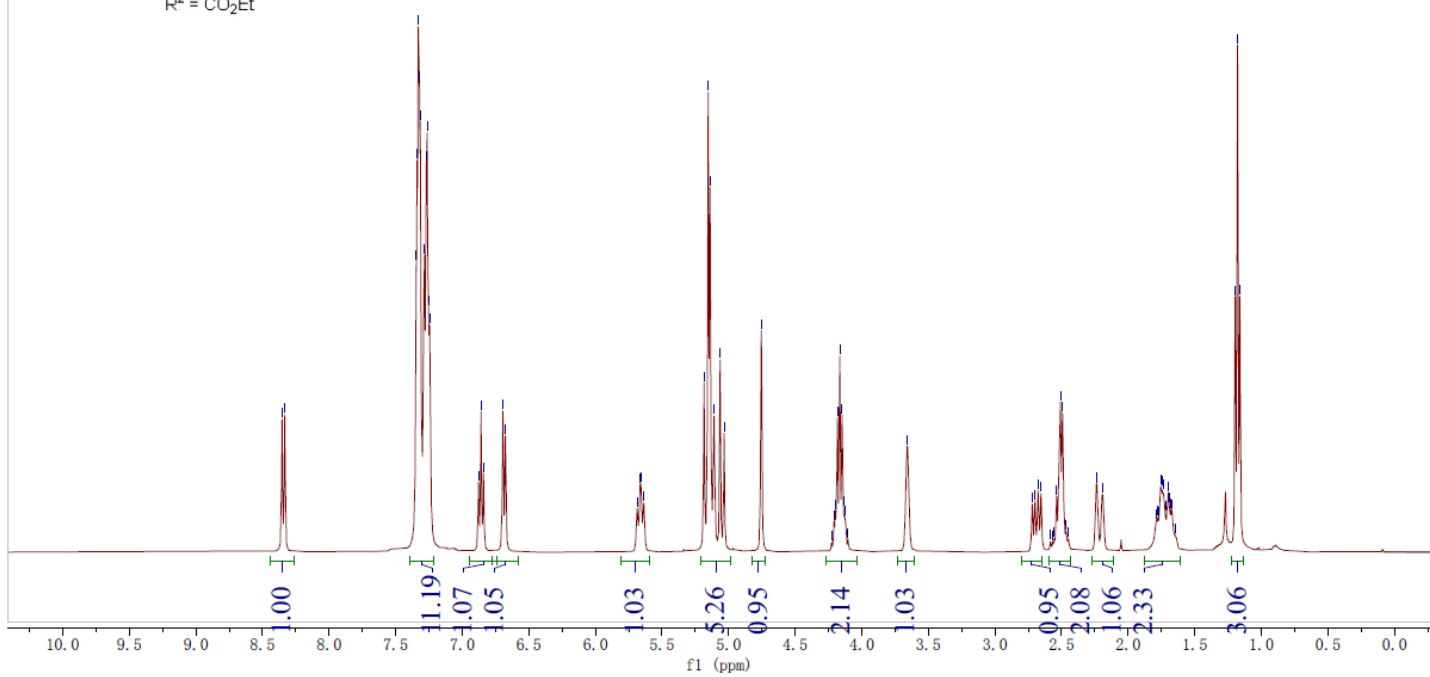

${ }^{13} \mathrm{C}$ NMR Spectrum of $\mathbf{2 t}$

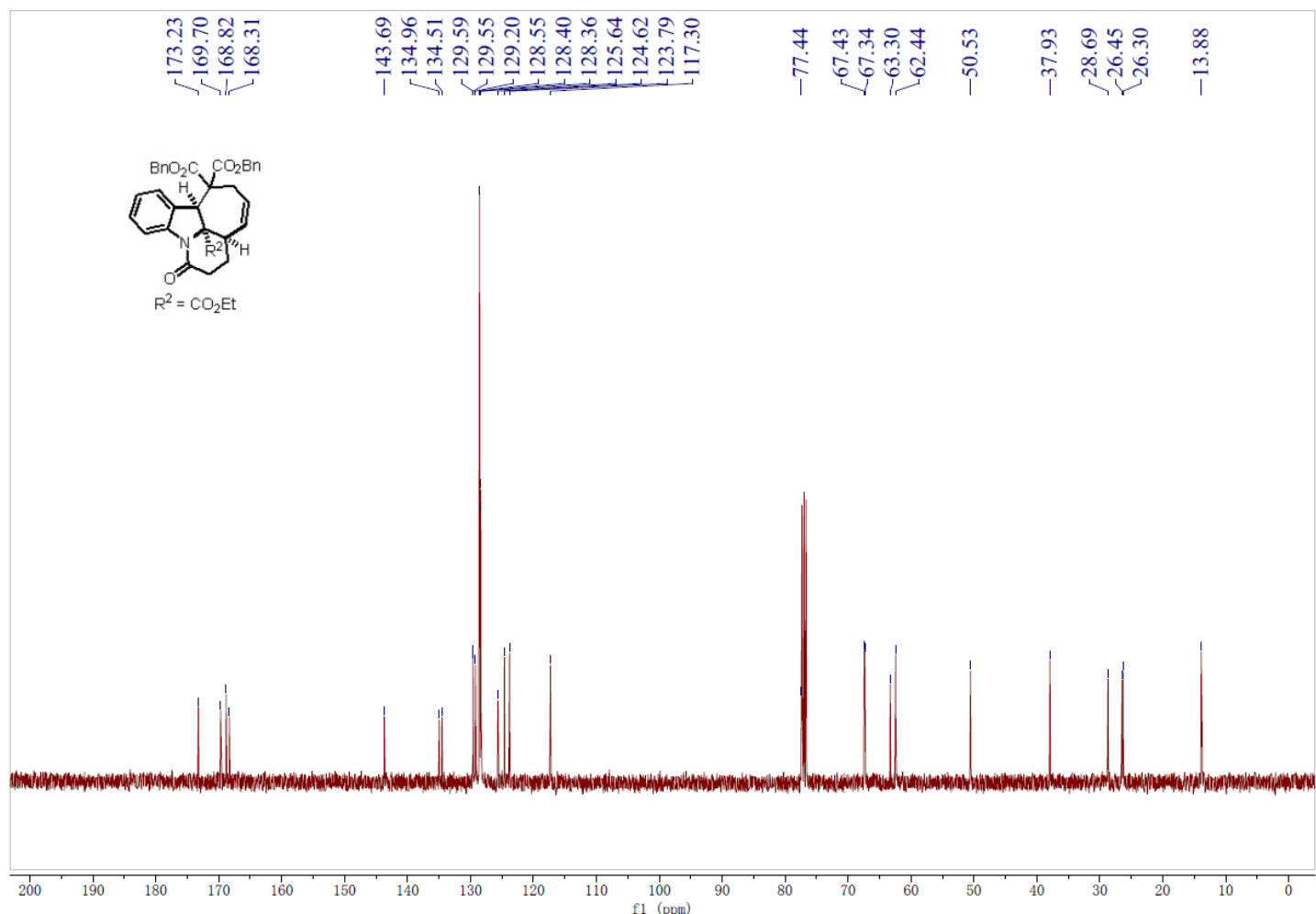


${ }^{1} \mathrm{H}$ NMR Spectrum of $\mathbf{2 u}$

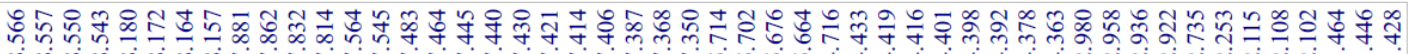

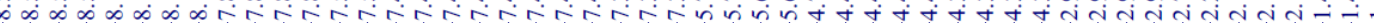

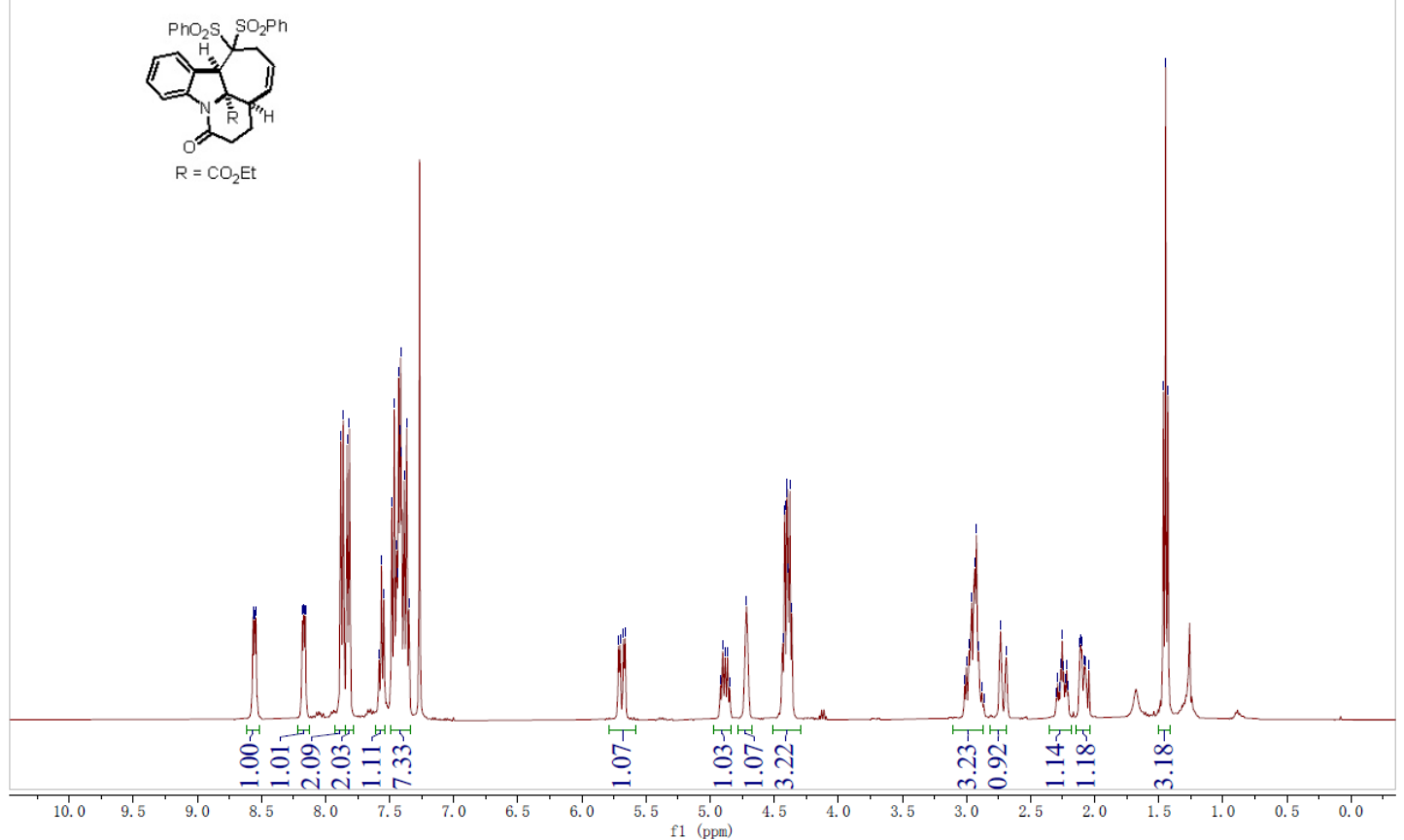

${ }^{13} \mathrm{C}$ NMR Spectrum of $\mathbf{2 u}$
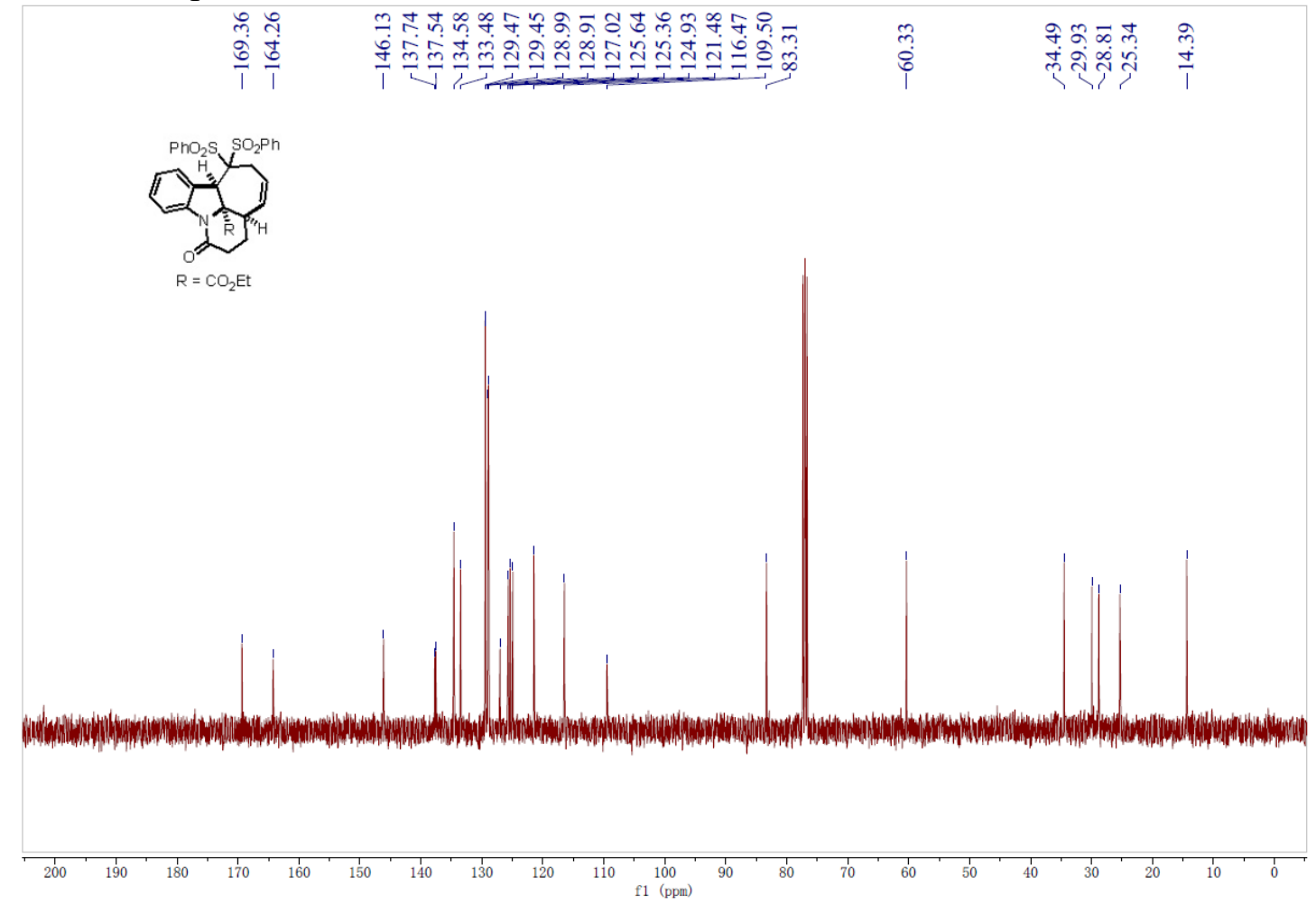
${ }^{1} \mathrm{H}$ NMR Spectrum of $\mathbf{2 v}$

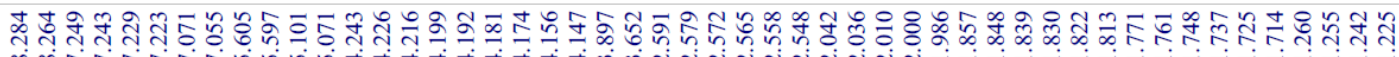
sontri,

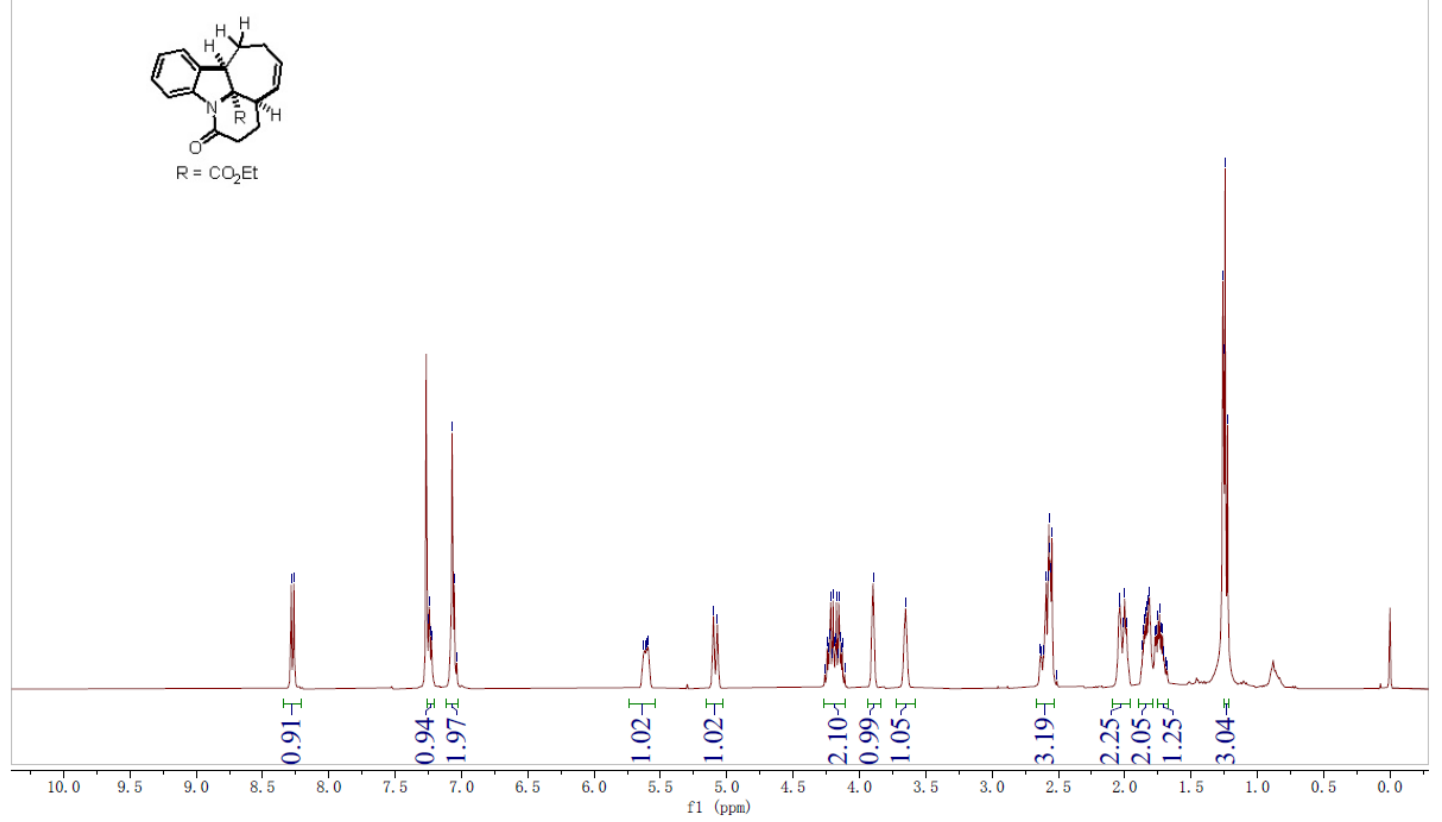

${ }^{13} \mathrm{C}$ NMR Spectrum of $\mathbf{2 v}$

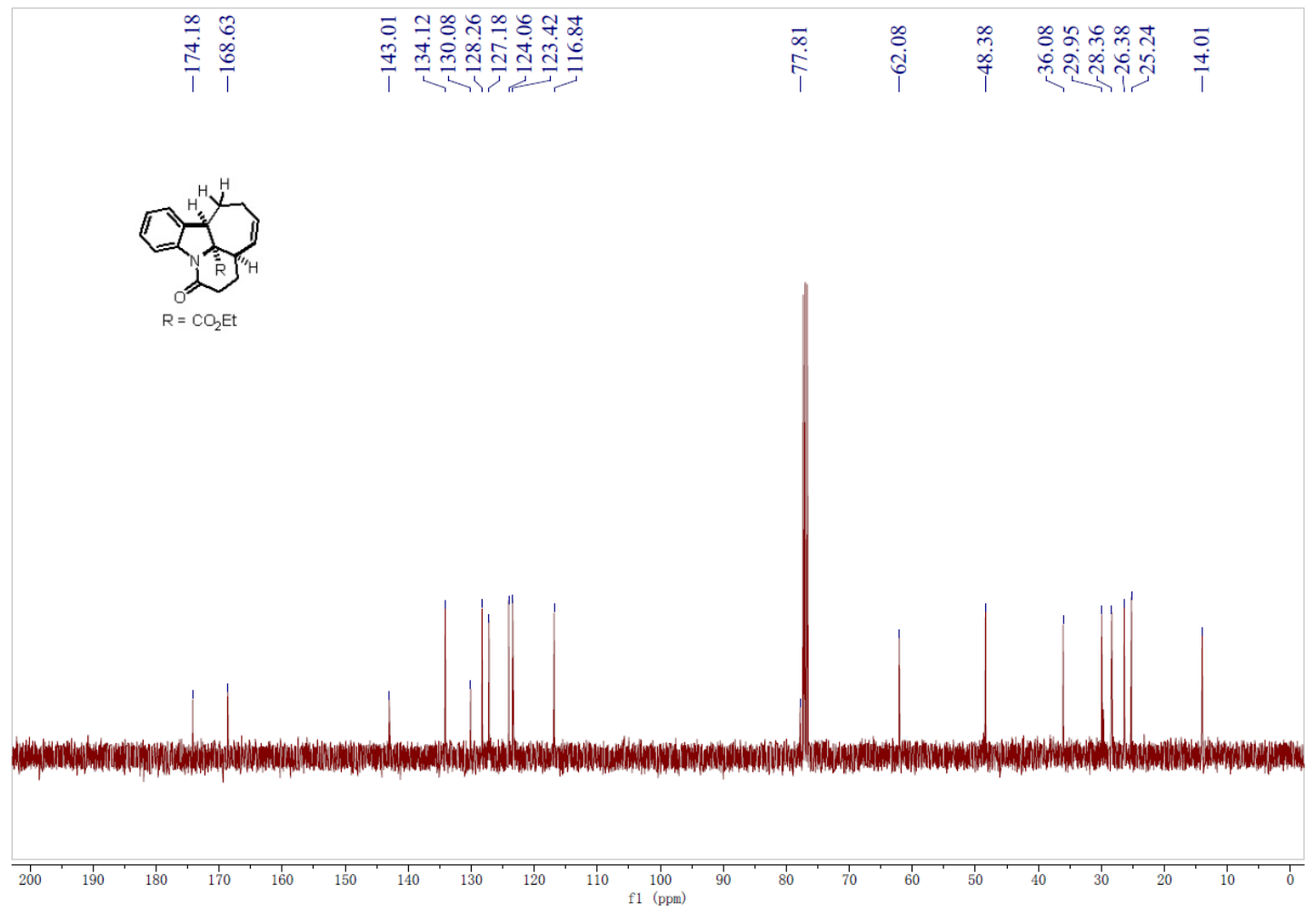


${ }^{1} \mathrm{H}$ NMR Spectrum of $\mathbf{2 w}$

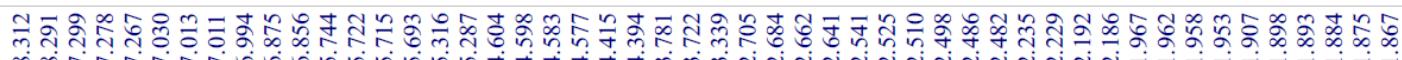
क l, 1,

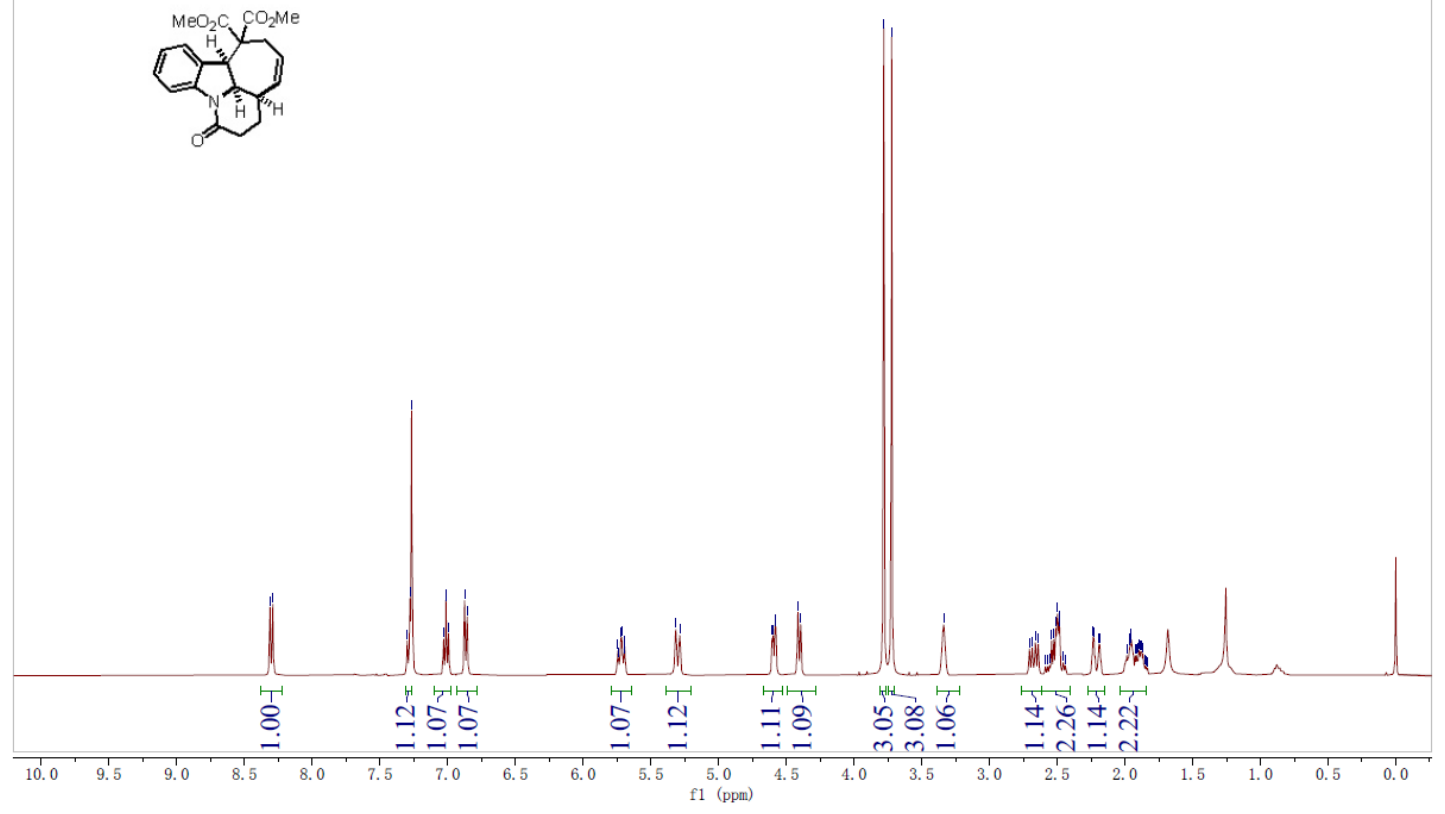

${ }^{13} \mathrm{C}$ NMR Spectrum of $\mathbf{2 w}$

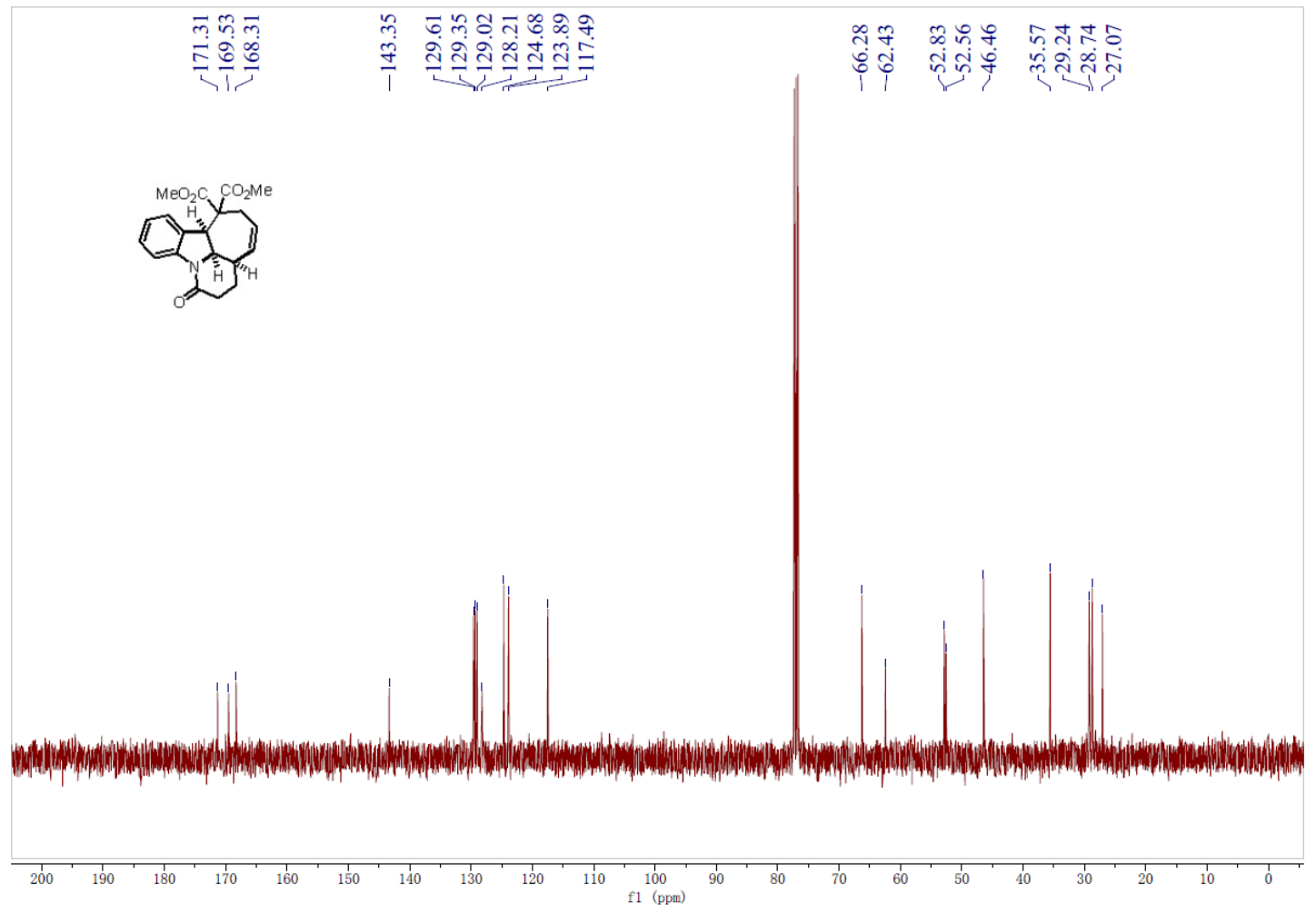


${ }^{1} \mathrm{H}$ NMR Spectrum of $\mathbf{2 x}$

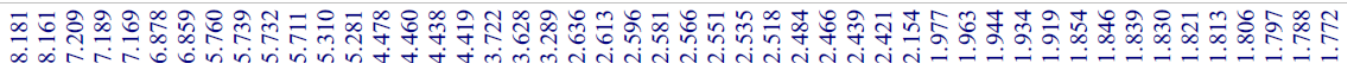

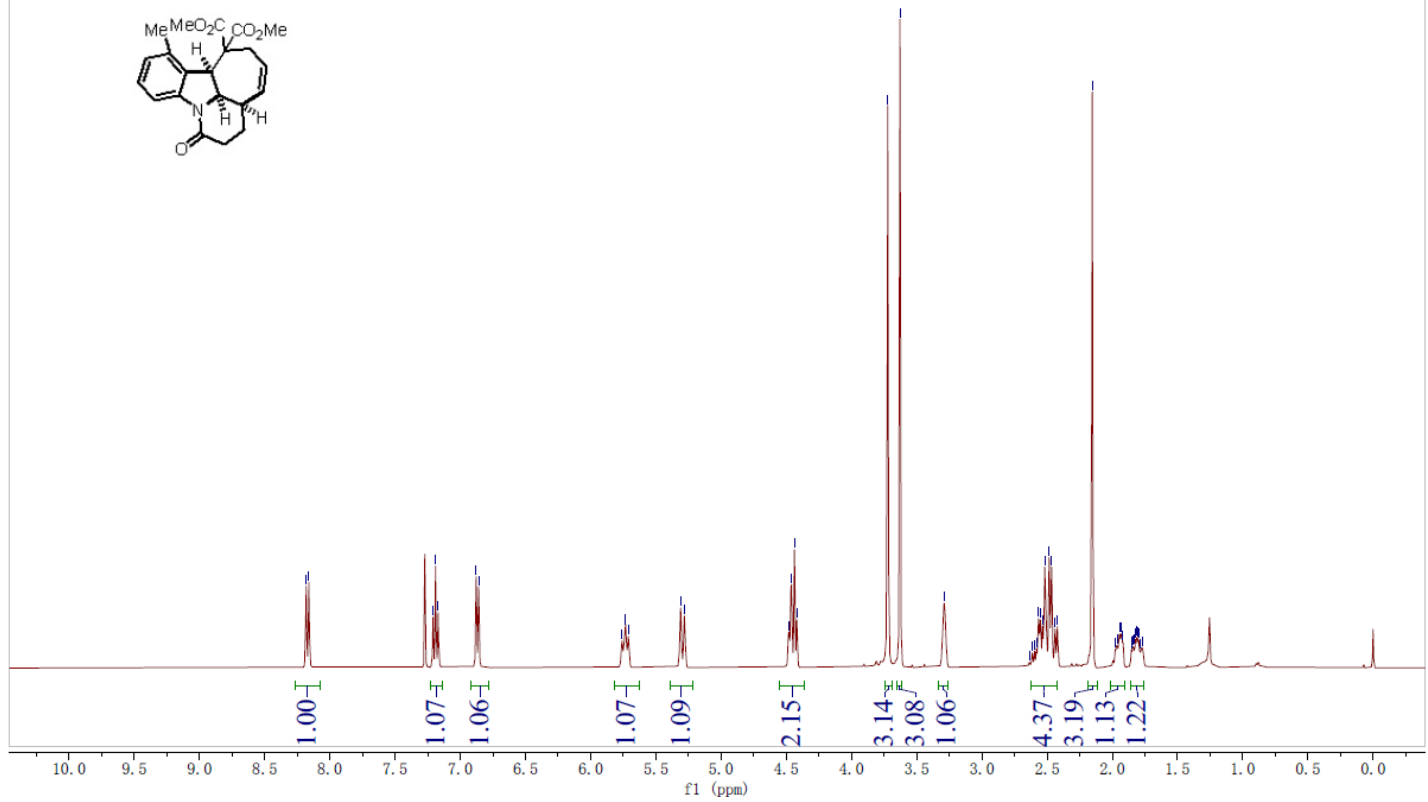

${ }^{13} \mathrm{C}$ NMR Spectrum of $\mathbf{2 x}$

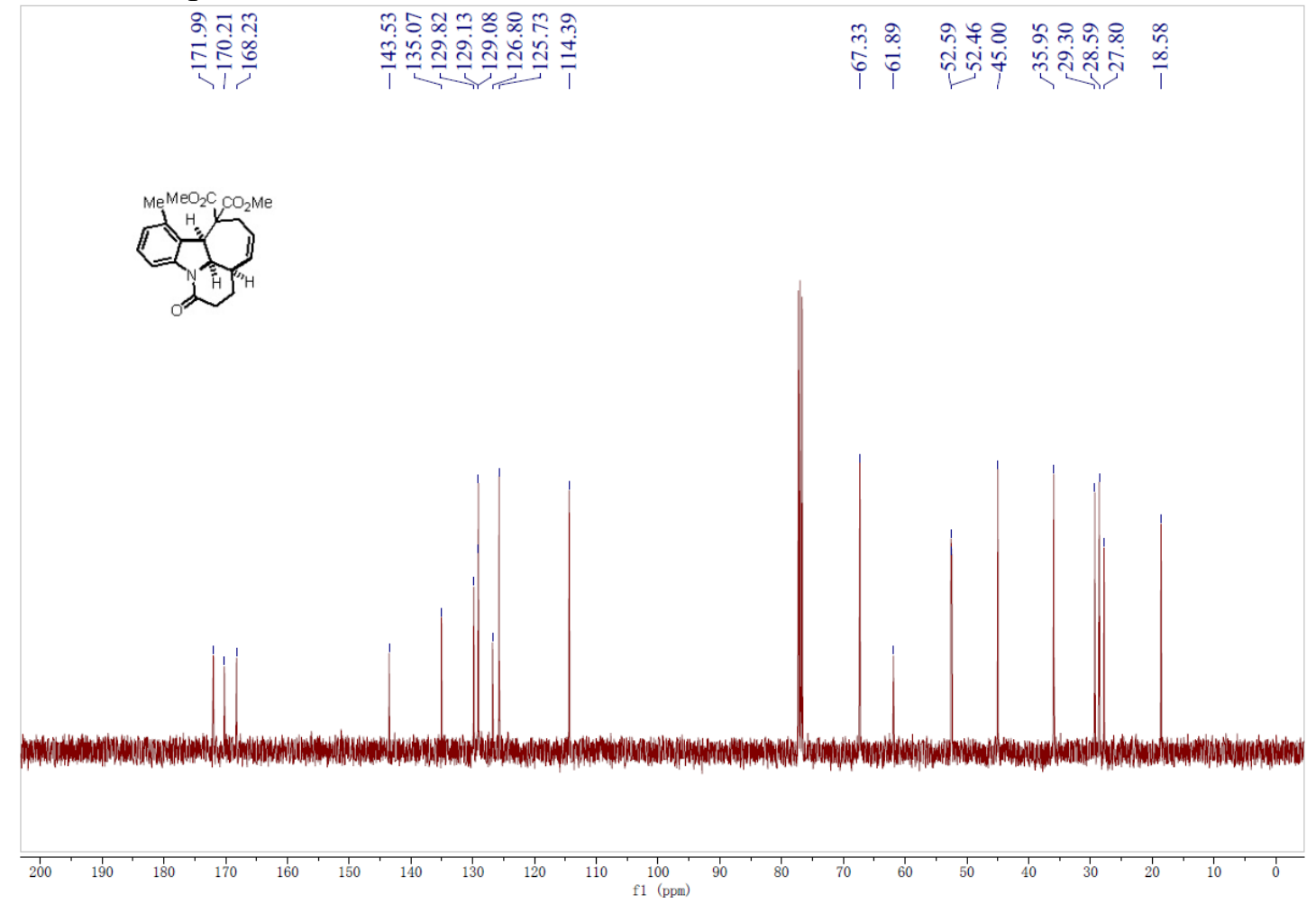


${ }^{1} \mathrm{H}$ NMR Spectrum of $\mathbf{2 y}$

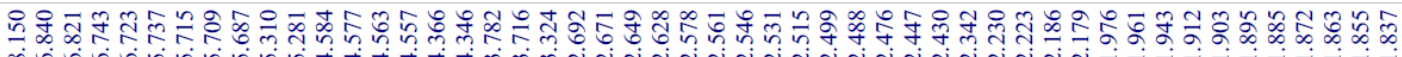

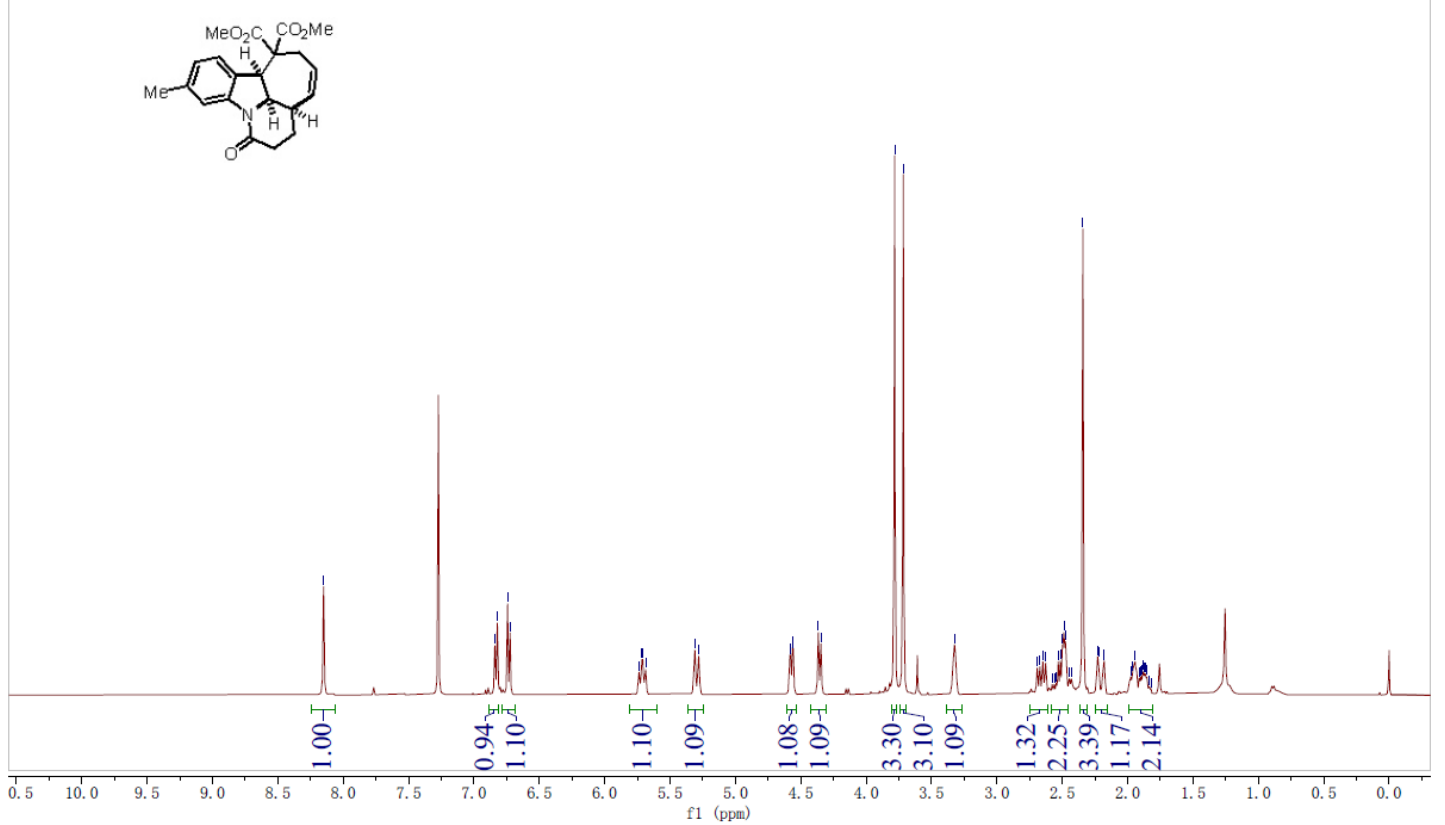

${ }^{13} \mathrm{C}$ NMR Spectrum of $\mathbf{2 y}$
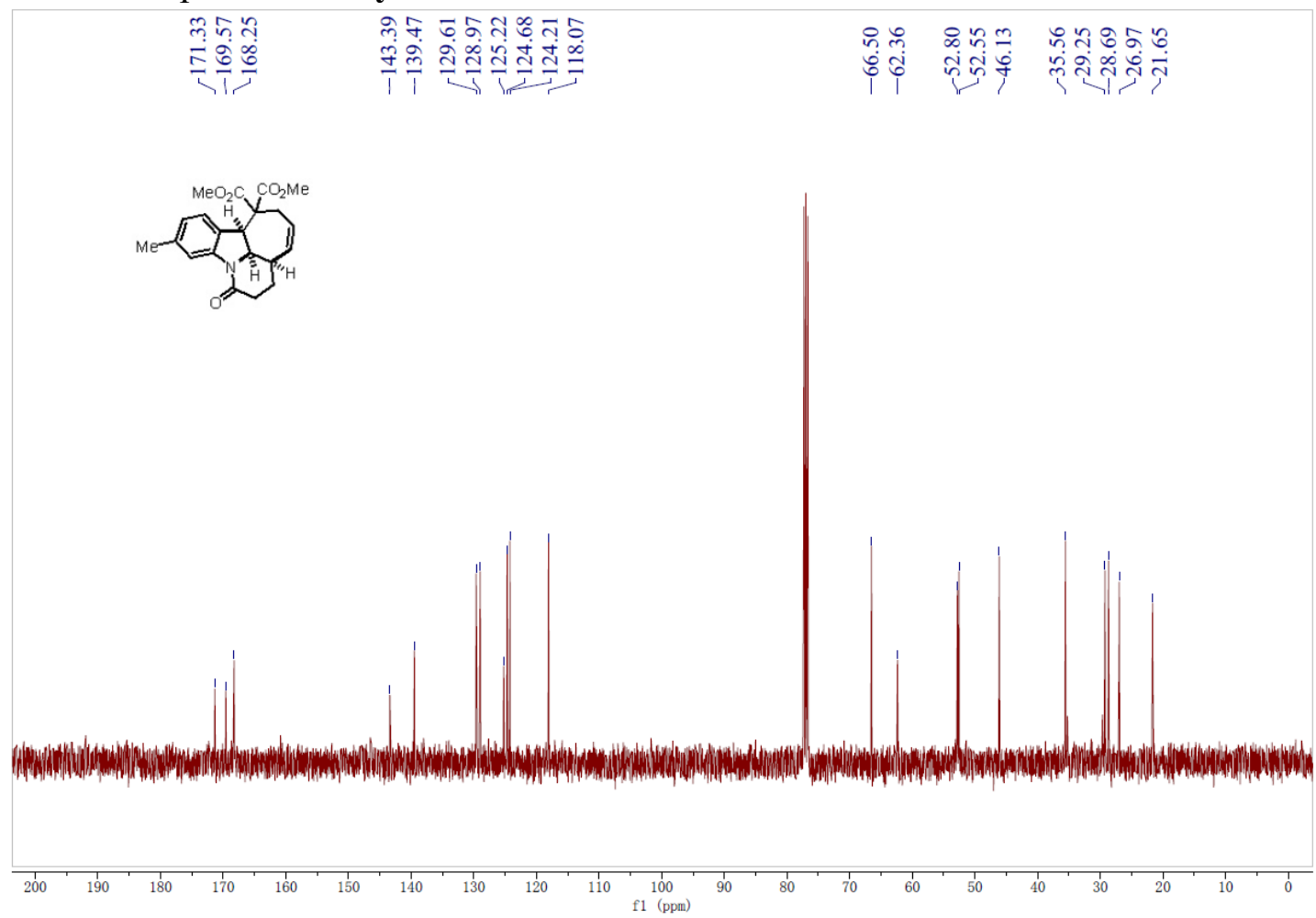
${ }^{1} \mathrm{H}$ NMR Spectrum of $\mathbf{2 z}$

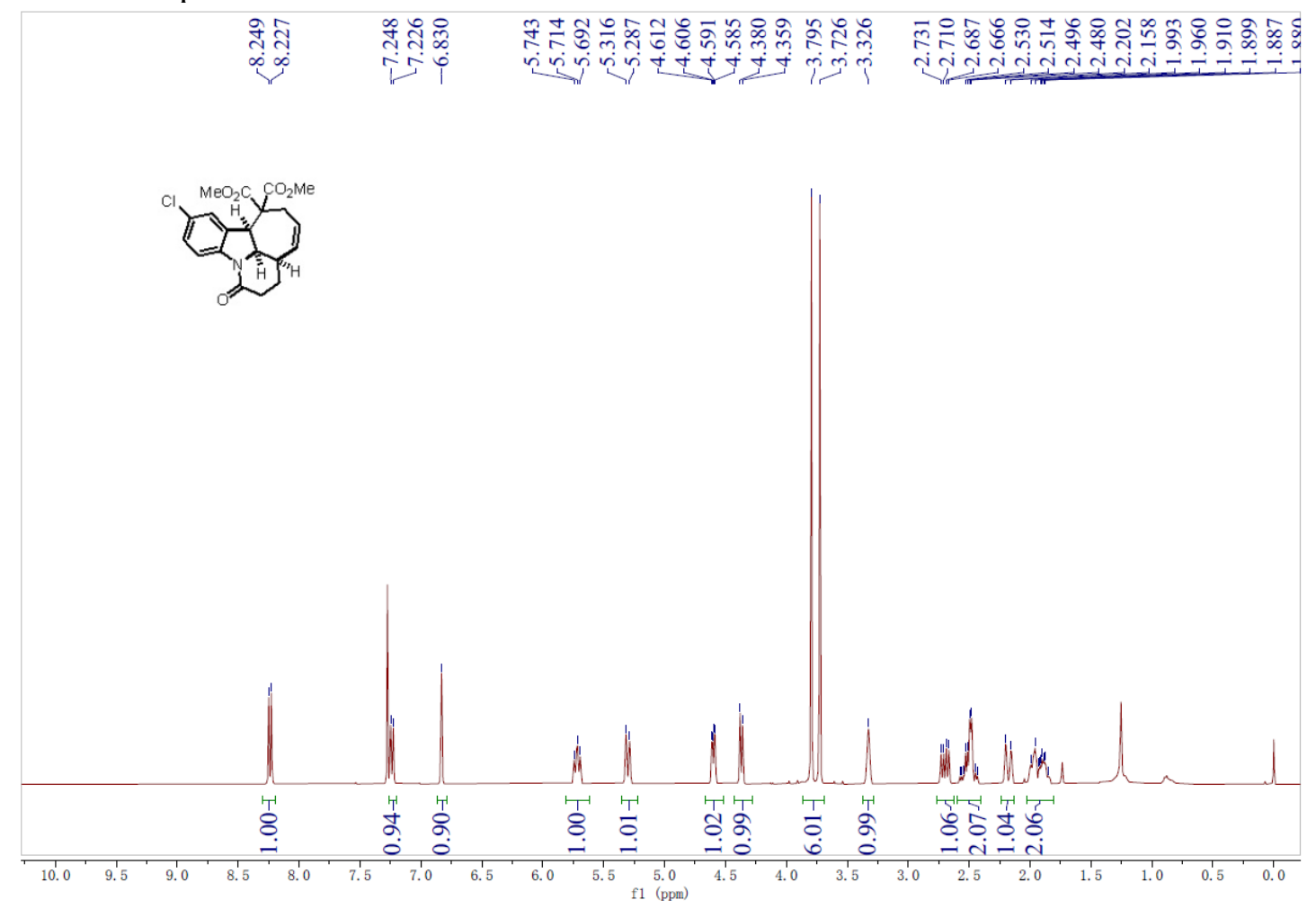

${ }^{13} \mathrm{C}$ NMR Spectrum of $\mathbf{2 z}$

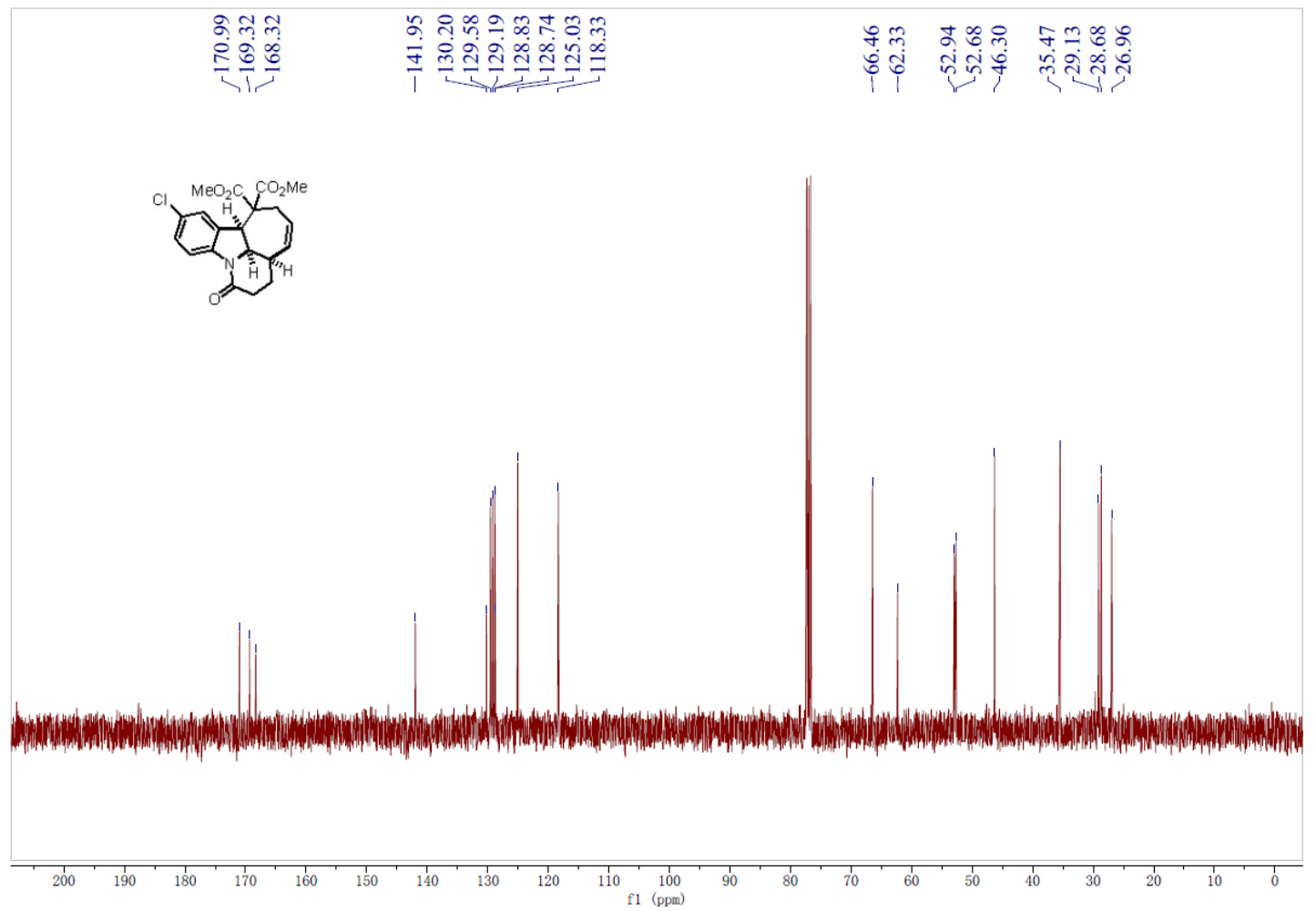


${ }^{1} \mathrm{H}$ NMR Spectrum of $\mathbf{2 a a}$

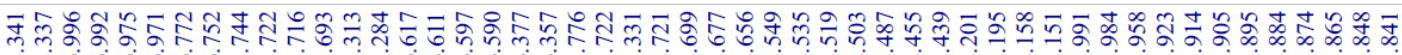

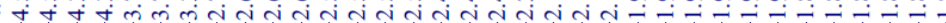

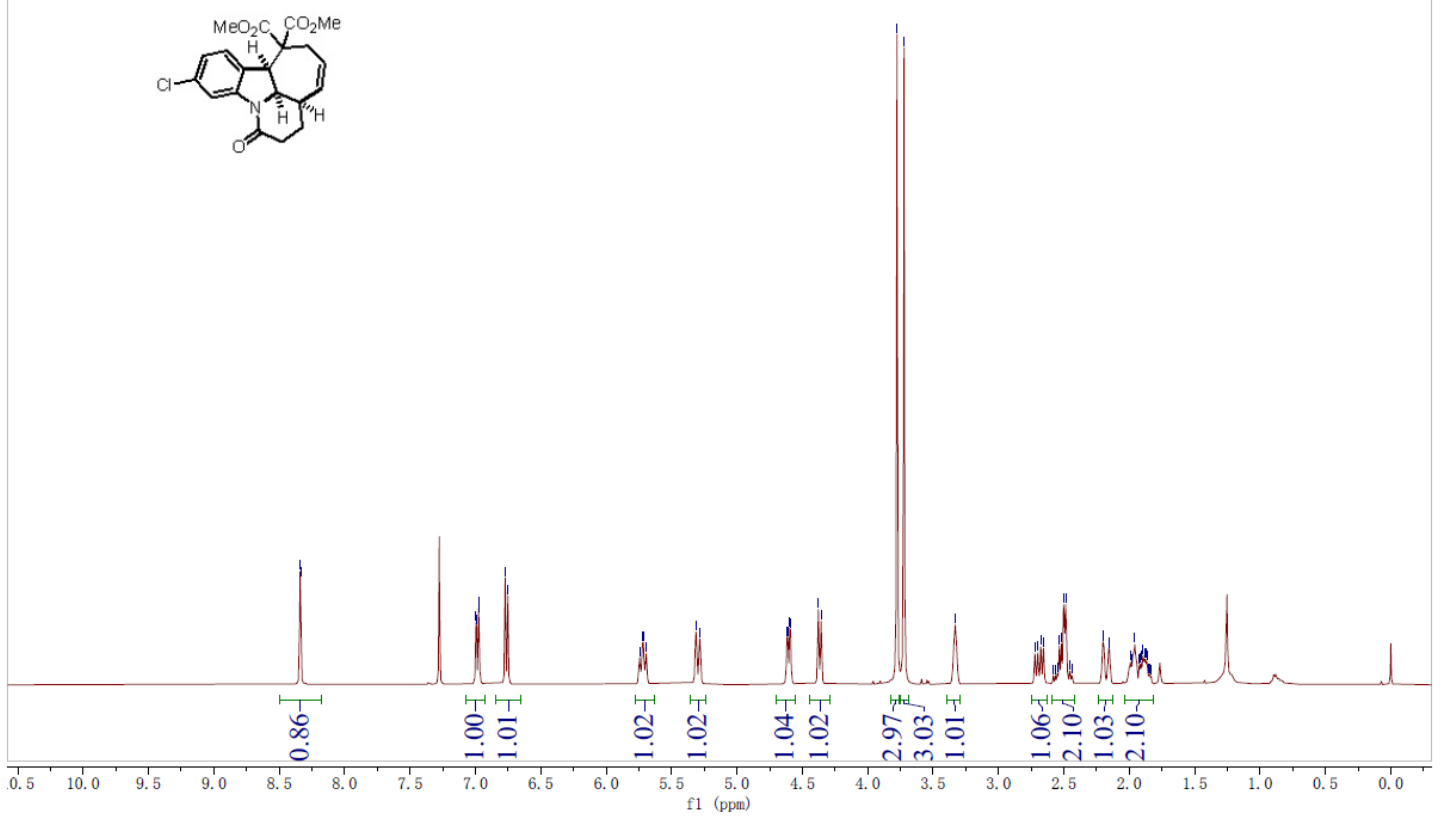

${ }^{13} \mathrm{C}$ NMR Spectrum of $2 \mathbf{a a}$
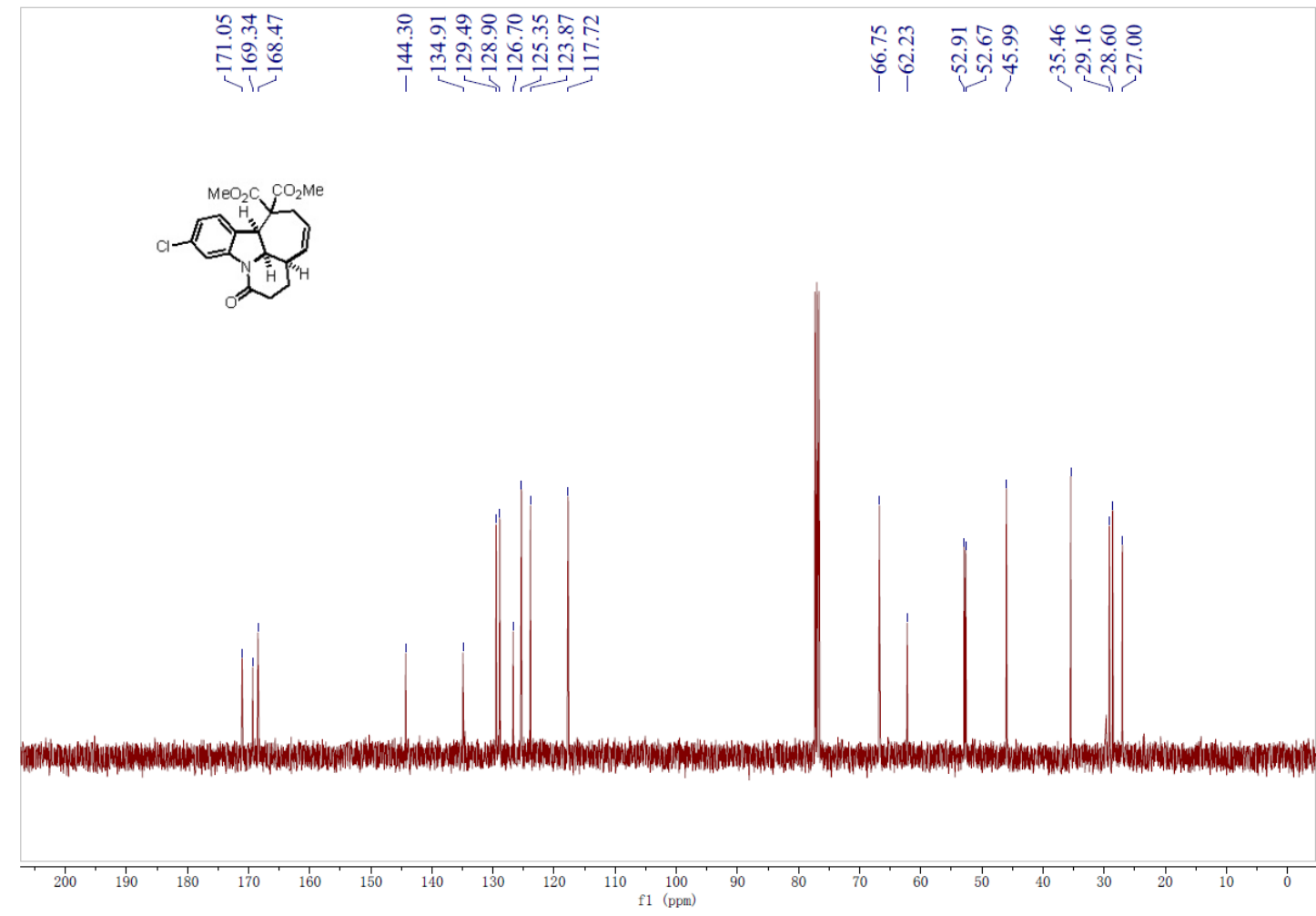
${ }^{1} \mathrm{H}$ NMR Spectrum of $\mathbf{2} \mathbf{a b}$

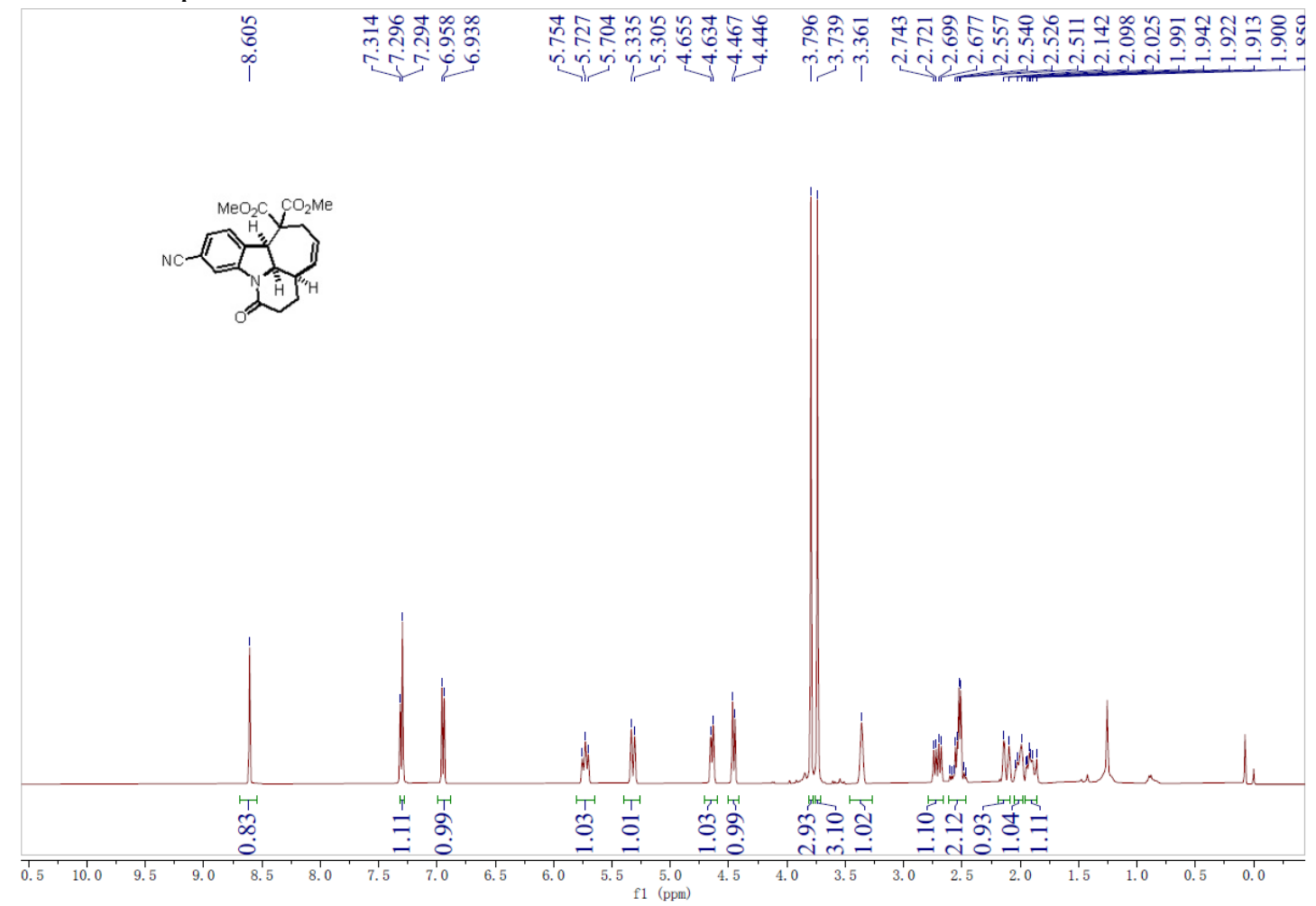

${ }^{13} \mathrm{C}$ NMR Spectrum of $\mathbf{2 a b}$

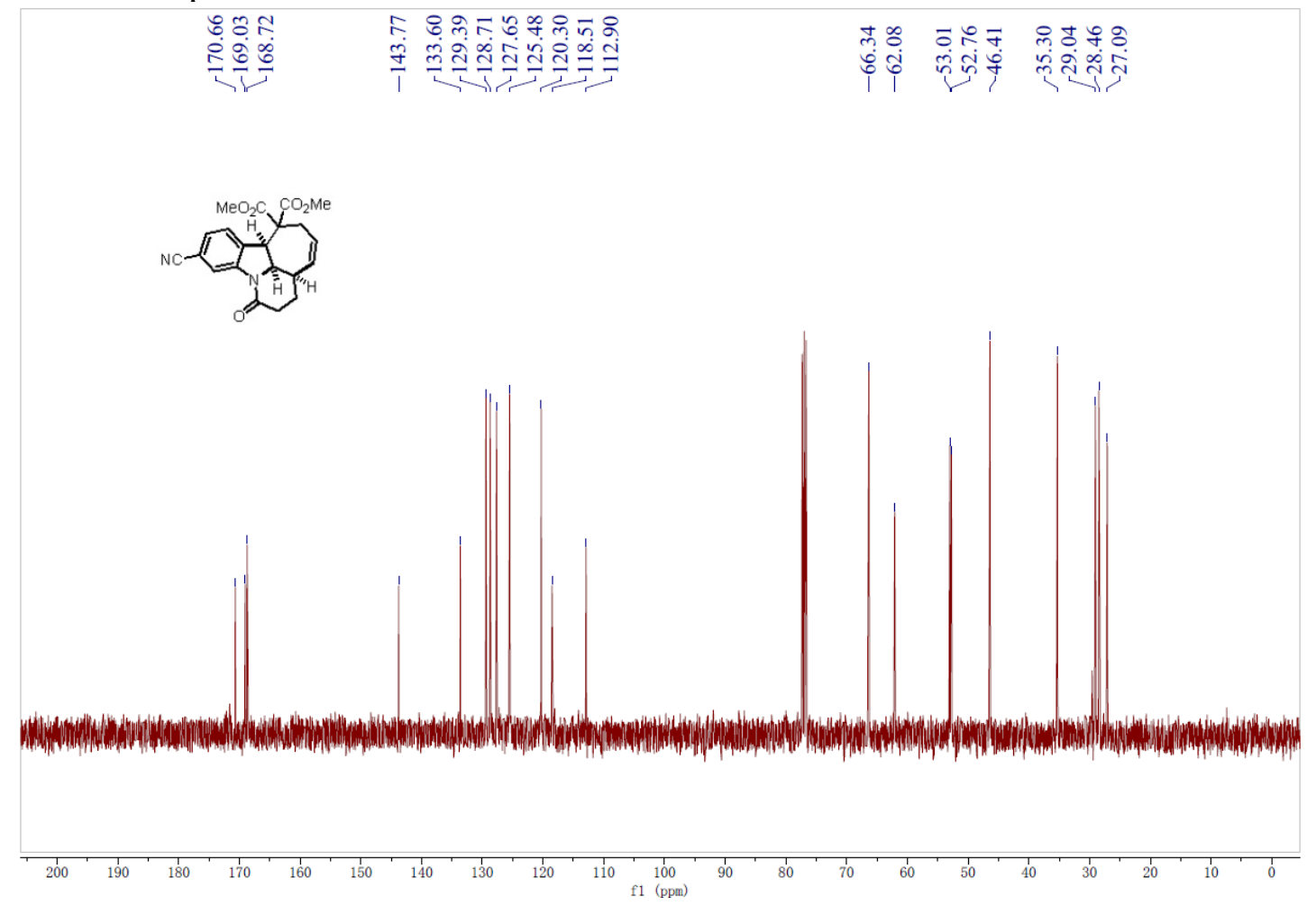


${ }^{1} \mathrm{H}$ NMR Spectrum of $\mathbf{2 a c}$

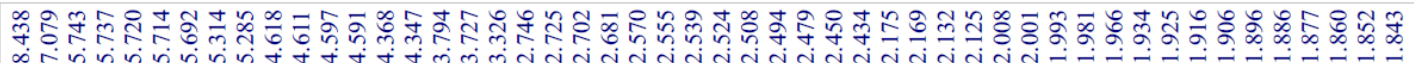

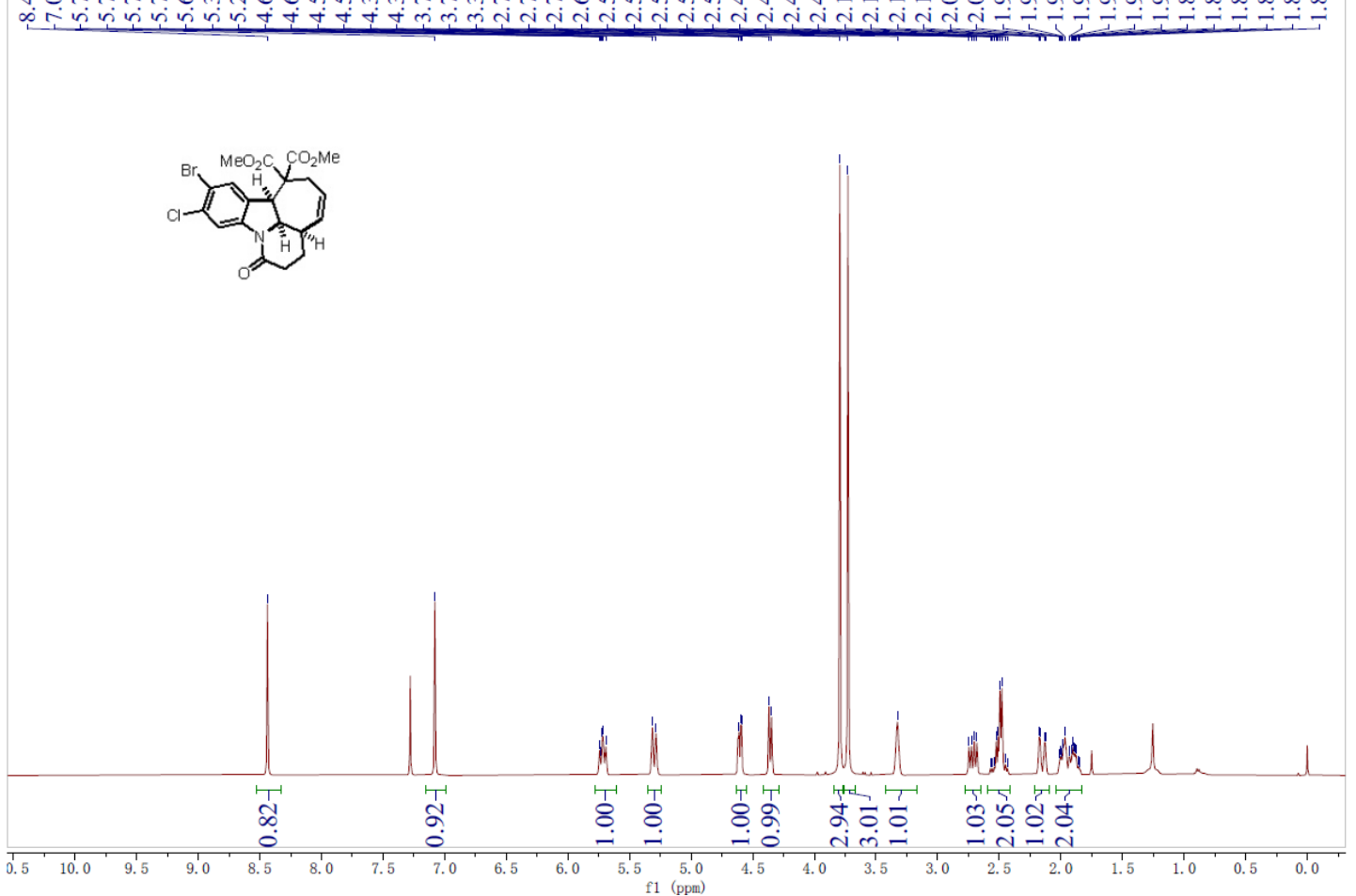

${ }^{13} \mathrm{C}$ NMR Spectrum of $\mathbf{2 a c}$

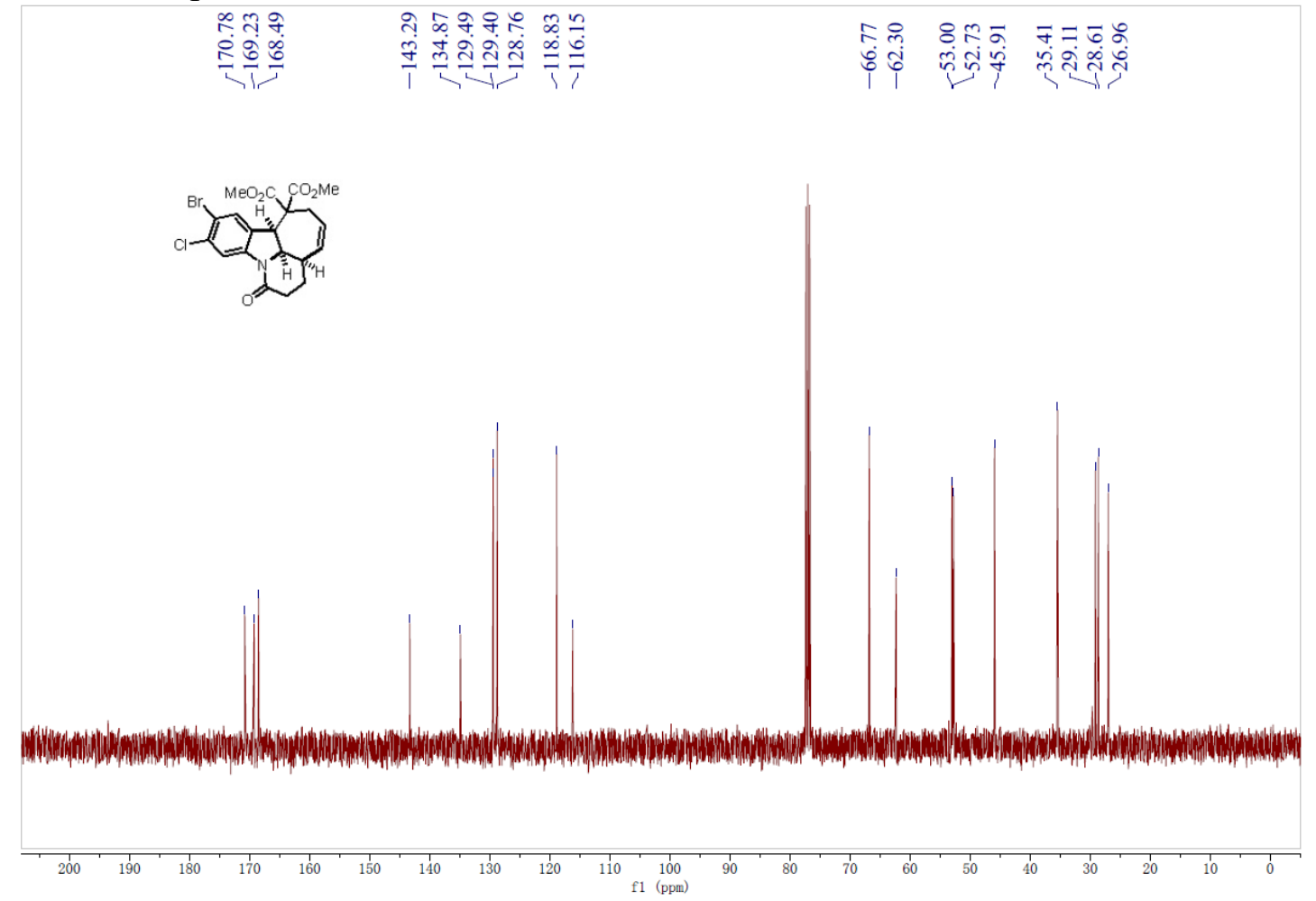


${ }^{1} \mathrm{H}$ NMR Spectrum of $\mathbf{2 a d}$

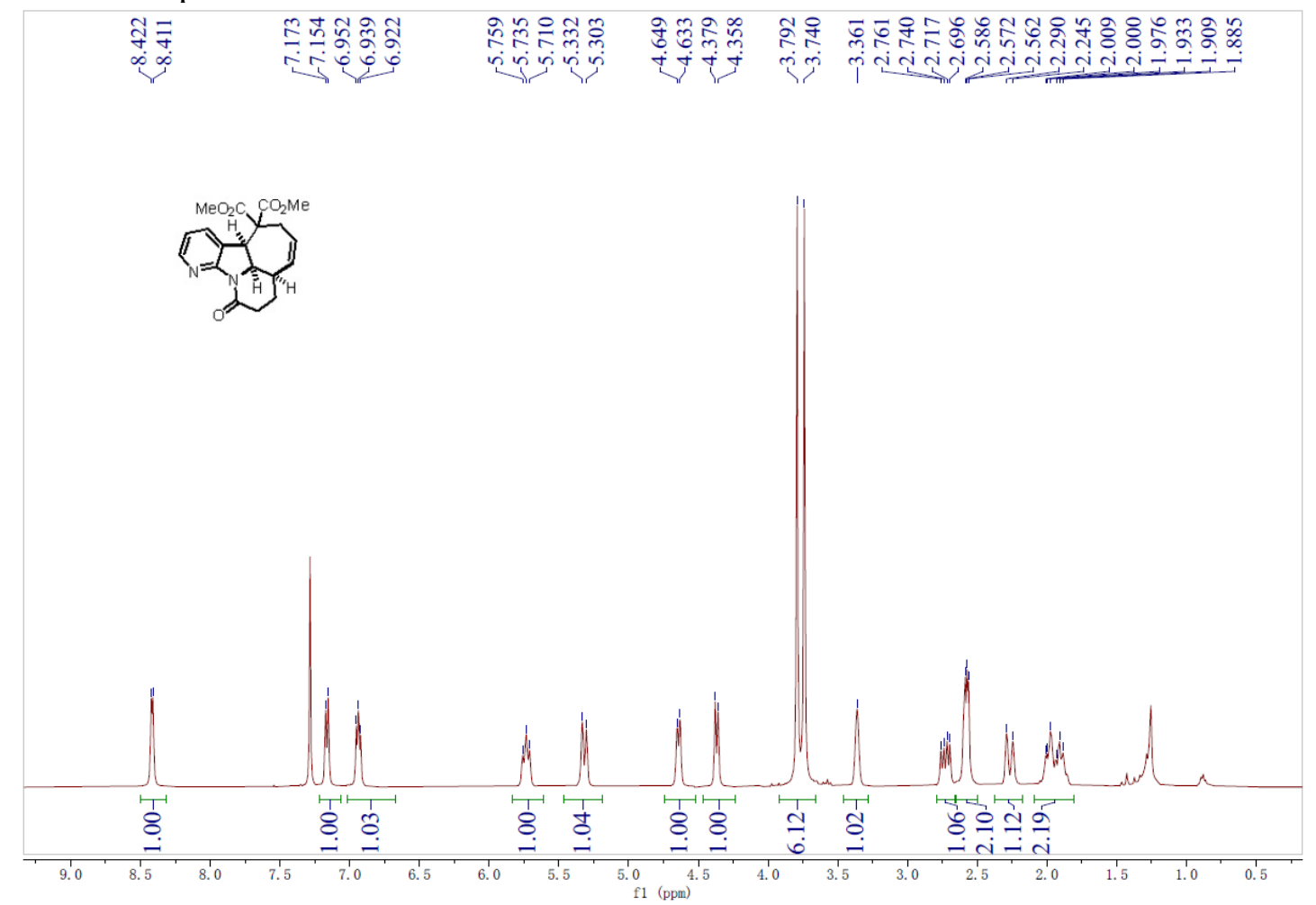

${ }^{13} \mathrm{C}$ NMR Spectrum of $\mathbf{2 a d}$

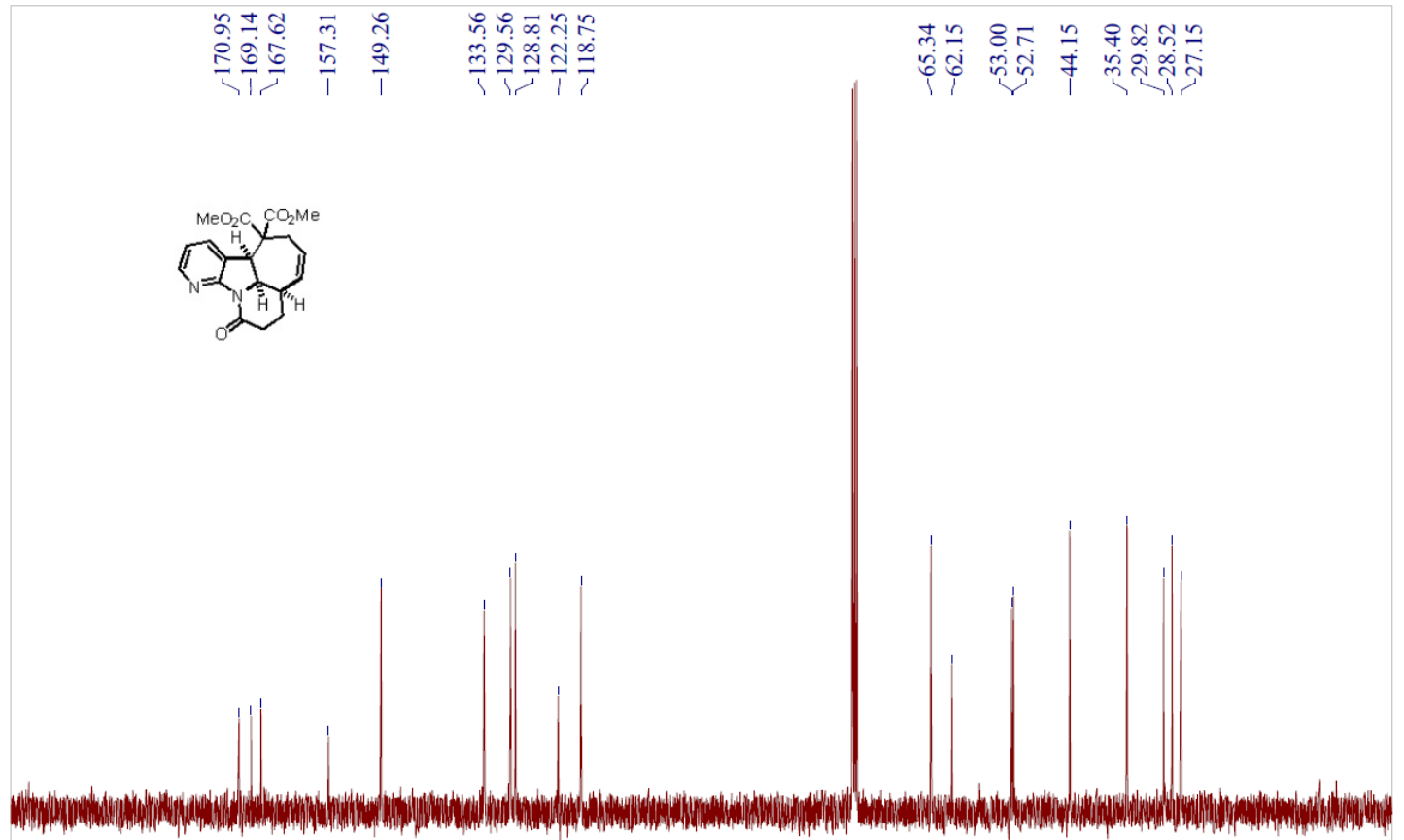


${ }^{1} \mathrm{H}$ NMR Spectrum of $\mathbf{4 a}$

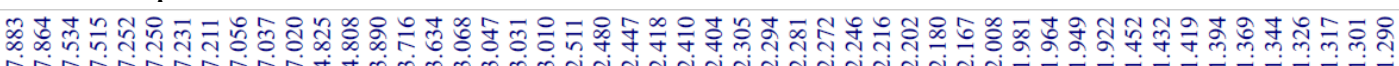

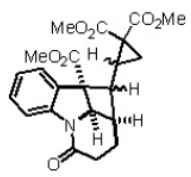

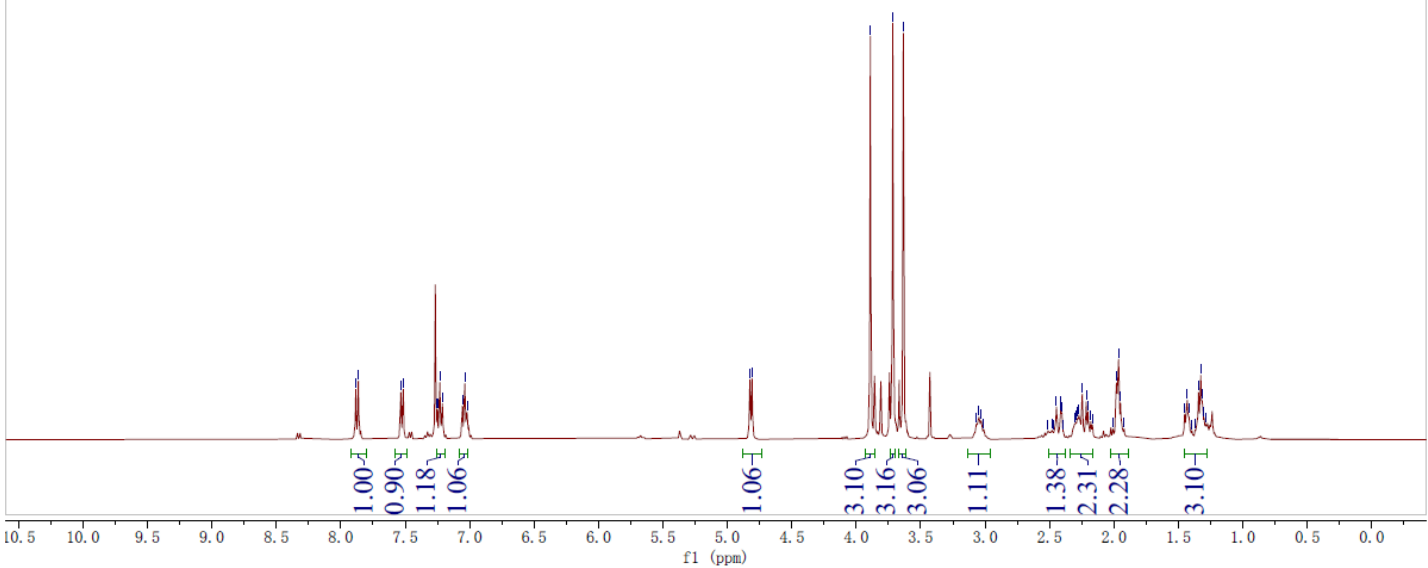

${ }^{13} \mathrm{C}$ NMR Spectrum of $\mathbf{4 a}$

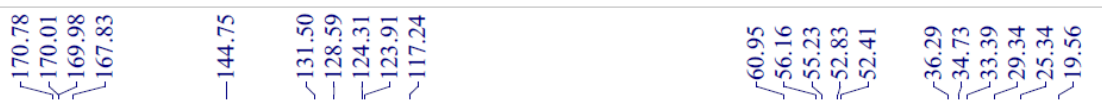

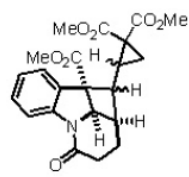
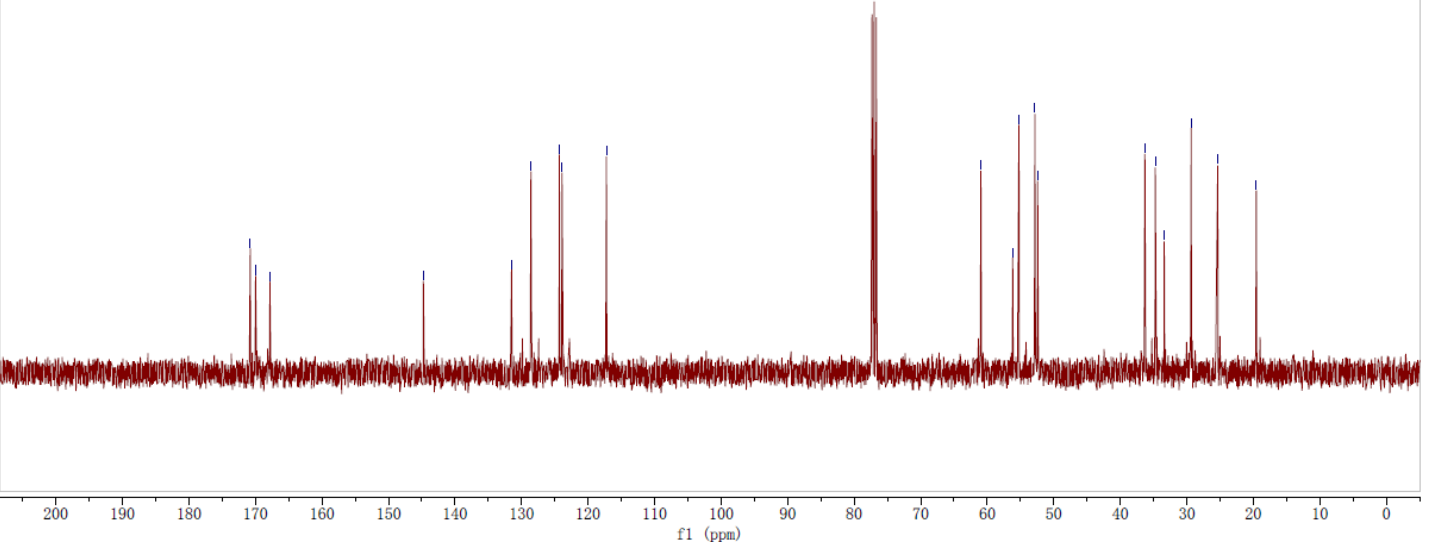
${ }^{1} \mathrm{H}$ NMR Spectrum of $\mathbf{4 b}$

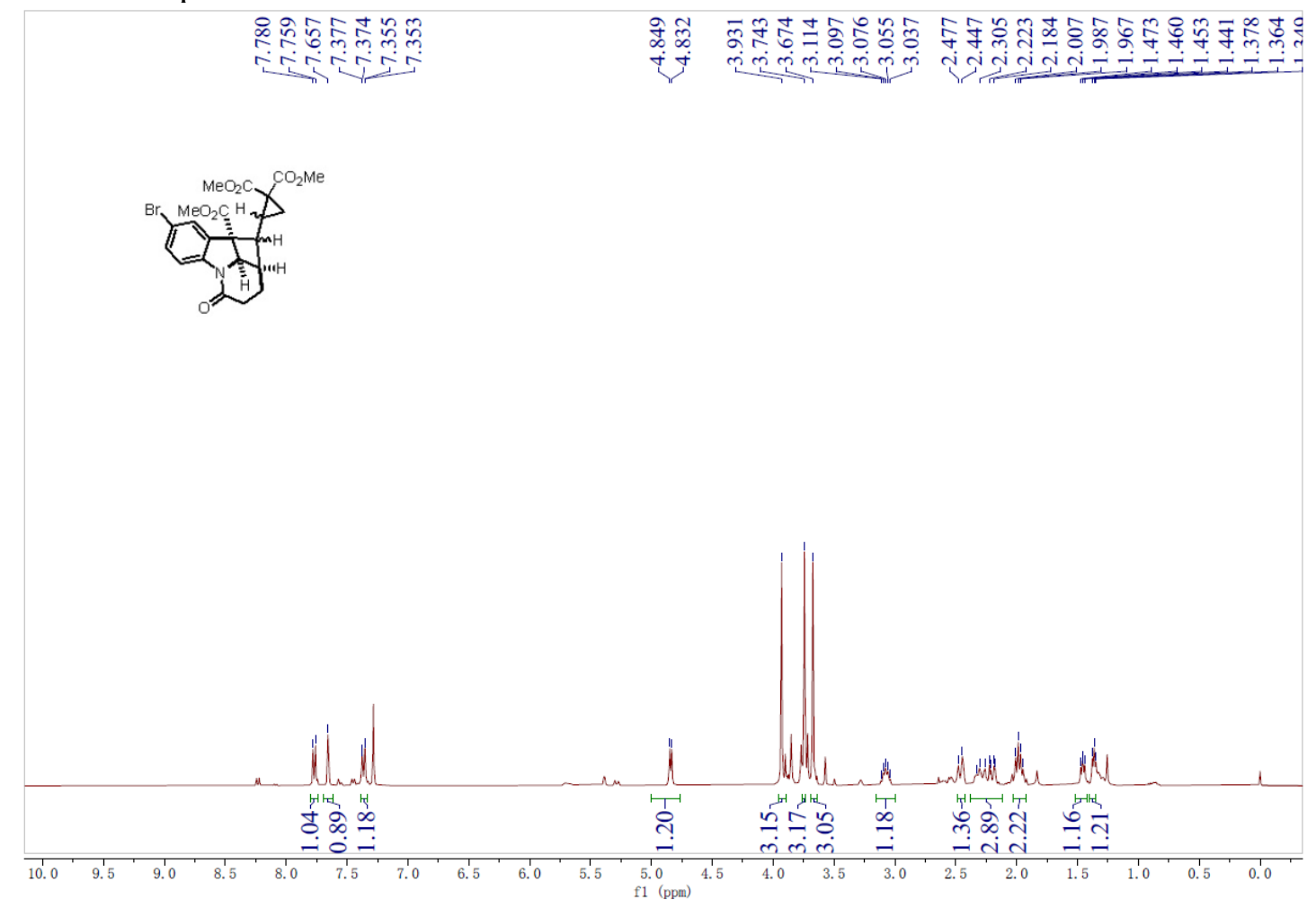

${ }^{13} \mathrm{C}$ NMR Spectrum of $\mathbf{4 b}$

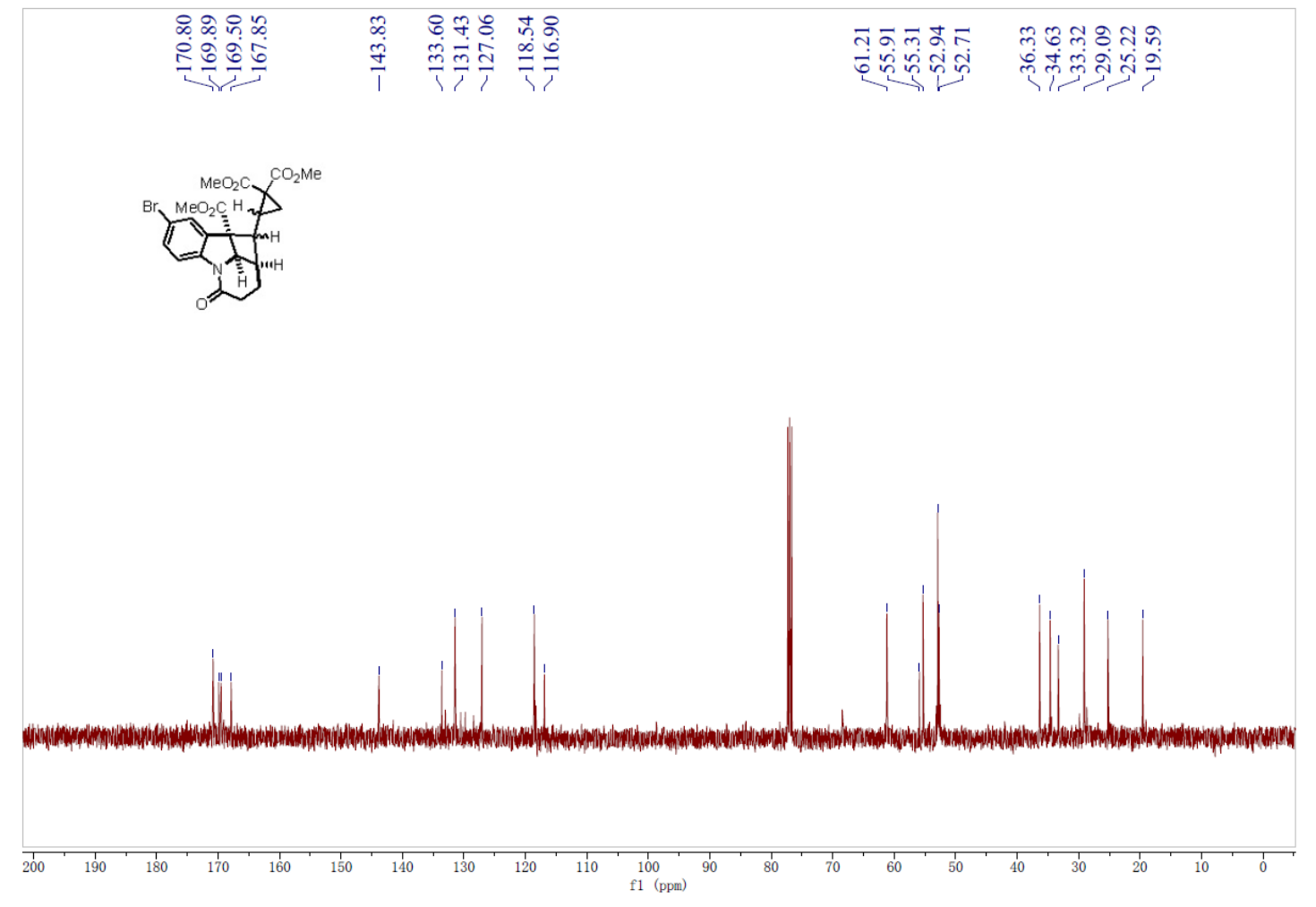


${ }^{1} \mathrm{H}$ NMR Spectrum of $\mathbf{4 c}$

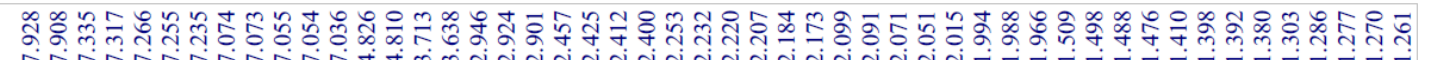

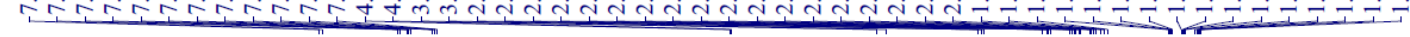

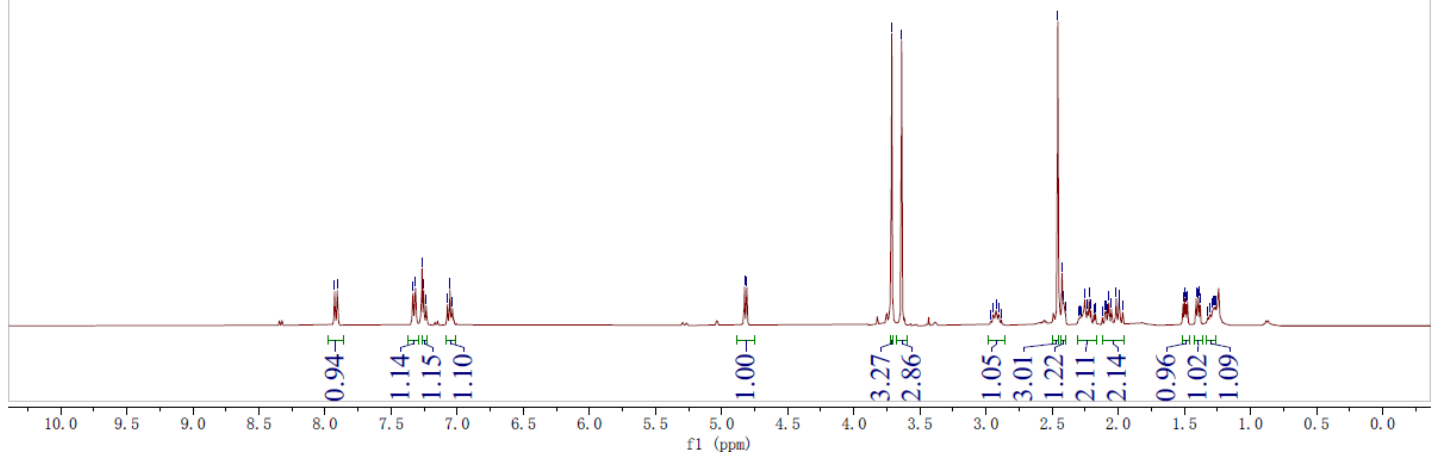

${ }^{13} \mathrm{C}$ NMR Spectrum of $\mathbf{4 c}$

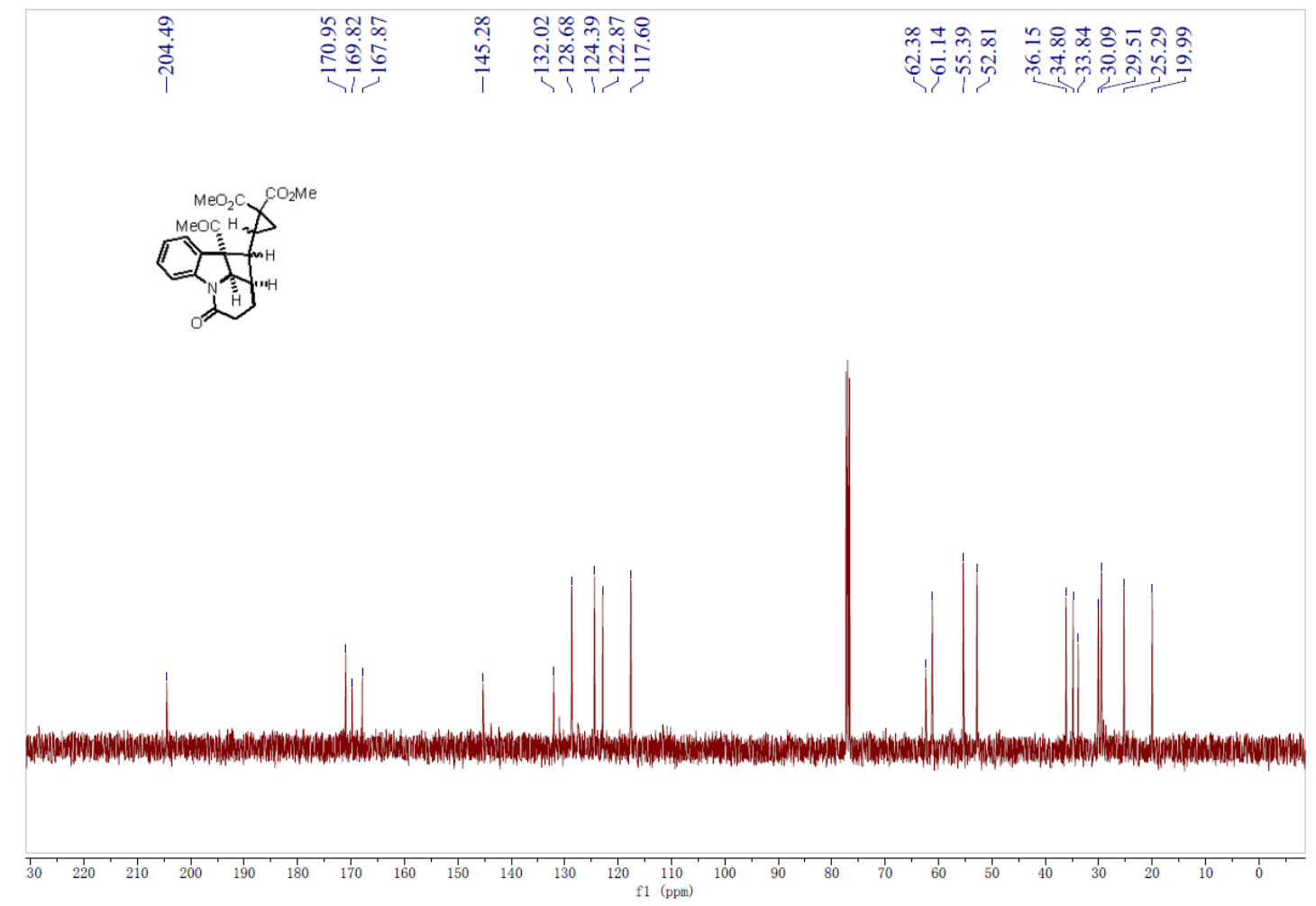


${ }^{1} \mathrm{H}$ NMR Spectrum of $\mathbf{4 d}$

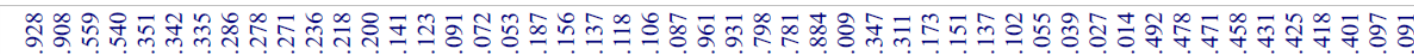

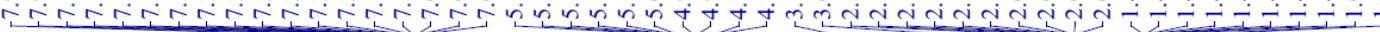

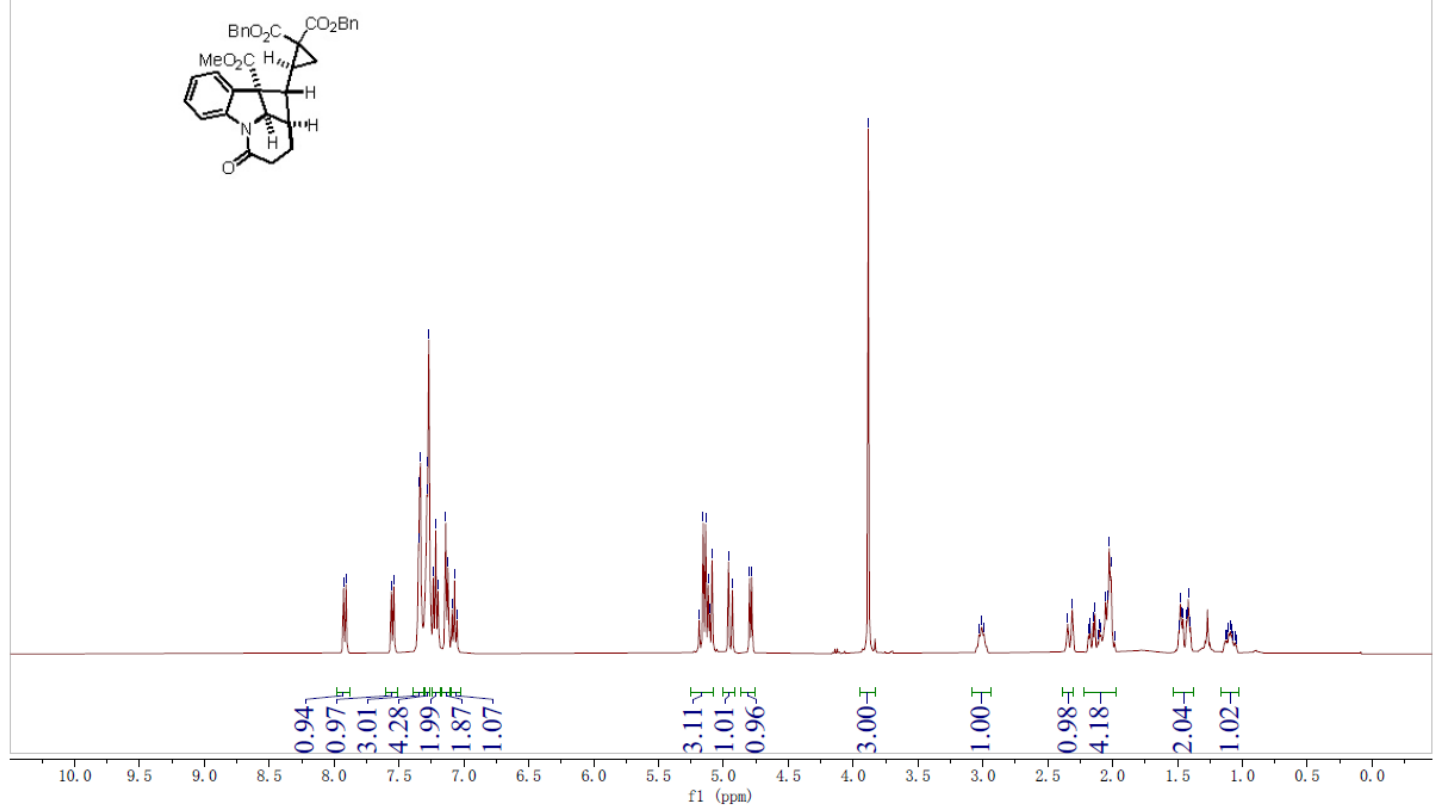

${ }^{13} \mathrm{C}$ NMR Spectrum of $\mathbf{4 d}$

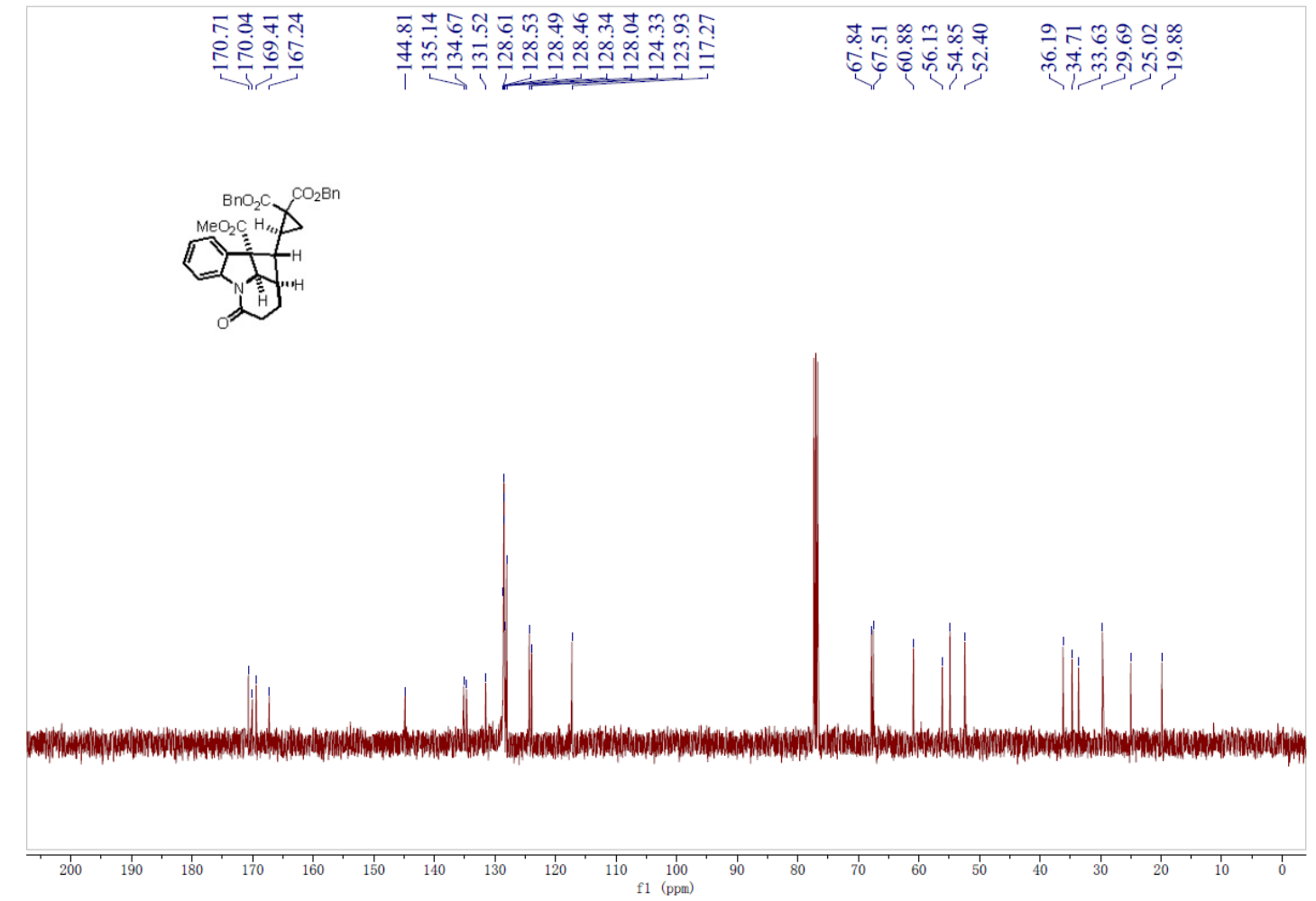


${ }^{1} \mathrm{H}$ NMR Spectrum of $\mathbf{6 a}$

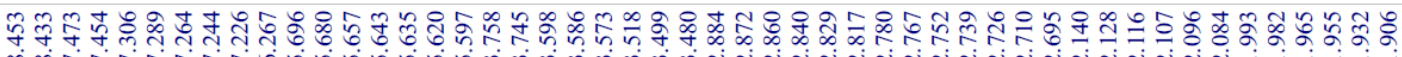

पin

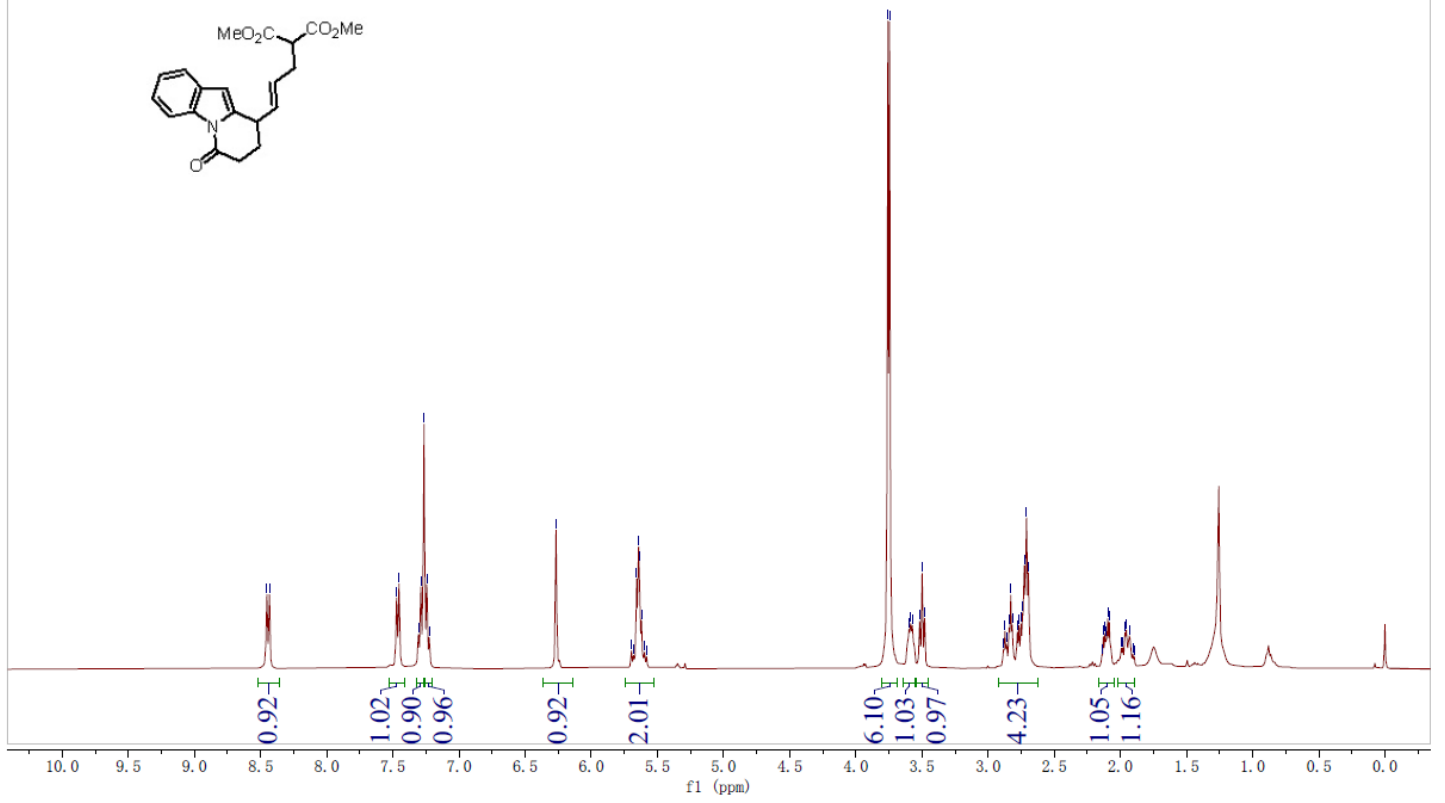

${ }^{13} \mathrm{C}$ NMR Spectrum of $\mathbf{6 a}$

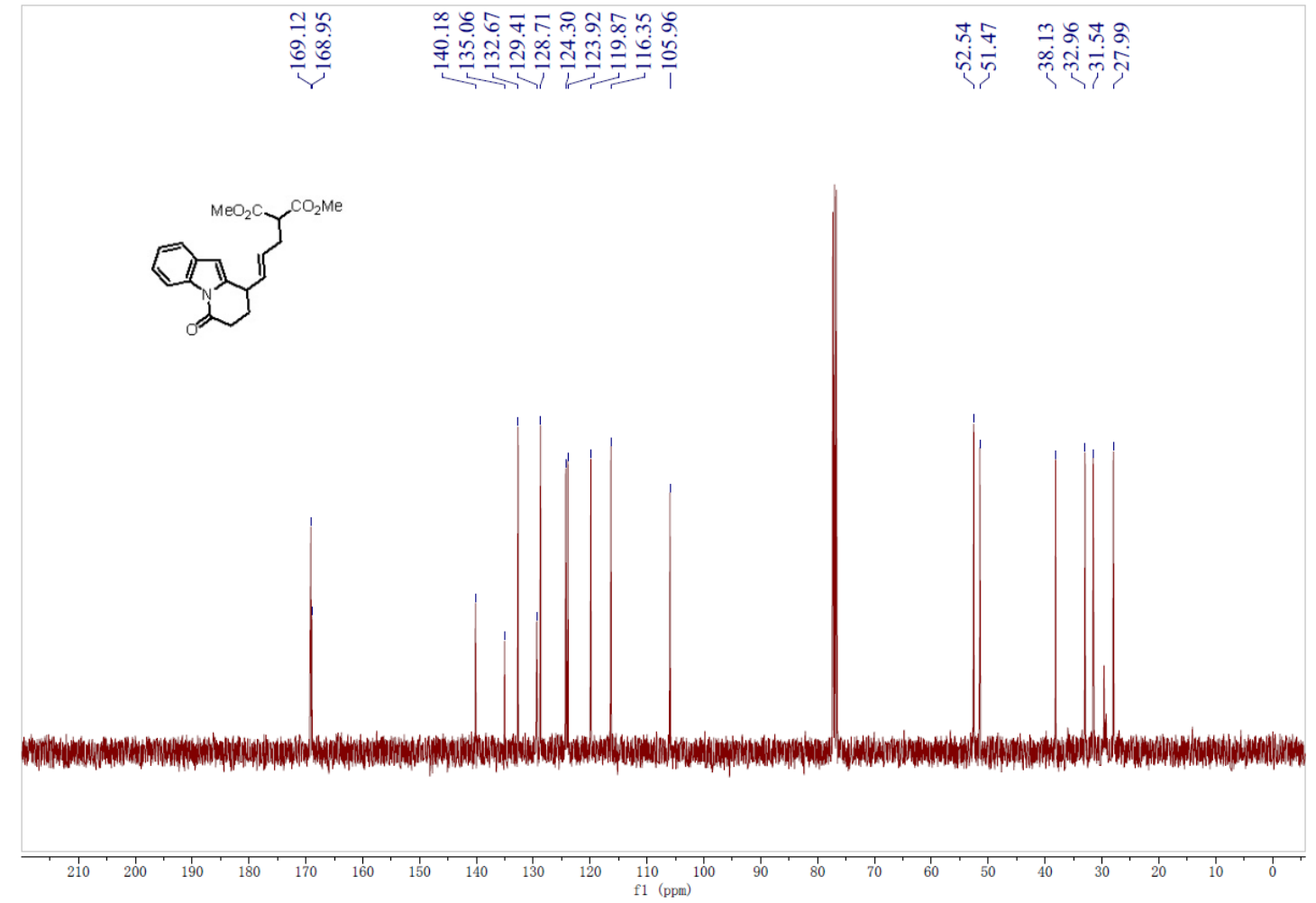


${ }^{1} \mathrm{H}$ NMR Spectrum of $\mathbf{6 b}$

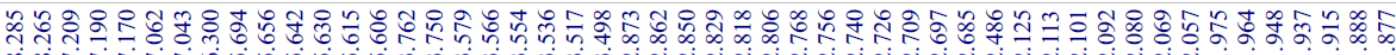

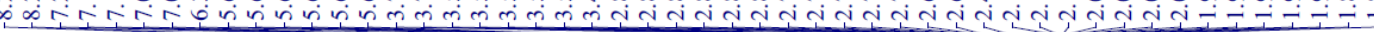

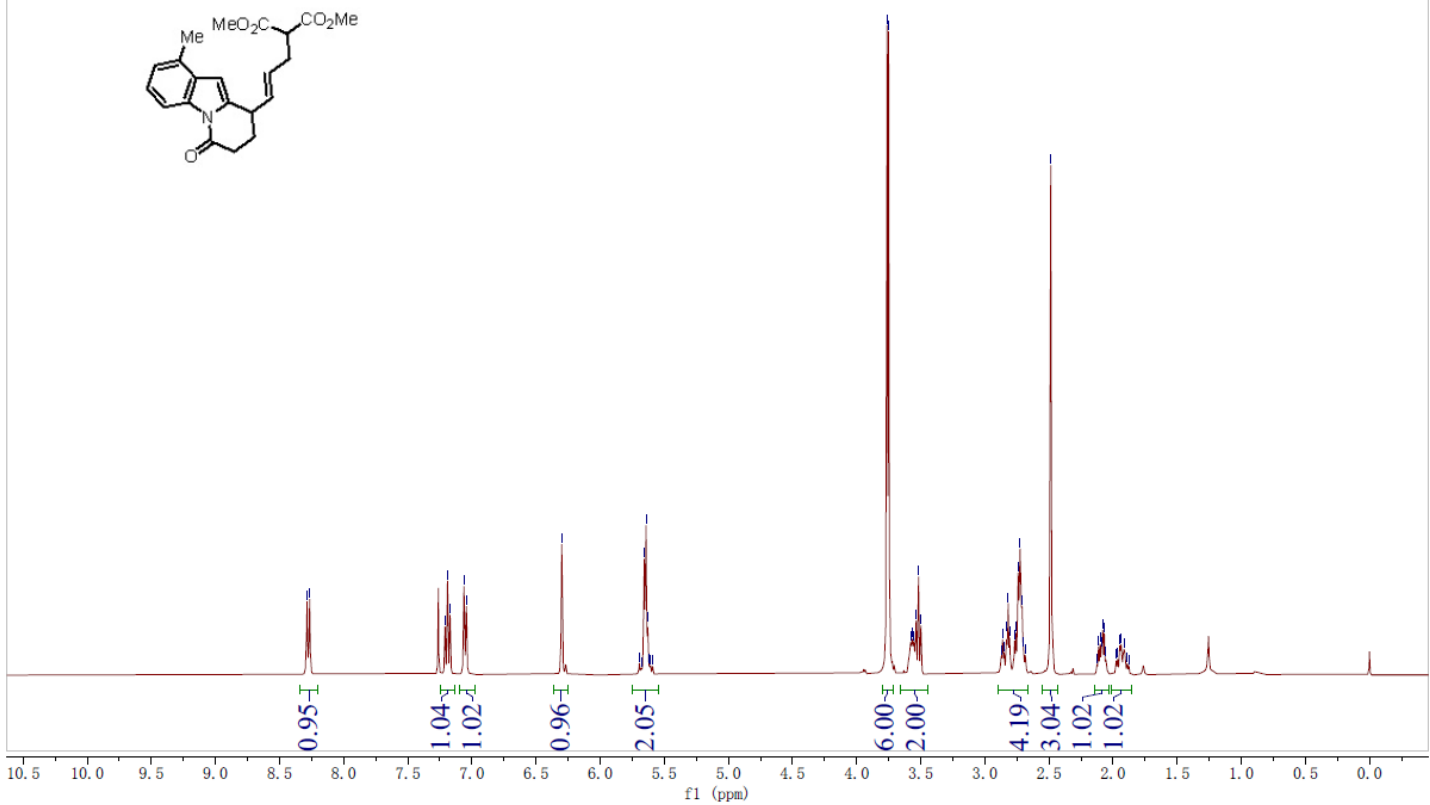

${ }^{13} \mathrm{C}$ NMR Spectrum of $\mathbf{6 b}$

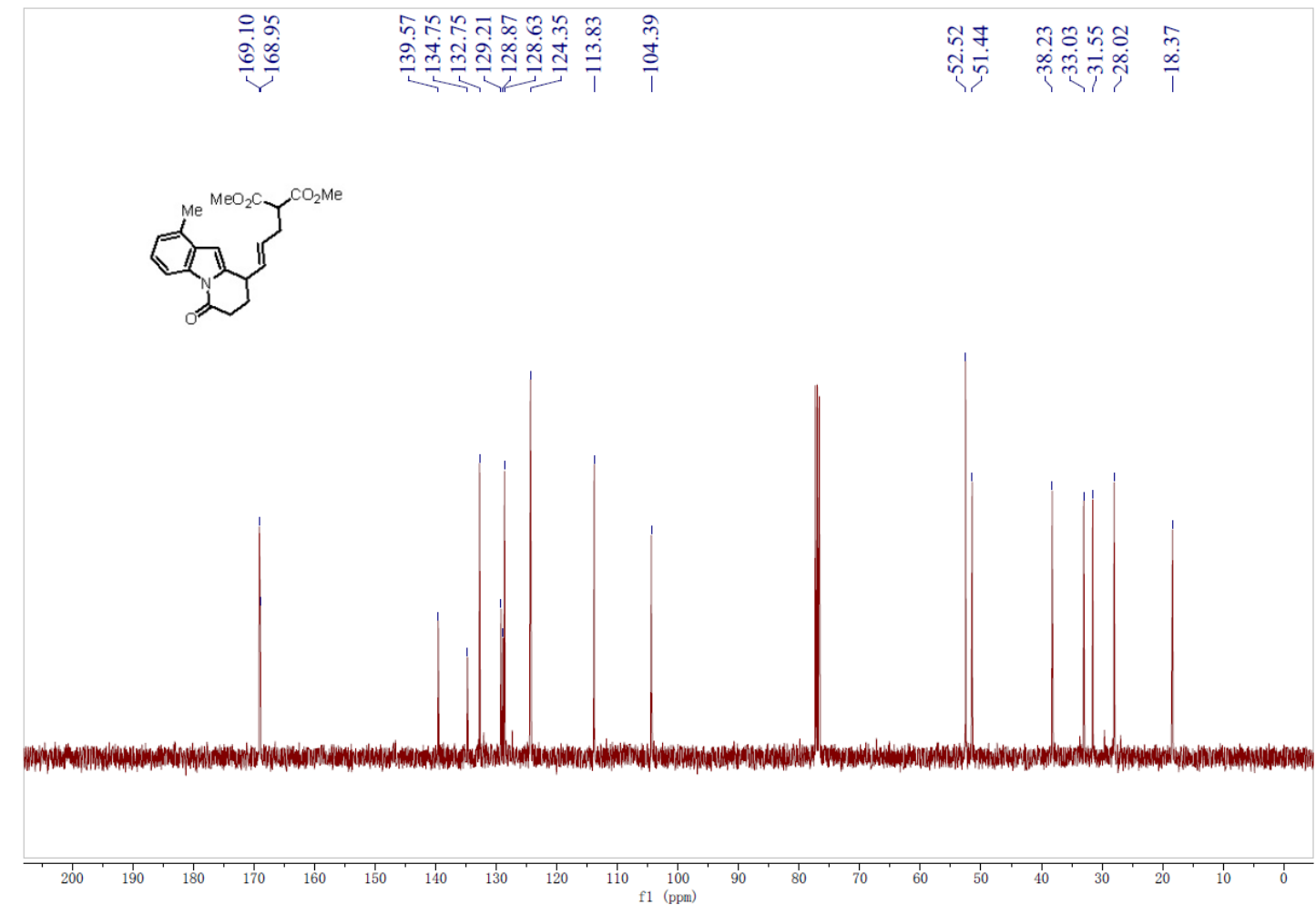


${ }^{1} \mathrm{H}$ NMR Spectrum of $\mathbf{6 c}$

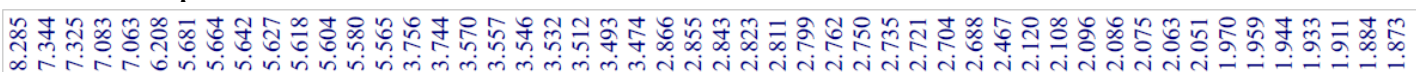

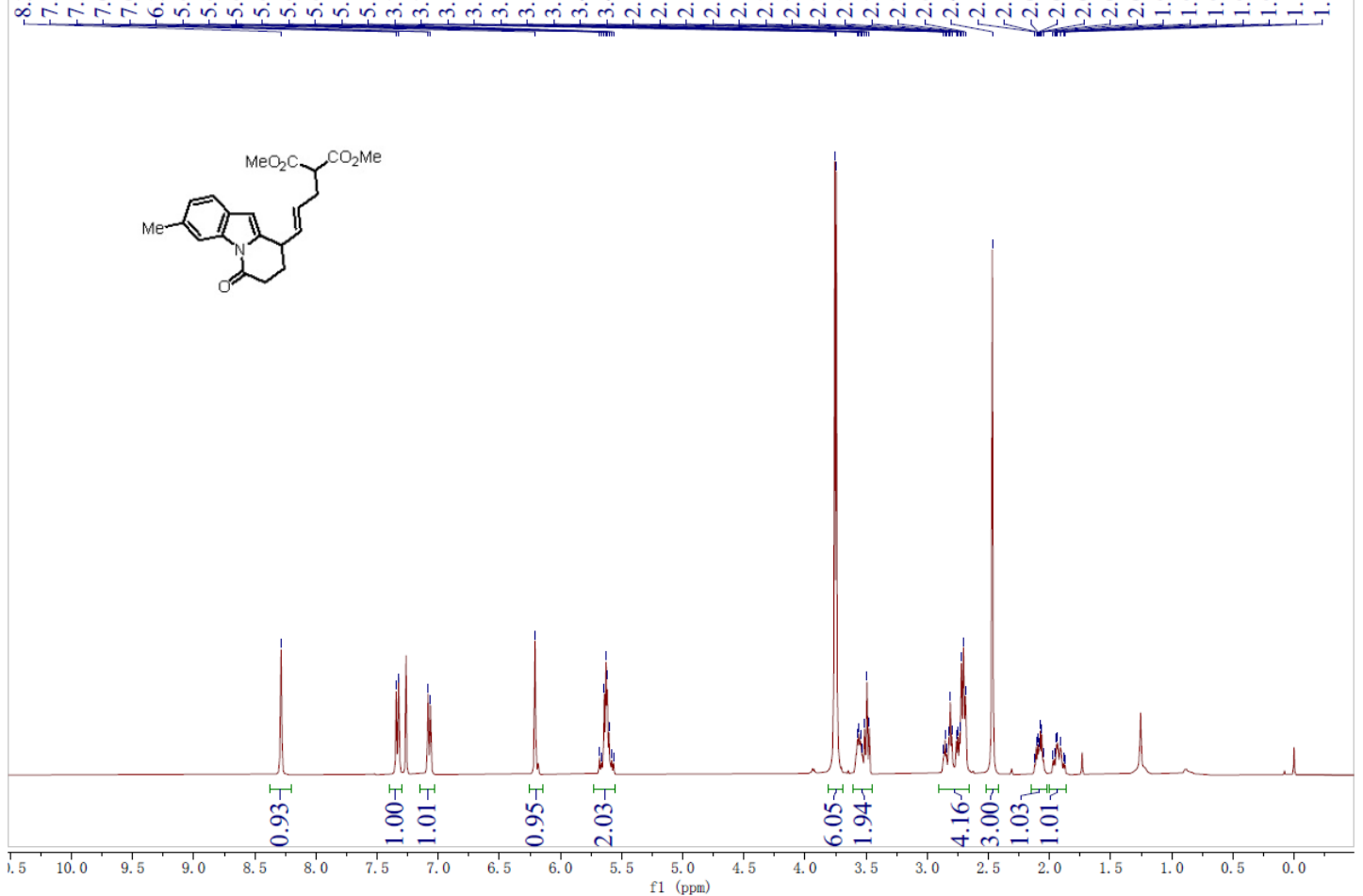

${ }^{13} \mathrm{C}$ NMR Spectrum of $\mathbf{6 c}$

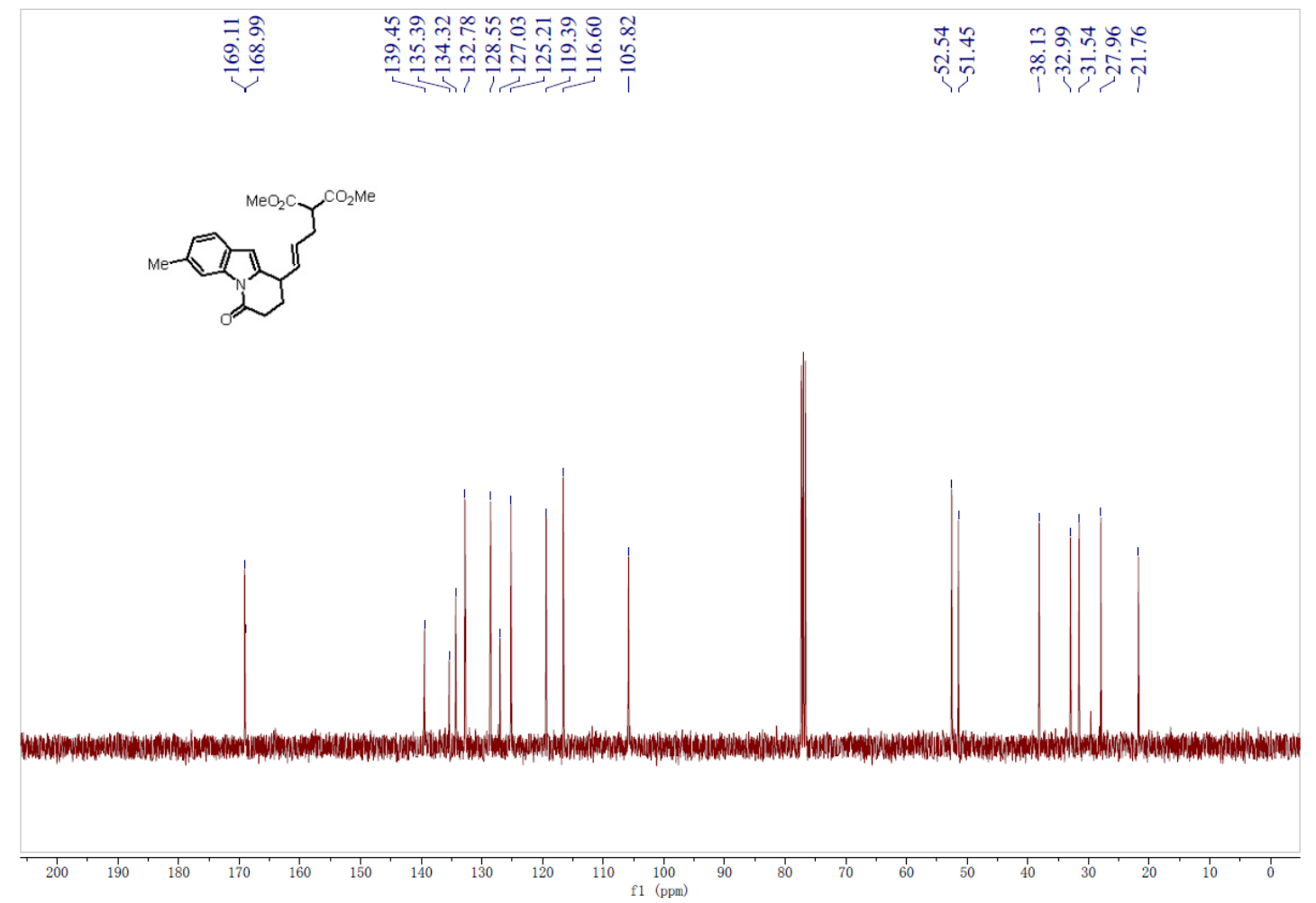


${ }^{1} \mathrm{H}$ NMR Spectrum of $\mathbf{6 d}$

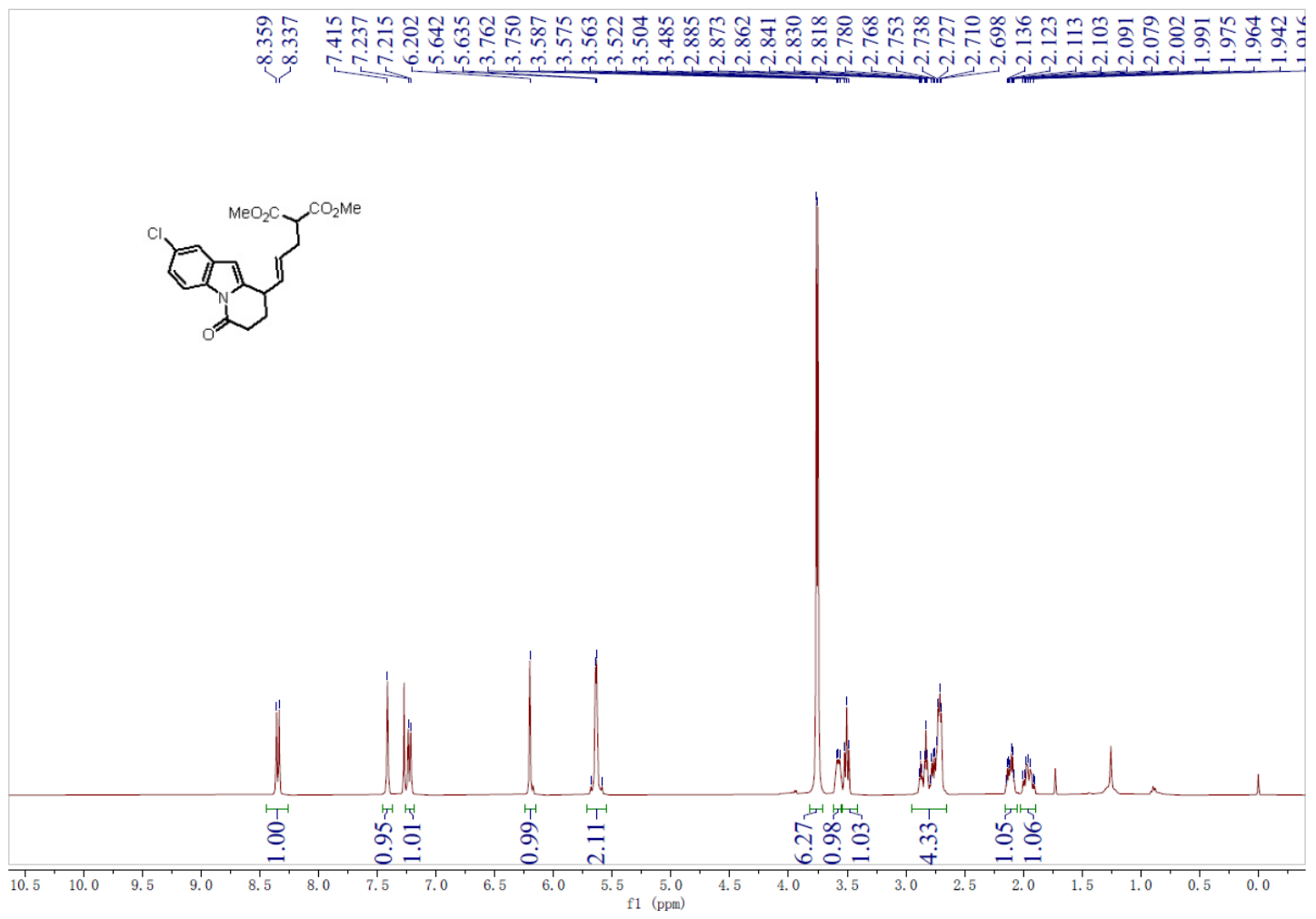

${ }^{13} \mathrm{C}$ NMR Spectrum of $\mathbf{6 d}$

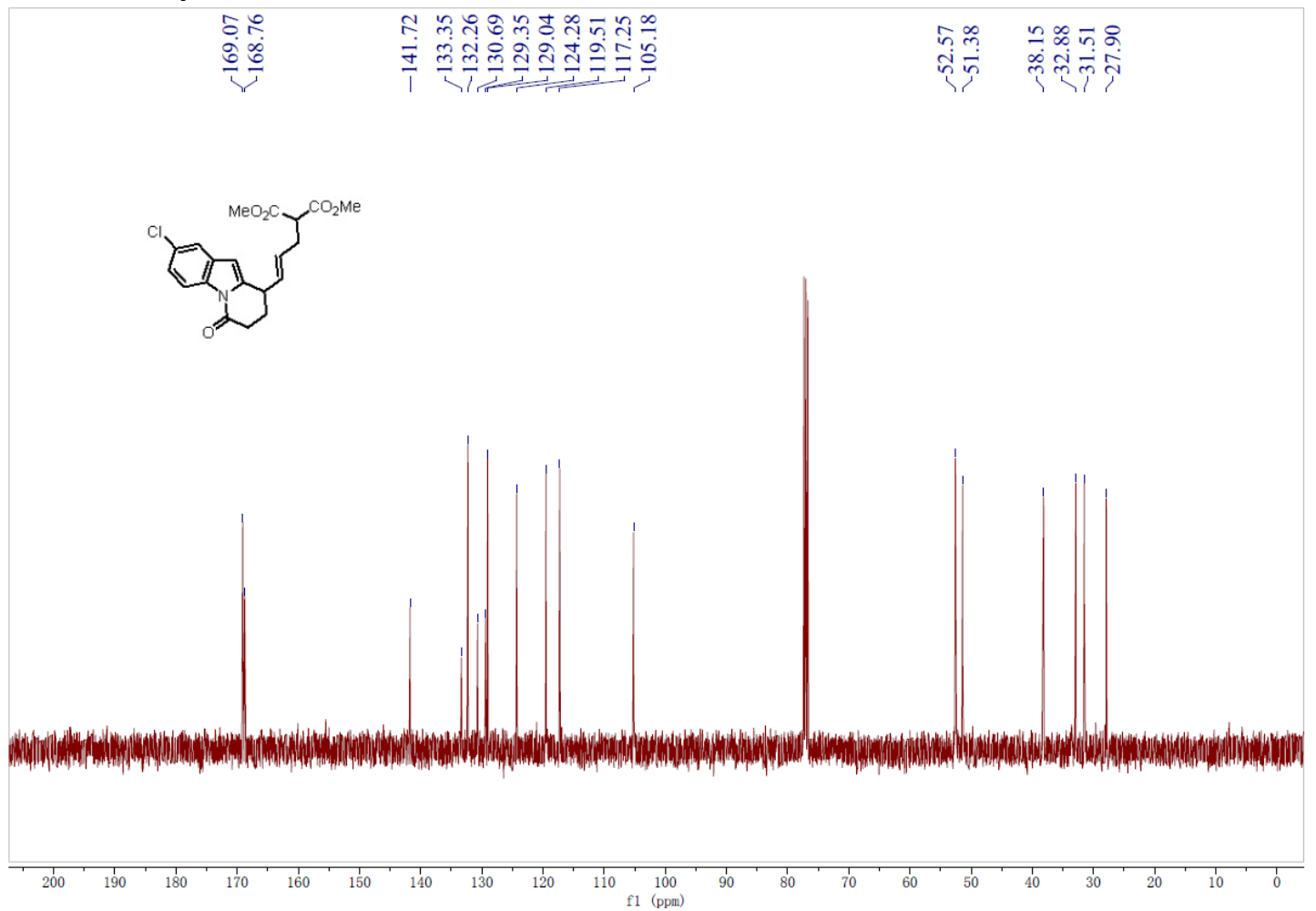


${ }^{1} \mathrm{H}$ NMR Spectrum of $\mathbf{6 e}$

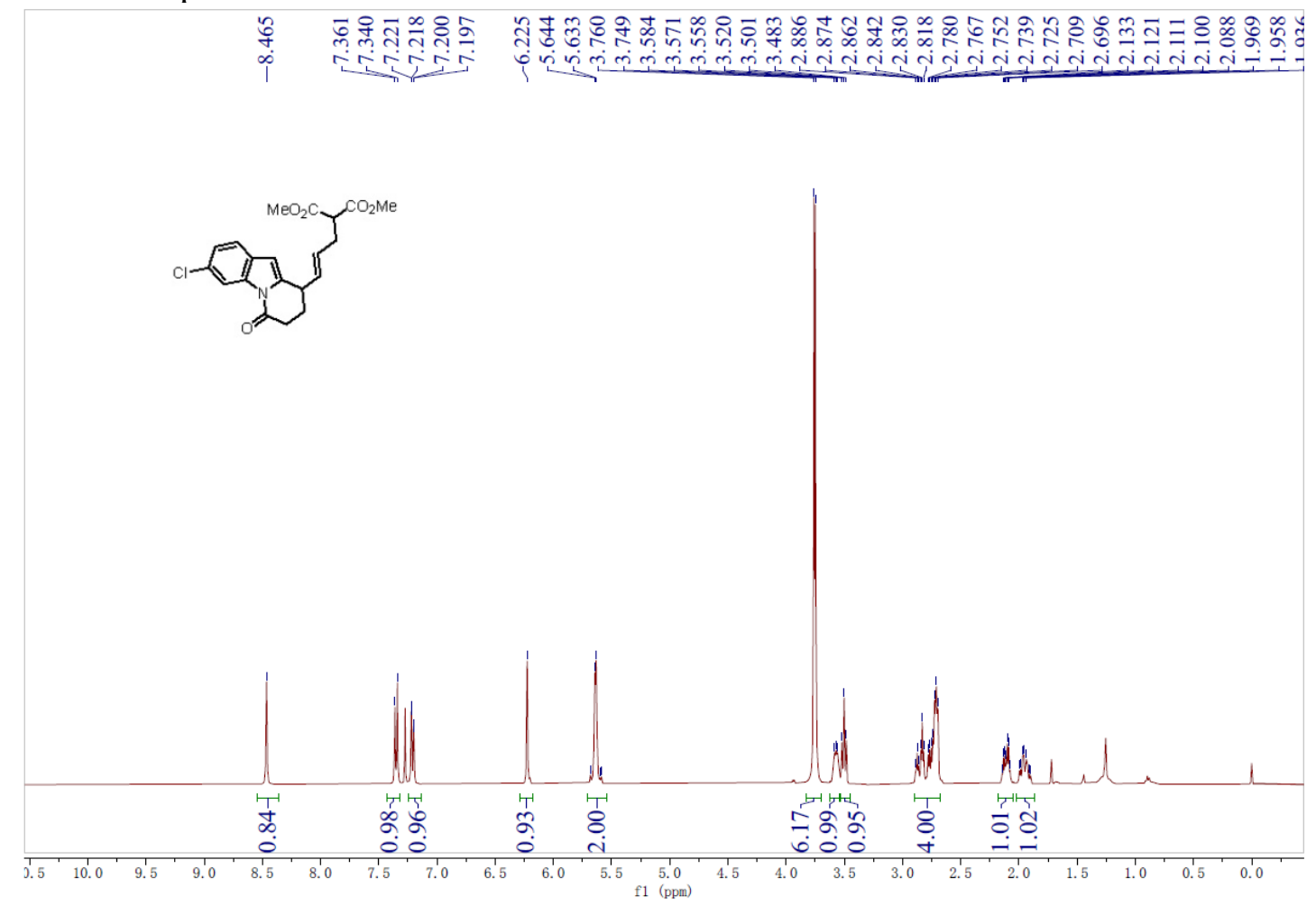

${ }^{13} \mathrm{C}$ NMR Spectrum of $\mathbf{6 e}$

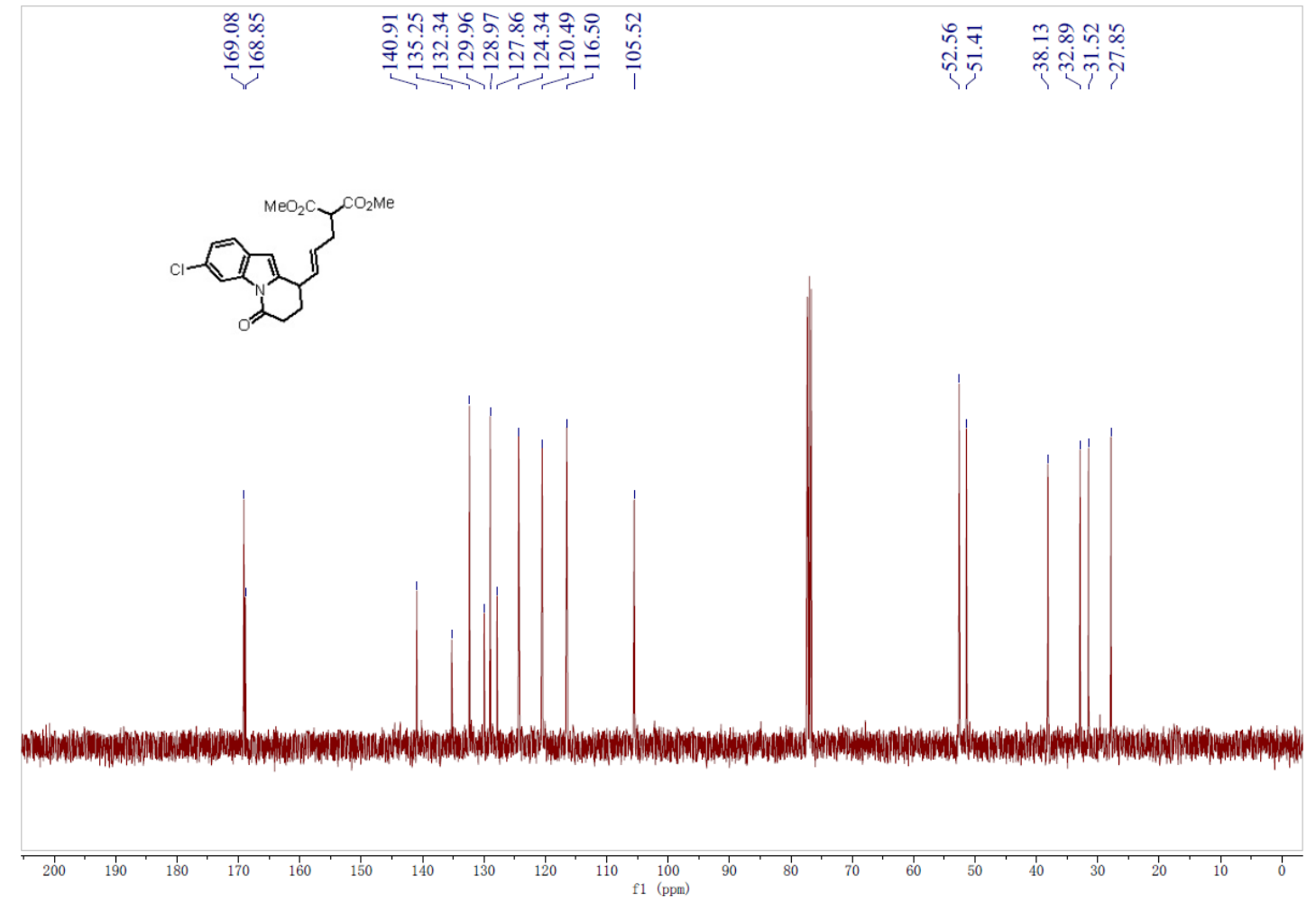


${ }^{1} \mathrm{H}$ NMR Spectrum of $\mathbf{6 f}$

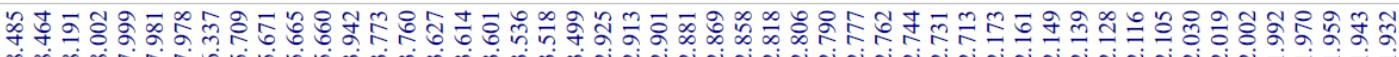
क क

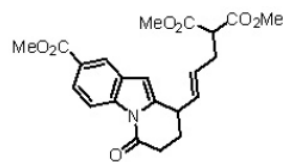

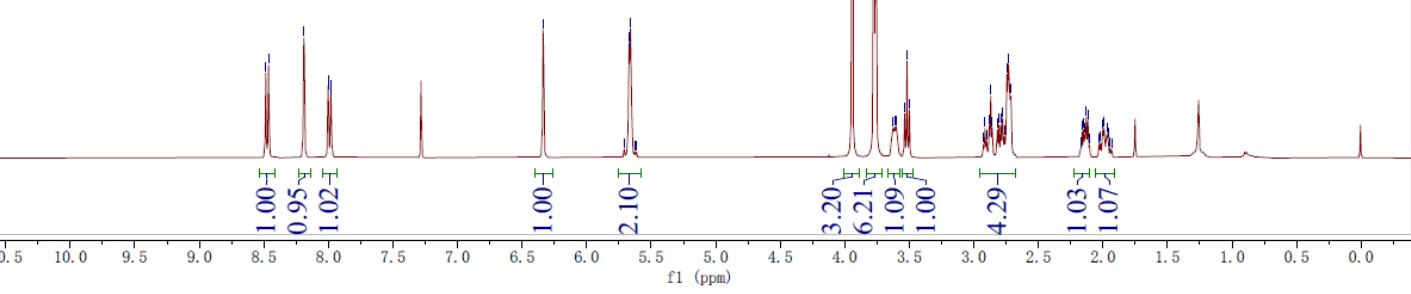

${ }^{13} \mathrm{C}$ NMR Spectrum of $\mathbf{6 f}$

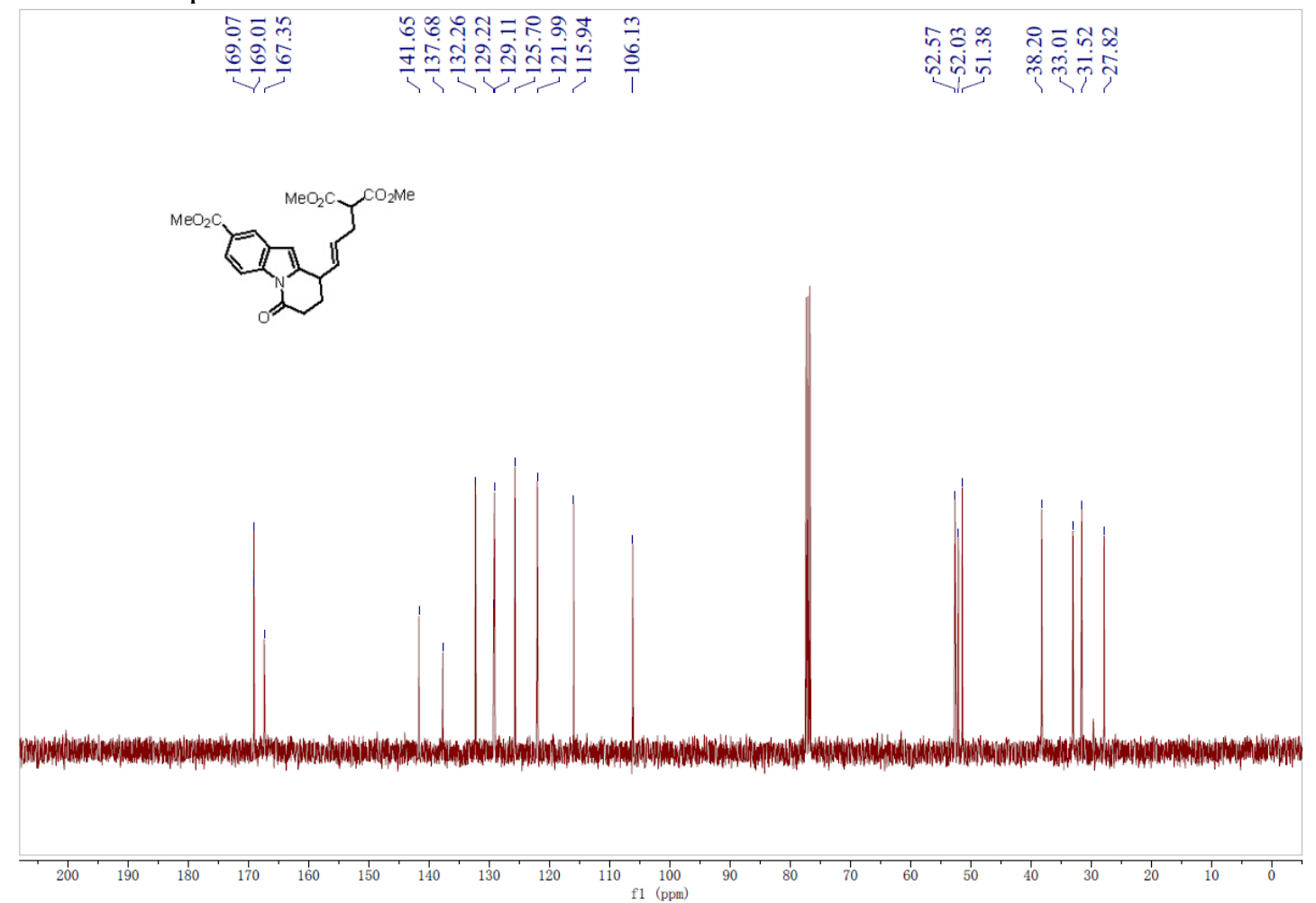


${ }^{1} \mathrm{H}$ NMR Spectrum of $\mathbf{6 g}$

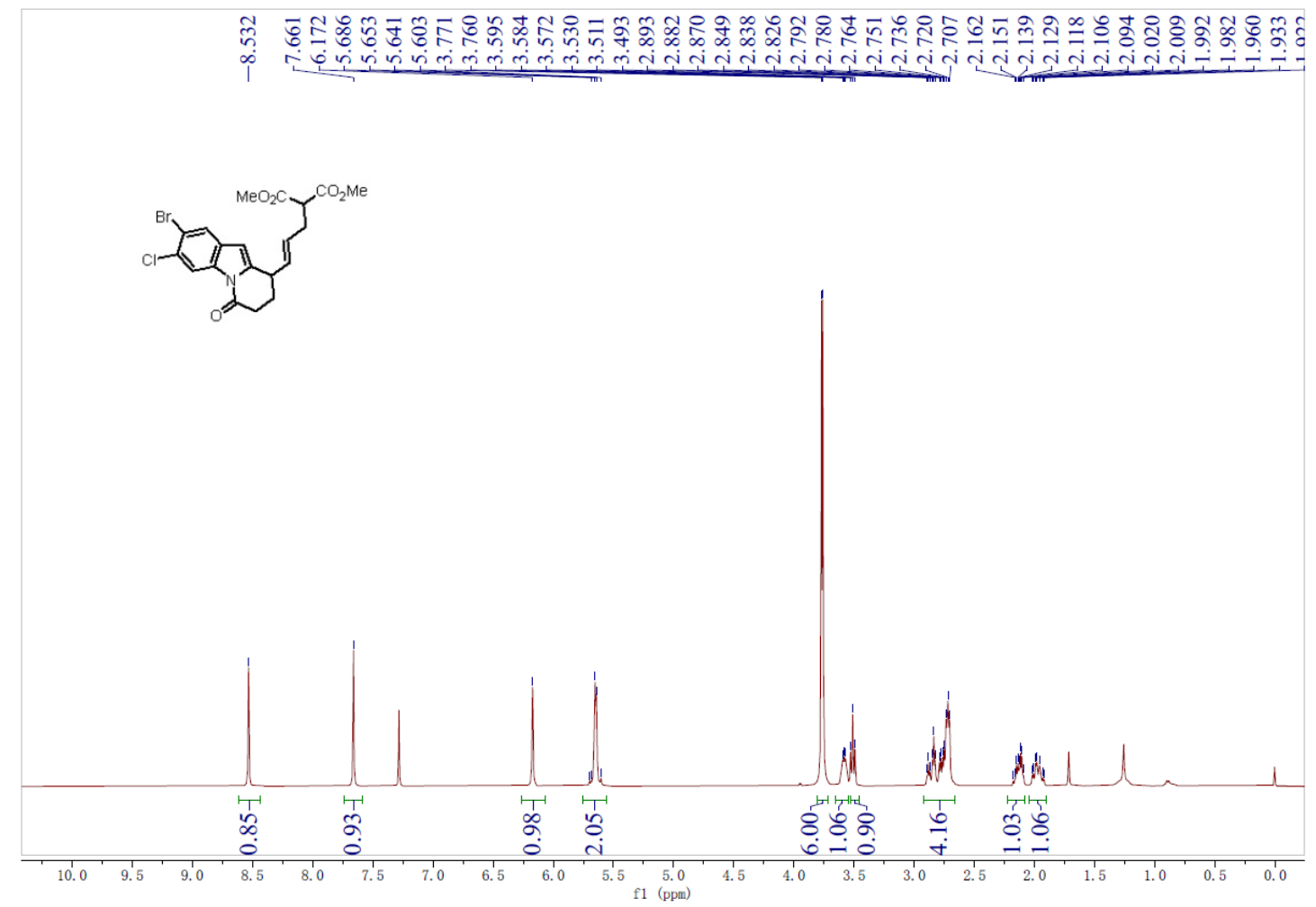

${ }^{13} \mathrm{C}$ NMR Spectrum of $\mathbf{6 g}$

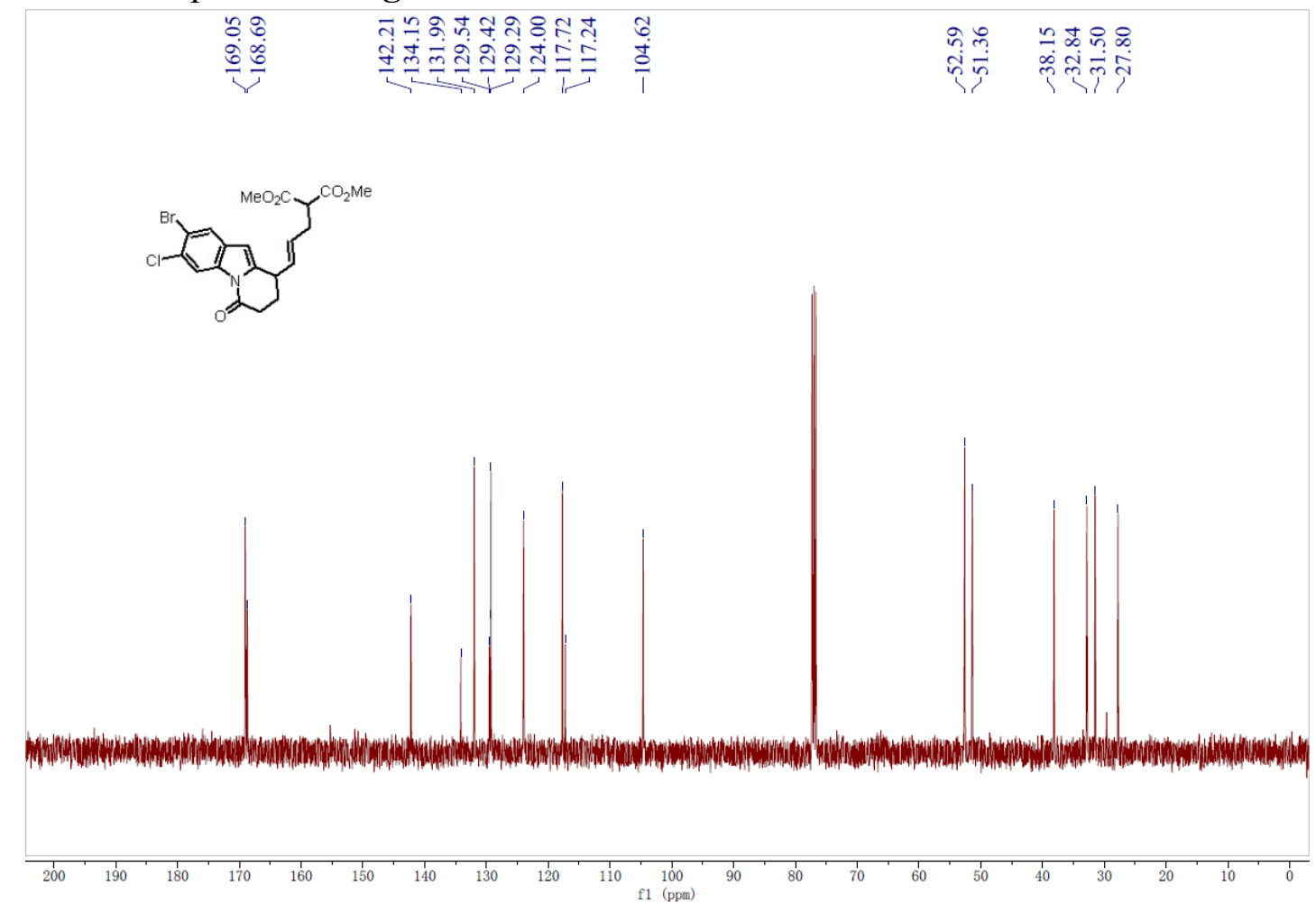


${ }^{1} \mathrm{H}$ NMR Spectrum of $\mathbf{6 a}-\boldsymbol{d}$

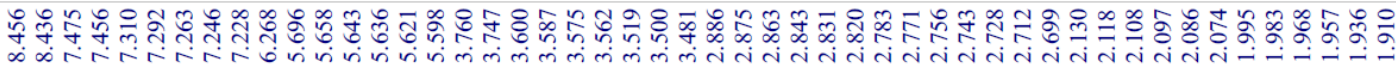

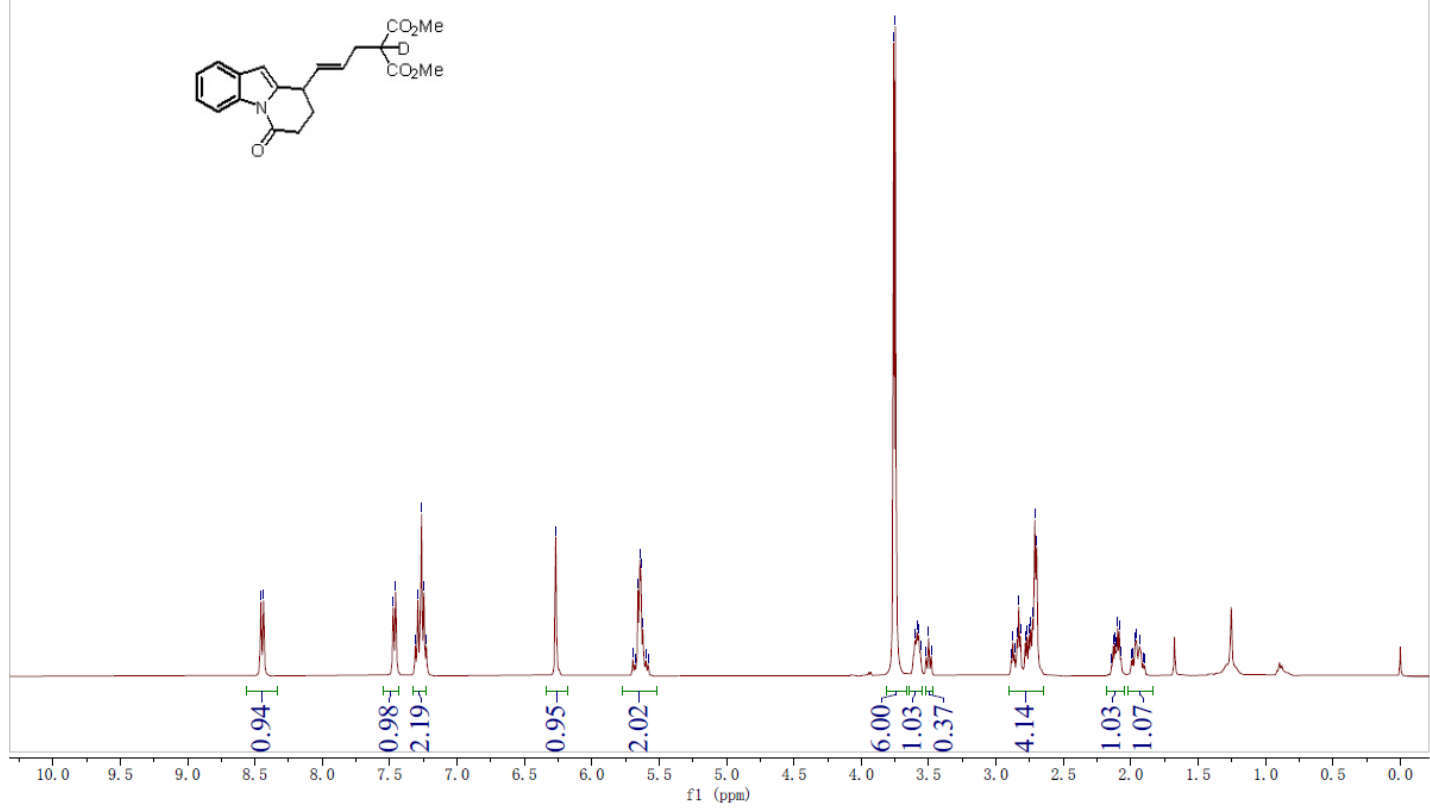

${ }^{1} \mathrm{H}$ NMR Spectrum of $\mathbf{6 b}-\boldsymbol{d}$

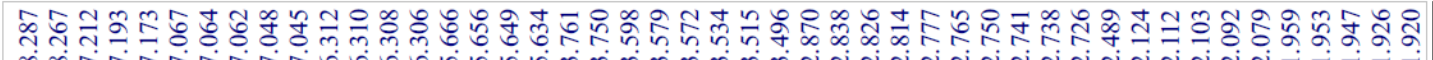

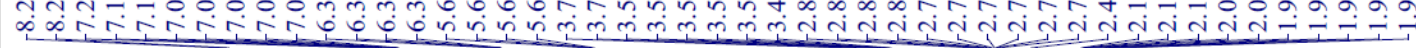

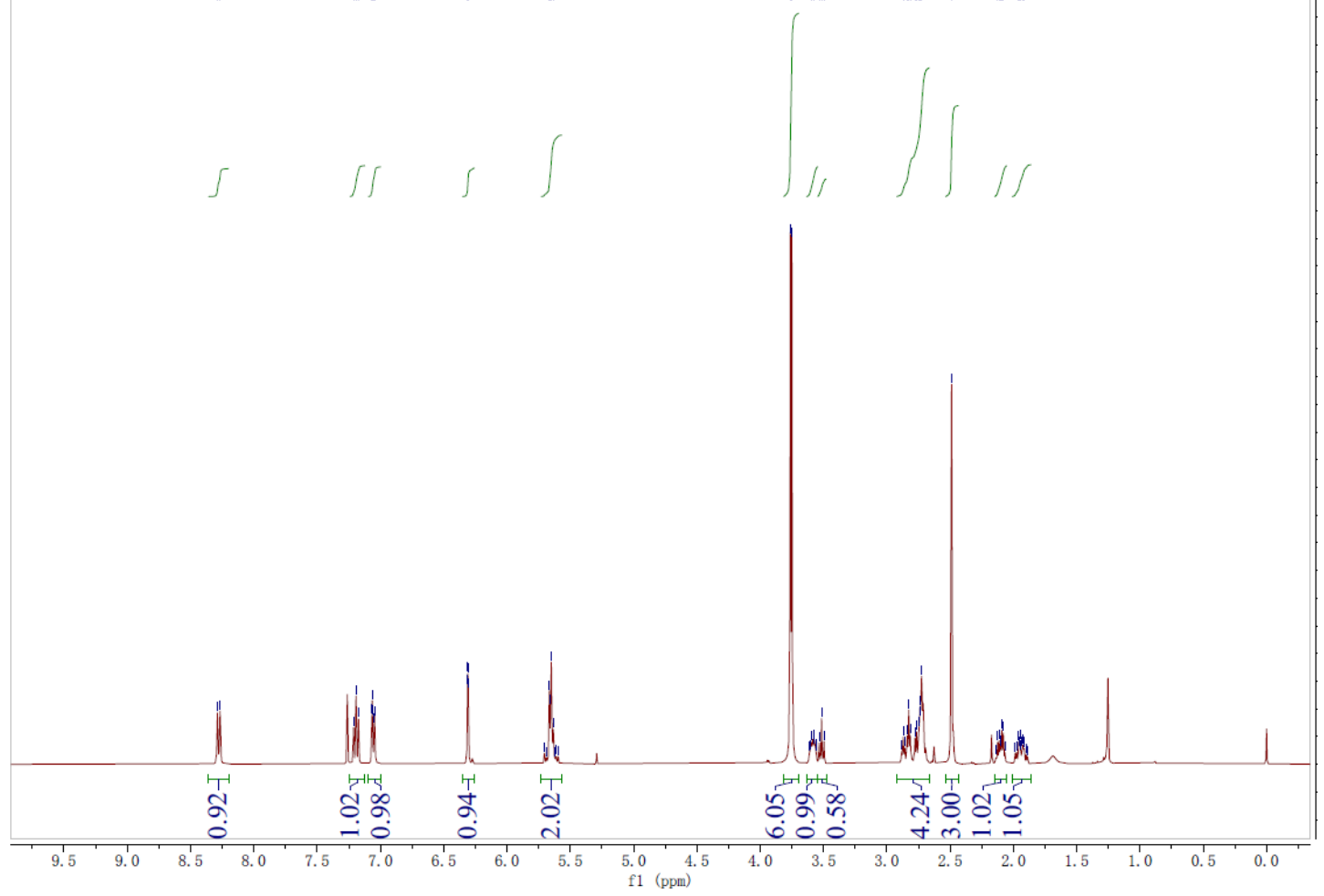


${ }^{1} \mathrm{H}$ NMR Spectrum of $\mathbf{8 a}$

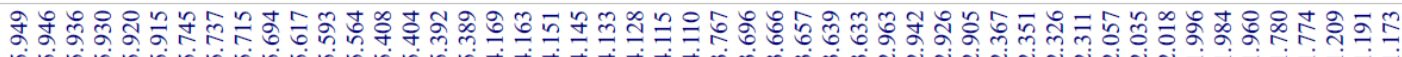

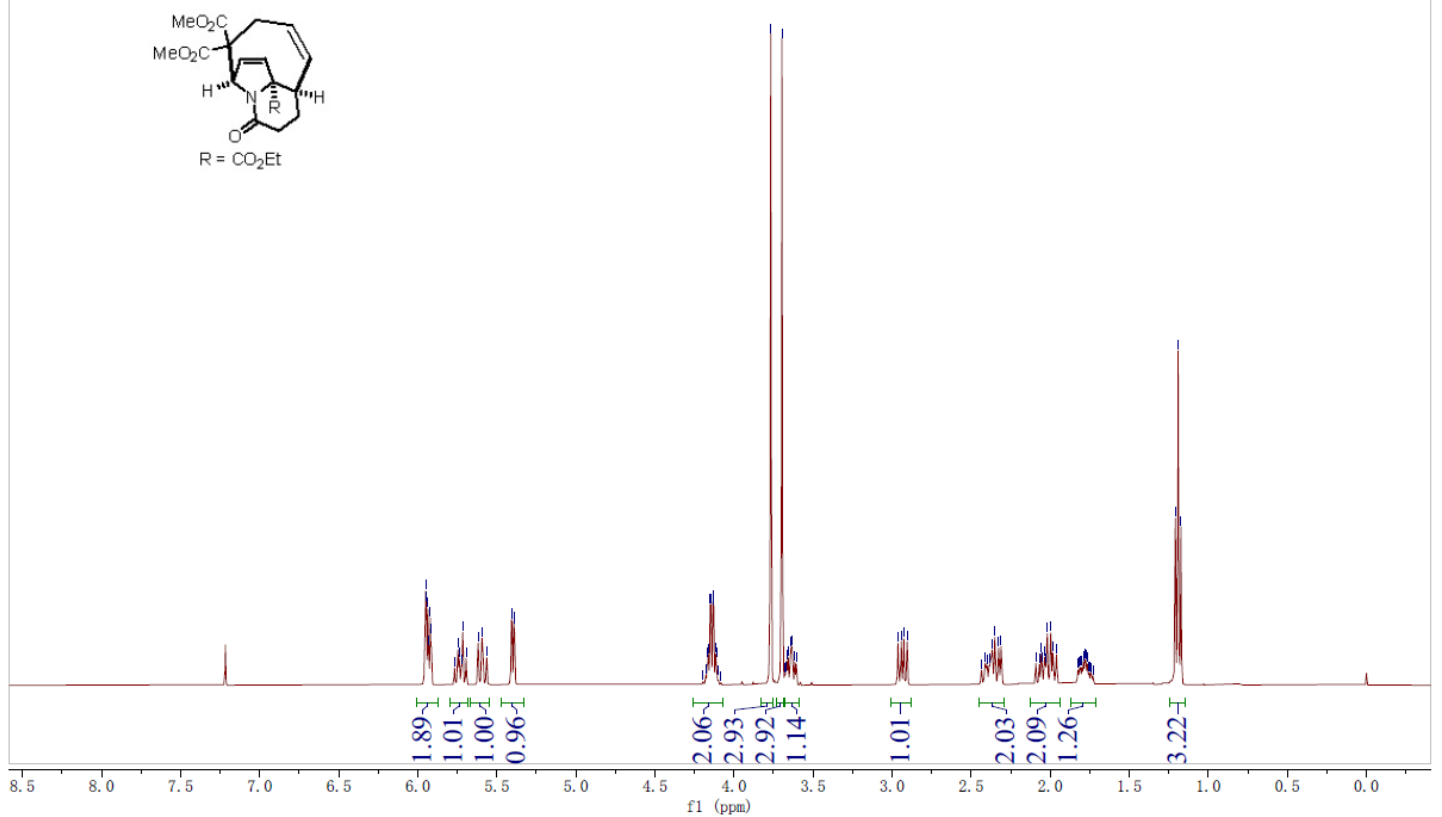

${ }^{13} \mathrm{C}$ NMR Spectrum of $\mathbf{8 a}$

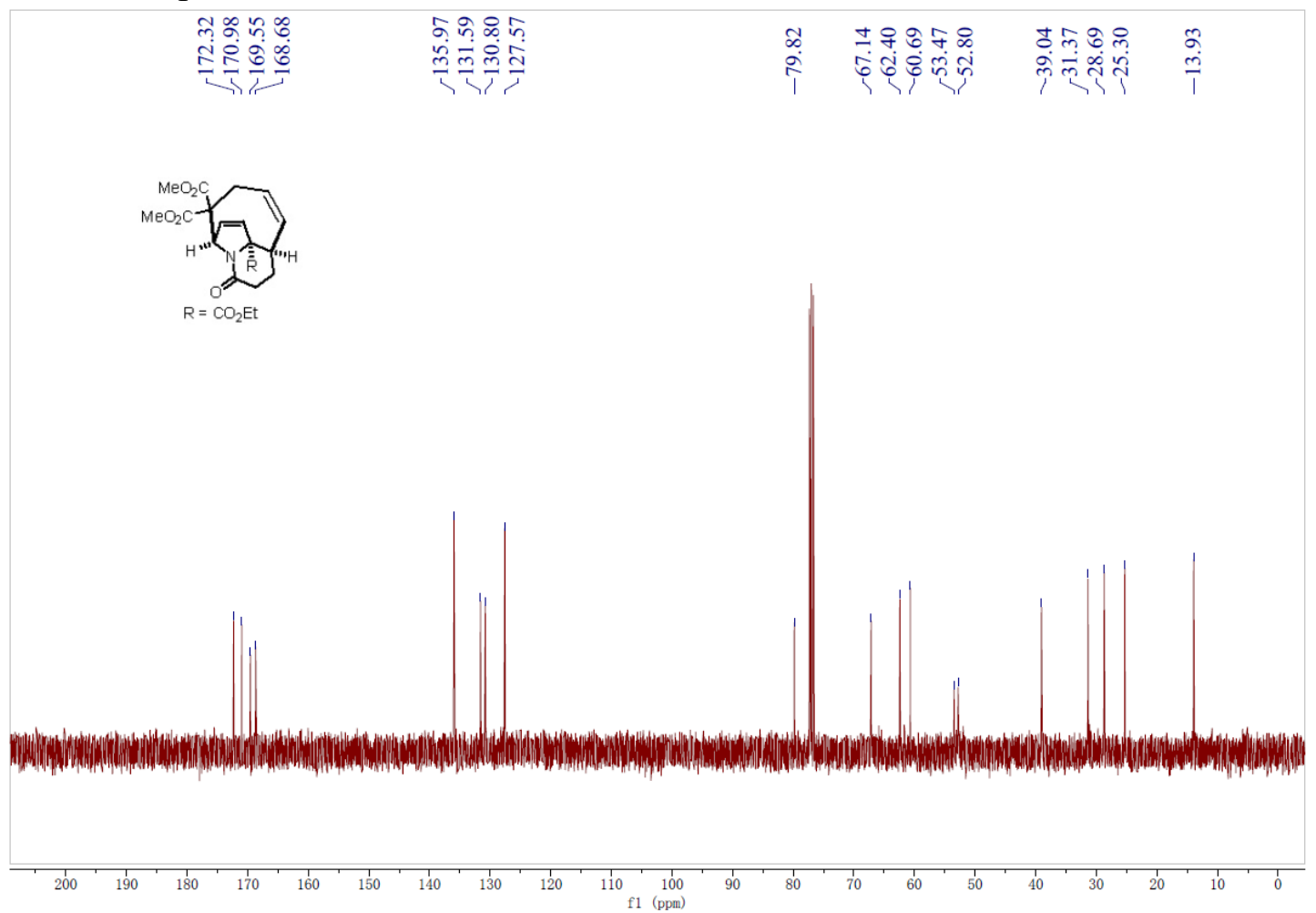


${ }^{1} \mathrm{H}$ NMR Spectrum of $\mathbf{8 b}$

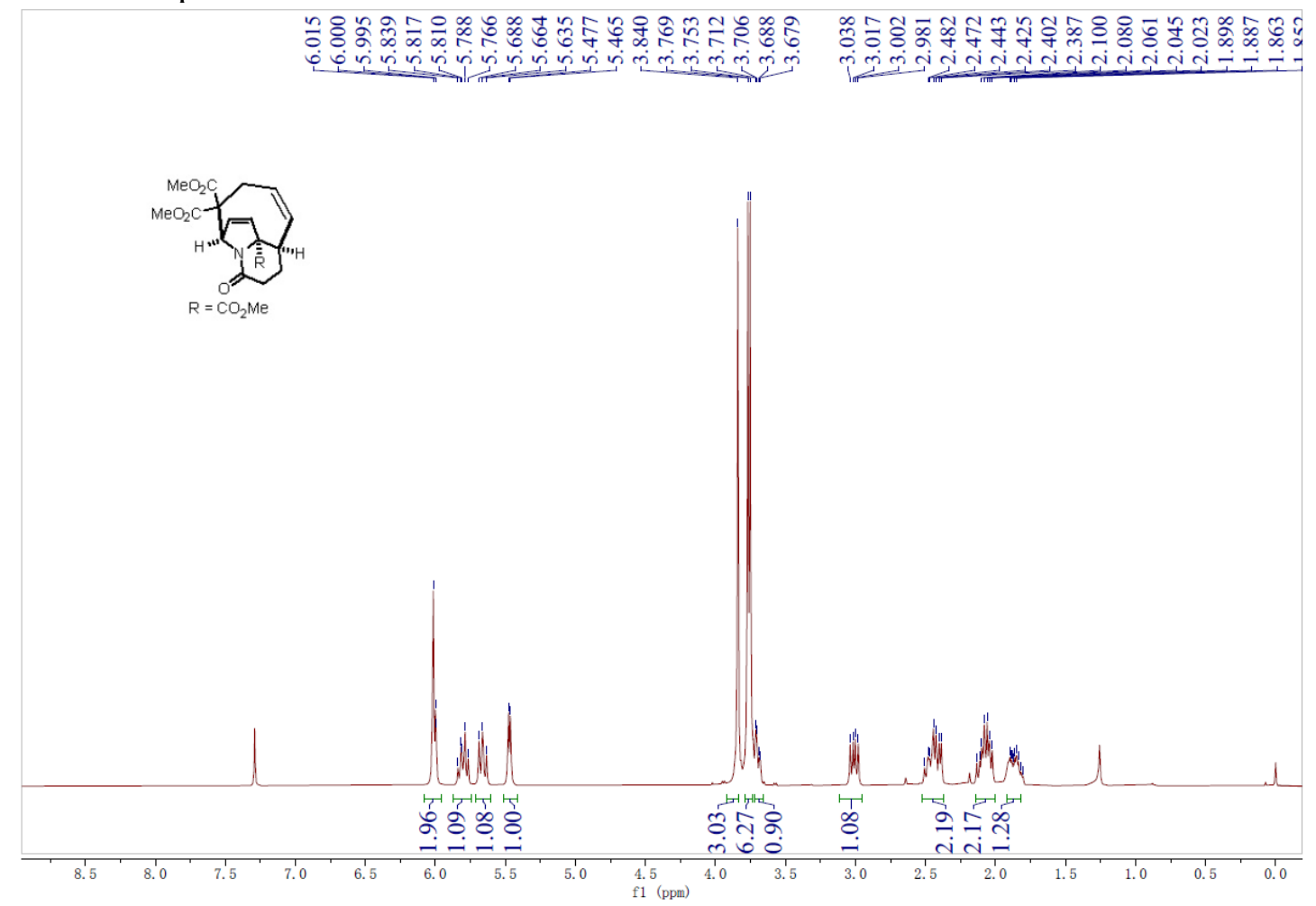

${ }^{13} \mathrm{C}$ NMR Spectrum of $\mathbf{8 b}$

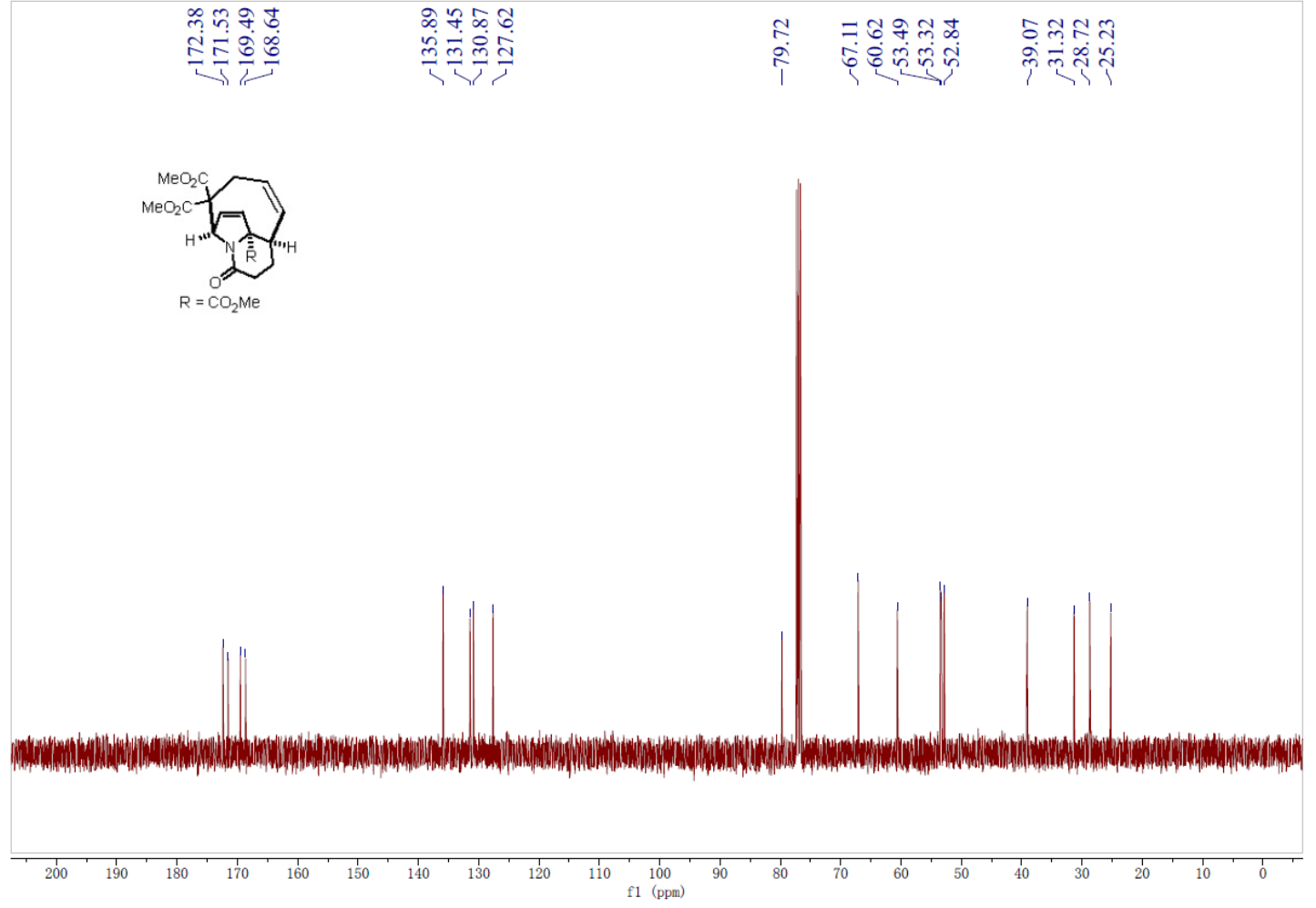


${ }^{1} \mathrm{H}$ NMR Spectrum of $\mathbf{8 c}$

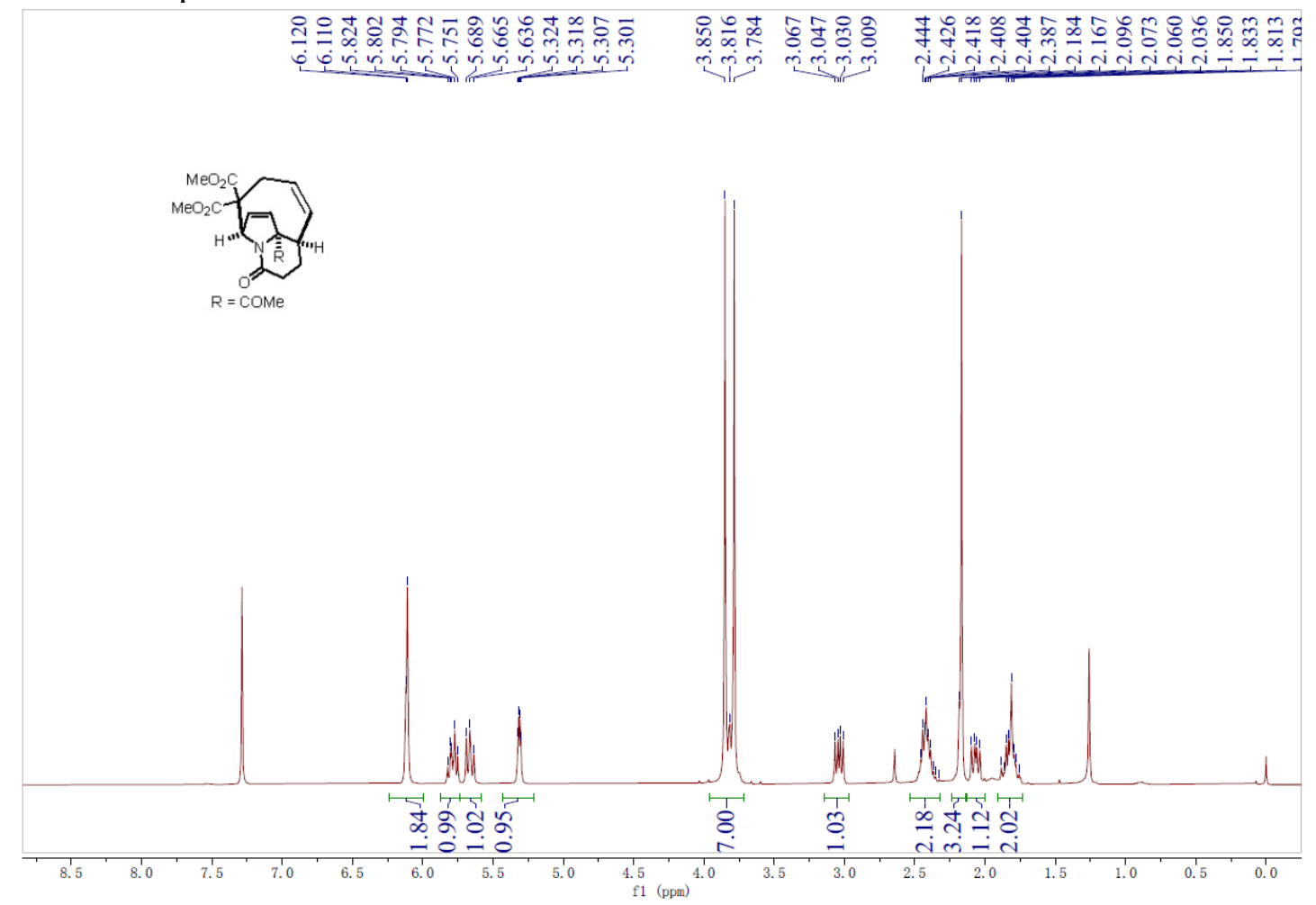

${ }^{13} \mathrm{C}$ NMR Spectrum of $\mathbf{8 c}$

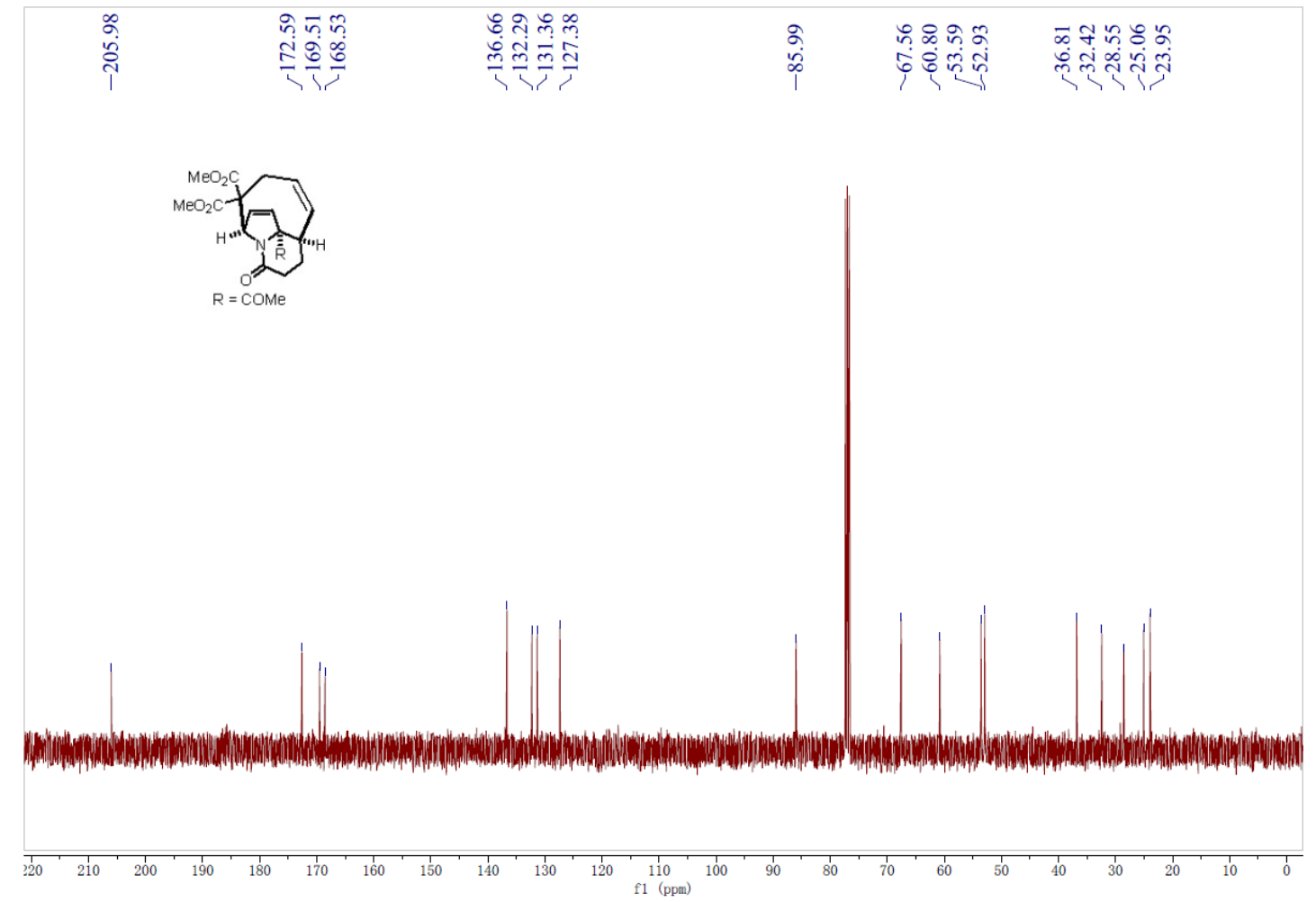


${ }^{1} \mathrm{H}$ NMR Spectrum of $\mathbf{8 d}$

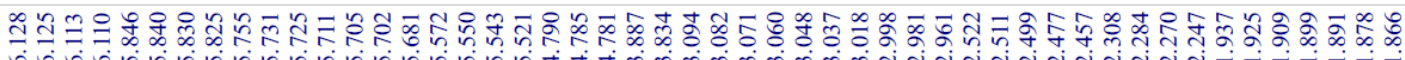

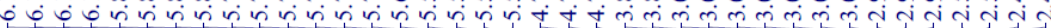

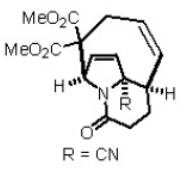
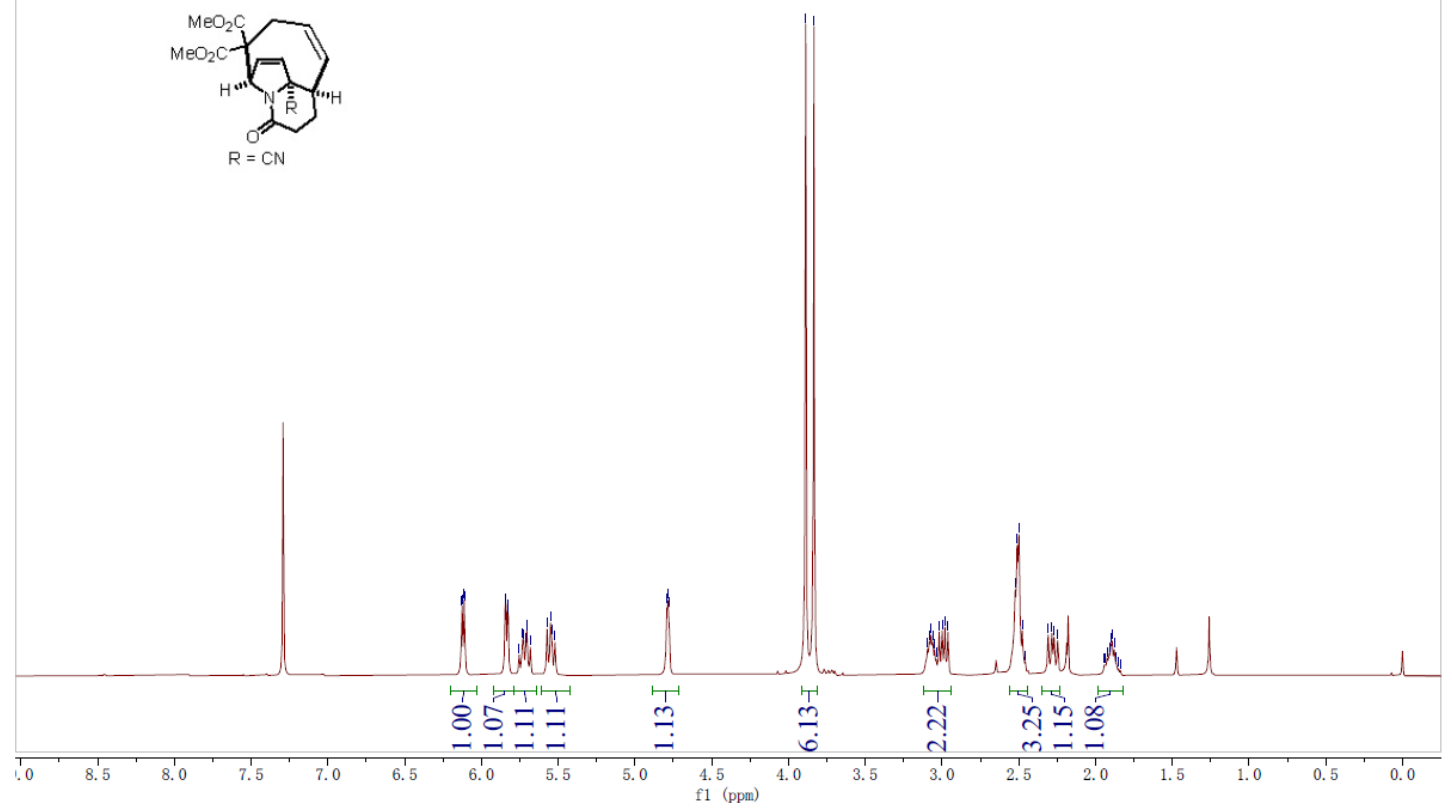

${ }^{13} \mathrm{C}$ NMR Spectrum of $\mathbf{8 d}$

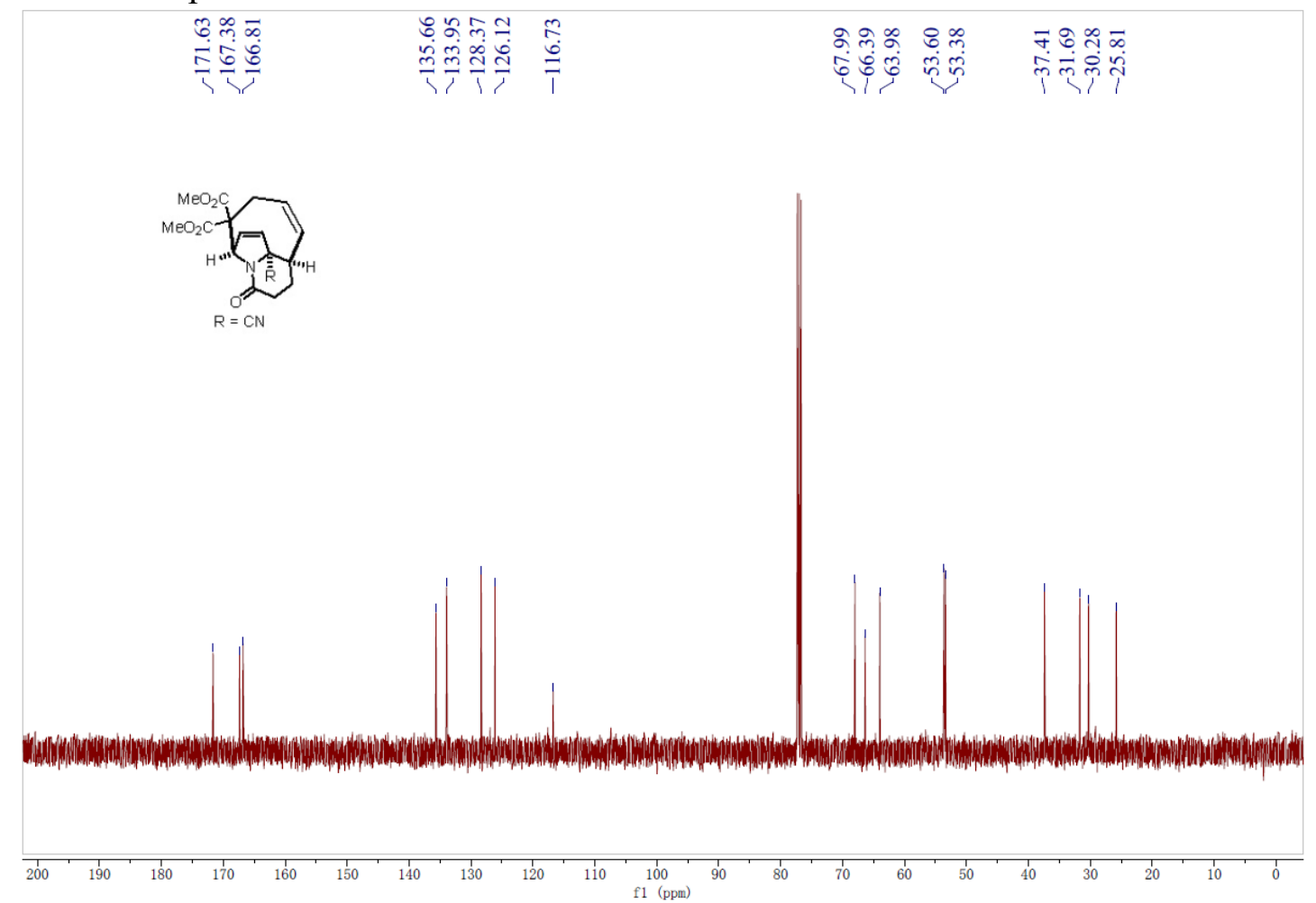


${ }^{1} \mathrm{H}$ NMR Spectrum of $8 \mathrm{e}$

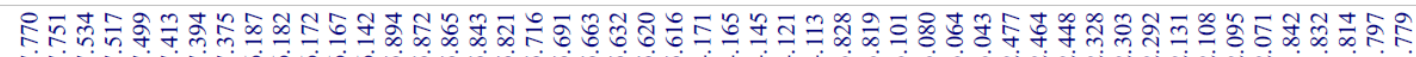

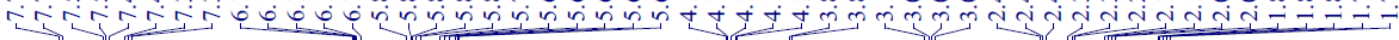

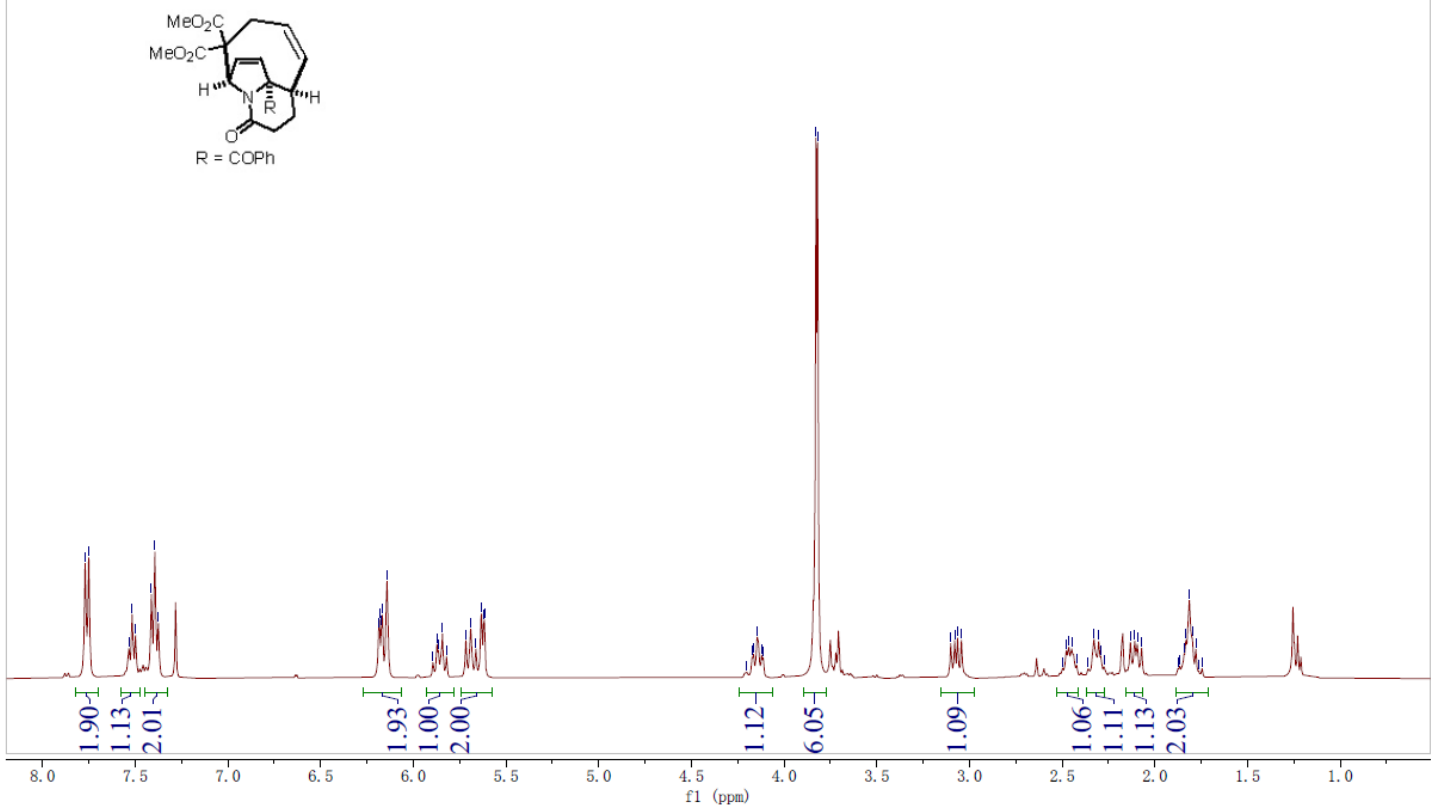

${ }^{13} \mathrm{C}$ NMR Spectrum of $\mathbf{8 e}$

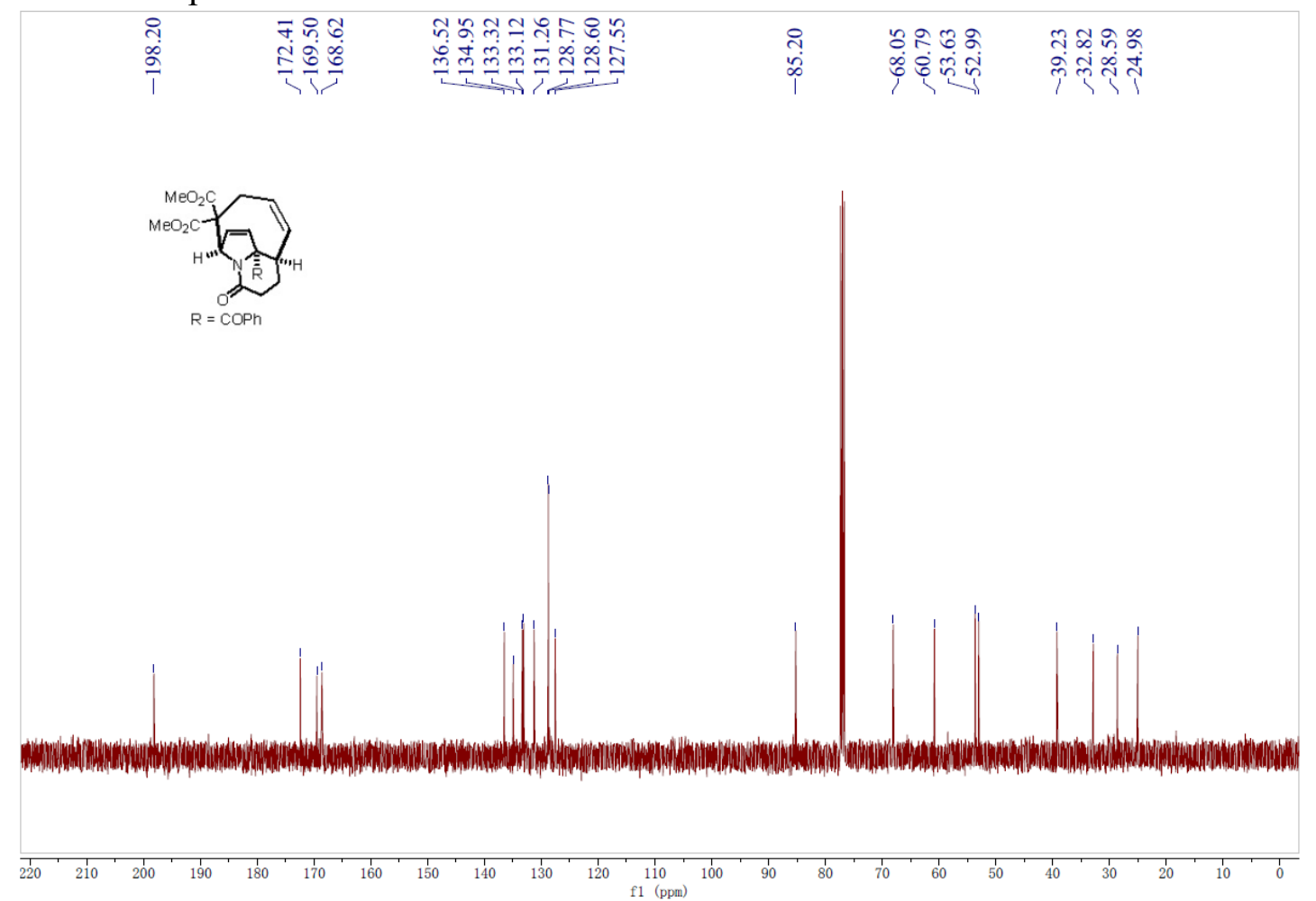


${ }^{1} \mathrm{H}$ NMR Spectrum of $\mathbf{8 f}$

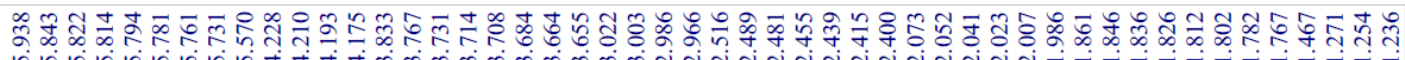
(n)

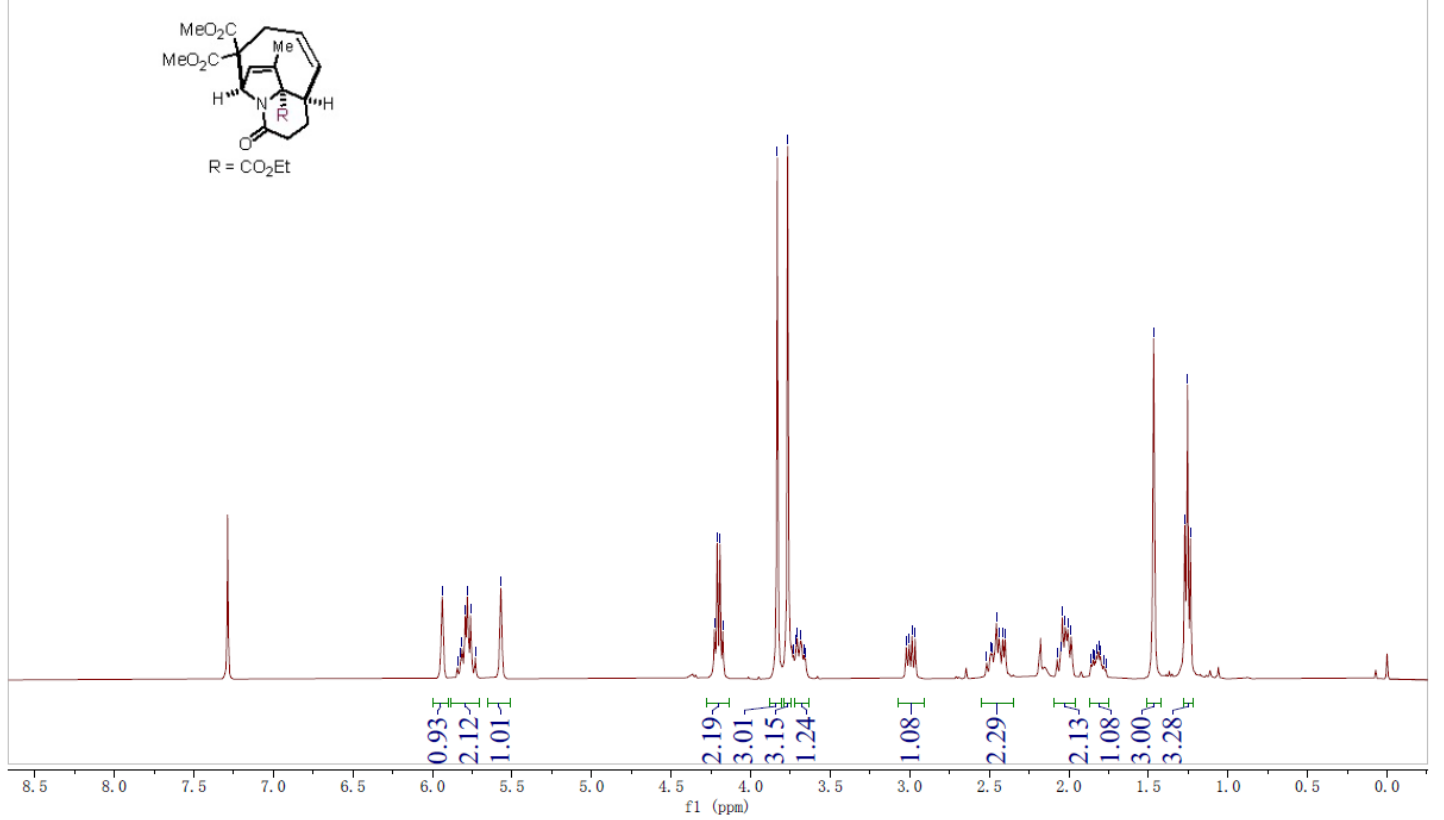

${ }^{13} \mathrm{C}$ NMR Spectrum of $\mathbf{8 f}$

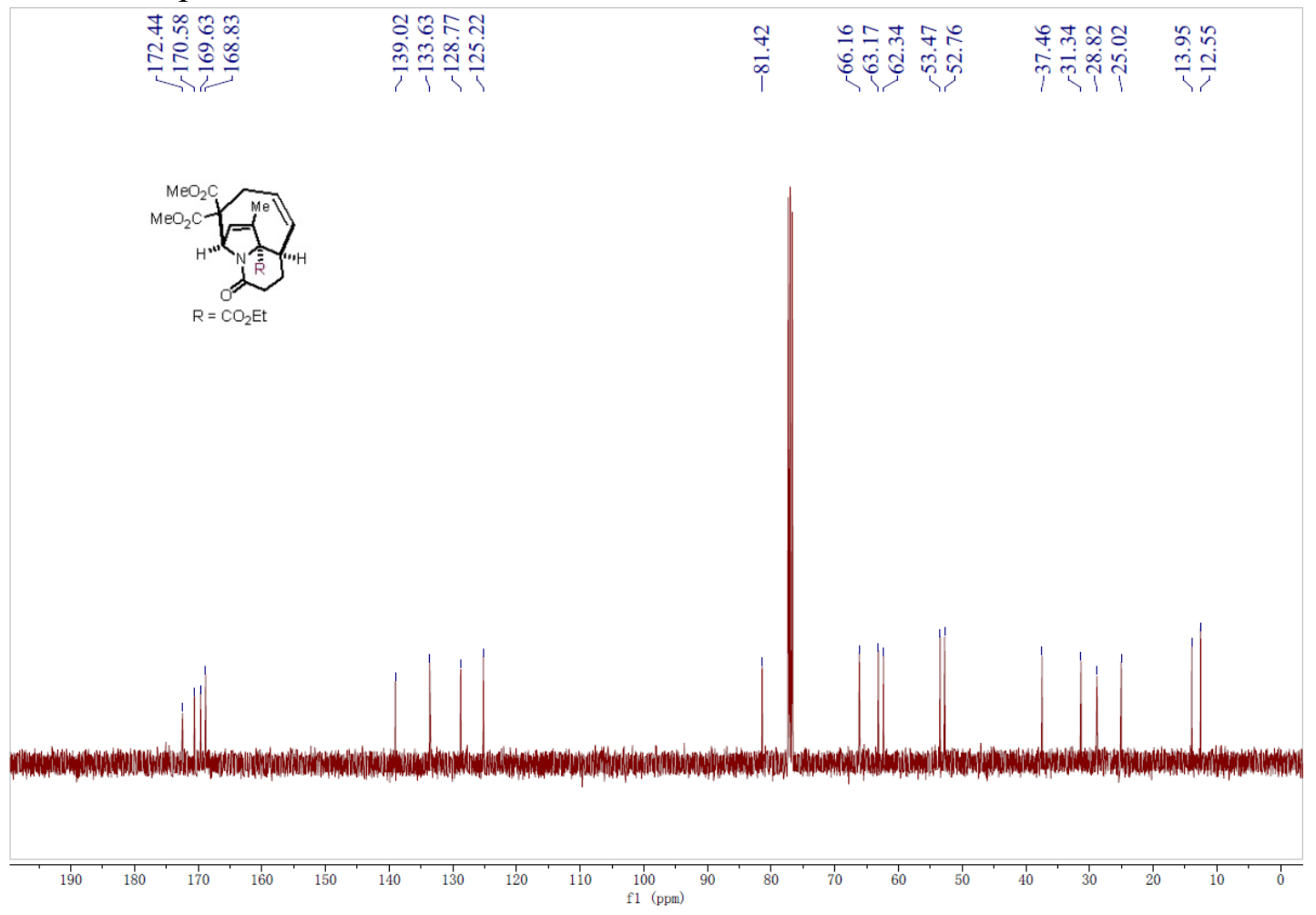


${ }^{1} \mathrm{H}$ NMR Spectrum of $\mathbf{8 g}$

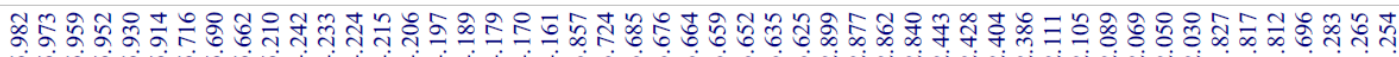

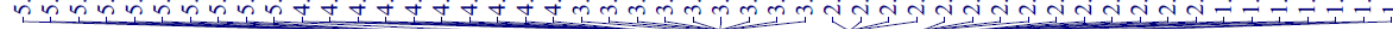
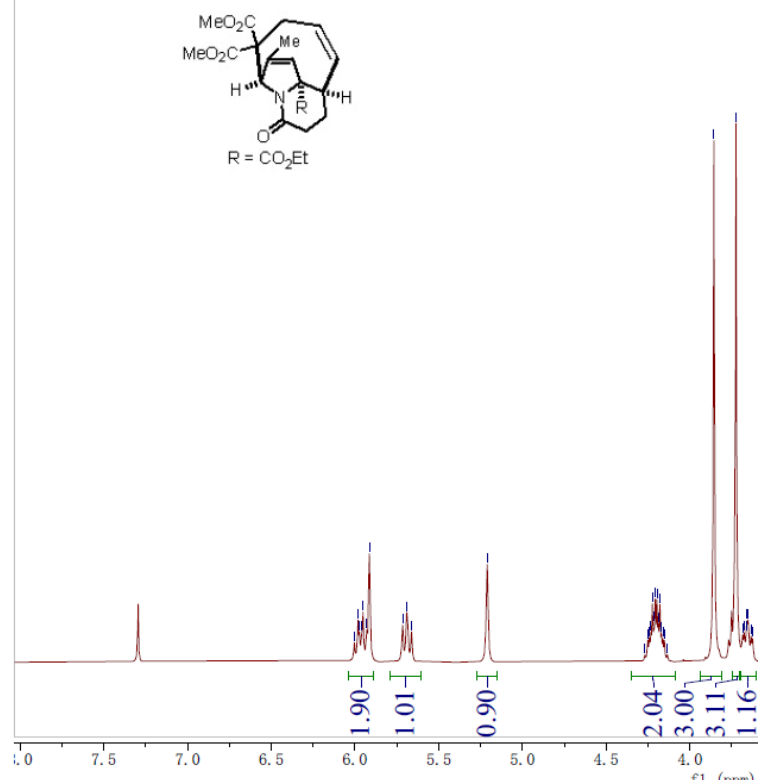

${ }^{13} \mathrm{C}$ NMR Spectrum of $\mathbf{8 g}$

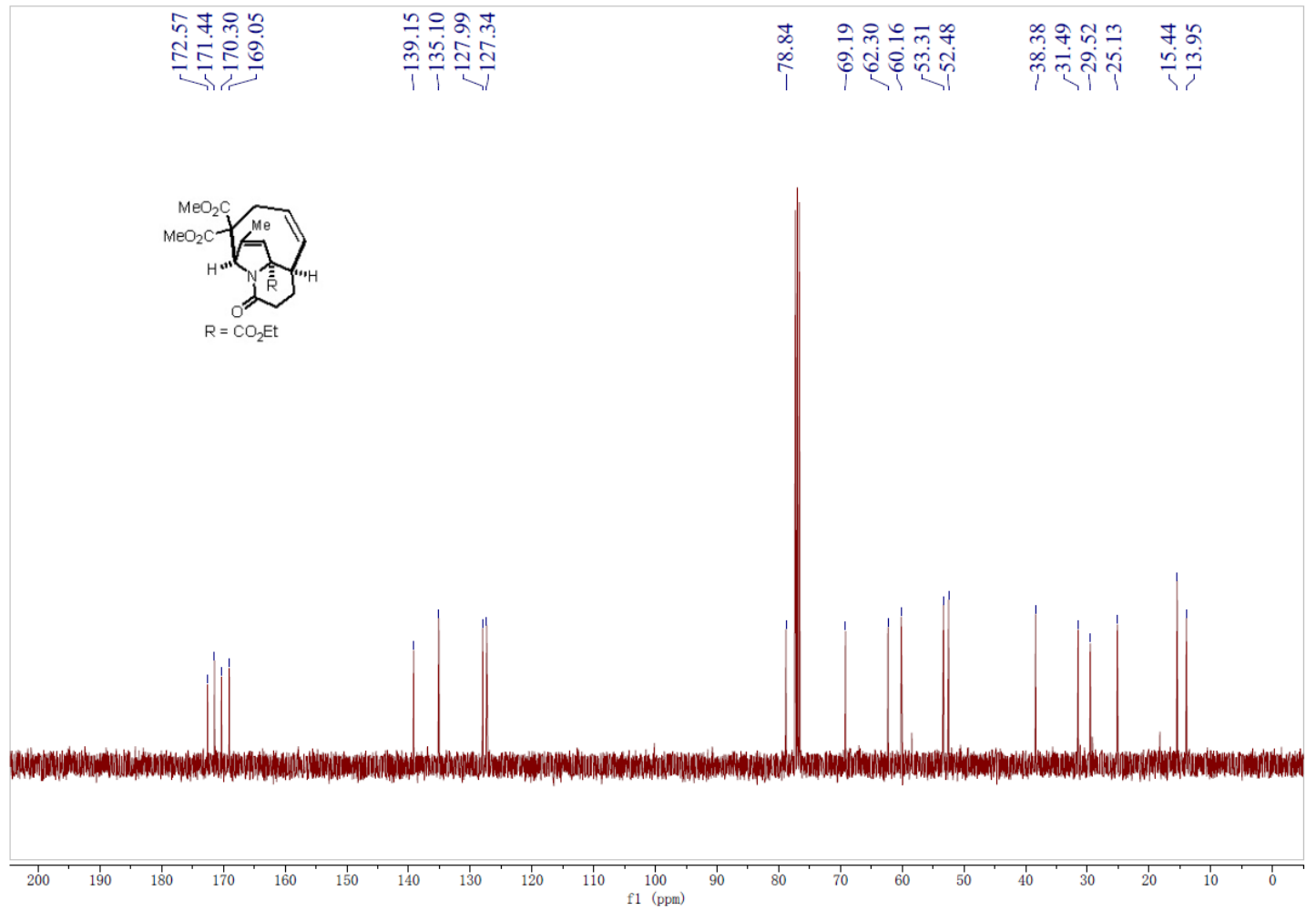


${ }^{1} \mathrm{H}$ NMR Spectrum of 9

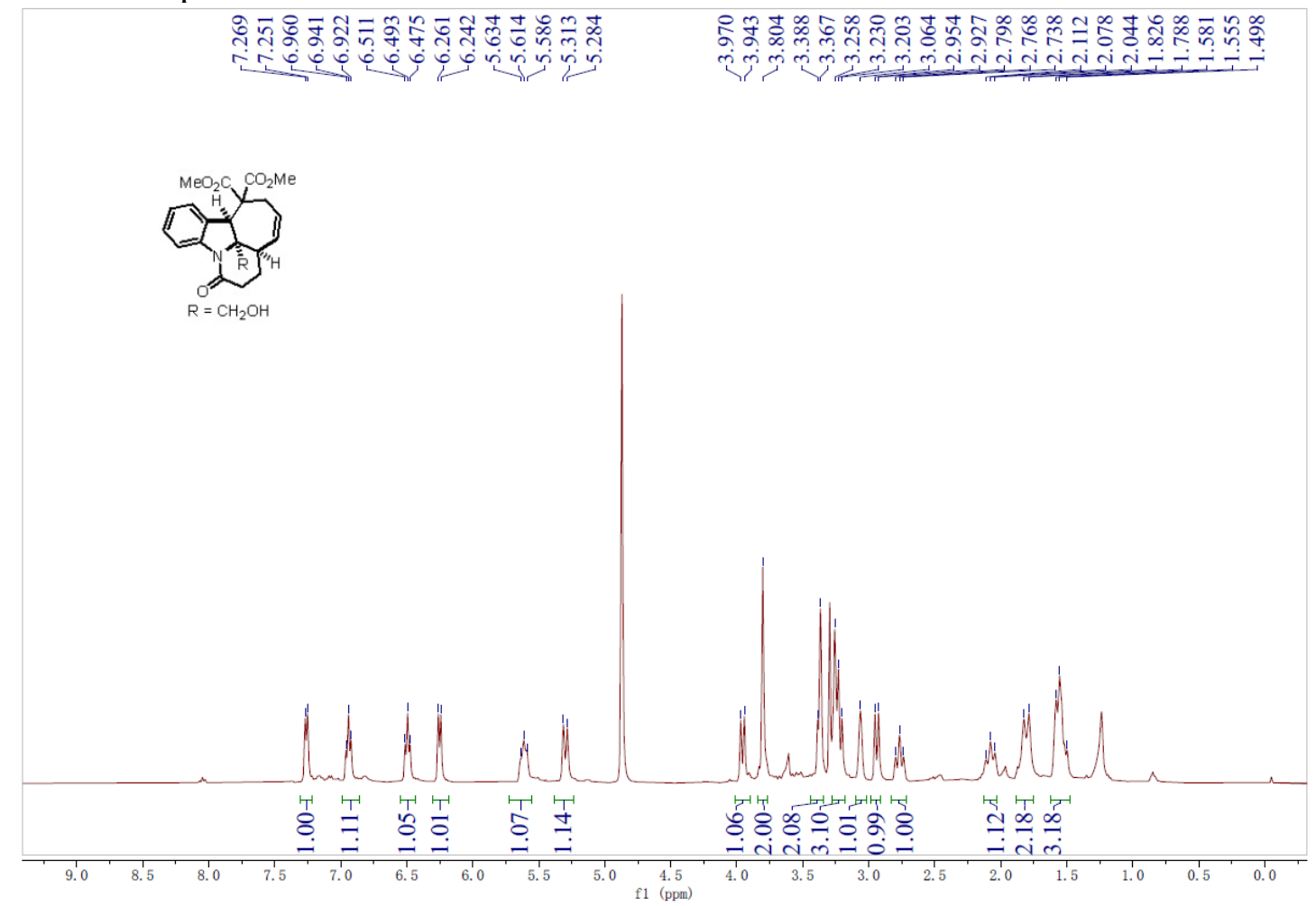

${ }^{13} \mathrm{C}$ NMR Spectrum of 9

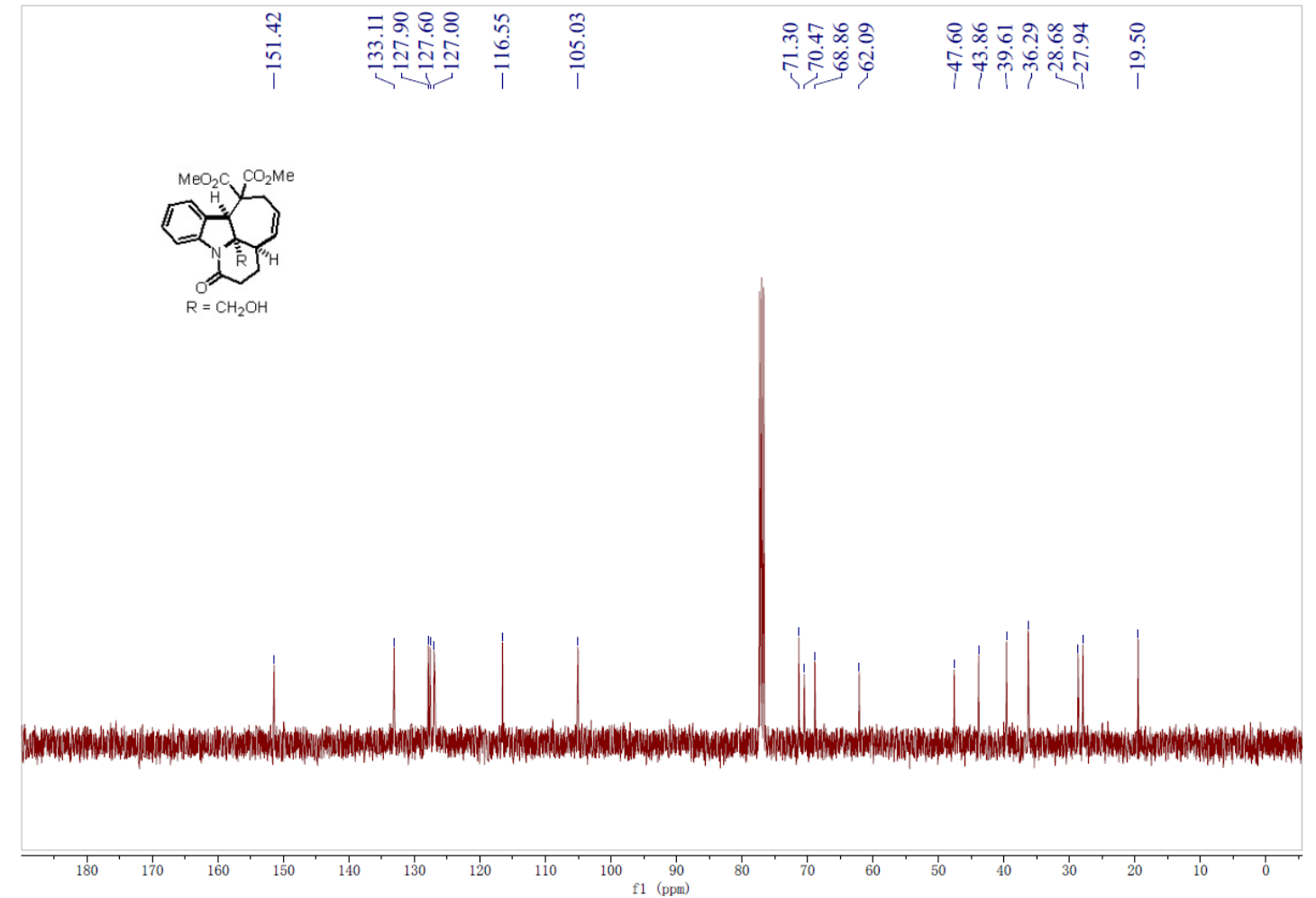


${ }^{1} \mathrm{H}$ NMR Spectrum of $\mathbf{1 0}$

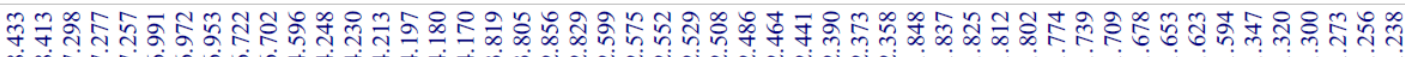

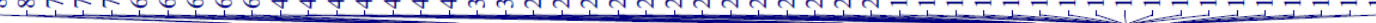
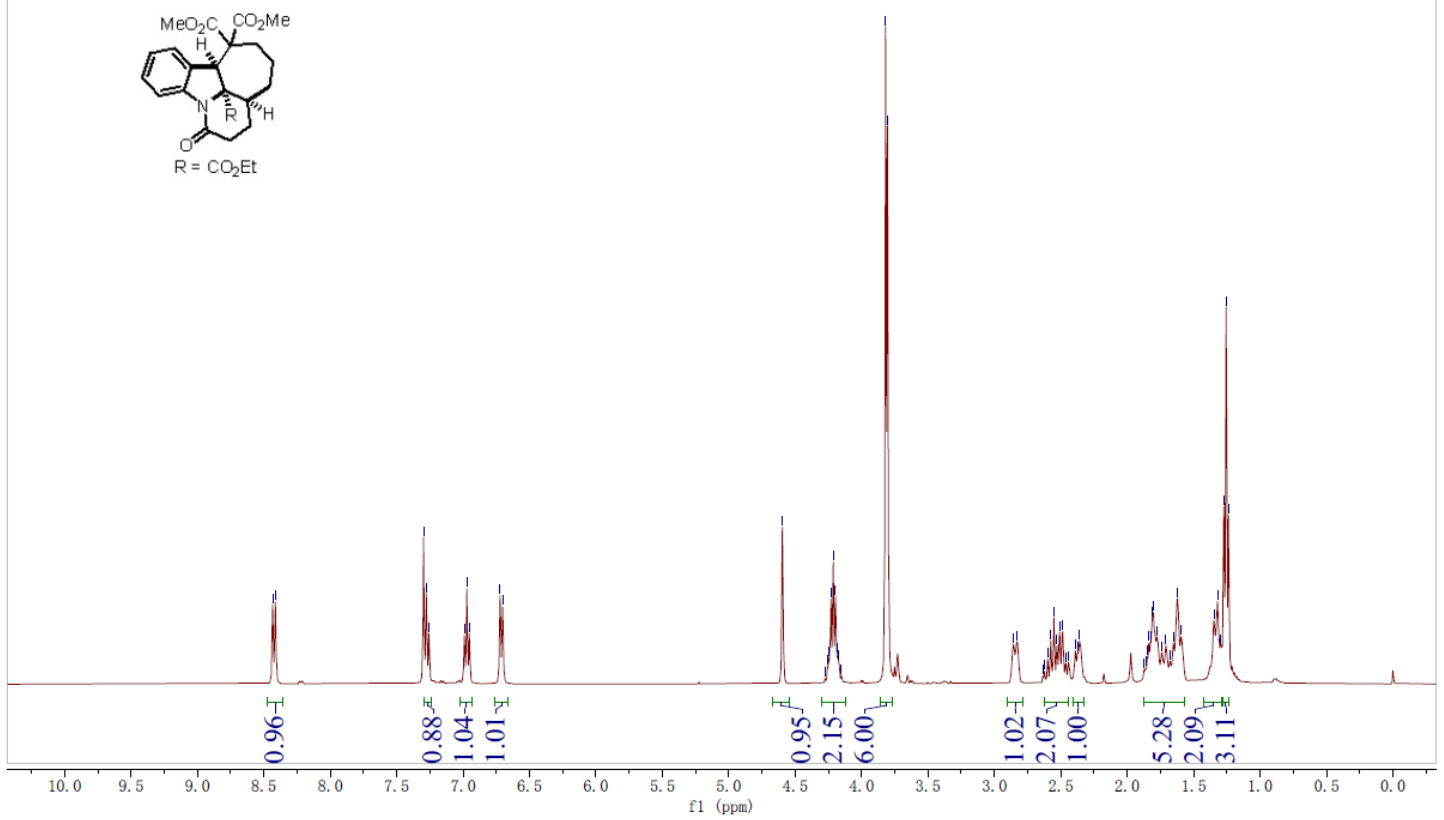

${ }^{13} \mathrm{C}$ NMR Spectrum of $\mathbf{1 0}$

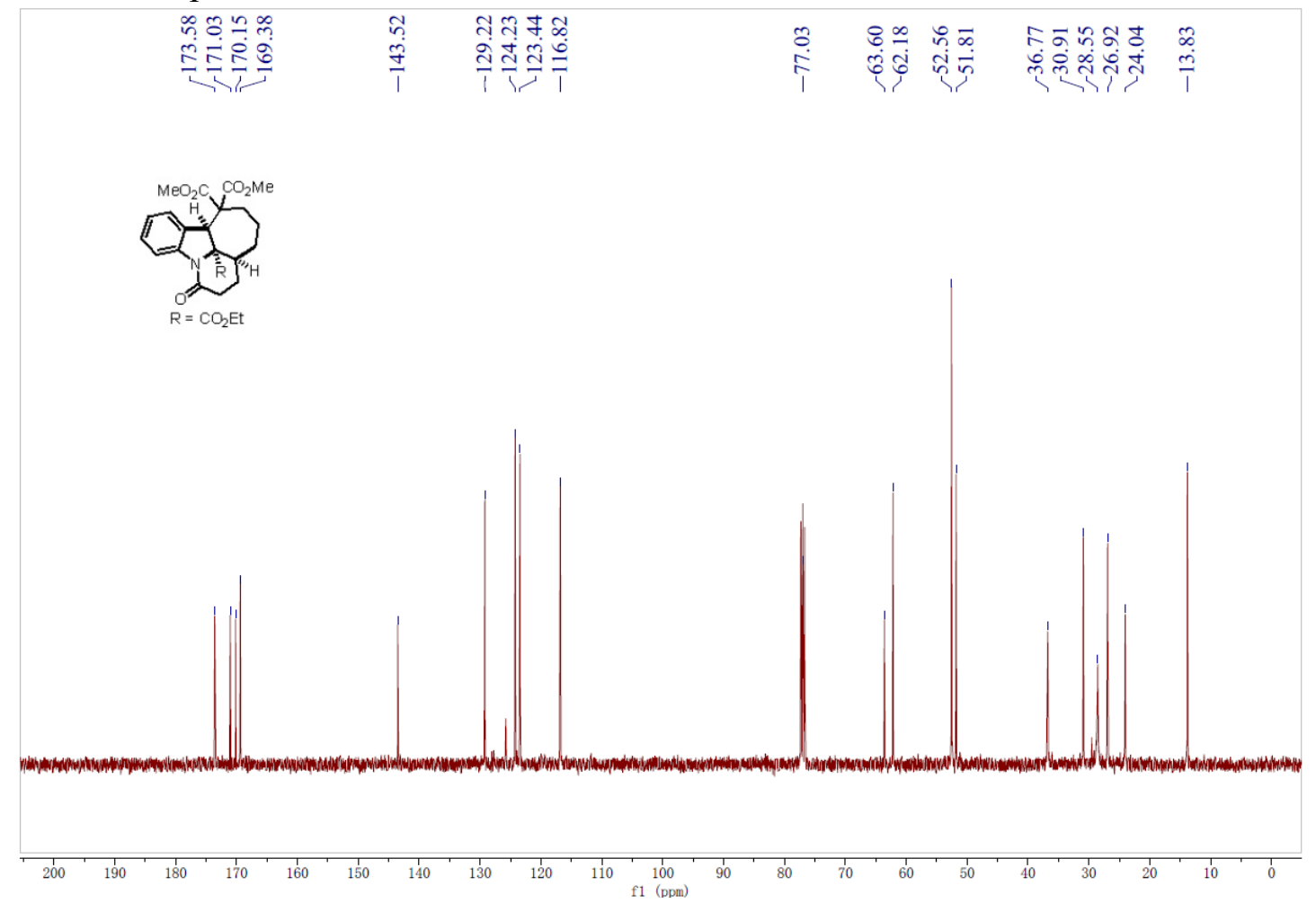


${ }^{1} \mathrm{H}$ NMR Spectrum of $\mathbf{1 1}$

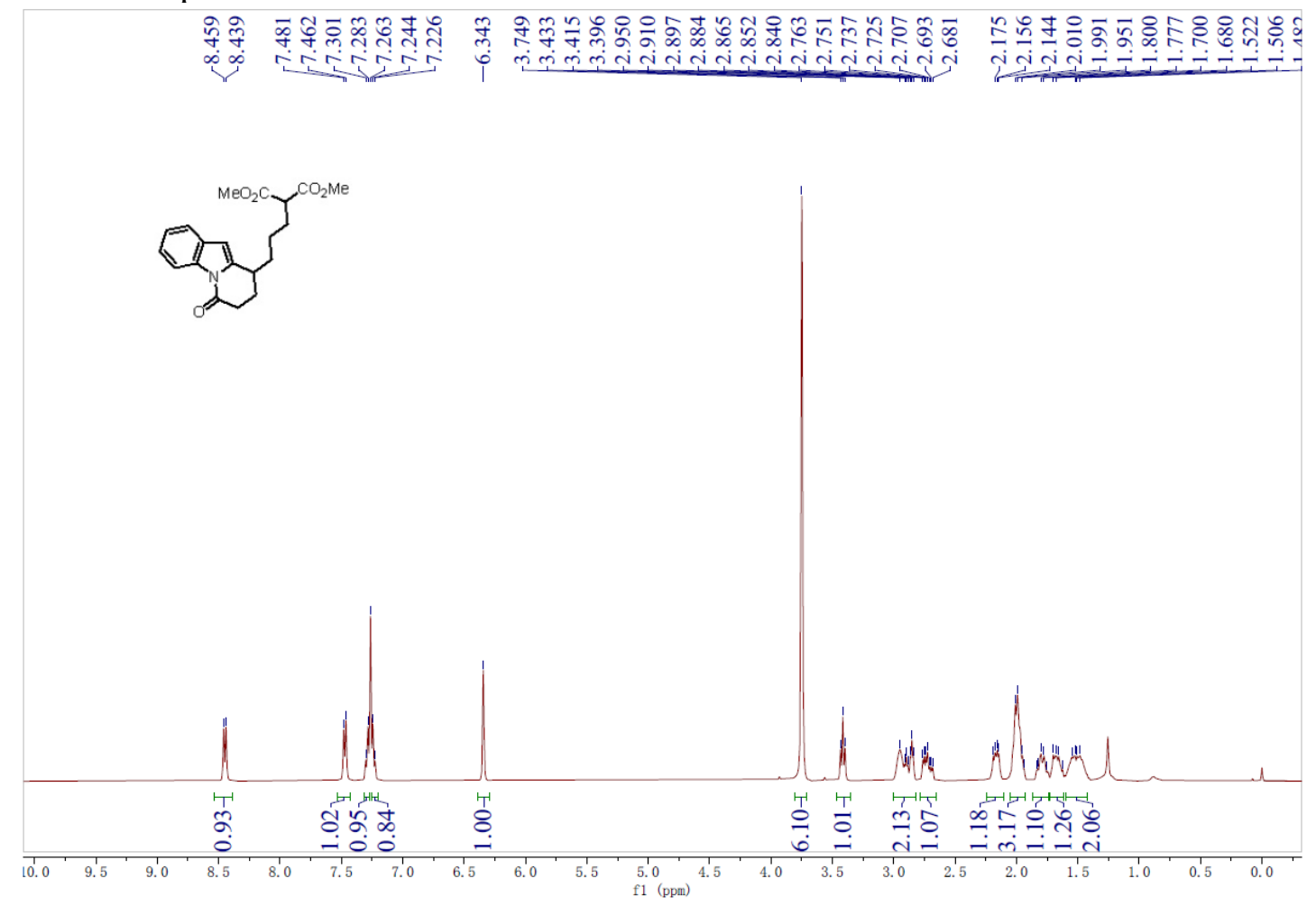

${ }^{13} \mathrm{C}$ NMR Spectrum of $\mathbf{1 1}$

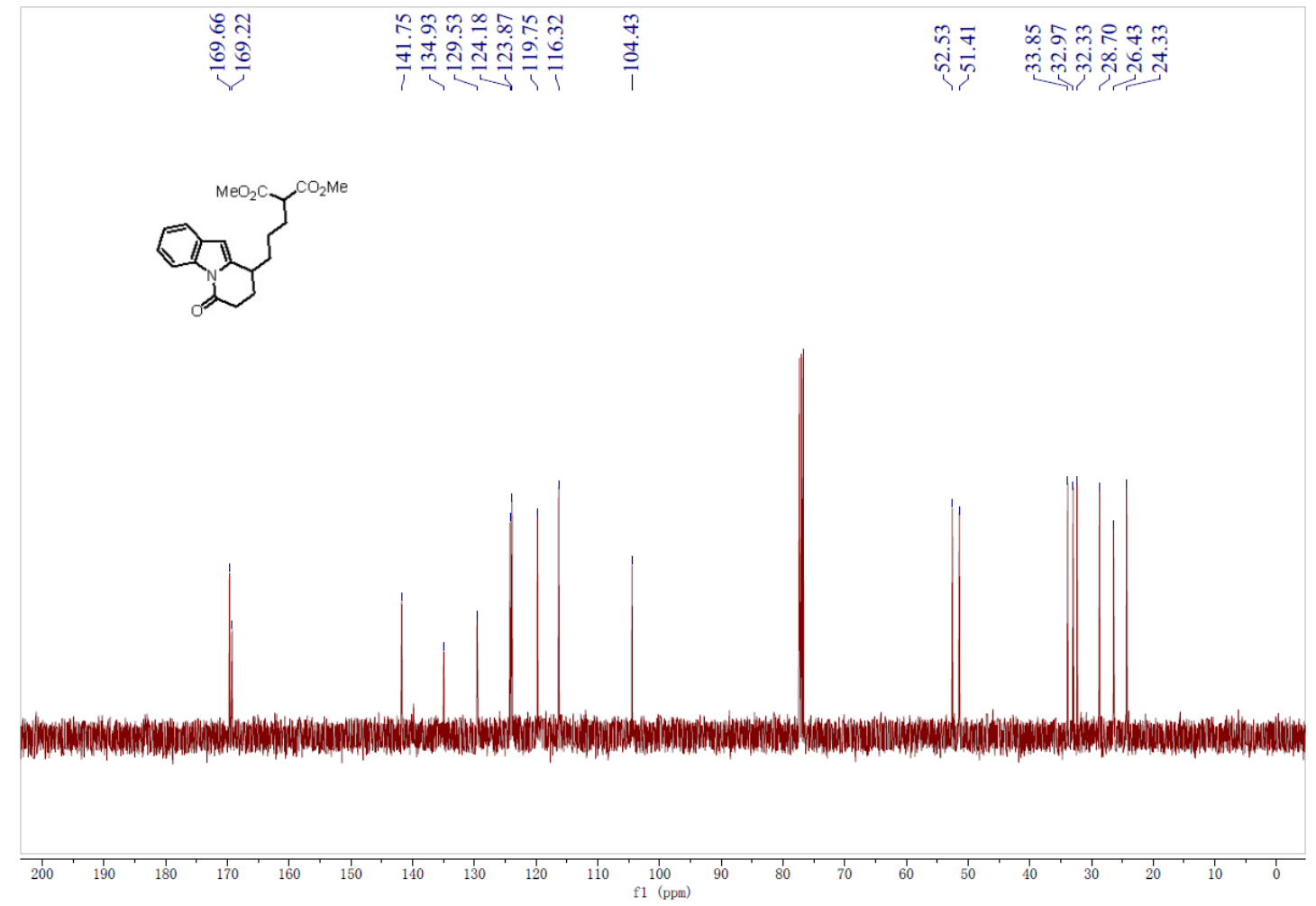


${ }^{1} \mathrm{H}$ NMR Spectrum of $\mathbf{1 2}$

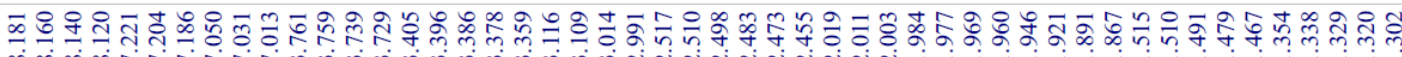

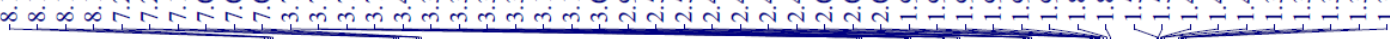<smiles>CC(=O)C(CCCC1CCC(=O)N2c3ccccc3CC12)C(C)=O</smiles>

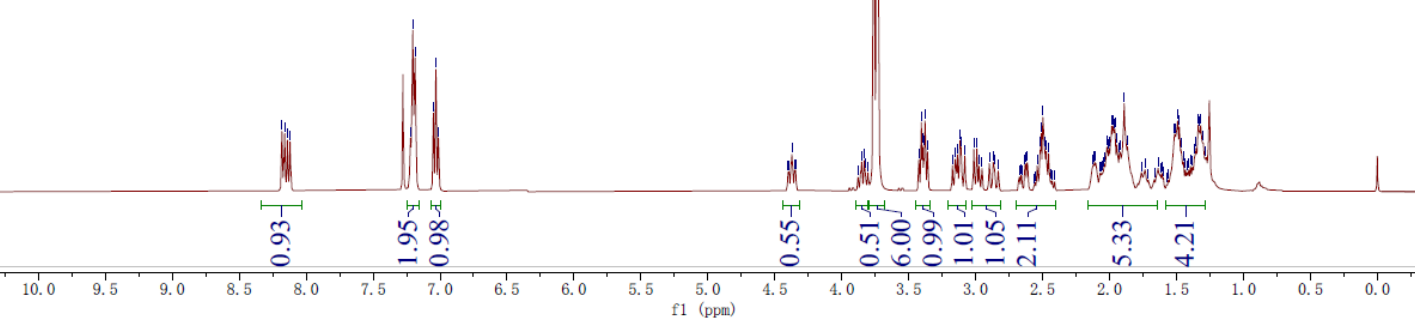

${ }^{13} \mathrm{C}$ NMR Spectrum of 12

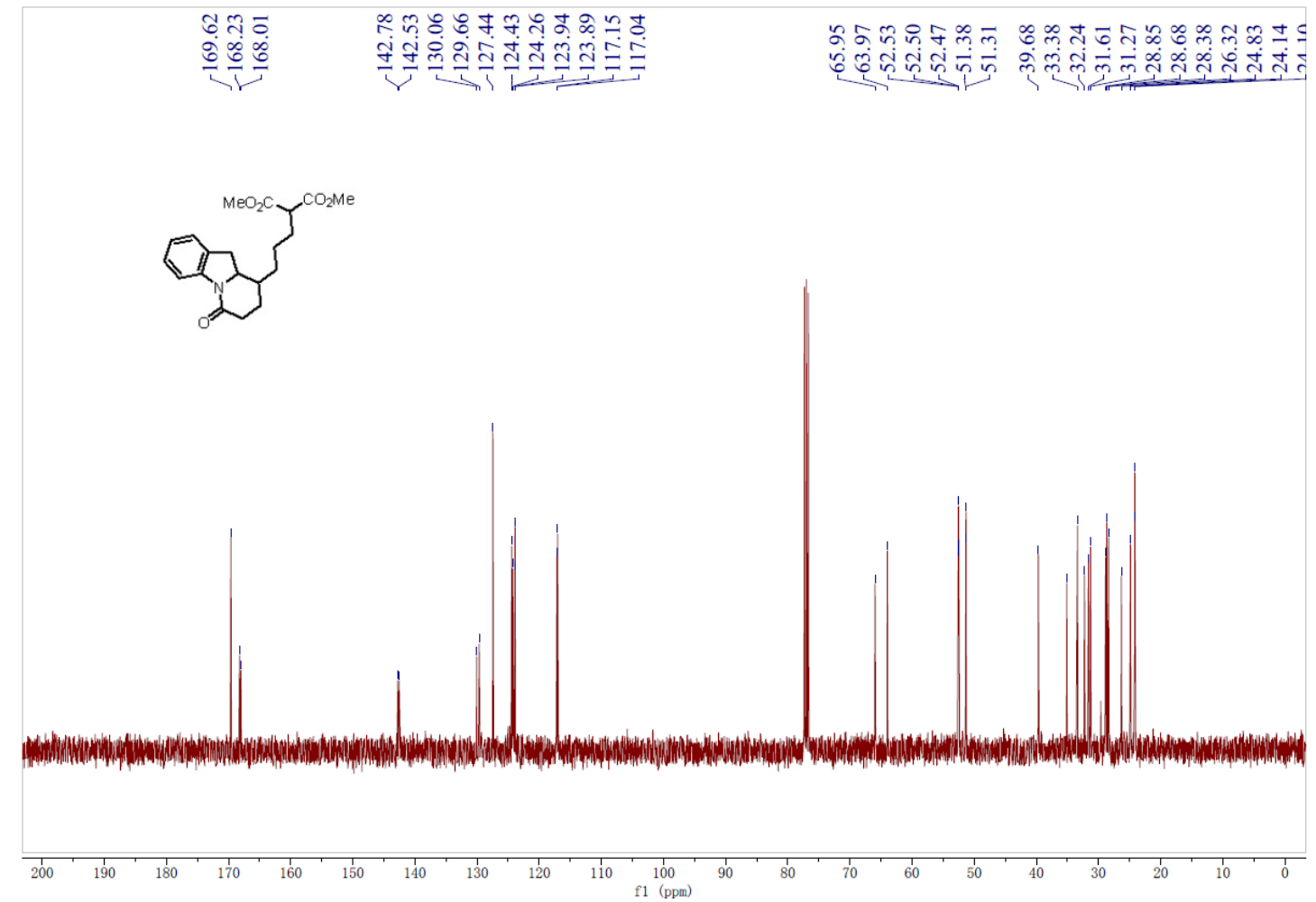

